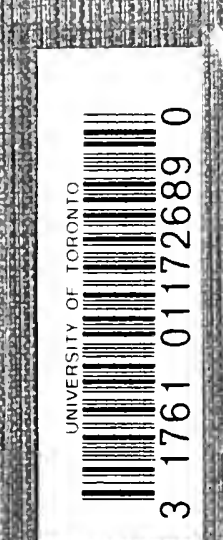




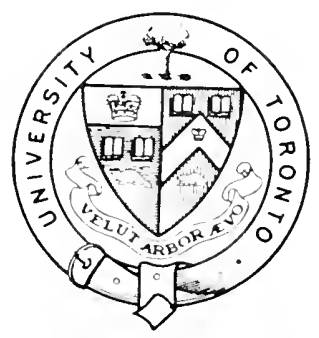

PUHCHASED TOR THE

(I)ITRSI) OI IORONTO LIBRARY

IHON1 1HI

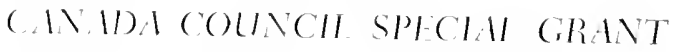

$10 R$ 






\section{BIBLIOTHËQUE}

I) IA

FACUITÉ DE PHILOSOPHIE ET LETTRES

DE L.UNIVERSITÉ DE LIËGE. 

BIBIIOIFE⿱亡⿴囗十⺀⿺乚一F

DF, I,A

\section{FACULTÉ}

J) F

\section{PHILOSOPHIE ET LETTRES}

1) $\mathrm{E}$

l'Université de Liège

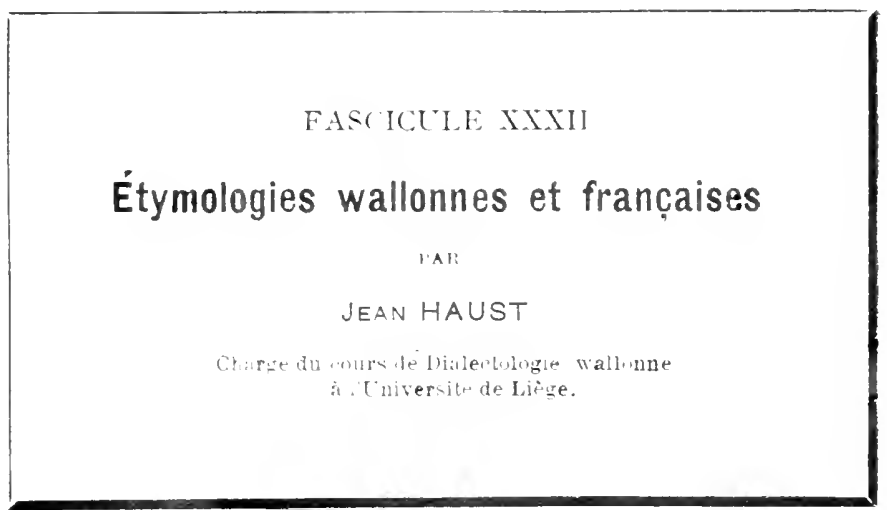

1923

Imp. H.VAILLANT-CARMANNE ÉDOUARD CHAMPION

Soriete Anenyme

Libiare-Editeur
4. Place St-Michel, 4
LIÈGE
5. Quai malaquais, 5
PARIS 
1 
A MONSIEUR

ANTOINE THOMAS

WIDEP DE LMTITIT 



\section{PRÉFACE}

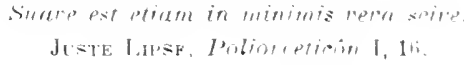

I)epuis plus de trente ans que jeétudie nos dialectes - et surtont depuis que la "Société de Littérature wallonne " mia charéc, arece mes amis Auguste Iontrepont et Jules Feller, de préparer le Dictionnate général des parlès mumas de la Bulgique, - je nai cessé de foniller les origines de nos curienx et savourenx rocables patois ( $\left.{ }^{1}\right)$. I'n premier résultat de ces investigations patientes tient dans ces quelques eentaines d'articles. C"est pen, sans donte. con comparaison du temps et de l'effort dépensés, et aussi an regard de tout ee qui reste ì ćlucider : je m'estimerai pourtaut très satisfait si les juges compétents trourent iei me utile contribution a l'étymologie romanc.

I“étude du wallon ma conduit, par me pente naturelle, i traiter de eertains mots francais. dont loorigine est temuc pour doutense et qui

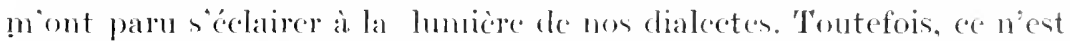
quavec une extrême prudance que jai tenté quelepues incoursions sur

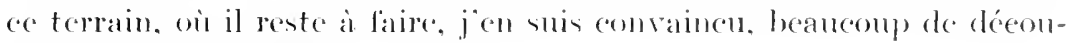
vertes intéressantes $\left({ }^{2}\right)$.

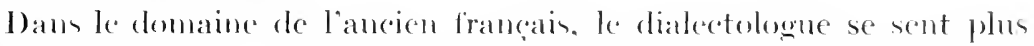
à latise. Presefue à chaque page du diretionmaire de Godefroy il pent noter des crreurs on des omissions et contribuer a parlaire l'aure du

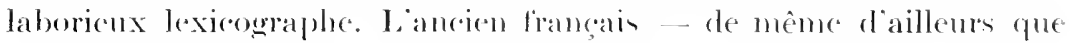

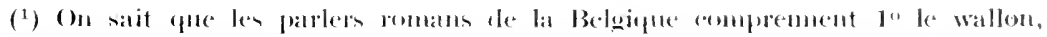
qui forme le grompe le plus impurtant. aver sen varietes prinejpales : le liégeois,

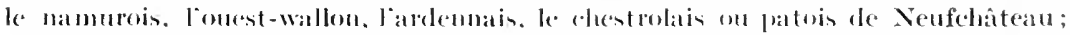

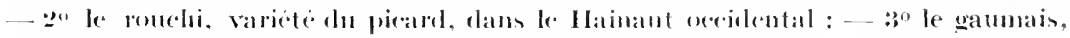
variete du lorrain, daus liaroubissenent de Virton.

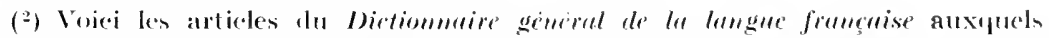

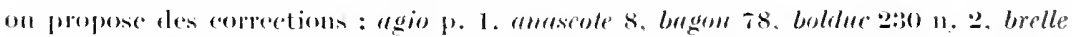

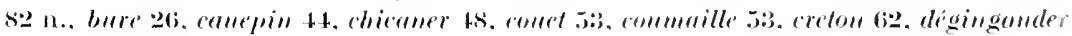

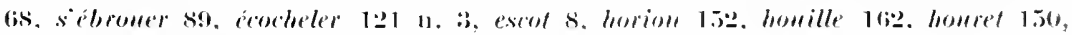

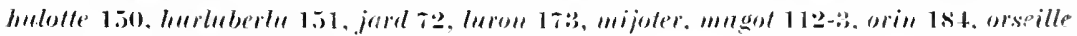

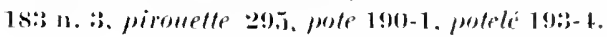




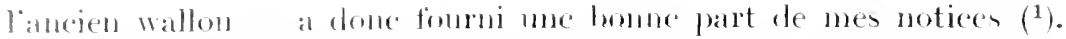
Eull général. cee meticen ont pour point de départ la reritique d'opi-

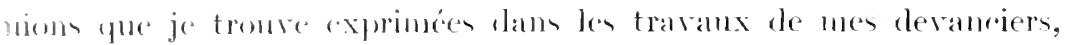

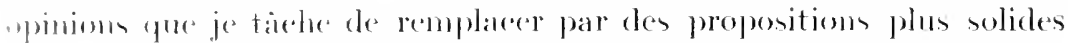
ou du moims phu plausibles. ("ent surtout Grandgagnage qui bait les

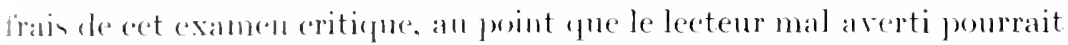

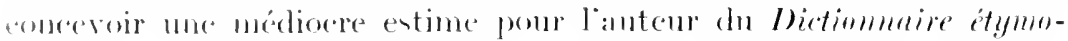

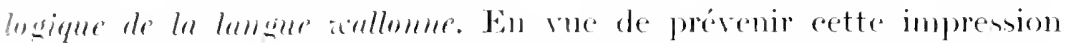
déavorable. je renroie anx pages:31\%-3:21, oil jexprime mon sentiment sur charles crandgaguage : 111 y vera la vénération que je professe pour linitiatenr de la philotogie wallonuce - Les antres philologues qüil mest arrive de enotredire rondront hien ne pas nien tenir rigueur. Il savent que la soience nest quime perpétuelle vérifieation

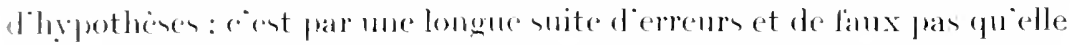
farrient a un hut : la vérité. Pour ma part, je suis loin de croire que partout jai touché juste: je ne me dimsimule pas la fablesse de certaines

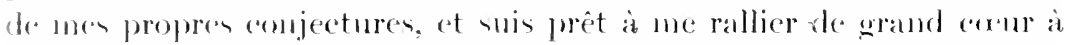

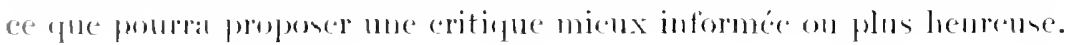

$$
\therefore \because ; \quad \therefore
$$

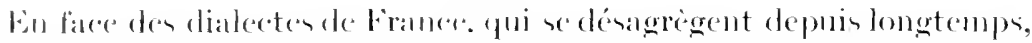
lat plupart de nou parlers septentrioname sont restés relativement sains

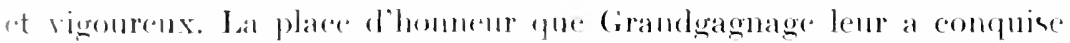
daus la philologere l'raneaine, serait phus romsidérable encore si les

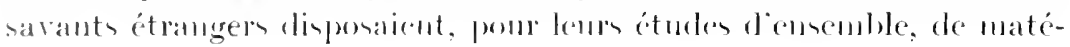

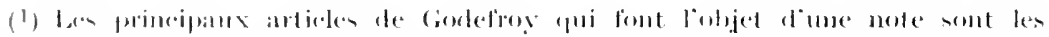

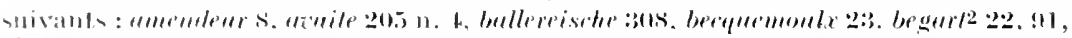

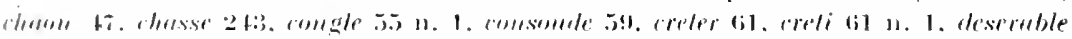

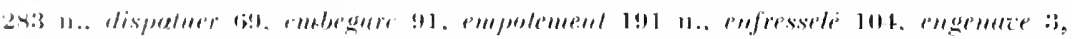

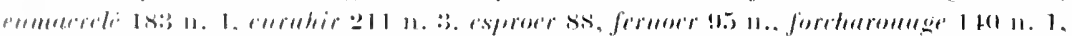

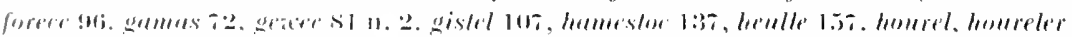

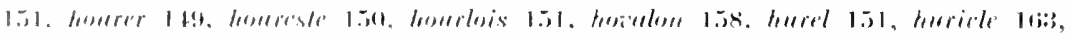

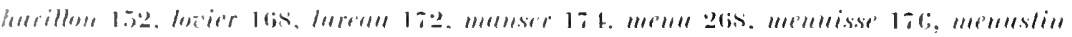

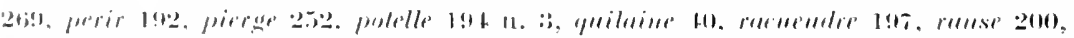

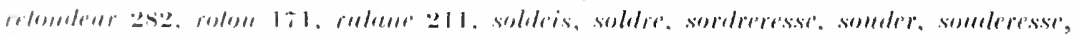

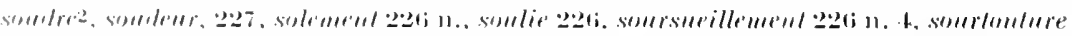

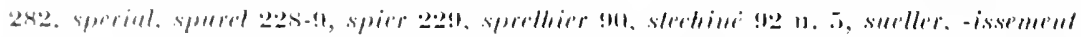

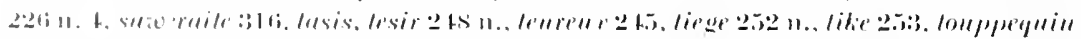

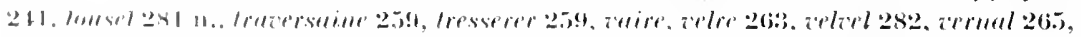

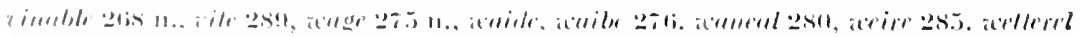
245. Encilial. 23:3.t. 
riaux plus abondants et mieux préparés. N"est-il pas remarquable, (ch effet, que Meyer-Lübke, dans son récent répertoire étymologique des langues romanes $\left({ }^{1}\right)$, aceorle une attention spéciale anx dialectes wallons et cite des termes liégeois on namurois bien plus fónuemument que ses devanciers I)ie\% et Körting ? Cette part légitime faite anx humbles parlers de Wallonie promet de s'élargir encore dans le dietionnaire étymologique de la langue lrançaise dont un philologne suisse vient ale publier la prenière livraison $\left({ }^{2}\right)$. En attendant le lutur I Dictionnaire avallon, des études préliminaires, eomme celles qu'on tronvera ici réunies, permettront amx romanistes de tous les pays de faire du wallon un usage plus étenchu et plus sîr.

Comparés à la langue francaise, nos dialectes, - et surtont ceux du Nord-Est, qui gravitent antour de Liège, - présentent m double caractère.

Lcur structure phonétique est phus archaigue : le fonds latin y transparait plus clairement. I)es mots liégeois tels que éie, far, stawn,

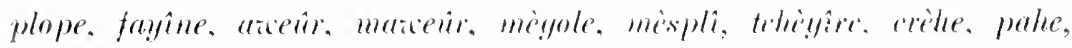
limole, sont plus voisins de la souehe latine que leurs eorrespondants français eun, fou (hètre), surean, penplier, fuint, heur. mir, moelle, néflier, chaise, côtre, paîtré. ammaitre. Certains types latins ne re rencontrent que cliez nous $\left({ }^{3}\right)$. Des expressions tontes latines, commes de l'ancien liançais et disparues de la langue moderne : adeser, musée,

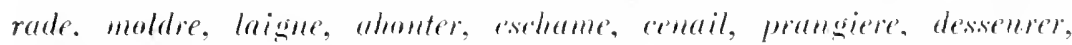
resprée, entait, cumbler. etr., survivent à Liège dans le langage quotidien $\left({ }^{4}\right)$; et que de jets noureaux poussés sur le vicux trone latin:

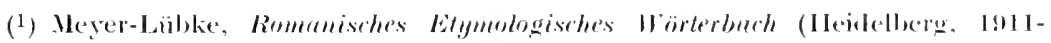

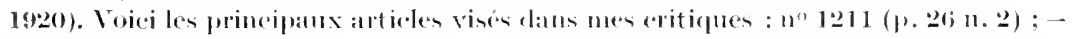

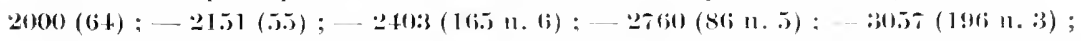

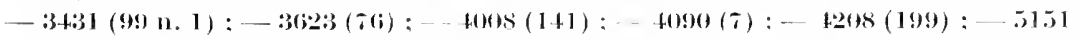

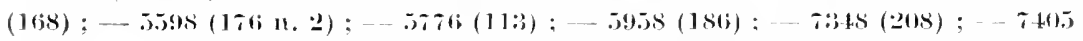

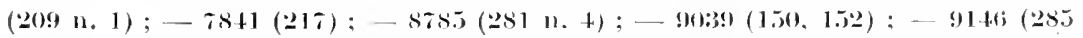

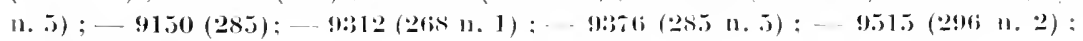
- 96036 (18:3).

(2) W. v. Warthurg, Franzäsisches Etymologisches IIörterboch (IBomn et Leipzig, 1re livatison, 19.2).

$\left({ }^{3}\right)$ Par excmple cupere (voy. kente, p. 16.5), terreum (voy. tidje, 1" 252), val rat (voy. were, p. 2s.5) : ce demier, inconnu en franģais, a survécu dans le provençal, lespagnol et le portugais; voyez Meser-löilke, no 9150. l'ent-être aussi gavia (voy. p. $\%$ ).

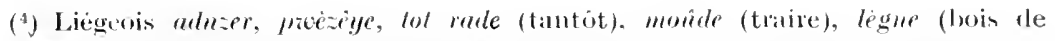
chanllage), ahonti, hame, cime, prandjire, dizmeirer (blesser), issprizge, étit (vovez p. 9:3). imâler. 


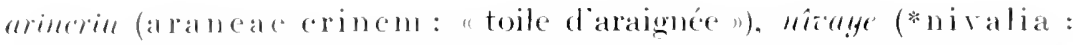
"neige"), mutaic (multum tastum: "pent-citres. littéralenent très tôt

si le fonds hé réditaire de nos patois, leur morphologie et leur syotaxe, sont essenticllement diorigine latine, il neen est pas moins rai qüil ont subi. surtont daus la phonétique ot le vocabulaire, unc forte influence germanique. ('ette inlluence, qui sallirme eomsidérable dans la bangue lrancaise clle-mêne, a dînaturellement agir bien darantage

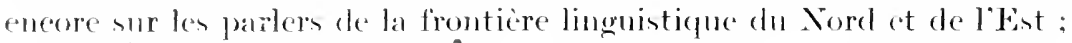
et, dams la contróe la plus soptentrionale qui ait adopté le langage des liomains, langle que forme le pays de Liege et de Malmedy s'est tromé le plus exposé à linvasion des mots tudesques : neest-il pas le poste avancé de la colture romane, dont la destinée séculaire fut de supporter,

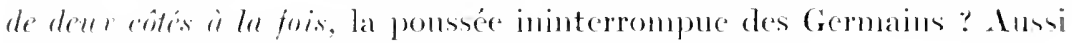
l. dialecte r est-il saturé d'éléments hétérogènes.

Il incombe it la philologic wallome de mesurer l'étendue et la profonderur de ces allurioms, de dater, si possible, chacun de ces emprunts, de laire le départ entre les roeables anciens et ceux dont lintroduction cot réconte. entre ceux qui ont lait souche et ceux qui sont cristallisés dam mor seule expression ( $\left.{ }^{1}\right)$, de déerire leur adaptation phonéticue

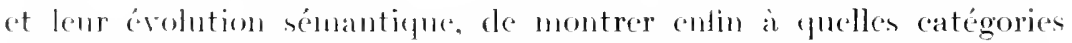
ideales appartiennent ces mots compruntés: vic rurale, travaux journaliers, instruments de méticus, ete. Il va de soi quime étude deensemble sur les élénents germaniques des dialecetes walloms serait prématurée anjourd honi : on ne jumra laborder que plus tard. lorsque l'enquête

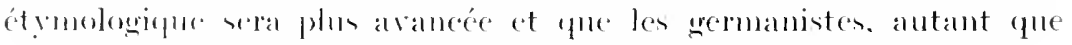
les romanister, uront pur con verilicer les rénultats.

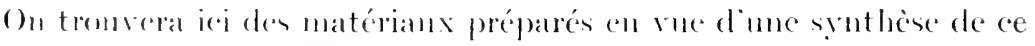

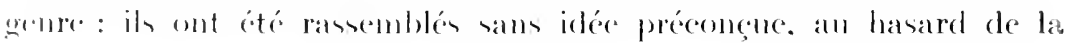

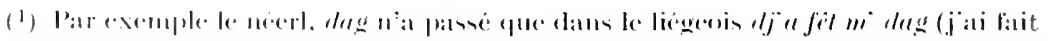

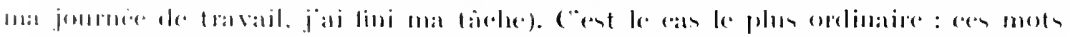

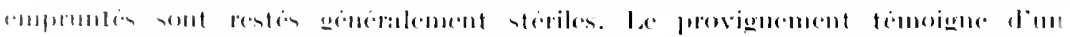

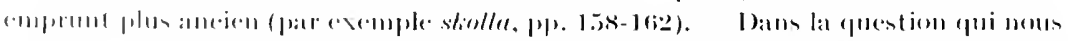

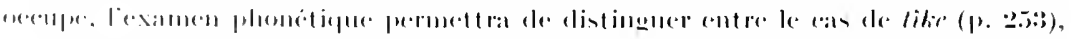

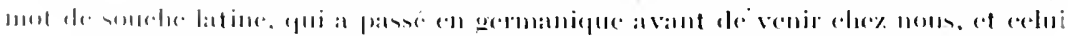

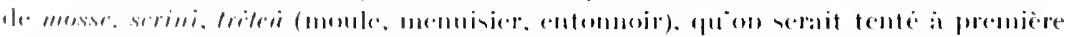

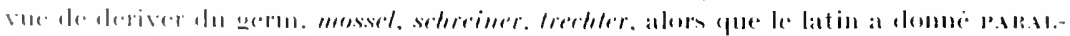

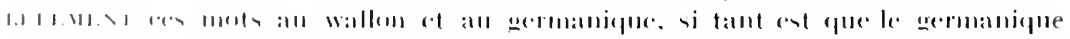

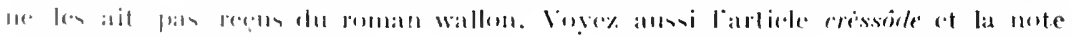

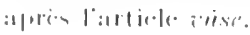




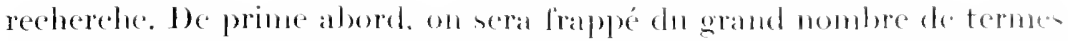
allemands on néerlandais qui ligurent dams ee reeneil. ('ertains, je nly attends, inelineront a me taxer dexagération, eomme on a pule faire, non sans raison, ponr Grandgagnage lui-mêne (1). J'ai conseience eependant diavoir été. dans mes conelusions. anssi prodent, ansei objectil que possible. 1)e phus, il serait illogigue de vouloir juger de lapport germanique en se fomblant miquement sur les guelpues centaines de temes qui sont étudies ied. C'e serait perelre de rue la masse des mots dialectaux qui ont des correspondants francais dont lorigine latine est solielement ćtablic et ne prête plus à discousion. C'e que l'étymologiste doit étudier et, s'il le peut, identifier, ee sont les termes obseurs, sams famille comme dans le domaine des langues romanes : mo examen attentil poura sams doute (en rattacher un eertain nombre an latiu $\left(^{2}\right)$ : mais, pour la majenre part dentre eux. il y aura présomption naturelle an farem d'mue origine étrangere.

Qnoi qüil en soit, il est nrenent, répétons-le. de reconeillir nou patois. Ils sont an train de périclitere tichoms tout an moins d'en saurer le souvenir préeis. 1) Hune part le progres constant de la langue francaine, réhiculée par les jourmanx ot par l'enseignemont, d'antre part le développenent industriel. qui modifie les anciennes lormes du travail humaliz en supprimant les méticrs of les ontils traditionncls, contin l'universel nivellement des conditioms soriales, - sams compter dautres factems

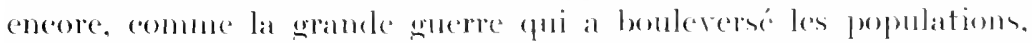

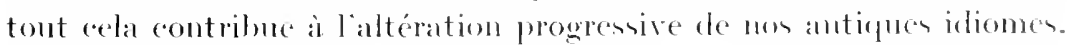
Tout vieillard qui disparait amporte arece lui de vicux mots, dont la génération suivante ne commait déji plus le som exact. Or. on laa dit

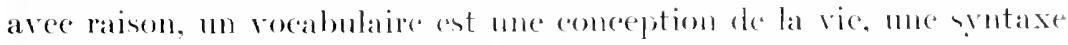

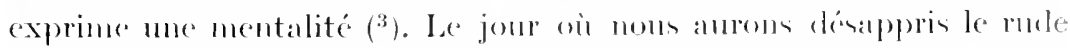
langage ele nos pères, notre horizon sera peut-être chargi et notre génie

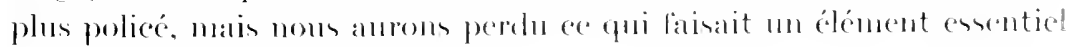
de notro persomalité.

$$
\therefore \quad \therefore \quad \therefore
$$

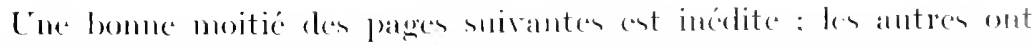

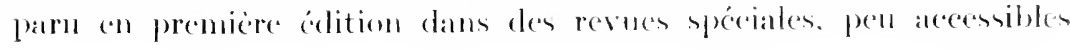

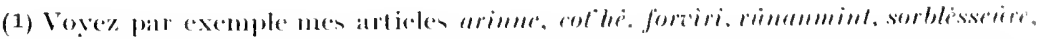

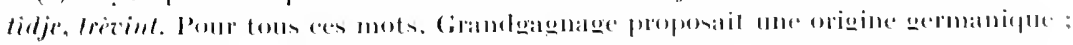
nous les rattachoms au latin.

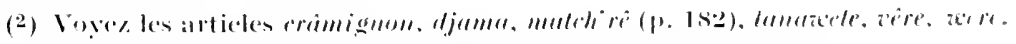

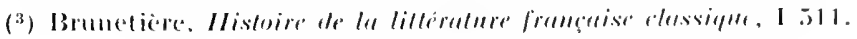


an public (1). Je les reprende ici pour ume domble misom. 1) abord. le sonci de corriger eertanes faiblesses de mes premiers essais : des

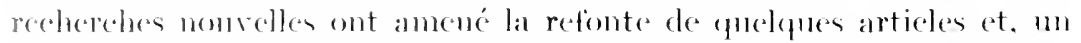
pen partont. des remaniements on des additions. En seeond lieu, si

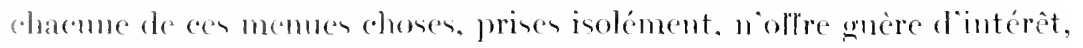

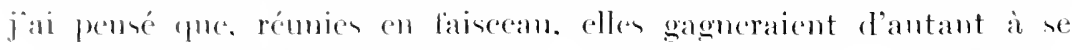

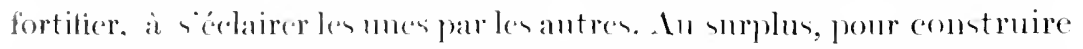
simement. la seience philolugigne réclame tont d'abord des groupement de matérian ansi mombremx. amsi comvemablement préparés ane pourible.

Il me rente i renereier les nombreux collaborateme correspondants qui mont fourni. aree tant de bomne grace, des remseignements de

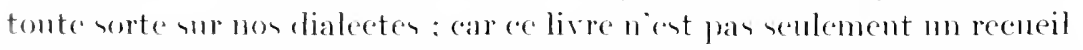
d'etyoulogien, il contiont ale plus les resultats partiels den imultiples

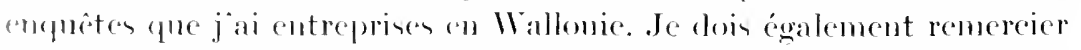
mes collingues at amis Augunte l)omtrepont et Jules Feller. que jai tomjum tromés prêts à midele de lemr savoir et de lenr expérience. Fit enlin je venx dire ici tout ee que je dois a l'éminent philologue, II. Antoine 'Thomas, membre de l'Iustitut. dont les brillanter etudes

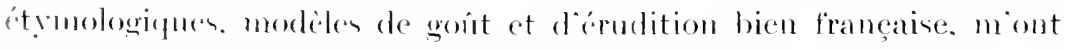

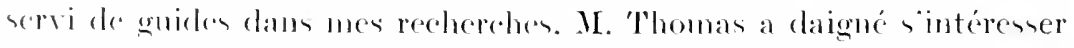
persomollement a mes modenter cssais: ses encomragements et ses

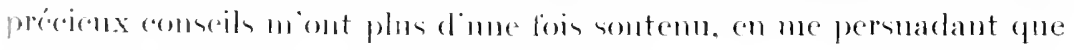

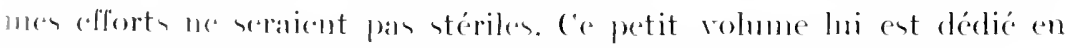

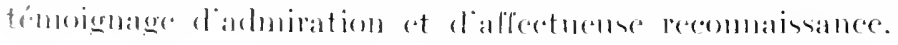

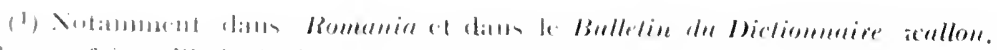

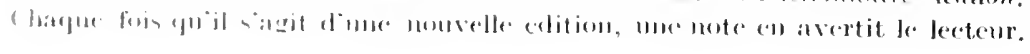




\section{Bibliographie}

Ponr ne pas allonger cette liste outre mesure. on ne reprend ici que le omvigen dont le titre est rité en abrege on mème simplement par le nom de lantenr.

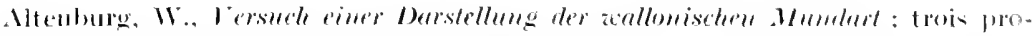

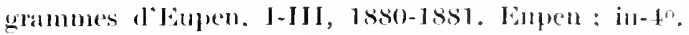

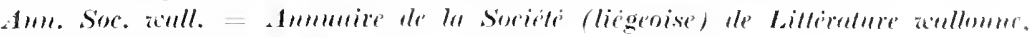
29) vol. in-12. I iege, 1s6:3-192:2.

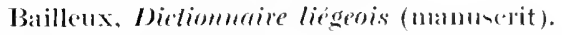

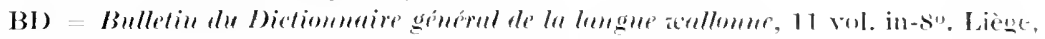
$190(i-1920$.$) .$

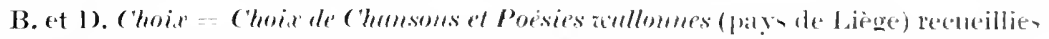

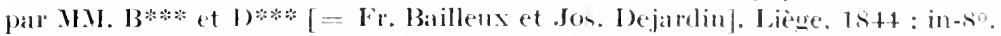

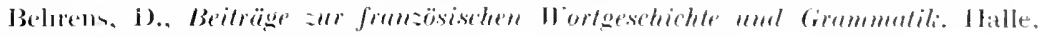
$1910:$ in-80.

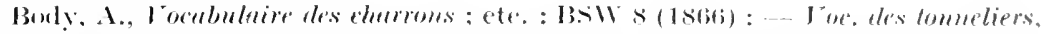

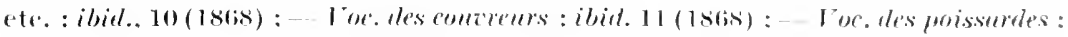
ibid. 11 (186s) : - Joc. lles ngriculturs : ibill. 20 (1885).

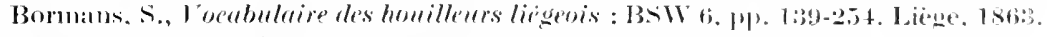

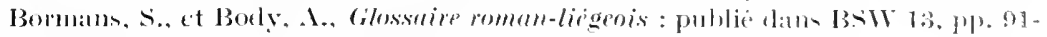

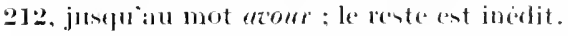

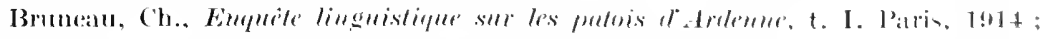
ill-80.

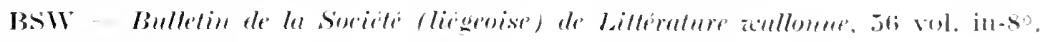
Liene. 1858-1922.

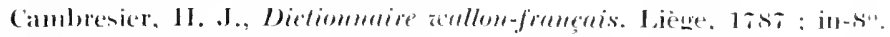

Choirt, voyez 13. et 1).. ('hrir.

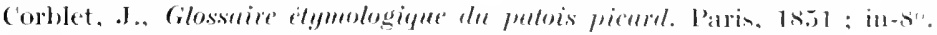

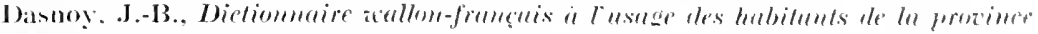
de Luxembourg. Neufchatean, 1 s.jol: : in-1:2.

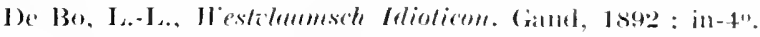

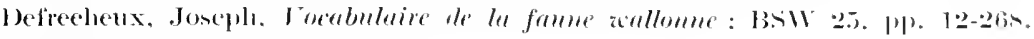
[L'ne 3e edition a parn en 18 s!s?]

1)(lfosie, voyez I'. 1).

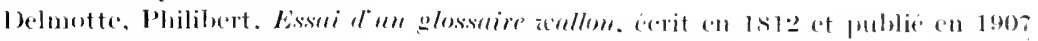
à Molls : in-8".

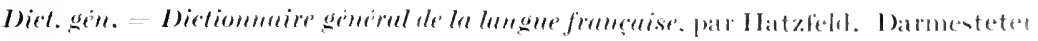

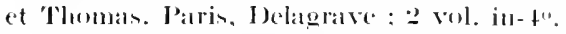

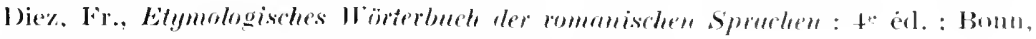
$1878:$ in-s".

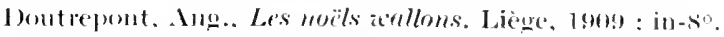

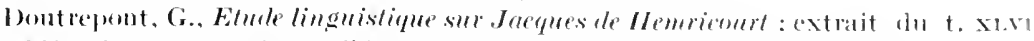

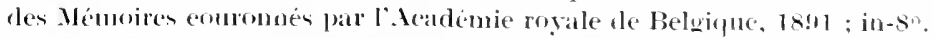

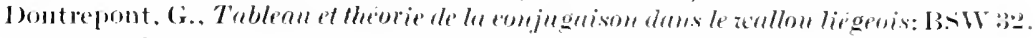

1) nviver. C., Dirtiommere liegenis (manuncrit). 


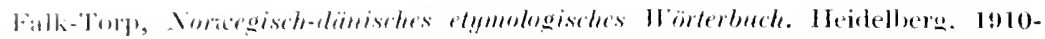
$1911: 2$ voll. in-1:2.

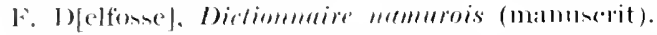

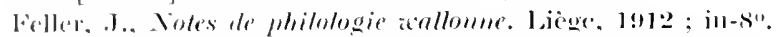

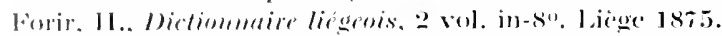

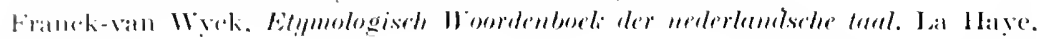
1912 : in-s".

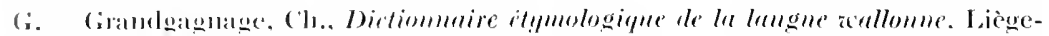

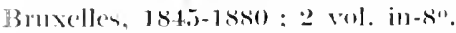

G.. Eidraits de lillers : voy. Villers.

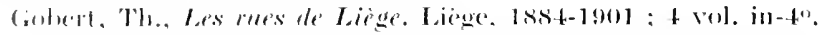

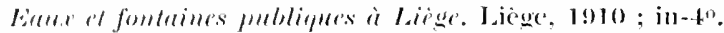

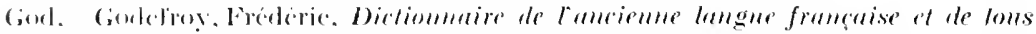

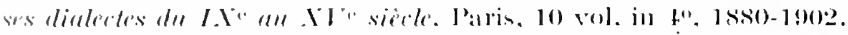

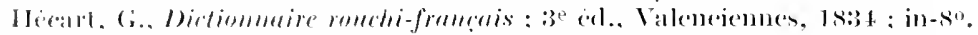

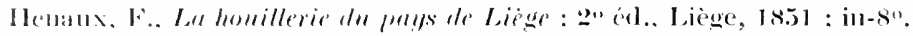

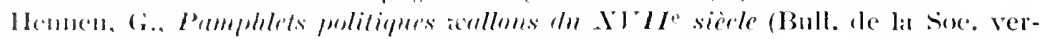

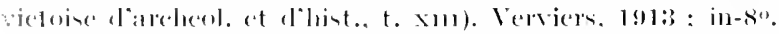

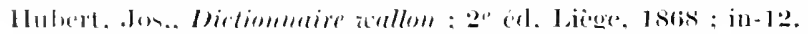

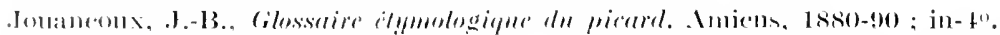

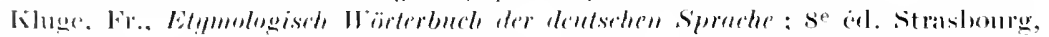
$131,5:$ in-s".

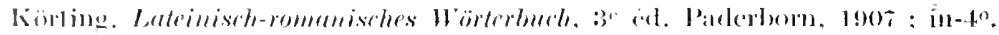

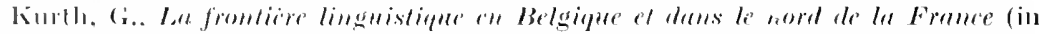

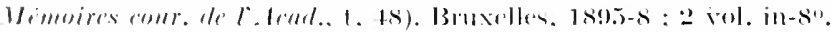

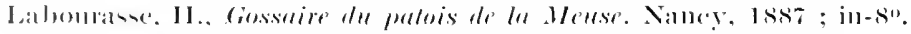

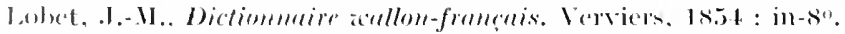

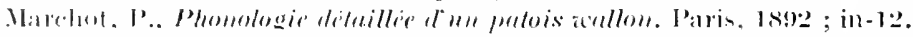

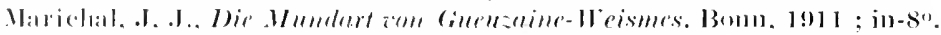

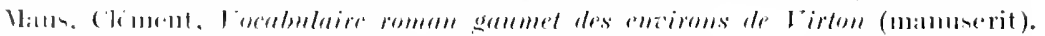

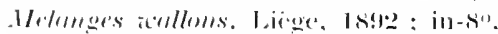

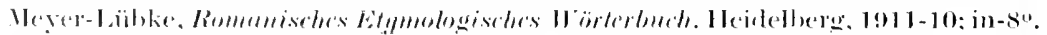

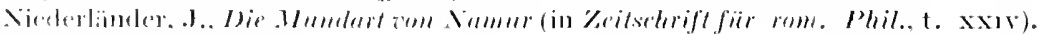

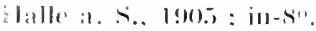

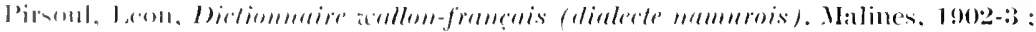
2 inl. in-12.

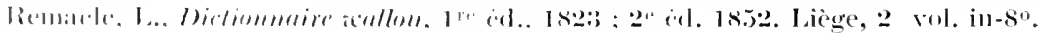

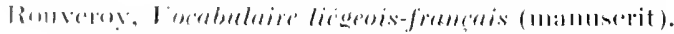

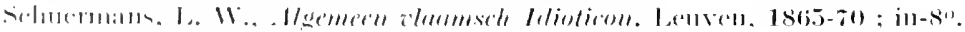

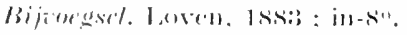

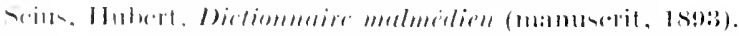

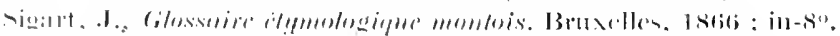

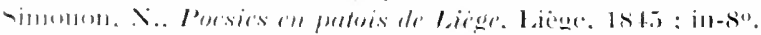

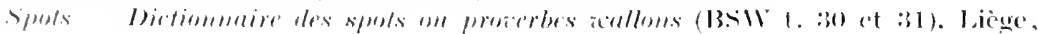

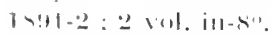

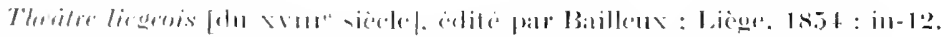

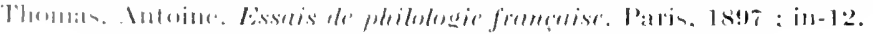

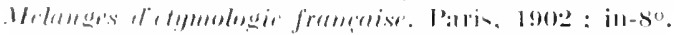

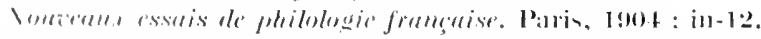

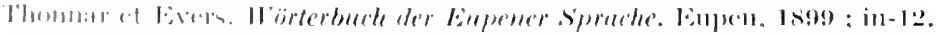

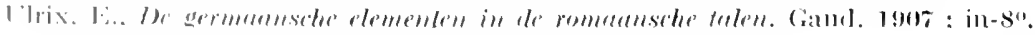

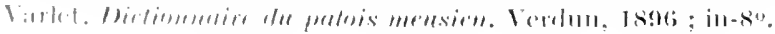




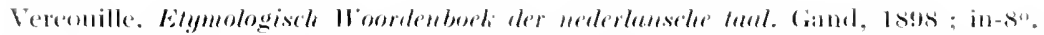

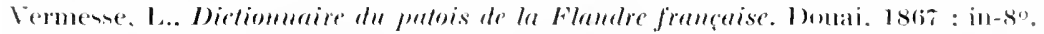

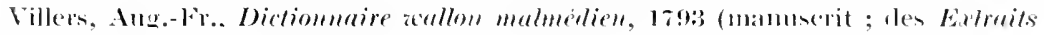

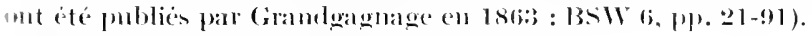

"lallowiu. Liege, 189:3-1914; 22.2 vol, in-s".

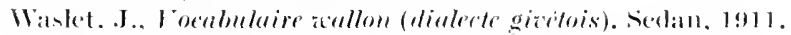

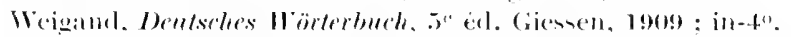

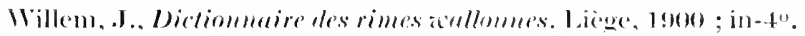

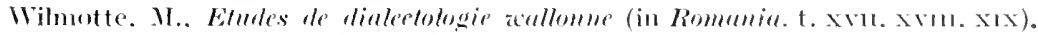
P'aris. 1sis-(0); ; in-son.

\section{Graphie}

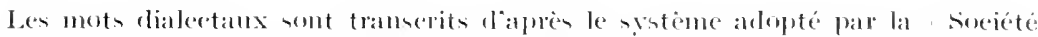

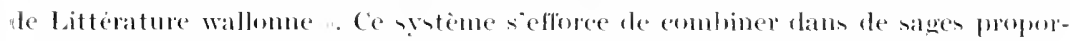

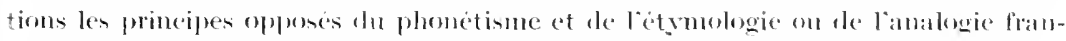
saise. Il mote exactement les som parles, tout en tenant eompte, antant gue possible. de lorigine des moth. de ha gammaire et de lohistore de la langue.

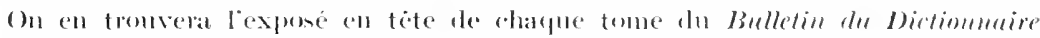

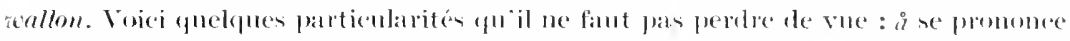

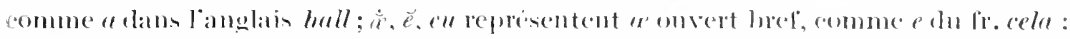

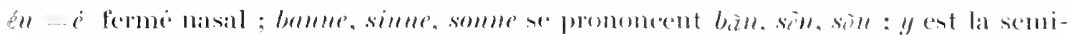

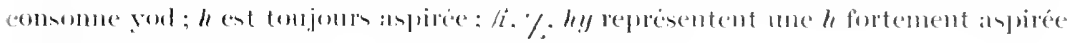
et légèrement munillée: $n-n g$ de laallemand loug.

\section{Abréviations}

\begin{tabular}{|c|c|c|c|c|c|}
\hline sll. & allentimul & fr. & tranceais & $111 \mathrm{~s}$ & matmm-rit \\
\hline ance. & ancien & Iaxm. & manmatis & 11. & noite \\
\hline ard. & arelennais & цеті1. & germanique & mam. & Ilantmorois \\
\hline art. & article & l. & ligne & néerl. & necrlandativ \\
\hline м.j. & aujouml'hui & liat. & lattin & lי. & pange \\
\hline hestr. & (rlecetrolatis & l. 1. & licoll dit & $r$. & romlelii \\
\hline (oml). & eompantez & licur. & licuenis & $\because$ & unbstantil \\
\hline dial. & dialecte & litt. & litteralement & -uff. & sulfixe \\
\hline él. & alition & $\mathrm{m}$ & minemlin & $\because n 11$. & ?rononymo \\
\hline esp. & (יp:agmol & malm. & malmérlien & t. & terme, tome \\
\hline f. & féminin & m. h. all. & moren lant allemand & & \\
\hline f & flankand & mole. & moderne & & \\
\hline
\end{tabular}

Lasterieque * infloge une forme hypothetique, restitue par induction. 



\title{
Etymologies wallonnes et françaises
}

\author{
w. abèrdondè, avèrdondé
}

Au sud-onest de Namur, à Starc et à Fosses. abèrdondè signifie: "plus porté au jeu qu’au travail " (BD 1913, p. 23). Hécart signale en rouchi le féminin ačerdondée "jeune folle. étourdie ". et j’ai noté. à Alle-sur-Semois, avérdondé "paresseux" ". avee une forme plus rare évèrdondé. Cela nous mène à l'ane. frr. eszergondé "sans vergogne, dérergondé ". dont le sens, dans les patois modernes, s'est restreint et l'aspect passablement défiguré. On renarque la même substitution du préfixe es-: t- dans le gammais arergougui (Ste-Maric-sur-Semois) " eonfus, éperdu. effaré ". qui reproduit l'anc. fr. estergoignié. doublet de estergondé.

\section{liég. ådios', an'tchou, verr. am'tchô}

Le liég. ådios", aidiyos" "façons cérémonieuses, salamalecs " (1) rient probablement, d'après G., I 8, de l'esp. a dios "adieu ". Cela n'est g'uère possible, vu l'aceent et la quantité de la protonique $a$-, qui derient même an- nasal (à Starelot), an'- (à Spa, Starelot, Sprimont), aw- (à Verviers. d'après Lobet). Il faut y roir l'altération du grec \&yos - prononcé âguiyos' $\left({ }^{2}\right)$ - qui est le premier mot et comme le refrain des rersets chantés à l'oflice du Vendredi saint: chaque tois que le ehour dit : "Agios o Thros (Sanctus Deus)", on fait une gémullexion.

La forme pure agios' s'emploie, toujours au pluriel, en picard (Corblet) et en montois (Delmotte) aree le même sens de "démonstrations d'amitié, eérémonies, salamalees ". Dans le fr. agio, tgiau $\left({ }^{3}\right)$, s final

(1) Voy. BD 1907, p. 84, où il fiut lire äw'dias' (Lobet) et an'doyes (Bailleux) [ce sont des altérations arbitraires, par intention sareastique]. Lobet donne aussi audioss, p. 6.17 (lire : ålios'), et non an'dios'.

${ }^{2}$ ) Dans les mots liégeois empruntés du latin, l'antépénultième a portant l'aceent tonique devient normalement å; comp. åsimus' (à larticle uruses, ci-après); åbarone, s. f., bannière carrée ; du lat. labarum (G.. I 3 et 3222).

$\left(^{3}\right)$ Voy. God., t. X, et Diet. gén. Ce dernier déclare le mot d'origine inconnue. Cependant, comparez, outre le fr. kyrielle, le picard faire des sanctus' on des adorémus" (Corblet) "des salamalees"; le montois des-ôrémus", des mittimus " des embarras, des objections" (Sigart, 256), le w. dès Jèsus" Harià, dès Matèr Dèi " des exclamations ». Pour arias (G., I 26), voy. Dict. gén. 
s'est amuï. Le liég. ádios' est remarquable par le changement de g dur cn $d\left({ }^{1}\right)$ : il a probablenent subi l'influence de adic, adiu "adieu ".

Dans le sen de "salannalees ". le liégeois emploie aussi an’tchô (BSW 39. 1. 23\%), -on (G.. II t97). altéré en am'tchô (Verviers : Lob., pp. 3\%. 38). -ou (Viclsalm : BSW 51, p. 236). Il faut rcconnaître dans antahô un durcissement de *andiô, forme empruntée du fr. agiau, agio, de mêne que an'dios' provient de andios', adios'. mis pour agios'.

\section{anc. liég. afahant}

Duvivier et G., I 9, traduisent ce mot par "affamé ». Le seul excmple commu ent de 1622: Toz estez oun gran afaxhan Aprè lè bin di no chimon' $\left({ }^{2}\right)$. G. II 266, note de plus. d'après Simonon, le composé rafahant "insatiable. glouton ". On n'a pas cneore expliqué ces deux mots, disparus de la tradition orale. - A première vue. on pense à faht (fasciare: (envelopper. entortiller ; hr. faisser); mais fahî n'a que le sem de : "mmailloter (un enfant). fagoter ", de même que l'anc. fr. enfaissier. L'all. lahen. qui est encore dans Luther et que l'all. moderne a déformé en fangen "prendre. saisir". conviendrait tout aussi bien pour la lettre ct beaveoup mieux pour le sens.

Le composé moyen-haut-all, ane zâhen signitie "revendiquer par voie de saisice sapproprier". Telle parait bien itre laeception de afahant, qui serait donc synonyme du w. agrafant, -pant, agrifant. -pant "rapace, eupide. aceapareur" ". ct formé de même du prélixe roman a- (lat. ad) et d'un radical gemanique. Daaprè cette eonjecture, la traduction "aflané " manquerait d'exactitude; "ctre afallant aprés qquh " répondrait au fr. familier " vouloir mettre le grappin sur qqch".

\section{w. aǵuèrôdî (Vielsalm)}

On lit dans he Bulletin de Dictiomaire acallon, 1911. p. 50 :

aguèrôdî (Viclsalm), v. intr., venir pour faire la guerre (?) : lís-annies

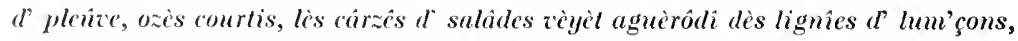
"les amnées de pluie, dans les jardins, les pares de laitues voient venir vers eux pour leur faire la guerre des lignées de limaces".

L'anteur de cette intéressante communication se trompe certainement : la traduction qu'il propose s'inspire du contexte (idée d'imra-

(1) Comp. le w. lidion "nielle des blés ", altéré de nîguion (G., II 25).

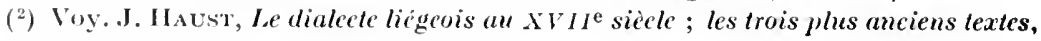
p. 28 (Liegre, 1921). 
sion) et d'une ressemblanee toute fortuite entre les sons. L'étymologie populaire ne proè̀de pas autrement ; souvent même, elle se contente de bien moins. En réalité. aguèrồi est composé d"un verbe guérôdî "réussir ", que nous arons relevé près de Vielsalm, à Petit-Thier : lésavonmes ont bin guèrôlî ciste ammêe. On connait aussi guèrồler à Malmedy, Faymonville. Stoumont. Moulin-du-Ruy; dans ee dernier village, on dit plus sourent djevinder $\left({ }^{1}\right)$. - La source est évidemment le luxembourgeois geroiden (= all. geraten) "tourner. tomber (bicn ou mal), réussir ". De là. notre composé aguèrôt doit se traduire par : " arriver" aceidentellement, tomber par hasard dans... " ; e'est, en fait, l'équivalent exaet de l'all. lingeraten.

liég. akinåve, nam. aginauve, anc. fr. engenave

G., II vm. enregistre simplement cette phrase de Simonon : il è-st-alinaze di mas d" dints "il est sujet aux maux de dents ". Récemment $\left({ }^{2}\right)$. on a rapproehé de alimâze le nam. asinauze "actif, énergique" (G., II 496) et l'ane. fr. engenare (Jean de Starelot, p. 75), que l'éditeur Borgnet glosait comme suit : "enclin ; probablement la forme aneiemne de alinaf signalé par Grandgagnage " $\left({ }^{3}\right)$.

Les trois mots présentent bicn le même suflixe (fr. -tlble); mais, à mon sens, nous avons affaire à deux radieanx différents. Le liég. alinàe parait ètre dérícé du lat. acelinare "incliner, pencher vers"s. Bien qu'on ne troure pas *aclimable dans Godefroy. on pent le supposer d'après aclim. acliner, entimable qui sy rencontrent. Le groupe al se sera simplifié en $l i\left({ }^{4}\right)$.

Quant à engenaże, Borgnet et Godefroy ont tort assurément d'y roir un synonyme de "enelin ». C"est la forme wallonne de l'anc. ir. enginable "qui a beaucoup de talent" (Gocl.). dérivé de ingenium : engin. La phrase de Jean de Stavelot : "il estoit mult engenave delle inquerir

(1) Toujours accompagné de bin on de mâ ; exemples : Su $l$ tins gnèrôde bin, fât soyî â stièrmint (Malmedy, Arm. dol Suméne, 1886, p. 18); çoula li-a mâ guèrôdé (Bastin, Voe. de Faymonville); il a bin guèrôdc, i guèrôde mà d"vins sès-afêres (Stoumont, Moulin-du-Ruy).

$\left(^{2}\right) \mathrm{BD} 1911, \mathrm{p} .36$. Corrigez, dans cet article, énergumène, agentue en : énergique, engenave.

$\left({ }^{3}\right)$ God., Engevare, reproduit le passage de J. de Stavelot et la détinition de Borgnet.

$\left({ }^{4}\right)$ Voyez ci-après les articles guingon, rakète $(=$ glingon, raclette). Il faut peut-être rapprocher le w. kègneter "taquiner" $(\mathrm{G}, \mathrm{I}$ 104) du vervietois eligneter "chercher noise pour des vétilles" (BSW, 53, p. 418). Forir donne kajo on klajo "toile d'emballage "; etc. 
comment ill acqueroit argens. sans estre honteux... "signifie qưil était très ingénicux pour reehercher les moyens d’acquérir de l'argent.

Enfin. dans le namurois aginaure - quon prononçait sans doute adjinauce - nous retrouvons un type primitif *idjinauve (= engenare: enginable). qui aura subi l'intluence analogique du préfixe $a$ - ${ }^{(1)}$ et eelle du verhe adji "agir ". Du sens propre : "habile, fertile en expédients", on passe sam effort à celui de : "actif, éncrgique".

\section{liég. ålon}

G.. I 18. donne sans explieation le liég. alế, cilon "échalas ", anc. liég. allon. La forme en - $\hat{e}$ ne s'entend jamais, je pense. à Liège $\left(^{2}\right)$; mais on comaît âlè à Fontin-Esneux. aulé dans la Famenne, aulia à Ciney. Le liégeois appelle alom la perche à haricots. Ces perches sont plantées isolément on bien par couples. sur deux lignes: dans ce dernier cas, elles sentrecroisent vers leur sommet et sont liées an point d'intersection. - La protonique a s'oppose à un rapprochement avec le liég. alite "ailette, aileron ". Il faut roir dans àlé. altom des diminntifs romans du moyen h. all. ahsel (all. mod. achsel "épaul:" : apparenté an lat. axilla) : le thène s'est réduit à ass-devant le suflixe $\left({ }^{3}\right)$. Tiéchalas est considéré comme une épaule sur layuelle s’appuie la plante grimpante (harieot. houblon, vigne).

\section{liéx. amå, må (" arant ")}

G.. I 20 et II +7 . signale ce mot qu'il juge ćnigmatique ; il mentionne bien un homonyme umâ "à moins ": djîrè, a mâ quéque astådje. "j"irai à moins d'un empecheryent ": toutcfois, non sculcment il ne suppose pas quo ecs denx ama penvent ître identiques, mais il se refuse même a roir daus le second lo même mot que mons (mo) "moins". Pour ma part. je suis d'mu aris opposá.

1) 'aberd. je crois que mo (noims). employé comme proclitique, est

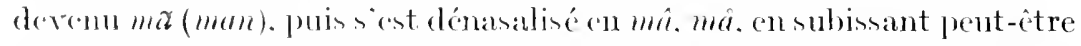

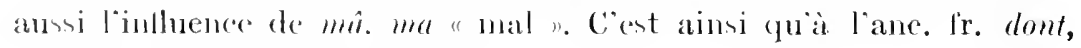

() Vor. ai-dessus l'art abredemdí.

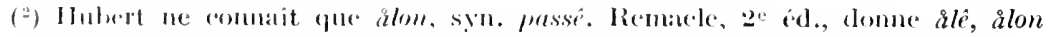

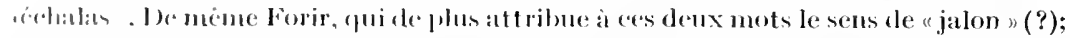

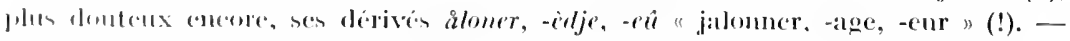

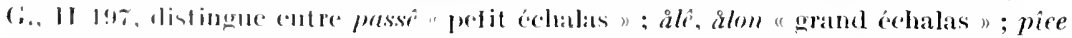

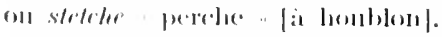

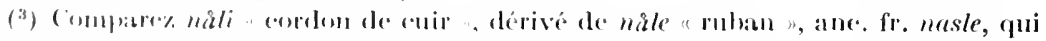

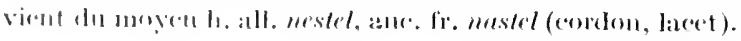


doint ("donne ", 3e pers. du subjonetif) réjoond (n anc. w. don. dan

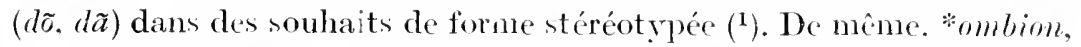
diminutif de ombe "ombre ", a passé par *ambion pour derenir le liég. moderne abbion "ombre d'une personne. d"un objet " (G., I 4), sous l'influence probable de abe "arbre". Le lieu dit C'rommonse ( $\left.{ }^{2}\right)$ est devenu Cramoûse à Jupille. Comparez encore lo liég. måquer " nanquer " et les formes arièye. aiagnî que le Dict. liégeois de Iubert dome pour anzèye "anguille ", rangnî "gagner ". - Dès lors, rien d’étomant que de vieux Liégeois prononeent, conme je l’ai entendu : djồì, a ma quéque (ou d'ine) astadje, au lieu de a mons.

D'autre part, le latin mimms triginta diebus (Cie., de Dizim. I 68) signifiant "dans moins de trente jours. avant trente jours " (telle chose arrivera), il paraît légitime de voir une syntaxe analogue dans le liég. ma (ou a ma) trinte djous : c'est à cause du eontcxte que "moins" a pris le sens de "avant". - Les plus aneiens exomples de cotte construction se reneontrent dans des pasquilles du xrire sic̀cle $\left({ }^{3}\right)$ où, deux fois, nous lisons aman qui " avant que "; on remarquera cette forme arehaïque man, qui répond à l'anc. fr. mans "moins " (rov. un exemple dans God.) et qui eonfirme ee que nous avons dit plus haut à propos de mó< má. - Dans le parler moderne. jai entendu mâ

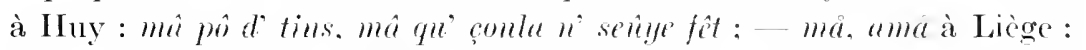
(a)må pô d' djunss ou d' tims "avant peu de jours. de temps " $\left({ }^{4}\right)$. zos-árez

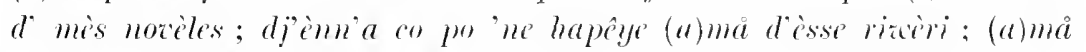
dè djomacer. i fat ozrer ; (a)ma qu' zos 'mialése. dji scè rim'non; il éstêut la (a)ma qui dì nî̀ fouhe.

J'cetime donc, contrairement à Grandgagnage, que ma (noins) est une forme variée de mons et que a ma (avant) ne diffère pas étymologiquement de a ma (à moins).

(1) Voici les quatre exemples que je connais : Dief don bon iour (1690: Ann. Soc. Wall., 19, p. 110); Dief dan boune mute et bomme santé (1631; l'éditeur du Choix, p. r9, a corrigé en donn' !); Dicf dan bonjour (1672: Hennen, Pamphlets, I,v.1); Dif dan bone mutte et bone santé (1700 : BSW 6, II, P. 18).

$\left({ }^{2}\right)$ Coude formé par la Neuse à Liège ; du néerl. krom, adj., courbe. Altéré en Coronmoûse sous l'influence de coron (bout) et francisé en "Coronmeuse ".

$\left.{ }^{3}\right)$ Vos-îrîz tot-avâ l' Holonde,

Aman qu' vos vièrìz fé ainsi. (Pièce inçlite, ms.).

A man qu' l-avint stu èlèvés,

Il a falou bin dès broûlés (1672: G. Hennen, Pumphlets, I, v. 103-4).

Comparez encore manrai dans la Geste de Liege, v. 600, qui répond au liég. mod. monrè " mènerai ".

$\left({ }^{4}\right)$ On trouve même dans H. Simon, Pun dè bon Diu, p. 114: ossu, so må pô d' tins, li tére ui sèrè pus... 


\section{w. amaule ( $\mathrm{S}^{\mathrm{t}}$-Hubert)}

Peur M. Marehot, amumle "importm. ennuyeux", qui se dit à St-Hubert en parlant surtout d'un enfant, représente le latin amabilem (aimable). employé par antiphrase $\left({ }^{1}\right)$. Le sulfixe équivaut évidemment au fr. -able: mais. si l'on dit également amâle à Fosset-Amberloup, on prononee hamâle à Laroehe. hamaule à Rossignol, et l'aspirée initiale sulfit ì indiquer une origine germanique. Dìs lors. il fant sans doute s'adresser à l'adjectif allemand hem qui. au xre sièele. signifiait "appliqué à nuire, rebelle. insoumis". et à l'all. hämisch "malin, malicieux". an xve siède hamisch "sommois. artificieux" $\left({ }^{2}\right)$.

A l'Fent du Brabant wallon. on relère amame (St-Géry, ChastreVilleroux. Ste-Maric-Geent) "rapaee, avide du bien d'autrui ". Il convient d'y voir le même mot. dont le sens a évolué différemment.

$$
\text { nam. am'bô, an'bô; lićg. hèn'vå }
$$

Grandgagnage a les articles suivants:

I, 20: ambau, nam. (hamgar). II, Ix: Ce mot parait êt re l'all. anbau, holl. anmbouc (construction ajoutće à une autre, bâtiment accessoire).

I1, xxxı et 535: hènevâ (soupente : petite pièce pratiquće dans une cuisine, ete.) Simoxox.

Je n'hésite pas à rapprocher ces deux mots: il ne peut done être question. ì mes yeux. de l'all. anbau.

Dans le Bull. du Dict.. 1914, p. 49, II. H. Gaillard note une forme nammroine albo "grosse pièce de bois dans le gerbier d'une grange ". 1 I) ence (Namur), à St-(iéry et à Chastre-Villeroux (Brabant), am bô dénigne nue espece de grenier situé au-dessus de l'aire de la grange: on y contance les gerbes quand les mafes (gerbiers à eoté de l'aire) sont remplis jusquan toit. Non loin de là, à Fosses-lez-Namur $\left({ }^{3}\right)$, à Meux et is 'Thorembais-St-T'rond. on prononee an'bô. Enlin. plus à l'Est. damb la région qui a gardé l'aspirée germanique $\left(^{4}\right)$. noms relerons

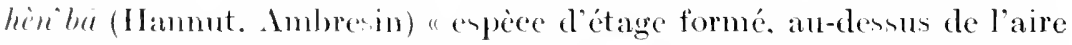
de la grange. an moyen de longues perehes, pour y mettre le foin et les gerber ". aimi que hinbi (Huy. Neuville-sous-IIuy). avee le sens plus

(1) Phonologie thu patois wallon (1892), Pp. 33, 57, 121.

(2) Voyez Wiegand nïnscit.

(3) BSW t. 52, 1. 110 .

(4) Cette aspirie tombe régulièrement en namurois. 
général et dépréeiatif de "bâtiment vieux et délabré" $\left.{ }^{1}\right)$; à Given (BenAhin): on vî ham'bår qui n'tint pö̌ "un vieux bâtiment qui ne tient plus». Ces formes asjirées rattachent elairement an'bô (et ses altérations $a m$ 'bô, al'bô) au flamand haneball" "traverse destinée notamment ì supporter les ais d'un plancher, par exemple dans une grange : de hanebalken in een sehuur " $\left({ }^{2}\right)$. Du hesb. hèn bå au liég. hèn'rả (auj. inusité), le passage ne fait pas diflieulté : la permutation de $b$ et de ctan nos dialectes n'est nullement isolée; elle s'explique de plus. cu l'espèce. par l'influence probable de cara (trappe, plancher mobile, fenil).

\section{wall. am'djoû (Charleroi), rouehi èm'djou (Mons)}

Le w. am'djoû " jour ouvrable, jour de la semaine " est bien connu dans l'Ouest de la Belgique romane (Charleroi, Viessille, Nivelles, Gcnappe). On dit am'jom à Maubenge. èmidjou à Mons $\left(^{3}\right)$. Pour expliquer la première syllabe im'djou. Sigart, p. 209. aree sa fantaisie coutumière. invoque tour à tour, et sans conelure d'ailleurs, l'all. heim. le breton pem. l'all. amt, le gree hebdomada! On est surpris de voir que Heser-Lübke. Rom. Etym. Wort. no 4090, adopte sans réserve cette dernière conjecture. - Notre explication sera autrement simple : ame, ème sont des altérations de ome (= homme). Dans son Glossaire des poésies de Froissart. Scheler a relevé deux fois l'expression "ne homme jour ne dimance ". c'est-i-dire ni jour ourrable ni dimanche $\left(^{4}\right)$; il voit dans homme jour: jour de l"homme, une "simple analogie aree domini dies: jour du Seigneur ". C'est exaet; mais il raut la peine d'ajouter que Froissart - né à Valeneiennes en 13:38 - n'a point créé cette expression, comme parait le croire Scheler ; il la tenait du parler populaire, où elle a survéeu jusquà nos jours.

[BD 1920, p. 3. - In exemple plus aneien de homme jour se lit dans les poésies de Gilles li Muisis (né à Tournai en 1272), éd. Kérvyn, t. II, p. 28, 1. 21. Voyez l'Écude lexicologique de šcheler sur ces poísies.]

(1) Exemple : dji n' mi sârê̂ plêre è ç' mohone là, c'èst co pîre qu'on hèn'bô ou $e^{\prime} \dot{e}$-st-ou tro grand hèu' bô (Henri Gaillard). Dans le BSW 53, p. 268, le même auteur a employé ce mot, par métaphore, en parlant d'une vieille cliarrette qui n'est plus qu'un hèn'bô désfoneé : un assemblage de planches défoncé, disloqué. Pour l'emploi péjoratif, comp. nos art. håbiêr, bèrôdh.

$\left({ }^{2}\right)$ De Bo : inanebalk et scheerbalk (voy. ci-après l'art. shèrbalik); comp. l'all. hahnenbalken. Pour la sémantique, comp. ci-après l'art. bèrôdi et le chestrolais travure (fenil; lat. trabatum). - On sait que le germ. balk a donné le fr. et lc rouchi bau "poutre" ; on le retrouve dans le w. inte-bi ; voy. ei-après l'art. $b \dot{a}$.

$\left({ }^{3}\right)$ Les graphies améjour (Hécart), hemme djou (Sigart) sont inexaetes.

(4) IIomme jour manque dans Godefroy. 


\section{w. amèder}

G.. I 20, signale le mot en liégeois-namurois avee cette explieation : "=fr. ancunder? on fr. émonder?" Pour M. Marehot, amidè (St-IIubert) est altéré du liég. ham let, all. hammeln $\left(^{1}\right)$ : erreur évidente. puinque amider et ham'ler eoexistent en liégeois. - Il est certain que amider répond au frr. amender (Meyer-Lübke. $n^{\circ} 2860$ ), qui a pris chez nous $\left({ }^{2}\right)$ le sens technique de "ehâtrer (m animal) ). Ameder ent la forme archaïque et purement wallomne $\left({ }^{3}\right)$. conservée dans une acception spéciale. Le doublet amiuler. qui a le sens général de "améliorer ". est pontérieur et refait sur le fr. amender. Au surplus, le composé raminder (liég.) "amender ", conserve la forme anciemue dans ramider (nam.), -i (Ciney) "réparer grossièrement".

Certes. il peut paraitre ctrange que la castration soit eonsidérée comme un "amendement "; mais. au point de vue de l'élereur qui choraisse le bétail. cette opération ancéliore l'animal. Des patois français (Nomantice. Anjou) domment de même à affranchir le sens de "chîtrer".

Godefroy cite deux excmples de. Valenciomes: "amendeur de bestes, de pourchiaux " (en 1+1+ et 1+19). sans roir qu'il faut traduire par "châtreur". W. "lmideù.

[BD 1914, p. 30.]

\section{fr. anacoste, r. anscote, w. hanscote}

Le Dirt. gín.. vo anacoste "espere de serue ". nous apprend quion dirait an xrefe siecle anaseot. aseot. arseot et qüll faut y voir "des

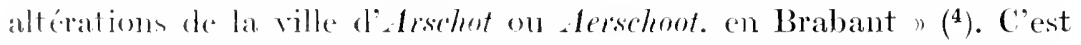
ambi l'ét yomologi que domme I'lrix ct que propose même le plus récent diectionnaire at ymokgigue de la langue française, echi de von Wartburg

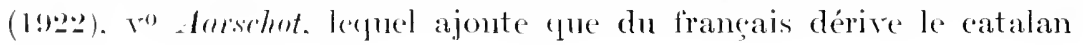
anciscot.

(1) Plomol. arm putois a. (1892), 1小. 1-2. - Niederliander, Mumlart zon Namur (19015), s : b, commut la móme crreur.

${ }^{2}$ ) Ameder Liege (G., Forir ; anj. dentet), Jeneffe (Hesbaye), Ben-Ahin, Meux, Numur, Johloguc, Camblonx, Chastre-Villeronx, ete. ; amèdè en Famenne, Saint-

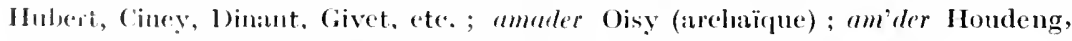
Viesville, Nivelles, Mons, etce se dit surtout du pore et de lat truie

(i) Pour i protonique, voy. labrt. garmiter.

(4) Gn renvoie a l'art. ascol (lire : eseot) sorte de serge "; cité pour la première foin dius m texte de 'Toulouse: "serges d'escot" (en 1568). 
Une note que j’ai insérée dans le $n^{\circ}$ d'octobre 1921 de Romania (t. Xlvir, p. 54\%) exprime une opinion différente. En roici le résumé : "Sans prétendre examiner par le menu les formes françaises du xrine siècle et leur authenticité. je crois utile de signaler un terme qui mérite d'entrer dans le débat et qui ne s'accommode guère de la dérivation proposée : c'est le w. hanscote (espèce d'étoffe : ancienncment bure ; aujourd'hui, tissu de coton durcté). G. I 27:, ne donne pas d'étymologie $\left.{ }^{1}\right)$. Bormans, Glosscire des drupiers (BSW 9, p. 260) cite des textes de 1589, 163\%, 1659. etc., oì le mot est écrit hansliotte. Enfin Hécart note le rouchi anscote (étolfe grossièrc en laine). - La forme liégeoise est assurément la plus pure : elle atteste que le primitif doit avoir à l'initiale une aspirée germanique. laquelle disparait normalement en français et en rouchi. Dès lors. je crois qu il faut remonter au flamand Hondschoote, nom diune petite ville du département du Nord, située sur la frontière non loin de Dunkerquc. Cette ville fut très florissante au xrie siècle ct comptait des filatures renommées."

Des recherches nourelles me permettent de reprendre aujourd'hui le problème, avec la conviction que l'histoire confirnc pleinenent les suggestions de la phonétique.

Pour les formes anciemnes que cite le Dictiomatre général, je dois à l'obligeante érudition de M. Antoine Thomas lindication des sources suivantes: "Serge d'Escosse demy-entroite... serge de seigneur et d'Ascot. L'Isle. Cipre, Angleterre ct autres païs estrangers " (tarif de 166\%. cité par Littré. ${ }^{\circ}$ escot. qui propose dubitatirement d’interpréter par "éeossais"); - "Serues d'Imiens facon d"Arscot... Serges appellees d'Ypres et d'Arsot..." : reglement de 1669. articles xiI et xiII ; cité par Sarary des Bruslous (2); - le Tarif de la Douane de Lyon (sans date, cité par Savary. II. 1533) mentionne "les sarges d'Ascot Françoises" ; - Savary. I 97. dit en 1723 que cette serge se fabrique notamment à Bruges ct à Aseot dans les Pays-Bas Espagnols. Le Dictionnaire de Tréroux (17\%1) le copie. mais imprime Arscot.

'Telle est la source de lopinion qui roit dans le fr. anacoste le nom d'Aerschot, petite ville au N.-E, de Louvain. Elle implique que cette localité aurait eu, dans les dernicrs sic̀cles, une industrie textile des

(1) Plus loin (H, 606), G. prétend que Lobet a une forme amascote, sans faire attention que Lobet, p. 236, vo lansliott, ne donne ce mot que comme trarluction française. Martin Lejeune, Voc, de lappriteur en draps du pays de I'criers (BSW 40, p. 431) insère bravement anascote, d'après Lobet, comme étant un terme verviétois ! En revanehe, il n'a pas d'article hanscotc!

$\left({ }^{2}\right)$ Dictionnaire du Commerce (172:3), II, 1525-6. 
plus considérables. Or aueun indice historique n’autorise cette présomption $\left({ }^{1}\right)$. En revanche. de môme que Lille. Bruges et Ypres dont les noms figurent dam les textes quon vient de lire. Hondschoote fut, anx $x^{2} I^{e}$ et $x^{*} I^{e}$ siecles, un centre manufacturier de premier ordre.

La draperie llamande avait dû surtout. au moyen âge. son étonnante vitalité à la confection cles drapo de luxe : mais la concurrence anglaise et la rancté croissante de la laine insulaire poussèrent lïindustrie rurale. opprimée par les eorporations urbaines, à chereher fortune dans la fabrication des tissus légers et à bon marché. A partir de la fin du xre siècle, les laines d'Espagne alluent en Flandre. Limion politique des Pays-Bas et de l'bspagne à partir du règne de Philippe-le-Bean (1+78-1506) en augmenta sensiblement l'exportation. Noins soyeuse que la laine anglaise. la laine espagnole ne pourait rivaliser avec elle dans la draperie tine: mais dle convenait parfaitenent pour les tissus légers. tels que les serges et les ontades $\left({ }^{2}\right)$. Si les draps que les artisans des villes sobstinent a fabriquer no trourent phus d'acheteurs, les serges et les ostades de Beronues at d'IIondschoote. les draph légers d'Amentières figurent, a partir des premières annéen du xve siècle parmi les principaux artieles d'exporta:ion des Pays-Bas. Cependant les troubles politipues et religicux porterent it cen villen un comp fatal. Les ourriers émigrèrent en masse rers l'Angleterre. Dès 158\%. ils proposaient à Elisabeth d"introduire dans son royaume la fabrivation des serges et des

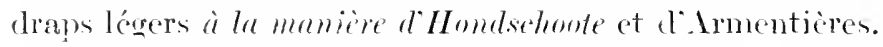

Ces détails historiques sont extraits de la remarquable étude de I Ienri Pirenne sur C'ne crise industrielle au xvI siècle $\left({ }^{3}\right)$. D'autre part, las arehives eommunales d'Hondsehoote eontiennent des doruments qui nous intéressent partiunliement $\left({ }^{4}\right)$. Nous y royons qu'en 1609 et de $16+6 ;$ à 1664 il y eut maints procés entre Bruges et Hondsehoote concermant la fabrieation des sayes. Des ouvriers émigrés de cette

(1) "A mia comnaissance, il n'y a pas eu de fabrication de serges à Aersehot" (Conmmication de M. Henri Pirenne, professeur à l'Université de Gand). - « Je n'a i découvert aucun indice de l'industrie des serges à Aerschot en Brabant ". (Communic“ation de M. H. Vander Linden, professeur à l'Université de Liège).

$\left.{ }^{2}{ }^{2}\right)$ Ostade vient de W'orsted, uom d'un gros bourg du eomté de Norfolk (A. Thomas, Nomitume esstis de phil. fr., p. 311). Voyez aussi Behrens, p. 2zog, sur l'ane. w. et fr. zicastarde.

(") Bull. de l'Aead. roygale de Belgique (Lettres), 1905, p. 489 et suiv.

(4) Département dn Nort. Ville dIIoudschoote. Incentaire sommaire des arehices communules amtéricures ì 1790 (Lille, $187(j)$, HH 18 et 19. - Je dois cette indication bibliographique à I. Antoine Thomas, qui ajoute: "Dès 1897, M. Morel-Fatio a attiré mon attention sur l'espangnol anaseote et son rapport probable avee la ville d'Iondschoote ". 
dernière ville avaient importé à Bruges cette fabrication, dont Hondschoote prétendait garder scule le privilege qui lui avait été accordé par lettres de Louis de Male en 1:373. Hondschoote protestait contre l'emploi de l'estampille portant les mots Fabrica Axascotes DE BRLG.t, empreinte qui signifie "fabrique de serges d'IIondschoote faites à Bruges ", et qui servait à marquer les étoffes expédiées en Espagne.

Il est temps de conclure. Le mot espagnol anascote na pas de racine dans cette langue ; les étymologistes le disent emprunté du fuancais. En réalité, e’est le nom même de la ville d'IIondschoote $\left(^{1}\right)$ : *an'scote a donné amascote par insertion de a. puis anacoste par métathèse. Qu on se rappelle le rouchi anscote et le liégeois hanscote qui. lui. doit nomalement garder l'aspirée germanique, et l'on jugera qu'il laut écarter du débat le nom de la ville d'Aerschot, les formen du xrine siècle (ascot. escot, arscot) étant manilestement de mauraises lectures on prononciations de anscote.

\section{chestr. anêvè, dusnêvè ; gaum, anâvèy, dènâvèy}

Dasnoy (p). 22. 172) et M. Liégeois (BSW 3\%. pp. 29+. 323) signalent ces mots à Neufchâteau et à Tintigny" ; de même Cl. Maus dans son vocabulaire des cuvirons de Virton (manuscrit. 1850). Voici. d'apres des enquêtes persomnciles, de quoj eompléter leurs domnées sommaires :

anêvè (Neufchàteau, Recogne), v. tr... engendrer, produire ou introduire (des êtres, plantes ou choses nuisibles) : ine pikêtre du gurzuliè [groseillier] ancite lu panaris ; lès nichitès $d$ la môjon [saletés de la maison] anếzant lès puees; lès prommes [ehiendents] s'ant ancivè par tont l tehamp.| De même le gaumais

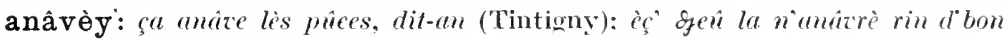
(Buzenol) ; v'èy [vous avez] anâtèy in tâs dè p'tites biètes, i n' fôt-m' lès layi s'anâzèy (Musson) "il ne faut pas les laisser se multiplier "; lès môtêses-yérbes s'anâvant da note mèje (Virton) "dans notre jarilin"."

dusnêvè (Neufchâteau, Recogne), dènâvèy (gaumais), v. tr., 1. détruire, extirper (une race nuisible, de mauvaises herbes) : dju $n^{\prime}$ su m’ foutu d' dènâtè lès pavines [ehiendents] du note tchamp (Buzenol); - 2. expulser : ljè lans dènâtè dè $d$ ' tchû nons (Musson) "nous lavons expulsé de chez nous "; - 3. faire disparaitre, escamoter : tu m'ès bintot ể dènâvé m' batan ! (Proury-Jamoigne : BSW 49, p. 150); - 4. v. réfl., se débarrasser (d'une chose nuisible, d'un importun) ; - 5. (réfl. ?) " émigrer, rassembler ce qu'on a et quitter un lieu, un pays " (Cl. Maus) $\left({ }^{2}\right)$.

(1) Comparez ostude à la page précédente et voyez ci-après l'article spini

${ }^{(2)}$ Varlet, Diet. du patois meusien, signale à Chattancourt se dénavi "se défaire, se dépouiller : $i n^{\prime}$ veut-m' se dénazi de s' bin. Etṛm. du lat. dehabere, avoir de moins, manquer $n(!)$. 
Si l'on détache les préfixes dí-, dus- (fr. dé-. lat. de-ex-) et a- (qui, dans cette région. représente le fr. en-, lat. in -. aussi bien que le fr. $a-$, lat. ad-). il reste un radical nêv-, nâv- ${ }^{1}$, où nous reconnaîtrons l’anc. fr. naif, naïf, lat, natirum.

Les formen *ematier. *desuaiver ne sont. je pense. signalées nulle part : Körting et Meyer-Lübke nöndiquent aneum dérivé verbal de nativus $(2)$ : co type latin a cependant. comme on le voit, provigné daus la région de Neufichâtean-Virton-Verdum.

\section{w. anô, ènåhe, ènèye, anspindje}

G.. I 19\%. donne sans explication : "enahe : bourre de chanrre ou de lin ". d'après Remacle. oe éd.. lequel ajoute que le mot ne se dit plus guère. Il l'écrit d’ailleurs inexactement. J'ai entendu dire : dès-ènåhes à Erezée ( $X$. de la prov. de Lux.), dès-anoyyes à Nourillers-Rceogne $\hat{\imath}^{3}$ ), dis-ènèyes à Compogne (S.-Wr de Houffalize). Plus an Sud. à la lisière du pays gaumais (Chiny). on dit. d'après une communication éerite, hunoche; mais h initial parait suspect. En namurois, nous relevons anô " tcille: écorec du ehanrre ou du lin. débris provenảnt du teillage " $\left(^{4}\right)$; en rouehi : ana. anô (Hécart). ana ì Luingue-lez-Mouscron.

Dans tous ces nots. nou reconnais: ons sans peine l’all. ahne "fétu de lin on de chanve ", moyen h. all. ane, aupuel se sont adaptés les suffixes -éye (lat. -ĭlia). -ihle. -ôje (lat. -ätia) : la finale de anôye. anô, anấ ent plus obscure et résulte sans doute d'influenees analogiques.

A Vielsalm, les débris de chèvenotte sappellent dés-anspintches (BD 1906, 1), :34). Ce terme curieux se compose apparemment de ân(e)

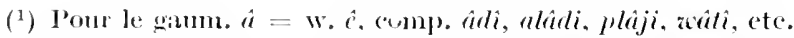

(2) Godlefroy dite l'ane. fr. mifzer (1660) "représenter naturellement", - La tonique $i$ de mif, devenant atone dans moiver, disparait néeessairement; eomjarez le romchi vïle (aicle, s. f.) a coté de aider.

$\left({ }^{3}\right)$ Région de Neuf(hâteam. Là graplie de Dasnoy, p. 74 : "anaie, chènevotte" est ingivougue.

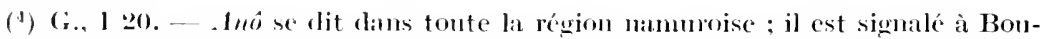
vigne-1)inant, Leignen. Menx, Thorembais-st-Trond, Ste-Harie-Gest, Chastre-

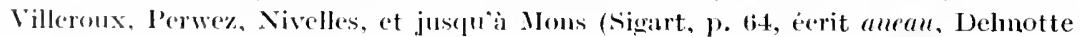

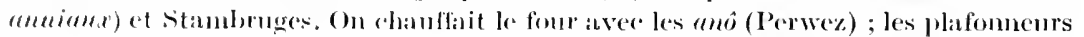

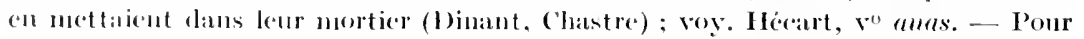

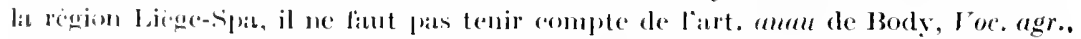

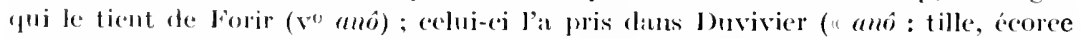

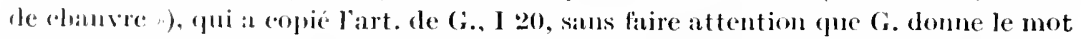
comme namurois : - A joutons que le milm. dit ariesses (à Ovifat : ériesses) et le frumain : arétles (a Ste-Mlarie-sur-semois), cest-i-dire "irêtes". 
et de spindie $\left({ }^{1}\right)$; toutcfois le procédé de composition a quelque chose d'insolite.

\section{liég. apotiker}

Le liég. apotilier signifie "classer. arranger. ageneer, ajuster. composer "; il a un petit air dironie familicre qui rappelle le fr. afistoler. Exemples : so 'ne munute. i r's-apotiliêye énula al'îdêye; i fat dè timps po-z-apotilier on diesionête; zosse rupote èst ma upotiliêye. - G.. II tos, le cite sans explication. On serait tenté d'y roir une création burlesclue, un terme d'argot. issu de potilict petit pot, ane. fr. potequin. du flam. pottelen). ou de apoticare (not de formation savante comme le fr. apothicaire). sous prétexte que les-apoticares ais-apotiliet des drougues divins des potiliets. Je proposerai pomrtant autre chose. A mon sens, e'est un composé du préfixe a et de botilie (bontique. lat. apot heca), dont l'aneicune forme poticlie se lit dans un texte liégeois du xre siècle $\left({ }^{2}\right)$. Au reste, apotilie'r peut provenir d'un primitif *abotilier, dont le $b$ s'est changé en $p$ sous l'influence de potilict. apoticare. Ce serait littéralement * aboutiquer. c.-ì-cl. "disposer en forme de boutique on pour la boutique. arranges. parer des objects pour les ctaler cu rente", doù en général : "ajuster soigneusement, aroe un gô̂t minutieux ». Pour la composition, comparez afagoter (Sery-Abée), afuh ner (Malmedy) "fagoter, accoutres": - apotchter on tiô d' somde ou ine mìne. t. de houillerie à Scraing. " disposer en forme de poehette un trou de sonde ou de mine. e.-i-d. commencer le tron de façon que l'outil ait prise et ne glisse pas " : - ane. fr. apotugié "arrangé eomme un potage ", ouestwallon et rouchi apotudị "arrangé ». toujours ironiquement.

w ârih, âr'hon, etc. (Malmeḍ̀)

M. l'abbé Bastin a reeneilli à Faymonville-Weismos (lez-Malnedy) l'adjeetif arih "grare, important. syn. de griyeks ". et l'adrerbe arihmint "gravement : il i-st-arihmint malade", oì il voit avec raison l'all. arg. Pour la forme wallonne, il suffit de comparer le malm. hìvếrith. emprunté de l'all. herberge (dial. d'Eupen : härbereg. auberge); Kahèbrih : Kalterherberg, village près de Montjoie; Limbourih :

( ${ }^{1}$ Le spindje est le battoir ou la dague dont on se sert pour frapper la filasse et la débarrasser des fragments de tige restés arhérents.

$\left(^{2}\right)$ "(Cn) hallier avoit empacke draps non sailleis en son stal et poticke " (1540: BSW 9, 255). - Le dialeete allemand il'Eupen a encore la forme pottick " hontique" (Wört. der Eupener Sprache, Eupen, 1899). 
Limbourg ( $\left.{ }^{1}\right)$. Quant au sens du mot wallon, on le retrouve dans les patois de l'Eifel ${ }^{2}$ ) et dans l'allemand moderne. où l'idée de "méehant, vilain" a produit eelle de "exeessif".

Il faut sams doute rattacher a eet ârih un mot verviétois qui ne se reneontre que dans une comédie inédite de 1759. Un père, apprenant que sa fille reut se marier, se lamente en ees termes:

Lu coûr nu d'hirive one saqwè,

Su n’ polèt-dj” même pinser poqwè

Quiile duvonéve ossi arrihce [lire ârihéye]

Qu'one vatche qui a stu må passéye $\left({ }^{3}\right)$.

Le sens est évidemment "méchante. diffieile. quinteuse, enragée". On peut admettre. d'après ce texte, qu'au xvmIe sièele un verbe Ariker ou - $i$ existait. dans la région de Verviers-Malmedy, au sens de l'all. ärgern (fâehes. contrarier, dépiter).

Il faut encore faire rentrer dans la même famille deux mots malmédiens restés jusqu'ici sans explieation : Ar'hon. s. m., "tâtillon, méticuleux. ladre, grippc-sou * (arehaique à Stavelot et à Malmedy : Villers, 1793) : Arkinc. s. m.. -ée, f'.. "avare" (Malmedy : Villers et Scius; d'après Body. Toe. des poissurdes, on dit surtout laide âr'hinée "vilaine solciere "). - Irhiné est dérivé de ârhon sur le type phonétique de bondon: burdiner. lumiẹn : lum'ciner : ar'hon lui-même dérive de orile i l'aide du sullixe -on. Ces termes mainédiens doivent être d'un age respectable, ear ce n'est qu'en aneien et en moyen haut allemand que arg signifie "avare" $\left(^{(1)}\right.$.

[Revne de Dialectologie romane, 1910, t. II, p. 375.]

(1) Voy. aussi les articles bêrih, mèu sik, skèrbalik. Cf. Küurth, Frontière linguistique, 1. 476, 11 .

$\left({ }^{2}\right)$ ('f. Heckimer, Die Eifel in ihrer Hundert (I'rïm, 1890), p. 17 : " arg, sehr : et deiht arg aih, es schmerzt selu" " - In west-flamand, arrig "âpre, rude, aigre ", se dit du temps ou du vent (1)e Bo).

${ }^{(3)}$ "Le cour me déchirait un je ne sais quoi (expression énergigue = j'avais le presncutiment d'un malheur); si ne pouvalis-je même penser ponrquoi (qu')elle deronait aussi dillieile qu'une valche qui a été mal passée ( = qui a mal passé l'hiver, (qui n'est pas en bon etat au printemus) ". J'ai rajeuni l'orthographe du texte cité, samf your arrikée. la pièce a pour titre : Le mayeur rniné par sa eharge ou Simon rescrini.

( $\left.{ }^{4}\right)$ Lane. J. all. are, arch, arg, arig = böse ; geizig, karg. 1)après Heyne, Deulsehes Wört., urg a chcore au xwe sichle le seus de "geizig ", mais plus souvent celni de "schlecht, nichts-wïrdig". - Voy. aussi Meyer-Lübke, n 591. 
liég. arinne, ane. liég. eraine ("araine, areine»)

Par ee terme très ancien et très important de l'exploitation houillère, on désigne, au pays de Lic̀ge. "une galerie d'écoulement ayant son orifiee (appelé "o.il ") au flane d'une eolline ou dans le fond d'une vallée, et pratiquée pour asséeher les travaux des mines ". C"est à peu près la définition de Littré. qui aceucille dans son supplément notre not liégeois.

Deux étymologies ont été proposées. Pour Morand (1), e'est peutètre une via arenata ou ex arena facta "une voie faite à ciment et à pierre"; mais le latin arenu ("sable ") n'a jamais en de vie dans notre région $\left(^{2}\right)$ et cette fantaisie n’a guère trouré d’écho. La proposition de G.. I 25, qui tire arêne de l'all. rime. rimen (rignole. conler) est d'allure plus sérieuse; aussi divers auteurs l'ont reproduite sans objection, notamment Bormans. Littré. Scheler et même Diez $\left({ }^{3}\right)$. Par malheur cette hypothène n'explique pas correctement l"initiale de eraine, arame $\left.{ }^{4}\right)$ : il faut done tenter une autre analyse.

C"est en 1278 quion relève eraime, ereme pour la première fois dans les arehives liégeoises $\left(^{5}\right)$. Iu xive siècle. ce type aree $e$ initial, eraine, erene, eraisne, erraine. heraine. herraine est de beaucoup le plus commun; haraine apparait dè̀s $131+\left({ }^{6}\right)$. ct l'a initial, rare au début. se généralise dans les sièes suivants. Le règlement de houillerie que Jean de Stavelot insère dans sa Chronique (pp.227-2333), conticnt 19 fois heraine et une seule fois haaraine (dans la marge). On en déduira que èraine est primitif; le changement de èr-en ar-à la protonique initiale ne manque d'ailleurs pas d'exemples $\left({ }^{7}\right)$.

(1) Art dexploiter les mines, 2"éd., Neuchatel, 1780.

${ }^{(2)}$ Les auteurs liégeois qui, dans les derniers siècles, ont écrit en latin sur eette matière désignent les araines par le mot arenae, mais ce fait est évidemment sans valeur.

${ }^{(3)}$ Bormans, Foe. des honilleurs; Littré, Suppl., Arense; Scheler, Gloss. de la Geste de Liege, eraine; I)iez, p. iro.

(4) Pour Scheler, il s'agit d'une simple prosthèse. Pour l'érudit archiviste liégeois Th. Gobert (Eaux et fontaines à Liège, p. 36), "c'est le préfixe e marquant idée d'extraction "; autant dire que le mot scrait de formation savante ou française.

$\left.{ }^{5}{ }^{5}\right)$ Henaux, La houillerie du pays de Liege, 2 e cd., 1861, p. 112.

$\left({ }^{6}\right)$ Gobert, l. e., p. 369. - L'initiale $h$ est purement ornementale, eomme il est arrivé fréquemment en français dès le xure siècle ; ff. Brunot, Ilist. de la langue fr., I, 498. - La graphie areine, qui parait être offieielle aujourd'hui, est relativement réeente; il faudrait en revenir à la désinence première -aine, qui, d'après moi, est étymologicue.

(7) Voyez ci-après l'article tèrouile. - Notre mot a formé le dérivé ernier, urnier, arenier, eonstructeur ou propriétaire de l'uraine. On trouve aussi le diminutif Ilerenalle en 1342 (Gobert, l. c., p. 249). 
Leetymologie par laall. rinne "rigole " rentre, à mes yeux, dans la catégoric de eelles qui sont dues à cette "obsession sémantique " dont je parle it l'artiele beur. De ee que l'araine. depuis que les textes en font mention. servit surtout à démerger les mines noyées et à doter Liège d'eau alimentaire. on a cru que ce roeable devait comporter l“idée d'éeoulement. Rien de moins certain eependant. si l'on se reporte à la manière dont furent entrepris les premiers travaux d'exploitation houillère.

I l'origine. on dut fouiller le sol aux endroits où la reine aflleurait. Sur les collines, on epérait par tranchées à ciel ouvert, en remuant le sol en tous points: quand la eonehe plongeait vers la profondeur, on établissait une galerie qui descendait suivant la pente; mais les éboulements et surtout lafllux des eaux entravèrent bientôt les travaux. D’autre part - et sans donte simultanément - sur le flane. des collines. on exploitait aussi les alllemrements; on y pratiquait des "baumes". des "dilatements" étendus et. en suivant la reine, des "roies de nivean "auxquelles on donnait mo pente ascensionnelle très faible, permettant l'élimination des eaux de suintement; ces voies finirent par reneontrer les premiers travans entrepsis au sommet des collines ct amenèrent dans la vallée les eaux qui les noyaient. Telle est l'origine des araines $\left({ }^{1}\right)$. Or. je pense que. dès l'origine. c'est-à-dire arant même que l'on eût. par co moyen fortuit. démergé les premiers travanx inondés, araine a désigné la voie ou galerie de nivean. Dans la suite. cette particularité si importante aura primé aux yeux de tous. Lorsque plus tard. éclairé par lexpérience. on pratiqua dans la roche des galeries qui deraient miquement servir à l'asséehement des travanx miniers. on réserva à ces galeries le nom d'araines; puis. le nom a pu même passer, par analogie. aux conduites d'eau qui. d'après Henaux (p. H). alimentaient les fontaines publiques de Liège bien longtemps arant le $\mathrm{x}^{\mathrm{e}}$ siecle.

Cela étant. je corois que eraine dérive de l'ane. fre erre "chemin, route, roie ". à l'aide dn sullixe -aine. qui a fait dans les patois, où l'on prononce d'ordinaire-ainne. une fortume plus eonsidérable qu'en français $\left.{ }^{2}\right)$.

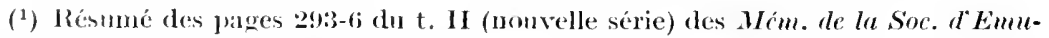
lation, contenant l'Mistorique de lexploitation de la houille dans le pays de Liège, patr lR. Mallaribe. Voyez aussi Gobert, l. c., p. 35.

(2) Le fre erre viont du latin iter "chemin") voy. le Dict. géll., ainsi que God. qui cite les formes ere, herre, "rre, harre. De lì, en ancien frauçais, errer "cheminer", chermin erreme grand chenin ". - Pour limportance du suff. -aine daus les dialectes, il sulfit de citer le: wo rolimne (Cincy) "ornieren", lès crechimes (Alle-sur-Semois) 
L'expression "une (voie) èraine» aurait signifié primitivement " une voie par où on ra (de niveau) ", une galerie horizontale ou de pente aseensionnelle très faible.

Il me reste à signaler l'emploi que Jean d'Outremeuse a fait de notre mot dans sa Geste de Liege : eraine figure dans neuf passages qu'on trouvera reproduits dans le Glossaire de Scheler; il est chaque fois à la rime et presque partout an sens métaphorique de "souree, origine ". L'ćditeur Bormans lidentifie aree orine (origine), mais Scheler y roit aree raison le w. arêne "canal d'écoulement". Pour la question d'étymologie que j'ai tâehé de résondre, ees textes me paraissent négligeables: depuis longtemps, au xive siècle. le sens premier avait fait place à l'aceeption spéeiale que le mot possède eneore aujourd'hui.

Ajoutons enfin que le liégeois arime a passé dans certaines régions roisines : il signifie "canal d'égoût " à Vielsalm ; "aquedue soutcrain " à Lesves-lez-Namur ; "drain dans un champ " à Solières ct à Hotton, de même à Melreux où l'on dit èrêne. Il est plus intéressant de constater qu'au charbonnage de Gives-lez-Andenne, outre le scns de "grande voie qui commence au jour ", arinne a le sens général de "galerie de mine $)$.

\section{liég. atîleûre}

G., II x. eite sans explication l'expression archaïque rimète in atîlếre "remettre en ordre, $\epsilon \mathrm{n}$ bon état ". Le subst. atîlểre est altéré. par dissimilation, de atîreûre; à Malmedy : atîrore "apprêt. assortiment; parure, accoutrement $\Rightarrow$ (Villers, 1793). Le verbe atîrer, qui existe eneore à Bra, ì Stavelot et aux environs de Malnedy, signifie. d'après Villers : "apprêter, assortir. parer, orner, accoutrer ". L'anc. fr. atirer a la même signification; il se rattache à tire, s. f.. "ordre, rang ; suite, file, rangée ; sorte, espèce, provenance " (Godefroy). en wallon tîre "espèce. sorte, race", qui vient du moyen bas all. tîre : sorte, manière, qualité ( $\left.{ }^{1}\right)$.

[Z. fïr franz. Spr. und Litt., 1909, t. xxxw, p. 155.]

" adénites de la croissance ", rucièrsimne (Stavelot) "versant d'ume colline ", doguimne (Verviers) "choc, heurt"; une gotterimme apparence d'awes (en 1556; Bormans, Voc. houill., côper) "une apparence de gouttes d'eau qui tombent "; al râyime dès crompîres (Wardin) "à l'arrachage des pommes de terre"; le picard couvraine " temps des semailles "; en Ard. franç., le mouzonnais fauchaime "fauchaison ", versainne " jachère ", etc.

(1) Cf. G., II 432 ; Falk-Torp, sir. 


\section{rouchi avèrlu}

Lierlu "résolu. guilleret. sćmillant. vif, alerte, turbulent, étourdi, ete. "ent très nsité dans le Hainaut. depuis Couvin jusqu'à Braine-leComte. Le Glossuire itymologique montois de Sigart ne trouve à comparer que laneien français arerlant "lourd, grossier ". rapprochement bien malheureux. ear un lourdaud est tout le contraire d'un averlu. Pour ma part. jy rois une altération de l'ancien trançais reveleus "disposé à sc rebeller" (anc. frane. rezeler = latin rebcll are): d'oì : "impétneux, vif. alurte. fringant". Le pieard a conservé eveleux. Notre acèrlu suppose une forme antéricure *arcèlu : le préfixe re-devient ar-en montois (areéroir. recevoir, atécer. relcver); la métathèse de $r$ est un phénomène des plus fréquents. surtout en contact avec une autre liquide : enfiu. il y a changement de suffixe (-u au lieu de-eus; eomparez liég. pinêts = nam. pinu. penaud. triste).

\section{liég. aw'hê, nam. aw'jale}

G.. I 36, note sans explication le liég. azelaai "fretin. alevin". qui sc dit ausuj de la jeune anguille tres mince ou de la petite lamproie. On yecommait sans peine un type acuecllum (diminntif de aeus "aiguille"). applicfué par métaphore aux petits poissons ténus eomme unc aiguille. puis. par extcmion. à la petite anguille $\left({ }^{1}\right)$. Le même nom, à Glons-sur-Gecer. désigne une plante. l'Aegopodimm podagraria L., égopole ou herbe aux guntenx. qui croit dans les lieux humides. -

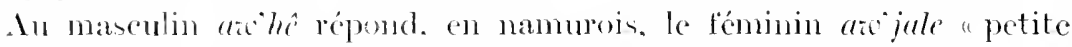
anguille " $\left({ }^{2}\right)$. qui présente toute une série de formes curieuses, propres ¿ montrer eomment les mots saltèrent dans lo langage du peuple : 1. ackaljule $\left({ }^{3}\right) .0$ i linfluenee du sullixe a amené l'insertion d'une syllabe parasite dams le eorps du mot:-- 2. avodiale. avee épaississement de $j$

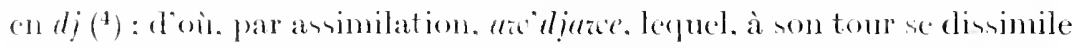

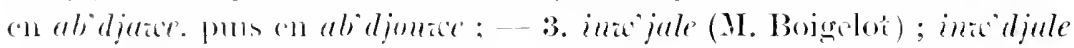

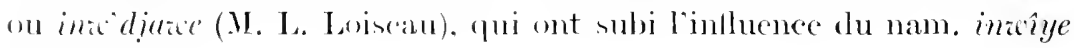
allgulle. $n$.

(1) A Vise, elle sappelle cowète (proprement "petite queue, cordonnet :).

(2) B1) 1910, p. 9. - I'n lieu dit de Wavre, la ferme de Lanzelle, se dit en w.

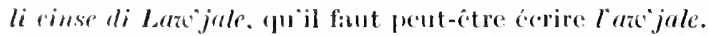

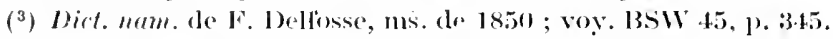

(4) comp. qui drljoz? (ciney) "que dites-vons ?" it côté du nam. qui drjoz?

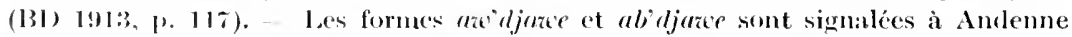
(131) 1909, p. 299); alidjourcer 'st daus le feuilleton de Li Ban-Cloque, no du + juin 1911 . 


\section{liég. åyehê}

Ce mot ne subsiste que comme nom de lieu aux environs de Liège. à Jupille notamment et à IIerstal, où il désigne une place publique, un terain communal, me aisanee : ecst en somme un synonyme de

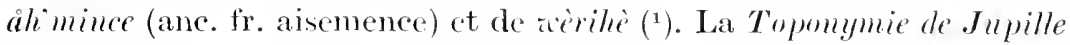
lui consacre un copicux article, plein de eitations d'archives (BSW 49. p. 226). On y relève : voye de Laiychea (1452), en Leyheal (1492), en Layhay (1498). Le mot s'est francisé en Laixhean, devenu aussi nom de famille. Bien que, dans tous les textes, l'article défini se soit soudé au substantif, il faut analyser l'ayehế. comme Lille (= l'île), la rue Lulay (=l'îlê. l'îlot) à Liège, ste.

La désinence est celle des diminutifs ôhhê (*aucellum: oiseau), wahê (vascellum : vaisseau). Elle est ici précédée d'un e muet ou, plus exactement, d'une protonique féminine, comme dans pan hê (*paniecllum, petit pain), caenhê (*cornicellum, bout de corne servant d'éteignoir ou de cornet is houdin), cothê (*oorticellum, petit contil), damhê (dominicellum: damoiseau), acthê (*acucellum), roy. cet article. Mais oì trourer le radical de ayelté?

Il y a quelques amnées (BI) 1910, p. 34), j’ai proposé le latin $\mathrm{a}(\mathrm{d}) \mathrm{jutum}$ "aide " : des textes parisiens du xII e sicele cités par Du Cange, portent en effet adjotum. ajoudum, au sens de terrain, terre. On pouvait rependant objecter qu'il n'y a pas trace, chez nous, d'un * ayou roman, employé conme substantif nuasculin au sons de "terrain d'aisance " $\left({ }^{2}\right)$. D'autre part. le latin adjacens "annexe" a domné le fr. aise, liég.ahe. de même que le phruel adjacentia a donné le fr. aisanee. liég. *ahinee, devenu ahèce sous l"iniluence du verbe ahècî (accommoder). J'estime à présent plus plausible l'hypothèse d'un diminutif qui se rattacherait à cette dernicre famille. Le trpe schématique serait done *adjace(ns) + ecllum, réduit en *ace-cellum. Comparez făy’hê (l. d. de Iormont lez krezée), diminutif de făyi (fagetum : hêtraie). comme le montre la forme făyîjé (l. d. de Moginont lez Ucimont).

\section{liég. bå}

On ne doit pas confondre, en liégeois, ba arec bi (voy. ect article); de plus, sous chacun de ces chefs, il faut distinguer deux mots différents.

(') On pourrait aussi reconnaitre notre $l$ 'a yehê dans le nam. laujø " endroit dans un bois où il y a de l'herbe ", qui ne m'est connu que par G., II 16.

$\left({ }^{2}\right)$ L'ancien wallon n'a que le féminin aiozve, aiouze (aide, action d'aider). 
1. A Visé. tout près de la frontière germanique. j'ai entendu l'adjeetif bå, fém. bade "hardi, eflronté " : bảde guêuye!, et, comme substantif : $\hat{a} ! l$ l ba ! "ah ! le brutal ! ". - C'est éridemment une surviranee de lane. fr. bull, baut "joyeux, hardi, fier, présomptueux ", qui vient du franeique bald "hardi. gai ".

2. Dautre part, le germ. balk - d'ou le fr. et ronehi bau "poutre "est devenu normalement en liégeois bi. que nous relevons dans le hesb. hin ba (= liég. hin'rà ; roy. ei-dessus l'art. am'bô). et dans int'bå (Liège. Verviors). t. de elarp.p. "entrait". littéralenent "entre-bau" $\left.{ }^{1}\right)$. Il faut $y^{r}$ ajouter une expression archaüque. inexpliquée jusqu'ici, qui se rencontre dans la Complainte de 1631 :

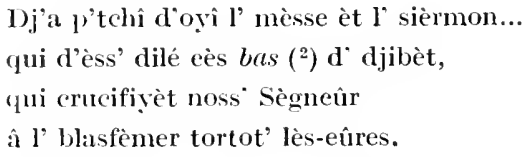

Lisez bâ (ou boi) d' diibit "poutre transversale de gibet", expression figuréc dont le peuple faisait jadis une injure analogue à potince, rocie ${ }^{3}$ ), c'est-à-dire "gibier de potence. pendard". Un mien ami, né vers 1870 à Milmort près de Liège, se rappelle que. dans son enfanee, sa grandmère le traitait parfois de bå-djubèt. J'y rois une altération de $b a ̊$ d' diubèt. par négligenee de prononeiation et. peut-être aussi, par fause étrmologie (influenee de ladjeetif $b a$ ).

\section{liég. bak'neûre}

Le Supplément de Littsé aceueille basmure ou baquenure, t. de mine, syn. bowzenn. Ce mot c st emprunté du liég. balineùre. syn. frintehe ("tranche") : e'est ainsi que nos houilleurs appellent une galerie menéc horizontalement ì travers banes de roche pour atteindre la

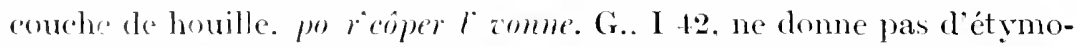
logic. Bormans. Voe. des hmilleurs liégeois. y roit un dérivé de bane (!). Il signale le yn. balin'mint (inusité) : mais il oublie le verbe bak'ner, qui est d'usage courant au bassin de Liège conme synonyme de trintebî ( trancher : pratiquer une baenurc). - On ne peut séparer

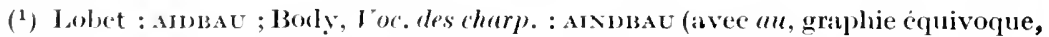
pour à).

(2) ('hoir, p. ro; orthographe de's éditeurs, qui ne paraissent pas avoir compris le mot que nous soulignons.

$\left({ }^{3}\right)$ Potenee, roue ", instruments de supplice. Ces mots féminins deviemnent mascoulins dauss a' seus liguré : on lêt (haid) potinee, on lè rozee. - Comp. lat. crux (esclate qui merite lat croix). 
bak'ver du néerl. bakenen "baliser. jalomner " (1) : dans le creusement d'une bak'neive. les ouvriers doivent fréquemment jalonner la galerie pour s'assurer qu'ils tiemnent la direetion roulue. Cette aetion particulière a domé son nom à l’ensemble des opérations.

\section{liég. bår, nam. baur, bôr}

Le Dict. liégeois de Forir tome laconiquement : "bô, hangar, abri "; mais il emprunte distraitement ee not à G.. I 49, qui l'attribue au dialecte namurois. Il faut rayer l'article de Forir et le remplacer par le liég. bair, s. m., que jai entendu à Liers au sens de : "ensemble des traverses qui supportent les gerbes au-dessus de l'aire " (roy. l'art. bìôdî). La forme namuroise est baur ou bôr, s. m., "chartil. hangar, remise pour chariots et instruments aratoires. abri léger formé d'un toit que supportent des piquets ou des piliers en maȩonnerie et qui s'adosse à un côté de la ferme " (Namur. Ciney. Dinant. ThorembaisSt-Trond). A Gives et à Solières, près d'Andemne, j'ai entendu : it í-st-è bâr. syn. è laangâr. Enfin, nu registre manuscrit des assemblées des manants et policiens de Sprimont porte ee qui suit pour l'an 1651: "faire nue espèee de bar ou chary afin d'y réfugier ses hernats... *.

Les formes bâr, bar prouvent que le nam. bôr n'a rien à démêler avec l'ane. h. all. bùr "demeure", ni aree l'all, bohren "percer" $\left({ }^{2}\right)$. Pour la lettre et pour le sens, le rapprochement s impose arec le liég. bare, nam. baure, bôre. s. f.. qui répond au fr. barte. d'un tỵe *barra (perche), d'origine inconnue. Un type *barrum (assemblage de perches, de poutres) expliquerait le maseulin bâr, bar, baur $\left({ }^{3}\right)$. A remarquer d'ailleurs qu'à l'ouest de Liège (Seraing. Iuy). on dit an masculin on bâr di fièr "une barre de fer" et, au jeu de barres : quiter. toutch on bîr.

\section{liég. bègå, bigå ; malnı. digâ}

G., I 51 et 54 , n'explique pas le liég. bègâ "fange. bourbe ". nam. bigan "vase, limon; jus de fumier». La forme bigi existe aussi à

(1) Dérivé du néerl. baken " balise, bouée ", d'où le w. bakìne, t. de bat., même sens (G., II 500). L'acception primitive de baken cst "signe " en général ; cf. Kluge BAKE; Franck-van Wyk BAAK; Schuermans, De Bo BAKE, BAAK.

$\left(^{2}\right)$ Voy. G.. I 49 ; Feller, Notes, p. 318. Comp. ci-après lart. beûr.

( $\left.{ }^{3}\right)$ D'après Meyer-Lübke, no 963 , l'anc. fr. bar "abattis d'arbres, château-fort ", qui survit dans de nombreux noms de licux, est probablement connexe avec * ba r ra. - Pour le rapport entre *barra et *barrum, comp. lat. vallus : pieu; vallum: palissade. 
Liège (Forir). à Jupille. Verviers. Sprimont, ete. $\left.{ }^{1}\right)$. Ailleurs, on dit bègau (Ciney). bigâ (Huy, Vielsalm). bigau (Awenne, Namur, Dinant), bigan on bigâr (Charleroi). bugau (Warre), begău (Jodoigne, Perwez, Chastre-Villeroux) et. avec $r$ épenthétique, brigau (Lavacherie. Orthenville). On voit que le mot n'appartient qu'an dialecte wallon proprement dit. Dan les villes, telles que Liège et Verviers. on lui attribue le sens général de "fange. bourbe.margonillis"; une faque d'eau répandue par mégarde sappelle om biga d'ête $\left.{ }^{2}\right)$. A la campagne. le mot a laceeption technique de "purin. cau de fumier". - ce qui est. pour moi. le sens étymologitue.

Je tiens en elfet beggá bigg pour un dérivédu moỵen h. all. bîge (all. mod. beige: anas. tas. monecan), formé à l'aide du sulfixe - à (-â. -ô, - $\hat{\text { ar }}$. fir. -ard. Le tas dont il s'agit, c'est pour les eampagnards le tas par excellenee. le fumicr. lieste à déterminer le sens du suffixe - a. Attaehé à des thimes nominaux, il a d'ordinaire me valeur angmentative. comme dans bîa "bière, eivière ", bora "trouée d"une haie " (dér. de bolie "bouche "), colea dr zê "jarret de vaun". propr. "cuissard". fisså. "fesier". fontia "feu en plein air ", hura "hure de sanglier ", ete. Plus rarement, il peut arrir un sens moins précis et marquer um simple rapport de proximité. de dépendance : le caici. c'est le palier de la cave, la trappe. le plancher mobile ou suspendu domnant acè̀s à la cave; le niya. e'est le nichet qu'on met dans le nid. Dans ce dernier eas. l'explication de biga est aisée : e'est la fosse adjacente au tas de fumier. Dans le premier eas. on admettra que biga a signifié d'abord "gros tas [de fumicr]" - sens disparu sans laisser de trace - ; d'oì. par extension : "le liquide qui sert de ce tas et qui est recueilli dans une fosse adjacenten (comp. fosseit de bigant : Jean d'Outremeuse); puis. en génćral : "au sale et bourbeuse . bourbier".

Ite liégeois liga est remplacé. dans la région de Malmedỵ par dighâ, qui signifie : 1. "bourbier" (Malmedy : Villers) ; 2. "purin " (dans les campagenes par exemple à Faymonville: trô d digh "fosse à purin "). C"est l'augnentatil de digue (pron. dze) "fose remplie d'ean. mare ", qui dérive de l’anc. I. all. dîeh "marais, étanug. réserroir (all. mod. teig : à kupen dëli : a Elberteld deeg : des dialeetes néerlandais ont de même conservé à dyli le sens de "fossé, mare. bourbier").

[Romaria, t. XLvi (1921), p. 548.]

(1) De mêne diuns Jean d'Outremense (Geste de Liege. II. 118.5) : "dedens un graut fosseit de bigaut les buttoit ". Godefroy a un article brgan's 2 , dont il ignore la signilication et qui est ćvidenunent notre mot. Voy. ei-après l'art. embegaré.

${ }^{(2)}$ Sỳn. måssî polí. - Entendu à Séraing : dè eufè quìst neûr come dè bigâ ("noir conune du purin"). 


\section{w. bêrih}

G.. Voc. des noms w. d'animaux. etc. (Liège. 185\%), p. 29, donne bairih "sol improduetif". Dans son Dirt. II 500. il eite le même mot d'après le verriétois Lobet $\left.{ }^{1}\right)$. et. à ee propos. Seheler pense à l'ane. fr. barraigne, fr. brehaigne (stérile) on à l'all. baar (mu) : propositions inaceptables. - Le not est ineonnu en liégeois. Nous ne l'arous entendu qüà Ferrières, où un bêrih désigne un terrain ineulte, courcrt de broussailles, de genĉts, ete. ; un trîh (moyen néerl. driesch) y désigne aussi un terrain ineulte et broussailleux. mais oì poussent quelques arbres. - On reconnaît sans peine dans bêrih un emprunt du germ. berg "montagne" (ane. h. all. bëreg. dial. luxemb. berch), qui a pris en wallon le sens général de "terrain impropre ì la culture. comme le sol d'une montagne ». Pour la forme. voy. l'article ârih.

\section{ane. w. berckmoese, ane. fr. becquemoulx, lerquenoux}

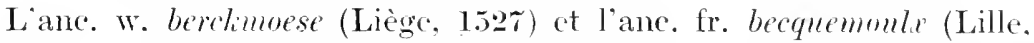
1461) figurent dans des textes qui contiennent l'énumération de matières tinctoriales ( $\left.{ }^{2}\right)$. Godefroy traduit raguement becquemoulu par : "sorte de teinture ". Pour Bormans (BSW 9. p. 24t). berclimoese est peut-être le même mot que le liégeois moterne lakmonise "bleu eorrosif qui sert aux maçons", cest-ì-dire "toumesol ". G.. II 557. enregistre dubitativement eette opinion; mais, se demande-t-il. eomment expliquer l'élément berck?

Si une seule des deus formes bercl-ou becque-était attestée. on pourrait supposer une graphic erronée ou une fausse lecture du moyen néerl. lecmoes. leecmoes. type primitif de lakmoes "tournesol" $\left({ }^{3}\right)$. Mais la même erreur a-t-elle pu se produire de deux eôtés si différents ? Ce n'est guère plausible. D’autre part, le réerlandais ne parait pas eomaitre berclimos. N’était eette difficulté. le premier élément pour-

( ${ }^{1}$ Lobet donne un sens 2 : "laine de moutons bruns employée dans sa couleur naturelle pour faire du drap de capucins ". De plus il fait de bruskène le syn. de bairih "lande, terre inculte". Chose curieuse, il n'attribue pas à bruskìne le sens 2 : "laine qui porte sa couleur ", sens qui est attesté par Remacle, G., I 84, Bormans (in BSW 9, p. 247), Villers, etc. Ce bruskène (laine) est inséparable de bruskin (espèce de drap : anc. w., G., II 562), anc. fr. broissequin (God.), qui est probablement d'origine germanique.

$\left({ }^{2}\right)$ Voyez ces textes ci-après à l'article oirzelle.

(') Franck-van Wyk tire lecmoes (vers 1500 ; west-flam. lekmoes) de ltkken, moyen néerl. lêken " égoutter ", altéré sous l’influence de lak "laque ". Nom d'une matière tinctoriale dont on fait une boullie (moes, pap), qu'on fait ensuite égoutter. 
rait être le moyen néerl. bercli "écoree". que possèdent eneore des dialectes modernes $\left({ }^{1}\right)$. D'après nos anciens textes. l'écorce de certains arbres (amne. noyer, pommior) fournissait une teinture $\left({ }^{2}\right)$.

Enfin un type lerquenome se rencontre dans un texte de $1+6+$ : "que doresnavant (les drapiers) pourront taindre tous petis draps non seellez, de ozeille ou de lerquenom, (Romania xxxin, 564). Il faut, je crois, lire berquemom. en rapprochant eette forme de berekmoese, becquemonler $\left({ }^{3}\right)$. Il est remarquable que. dans les trois textes, la matiore ainsi dénommée ligure à eôté de l'orscille.

[Romania, t. xLv11 (1921), p. 549.]

\section{liég. bèrôdî}

G.. I 52. 3333. a ces deux articles :

1. bèraudi ("age servant à clever les badigeonneurs, ete.). Nam. id. (baseule d’un puits) [\%]. Comp. dial. de Bayeux bèren (tuyau qui sert à déposer le cidre) ?

2. bèraudi (1. grenier situé au-dessus de l'aire d’une grange; 2. en général tout grenier formé de perehes ou de poutrelles et servant à mettre de la paille, du foin, des fagots, etc.). Nim. bircudi (au sens 1 ) ( ${ }^{*}$ ).

Pour le lecteur habitué aux analyses rerbales, és deux articles se raminent sans ellort à l'idce commune d' "ćchafaudage ". Les formes anciennes beroudie 1208. berrodier 1273, berodier 1275, -ire. 1326, - $i$ 1394 $\left({ }^{5}\right)$. ne nous apprennent pas grand' chose. Il fant poursuirre notre enquête à trarers les rocabulaires régionaux et, autant que possible. sur le terrain.

Le sens de " cage... " est signalé en outre par Body. Toeal. des eouarems (BSW 11, p. 14t), A. Bomhon, roc.du peintre (ib., 39. p. 14t), et Pirsoul, Dict. nammoris, qui le donnc eomme terme de fondeur. pour

(1) Les dialcertes de Groningue et de la Drenthe lui doment le sens spécial de "écorce de chêne, tan ". Le west-llam. bark, bork signilie " croùte d'éeoree, épiphloum, enveloppe suberearse ".

(2) Voy. G., II t;2:3 ; Godefroy, bntgnter.

${ }^{(3)}$.I. Belurens, Beiträgge, 1\% 150, fait le même rapprochement, mais il paraît admettre l'opinion de Bormans qui voit dans ces mots des variantes de lakmoûse. - Ir. Roques (Rommia, xxxvi, 276) dit que lerquenoux est pent-être lorcanc̀te en supposant lagglutination de larticle et me substitution de désinence. Cette anjecture n'est guère admissible.

(1) De même Forir : bèradi [?], birôdi ; bèrondî. - Voy. auısi G., I 55 : "biraudi, sorte de jall ".

${ }^{(5)}$ J. Cinvelier, Inemetrire des arch. de labbaye du I'ab-Benồt (in Bull. Inst. archíol. lieg., t. xxx, p. 58.5) ; Cartuluire de l'abbaye du Val-Benoit, pp. 188, 206, 212, 375, 708 (13ruxelles, 1906). 
désigner le plancher sur lequel se tient le ehargeur. - L'autre signifieation "faux plancher au-dessus de l'aire d'une grange " est de loin la plus répandue. Voiei les formes recueillies : bèrôdî, -i Liège, Verviers (1), Spa, Sprimont, Cherain. Villers-Ste-Gertrude, Wellin. St-Hubert, Lavaeherie, Sibret. Huy. Cincy ; Namur (Pirs.) ; Philipperille, Dorinne, Stave, Vonêche, Chiny, Florenville, Buzenol, Ste-Mariesur-Semois, ete. ; bèroûdî Dinant, Bourignes ; birôdi Malmedy, Stavelot, Franeorehamps; Namur (G.) ; bèrôdể Mont-le-Ban ; - $\hat{\imath}$ Vielsalm, Borigny ; birlôd̂̀ Glons, Roclenge-sur-Geer (épenthèse de $l$ ); barôdi gaumais (Maus, rocab. de rirton. ms. de 1850).

L'aire géographique de ce mot comprend dons Mahmedy. la majeure partie des provinees de Liège, de Luxembourg et de Namur : l'Enquête sur les patois d'Ardenne de II. Ch. Bruneau (1914; t. I, p. 45t) nous apprend qu'elle s’étend même au nord du département des Ardemnes. La variété des formes porte sur la désinence $(-\hat{\imath}$ ou $-i$, rarement - $e \hat{u}$, - $\hat{\imath}$ par confusion de suffixes) et sur la première syllabe bì-, bi-, ba-, où l'on soupçonnera la présence du préfixe latin bis- (roy. BD 190\%, p. 13s). dautant plus qu à Rethel (Ard. franç.) nous trourons arraudi $\left({ }^{2}\right.$ ) et que M. Bruneau, dans son Enquête relère érôdi sur quelques points de la même région. Mais que signifie le radical -roj- ?

Si nous interrogeons les synonymes de bèrôli "faux planeher audessus de l'aire d'une grange ", nous retiendrons bihoîle, s. f., à Sart-

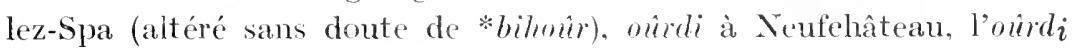
ou lé(s) oûudis à Alle-sur-Semois, l' hoûr ou lé(s) honirs à Oisy, oûr à Pecq. ourdege à Wiers. ordiu à Marbais. qui nous ramènent à l'ane. h. all. hurd (all. mod. hürde, elaie), désignant tout ouvrage fait par entrelaecment de branches, assemblage de poutres. etc. $\left({ }^{3}\right)$. Dis lors. nous poserons en fait que bèrôdî est altéré de *bèhôrdî. devenu par néta-

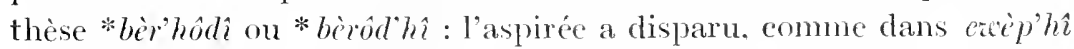
cordubisarium > excèt. Le sulfixe est - $\hat{\imath}$ (-ier, -arium) comme dans plantchî, plancher $\left(^{4}\right)$. Quant à la première syllabe, on peut y roir

(1) On signale à Verviers l'expression : e’⿳亠े-st-ô graml bèrôdli " èest un grand fainéant, un grand eorps sans énergie " (JSW 53, p. 41\%). - Le même sens de dénigrement est attribué, par métaphore, à lièn'bô (voy. l'art. am'bồ) et à hảbiêr (voý. cet article).

$\left.{ }^{2}\right)$ H. Baudon, Le patois des environs de Rethel, donne arraudi " espèce de planeher formé de perches où l'on place des bottes de paiłle ".

(3) Voy. Diet. géu. hourd ; Hécart mourdage ; Corblet hourds ; Bruneau, op. cit., pp. 454-5.

(4) Dans le fr. hourdis et l'ane. fr. behordis, le suffixe est -is, qui se prononcerait en wallon is' (taillis : tèyis'). Les formes du xu1 ${ }^{\mathrm{e}}$ siècle citées plus haut montrent aussi que le suffixe est bien -arium. 
soit le prétixe latin bis-. soit le germ. bi-; dans ce demier cas. bèrôdi se rattache directement à l'anc. fr. behourder, que Meyer-Lübke, $n^{\circ}$ 109s. dérive du francique bihurdan "enclore. palissader" $\left.{ }^{1}\right)$.

\section{liéğ. beûr, fr. bure}

1) apres le Dictionnaire général. le fr. bure. s. f.. puits de mine, " est emprunté du flam. booren (sic). all. bohene percer". C"est lopinion communément recue : les auteurs la répètent. arec plus ou moins d'assurance et toujours sans l'ombre d'unc démonstration (2). Pour ma part. je ne puis n'y rallier. Dabord il eomvent. en toute justice. de restituer ce rocable au dialecte liégeois, qui a domé au trançais tant de termes de houillerie. à commencer par houille même. Le fr. bure, devenu féminin en vertu de fanses analogies $\left({ }^{3}\right)$. vient d'un vieux not liegeois. toujours maseulin. que les actes anciens écrivent bur ou bure

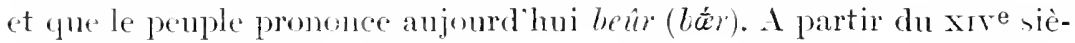
cle $\left({ }^{1}\right)$. on le reneontre fréquemment dans nos archives, par exemple en $1: 316$ : " et ne poront parmi le fosse et le bure fait clle terre... traire nulle hulhe " $\left(^{5}\right)$ : en 13:3t : "ilh dercont les bures remplir $"\left({ }^{6}\right)$ : 11 1358: "faire burs. fosses. voies ne paires" $\left({ }^{7}\right)$; ete.

C'e point ćtabli. d'oì rient le mot liégeois" Il apparait isolé. sans ascendant. sans fanille. Close étrange : si. comme on le prétend. il est né d'un rerbe. d'un nom d'action. il n'a lui-même produit qu'um dininutif : burtê "

(1) Codefroy, il l'art. behordeis, cite la forme borordeis, qui se rapproche singulièrement de notre bèrôli.

(2) C.. I $5: 3$; Scheler ; Bormans, V'oetub. des houilleurs liég.: I lrix. no 228 ; MeyerLübke, no 1211. - Norand, Art dexploiter les mines, invoquait en 1768 l'anglais bore, trou. Delmotte, en 181:2, remontait au celtique bor, puits (!). Sigart ne parle qu incidenment de bure, à propos de Borain, Borinage. Soit dit en passant, il est certain que bure et Bornin nont aucun lien de parenté.

${ }^{3}$ ) Influence de la linale -ure et de Thomonyme bure, étoffe.

(') On ne peut faire fond sur le prétendu cheans de (ou del) bur, (pui, d'après F. Henaux (La Inonillerie du prugs de Liège. pp. 35 et :36; Liège, 1861), figurerait dans une (harte de 1202. Grandgagliage ne connait que les formes cheans do (ou del) bu (I'oe. des ancieus noms de lieux, p. 15).

$\left.{ }^{5}\right)$ Acte du Val-St-lanmbert. cité par F. Henaux, p. 116.

(6) Curelies, Cartul. de labbaye du Val-Bewoît, P. 4 i2: item en 1379 et 1394, Hp. 6332 et 660 .

(i) Hicl., P. 48.; ; item en 1365, p. 512.

$\left.{ }^{(}\right)$. le laisse de coté buriu, qui nest pas, je crois, foncièrement wallon. On tire d'ordinatire le fr. burin du germ. boro (all. mod. bohrer : perçoir, tarière); mais e'ette derivation ent tres doutense pour la forme et pour le sens; voy. Körting.

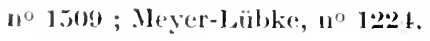


nique nous aurait donné directement un substantif. Enfin, il me paraît phonétiquement impossible d'associer le germ. boren (bohren) et la forme francisée bur(e). Il faut done ehereher autre chose.

Le dialecte liégeois confond aujourd'hui deux eatégories différentes de mots dans la même prononciation -ềr (-ter formé long) : $1^{\circ}$ ceux qui

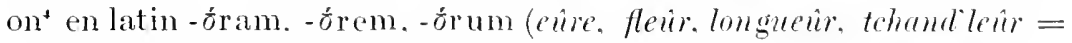

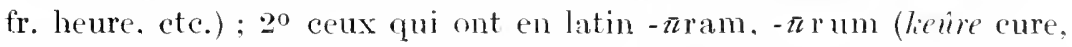
doble tere doublure, matevir mûr, meîr mur. dềr dur) on en ancien germanique -ûr (sềr sur. aigre. hềre grange). Wais d'autres dialectes conservent une distinction qui s'est effacée en liégeois moderne. A Seraing-sur-Meuse par exemple, si l'on dit comme à Liège cû̀re. flềr, etc. on prononce lieure. dobleure. etc.. avee ä ourcert bref. ainsi que beur, puits de mine. Notre mot rentre donc bien dans la seconde catégorie, et la forme francisée bur(e) est correcte. Une origine latine ne pouvant ici être inroquée, il sulfit d’interroger eeux de ces mots qui viement du germanique, à savoir sethr, seur (sur. aigre; de l'ane. h. all. sûr. all. mod. sauer). hethe heure (grange; de l'ane. h. all. sciur', all. mod. schauer, scheuer). pour se convaincre que beur. beur postule nécessaircment un type ane. h. all. bûr. Ce type existe en effet. - mais arec le sens de "maison " (all. mod. baner : volière).

A première vue. on se croira sur une fausse piste : comment une "maison " peut-elle se muer en "puits"; ? On fera bien ecpendant de ne pas trop sarrêter a l'objection. Ce que nous pourrions appeler "l'obsession sémantique " est sourent un écueil pour l'étymologiste. Celui qui. par exemple, guidé par l'analogie des significations. reut dériver boucher (s. m.) de bouche, houille de kohl, bure de bohren. se laisse égarer par cette obsession. Poursuivons done nos recherches, pleins de foi dans la constance des lois phonétiques, et nous trourerons ceci. Jadis, au pays de Liège. sur la bouche du puits de mine. s'élevait un hangar ou unc baraque, abritant la machine d'extraction. Pour les petits puits, c'était une cabane de planches ou de elayonnage. appelée hutte, en w. houte. Pour les grands puits. on établissait une enceinte plus solide : de fortes pièces de bois formaicnt une cage à claire-roie, couverte de chaume et garnie sur trois côtés de planches a hauteur d'appui. Sous ce toit. au-dessus de la bouche du puits, étaient suspendues les deux poulies ou rôles du bur. Un savant allemand du xre siècle (Agricola, de Re metallica. 1546) appelle cette constmution casa putealis. - J'emprunte ces détails d'histoire à l'ourrage de Morand (1) : ils

( $\left.{ }^{1}\right)$ Art d'exploiter les mines de charbon de terre (2e éd., Neuchatel, 1780), pp. 37-38. - Sur cet ouvrage important, voy. F. Henaux, op. cit., p. 15. 
corroborent singulièrement les données de la phonétique et légitiment la conchusion suivante. Dans les premiers temps de la houillerie liégeoise, on appelait *bur de fosse la hutte élerée sur le puits. Le bur de eette époque lointaine. avant de donner son nom à la fosse même, c'était une très simple ébauche du "beffroi" de la houillère moderne $\left({ }^{1}\right)$.

Il est certain que les dialectes du nord - y compris le liégeois ont connu bur au sens général de "maison ". Le normand le connaît encore (Meyer-Lübke, $n^{\circ}$ 139\%); l'ancien liégeois et lo français en ont tiré le diminutif buron "cabane " (roy. ci-après l'article hî̀ê). Il est non moins avéré que, chez nous. bur a perdu depuis des siècles le sens de "maison ". On peut conceroir plusieurs raisons de ce fait. Le mot étant d'origine étrangère, il s'y attachait une nuance de dédain : c'était une maison de clayomage, une chaumière. Il trourait aussi un coneurrent dans le dérivé buron, qui arait plus de corps. Enfin il s'employait pour désigner la construetion rudimentaire qui surmontait le puits de mine et eette fonction spéciale fit oublier l'aceeption générale. Seuls les houilleurs conservèrent ce terme archä̈que, mais en le détournant bientôt de sa signification propre. Grâce aux expressions courantes monssî. dihinde. orrè beur (entrer, deseendre, travailler dans le bure). li trô. les rôles di beur (le trou. les molettes du bure). ete.. on le prit naturellement pour le synonyme de fosse. L'évolution sémantique dut s'accomplir de bome heure. probablement au xir e siècle (roir les textes cités plus haut).

En r'ésumé, ]e liégeois bur(e) a passé par les étapes suivantes : 1.t. gén., maison : surtont maison chétive. sron. burm: - z. spécialement. t. de houill. bur (de fosse). construction élevée sur la buuche du puits d'extraction :-...3. par eonfusion : puits d'extraction : - t. par extension : tout puits de mine sourrant au jour, uon seulement le puits d'extraction (bene a trên), mais aussi le puits d'aérage (bềr d'êr). le puits d'exhaure (beîr as colomes) et le puits aux echelles (bêur as håles). Le sens t est le seml commu aujourd'hui.

[BII) $1920, \mathrm{PH}, 4-\mathrm{T}$.

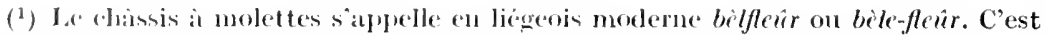
une altination, par etymologie populaire, de belfreude, fue Iorand donne au xw11e sicele (op, rit., p. 38). Liane. w. bellefroit equivaut ì l'ane. fr. berfroi (= fr. modl. boffroi), emprunté de liane. germ. bergfrid "(tour) qui protège la sûreté) ". Le fr. beffroi a aussi, par exteusion, le sens de: "clarpente supportant les cloehes d'un elecher ou fe mécanisme d'un moulin"; voy. G., II 502, 557. 


\section{w. bihot}

Aucun dietionnaire ne signale le w. biht "vase de ménage, bidon, réeipient queleonque servant à la euisine ", qui existe notamment à Crehen (IIesbayc), à Huy ct à Melreux. surtout au pluriel. Nous y reeonnaissons le moyen néerl. behôf. néerl. behoéf (all. behuf) "lyesoin, nécessité; au plur., ustensiles ". Le sens concorde pleinement. Pour la protonique $e>i$, comparez l'art. gistel. La finale seule peut faire diflieulté: on l'expliquera par l'influence analogique du suffixe -ot et surtout en comparant le liég. rö. t. de batellerie, "cabine plaeée au milieu du bateau " (G., II 319), emprunté du moyen néerl. rôf, néerl. roef (angl. roof, d'où le fr. rouf). - Pour désigner le coflin du faucheur. on dit au sud bihŏ (La Cuisine. Chassepierre, ete.). biyo (IIerbeumont. Stare, etc.). C'est peut-être le même mot pris lans une aeeeption spéciale, mais plus probablement une altération du fr. ane. et mod. buhot "tuyau, gaine" $\left({ }^{1}\right)$.

\section{fr. bleime, w. blème, blène}

G., II 504, emregistre, d’après Simonon. le liégeois blem (sie) " eor au pied d'un cheral " et, d'autre part, M. W. Gorrissen signale à Huy blëme. s. f., "bleime du cheval ". Ce mot technique nous vient apparemment, par roie lirresque ou sarante, du fr. bleime,s. f.. t. de rétérinaire, "irritation qui attaque la sole des talons du eheral ". Mais d'où sort le fr. bleime? On le trouve poun la première fois en 1690, dans Furetière $\left({ }^{2}\right)$; il est d'origine incertaine ; peut-être a-t-il le mêne radical que blême. C'est tout ee que nous apprend une note concise du Dict. gén.. qui atténue sagement l'opinion de Littré $\left({ }^{3}\right)$.

En réalité, bleime, s. t., n'a rien de eommun avec l'adjectif blême. Pour l'expliquer, je m'adresserai au néerl. blein "ressie, ampoule ", qui est bien connu en payss flamand $\left({ }^{4}\right)$, d'où il a pénétré dans l'est du Brabant wallon : nous y relevons en eflet blène, blème. s. f., avec le

(1) Sur buhot, voy. God. et le Diet. gén. - Les formes ard. et gaum. bỹè, buxiè, buж, bua, b̆̈ya, ete. (voy. Brunean, Enquête, I 194), plaident pour buhot.

${ }^{(2)}$ M. Ant. Thomas me signale un exemple de 1665 cité dans le Dict. gén., semne.

${ }^{(3)}$ Ulrix, Germ. Elementen in de Rom. talen, $n^{\circ} 188$, aurait dì imiter cette réserve prudente. - Voyez l'art. de Littré. On ne trouve rien dans Diez, Körting, MeyerLübke.

(4) Voyez une note intéressante dans De Bo sur le west-fl. blein(e). Vereoullie tire le néerl. blein du moyen néerl. blaeien "souffler ", sur le type du syn. blaar, dérivé de blazen. Franck-van Wyk n'en parle pas. 
sens général de: " contusion. blessure plus ou moins grave " $\left.{ }^{1}\right)$. Le ehangenent de la finale -ne en -me est assez fréquent dans nos patois $\left(^{2}\right)$; lc fr. bleime dérive sam donte d’une forme dialectale qui arait subi eette altération. Enfin. la graphic -ei(me). insolite en français, pourrait être un somrenir du germ. -ei(n).

[Romania, t. XLY (1919), p. 179. - Ajoutons que la bleime du eheval se dit à Thimister-Clermont stếgal, emprunté de laall. steingalle.]

\section{liẹ̣. bô, ard. bôkê ; liég. bôkê, bôkî}

Nour arous clit. à l'article ba qu il ne faut pas confondre ba arce bô. Ce dernier représente doux termes techniques différents. d'ailleurs peu connus en liégeosis.

1. Le batelier de la Meuse appelle bô un boulon-tirant à tête très laroe. traversant les hanehe's du bateau et venant se boulonner sur les traverses $\left({ }^{3}\right)$. Nous y royoms le néerl. bout. all. bolz "boulon. eheville "; comparez hout. holz (bois). qui a donné hô dans des noms de lieu. par exemple : Bèhó. près de Vielsahn. altéré de *Boho : all. Boeli(h)olz. sur la cartede leprarie $\left({ }^{4}\right)$.

״. I'n autre bij. an X.-E. et an S.-E. de Liège, désigne une sorte d’anncau, dans len quatre eas suivants : $1^{\circ}$ anneau de fer adapté aux extrémités du carlec de la charrette a ridelles ot aux denx bouts du hame ou banc (j):-- 2o lien en fer qui assemblait la botte (bocèrê) des rirger de fer dont le cloutier à la main faisait naguere des elous, au pays de Fléron-liomsée $\left(^{6}\right)$ : — 30 a Thimister-Clemont, anneau de chêse tordu ou de fer qui glisso dans le poteau (le staminî) de la crêehe ou mangeoire de la vache : une chaine. passée dans le bô. retient par le eon lanimal gui peut ainsi lever et baisser la tête $\left({ }^{7}\right)$; - $4^{0}$ rers

(") l'all excmple it (hastre-Villeronx (Sud de Wavre) : djĕ m'a fouteu one blène; el a me líle bline "s ljambe ; de même à Ste-Marie-Geest (lez Joloigne), où l'on

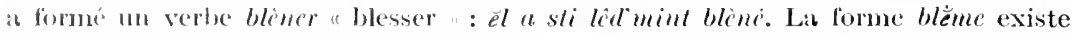

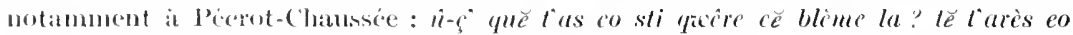
buter, bi serier!

(2) Lietg. strime, itreme; ard. preme (Bovigny), prune; rouchi time (Ions), timu, mince ; ete.

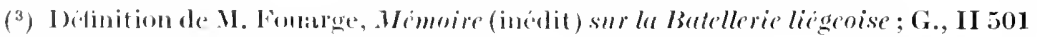

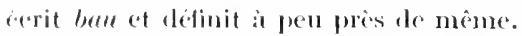

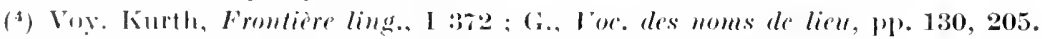

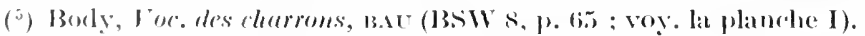

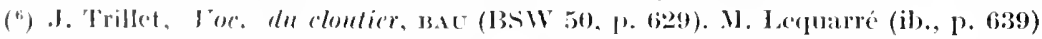

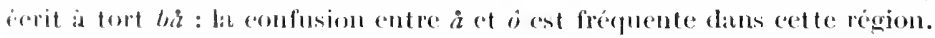

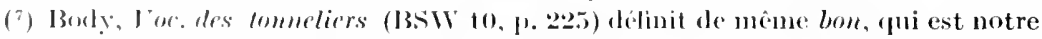

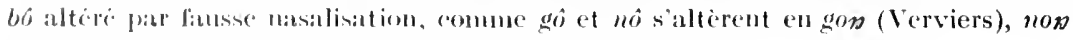
(Ayeneux) ; voy. ci-itprès l'atrticle gô. 
Villettes-Bra : baguette ployée en un cercle dans lequel on insère le lacet à prendre les grives.

Nous reeonnaissons iei l'ane. fr. bon (bracelet), qui vient du francique baug. m. s. : comparez *traucum : fr. trou. w. trô : paueum : ane. fr. pou (peu), w. pô.

De lì, dans les Ardennes, le diminutif bôk $\hat{e}$. aeceptions où reparait le sens fondamental de "anneau, lien circulaire". M. Ch. Brunean a recucilli, dans la région de Givet-Dinant, les notes suivantes :

bôlé (Hargnies) "annean formé d'une hart tordue, qui monte et descend le long du poteau de l'étable "; - bôkia (Félenne), m. s. ; - bôké (BourseigneNeuve) " collier de veau "; - bôkia (Givet, Agimont) " anncau qui maintient rapprochés, à leur partie supérieure, le montant d"une porte de jardin et le poteau qui la tient fermée ; - bôkia (Chooz, Ham-sur--lleuse) " entrave, pièce de bois suspendue au con des vaches " $\left.{ }^{1}\right)$.

De mon côté, j'ai noté au N.-E. de l'Ardenne: bôliè : 1. (Borignny) "baguette ployée en forme de eollier et suspendue à une branche horizontale: on insère dans ce cercle le lacet a prendre les grives " $\left.{ }^{2}\right)$ 2. (Villers-Ste-Gertrude) "jarretière ancienne faite de lisière tournée autour du bas sous le genou ": il sagit sans doute ici d'une allusion plaisante ou dénigrante.

3. Signalons encore lo liég. bôliê "hausse de larchct, chevalet de violon " $\left({ }^{3}\right)$. Ce sens a disparu de l"usage moderne; mais je tiens d'une vieille famille de Liège (Cointe) les renseignements inédits suirants : "on p’tit bôliê se dit d'une petite personne massire : syn. "m p’tit bôlî ome, ine pitite bôlìye feume". On peut done présumer que ce bôliề a le même radical que le verbe boki " bourrer ", et que le sens premicr est : "petite masse bourrée, serrée, tassée "; d'où le sens archaïque "chevalet de riolon".

(1) Bruneau, Enquite, I 322-3. La dernière acception pourrait faire penser an germ. balk (poutre); mais, en réalité, la dite pièce de bois cst suspendue à un collier ou à une corde passée au cou de la vilche. - Comp. l'ane. fr. baucuel, que Godefroy traduit par " bride" (?).

$\left.{ }^{2}\right)$ On distingue à Bovigny le bôkê et le plöyerồ "baguette pliée en are de cercle et dont les deux bouts sont insérés dans le trone d'un arbre ". Body, Voc. des tomeliers (BSW 10, p. 220), ignore cette distinction.

${ }^{3}$ ) G., I 59, II 504, le dérive tantôt de bôkî " bourrer ", tantôt de l'all. ballie " poutre ". Ce demier, (qui aurait donné * biakê, doit être évidemment écarté. - Voici un exemple tiré d'une pasquille de 1792: qui ioste êrçon (arehet) djusqu'a bôkê Fasse rêzoner tos zos boyês (boyaux : cordes de violon). 
4. Nous sommes ainsi amené ì parler de bôkî. Etant donné que bô et bolkê d'une part. bôk $\hat{e}$ et bohki d'autre part, sont intimement umis, il parait assez naturel de rattacher bôkî au primitif $b \hat{o}$ "lien eireulaire, anneau \%. Pour la forme, bôkî, aree ses dérivés bôk ner, bôk'ler ${ }^{(1)}$, ferait le pendant de ritroklit. -(i)uer, -(e)ler, dérivés de tiô "trou " (ef. G.. II 296). Le sens premier serait : "bourrer, tasser, serrer une masse compacte à l"aide de liens cireulaires "; d'où les sens dérivés : 2 . "bourrer. gaver. bonder "; 3. " cmmitouller, bourrer le cou et le crâne, affubles" $)$.

La question eependant n'est pas aussi simple qu'on pourrait le eroire, Pour expliquer bôkî. G., I 62, proposait dubitativement l'all. poelen, nécrl. beulien "frapper". Il existe en effet de ee verbe une forme bolieu, en mộen néerl. et en moỵen bas all.. dont notre bôkî pourrait s'aecommodes, d'autant plus que le dialeete allemand du Grand-Duehé de Luxembourg possède une série de mots qui méritent d'être pris en considération : bôken "frapper sur la tête "; bôk, m.. " eoiffe"; bôkemanl "masque"; aboblien, -elen "emmitoufler. masquer".

Fn somme. il est probable que notre bôkî vient du germ. bôken "frapper, éeraser. boearder" et que le liég. bôkî̀ a done une migine différente de l'ard. bôliê. D'autre part, il n'est pas impossible que bô ait aussi produit un verbe bollî. lequel, pour le sens eomme pour la forme, se sera aisément confondu aree le préédent.

\section{liég. bodje}

L'aneicn liégeois boigge "fût. trone " se lit dans le Myreur des Histors, I. 640. En liégenis modeme bodje. s. m.. signifie : $1^{0}$ trone du corps hmmain (G.. I 60) ; sens dimparu aujourd'hui mais dont la trace subsiste dans lo suivant; $2^{\circ}$ corps d'une chemise d'homme; $3^{\circ}$ trone d'un arbre virant on ciu moins eneore debout (seul sens donné par Renacle, le éd.. et par Inbert) : aspoŷ̉ 'ne heile so l' bodje ; on-z-a côpé l' tiésse

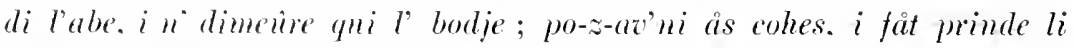
bodje; spécialement. endroit oì naissent les branehes : i-n-a on nid ¿ bedie (2).

Ponr l'étymologic, l'artiele de G., I 60, est indécis. Il nous dit que le nam. appelle buc le trone d'un arbre ou du eorps humain ; il suggère

(1) Et ses composés abôkî, -eler, -ener ; voy. BD) 1906, p. 100.

(2) Il a douné le dérivé boudjèye (liég.), bodjêye (verv.), s. f., touffe, ensemble des rejctons sortant d'me mêne souche : ine b. di erompires, di zoazon, di gruzali, ete. Le sulfixe répond atu fr. -ille. synonyme bouhèye. - Voy. ci-après l'art. manote. 
suceessivement l'anc. h. all. pûh et l'ane. h. all. botah. sans s'arrêter à une conclusion solide.

Il est pourtant bien elair que le nam. buc représente le néerl. buik, flam. beuk. all. bauch (rentre: anciemement : trone). Quant au liég. bodje, ane. liég. boigge cest le diminutif beulije qui. d'aprés V'reoullie, signifie aujourdhui "ehemise sans manches". Pour la phonétigue. comp. $1^{o}$ le néerl. huik (manteau. capuchon), ane. fr. hucque, hencque, ane. w. hoike (1+15). heule (1+20). "eape. capuchon ". qui subsiste notamment dans le w. s' mite a hok (Pellaines). a ynk (Givet) "se mettre à l'abri de la pluie " (BD 1911, p. 90; G.. II 688); - 2o le flam. fuik (blouse) : ane. liég. focke (roy. ei-aprìs l'art, coxhe).

[Romania, t. Xlvil (1921), p. 550.]

ouest-w. boughnèt; nam. bougnote

Dans l'Ouest-wallon. buuguèt signifie "boulet formé de poussier de houille mélangé d’argile" (Fosses-lez-Namur : BSW 52. p. 116: Stave, Charleroi, Viesville). Nous y reconnaisons * boutyet alt érépar épaississement de $y$ en gn $\left({ }^{1}\right)$. C"est le diminutif du nam. bouye "bulle, ampoule. bosse à la tête. bosschure. noud dams le bois". qui représente le lat. bulla (boule, bulle). comme le nam. pouye "poule" reproduit le lat. pulla. Un bougnèt est done proprement un "boulet " $\left({ }^{2}\right)$.

Bonye a produit un autre rejeton, bouyote, qui a le mêne sens que le primitif et qui, pour cette raison, en a eonservé le radical pur. Supposez que le sens du dérivé s'éloigne de celui du prinitif. l’altération phonétique en sera d'autant facilitée. C"ent ee qui ent arriré. je crois, pour le nam. bouguote "petite aisance pratiquée sur le banc des verriers, à gauche, pour y remettre leurs mesures. leurs mouehoirs. ete. " ( $\left.{ }^{3}\right)$. Il est probable que ce mot se ramène à bouyote.

\section{liég. bougnou, bougnet (:)}

On lit dans G.. I 66 : "bougnou, t. de min. (puits creusé au fond de la bure pour recueillir les eaux). Remacle, ze éd.. a la forme boughè (puits d'une bure )". - Pour toute explieation. G., I 1.58, dit quion pourrait comparer l'italien bugno (ruche).

Les auteurs qui ont étudié le roeabulaire du houlleur liégeois ne

(1) Voy. l'article bougnou.

${ }^{(2)}$ Comp. les synonymes clûte, hotchèt, expliqués à l'article hotchèt.

$\left({ }^{3}\right)$ Ce mot ne nous est connu que par G., II, 505, qui le donne sans explication. 
signalent que bougnô. -ou (1). En réalité. ce mot n'est pas restreint au parler du mmeur; il a le sens général de "réservoir, citerne. puisard". Hubert et Forir en tont le synonyme de pisseroû (ef. G.. II 227). Au xire siècle. les eaux de la Légia se jetaient. près de Hors-Château (rue de Liège). "dans un vaste et long réservoir, nommé Bougnoux, le long des remparts. qu il aidait à défendre " $\left({ }^{2}\right)$. Rouveroy a un artiele: "bräou. brüuou. puisard pour receroir les caus des eombles; bétoire dans les ehamps; poêle d’un étang ". Ces deux dornières significations paraissent rares ou peu sûres: Rouveroy aurait micux fait de ne pas oublier l'aeeeption la plus fréquente du mot. Quoi qu'il en soit. les formes boyou. bouyou sont remarquables. J'ai eonstaté qu'à la houillère de Gives (a l'Est d'Andenne) on ne eonnaît que bouyou ; à Liège même, jai parfois entendu prononeer böyou. Tel est sûrement le type primitif : bouguou en provient par épaississement de y en go, phénomène dont nos dialectes foumissent de nombreux exemples $\left(^{3}\right)$. Le suffixe dimimutif -ồ (lat. - eolum), abrégé souvent en ou moyen ou bref, s'est ajouté au thème quion retrouve dans le nam. bouye "bulle (d'air. d'eau, de savon), ampoule, bosse à la tête. bosselure. ete. ". du lat. bulla "boule, bulle (roy. l'art. bougnè). I'n bayoù (bouyoù, bougnoû. -ou), c'est proprement l'endroit qui bouillomne et pétille' par suite de la chute continuelle des gouttes d'cau. Lialtération de $y$ en gn s'est faite probablement sous l’influence de cougnori. pauguon.

La forme bougnet. que Grandgagnage cite daprès Romacle, mérite une étude particulière. Nous avons vau que Morand. Brixhe et Bormans lignorent: nous ne l'arons pas non plus relevée dans nos enquêtes et nous doutons fort de son existenee. Trois auteurs cependant la signalent; mais les déponitions de ees témoins sont bien faites pour déconcerter.

(1) Morand, Art trexploiter les mines de ehurbon de lerre (20 ed., 1780): Brixhe, Essai dun répertoire de ligistation en matieres de mines, II 4it (18:3) : Bormans, Vocrab. des houlleurs liégeois (1864). - Brixhe (ef. G., II xiv) a maladroitenent francisé bougnoil en bonim : ees deux formes ont passe dans le Supplement de littre et aans le Larousse illustri. Je relève "bougnou : puisard "diuns le Vocub. des mineurs du Nord at du Pas-de-Calthis pas .J. Bovio (J)ouali, 1906). On roit que notre mot liégeois a fatit du chemin.

(2) Gohert, Eanx ef Fontaines publiques a Liege (1910), p. 124. Cf. anssi BsW 9, 1. 31:. Le rize de Bongunle ent cité en 1:37s dams le Cartulaire de labbaye du ValBemoil. p. 6i2t. et le "lieu condist an Buguoilhe an Geron , en 1365, ibid., p. 512. bans des comptes duxvile siecle, "lit cité ordonne le nettoyement des bougnoux

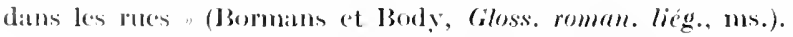

$\left({ }^{3}\right)$ Voir les articles bonguet, cromignon. dognon, hoye, sprogni, tongnoûle. 
Remaele (e éd., 1839): bouniot, puits d’une bure: toumer è bouniet, tomber dans la bure. [Remacle n'a pas d'article bougnou; il définit beur " bure des mines de charbon minéral \%.]

Lobet (1854) : bouniet. ouverture d'un puits, d'une bure, ouverture de la terre en rond pour l'extraction des minerais, ete. [Lohet a un art. beurr " bure, puits profond des mines, ete. "et un art. bougniou "puits de mine, puits ereusé au fond de la bure pour recueillir les eaux ».]

Forir (1866) : bougnet et bougnou, puits creusé au fond de la bure pour reeneillir les eaux; puisard : toumer è bougnou, tomber dans le puisard.

'Tout pesé. voiei nos conelusions. En réalité, Remacle seul atteste l'existence de bougnèt ct, comme il n'a pas - chose étrange - d'article bougnou. on est en droit de le soupecommer d'erreur. Au surphus, sa rédaction manque de clarté : un leeteur non prévenu prendra bouniet pour le synonyme de beir. - Il est manifeste que Lobet emprunte à son devancier le mot bouniet et qu'en domant à ee terme le sens de beur, il pèche par inattention. Son artiele n'a done aucunc valeur. C'e qui prouve l'emprunt, éest que Lobet eonserve ici la graphic de Remaele. alors que, d'après son système, il écrit bougnimu comme dosnion. kougniou, pougniet. Au reste. noublions pas que Terviers, oi vivaient Remacle et Lobet, se trouve en dehor's de la régrion houillere. - Forir a tiré la forme bougnèt de Grandgagnage (qui la donne sous la i'esponsabilité de Remacle) : dans l'exomple eité. il ne eomnait plus que bongnou, ainsi qu'aux articles pis'rax. et tro-plin. - Théoricuement. les suffixes -ok, -èt ayant môme valeur diminutive, rien ne soppose à co que ega et lì. par exemple dans me homillere du pays de Herre. bungnèt ait surgi à côté de bougnoû (ermp. enusnoû. congnit). Mais. con l'espèce, - que ce soit le résultat d'mne bérne ou le fruit d'une création isolće. on doit tenir le mot pour suspeet, en attendant un témoignage phs probant que celni de Remacle.

\section{w. boût'ner, poût'ner}

Le verbe intransitif bout ner - ou pout ne', forme ordinaire i Liege s'emploie toujours unipersomellement : i bonitine ou $i$ brit'néye se traduit en français du terroir par : "il bitume net correctement par : "il se répand une odeur de bitme " : cela se dit surtout de l'odeur que produit un foyer où l'on brûle de la houille. quand le rent refoule les vapeurs dans l'appartement : quaml li tch'minêye rebat'. On dit aussi : $i$ flêre li bont'né ; quéle flêrante bont'nêue! Vogez ci-après l'anticle sur le synonyme tèzi. 
urandgagnage en parle deux fois (1 72. II 254) sans jouroir dégager une explication satisfaisante. Prenant ponther pour ta forme première. il propone d’y roir $m$ dérivé du latin putêre "puer". Son erreur provient de ce qüil range poutềr (qu'il écrit ponteure) à coté de pout neure. Or il n'y a rien de commum entre ces deux termes.

Le w. poutête. s. f. arec on bref. répond exactenent à l'ane. fr. puteur (junanteur). dérivé de l’ane. fr. put. lat. put idus (puant. infect). Il est bien eomnu de nos honilleurs. qui entendent par là lanhydride carbonique. gaz asphyxiant. mais non inflammable diflérent du grisou. On troure le not altéré en poutéie (sous l’influence de pout néue? ?). dans le dictionnaire de Forir par exemple. qui eite lexpression arehaï-

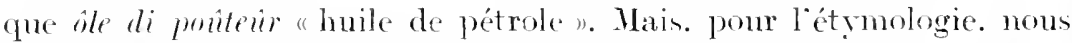
sépareroms nettement ponit nevie de poutêu et nous verrons dans le premier me altération de lont nevêre due à loinfluenee de poutêu.

En réalité. le sentiment populaire qui met en rapport boût'ner et bitmme a pleinement raison. Au licu de bitume. forme savante empruntée du latin bit mmen. l’ancien français disait betun (an sens de : boue, gravois. doin le fr. béton). Nous tromvons d'antre part dans ce texte d'archives liégeoisen: "entoit lais de colle et de butyue", une forme qui provient de *bitune par nuétathèse: lane. fr. betumei "fondrière "se rencontre aussi sous la forme butemei (God.). De mème le verbe *bituner a domné *butiner. *butener. cest-ì-dire bout ner. devenu bout ner par un allongenent de la voyelle initiale dont le liégeois présente de nombreux exemples (roy. l'art. crumignmu).

\section{ličg. branvolé, brivolé}

Forir seul signale benzolés -fye "écervelé. volagge. celui qui piaffe, qui montre she grande somptuosité en habits. en meubles. ete. ; fél bramolé piafler. faire piafle ". Ce terme archaigque est eneore comnu des rienx Liégeois. Volei ce que jai recuedlli à ee sujet. In beanowé, cest un boute-en-train. mu meneur de elanses. mo joyenx drille qui

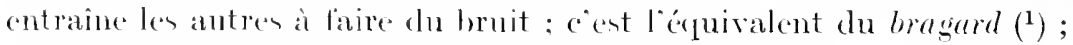

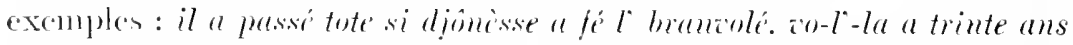

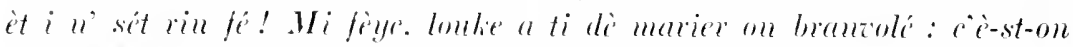
ziolon a-d foni. an sorogmm a-dizins. Le féminin est imusité.

Je ne combain que deux exemples anciens. D)ans me picee manus-

(1) "Dircteu de fetc, porte-drapean; hableur" (Forir) : cf. G., I it. Ce terme est plutiot rural. 
erite de 1759 . con dialecte de Verviers, mo femme reproelse à som mari les prodigalités qui les ont ruinés:
Esteuse a ti monseu el hause
[ = Esteǹt-ce a ti, monseù èl-håsse,
du tinre on jvau so lu stauve du tinre on rlj’va so lu ståve,
du tel fez tos les jous selez
po zalez fez l' branrolez ? (')
du tèl fé tos lès ajoùs sèler
po-z-aler fé l' branvolé ?]

L'antre exemple se décourpe. non sans peine, dans un dialogue liégeois de $16 r 6$, publié récemment :
Pens tu kly roy nay nin rmareque
[= Pinses-tu qu' li rwè̀ u’àye nin
[r'marqué
ki sestin ton bron volez (2).
'püis-èstint turtos bronvolés :]

La Soeiété de Littérature wallonne posside, du début de ec lialogue, une copic anonyme. aree cette mention: "Extrait d"un pet it reoristre aux rentes particulières de l'an 1645 . Copie médincre. qui donne cependant quelques rariantes remarquables. entre antres le v. 16:

'qui see nesten ki tiertout brivolez $\quad$ [ = qui ç" n'èsten̂t qu’ turtos lorivolés].

Ce brivolé nest guère nieux comnu ni phu elair. Il est signale par Ch.-N. Simonon arece le sems de "rolage" (G.. II xr et 5obi). et par

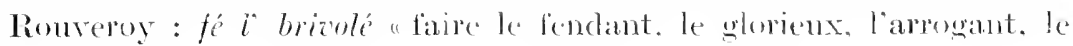
fanlaron, ctc." $\left({ }^{3}\right)$

Dexplication, rien on plespue rien : G. compare a brizold le nam. brif-braf "inconsidérément. ‘ans rélexion "et le liég. aronlou "brusque ". Ce dernier n'a que faire ici $\left({ }^{4}\right)$. (2uant a brif-braf. il se rattache peut-itre à l'ane. fr. bricer "courir aree rapiclité \% On peut conjecturer que brizolé est altéré de branoolé. - som lintluenec de co brizer ou pour

(') Le Mayeur ruiné par sa charge, v. 997-1000. Voy. l'article Arih.

(2) Discours entre Jollet et Wustay, v. 15-16, in Bull. Soc. vervistoise ditrchéol. et dritist., xum (1913), p. 221. L'éditem conjeeture : "qu"is estint [Hons po nos voler] ? " mais lab seule dilliculté du vers est ton, qu'il faut eorriger cn turto ou en tote $(=$ tos dès $)$. Bron, pour bran, est la prononeiation de Montegnée-lez-Liège ; comp., dans la pièce, v. 14, on pour an (ammie), rimant avee déaront (deviendront).

${ }^{(3)}$ Le Diet. liég. manuscrit de Baillenx dome : "brizoler, v. a. [sic], faire grand train, vouloir éelipser les autres ". Cet infinitif nous parait très suspect.

(4) G., I 28, If Ix, n'explique pas ärolou. ("est l'anc. fr. arrolut "vouté en forme d'are "; d'où, en liégeois, les sens de : "baneal " (Forir) et, au moral, de : "anrogant, impérieux, brusque". - On ne pourrait pas non plus rapproeher intribolé "étourdi, effronté " (Forir), qui est singulièrement écrit intrizolct dans BSW 22, 1. 100. C'est probablement l'ane. fr. entriboulé "troublé, affligé, désolć ", altéré sous l'influence du w. rèzolé " éeervelé ". 
une autre raison $\left({ }^{1}\right)$. Nous n'aurions, dans ce cas, à tenir eompte que de braniolé. dont l'étymologie, au surplus, reste obscure. l'ancien français noffrant rien de semblable.

Lne chose eependant paraît assurée : e'est que branoolé renferme le substantif $\operatorname{br}(m(d)$. Cette présomption se fortifie si l'on examine ee mot et ses dérivés dans nos dialecten $\left({ }^{2}\right)$.

On sait que le germ. brand (1. tison : 2. épée) a donné en français ancien ou moderne : $1^{0}$ brander "sembraser. brûler " brande " embrasement. flamme : fig.. agitation, ineertitude. tourment " : brandon "tison ": : o brand "lourde épée quion maniait à deux mains ". brandir brandeler. branler. brandiller. brandoyer "agiter. remuer. faire oseiller " $\left({ }^{3}\right)$. - En wallon du Nord-Est. nou relevons les verbes intransitifs brendi (Verviers: Lobet) "bondir. crier. rugir. luurler " $\left(^{4}\right)$; ribramdi (G.. II 300). rèsbandi (à Fontin-Emneux) "retentir. résonner $\left({ }^{5}\right)$ : diminutif : brandiner (Malmedy) "brandiller " d'après un rocabulaire anonyme du xrme siecle. "faire du rodomont, fanfaromner" d'après Viller $\left({ }^{6}\right)$. ce qui est le sons du liég. fé l' branzolé. Quant au primitif bran $(d)$. il a revetu chez nous les aceeptions suivantes: 1. "bruit. tumulte. tapage. tintamarre" (Mamedy : Villers) : 2. "mourement" : mite i bran (ib. : Scius): duner wh bran (Stavelot) "domner un élan pour mettre en branle : d'où. au fig.. aceut an bon bran. on maza bran (Villettes-Bra) "avoir un bon. un maurais mourement. itre bien ou mal dieposé : — 3. "branle : danse populaire" (différente du crâmignon), qui se fait dans eertains villages an Nord de Liège (IIermalle. Visé. vallée du Geer, ete.). lors de la fëte paroissiale: danser on bran d fiesse (Forir). miner l bran "mener le bran ". mineu

(1) Duvivier donne le liég. brigoler " earaeoler , qui est lane. fr. brioler " courir avec agitation . Ie croisement de bramolé et de brigolé expliquerait bricolé. Bripourrat aussi être dû à l’influence de brigosse "vaurien".

${ }^{2}{ }^{2}$ (Cettétude a été esquissiè dans le Projet de Diet. zéallon (1903-4), 1) 16. Nous la completons et corrigeons dans ce qui suit : il faut notamment supprimer le $4^{\circ}$ de larticle bran; woy., ci-après, l'article selanbran.

$\left({ }^{3}\right)$ Voy. Godefroy : Meyer-Lübke, no 1273 . - Lall. mod. brand signifie "incendie".

( ${ }^{4}$ Ou mieux sans doute : "bondir et luurler à la fois ". Lobet dome aussi brandihèdje "lurlement" Pent-être le verv. braidi ou brêdi a-t-il déteint sur brandi; comp. le fr. brandi dans le Dict. général.

$\left({ }^{5}\right)$ Scheler à ce propos rappelle que l'all. branden "falaiser "se dit des vagues qui se brinent eontre les falaises.

$\left.{ }^{6}\right)$ Existe encore, avec (e dernier sens, à Ligneuville-lez-Malmedy (.J. Bastin).

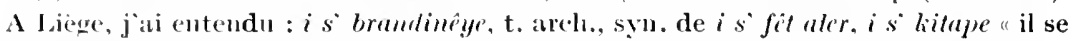
démène. il prend des airs dimportance "; aujourd'hui, on dit plutôt dans ee eas : i s' daurlinéye. 
d' bran "meneur de bran ". Cette danse est ainsi déerite dans IVallonia, xı, 193 : "Le eortège. formé de eouples qui se domnent la main, sautille aree des cris et des rires, au rythme de la musique qui le précède de quelques pas. Parfois, devant les cabarets où l'on va se rafraîchir. le mourement s'aceélère, les danseurs sautent de plus belle... " ( $\left.{ }^{\mathbf{1}}\right)$. Le sens qui ressort de tout cet ensemble est eelui dagitation violente, bruyante, tumultueuse.

Pour en revenir à branoolé, si la premicre syllabe parait séclairer. comment expliquer le reste? Je ne vois qu'une eonjeeture possible, eneore qu'elle soit assez hardie. Le substantif branoolé résulterait, par étymologic populaire, d'une expression archaïque *fé l' bran coter ( $\left.{ }^{2}\right)$ - e'est-a-dire "faire voler le brand" - laquelle se serait dite. au propre, du meneur de danse qui fait santer, comir. éroluer arec rapidité le cortige appelé bran(d). Le sens primitif s'étant oblitéré et l'expression n'ayant surréen que grâce à la métaphore, on aurait modelé "faire le branzolé "sur le type de "faire le fanfaron ". Mais. je le répète, ce n’est là qu'une hypothèse. en attendant que des témoignages plus explieites riennent éelairer la question.

\section{w. brigale, briguèle}

G., II 506, donne sans explication le w. brigale "soupe on brouet fait avee des pommes douces pelées. enites dans de la bière ". Le mot est en italique. ee qui signifie qu'il appartient au dialecte namurois. Cependant, à l'Ouest de Liège, on ne le troure signalé qu’à IIrntSt-Guibert (au Sud de Warre), oì dèl brigale désigne, parait-il, une "éturée de pommes de terre et de légumes ". Ce serait done plutôt un terme brabanẹon. - D'autre part, nous eomnaissons briguile 1. "soupe à la bière ", à Verviers et Tlumister $\left({ }^{3}\right)$; : " "bouillie, cataplasme ", à Fexhe-Slins $\left({ }^{4}\right)$. Ce mot, qui napparait que rers la frontiere linguistique, est emprunté de l'allemand dialectal brägel (Grimm) "soupe aux fruits, bouillie ", auquel se rattaehe le verbe brägeln (Weigand) "bouillir bruyamment. euire dans son jus".

(1) Voy aussi deux études folkloriques de Martin Lejeune, BSW 39, 1\%. 171-5, 191-203. - Aujourd hui, à Glons, miner lès brons est sỵn. de fé dès cråmignons.

$\left({ }^{2}\right)$ Pour l'inversion du complément direct, comparez en wallon : fé l' trôye danser (Spots, $\mathrm{n}^{\circ}$ 3015) ; djèl cèya a pan briber (Remacle, 2e éd., pârti) " je le vis (réduit) à mendier (son) pain "; en anc. fr. : faire le poce baler "faire danser le pouce, donner de l'argent "(God.) ; en rouchi : faire èl poûre voler (Wiers) "laire des embarras".

$\left({ }^{3}\right)$ Fé dèl b., magni dèl $b$. Voyez un exemple dans A. Doutrepont, Noëls zeallons, p. 231, et la note, p. 262, où l'éditeur hésite sur le sens exact de ce terme archaïque.

${ }^{(4)}$ Exemple : il a l' brès' tot tchèreljî d' cliss, on l'a tot coziè d' briguèle. 
anc. fr.-w. bulaine (?), quilaine (?)

Bulaine ne se reneontre que dans.Jean d'Outremeuse, Geste de Liege:

Cascun l'escarnissoit : les femmes de bulaine,

Femmes aux chevalirs et princesse hautaine... (v. 8991-2).

L'editeur Borgnet laisse passer le mot sans soureiller. Dans son excellent Glossaire de la Geste $\left.{ }^{1}\right)$. Scheler relève ce terme insolite. mais sans pouroir en pénétrer la signifieation. Le passage s'éclaire si on lit butaine, qui est une ancienne forme wallonne de lane. fr. butteme, bustame "sorte d'étoffe fabriquée jadis à Valenciennes" (2). Le sens serait : "les femmen rêtucs d'étoffe commune ". par opposition aux dames de haut parage.

Ene correction analogue permet d'élucider un antre endroit obscur de la Geste, r. 3s389. Il s'agit du récit d'une bataille:

L'endemain fait drechier une bele quilaine

(“on ayelle espringalle en paiis d'Aquitaine ( $\left.{ }^{3}\right)$.

Le Glossrime de Scheler dit à ee propos : "Je ne comnais pas ee nom de baliste et ne mon explique path lorigine : il doit ecpendant appartenir au domaine wallon puisqu"il est opposc au mot étranger espringale ». Le not étant incommu en wallon. je lis quitaine, forme variée de quintaine (') qui, an sens propre. désigne certain appareil serrant dans un exereice militaire du moyen âge. Iei, le rimeur liégeois, par unc de ces hardiesses qui caractérient hon style et sa versification, lui assigne le sens très génćral de "merveillenx engin de guerre ". Je rangerai done quitrine parmi les innombrables mots que Jcan d'Outremeuse revet arbitrairement dacecptions extraordinaires et dont le paticnt seheler a drensé la liste forément incomplete.

[Romaniu, t. XLV11 (1921), p. 551.]

\section{licog. busticlape}

Le liég. lusstirlup "plastron (de cordomnier) " est signalé seulement par lourir ; Lobet écrit bustelap en rerviétois. ("est évidemment un

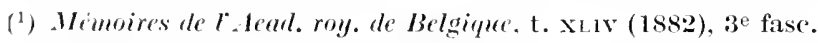

(2) God. BUTtexie ; llécart bustrixe. - En anc. w., nous tronvons : " ung costreal de futaine et ung de butane "(testament de 1+202, cité dans Bull. Soc. zoalh.,

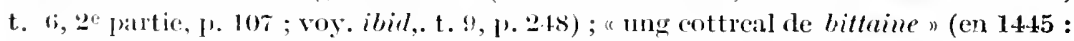

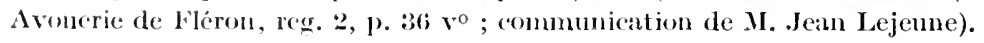

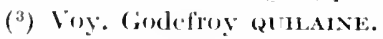

(4) Ia Geste donnc une fois quitaine et huit tois quintuine; voy. Scheler, $l$. $c$., et Godefroy. 
emprunt du néerl. borstlap, dont le second terme lap s'est altéré en clape sous l'influence du verbe claper (émettre un bruit sece, faire clap clap).

II. Léon Simon me signale à Ciney michitelape. s. m., "sorte de pince de bois, formée d'une pièce rigide et d'me pièce à charnière ; le cordomier sen servait pour maintenir la botte sur ses genoux ". C"est notre busticlape altéré de forme et de sens.

\section{nam. butin, blèti}

I Bourignes-Dinant, d'apres M. J. Nollet. butin signifie "glaęon flottant sur une rivière " a Xamur également (1). Pour le Mainaut, on possèle le témoignage de Sigart : "beutin. s. m. glaẹons qui, selon l'opinion des bateliers, sélerent du fond des rivières dans les fortes gelées ". - Nous rattacheroms ce mot a lanc. fr. beter "se figer. se coaguler, en parlant du sang; geler, en parlant de l'au s. Pour u protonique, eomparez le nam. fumile. strumer. mumute purdans (femelle, étremner, minute, prenons). oì le voisinage d'une labiale détermine de même le changement de e formé en u. Le suffixe est -in (lat. -amen ou -imen), qui a domé dans nos dialectes maint dériré indiquant le résultat de l'action $\left({ }^{2}\right)$. En somme. un butin c'est une masse coagulée, congelée, de même que le rerriétois blitin dévigne une masse blettie, un tas de fruits blets.

D'antre part. G.. I 5 s, cite le nam. bliti (lire -i). "1. derenir blet; ?. figé. caillé ". Forir donne ausi bleti song" "sang caillé ". expression que je n’ai pas retromrée a Liène. maic qui existait à Mahnedy en 1793. d'après Villers. Or un autre manuscrit malmédien du xrme siècle porte : do betti son, du sang grumclé ". Il est dè lor manifeste. conme le leeteur a déja pu le deviner. que bliti dans cette expression est mis pour beti (= ane. fr. betir. plus rare que beter).

P.-S. - Ce qui précède était écrit quand, après la télivmanee de Liège (2.4 novembre 191s), j’ai cu eomaissanee d'un article de M. Ant. 'Thomas sur l'anc. fr. bet, beter (Romania. 1916. p. 330). On me permettra de réstmer ici cot artiele qui intérense le w. bè (G.. II 502). Le savant français proteste contre l'hypothese de Mackel. Körting. Meyer-

(1) D’après G., I 87, et Delfosse (Dict. nam., manuscrit, 1850).

(2) Comparez inflin (Villers-st"-Gertrude) "enflure (a la suite d'me piqûre)"; foliu (Malmedy) "partie fonlée au milieu d'un chemin de voiture " ; ployin (Malmedy) "jointure (de la jambe)" : soyén (Faymonville) "terrain où la litière a été fauchée "; såclin (liég.) "sarelure "; ete. Voy. l'art. tièssèrin. - Pour le français, voy. Thomas, Essais, p. 371. 
Lübke, qui tirent beter de bet "colostrum " et ce dernier du germ. beost (all. moderne biest). Il affrme, au nom de la phonétique, que beter " se coaguler "smppose un type *bettare, d'origine incertaine, et que bet (d'oir le moderne béton) est un dérerbal masculin de beter. Il cite un texte de 1309 qui établit l'existence d'un déverbal féminin bete au sens de "congélation". et nous apprend. entre autres choses, que bete (lire bite?) survit dans les patois modernes, au sens de "onglée" (dép. de la Marne. ete.) et de "glaçon flottant sur un eours d'eau " (eonfluent de la Semois). - On roit que le nam. butin a de qui tenir.

\section{w. cakèdô et heûpon "gratte-cul "}

J'ai relevé calièdô (églantier : gratte-cul) dans l'Est du Brabant (Noduwez. Marilles. Jodoigne. Gistoux. Chastre. Perwez) et au Nord de Namur (Gembloux. Meux). Ce terme est inédit : Rolland lui-même ne domne rien d'analogue dans sa Flore populaire. Y 229. On y reconnaîtra le flam. et nécrl. hagedoom (aubépine). qui a passé par les étapes *hagèdor. *haliedó. pour aboutir à eakidô par assimilation régressire ${ }^{1}$ ).

Entre l’églantier et l'aubépine. la confusion n’a rien de surprenant: ces deux arbustes sont eommuns dans les haies : ilis ont tous deux des épines et des fruits rouges. Iu reste. l'all. hagedorn (littéralement "épine de haic") se dit à la fois de l'aubépine et de l'églantier. D'autre part. si le west-fl. hiepe signific "baie d'aubépine" $\left({ }^{2}\right)$, l'all. dialeetal hiefe est synonvme de hesgebutte "fruit de l'églantier. grattecul ". La divergence s'explique ici eneore par le sens général de "épine ", qui est celui du primitif anc. saxon hiopo. anc. h. all. himpo $\left({ }^{3}\right)$.

G., I 293. propose dubitativement de rattacher à ce primitif le liég. henpon "gratte-cul». Je tiens cette étymologie pour assurée. A première vue. la protonique en pourrait faire diffieulté $\left({ }^{4}\right)$; mais. si l'on compare le lién. reuipe. -er (rot. -er). qui est emprunté de l'all. bavarois rïlp, et si l'on tient eompte de ce que des dialectes du haut all. moderne ont hiefen, hüfen (uratte-eul), on en conclura que le liég. heîpon provient d'une forme bas-all. *hüpen.

(1) Comp. le fr. dégingandé pour déhingande. - Pour le traitement de la finale, comp. le liég. bolkhô hareng salur "dı moyen néerl. boxhôren ; ef. UTrix 253,

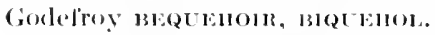

(2) Cr. De Bo, West-zl. Itlot. : "hiepe, fr. cenclle, péchalle (!), baje d'aubépine n. I'anteur fat an liég. pitthale llonneur de le prendre pour du français.

(3) Voy. Weigand unlw, lialk-Torp xye.

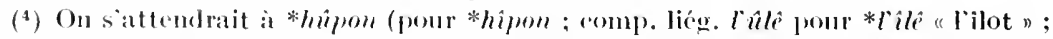

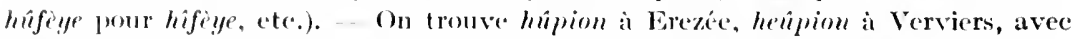
le sulfixe dimimutif -ion, fr. -illon. (comp. Fart. horon (à IIalleux horion). 


\section{liég. camatche}

Le liégeois camatche, s. m.. signifie "objet" en général : qu'èst-ce çoula po on - ? "quel objet est-ce lì ? ". Il s'emploie d'ordinaire au pluriel : dès bês p'tits - . "de jolis bibelots. des jouets"; wistez tos zos —. "ôtez, enlerez tous ros objets"; mite tos lìs z'îs - a grinî. "mettre au grenier toutes les vieilleries "; mite sis bons - ses bons vêtements.ete.

Le mot est inconnu en namurois. Plus à l'Ouest. à Houdeng (Hainaut), on nous signale gamache "tohu-bohu. embrouillamini " ${ }^{1}$ ); à rapprocher de notre camatche qui. à Crehen (Hesbaye) et en Famenne, ne se dit qu'au singulier et aree le sens de : "embarras. eonfusion d'objets " : qué camatche! "quel désordre !".

Delbœuf (BSIV 10, p. 97) y roit le fr. camail appliqué aux rêtements de fenme, ce qui se passe de réfutation. G.. I !s. propose lane. fr. gamache, all. kamasche "guctre, ; plus loin. II xrr. il retire arce raison $\left(^{2}\right)$ cette eonjecture. mais sans rien mettre à la plaee. - Pour moi. je suppose une forme première *gamatch $\left({ }^{3}\right)$, qui serait empruntée de l'all. gemacht. néerl. gemaalit, participe de machen. maken "faire". Le préfixe ge- a été traité comme dans gamin. que l'on tire du germ. gemein "commun" $\left.{ }^{4}\right)$. Pour la finale, eomparez batch, bae, hatche, hache, ratche. vaehe, et l'expression liégeoise taper la hatch it match "jeter ses outils, renoncer à la besogne ", proprement : "jeter (tout) pêle-mêle. en un tas ", de l'all. hack und mack "mélange confus " $\left({ }^{5}\right)$. Le sens primitif de camatche serait done : "objet fabriqué " (etwas gemachtes, ein Gemächt ; comp. l'all. gemaehte Blumen : fleurs artificielles) ; d'où : "objet " en général.

\section{liég. canabûse}

Ce mot signifie "sarbaeane" : lis-ifants soflet dis peûs arou 'ne canabûse (syn. soflète). G.. I 99. éerit cane-ì-bìse. eomme si c'était proprement une "canne à turau ". Cette explieation n'a manifestement que la valeur d'une étymologic populaire. Le néerlandais appelle

(1) Communication de II. l'avocat Hubaut.

(2) A l'anc. fr. gamache répond le w. gamasse (G., II 528); de même à l'anc. fr. flache, all. flasche, répond le w. flasse à poûre, poire à poudre.

$\left(^{3}\right)$ Pour $g>c$, comp. les articles kich'tôn', gistèl, spricatzuére.

${ }^{4}$ ) Pour $e$ protonique $+m>a$, comp. cramer, écrémer, tamon, lat. t emonem, et voy. l'art. djama.

$\left.{ }^{5}\right)$ Ou mieux de l'all. hack und pack hinwerfen, en admettant que pack aurait donné * patch, qui se serait ensuite altéré en match. 
linapbus (1) m autre jouet qui ressemble à la sarbaeane. à savour la canomière. - que le liégeois appelle bouhale. Malgré la différence de signification, il y a me telle ressemblance de forme entre linapbus et eanabise que je n'hésite pas à voir dans le second un emprunt du premier. Pour l“insertion de a dans le groupe initial lin-. comparez le fr. cumapa (de l'all. limappsach). canif et comizet. La finale s'est allongée sour l’influence de biise (tuyan); comp. harlibitse (arquebuse).

\section{fr. canepin, w. ard. kèn'pin}

Le tr. entepin signifie, a après le Dict. gén. "peau fine d'agneau ou de chrreau dont on se sert pour essaycr la pointe des laneettes, bistouris. etc..." Lo Ligine en est incomnue. Il faut remarquer. comme le dit Littré. que eanepin a aussi signifié la pellicule prise au dedans du tilleul. C"est même le seul sens que dome, en 1715. le Grand Dictionnaire royul du P. Pomai : "peau d’arbre fort déliée dessus ou dessous de l'écorce. lat. philyra. cuticula pertenuis arborum ". D'autre part, Godefroy a cet exemple : "Du fust (du papyrus) on cn fait des barqueroller. et de sa teille. de la pelure ou camepin. on en fait des roiles. nattes, linges. etc. "(E. Binct. Merr. de mat.. p. 369) $\left({ }^{2}\right)$. On peut se demankler si le mot ne sest pas dit d'abord de la pellicule du paprous. Dans ce cas. Ie teme de mégisnerie penu de eanepin, qu'on trouve dès 1310. sinnificrait "p pau ausi finc que du eanepin ", ct eanepin. au sens domé par l. l)ict. gón.. serait proprement une métaphore. S’il m'était permis d’émettre une conjecture étymolugique. je rerrais dans ce mot une altération de *amopin. dérivé de Camope. Kx́wpos, ville du Delta. Pour la dérivation et pour la sémantique. comparez godemetin (Ant. Thomas. Wélunges d'itym. fr. p. 85 : "cuir de Gadamess "), marocain, clusirin, ane. fr. bundequin, ate.

(2uoi qüil con scat. si le mot. ch France. n’apparticnt qüà la langue technique. il est intéressant de constater que. dans un eoin ì l'bst de la lbelgique. le langage courant le prend au sens figuré de "aeabit, genre. cypece. earactère ". J'ai relcré en effet dans nos Ardemes (au Sud de la provine de liege les exemples suirants de ee terme inédit :

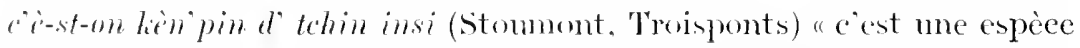

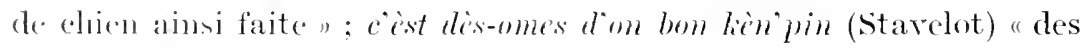

(1) Compone de linap. arace, at de bus, boite. canon (de fusil).

$\left.{ }^{2}\right)$ (ionl. (I is6, VIII 419) donne les formes cancpin, eanequin, quenepin. Joignez-y carpin (:) dans Jean d'Outremense, que scheler, Glos). te la Geste de Liege, ne peut détinir. 
hommes bien portants et d'humeur joviale". De mêne, à Erezée (au Ford de la pror. de Luxembourg) : on fièrt lien" pin " un solide gaillard"; on drole di lien"pin "un original " cist dis cis quent on drole di lien pin "des gens qui ont un singulier genre de rie". - Pour la métaphore. comparez le w. coyin "caractere" $\left({ }^{1}\right)$. dont le sens propre "peau [de mouton], cuir [de porc] "survit à Malmedy $\left({ }^{2}\right)$.

[Romania, t. xivil (1921), p. 552.]

\section{w. caribôdèdje, caribôdion (Verriers)}

Ces mots sont inusités à Liège. Lc premier est dans Remacle, ge éfl. qui le définit : "patarafe. traits informes, lettres embrouillées. syn. grabouyidie : Serait-ce, demande G.. I 101. une forme déreloppée de crabomŷ, gibouller" - L'explication est autrement simple. Le

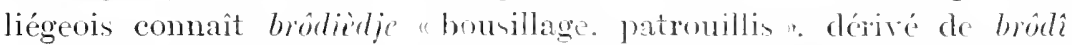
"bousillex. travaill"r mal ". De là, le composé curibrodicdie. qui kevient par dissimilation caribródidje (Lobet, p. 26s), puis curibôdidie. De mênc le synonyme curibôdion vient de caribrôdion. connosé de brôdim. "petit objet embrouillé. gribouillis". - Le préfixe cari- a unc valeur péjorative, comme le fr. chari-(charisari), cali- (califourchon), coli(colimaçon), gali-(galimatias. galimafrée). ca- (ealoosser). En wallon, nous relevons des formes tout ansi variées : cari-. cara-, car-. cra(voy. l'art. carimadjôye), cal-, cas- (calmoussî. casmoussî). ca- (caboûre. cafouguî, cte.). $\left({ }^{3}\right)$.

[Mélanges Kurth (1908), t. 11, P. 31\%.]

\section{lićg. carimadjôye}

Le liég. carimadjôye. carmadiôye désigne. d’une faẹon générale, une "confusion bizarre (de conleurs, de traits, de sons, de gestes ou de paroles) ". L'exemple le plus ancien. daté de 1758. se lit dans le Théritre liégeois. p. 13r : un hypocondre critique arec humeur la mode musitale du temps. "Il faut aujourd 'hni, dit-il. des airs italiens,

(1) A Liège et Verviers : il c̀st don maiva coyin, don si bon coyin : aji "l" so nin di $c$ ' coyin la ; c’è-st-on si bon coyin; métaphore analogue au français : "cest une si bonne pâte $n$.

(2) Daprès une note de l'Armonac', Jalmedy, 1911, p. 66; à propos du texte : on bouquin r'loyi d' coyin. Villers, Dict. malmédien (1793), donne seulement : coyin " eouenne ou eoine, peau de lard ". - On ne trouve rien dans Grandgagnage sur ee mot, non plus que sur la forme féminine coyimne (küyẽu") "conenne " (Liège, Verviers, Stavelot), -ine (Wardin), -ème (Stave), - öne (Péerot-Chaussée, au N. de Wavre). Sur l'étym. lat. eutina, cf. Meyer-LïJ)ke, $n^{\circ} \mathbf{2} 431$.

${ }^{(3)}$ Sur le préfixe ca, voy, les Mélanges Kern (Leide 190:3), pp. 12:3-6; Feller, Notes de phil. w., pp. 222-237. 
Dès carimadjôyes, dès firdaines

Èt tos erinèdjes qui d'nèt l' migraine,

c'est-à-dire "des fioritures. des fredons (1) et tous grineements qui donnent la migraine ". Voiei des phrases que j’ai entendues à Liège : si moussemint n'èst qu'ine car(i)madjôye (une réunion bizarre de couleurs) : ile arente dés c. a s’tchapê (des enjolivures multicolores) ; fé dès $c$. azom dèl crôye (des dessins connus à la craic) ; èle mi fêt ne tiesse come on sèyê acoul totes sès c. (ses propos sots ct déeousus) ; ni fes nim tant dès e. (des politesses exagérées en gestes et en paroles).

Sous des influences diverses. la forme et le sens ont subi en liégeois des altérations isolées. La finale, confondue avec diôye (joie), a ralu à carimadjôye l'acception de "divertissement, réjouissance " (Remaele, $2^{\mathrm{e}}$ éd. ; Forir). L’analogie de crama (crémaillère) et de crås (gras) a dommé cramadjôye, cramadjôye "fête, festin ". D'autre part, caramadjôye ("bigarrure": Dur.) paraît aroir subi l“influence de ramalje (" ramage "). influenee qui est manifeste dans la forme simplifiée ramadjôye ("suite de paroles vides de sens": Hubert. ap. G., II 273).

Ailleurs. il faut noter les variations suivantes : caramidjoyes (" enjolivures extravagantes " : à Marche-en-Famenne) ; carabadjôye ("griffonnage : a Malmedy. Stavelot); caraboutcha (id. : à Herve); carimadja, carimudion (à Glons-sur-Geer, au sens général du liég. carimadiôye).

Nous eomnaissonu de phus deux dérivés : l'ard. carimadjôréces (" dessime confus : politeres excessives " : à Erezée), s. f. pl.. qui paraît être mis pour -djôyerices. avec le suffixe -eréce (-aricius) : — et le liég. calpumaljôyelé (" Jigarré bariolé : Dur.), dî au croisement de caróyeler (rạer) et de caramadjôye, de même que le liégg. caramarôyeler "chamarrere): Rour. a $)$. (... II 509).

G.. I 101. ne domne pas d'explication. Pour ma part me fondant surtout sur la forme dialectale crmimadia (Giloms), je suis porté à rattachere curimadjoyge an ir. salimatios. Ce dernier, d'apres MeserLiblke. $1^{0}: 38: 3 \%$. vicudrait du latin grammatica. par l"intermédiaire

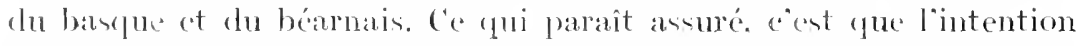
satiripue et l'étymologe populaire (influence notamment du préfixe péjoratif cari-. salli-) ont dî anuence des déformations bizarres comme celles fue nows aroms signalées dams nos patois. comparez aus surplus,

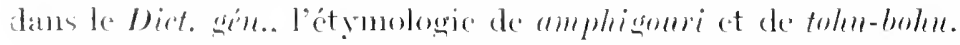

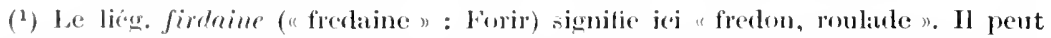
servir d':urgument a ceux qui, conme Littre, pensent que fredaine et fredon ont une origine conmune. 


\section{w. cåveler, rucåveler (Verviers)}

G., II 301, enregistre le verv. reciceler "abuter [de nouveau]" (Lobet). et le mahm. rềceler "réitérer, faire da capo ". rễelèdje $\left.{ }^{(}\right)$ "l'iterum. la table de multiplication " (Villers). qu'il dérive du provençal rechap, fr. rechef. Cette explication est inadmissible.

Outre le composé, Lobet. p. 2r1, a le simple careler "abuter ", dont G. aurait dî faire état. En terme de jeu. e'cont lancer des billes, des palcts, des quilles vers le hut pour déterminer quel doit être l'ordre des joueurs. Il importe de ne pas le confondre, comme parait le faire Lobet, avee caiveler " eneaver, eneuver. enchanteler ", dérivé de caive "cave ". Le terme de jeu (m)coiceler, qui n'existe que près de la frontic̀re linguistique, reproduit le néerl. licelen "tirer au sort". Dans le llamand de 'Tongres. licielen a le même sens spécial que le mot verviétois.

Le w. caiveler, t. de jeu, parait avoir disparu. Du moins, un auteur verviétois (BSW 53, p. 418) donne ì la place : cosseler "abuter". rucôsseler "abuter de reehef " $\left({ }^{2}\right)$, tout en attribuant à rucizeler le sens de "remettre (de l'argent ou des billes) au jeu " $\left({ }^{3}\right)$. — I FaymonvilleWeismes. rëcâteler c'est "faire une seconde séanee de jeu dans une autre maison, après les sîses (soirée. veillée). au lieu de rentror ehez soi ";

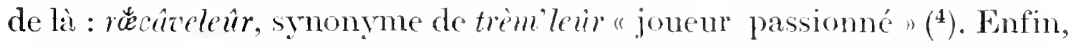
à Petit-Thier lez-Vichahm, richeler. v. tr.. n’a plus que l'aeception métaphorique de "ressasser. rabâcher "i ŕchòle toudi sès vês mèssèdjes. Ainsi s'explique le dérivé ruck̂́cledje "table de multiplication ", que Viller's seul signale et qui est anjourd'hui inconnu à Ialmedy : ècst l'action de répétër la même formule : (comp. l’all. das Finmaleins.

[Remaniement d'un artiele du BD 1907, p. 142.]

anc. fr. chaon

Godefroy définit cham : "partie du lard qui ne se fond pas à la poêle et se grille, grésillon ". II ne cite que deux excmples. l'un tiré du

(1) Et non rácéclêie, eomme écrit G. : loriginal de Villers porte rä̀leje.

( ${ }^{8}$ Dorigine ineonnue. Comparez le ncerl. kcuzelen jouer aux lilles (voy. Franckvan Wyk, s. vo ; Schuermans limizel, -cn). - En liégeois, "abuter " se dit samer (ex-aestimare).

$\left({ }^{3}\right)$ De même rucãcler (Trembleur), ricâceler (Ferrières) signifient "remettre de l'argent au jeu de bouchon pour reeommencer la partie ".

(4) Dans les environs (Sourbrodt, Ovifat, Robertville), on emploie dans le même sens r'tchacer, -ê̂r, qui se rattache à l'anc. fr. chuncer "jouer à un jeu de hasard". 
Ménagier. l'autre de la traduction des Psaumes (dćbut du xir siècle) : " ct mis os cum chaons sechirent" (God.. t. II. Errata). Il faut y ajouter cet article du Catholicon de Lille. éd. Seheler, p. 49: "creminm. chaon. creton. cest la char qui demomre apris la craisse ". - Je vois dans ce mot un diminutif en -on du moyen néerlandais côde "crôton de graisse grillée " mogen bas all. litide ; néerl. laum (eontraction du pluriel kacyen), que Plantin traduit par "ratons du sain. de poureeau ". Le flamand liale. liaai existe eneore aujourd'hui avec le même sens en Campine et dans les provinces belges d'Anvers et de Brabant (1). Lanc. fr. chan se retroure dans les patois modernes de Metz et du département de la Meuse. sous lws formes chä̈on. chö̈nn, chaon. chom $\left.{ }^{2}\right)$ : je ne sache pas quion ait déjà proposé une explication de ce mot rlialectal.

[Romanie, t. XLv1 (1921), ץ. 554.]

\section{fr. chicaner, w. chakiner, tchakiner}

Pour le Dict. général. le fr. chicaner est d'origine inconmue. A côté des propositions qu'on trouvera dans Littré, Scheler, Körting, ete. le wallon suggère une explication des plus simples : clicaner serait altéré de (t)elualiner. forme conservée dans nos dialectes et dérivant, à l'aide du sullixe diminutif -iner. de lonomatopée (t)eleac. qui exprime un petit choc brusque.

La forme wallonne existe sur des points très divers : à Malmedy, tchakiner. -eur (Villers. 1793) "chieaner, -eur"; - dans les Ardennes, chalimer "tricher au jeu s $\left({ }^{3}\right)$ : — à Givet, chakini" 1 . chicaner, tracasser : i m' chaline tomdi : 2. v. intr., tromper an jen "(J. Waslet) ; dans le Brabant. chaline: (Nivelles. Bornival) "1. chicaner. quereller : il ont enuminchi acheliner inté yexis" : 2. tricher au jen "; chaline (ibid.),

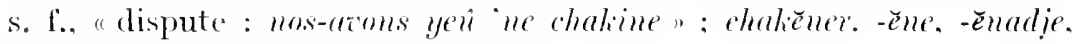
-ĕchì (Chastre-Villeroux) ; etc. $\left(^{4}\right)$.

Nous trourons le primitif à Awenne (pror. de Luxembourg, entre Grupont (t St-Hubert) dans l'expression chalie lès moins "battre des

(1) (f. Schucrmans, Franck-ran Wyk. - Voy. ci-après l'article erèton.

$\left({ }^{2}\right)$ Voy. les glossaires de M. Lorrain, de Labourasse et de Varlet.

${ }^{(3)}$ Ce sens dérive naturellement de celui de "chieaner, vétiller"; eomp. étricer "tricher au jeu "(Watteeuw, Tocab. de Tourcoing).

(4) (.., I 1.t9, donne simplement le nam. chakine "chieane". - Le liégeois ne connait que (t)chicume, -er, -êt, -ìlje, -erìye et même (t)chicunier : e'est du français à peine wallonisé. 
mains, applaudir"; à Stave (Namur), tchakè "trotter brusquement (par ex. une allumette) ". tchake-fen "briquet avee silex et amadou "; à Buzeno] (près de Virton), tchaker "faile du bruit (en mangeant)": lès pouchés (pores) tchakant acon lề suemue. A Faymonville-lez-Malmedy, tchacant se dit de l'ocil vif. brillant de joie, dont le regard frappe, vous donne un ehoe quand vous le rencontrez: il a les-lis tchacants; come i loulie tchaeant! - Lonomatopéc tehae entre dans les expressions suivantes : çoula tome "thar (Fontin-Esneux) " eela tombe à point"; avou çoula, ci sèrè l' tchac (ib.) "avec cela, ce sera parfait " ;'śstent dès tchic èt dès tchac a n' nin fini (Liège) " e"étaient des comps de langue, des pointes, de vertes ripostes à ne pas finir " $\left.{ }^{1}{ }^{1}\right)$; dinaer al tchac (Verviers : Lobet). espèee de jeu de billes; d'où tchaliète tehaliter', cte. $\left({ }^{2}\right)$.

De chaliner à chicaner le passage est faeile : il s'explique par une métathèse naturelle et par l'influence de chiche. chiquet. chicoter, ete. On objectera $1^{\circ}$ qu'un primitif chicaner a pu lui-même s'altérer ehez nous en (t)chakiner sous l'influence de (t)ehac et de taquiner : : ${ }^{\circ}$ qu'il peut ne pas exister de rapport d'origine entre chicaner et (t)ehaliner, dont la ressemblance serait done purement fortuite. Sans doute ; mais je me persuade que l'autre thèse est plus rraisemblable et quelle mérite tout au moins d'être prise en considération.

[Romania, t. XlviI (1921), p. 55t].

\section{w. cîçale, cinçale}

Forir est le seul qui domne cîcale. s. f.. "furonele ". Les Liégeris que j'ai interrogés ne connaissent pas ce mot ; mais, à Glons-sur-Geer, d'après M. Mathieu Fréson, des "inflammations sur la peau "s'appellent des cinçales. Cette dernière forme est mieux eonservée, et la signnification qu'on lui attribue, eneore qu'assez vague. me parait plus sûre et plus exaete. Partant d'un type supposé cinctum + -ieella, je pense que, primitirement, les cinçales désignaient l'herpès zoster ou zona, éruption vésieuleuse que le liégeois et le namurois dénomment li cingue ou lès cingues (ceinture: eingulum). Pour la protonique - $i n=\hat{\imath}$, comparez cintrồ. cîtrồ (lisière de drap) : einetorium (ou cinctura) + -eolum: cincième, cîczème (Pentceôte) : quinquagesima. Pour le suffixe, comparez monçule (à Harzé : tas de gazon d'essartage) : *monticella ; nècale (nacelle) : narieclla ; saçale

(1) Le liégeois, en français familier, dira de même : ils sont toujours en chic-chac".

${ }^{2}{ }^{2}$ Sur le dérivé tchalitrèce, voy. Feller, Notes de philol. zall., 1. 219. 
("espèce de plante saurage " : G.. II 342) : * salicella ? ; queçale (dans des lieux dits; anc. fr. raucele. vallon): * vallicella.

$$
\text { anc. liég. cincque (= singue) }
$$

Le passage suivant de la Charte liégeoise du Métier des Fèrres. 1+18, contient trois expressions que G., II 550, 56\%, 63\% signale sans pouvoir les expliquer :

Quclconques dorsenavant metterat a esscience ou de certaine science en anvre fiere embleit, ou qui fache faire cincque contre cincque cleiffs encontre enscingne de paest, de chirre ne d'aultres semblables cas, ne quy achapterat koemme ne anko embleit, et pronveit soit suffisamment que ce ayet esteit à scicnce, icy perderat ledit Mestier... $\left.{ }^{1}\right)$.

Le règlement défend de mettre sciemment en cuvre du fer volé (embleit) et de commander sans témoin qu'un forgeron fasse des clefs d'après une empreinte de pâte, de cire, ete. Cincque devrait s'éerire singue. car il répond à l’anc. fr. sengle. single. sangle (lat. singulum) "chacun en particulier. seul. isolé ". La curieuse expression singue contie singue (en tête-à-tête. cntre quatre reux) ne se rencontre pas ailleurs. La finale. que nous éerivon -gue d'après l'étymologie, se prononce -li en wallon; de même dans tringue "tringle " cingue "sangle, ceinture ".

Les mots loomne et anko sont des noms de métaux, défigurés par la négligence du copiste ou de l’imprimeur. Il faut corriger le premier en koenne. lioence : c'est le liég. mod. ken̂ze "cuirre ". Pour l'autre, je conjecture une fause lecture de arto. Godefroy. vo archal, cite les formes arcon. archout, archant. Le liés. mod. ne connait que airca. mais areô existe encore dans le centre du Hainaut. par exemple à La Louvière: voy. Irojet de Dirt. wallon. p. 15.

\section{w. coper (Liège. Huy), ècoper (Terviers)}

Dans certain jeu d'enfants. toneluer un joueur quion atteint à la eourse se dit cöper à Liège. Jupille. Jemeppe. Huy : dii r's-a copé ; ou écoper à Verviers. Starelot : din r's-a ceopé. c’èst ros qu'èm" ist " qui en est ": qui devez poursuire ì rotre tour). Le jeu comporte plusicurs variétés: la plus simple ressemble au jeu que le Larousse illustré appelle "chat" (on dit à Jupille et à Seraing : djonacer al pouce "à la puce" ou al cöpe. déverbal de cơper : a lluy : djouwer a cöper); celle

(1) Chartes ct Privileges, I 29. 
qu'il dénomme "chat coupé "s'appelle à Huy le cò̀per r"côpé ; une autre, le "chat perché ", se dit à Jomeppe le cŏper pirché. On cŏpe aussi au jeu de barres. Les verbes coper, écoper (qui nont évidemment rien ì roir avec côper : comper) ne figurent pas dans nos dictiomnaires $\left({ }^{1}\right)$. Ils ont cependant du prix a nos yeux, car nom y reconnaissons l'anc. fr. cou(l)per, encou(l)per, encoper "inculper, accuser", lat. culpare. inculpare : colui qui est ç̋pé ou ćcơpé est mis en faute ; c'est l'inculpé. le coupable. qui doit travaillex à se libérer. Les enfants ont conscrvé ces termes quils tiennent sans doute du langage ecclésiastique ou judiciaire.

\section{liég. cot'hê. cotî}

Le liég. cothê " closeau. jardin potager "se rattache à cotî "maratcher " [nam. cot'lî], fém. cotiyerèsse "maraîchère ". cotiedje [franeisé à Liège en "cotillage $]$ "terrain de la banlieue dans lequel on cultive des légumes pour les veudre ". Ces mots, f’après G.. I 129. 342. viennent de l'all. liothe (chammière, petite métairie), köther (nmanant), lötherei (petite métairie). Notre anteur invoque le bas latin cotasium. l'anc. fr. cotier (tenant d'une coterie ou terre roturiere). et il ajoute : La ressemblance de l'anc. fr. courtilier, -age, m. signif. que cotî. -iedje ne saurait faire conchure à l'identité de ces mots, de mòme que le ronchi courtisean (petit courtil) ne pourrait être le même nut que le liég. coth hê : comment, en effet. cotî serait-il dérivé de corti ?"

En réalité. l'all. kothe n'entre pour rien dans l'origine des mots wallons ct cot'hê est bicn l'anc. fr. cortisel; coth. s. m.. est l'ane. fr. cortillier, et cotièdje l'ane. fr. cortillase. A l'antépénultième. $r$ suivi de deux consonnes disparait de mème dans tot'ict (= fr. tonrtelet : G., II 437. n.), mascacer (= anc. fr. mareschancier). Hesta (= Haristallium : Herstal). Ross"ner (Faymonville: "trousser", dér. de leoser à Weismes; all. schürzen) ; etc. Le nom propre Cortchai existe encore aujourd'hui, comme nom de famille, dans la vallée du Geer. Les archives liégeoises foisoment de cortiheal, cortiseau. ete. pour dénommer des lieux qui s'appellent de nos jours cot hê. Enfin, cot hề désignant le potager attenant

(1) Grandgagnage lui-même les ignore. Il note seulement, sans explieation, dans ses Extraits de V'illers, le malmédien ècope "se dit quand on saisit qun, dans le sens : vous êtes à moi, vous êtes pris". C*est apparemment l"ahrégé de dju v"s-ècope, plutôt que è cope! (en faute !). - A Robertville-lez-Malmedy, eoper, v. intr., a pris le sens de : "jouer à cache-cache, appeler en étant eaché " (131) 1908, 1). 32). - A Glons-sur-Geer copler "atteindre à la course ", t. du jeu de "puce ": dji l'a coplé. 
à la maison. il est naturel d'y voir un diminutif de eorti (terrain elos, assez étendu. contigu à l'habitation) plutôt que le dérivé d'un primitif germ. liothe $\left({ }^{1}\right)$. Les mots liégeois sont donc bien distinets du fr. eotier, eoterie, cottage et de lane. fr. eotin (eabane, maisonnette ; voy. MeyerLülske, $\left.n^{0}+7+6\right)$.

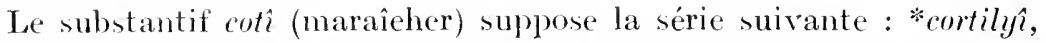

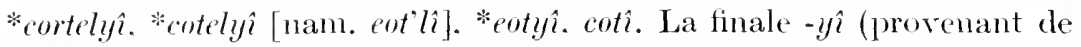

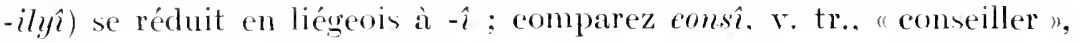
trầ. ane. fir. traveillier (être en travail d'enfant). Le nam. eot'lî a conservé l que le mouillement a fait tomber en liégeois. mais dont le fém. cotiresse (provenant de eotiyerèsse) atteste la présence à l'origine ; comp. cinsî. cins'rèsé ; botî. but ièsse.

Enfin le verbe cotî lui-mîme (marcher. se promener : G., I 130) représente l'anc. fr. cortillier (cultiver un jardin). Le sens propre " eultiver, cest-à-dire circuler daus son jardin et conduire au marehé les produits de son eoticldie ou cortillage "n’est pas noté, que je sache; j'ai eependant entendu à Liège : i eotèye a Hacou "il fait le maraîcher à I Iaccourt ". Nos dictionmaires n’attribuent à ce verbe que des sens dérivés: 1. circuler, marcher, aller et renir: i rataque a cotî $\left(^{2}\right)$, dit-on d'un convalescent : - 2. flâner. baguenauder : come i n'a rin a fé, i cotèye tot-aial mohone; èle cotèye tot-äa s' manèdje èt èle n'avance nim. dit-on d’une mauraise ménagère. D’où le dérivé eotient, -eûse "flâneur. -euse ".- Par une métaphore contraire. eotier à Faymonville signifie "circuler aetivement. se dépêcher au travail. marcher vite ": i eotèye tote djôr : i $t$ fầe cotier, si tö arous arizer a tins. Un composé sü d'cotier s’y est mîme formé sur le tỵpe de sǘ d'hombrer "se dépêcher" (BSW 50, p. 555$)$.

[Hélanges Kurth, II :318 (1908). La fin surtout est remaniée.]

\section{fr. couet}

Le fr. comet, terme de marine, désigne une "grosse corde qui s'amarre au has d'me roile de navire ". Körting (Etym. Wört. der fr. Spr., 1908), so fomdant sur le nom allemand de ee eordage (Hals eines Segels), y

(1) Dn germ. kot, moyen hant all. kote. kole "lutte, maisonnette de paysan" dérive le flan. Loterij (ensemble de taudis, de petites remises pour tonte espèce

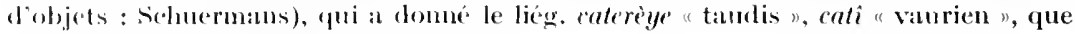
G., I 10:3, ne réussit pats à expliefuer.

(2) Symonyme : i roblye, d'un verbe ricoti, qui manque dans nos dietionnaires. A . Nle-sur-somois, on dit de mêne : i retcampil. 
voit un diminutif de con ; mais un tel dérivé ferait singuliere figure à eôté de collet. Daprèn le Dictimnuire général. conct est me autre forme de écoute : Cotgrave domne en effet esconctte pour ćcoute. Toutefois l'argmment ne parait pas décisif. éscomette pouvant ctre unc creur, ou une contamination de écoute et de couct. D'autres dictionnaires ont bien écouet pour eouet : mais c’est sans donte le rénultat d'mne fausse perception : le phuriel les conets est devenu l'éenuct. - A mon aris. conct représente le masculin de conette (petite quene). La définition de Godefroy (comet : "un cordage qui ra dimimuant par un bout ") me paraît suggestive à cet égard, surtout si on la compare à celle du liégeois cowète, terme de houilleric. "partic du eâble reliéc à la cage ": cette partic inférieure est d'ordinaire en seetion décroissante; de là son nom, qui signific proprement : "petite quene, petit bout".

[Romanit, t. xuril (1921), 1. 555.]

\section{liég. coumê, coumaye, fr. coumaille}

Il fant réunir ces trois articles d. G., I, 122. 131 ; II. 529 :

comai (petite enchme sur laquelle les faucheurs battent lemrs fux) B.

coumai (erosse petite lemme mal bâtie) Hêv. Comprarez commi.

goumai (dégorgeoir: outil de maréchal ferrant ; tasseaur: petite enclume ;

ponme d'Adim) Loв. Comprarez gomi.

Il s'agit d'un même mot (comê à Glons-sur-Gecr, eommê on plus souvent goumê à Liège). qui répond littéralement au fr. "enclumcau "; coumê provient. par aphérèse, de ${ }^{2} \dot{e} e m m \hat{c}$. diminutif de ècome (Glons, Trooz. Mahmedy). liégeois èglome. enchume, latin incudinem (I). Du sens premier "enclumeau "dérivent, par métaphore naturelle, ceux de "personne courtaude et massive " $(2)$ et de "pomme d'Adam".

Le fr. dial. et techn. conmaille, terme de minéralogie. a reçu les honneurs du Dictiomaire géméral ; mais l’article qu'on lui comsacre laisse bien à désirer. La définition : "roehes $\left({ }^{3}\right)$ des mines oì la houille est divisée "manque de elarté. La plus ancienne mention scrait de 1818 ; or nous trouroms le mot cité dès 1768. Enfin on le déclare d'origine inconnue ; nous pouvons assurer qu'il est emprunté du dialecte liégeois, comme beancoup d'autres termes de "houillerie ". Une description de

(i) Comparez le heshignon ègloumiu (Cras-Avernas), gloumiu (Darion) " enclumeau de funcheur ", et roy. BD 1911, p. 36 .

( ${ }^{2}$ Comp. dans Forir magoumi" "magot, petit homme mal bâti ", qui résulte du croisement de magot et de goumê.

$\left.{ }^{3}\right)$ Corrigez roche. MIême dléfinition dans Littré et dians Lobet, d. 303. 
cette industrie au paỳs de Liège, faite en 1768 par Morand et rééditét en 1780 ì Neufchâtel. porte ce qui suit :

Dans le dric [ = deye, sol de la galerie] et de tems en tems dans le toit, se reneontrent des marons, gros et petits, bien polis, de conlenr noirâtre, qui font feu contre lacier et gâtent les outils ; ces clors... sont appellés à House,

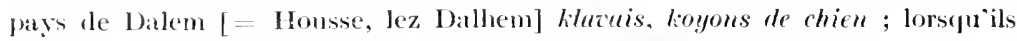
sont d'un très grand volume, on les y nomme koumuilles ( ${ }^{2}$ ).

Le liégeois commaye ou. phus somvent. gommaye a. d’après G.. I 131. trois significations: " 1 . bloe de briques réunies par un commencement de fusion ; 2. mâchefer : 3. t. de min., rognon arrondi, très pesant et très dur. de chaux carbonaté fétide". Le sens 1 est le plus eomu du vulgaire et de nos lexicographes. Hubert et Forir ajoutent une aceeption figuréc: "femme eourtaude et indolente", qui rappelle notre gommê de tantôt. Et. de fait. nous trourons dans gommăye le même radieal avec le suffixe collectif -aye. lat. -alia (comp. le fr. ferraille, pierraille. rocaille). Le sens étymologique cst done : "agglomérat de substances (minerai. argile dureie au feu. etc.). dont la masse et la dureté rappellent me enclume".

[BD 1920, p. 7.]

\section{w. ard. coyonke, coyongue}

La cöyonlee. dans nos Ardennes (Stavelot, Bovigny, Villers-SteGertrude). cest la longue courroie qui fixe le joug sur la tête du boeuf. II. Ch. Bruncau a relevé cette même forme au Sud de Givet, à Harguies et à Sérigny-la-Forêt $\left({ }^{2}\right)$. Par eontraction, à Faymonville-Weismes, le mot devient côlie. arec " mi-nasal $\left({ }^{3}\right)$. On dit cöyonpe à Cherain. Lutrebois, Ortheuville, II , ufalize. Reeogne. Neufchâtean et aussi, d'après II. ('h. Bruneau, à Cugnom-sur-Semois et à Louette-St-Pierre. J'ai relevé de plus : 1. clöymbe, à Alle-sur-Semois, avee l épenthéticpue sous linfluence probable de clôre " elore " et de clôye "claie " : 2. le

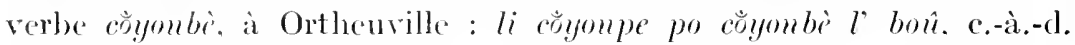
po loyge l' bon après l' djề (au joug) ; à Bonnerue-lez-Houffalize. le

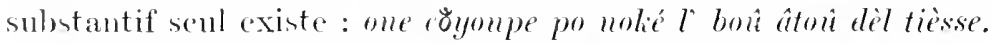

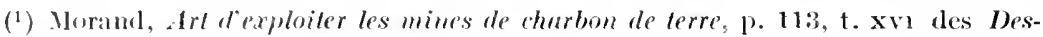
cripliosis des arts el méliers, nouv. éd., Neurchatel, 1780, in-4o ; 1 re éd., 1768,2 vol. in-folio. Bormans, Ioc. des homilleurs liegeois, dedinit commaille "pierre plus dure

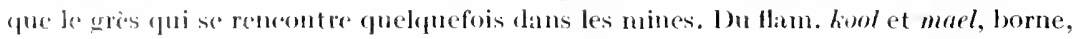
limite, etc. (!).

$\left(^{2}\right)(1$. Brumsitu, Emquôte sur les putois d Ardeme (1914), I, p. 499.

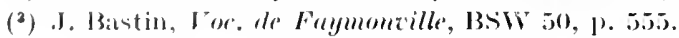


L'origine de ce mot intéressant est pleinement assurét : il reproduit le lat. co(n)jungula (petite chose servant à conjoindre), qui a domné l'ane. fr. co(n)jongle. d'où la forme contracte congle dans une eharte namuroise de $\left.1265{ }^{1}\right)$. Le w. coyoulie - ou. étymologiquement, coyongue, - s'est altéré en coyoupe (-he). comme ranonke "renoncule" en ranonpe (G., II 279).

Meyer-Luibke, $n^{\circ}$ 2151, énumère des représentants du tỵe conjungula en italien, en espagnol et en ancien français. Il conviendrait d'y ajouter les formes wallonnes que nous renons d'étudier.

[Romania, t. xLYH (1921), P. 556.]

anc. liég. coxhe, fohe

G., II $5 \% 1$ : coxhes Chartes [des Mitiers], I, 2333 (152\%): an fait des draps qui seront drapues de vilaines coxhes et pellins tondus entre le mois de may et s't Reny. [Note de Scheler : "Voy. Bormans, Drapiers (BSW. t. 9. 1. 25:3), qui assimile corhc à cote, ce qui n’est guère admissible. 川

G., II 593 : iohes Chartes [des IIétiers] I, 305-10 (1575) : pourpoint, ehansses, henches $\left({ }^{2}\right)$, fohes, cottreaux (jupons), golliers et autres habillements d"hommes ou de femmes. - Quicl ? [Note de Sicheler : "s"il y avait flohes, on pourrait songer à floscus, autre forme du b. lat. floccus, froccus. n]

Le Glossaire de Reichenau a un article lena : tora (= lat. rulg. tosca, lat. tusca ; d'après Hetzer, Reich. Glossen. "étofle grossière. manteau de cette étoffe »). M. Marchot. à qui jemprunte ees détails $\left({ }^{3}\right)$. prétend retrouver le même mot, estropié par l'éditeur. dans les deux textes liégeois du xvie siècle que cite Grandgagnage. I'estime qu'il se trompe doublement et qu'on doit tenir coxhe et fohe pour des types correets.

I. Si le texte de 1527 était isolé, on pourrait à la rigueur suspecter la forme coxhe; mais une charte de 1435 ne laisse aucun doute: "pour eascun drap fait de grayt mons (?), de fleur. de koxhe, de simple gris" ( ${ }^{4}$ ). Nous y royons le liég. cohe. qui aujourd'hui a seulement le sens figuré

(1) "Les eongles dont on joint les buves ki mainent le laigne el eastiel de Namur"; texte eité par Du Cange, consugla, et reprodnit par Godefroy, qui rléfinit congle: " joug (!) pour les Jurufs ". - II. A. Thomas eite lanc. fr. cojongle dans Romania, 1910, ए. 237 ; cf. Meyer-Lübke, $n^{\text {ns }} 4621$ et 4646 .

${ }^{(2)}$ Corrigez heuclies, et voy. ei-dessus l'art. bodje.

( $\left.{ }^{3}\right)$ Zeitschrift für framz. Spr. und Litt., xxxix (1912), p. 148. - Cf. Diez, Anciens gloss. romans, trad. Baner, $\mathrm{P}$. 40.

(4) Texte eité par Bormans (BSW 9, p. 213). Dans son Gloss. des Drapiers (ib., p. 265), Bormans eonfond koxhe avec cote! 
de "branche", mais qui jadis a signifié "cuisse ", lat. cowa (cef. G., II 5\%1). Du diap de coxhe (prononeé $c \ddot{\%} \%$ ) désigne du drap fait avec la laine prise aux euissen des moutons $\left({ }^{1}\right)$. Comparez lanc. fr. cuissete "sorte d'étoffen: mantean de cuissettes noires (1486: God.).

II. Les arehives liégeoises de 1530-33 portent à plusieurs reprises : " une focke de drap noire foree de penne condist fin gry ", " une foucke de drap ". "une fock de drap sanguinne foree " $\left({ }^{2}\right)$. ete. On y reconnaîtra le moyen néerl. focke. que Kilian traduit par "superior tunica " et qui subsiste en flamand moderne (Schuermans) sous la forme fuik "blouse. sarrau ". De folie ì folle le passage ne fait pas difliculté $\left({ }^{3}\right)$.

[Romanit, t. xivn (1921), p. 557.]

\section{liég. cråmignon}

Le " eramignon " ent une danse populaire propre an pays liégeois. Aux fêten de paroisse surtout, danseurs et danseuses, placés alternativement, forment une longue chaîne qui. sous la conduite d"un "meneur ", se déroule et s'enroule, se tord. se pelotonne. ct promène ses détours capricieux à travers les rues du quartier. tout con répétant les couplets que chante le meneur du branle.

G.. I 135. nexplique pas ce mot, qui a pourtant sollicité maint amateur d'étymologie. On a invoqué le latin carmen. la sinistre carmagnole et d'antres analogies non moins fantaisistes. Pour ma part, je crois que cramignom, ou mieux cramiyon. est altéré de crămiyon. Lia bref ch type primitif s"est allongé. probablement sous l’influence de cras. gras ( $\left.{ }^{4}\right)$. Le suflixe -illon. -iyon. qui aurait dî normalement se réduire à -yom. seest maintenu, grâce ì la mesure du vers, dans des

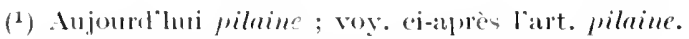

(2) Registres at1x Arrets (Auchives de Liege).

$\left({ }^{3}\right)$ (onnp. solie (Forir) "socle" $=$ sohe (Hub. ap. (i.) : take (G.) "cadenas" = take (lorir); voy. latut. arombe ei-après.

${ }^{4}$ Le liegeois allonge souvent la voyclle de la syllabe initiale. Le joli nom de la pinfurette : măgrigite (Verviers ; litt. *margueriette) s'est cléfiguré en mågriyète (lieg.). a cause de miggrigi, mangréer, ct des composés où cutre le prélixe mä, mal.

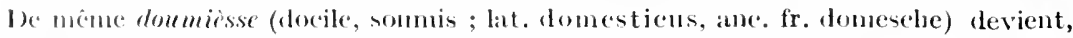

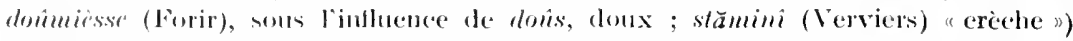

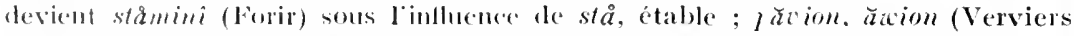

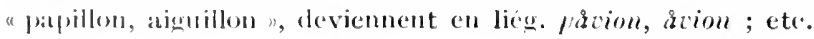


refrains traditionnels $\left({ }^{1}\right)$; puis, il s'est d'ordinaire épaissi en -ignon $\left({ }^{2}\right)$.

Le mot liégrois, nettoyé de ses sureharges, ne fait qu'un avec crămyon "zigzag, détour " (Robertville lez-Malmedy), "objet embrouillé» (Stoumont ; syn. kumuhèdje, vôtion). fig. "affaire eompliquée, querelle" (Dinant, Bourignes) $\left(^{3}\right)$. Il appartient à la même famille que les termes suivants : déscrami (Huy). discramî (nam.). -yè (Fameme), -ŷ̂ (ard.), dècrŏmồ (gaum.) "débrouiller, démêler, dépêtrer"); - èserami (Huy),

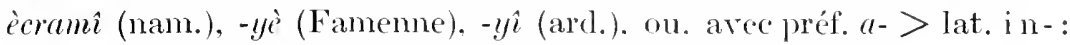
acramŷ̉ (Givet, Couvin), -yè (Neufchâteau), acrĕmîr (gaum.) " embrouiller, eneherêtrer. entortiller s: - de là, des dérivés, notamment en Famenne : ècramyis" "sujet à s'enchevêtrer ", discramycû "démêloir ". Cette famille pénètre au sud jusque dans le patois meusien (acramilli, dècramilli: Varlet) et dans colui de Metz (ancrèmié : Jaclot). On ne trouve rien de semblable en ancien français.

Quelle est l'origine de ee groupe ?

Eeartons d'abord le radical germanique *kram-. par lequel FalkTorp explique le bas all. kramme (crampon ; griffe) et le holl. kram (crampon) : le messin ancrèmié et le gaumais acrö́mîr, dècröemîr (à Ste-Marie-sur-Semois) ne peurent s'en accommoder. En revanche, il y a concordance entre ces dernières formes et le messin crèmô, gaulmais crăımă. (qui répondent au fr. cramail, w. crămă "crémaillère ", du lat. *eremaclum : moyen de suspendre. De plus, l'aneien français offre un dérivé cramillon ("crémaillère"), qui survit, avec le même sens, au Sud de Givet, et sur divers points de la Wallonie : crămyon à Vielsalm, Charleroi, -iym à Bourlers, -(i)gnon à Couvin. Hondeng $\left(^{4}\right)$. C"est le diminutif de *eramil (*eremiclum : en Art. fr. crami); d'où cet autre diminutif : *cramillette $(=$ w. crămyite, gaum. crö̌myète,

(1) Par exemple le relimin si commu :

Princlez vosse bordon, simon,

Et is minez nosse cramizon.

(2) Le Thétrc liégcois du xvur siècle ne eomnait que crimigon (écrit crâmion dans l'édition de 1854: pp. 27, 29, 34, 1299). C"est aussi la seule forme connue à Huy de nos jours et ha seule domnée en liégeois par Cambresier, Inbert, en verviétois frar Lobet. - Pour $y>g n$, voy. les articles bougnou, doguon, sprogni, etc. Comp. fe plus franskilion (G., II 52\%) > franskignon (Forir): lanc. fr. manuillon $>$ fr. maquignon ; l'ane. fr. escufillon, -ignon, w. scufiguon "escarpin " (G., II 345) ; le liég. ahàyi, houyot, qui peut s"altérer en ahågnh, hougnot : etc. (Voy. rart. hoyc).

(3) Lobet, p. 310, donne en verviétois : cro̊mion "zigzalg... : branle, danse gaie en rond et en zigzag ". - On pourrait croire que le sens "zigzag "dérive du seeond; mais les termes que nous citons ensuite (déscrami, etc.) écartent l'objection.

(4) Voyez aussi Bruncau, Enquête, 1, Pl). 229-230. 
messin crèmyote) "petit appareil eomposé d'une tigc courbe terminée par un double crochet, qui sert à retirer le pot de la erémaillère ".

Il est visible que *encramiller, *descramiller viennent de là, et que cramyon "détour " ne peut se séparer de cramyon "erémaillère ". Le cramillom. qui est proprement une tige dentée servant i acerocher, et la *eramillette. qui présente quatre eourbes rapprochées, ont éreillé l'idée de : "ligne brisée on eourbe, erochet, détour. tortillement ".

Pour en revenir an cramignom liégesis. nous pourons maintenant conchure arec assuranec. (Quand il a ru les troupes joyeuses dessiner leurs zigzags fantaisistes (1), le peuple a bien saisi le earactìre pittoresque te ee dirertissenent : la ligne sinueuse de la crémaillère s'est offerte a son esprit at il a créé cette image : "mener un eramillon". Puis, de l'espèce de danse, le nom a passé au chant qui toujours aceompagne les évolutions de la eluâne dansante ; de la l'expression braehylogique : "ehanter un cramignon" (2).

Ainsi le eramignon tire son nom de son principal earaetère : le mouvement. - tout comme les trihes de Malmedy $\left({ }^{3}\right)$, le branle français et sans donte aussi la farandole de Provenee ta bourvée d'Aurergine.

[Remaniement de BI) 1910, p. 65.]

\section{w. crasse (Verviers, Nalmedy)}

Autrefois. à Verriers. on employait, pour carder la laine ì la main. une sorte de peigne on séran appelé crasse. s. f. : cet instrument eomprenait de nombreux croes en fer qui hérissaient la tête d'une table montée obliquement sur quatre pieds et appelée "baudet ". La mention la plus ancienne que je connaisse figure dans une eomédie inédite de 1759. en dialecte verviétois : $i-i-s t-o s s i$ sitch qu'on badlet d crasse " il est see et décharné eomme un ehevalet de eardeur". Villers et Seius à Mahmedy. Remacle et Lobet i Vervicrs, signalent crasse "earde". crassî "carder". crasserèse " onvière qui carde" $\left(^{4}\right)$. Grandgagnage enregistre ees mots sans domner d'explication valable $\left.{ }^{5}\right)$. Ce groupe

(1) Cf. Thicitre lirgeois, p. 129 :

Qui tos cès k’twèrdous crâmiyons

On-ce gulon s’kiméle onk arî l'aute.

(2) Pour Littré, Suppl., "cramignon " désigne une "chanson populaire en Belgigue ". Il ent inutilo de souligner les inexactifudes de eette définition.

(3) Comp. l'ance fr. trescher: 1)iez, 1. 327 : Wilmotte, Le zoullon, p. 89-90.

$\left.{ }^{4}\right)$ (f. Henier, Hist. de l'iml. drapière un pays de Liège, p. 145. - Dans le BSW 39,

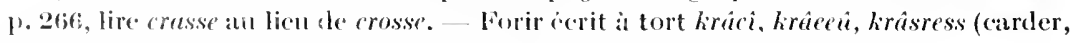
-(ur, -rouse) : res mots sont d'ailleurs inconnuss à Liège.

(5) G., I 13\%, 11 515, et Extruits de rillets. 
dérive clairement du néerl. et bas all. lirassen (rayer. raeler). all. kratien ( $\left.{ }^{1}\right)$.

\section{liég. crèssôde}

G., I 139, roit dans erèssôte "pâquerette à fleur's doubles " un dérivé de crèsse " erête ". Conjecture inadmissible, puisque les dérivés de erèsse (crèstê, ècrèster) reproduisent le tỵe primitif erèste. - Le liég. crèssôde (verr.-malm. crissôde. nam. crussôde) ne peut être séparé du flamand kersoude, larsoude (aneiemnement liassoude. liessourie; moyen néerl. corsoude. carsoude. lersoude; néerl. mod. liersoucis). syn. de madeliefje "pâquerette ou petite marguerite, bellis perennis L. " ( $\left.{ }^{2}\right)$. Toutes ees formes se ramènent à l'anc. fr. consaude. fr. ane. et mod. consoude : lat. consolida. L'épenthèse de $r$ a pu se produire simultanément en wallon et en flamand, à moins qu'il n'y ait eu emprunt de l'un à l'autre; pour le wallon. on trouve déjà crksssode an xume siécle.

Froissart. dans ses poésies, fait de consunde le synonyme de marguerite $\left({ }^{3}\right)$ et déja le moyen latin eumulait les sens de "consonde " ct de "marguerite". On ne doit pas s"étonner de voir le même terme appliqué à des plantes d'aspect si différent, puisqu'il rappelle une simple propriété médicinale : eonsolida (qui consolide. raffermit les chairs. arrête l'hémorrhagie) a pu désigner des plantes très diverses. dont les feuilles, les racines ou les graines serrent à tel usage; le nom est. on réalité, aussi peu spécificatif que l'expression populaire "herbe à coupures". Notre crèssôde ou pâquerette double a joué un rôle dans l'ancienne thérapeutique témoin cette recette liégeoise du xin ${ }^{\mathrm{e}}$ sièele: "Por estinde le fowe de saint Anthone u atre fowe, prendeis de loges flours de crussode ki soient couloutes [eueillies] en seece teins devant laseension a erous " $\left(^{\mathbf{4}}\right)$. Au surplus. le français moderne appelle du même nom consoude une renoneulacée (la c. royale) et une borraginée (la e. proprement dite).

[BD 1921, 1. 9.]

(1) L'all. kratzen, qui a donné le fr. gratter, a encore le sens technique de "carder ".

$\left({ }^{2}\right)$ Voy. Schuermans, De Bo, Franck-van Wyk.

${ }^{(3)}$ Scheler, Gloss. des poésies de Froissart (Brux., 1872). Le passage est cité daus le Compl. de Godefioy, cossovde, oì il mériterait une rubrique spéeiale.

$\left({ }^{4}\right)$ Texte eité dans le Bull. de Folklore (Liège, 1891), I, 153. — La fenille de la crèssôdé est encore employée aujourd hui à Liège pour arrêter une légère hémorrhagie; on fait deux ou trois incisions dans la feuille que lon applique sur la coupure. 


\section{w. crète et dérivés}

Il existe, dans les dialectes wallons, au moins six substantifs féminins crète. dorigine et de sens différents. Les deux premiers n'offrent guère d'intérêt : le troisième a été expliqué de façon très plausible ; nons nous étendrons davantage sur les trois derniers, qui sont moins connus.

1. crète (Malmedy : Villers, 1793 ; Houdeng) "crèche "; altération isolér du liég. crêpe : all. lirippe.

2. crète (Liège : BSW 34. p. 189), terme d'armurerie : probablement emprunté du fr. crête.

3. crète di mitches (Verviers ; anc. liég.) "carré de petits pains cuits ensenble"); emprunté de l'ane. h. all. cretto. moyen h. all. grette "corbeille, panier " $\left.{ }^{1}\right)$. - Dérivé : crètin (Erezée) "grand panier de paille tressée, pourant contenir quatre setiers de blé et pourvu d'une petite ouverture en haut"; rouchi kèrtin (Hécart, Sigart) "panier d'osier à anse" ; anc. fr. cretin (God.) "sorte de hotte" $\left({ }^{2}\right)$.

t. crète du bicas (Mahmedy : Villers. 1793) "moncean de bois arrangés, pile, bûcher "; à Faymonville erète de bac̀s ; isse broûlé sol crète du breas (Malmedy. Irm.. 1906. p. 49-50). Un vocabulaire françaismalmédien. manuserit du xvine siècle. dome "crèpe du bois : pile de bois " (par confusion avec crèpe : crèche). - G.. I 140. note le nam. crite "pile de bûches disposées par lits croisés "; l'explication qu'il en donne est sans valeur. Nous savons de plus que crète à Neuville-sousHuy. est un terme de bûcherons désignant "un tas de einquante facots" (H. Gaillard) ; à Liège, pour les meuniers. c'est " un tas de sacs superposés contre $u$ mur " (Ed. Remouchamps) ; enfin. à Stave au Sud de Nammr. c'est " mm amas de dix à vingt gerbes placées debout " (I. Ioisean). - - Les langnes germaniques noffrent rien d'analogue, tandis que Littré et le Dictiommaire général ont l'expression : "mettre dı blé en crête : l'entasser en hui domnant une forme pyramidale ". Le walkon. qui derrait dire crèse dans ce cas. parait avoir emprunté le not francearas.

1 Lenze (Hainant), le talus on la berese d'un fossé s'appelle : ène crète,

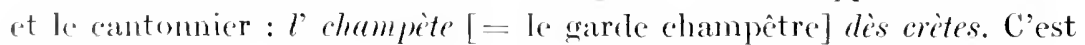
évidemuncht le fr. crête (roỵ. Littré).

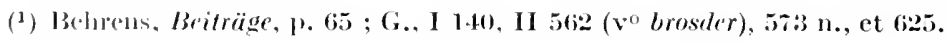

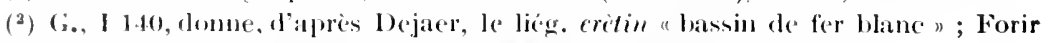
reproduit cet article. En réalité, le mot est incommu ì Liège. 
5. crète ("frette": eercle de fer dont on garnit le moyeu l'une roue et, en général. l'extrémité d'une pièce de bois pour l'empêeher de se fendre) est signalé à Verviers (Lobet), à Spa (Body. Toc. des charrons) et à Neufehâteau (Dasnoy. Pp. 80, s6). ('e terme technique a échappé à nos autres lexicographes. Je l'ai entendu ì Glons-sur-Geor, à Jupille (près de Liège), à Ben-Ahin. Gives, Solières, Yvoir. Andenne et à Neuvillers-Recogne ; le dérivé crit'le "fretter "existe dans ectte dernière localité. - Nous y verrons un emprunt fait par le wallon aux dialectes germaniques : le luxembourgeois liratt et le west-flamand kerte, harte, ont en effet le même sens ( $\left.{ }^{1}\right)$.

On doit sans doute rapporter ici larticle suirant tu dictionnaire liégeois manuserit de Rouveroy : "crette. s. f.. déchargenir. pièce de bois rond, autour de laquetle le tisserand roule la besogne fu'il lève de dessus la poitrinière $"\left({ }^{2}\right)$. La frette de cette pièce te bois aura donné son nom à l"ensemble; cef. Lobet, vo lirett.

Godefroy a l'anc. fr. creter aree cet exemple : "pour creter l'arbre sour quoi on fist le dist molle " (Valencienmes. 1358). Il faut éridemment traduire par "fretter, garnir d'une frette" _ ct non par "entailler ", comme le propose Godefroy et comme l'admettent Bonnard et Salmon ( $\left.{ }^{3}\right)$.

6. crète enfin existe comme nom de lieu, dans le voisinage de Liège : $1^{\circ}$ à Esneux : lès crètes en amont d'un ravin; 20 à Vaux-sous-Chèvremont : èl crìte "en la crette " : ee nom désigne un fond. M. Jean Lejeme l'a rencontré plusieurs fois dans les archives de l'Arouerie de Fléron eoncernant cette eommune : "terre gissant en le crete desem les mavais preis " $(1+18$ et $1+60)$; "en le erette dessonlx (hamont " $(1+79$ et 1505) ; "preit qdist les erettes" (1549) ; "en la erete en Vaulx" $(162+)$, etc. - J'ai relevé anssi le diminutif lis crètales, lieu dit de Ferrières; dans les crètales. nom d'un ravin à Esneux : sur les crètales.

(1) Lorigine du mot germanique est, je crois, incounue. De Bo $1^{\circ}$ en parle pas. Le Wört. der luxemb. Mumdart (1906) a cet article : "krutt, f., eiserner Reifen, Zwinge aus Metall ; fr. crairate \#. si ce dernier terme est allégné comme étymologie, il y a sîrement erreur.

${ }^{(2)}$ Le mot ne figure pas dans le Voe. du tisseraml, par $\mathrm{V}$. Willem, de Dison (BSW 38, ए. 193).

( $\left.{ }^{3}\right)$ A Houdeng (Hainaut), un gourdin s'appelle un crèti a Braine-le-Comte crèti. On pourrait y voir un [bàton] ferré ou fretté : mais il vaut mienx en rapprocher l'anc. fr. cretu "bâton dont l'extrémité supérienre est en forme le crête " (God., CRESTU) ; comp. crêtu dans le Larousse illustré. - A Braine-le-Comte, on connaît de plus un verbe crèter "travailler ferme, marcher très vite "(proprement : manier énergiquement le crèti ?). 
nom diun raidillon à Erezée; ainsi que "sortie des crétias". sur une earte-rne de Waulsort.

Le namurois crètia est bien eomu pour désigner me fronce, un pli dans une robe (Vezin). wne ride au front (Iny). Il répond au rouchi kèrtiau (Ions: Siggart) "pli fait au linge par le fer à repasser ". lï̌rtiau (Ellezelles) "fanx pli dans me étoffe"; - et an verviétois crètê (Dison : BSW 53. p. 418 : Thimister, Trembleur) "ribaudure, mamrais pli dans une ćtoffe : ride du visage ". De là le double dimimutif crèt'lê (Liège : mêmen semu) et le verbe crèt'ler (ibid.) "rider. crisper. plisser. froncer, goder "qưon retromve jusqüà Fonses-lez-Namur (dés canadas erètlés : pommes de terre à peau ruguense) et à Domr-lez-Mons (lictelé : froissé, chiffomé. en parlant d'me robe) $\left.{ }^{1}\right)$.

Pour expliquer crit lé. cret'ler. M. Behrens, faisant table rase des conjectures de Grandgagnage et de Bormans $\left({ }^{2}\right)$, sadresse an bas all. liäte. lerete "ride. sillon. pli. fronce. coche. entaille. éraillure. ete. ". On ne pent que lui domner raison. d'autant plus que les dialectes flamands possèdent ausi lierte "entaille. fente. erevanse ". liertelen "se crevasser" (voy. Schnermans et De Bo). - Nous étendrons la mome explication aux noms de lien crite et critale. Entin nous verrons dans criton mu autre dérivé de la même suree; voy. l'article sivivant.

[Romania. t. xLvin (1921), 1. 558.]

\section{w. crèton, fr. creton}

Diaprèn Littré et le Dictiommaire gćnéral. le fr. creton "résidu de araise fondue". qui se reneontre dè le xine sièle. est d'origine inconnue. On ne pent admettre en effet. comme certaim l'ont proposé $\left({ }^{3}\right)$, quil dérive de crite par un type *ereston. on de crotte en se fondant sur le picard croton "graillon" $\left.{ }^{4}\right)$. Lancien franceais, qui a plusieurs fois

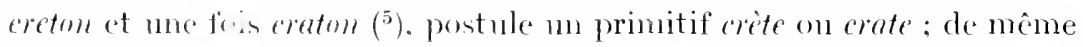
les dérivés eretommé. eretmmée (God.. t. II).

(1) Comparez lane. fr. areti (que Godefroy ne preut traduire dans cet exemple : "jupes et grailles cretis") et le lorrain liroti, dans Zeligzon, Lothring. Mundarten, 1). (3).

(2) Behreus, Beiträge, p. 6.t ; G., I $1 . t 0$; Bormans, Gloss. des Drapiers (BSW 9, 3.. 2.5.5).

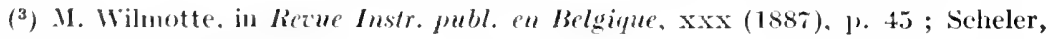
Diet. détym. frençuise.

(4) Le picard croton et le rouchi erotelin (Hécart) peusent se rattacher à crotte, soit directement, soit far le croisement de eretou et de erolte.

(5) Voy. Godkfroy, Compl., qui dite, contre autres, cet exemple significatif : "crever et defrire et dessechier comme ung cratom ". Ajoutez l'article du Catholicon de Lille due nous citous à laurt. chaon. 
Selon toute rraisemblance. le mot franęais est venu de la frontière germanique du Nord-Est. par l'intermédiaire des dialcetes rouchi, wallon et gaumais : kìton (Chimay, Givet ; rouchi : Héeart ; altéré en guèrdon à Eugies et à Moms : Letellier, Sigart) : lètun (gammais); curton (Bastogne) ; crèton (Dinant. Namur. Marche-cn-Famenne, Liège. Malmedy, etc.). On entend par là. en général, un petit moreeau de lard frit qui sert à préparer certains mets $\left({ }^{1}\right)$. En attribuant au mot le sens originel de "petit objet reeroquevillé ". j'estime qu il appartient à la même famille que crètê, hivtian. crètler. liètelé. et qu'il représente un diminutif du bas all. krëte. livete "ride, fronee. pli s. flan, kerte "erevasse " $\left({ }^{2}\right)$. dont nous avons parté à la fin de l’article précédent. - Au surplus, deux autres termen français clésignant le même objet sont également dérivés d'un radieal sermanique à l'aide du suffixe -on : l'anc. fr. chaon (roy. eet artiele), et le génerois greubon, de l’all. griebe.

[Rommia, t. XLY11 (1921). 1. 561.]

\section{gaumais cuchale}

Le gaumais cuchele (Virton : -èle à Proury-Jamoigne) "espèce de ruelle qui sépare deux bâtiments et où l"on met le bois à brûler ". viendrait, dit-on. de cuche. w. cohe "branchage " (BSW 49, p. 149). Cette étymologie a le tort de reposer sur une seule forme dialeetale et de s'inspirer d'une définition oì l'aeensoire a fait perdre de rue l'essentiel. Près de Virton. à Musson et à Ruette, jaa noté conchale. forme qui se retrouve dans le meusien conchele "cour d'une habitation; à Metz cochèle " (Labourasse). C'ent l'ane. fr. courcelle, diminutif de cour ( ${ }^{3}$ ). Linfluence de euche a déformé le mot en cuchale. qui derient même cruchale à Saint-Léger. avee r épenthétique.

\section{liérir. cwasse}

G. I 120 et 146, donne d'après Dejaer et sams explication : "coise ou quase, iagoue. thymus, ris de veau ". Hubert. p. 146, et Durivicr

(1) Villers (Malmedy, 1793) ajoute: crèton d hate "chiquet d'écorce "et crìton, au fig., "trésor, amas cargent que l'on conserve".

(2) Le Norw.-dän. etym. Wört. de Falk et Torp assigne de même an norvégiensuédois liart "fruit vert "et à l'est-frison kret "fruit ratatiné ", le sens fondaunental de "rugosité, objet recroquevillé ".

$\left({ }^{3}\right)$ Pour $r c>c h$ en gaumais, comparez foch'lète "perce-oreille, forficule $"\left(={ }^{*}\right.$ forcelette, diminutif de force: forficem); pouché " pourceau"; afoch'ué "triste n (= ane. fr. enforcené " furieux "). De même, rs dans pachône "personne ", r'véchi "renverser". 
ont un article cicuss" di $z \hat{e}$ " ris de reau ". - Ce mot est emprunté du flamand kicast "tumeur. excroissance" (Schuermans. p. 31t). On sait que le ris de reau est un corps glanduleux placé sous la gorge du veau. Pour la forme. comparez lanc. w. lasse (= all. last: G., II 613) et, ci-après. l'art. toûlasse. — Le mot est féminin : del blanke criasse. dèl neire cicasse, daprès Semertier (BSW 35. pp. 32 et Si).

Du néerl. liatast "brone". vient le liég. eacasse. f.. t. de batellerie, a brosse à goudronner ": li criasse à daguèt.

\section{liég. cwassî, cwahî, cwahe}

I. Meyer-Lübke. $n^{\circ} 2000$, tire l'anc. fr. quacier (serrer. comprimer, coaguler) du lat. *coactiare (dérivé de coactus : pressé). Il faut ! ajouter le w. caussîn ou caci. qui se rencontre dams le coin X.-E. de la Wallonie, limité par Glons-sur-Geer. Liège. Starelot, Malmedy $\left.{ }^{1}\right)$. Pour l'expliquer. G.. I 146. hésitait entre l'all. quetschen et le lat. quassare. La phonétique n'admet ni l'un ni l'autre. tandis qu'elle recommande pleinement *oactiare. Pour coa- $>$ cia-, comp. cicayou ; pour le traitement de la finale, comp. *suctiare $>$ such, sucer ; *addirectiare $>$ adrisit. adresser.

Au sens propre. caisst. v. tr.. signifie "soumettre à une pression violente : froisser. meurtrir : piler. écraser (du sucre. des drogues. ete.) ; éculer (des souliers) ; aplatir (un chapeau) ; serrer (qqu contre un mur) ", etc. $\left({ }^{2}\right)$. - D'où le dérivé cicassore (Malmedy) "durillon ". -are (Verviers : Lob.) " ampoule, fourche. abcès aux mains des gens de trarail"; et le composé acioussi (Malmedy : BI) 1906. p. 139) "froisser, écraser, broyer $"$.

(1) Le liég. -i devient normalement -i à Malmedy-Stavelot, -er à Faymonville. Dans rette dernière localite, on dit caiesser.

${ }^{2}{ }^{2}$ La Complainte liégeoise de 16331 (Choix. p. 73: et p. xxi) porte dans un passage, dailleurs peu sur : quasse. Crespou fi. Léditeur Bailleux traduit : Trinque, mon fils Crespou ". - G. II 5it, a un artiele : "quase (fé quase : trinquer), pièces de 1631 et 1710, d'après Simonon . . Je trouve en elfet, dans une liasse de chansons du début

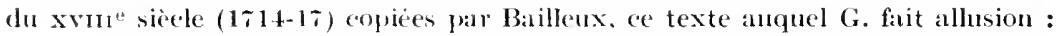

\footnotetext{
Vocial li pus bell chanson

Qui Baptiste a faict di s' veie,

Cest dè fé quass et raison

Al sante des jone's feyes.
}

Il faut saun doute lire croasse, impératif de notre czossî, qui aurait ici le sens fipécial de "chopuer les verres, trinquer". 
Cwassî est aussi intransitif et signifie 1. "mâeher ", t. de drapicr et de conturier, en parlant des forees ou ciseanx dont les deux branches, au lieu de couper, s'écartent et laissent passer l’étoffe ( $\left.{ }^{1}\right)$ : li çuìte

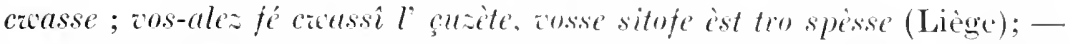
2. à Stavelot-Malmedy : "plier (soms le faix) " : i catasse dusos l' paquet ; one tchèdje qui frent cáassi on bâdet. - Le Dict. malmidien de Villers (1793) a eet article: "cicassi. v. n.. biaiser, caracoler. gauchir, vaeiller ", qui n'est pas repris par Seius (1893). On pent supposer que "biaiser, gauchir ", s'applique aux forees ou eiseaux qui ne compent pas nettement, et que "vaciller " répond à "plier sous le faix" ; entin "caracoler "serait tout à fait impropre $\left({ }^{2}\right)$.

Il. On ne duit pas confondre ewassì avec le suivant, dont l'aire est beaucoup plus étendue :

rouchi : ciassier "blesser" (II'cart : coissicr, quoissier) :

Tournai : caucher "blesser, mentrir, estropier" :

Viesville, Genappe : czuchi : (harleroi : cochi "blesser" :

gaumais : czachi "aplatir dun coup violent":

ehestrolais : cauchè "écraser ": intr.. fléchir " (I)asnoy : coicher) ;

Famenne, St-Hubert : crochè "meurtrir" ;

Namur : crachi ; Liège cataki, anc. liég. truetsseir (14:24), quaxheir (1431)

"blesser avee un instrument tranchant ", surtout au réfléchi "se couper".

C'est l'équivalent de l'ane. fr. eaissier. quaissier. que Meyer-Lübke. no 6940 , rattache au lat. *quassiare $\left({ }^{3}\right)$ " easscr, rompre. briscre, dérivé de quassare $(>\mathrm{fr}$. casser). Pour la forme, comp. *hassiare $>$ baisser, bathi, bahî; *incrassiare > engraisser, écrachê, écahî.

Nous rattacherons à ce groupe ladjectif cache (Liège : G.. I 14t), cwache (Namur). coche (Charleroi), yui signifie : "sensible. endolori, douloureux" : d'où, au moral : "timoré " (Liège), "mou, sans énergie"

(1) Le Lurousse illustrí dome "mâcher, t. techn., couper anns netteté et en dechirant les fibres au lieu de les trancher ; michure. partic du drap ou les forces ont mal eoupé et, prour ainsi dire, mâché le poil \%. Il fant expriquer de la sorte l'article ciussìdje le Lobet, p. 32:2, que des coquilles rendent inintelligihle à jremière vue.Dans G., II 516, il faut lire czoussi an lieu de quuscr.

${ }^{(2)}$ L'artiele de G. dans ses Extraits de V'illers. 1. 36 : "coise, cote ; coisi, coiseler, biaiser, ganchir ", contient deux erreurs : le mahm. czucsse (côte, liég. czè̀sse) n’a rien à démêler avec cáassi (liég. -î), que nous venons d'expliquer, ni aver le mahm. qwas'ler " ehanceler, biaiser" (V'illers : à Troisponts azcuns"ler), dhiminutil de qzousi (à Stavelot) $=$ fé lès qrounses, faire semblant, hésiter, tergiverser.

${ }^{(3)}$ Grignard, Worphologie de l'Ouest-zullou, \$6. (BSW 50, p. 4:37) explique correctement cochî, crocthi, crochî par *quassiare. Il faut écarter les propositions de G., I 144, et de IIarchot, Phonol., p. 42 (néerl. kzetsen, all. quetschen); de Niederländer, Mundart zon Namur, \$8 (lat. *coactiure), de Hécart, p. 381 (lat. quassure). 
(Starelot). "susceptible" (Namur, Charleroi). C'est un dérerbal de catahî, cwachî, cochî $\left(^{1}\right)$ et non, comme l'anc. fr. cas, un produit direct du lat. quassus.

\section{liérg. cwate, cwatê}

Le liég. căăte. s. f., signifie "flache" $(=1$. inégalité dans le pavage d'une rue par suite de l'enfoncement d'un paré ; 2 . ereux où l'eau s’amasse. flaque : Diet. gén.) : ine tchèrète sitantchêye divins 'ne cuate "une charrette arrêtée dans une ornière profonde"; au fig.. il $\grave{e}$-st-èl carate "il est cmbourbé, dans le pétrin". De même le houilleur de Liège. Jupille, Fléron, Seraing. cte.. appellc cacatê (s. m., diminutif en -ellum) un enfoneement dans une voie souterraine, où l'eau peut s'amasser de façon ì gêner la circulation : ine fossale wiee qui l'êrue lêû̃e ("couve" : eroupit). - Bormans tire ce mot du flam. kot (eavité), ce qui est phonétiquenent impossible $\left({ }^{2}\right)$. G.. I 146, se demande s'il faut comparer cacate avec le rouchi escuater (écraser). Ce rapport est, en effet, des plus raisemblables $\left({ }^{3}\right)$. Nous voyons dans ercate le déverbal de *cati " presser. enfomeer" (= anc. fr. quatil. fr. catir : lat. *coactire?). qui est resté dans s'acicuti "se tapir". Le sens propre est "enfoncement" $\left.{ }^{4}\right)$. Pour la dérivation postrerbalc. emp. le w. transe, minte (nensonge, ane. fr. mente).

\section{w. cwayoû (Stavelot. Mahnedỵ)}

G. ne parle de cäboni que dans ses Extraits du Dict. malm. de Tillers, 1. 38. et ee cn termes trés cubarrassés. Le mot. dans la région de Mahmedy, désigne un panneau de muraille en torchis. I Starelot, ee sens s'appliane à pariont. et catayon désigne une partie oni se détaehe du mur en torchis $\left({ }^{5}\right)$.

(1) Comp. le fr. blime. ganche.

(2) Voy. Franck-van Wyk: кот. - On ne peut pus non plus invoquer le moven

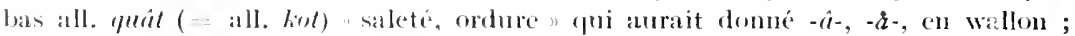
voy. Fanck-valn Wyk : kw.1D ; Falk-Torp : nvamber.

(3) En alduettant que le ronchi escacaler (Sigart : skoiter), ance fr. esquater (God.).

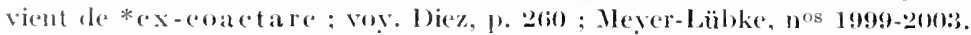

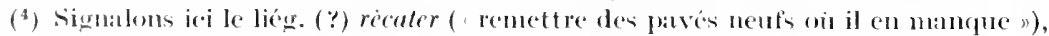

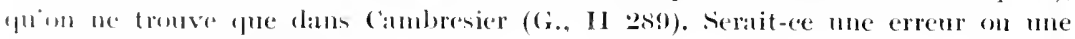
altiration, prom *recioter?

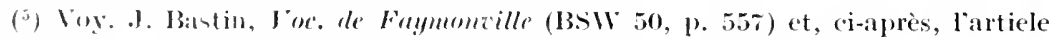
parimete. 
G. ne croit pas qu'il y ait un rapproehement possible entre ewayou et le verviétois cicayot (" caillot [de sang] ; motte de terre corroyée ": G. II 516. d'aprìs Lobet ; ef. Forir : licaüntt "motte de terre"). Fn réalité. ces deux mots dérivent du lat. coagulum à l’aide des suffixes diminutifs -ot et -ou (fr. -eul. lat. - eolum). Lïinitiale cro le lat. eoa-, devenu en fr. ca-dans cailler. caillot (comp. coaeula: fr. caille. w. exaye). Le sens que Lobet. p. 322. attribue à cricayot (" motte de terre corroyée et prête à être mise en moule pour la fabrication des briques et des tuiles ") rend bien visible le lien entre ec mot et notre ezouyoû. Je définirai donc ee dernicr : 1. * [torchis (scns général, rétabli d'après l'étymologie)] ; 2. (Malmedy. Faymonville) panneau de mur en torchis ; 3. (Stavelot) partie qui tombe de ce panneau. débris de torehis desséehé: plâtras. - Comparez pariou. payou.

\section{malm. dêve, dève}

Villers (1793) donne, en dialecte de Malmedy. dêtes. s. f.. "écorce de

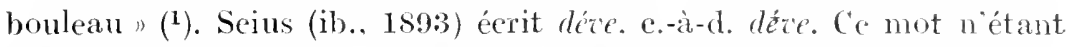
pas eomnu ailleurs ehez nous, on lui supposera de prime abord une origime germanique et, de fait, nous an retrourons l'équivalent dans le bas allemand d'Eupen, tout proche de Malmedy. Le Ẅ̈rterbuch der Eupener sprache a un article : daver. m.. "éeorce siche du boulcau", davere "l'aetion d'écorcer le bouleau ". T'ontefois. l'on y déclare que ces mots se dérobent à toute explieation satinfainante. Si l'eupenois daver est aussi isolé dans le bas allemand que le malmédien dễé dans le wallon, la question en devient plus obscure. Die lors. il est bicon tentant de s'adresser au celtique dera "chêne" $\left(^{2}\right)$. Lécorce du boulcau servant a tamner comme celle du chêne, une contusion a pu se probluire entre les deux essences.

\section{w. dihâhiné, frr. dégingandé}

G., II 520. signale sans éclairciscontent : dihrhiné "dégingandé ", d'apres Remaele. thez qui le mot ne ligure que dans cet exemple:

(1) G., daus ses Earrails de rillers, écrit à tort dèzé. Liecorce blanche du bouleau est appelce déve. Elle se detache fiteilement par lanièren et servait anciennement à allumer le feu... De déze vient le verhe dizer. Les chênes (á écorcer) dizèt, lorsque la pellicule supérienre de lécorce se détache d’elle-ntêne : cela arrive à la seeonde poussée de la sève et, alors. l"éporfuc de l'écorcuge ent linie ". (Folklore Eupen-Malmedy-St-Vith, no 1, juin 1922, p. 24; article de J. Bistin sur "les I'lantes dans la Wallonie malmédienne ).

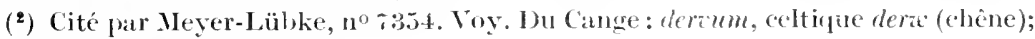
Godefroy : arcee (chênaic). 
c'è-st-ine grosse dihâhinêye zatche "c'est une grosse rache dégingandée " $\left.{ }^{1}\right)$. Nous trourons d'autre part :

håhiner (Blequy-Trembleur') "vaciller " : i hahinêye so sès djambes. | su likghiner (ib.) "se dandiner, se balancer en marehant ". kuhahiner (Verviers)

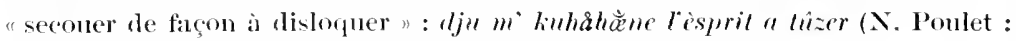

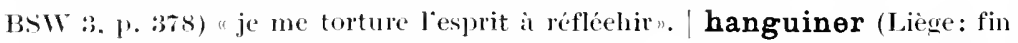
du xvule siècle), v. tr., "pendre (a la potence)" : zola quon $r$ hanguime (ASW 9. p. 160) $\left({ }^{2}\right)$. higniner (Verviers), v. intr., "valeilles, chanceler ":

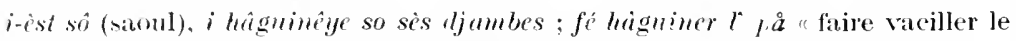

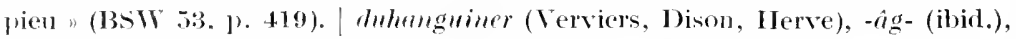

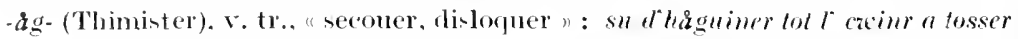
"se seconer tont le corps il tousser" ; èsse tot ahaguimé "ivoir les vêtements en désordre " (B.W 53. P. 419) ; one houlêye passète tote duhangninêye (ib., 54, p. 10) " un tabouret boiteux tout disloqué ".

Cotte famille est propre au pays de Verviers et de Herve ; elle présente le suffixe diminutif -iner. le's préfixes du-. ku- (lat. de-, com-) et $u$ radical hahl- (hah-) ou hâg- (hang-. hâg-). où l'on recomnaît sans peine le moven haut all. hôhen. hangen (tr.. "suspendeve"; intr., "être suspendu").

Liane. fr. dehingander. qui se reneontre pour la première fois dans Rabelais. est derenu. eomme on sait. en fr. mod. dégingander. Pour l'expliquer, on poutrait songer. dit le Dict. général. i l'angl. hinge "gond". Ne serait-il pas ausi naturel d'y voir l'all. hangen. hängen ou. ce fui revient à peu près au meme. le moven h. all. hengen (all. mod. henlien "pendre")" De la sorte. dehingander répondrait — sauf pour la finale qui reste obseure - a notre duhahiner. duhanguiner. Il y réponulrait mieux encore si l'on royait dans dehingander une métathese pour *hehanguin(d)er.

\section{anc. nam. dispatuer. w. däspatouwer (Brabant)}

J'ai entendu a Ste-Marie-Geest (près de Jodoigne. à l'Est du Brabant) l'expresuion inédite : dăespatmuacer dès côrts ${ }^{3}$ ) "dépenser, distri-

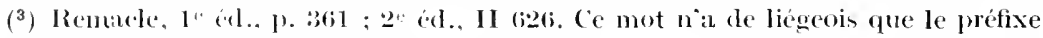
di- all lien du vervictoin der-.

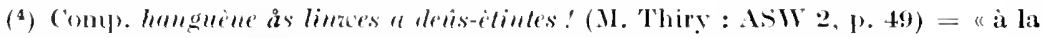
pretence les hommes astuciems an lingage ciquivoque !".

${ }^{3}$ ) le nam. côt on, itymologiguement, quenrt, cest proprement le "quart"

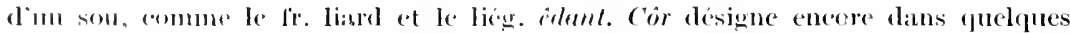

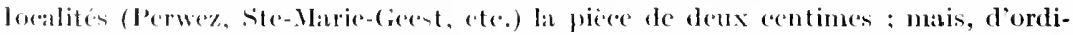

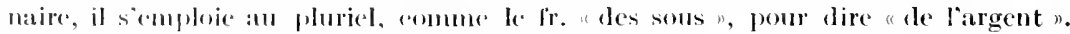
(omp) lespagnel cuartes, mence sems. 
buer de l'argent \%. Nous y trouroms une eurieuse survivance de l'ancien namurois dispatuer. que Godefroy traduit par "écarter, détourner" sans autre explication (1). Il faut se reporter à l’ane. tir. despostuer "déposséder" (trois exemples dans Gord.). altéré de desponester (ibid.), par l'intermédiaire d'une forme *despoüster. C"est proprement 1. dé-

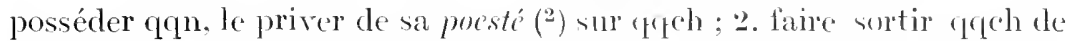
la propriété de qunn. e.-i-d. déménager $\left({ }^{3}\right)$ ou aliéncr pyels. De ce dernier sems, le wallon a passé naturellement à celui de "clépenser ". Pour la forme wallonne. on notera dans dispatuer la chnte de s par dissimilation et le changement si fréepuent de a protonique en $a$.

[Romania, t. Xurir (1921), p. 562.]

\section{liég. dital'té, rital'té}

G., I 17\%, note sans explication le liég. ditalté " fatigné. las. ineommodé, souflrant". Hubert écrit distalté forme régulière, mais qu'on n'entend jamais. Remacle. Forir ont dli)talte; à Malmedy et à Verviers d(u)talté (Lobet, Scius). Exemples : ros-ares laair ditulté (175s : Théatre liég. p. 120); dii so tote ditaltéye oniy. - Dans la vallée du (fecer (Gloms). dital'té a le même sens. mais il signilie aussi, et plus sourent. en parlant

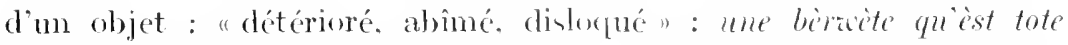
ditaltêye. dés solés qui sont tot d'tal'tés. - ("ent le participe de l'ane. fre, destalenter "prirer du talent (= du désir. de l'onvic de faire me chose), dégonter. déeourager" $\left(^{4}\right)$. Ponr la syneope de la protonique en wallon, comparez agaimiter "soutirer (de laargent) ". componé de l'anc. fr. gaimenter (G.. I 1:. 3:2t); upaliter, anc. fr. apaisenter; fiem'tê (serpette), diminutif de fier"mint: lat. fer'ramentum; tehip'ter

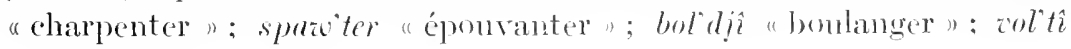
"rolontiers"s" ( $\left.{ }^{5}\right)$.

J'ai noté aussi à Verriers li(u)tal't'. à Robertville-lez-Malmedy li(i)tal tó " affaissé, abattu. détait (à la stuite d’une orowe, d’une insomnie, d'une indisposition)". 11 fant y roir une dismimilation annchée par le

(1) Dans ce scul texte : "Comme ammi seroit si, apres toutes les dites solemnitez achevees, estoit trouvee ieelle vefve aroir dispatue, ahomsé, on recelé. faict dispatuer, absconser, receler ou porter dehors lat susdite maison " (Cout. de Nemur. Nouv. Cout. gén., I, 886).

(2) Lat. potestatem, puissance. La forme ponsti est dans cinlefroy.

(3) God, attribue ce sens à l'anc. fir. desproestir.

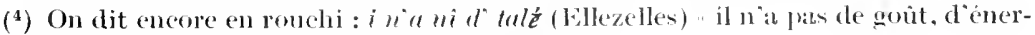
gie "; voy. G., I $16 ; 2$ : drelunt : II 415 : talumt.

(5) Voyez d'autres exemples à l'art. mayeté. 
grand nombre des verbes à préfixe ki- (lat. com-) et la difficulté de prononcer tot d'tal'té.

Lanc. fr. connait encore atalenter, entalenter, ratalenter. Nous retrourons en wallon :

atal tiné (Comblain-la-Tour) "attité, aecontré * : ile èst drol'dimint atal'tinçye. Ce diminutif suppose quion a dit aneiennement *s'atal'ter "s'arranger aree goût. se parer".

ratal"ter (Glons) "rafistoler. réparer" (un vêtenent, des souliers, une brouctte. etc.).

ritalté (Liège) "remis (d'une indisposition)": zola l'èfant r'tal'té ; zo-t"-la r'tal'têye tot l' mimme! - C'c mot. que nos dietionnaires ignorent. réponel à une forme franęaise *retalenté.

[BD 1911, 1. 98 ; remanié.]

\section{w. djama}

"Denx on plusieurs jours de fête qui se suivent, soit à canse de la solemité. comme à Nö̈l. à Pâques, cte., ou parce qu'une fîte conservée tombe la veille on le lendemain d"un dimanehe n. Cette délinition de G.. I 250. parait donteuse a Scheler. ibiel.. II 600) : cest pourtant la meillenre quon puisse domer. arec eette légère modification : "denx (on, par extension, plusieurs) jours... ). (2uant aux étymologies tentées par G. à propos de ce mot. "elles ont peu de probabilité " anx youx de Scheler, qui risque de son côté. ct sans grande convietion. une proposition nowrelle (dies magnll!). daalleurs inadmissible.

Je pense que djamă dérive d'un verbe djamer. à l'aide du suflixe -ă, fr. -ail. lat. - a culum. Le rerbe djemer a dû exister jadis (1) dans notre Est-wallon. ainsi quen témoigne la survivance prés de Malmedy. à l'extreme frontière linguistique. chu partieipe diamé $\left({ }^{2}\right)$ et du diminutif

(1) 111 existe enore. Depuis lis 1 re édition de eet article (B1) 1910, p. 6i3), j’ai releve 1" it Erezie, djămer aceonpler ", dans le "als suivant : pour faire une djanme (jambe, botte) l'oignons, m lic les oignons deux a denx par les tiges et on les djumige a "alifourchon sur une fieclle ; - 2" a Malmedy, djame, s. m., "arlore fourché, deux arbres sur le même trone" : - - $3^{\circ}$ à Berzée (S.-W. de (harleroi), ène djomilige ou troupleyge de mochites "un trochet de noisettes " - to a Wiers (S.-E. de Tournai), ringemiler "pousser deux on phusieurs jets an lieu d"un seul": lavinue ringemirle ou "l ringivili (ce qui est un mal) ; au lig.. "tramer, eombiner

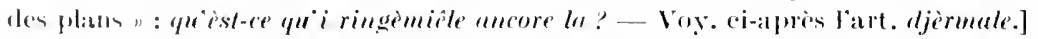

$\left({ }^{2}\right)$ A Roblertville : deus-abes qui somt djamés, devis neuss qui sont ajamées, denx arbres, deax noix qui ont grandi ensemble de laşon a former bloce. 
se djam'ler $\left(^{1}\right)$. que le français derrait pouvoir traduire par " se gémeller, se jumeler $n\left({ }^{2}\right)$.

C'est en effet du lat. geminare, * gemellare, que proviemment djamer, djam'ler : le sens impose d'emblée le rapprochement. Phonétiquement, geminat dome djame comme seminat : same $\left.{ }^{3}\right)$ : geminare domne djaner comme septimana: samaine.

Le w. djama a dû désigner primitivement un instrument qui sert à doubler. unir, accompler deux ctres ou deux objets semblables et semblablement disposés (par exemple un joug) ; puis, an sens passif, ce qui est de la sorte doublé, uni. aceomplé (comp. ad'rina, hagna). Lienploi général a pu disparaitre assez tôt $\left({ }^{4}\right)$, et notre mot n’aura survéeu que grâce au sens spécial et folklorique de "fête double \#. Li djama dèl C'iquìme dit plus que "la fête de la Pentecôte " c'est la. Pcutecôte comprenant, outre le dimanche, le lundi, fête chômée, qui en est le double ou la répétition. Suivant de pittorespues expressions liégeoises, e'est un "dimanche à fourche " ou "à deux coups". — S"étomnera-t-on que du sens de "jong" (ou tout autre objet analogue), on ait pu passer à eelui de "fête géminée " Mais l’argot foisonne d’imagges du même gout ; il s'en erée aujourd'hui même sous nos yeux. L'étudiant appelle "fourche " une hemre vide ou libre entre deus cours. Lorsqu'une fìte chômée tombe le vendredi ou le mardi. certains fonctionnaires réelantent la faveur de "faire le pont", e'est-ì-dire de chômer également le samedi ou le lundi.

L'association des idées est le facteur le plus actif de l'enrichissenent, mais aussi de l'altération sémantique : si elle multiplie les jets autour d'une sonche commune. la plupart de ces jets sont destinés à périr, quelques-uns seuls survirent. Ou, plus exactement, étant domé une souche mère, des racines peurent se projeter en tout sens et produire çà et là de nouveaux jets. entre lesquels l'oeil ne déeourrira tout d'abord aueun lien de parenté : il faudra mettre à nu les racines et les suirre jusqu'au point d'origine commune. Cela est surtout rrai dans les parlers populaires et djama en est un exemple caractéristique.

Pas de grande fîte sans bon dîner : ainsi, à Erezce notanmont, on-z-a fêt on bê djama quand on a fait un beau "dincr de fête ", et

(1) "S'unifier par la croissanee : dès cohes, dès crốpires qui s" djum"lèt ".(J. Bastin.)

(2) Le Dict. gén. donne "jumeler ", mais seulement comme terme techni(jue.

(3) L'ane. w. same est eité par Wilmotte, Notes d'uncien zuallon (189r), p. 18, n.

(4) A Malmedy on appelle encore on djama deux noşaux dans la même ceale de noisette (J Bastin). 
le mot s'est vidé de toute autre aceeption. - C'est aux djamas, surtout à celui de Pâques. que l'on étremne de nouveaux rêtements ; de là : dịa miton m' diama. j’ai mis mon "labit de fête " (Forir) ; djennè fren co mes djamas, [rons arez tort de jeter cela an rebut, moi] j'en ferais eneore mes "habits de fîte "(IIerve. Liège, cte.). - Djama est ineomm anjourd hui au pays de Charleroi ; mais on le trouve dans un texte de 1568 : les habitants de Jamioulx remettaient ehaque année au curé de Nalimnes 18 deniers de Brabant pour ses jamas de Pâques $\left({ }^{1}\right)$, ést-a-rlire pour son "catean de fète ". En namurois. djama signifiait ausi naguère: "ce qui se paic au curé à Pâques et à la Noël " $\left({ }^{2}\right)$. Le sens do "cadeau [de fiete]" est également comm en liégeois (Bull. de Follil., I t6). - Cette idéc arehä̈que de "rederanee "xplique qu'à Ciney le mot ne subsiste plus que dans: des riss djamas "de vieilles histoires $"$.

Pour l'ancienne langue. Godefroy ne tomme que gamas (lire djamas, fètes) dans Jean de Stavelot. Il fant ajomter les exemples surants: "por le reverenee des hans iamas, a savoin est le Noel. le Pasque, l'Ascension. le Penthecomste. les Tousains. Ies fjestes Nostre Dame, S. Lambert et li dedieasse... " (135:) : Ord. somptuaire ; in Leodium, 1903. p. 1.1); "az f'estes ct jamas" (1435: BSW 6. p. 102); "a hault jamae (qui y sont estaubly " (125\%: (7. des Métiers, I 97) ; les quattre jamas de l'an " (1573 : ibid.. P. 11s) ; roy. G., II 600. - Encore aujourd'hni. lis quate (gramds) djamas est une expression eomrante à Liege, comme en témoigne eette croyance populaire : Po s' sipargni lis mas d' dints et co tot plin dautes mihins, i fat promite di n' nin magnê dèl trhar as quate (arands) djamas. 1)'me persomne qui ne rit gुuère, on dit: ile ni rity quisis quarte diamas $\left({ }^{3}\right)$.

\section{w. djårdeus "ladre " ; fr. jard "poil"}

Dapres le Dictionmate gémóral. le frane. jard "poil long et dur dans la laine n est d'origine inconnue. Ce mot a des formes variées dans lancieme langue : jar. jard. jart, jars. gart, gard (Godefroy. IV, 202, 23:s. (1335-s; X. 3s) : mais le type primitif est surement jurd, gard,

(1) Vur. Lejeme, Hist. de Nelimes. 1) (is.

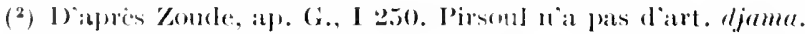

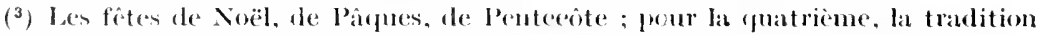

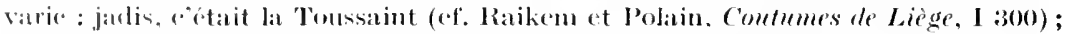
anjourllui, rent l'Assomption, sans doute parce que djamo emporte lidée de fête joyeune. - (On peut voir à a propos une dissertation intéressante sur les quatre

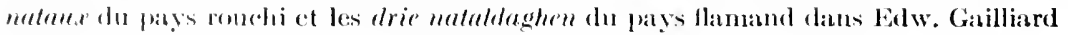

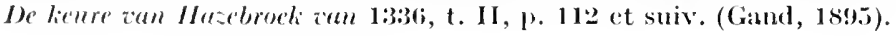


eomme l'atteste le térivé jurdens. gardeus, supplanté aujourd'hui par jarreux.

Une étude récente de II. J. Feller (1) a mis en lumière le rapport qui mit le franeg. jard (en wallon : djar. t. de tisserand a Verviers, "poil long et dur qu"on enleve du drap arec des pincettes") et le groupe wallon djardetis "ladre, en parlant du pore ", dicirder "langueyer ", djaide "ladrerie". Aree raison. M. Feller recomait dans ees mots un radieal commum jard- (2). Son étude. très intéressante et très documentée. n'apporte point eependant de solution définitive. Près de conclure, l'auteur verse au dossier l'ane. fr. gordement "rilement", gordin "niais", gordine "dćbanchée ". qui lui paraissent se rattacher à la même raeine. Du germani(que. il eite. mais pour les éearter aussitôt, le flamand gortig. le luxembourgeois gorseg. le has allemand d'Eupen gareteg, qui signifient "ladre". Le dictionnaire allemand. assure-t-il, ne donne rien qui rappelle la racine jard-; il ne s"étonnerait pas qu'elle appartint au domaine celtique.

Je crois, pour ma part, a l'origine germanique de jard, jardeus, djardens. Non pas qu'on doire poner comme antérieurs gortig. gareteg. garagg. Ces mots nont rien de eommun arec la famille jard, ni même, malgré les apparences, rien de eommun entre cux. - Grandgagnage (I 251) tirait djardeks de gortig; c'était s'enfermer dans une impasse. D'après Vereoullie $\left({ }^{3}\right)$, le nécrlandais gort "ladrerie " est le même que gort, grut "gruau " : la maladie est ainsi nommée à cause des gramulations qui se forment dans le tisu graisseux; gortig signifie done proprement "granuleux" $\left.{ }^{(}\right)$. - Le Wörterbuch der Enpener Sprache (Eupen, 1899) traduit gareteg par "garnartign (filamenteux), "faserig" (fibreux). "fimnig " (ladre); cent l'adjectif de gare (all. Garn). de même que son synonyme horeteg (hatrig. faserig) est celui de hor (all. Haar). La ladrerie, II. Feller l’a très bien montré, présente des caractères multiformes; rien d'étonnant qu'elle soit, dans des régions diverses,

(1) Bulletin all Dicfiommate atullon, 1914, 191, 21-30.

(1) .11. Feller rattable au même radical d'autres mots gui nonterensent pas direetement mon sujet et que, pour acte lasisn notamment. jerarte du debat présent. Ce sont : $1^{\circ}$ le franç jurde, jordom tumenr calleuse qui vient aux jambes du cheval,

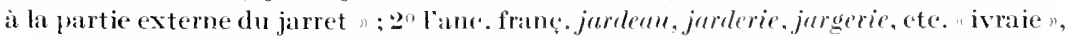
et des noms dialectaux de divornes varietés de gense et de vesce.

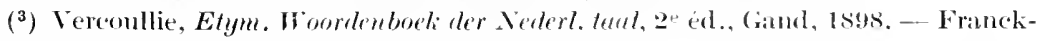
van Wyli ne parle pas de gort " ladreric ".

(4) Comparez : "Porecau ladre ct surecune : stis grandinosus ", citć par Godefroy, soursame. Voy. le Dict. gén., slresmel. 
dénommée diversement. - Quant au luxembourgeois garz, garzeg, ou mieux gârz, gârzech. (1). il répond, si je ne me trompe, au moyen haut all. garst "goût ou odeur ranee, féticle " et ì l'all. garstig. Ici encore, on aurait affaire à un autre caractère de la ladrerie.

Après cette élimination. une autre s’impose : celle des mots qui, comme gordement, gordin, gore, etc., ont o bref au radical. En effet. le thème que nous étudions est jârd- (plutôt que jard-) ; la roỵelle est longue comme en témoigne le a du wallon. Dans le fr. jardin, garder, barbier. la protonique n'a plus qu'une longueur moyenne ; mais. dans le wallon djardin, acirder, bairbî, elle eonserve toute sa quantité.

Quant au sens. le point commun entre jard, dijar " poil long et dur..." et djardeiss "ladre " mªpparaît très elair : e'est l’idée de hérissement, d'aspect rude et hirsute. Pour jard, djar, cela va de soi ; pour djairdêts. on a signalé just(ment, parmi les caraetères que présente le pore malade, "l'aspect spéeial du poil, rigide et gâté à sa racine".

Cela posé, sst-il rrai que le dictiomaire allemand ne dome rien qui rappelle notre thème jârd- ? Le type germanique correspondant serait gard-; comparez djabe. jarbe (gerbe). de l'ane. h. all. garba ; wairder, gaterder, du germ. awdon : et surtout l'ane. franeg. jart, jard (jardin, wallon djärdin). de l'ane. l. all. garto (auj. Garten). Il convient de ne pas eonfondre ce jard avee son homonyme dont il est iei question ; mais n'y a-t-il pas lì une indication?

Une fois sur la voie, on ira les yeux fermés il l'ane. h. all. gart "pointe, piquant, aiguillon " (gothique gazds, même sign., all. Stachel). Le germ. gart. gard - d'où l'all. Gerte, néerl. gard. garde "verge, baguette nexplique. pour la lettre et pour le sens. le franç. jard et le wallon djair, qui signilient done proprement "pointe, piquant". L'adjeetif jardens. djardens "plein de piquants " a pris en wallon le sens spéeial de "ladre". (Quant au substantif féminin djade "ladrerie ". c'est un déverbal tiré de djarder "langueyer ", suivant l'analogie de warder, wade, "garder, garde ", sirder, gade " carder, carde".

[Romania, 1914, t. xum, p. 432.]

\section{w. djèrmale}

G.. I "253, domne le masc. germal (jumeau). qui est assurément une crreur d'impression pour germai (lire diermê, qui existe eneore dans nos Irdennes : Stavelot. Vielsalm, Erezée. etc.). En liégeois, il a dis-

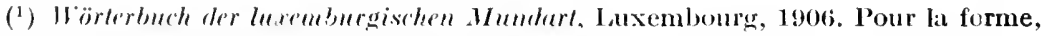
comparez ibid. hârz, harzedh (all. Itarz, hurzig). 
paru - sauf comme nom de famille : Germay - et le féminin djèrmale a pris le sens collectif du smonyme troliète, trolilete ("trochet") : c'è-st-ine djèrmale "ce sont des jumeaux" ou "des jumelles "; pour distinguer le sexe, on dit ine djèmale di zalets ou di baciles. ('et emploi remarquable du féminin arec le sens eollect if existe dans la plus grande partie du domaine proprement wallon ${ }^{1}$ ) et coneorde aver celui de djumèle à Nivelles et de hmèle dans les Vosges $\left({ }^{2}\right)$. On dit de même une doublée dans les Artennes françaises $\left({ }^{3}\right)$. Il y a sans donte ellipse d'un substantif telle que "portée ".

Il ra de soi que diermale pourra en outre se dire de tout re qui ust géminé, par exemple de deux fruits jumeanx: aux Encilles, c'est le double épi que porte parfois la même tige de seigle. ou cncore denx gerbes on copales dressées ot liées par la tête.

G. tire notre mot du lat. gemellus. avee épenthétique : de même Scheler, Niederländer. Pietkin $\left(^{4}\right)$. qui justifient l'insertion de $r$ en invoquant l'influence de germain. Pour ma part. jai autrefois combattu l'opinion de G. paree que gemellare a domné en wallon diămilé et que gemellus doit normalement devenir djamê $\left(^{5}\right)$. Aujourd'hui. je crois que. si djèrmê se rattache à gemellus, ce n'est pas par roite directe (eomme l'ane. fr. gemel, jumel). nais par l'intermédiaire d'une forme *aemerel, le suffixe -el ayant cédé la place au suffixe plus plein -erel $\left({ }^{6}\right)$ : djèmê résultrait de la métathèse dont nous citons de nombreux exemples à l'article gorlète. Cotte hỵpothèse se fonde sur le rouchi jum'rîle. s. t'., "épi double ", mot inédit que je déenure à Ellezelles. En déjit de la différence d'aspeet, djèrmale et jum'rîle sont bel et bien. si jose thre, des sours jumelles.

(1) Liège, la Famenne, Jodoigne, Namur, Dinant: djèmnle ; Stave, Givet: djèrmèle. Les exceptions se rencontrent surtout dans la province de luxembourg : on ne eomnait pas le sens eollectif à Erezée, Odeigne, Ortheuville. Lavacherie. Une fenme de Compogne (au Sud de Houflalize), qui ne comaissait ni djèrme ni djèrmnle m’a dit : dui n'é jamins arou dès djèrmalés. Ce curieux dérivé suppose l'existence antérieure de djèrmale an sens eollectif.

${ }^{(2)}$ Horning, in Mélanges IVilmotte, I 234. Lauteur se demande sil faut y voir un anejen neutre.

(3) Goflart, Giloss. du Monzomuris, supplément.

(4) Seheler, Gloss. de la Geste de Liege, p. 167 ; Niederländer, Inndurt von Nomur, $\S \S 23$ et 7t: Pietkiu, Orthogr. du we. malmédien, 1) 7 .

$\left.{ }^{5}\right)$ BD 1910, p. (62. - Depuis lors, on ma signalé que djamé "xiste réellement à Malmedy, ou il signifie: "arbre fourché, deux arbres sur le mêne trone ". Voy. ci-dessus lart. djamu.

${ }^{6}$ ) Comp. le fr. passerelle, tomberean et, ei-après, l'art. hèrmale. 


\section{liég. djèrson, djèr'çon}

Ce mot désigne une partie de la gorge : le pharynx ou gosier ; on ne l'emploie guère que dans certaines locutions : si ramouŷ̀ l' djèrson "s'humecter le gosicr " dè pèlèt qui " pice li djèrson "du genièrre qui rous pinee le gosier". Remacle et Forir hésitent entre les graphies dièrson. gèrwon ; de même G.. I 168, qui ne donne pas d'étymologie. J'ai contendu à Glons-sur-Geer guèrsm (avee g dur). A première rue, on peut y soupesonner la présenee du suffixe diminutif -con (roy. l'article omg'con); mais le radieal reste obseur. parce que le mot paraît être isolé dans nos dialectes.

Cependant il existe. an Sud de la province de Luxmbourg. nn terme qui présente aree le nôtre des analogies de sens et de forme : e'est le chestrolais-daumais gorgossom "trachée-artère, gorge" (1). Il nous suffira de déeourrir l'anc. nam. garguechon (2) "gorge". pour que la présomption se change en certitude.

On admet que l'onomatopéc gorg- a donné aux langues romanes un certain nombre de termes, tels que le fr. gargamelle, gargote. gargoniller et l'ane. fr. garguette "gorge " (Meyer-Liibke, no 3685 ). Le namurois moderne comnait encore ce dernier mot. qui a pris con liégeois la forme adoncie djerdjete (G.. I :331. II xxvi). L'ancien namurois garguechon est un climinutil de la mòme raeine $\left({ }^{3}\right)$. En liégeois. par syneope régulìre de la protonique non-initiale, il cut devenu *guergogon ; d'oì. par disimilation : guèregn (Glons). et. par adoucissement normal de la gutturale : dier çon.

\section{w. djêve, gawe, badjawe}

Sous le type * gaba "jabut". Meycr-Lübke cite quelques formes dialectales nuslernes. notamment le picard gaz'. le wallon saf ct le namurois djef [sic] "jahost du pigeon". Ces indieations. en ce qui eoncerne nos dialectes. ne sont pas des plus exactes. Quoi quen disent G. I 22s. II 508. Forir et Sigart. gaf-oumieux gaze comme en aneicn francais - lla pas cours an pays liégeois (le jabot du pigeon s'y appelle foce) : ce niest même pas du wallom proprement dit. Giàe appartient

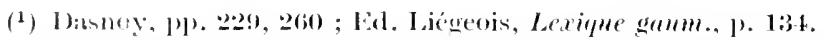

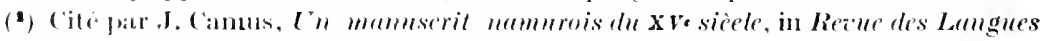
romenes, t. xxxvu (1895). 1). 1633. - I'n trouvère hrabançon enploie de même

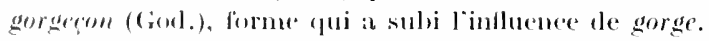

(3) Ice chentrolatis-ganmatis gargogon ent mis pour gergueģon; gargossi (Marche(th-fanchne), gargozin (Vonêthe) ont subi linfluenee du sullixe -ellum. 
au pieard et au messin. De là il pénètre en Wallonie dans le gaumais, le chestrolais. le givétois (sous la forme gaze, qui se prononee găf). et dans le namurois, où il derient djafe (Namur. Stare, (iney, ete.). Il y donne plusieurs dérivés. tels que gare (Givet) "gaver"; gaciot ou gavion (Fosse-la-Ville) "larynx, gorge " savéye (Neulchâteau) "jabot d'oiscau, devant de chemise, gorge ", proprement "gongée. plein la

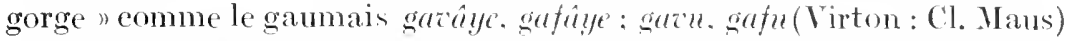
"qui a un gros jabot" : ete. Quant à dicf. qui noms est donné comme namurois, il ne me parait pas des plus sûrs. Je n"y rois. pour ma part. qu'une graphie approximative de diâe qui existe notamment à Fossela-Ville au sens de "gésier", à Gembloux, en Hesbaye, en Famenne et dans la province de Liège an sens péjoratiö de "gueule". en parlant d'une persome ${ }^{1}$ ). Reste à voir si ee mot bien wallon diếe sexplique par le type proposé.

M. Dauzat $\left({ }^{2}\right)$ a publié récemment une ét ude approfondie sur * ga ba et ses dérivés. Il admet plutôt un type primitif * gava. d'origine probablement celtique, et démontre que *gatut"a "joue postule un intermédiaire *garita (et non * gabat a comme "jatte "). Trompé par la mauraise graphie dicf de Meyer-Lübke. il entime que "seul, ce mot namurois [lisez plutôt : "liégeois") cst phonétique ". Je ne puis partager son aris: *gaba ou *gara aurait domé en liégeois diête. comme faba $>$ féce et l'imparfait - abam $>$-etee. Pour expliquer diêze, il faut comparer cavea > tchểe "grande eage pour transporter les pigeons. les poulets. ete. "*sapius> sềe "sage, lueide". et partir d'un dérivé *gabia (que Meyer-Lübke ne donne pas) ou mieux gavia. Dans l'étude intéressante que j’ai signalée. II. Danzat admet que garia. mot rare et isolé en latin. attesté seulement par Pline l'Aneien au sens de "mouette " (cf. Meyer-Liubke. no 3708). se rattache nécessairement ì *gava. la monette étant remarquable par la grosseur extérieur de son jabot. Le wallon djếe attesterait que ga ria a survéeu à l'extrême Nord-Est du domaine roman.

Au reste, ce n'est pas le seul dérivé important que le wallon a conservé sous une forme originale.

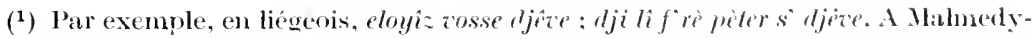
Stavelot, djête signifie "bouche bavarde, caquet ", d'ou les dérivés djêtè djêtler "jacasser ". A Dinant, Cembloux et en Fameme, le son $\dot{c}$. tout en restant long, n'est pas aussi ouvert qu"en liégeois. A C'harleroi, daus le Coq d' twous' du 13 mars 1909 , on signale bien djère " aquet " fe uler s" ljèce su tot $l$ momde ; mais il faut probablement lire djềve, à moins que $\hat{e}$ ne se soit réellement alrégé par altération.

(2) Romania, t. xLy (1919), 1p. 250-258. 
A propos du liégeois-namurois gaze. s. f.. "guimbarde, petit instrument sonore...". G.. I 233. pour toute explication, se demande s'il faut y voir une onomatopée. En réalité, le sens de "guimbarde ", aujourd'huile seul comnu, est secondaire. Il est certain que gawe répond littéralement au fr. joue et vient, comme ee dernier, de *gauta, dont nous parlons ci-clessus ; comparez eare, anc. fr. eone, queue ; haze, hone, mai'e. mone. etc. Le sens primitif se perȩoit eneore dans l'expression ironique fé aler s'gazé "faire aller sa bouche, pour manger ou pour parler". et dans le dérivé gacî (Namur) "brifer, gruger ", djazŷt (Luttre) "bavarder".

On notera dans ces deux derniers termes $g=d j$. comme dans găve, djăie. que nous arons cités plus haut; de même le fr. s'engouer a la mòme origine que joue. On ne s'étomnera done pas si nous royons dans baljaze un composé de gaze. G.. I t2, ne connaissait en liégeois que la forme badjowe et le sens de "babil. caquet, bagou ". En fait. badjaze est la forme prenière, commue aux environs de Liège et encore à Liège même (comparez le w. caže "queue", à Liège coze). Ce mot signifie 1. "bajoue ", équiralent littéral. qui subsiste dans l'expression on li a fêt pèter s' badjuice "on l'a soullleté" ; - 2. bouche bavarde : cloyiz onsise badjace. fé aler s' badjacie ; - 3 . persomne bavarde ; - 4. carpuet, hagou : il a "ne famenise badjacie.

Le liégeois badjarie répond donc à deux mots franęais bajoue et bagou, auxquels les étymologistes nassignent pas la mème origine. Ont-ils raison? Il cot permis d'en douter. Le fr. locosou. pour clédat. est un terme d'argot. ce qui dispense apparemment de plus amples explications. Diez. Scheler. Körting. Meyer-Lübke ne le signalent pas. Pour le Dictionudire sénéral - qui reprend en somme. arec des attématioms pruclentes. lopinion de Littré, - "bagou est pent-ĉtre substantif rerk.ul d. l'anc. fr. basonler. parler incomsidérément, lequel scmble composé avec la particule péjorative ba ct goule pour gueule, c.f. débagmeler". On peut se demander si bagouler nest pas un aneien *bagoner influencé par goule. L. w. badiace appuie cette présomption. Ajoutoms que "bajoute" se dit bajole en rouchi. bajote à Toumai

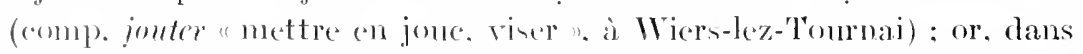
ces mots. la finale ne pent sexplipuer que par des influenees analogुiquiles.

\section{liég. djihan}

G.. I 2.52, distingue antre

1. Gehan, gihan (Jeaus)... [Lire Djhm, Dihrom.]

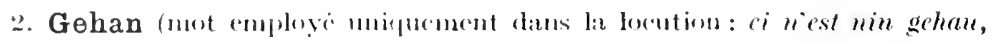
cest costrent, qui, actucllement, signilie simplement : cest (her ou très cher). 
Cette distinction est mal fondée. Imaginons la scène suivante. Un enfant prie ses parents de lui acheter un objet et répète ses instances:

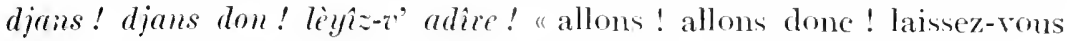
fléchir !" Au lieu de lui dire sérieusement : "Ninsistez pas ; ee que vous roulez eônte trop eher ". on lui répond plaisamment par la phrase susdite, qui eontient un double calembour : on joue sur le sens de djans (G., I 251), que I'on feint de comprendre Djham, et sur le sens de costant (eoûtant, eouteux; ou Constant. nom propre ; roy. des exemples dans Remaele. $2^{\mathrm{e}}$ éd., pp. 40 et 565 ).

Ce genre de réponse par équiroque plaisante est bien eonforme au tour d'esprit du peuple en général et surtout de nos Wallons ; j'en ai réuni une foule d'exemples. En roici deux, eueillis dans la eomédie fameuse de Remouchamps. Trît l’ pèriquî, v. 51 et 548 (BSW 48) :

Este z-v" la? "Etes-rous là ? " Linterloeuteur feint de comprendre l'a "l’ail " ct répond: Nèui, dji sol'osnon!

Qui i" somne-t-i don? "que rous semble-t-il done?". L'autre feint de comprendre somer (saigner) et répond : Dii $n$ somme nin. dj rètche tot blane "je ne saigne pas. je crache tout blane".

De même. ee serait un tort de rouloir explicjuer grarement l'imprécation : qui l' diâle èl possîhe! C'e n'est qu'uné atténuation de : qui l' diâle 'l possite! (= possède). par jeu de mots entre sèt'. sept. et sîh. six. - De même encore, dans dï lî liề à dobe fi (Remaele, II 15s: "je le lui souhaite de tout ecur"), il y a confusion voulue entre liête (roy. l'art. ci-après) et liehse a dobe $f i$ "eoudre an donble fil " : - $i$ r'grèt'rè s' mérè azou dès-ongues di fiér : confusion voulue antre rigrèter " regretter " et rigrèter avou dès... "regratter arec des ongles de fer ".

[Romania, 1911, t. xL, P. 323. - En qúnéral, ces éfuiroques se comprennent d'emblée, l'allusion conservant sa force lumoristique. Inus : ésse a Siml-Pô "avoir le gousset vide "tout Liegenis saisit le jeu de mots entre po, pesu, et

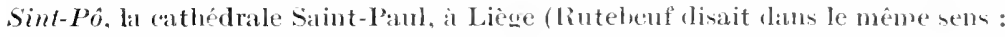
"être de la paroisse saint Pou : Goul., pot). Parfois cependant labllusion, moins transparente. nest plus saisie par le jexple, qui comtinne a ascle de lexpression toute faite. Comment expliquer : aceur li tiesse fon des strins (uvoir la tête hors de la paille $n=$ être sorti dembarlas) ? Pour ma part, après hien des recherches infructuenses, je pense qu'il y a. ici cucore, mo trait d"humur : on a rapproché strin (étrain, paille) de l’impersonnel $i$ strint (" il ctreint " = la situation est eritique). Ene locution inclite du vocabulaire de bu intellerie liégeoje confirme cette hypothèse : si mite a strim. -e coincer, conncer son batean entre deax antres par suite d"une fausbe mancenve, ne plus pouvoir avancer ni reculer.] 


\section{liég. djîvå}

Le djiâa. c'est la tablette de la eheminée. La ménagère y étale des bibelots. des statuettes. deux chandeliers et le grand crucifix de cuivre nommé en liégeois bou Diu d" djižai on y met les brocales (allumettes) et Famamar (almanath). Un petit rideau tombe dordinaire de ect entablement : on l'appelle beaye. brayie (Liège : Forir). rabat do djî̃au a Bande lez-Marehe. gudine do djî̀tu à Orthenville. Aujourd'hui, ee vieux terme tend à disparaitre arec l'antique cheminée ardemaise à feu onvert: on dira de plus en plus so li tch'minêye an lieu de so l'djî̀i ${ }^{(1)}$.

Laire d'emploi de ce mot représente un vaste quadrilatère. On dit

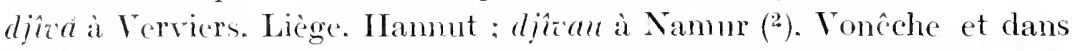

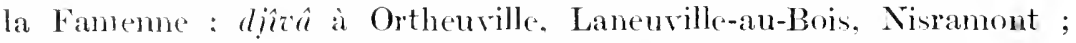
puis. en remontant vers le Nord. à Cherain. Malmedy, Spa. Seraing. Partout le sens est le même, sanf à Vonêche. ò̀ dî̃zan désigne la "corniche d'un meuble ". et aux usines de Corphalie lez-Huy oì djîzâ désigne le "dessus du four à zine".

Pour expliquer dîna. G.. I 255, propose le fr. ogize aree suffixe augmentatil -a et suppression de la syllabe initiale. J'y rois. pour ma part. le néerl. gezel, ab. giebel "fronton, pignon ". La forme remarquable du luxembourgeois giezel et du westphalien giewel convient pour le radical et même pour la désinence de djỗa : comp. l'anc. w. stivanz (BSW 5, p. 386) = néerl. stierel. all. stiefel ; tet le liég. djérdja. nam. djordjun "jable " = néerl. gérgel. all. gargel $\left({ }^{3}\right)$. Tont au moins le radical djî̀- pent en être issu. ot sètre combiné avec le suff. -a. sur le type de càce : caza. - Quant au sens, diüa. après avoir tout d'abord désigné le fronton (de la eleminée). s'est appliqué spécialement à la partie inféricure de ce fronton: la cimaise on tablette. Ce qui a pu faroriser le changement te sens. c'ent l'expression ordinaire "mettre qqeh sur le diäa ". cest-à-dire. dans mon hypothèse : "sur la tablette (du froston) do la cheminée " $\left({ }^{4}\right)$.

[13I) 1912. 1., 93..]

(1) Nons trouxoms gyzaz (1'mne chemince) en 1414, Cartul. Ste-Croix, et dans Hemricourt, p. 34. - Cette tablette saplelle aussi cimd (liège : Simonon, Forir), rimumlje (Nimur, Wavre), cĕmumlje (Perwez, St-Géry, en Brabant). C'est le fr. cimaise ; voy. G., II :361.

$\left({ }^{2}\right)$ Dipres (x. et l'irsoul. Ce dernicr ajoute : mot très peu usité et de provenance

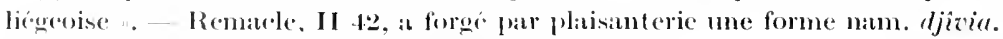

(3) G., I 2.5.3, 11 601. On dit djordjé a Faymonville.

(4) llene brachylogie dans le fr. fimilier : "mettre afeh sur ha fenctre " (= sur

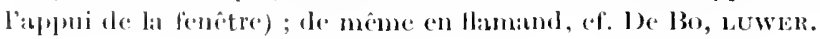




\section{w. djîvèye (Lìgge), -éye (Namur, Giret)}

Terme archaïque de la batelleric mosane. qui désignait un " train de bois llotté " $\left.{ }^{1}\right)$. Un article du Couquen. sazette de Namur. 10e année,

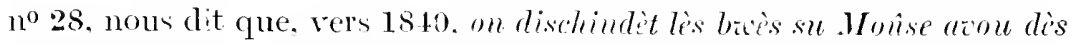
grandès dị̂zéyes. Le Tocabulaire wallon (dialecte gizétois) de M. .J. Waslet signale aussi djû́éye, f.. "train de bois monté sur des tonncaux vides et servant au transport, par eau. des perehes, des étancons, ete. ; on disait également pouris-talons". Je tiens de l'auteur la note complémentaire que voici : "dî̄éye se dit cncore à Givet pour désignor, par métaphore, de grandes quantités de joncs. d'herbes. que l'on coupe sur le bord de l'eau et qui partent d'un seul coup. Le sen propre n'est plus eonnu : depuis longtemps. on n'use p'us de ce genre de transport pour le bois ". - En liégeois. G.. I 2.5.5. II 601, note sans explication :

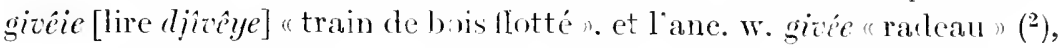
dans ce texte de 1568: "arhepter de toutes sortes de rond bois à borhea et givie " (Chartes des Métiers. I s.). Il domne aussi l'ane. w. "gymeth (bois flottant) ", en 15.8 : "le tonlieu que l'on liere i Iruy sur les Mairines [= merrains] et gyneth ou bois flottant " (Lourrex, I 220], où il faut sûrement lire gyeees. Du reste. notre mot a subi d'autres mésaventures. Dans des eomptes de 1 tro. relatif's au tonlieu du Pont des Arches, publiés récemment $\left({ }^{3}\right)$. on lit ginée ( 1.5 fois) et même

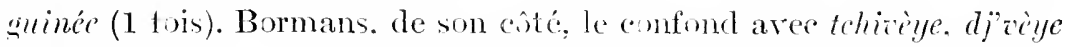

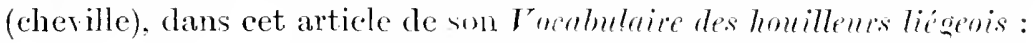

chiveie on giveie s.f. Rivlean frim: l'me centaine de pienes de bois chevilles ["] les mes aux autres et que lon amene flottant par les rivieres pour l'usage des fosses. "Retenant lemr prate de tontes ustensiles et de toutes

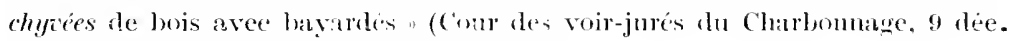
1666i). - signifie anssi une broche de buis.

Il ra de soi que tchiziye, di'itye (cheville. broche de bois) n'a rien à démêler aree dijồ̂ye; mais la forme ancienne dhyze'e est sllggestive. Nous la lisons *tehîêye et nour y royons un dérivé de tehif. s. m. (ane. fr. chief, fr. chef : *apum). formé à laide du sullixe -îye (fr. -ée:

(1) Symonymes bosséye. bosselize (G.. Il xiv).

$\left.{ }^{2}\right) \mathrm{G}$. dit que gizé est ansis anc. fr. : je ne tronve pourtant rien de ce genre

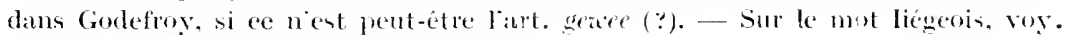
eqalement Body, Toe. des charms. 1). 10.

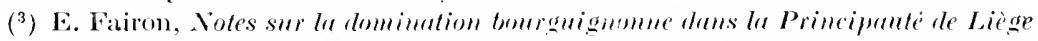

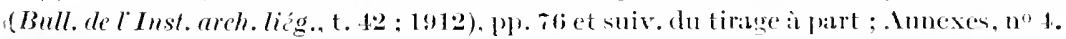


lat. - at a). marquant la quantité eontenue dans le prinitif. Le w. tchîf (proprement : "bout [de eorde] ) survit dans les houillères liégreoises pour désigner le câble qui amène la cagse à la surface, ou la "berlaine " au haut dime pente. La masse de bois llottant était liée par une corde qui l'empechait de se disloquer ${ }^{1}$ ) ; cette corde avait même probablement une longueur constante et servait de mesure. Sur le type de naiêye (naréc : contenu de la nef'). on a formé *tchîcêye (contenu de la corde. masse liée par des cordes). - Pour l'adoucissement de l’initiale. favorisé iei par le à qui suit, comp. diontî (chantier), djalila (G. II 530).

\section{namı. djôguîye}

G.. II 5:32. eite sans cxplication le nam. jôgute " jeu che quilles".

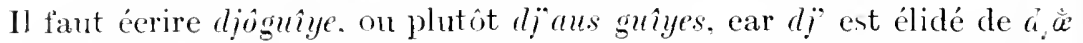
a jeu,; comp. dju d guiges à Warre, et ome bole aus guîyes (Ste-MarieGeest) " whe boule qui sert au jeu de quilles".

Voici diautres expressions où se remarque une élicion analogue:

lizrihaye. ৬. m., "chef d'une brigarle de briquetier's" (G., II 30 ;

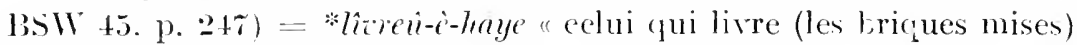
en haie ". c.-i-d. séchées et prêtes pour la chisson. - Comparez paibtasitele ci-après.

mal-guenye "souflets (Verviers: Lobet) = main-al-guenye "main à la gueule $\%$ [G. II 69 et $5+0$. écrit à tort mêle-guéuye en comprenant mule "male, manvaise, mais. dans ce cas, Lobet aurait écrit maull.

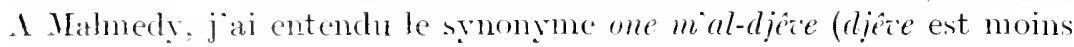

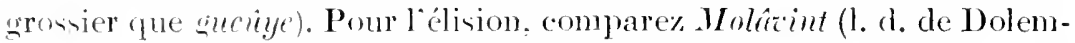
brens) = molin $\hat{t}$ int "moulin an rent".

maulié (nam. : (i.. II 5.2 ) = mun aulié "mal coiffé n; royez ei-après larticle icelit.

montenss cinonté, (arel.: Ille-snr-Semois). = man onteus " mal

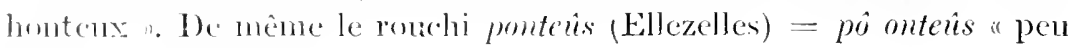
hontens. inupudent n.

Imerace "setit pocton de terre, (Lobet; $\mathrm{G}$., II 189$)=$ po-a-caie

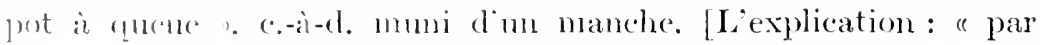

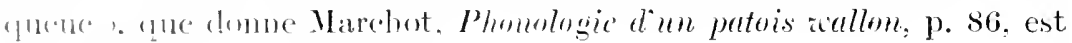
incracte ; roy. le suivant.|

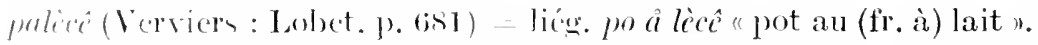

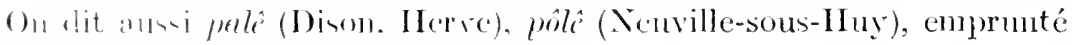

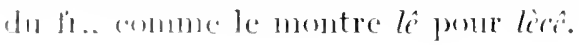

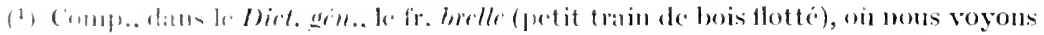

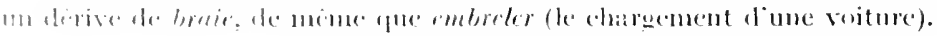


pivèrtasèteh (Verviers) = liég. prièten-a-sèteh "porteur-au-sac", = fr. "portefaix".

s'lonbran = s'lo ombrant; roy. l'article sclanbran.

t-infini "temps intini " (Neuville-sous-Huy: 131) 191: p. 4s) = tin infini : lé mèsse a duré on t-infini.

Les noms de lieus foumiraient ansi me foule dexemples de ee genre de eontraction. Le nom wallon de Villers-aus-Tours est riy-astoûrs (prononcé Viyatồr). I Esneux. II. Edgar Ronard a relevé les lieux dits cratomke, è faleôr, al haminire, qu'il explique par rrè a Tongue, $\dot{e}$ fond al être, al hè às mimînes.

D'autres expressions encore s'expliquent par l'ellipse de la conjonction it ; par exemple : 1. hoter à ma 'me aw'ye (t. de homill. à Seraime) "fissurer (la roche ou la veine) arec un mail et me aignille "; - ?. dutindelure (Malnedy-Stavelot) "de bonme heure ». (r.. dams ses Eitraits de Villers, p. 26, y royait "de tendre heure" ; mais l'expresion complète di tins ìt d'eire (= de temps ct d'heuren) se lit dans une pièce liégeoise de 1634 (Choix. p. 109) et dams la Moralité de 1623 (roy. mon étude sur le Dialecte liégeois an XVII sicule. p. (66) ; comp. Exse prèt" a tin a ềre (Fr. Renkin, Ecrits iallons, p. 69).

\section{W. djohe (klćrom)}

Dans les houillères à l'Est de Liege (Fléron), une d óte c'est me des quatre grosses pièces de bois qui forment l'encarlrement du puits de mine; d'où djoliler "mettre des djolees" (1). La proposition de G., II 532 : "djol, = fr. joug ? " est inaeeptable, si clle signifie que le mot wallon est une corruption du français. On ne peut pas non plus penser au lat. jugum, qui a domné djổ (Vielsalm, Moulin-du-Ruý, Weismen), djề (Villettes-Bra, Wardin, Gros-Fays, ete. ; royez aussi Brumeau, Enquête, I, 498). Le wallon eomnait djoli (se mettre ì djoli : se percher, en parlant des poules) qui, comme l'anc. fr. juc et le fir. jucher. vient du franeique juk " joug, traverse de bois s $\left(^{2}\right)$. An point de rue phonétique, le passage de djok à dijol se justitierait à la rigueur. 'Toutefois, étant domé l'aire restreinte de djolie à proxinitć de la frontière germanique et l'emploi spécial de ee mot, nons y verrons un emprunt direet de l'all. joch " joug, smpport ", qui a la mème aeception tech-

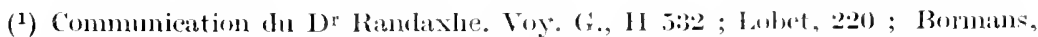
Voc, des houill. liégeois. - I ronest de Liège (Seraing), lit piones sippelle : mimbe di cope, et lencaullement susdit: une cope dissise "couple alisise [sur laupulle s'ippoient les taquets] \#.

(2) Meyer-Lübke, $n^{\prime 2} 4611$. 
nique (1). Le yod ient ápaissi en di, comme dans sardiete (Stave) "sarrictte. Pour la finale. royez lauticle solie.

\section{w. dognnon}

Ponr G.. I 1sio. dimim (Licge, Namur) "enfhure on eallosité qui se produit i la naisanee du gros orteil "est probablement le même mot que le fr. aisnon. qui a la môme signification.

Le sems de "oignon. durillon. callosité domboneuse au pied " est donné par Forir (Licose). Tobet (Vervicrs). Scius (Malmedr): mais laclée de "callosité n napparait plus dam les définitions suivantes: "iointure du gros orteil "(Malmedy : Villers, 169:3), "gros orteil " (1)ur.). ( excroissance onseuse eontre nature aux pieds " (Lobet), "saillie exagéce de la tête du premier métatarsien. produite à la face interne par la deviation du gros orteil " (1) Randaxhe: Thimister-

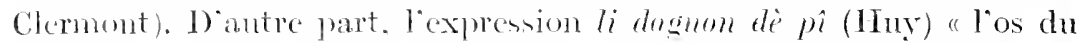
gros orteil " montre gue notre mot a un sens général, puisquion a besoin de le détorminer quand il s’agit de l'orteil. - Plus à l’Onest. il désigne mingument me articulation de la main : a Ben-1hin et à Gives (lez Huy). jai noté : les dosnmus del main "les arites osseuses

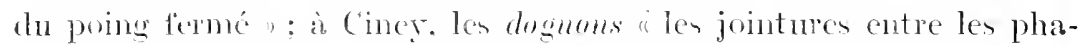
langer des Hoints de la main ou du pied ": a Pellaines (Hebbaye). les enfants joment anx billes pour des chinolies: coups de bille sur le dosum. c.-ited. sur larticnlation da métacarpe et de la grande phalange : a Namur. le dognom cest le "dos du ponce n (F. D.. Diet. ms., $1850)$; i Warre, le "dos des doigts " ; Charleroi, l" "éminence articulaire entre la preniere et la deuxieme phalange du doigt ".

Licxplication de $\mathrm{G}$ est inadmissible. Phonétiquencnt, il faudrait justilier la prosthene anomale de d. Ponr le sens, lidée de "oignon, "allosite" ent risiblement secondaire; elle n'apparait d'ailleurs que dans un coin du domaine walton. Pour noi. dognon provient de *doyon,

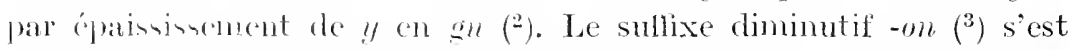
ajoulé an primitif dhige. ane. fr. doie, du lat. *dǔta (eomp. w. deû, fr. doigh. du lat. *atum, class. digitus). Notre mot désigne done propecuncul unc partic du doint, la partie forte et saillante. Comme le desumb. surtout au pieri, pent derenir callenx, cette particularité,

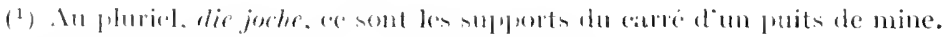

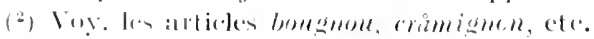

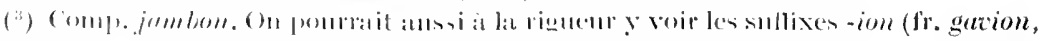

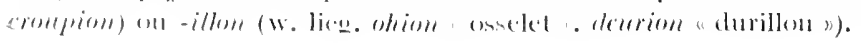


jointe à la ressemblance avee aginon, a modifić et restreint le sens propre sur certains points du Nord-E,t $\left({ }^{1}\right)$. - Le primitif dôye. qui manque au Nord de la Wallonie. se rencontre en Famenne : aceur lis dôyes idjaleyes "aroir les doigts de pied unclés"; de mene à Ciney : lis dôyes (doigts de pied), li grosse dôye (l'orteil). Sur la Semois et dans l'Ardeme française, doye, dok̂ye a le mênc sens $\left({ }^{2}\right)$. En gammais. doutyon "petit doigt de pied " existe à côté de douye "doigt de pied " $\left({ }^{3}\right)$. Pour la formation, sinon pour le sens. ce douyon ne fait qu'un avec notre dognon.

\section{rouchi dona (Mons)}

Sigart dome le montois "dma, imbécile. dupe ». M. Behrens a eru voir dans ee mot un don-a(rl) primitif qu'il rattache au gaumais dône et au radieal dam- $\left({ }^{4}\right)$. Au point de vue phonétique. cette explieation prête le flane à me double objection. Le radical durn-. s’il existait en montois, y garderait cette mime forme; comp. fr. mame, montois marle, gaum. môle. De plus. le sullixe -ard donne, suivant les localités, $-a r,-\hat{a},-a$ dans la région montoise. jamais a bref: on tronve par exemple dans Sigart dudlar, tufiar. lomgnar, macard, à côté de macĥ, hougniâ, hulau. - On recomnaitra plutôt dans ce mot le prénom Donat, employé, comme tant d'autres, dans un sens sarcastique. L'exemple domé par Sigart : il a sté dlmu del frerce. signitie : il a été (le) Donat de la farce; comp. le fr. gille, niais, et le montois jacqué, dupe. La même explication, s’appliquera au montois sark. s. m., fille étourdie, remuante, espiegle (Sigart). - A remarquer que l'expression de Sigart n'est plus commue aujomrl'hui à Mons. On y commait seulement, comme dans toute la Wallonie. le prénom Dónat, qui cutre en liégeois dans une eomparaison plaisante : on dîreût on p’tit saint Dônat, comme en fr. : "on dirait un petit saint".

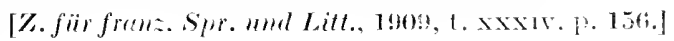

(1) Cette altération sémantique doit ître de date récente : à Liège même, on m’a

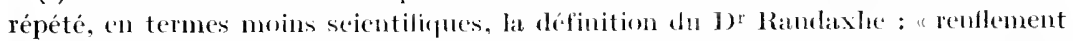

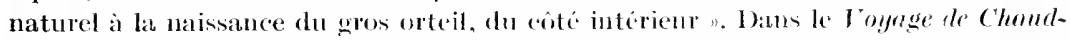
foutaine, IlI 1 : atot tès dames scafignoms, ti mias crisi spute $l$ dognom, il faut done comprendre : orteil ", et non "ognon ", comme fait Batleux datus son dobition du Thêitre liégeois, p. :-29.

(2) Brmean, Enquête, I, p. viri.

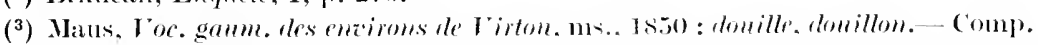

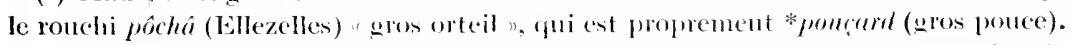

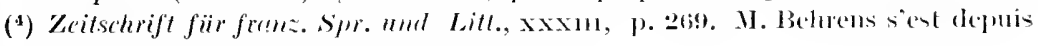
lors rangé à uotre avis ; voy. ses Beiträge, p. 


\section{W. d'ploustrer, d'poûstrer (V'criers)}

G.. II 520, cite daprès Lobet (elploustré, p. 159) ce mot verviétois. qui signifie "dévaliser ". Lobet enregistre également dpoustré "dépou(lrer (les chereux), désargenter. dégarnir (qgn) de son argent ". On aurait tort de voir entre ces deux termes un rapport de parenté. - Le verbe simple dont dploustrer est eomposé se retrouve dans le moyen has all. plnsteren. ane. flam. pluysteren "piller "; eneore aujourd'hui, le west-flamand commait pluistere" "éplucher " $\left.{ }^{1}\right)$. - Au lieu de d'ponstrer, ou mieux d'pot̂strer, on sattendrait à d'poûtrer, puisque le simple est poûtrer "poudrer" $\left(^{2}\right)$. Pour expliquer l's anormale, on peut inroquer lintluenee de dpons'ler "épousseter "; mais il raut mieux. je erois, y voir le résultat d'une métathèse $: d(u s)$ poutrer $=$ d'pôtstrer. Jexplique de môme $d(i s) w \hat{k} b i=d$ wêsbi (G., I, 178; roy. ci-après. l'artiele $a \hat{e} b i), d(i s) f r \hat{t} t i=d$ frếstî (Duvivier : "défrayer r).

[BI) 1920. p. 10.]

\section{nam. dronke}

Lieczéma infantile, en fr. les erốtes de lait. sappelle li dronhe à louest et au Sud de Liège (Engis, Huy. Harzè). à Erezée (Lux.). à Noiseux (Namur) ; li dromle en namurois (Namur. Ciney: Dorinne, Stare; Gembloux. Forville : prov. de Brabant). lès dronlies à St-Géry, (Brab.) et à Vicsville (près de Luttre: Hainaut). G., I 183, donne sans explication le nam. drongue $\left({ }^{3}\right)$. C'e mot, en dépit de son aspect germanique. a une origine romane bien assurée. Il reproduit le moyen latin dracunculus "aposthème. ulcère" $\left({ }^{4}\right)$. d'où l'anc. fr. draoncle "apostène, éruption eutanée, ete. ". Un texte namurois du xve siècle porte cette recette: "Pour drongles, R. de la farine de soile [seigle] pillée en moitict vin et moitiet yawe" $\left({ }^{5}\right)$. Au point de vue phonétique,

(1) Voy. I) Bo Bo, ansi (fuc Franck-ran Wyk, pucrs.

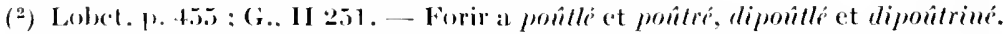

( ${ }^{3}$ ) Plus loin. II xxi. il enregintre, d'après Simonon, le s. f. dronhe. De là, cette forme a pascé daus le dictiomnaire de Forir : mais elle n'est pas connue à Liège, du moins anjourl'hui. Le lieg. dit lis senyyes (esoies ").

(4) (f. 1)" (ange, drumnculus on drumenlus (ulceris vel cancri sprecies). D'après 11. Ant. Thomils, le gr. bpxxivasoy "rer qui siengendre sous la peau "prouve que

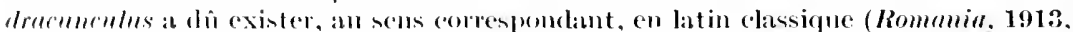

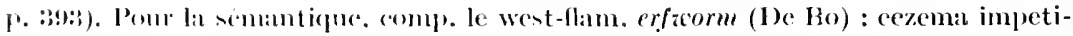
ginoïles.

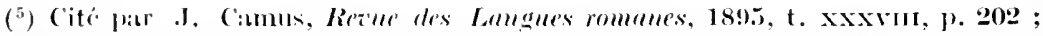
rf. ibill. p. 160 : drangler, dromglure (apostime). - liarticle de Meger-läbke,

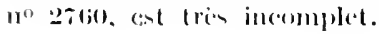


comparez le liég. ronhe (rancher ; G., II :32t). altération du nam, ronlie (= ane. fr. ronghe. dans trois textes tournaisiens cités par Godefroy"). [BD 1920, p. 10.]

\section{w. d(u) grâde (Verviers, Malmedry)}

G., I 171, se contente d'enregistrer, d'apr's Remacle. „2e éd. : "digrîte, probablement, assurément : $i$ rimit, d'grite : il reviendra, probable-

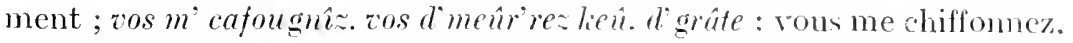
je suppose que vous allez rester tranquillen. - Lobet (Verviers. 18.j.4) écrit : dgrande "probablement. rraisemblablement ». De même, Villers (Malmedy, 1793) : dugrande "sans doute, eertainement " (G., Eutraits de Villers). J'ai noté aussi à Stavelot : ros n' mâcèyeroz nin. d' grude du v's-è sov'ni "vous noublierez pas. j’espère. de fous en sourenir ».Cette expression arehaïque. qui est ineonnue il Liège. n'a pas cncore reęu d'explication.

Disons tout de suite que les graphies $\hat{\imath}$, un sont an fond identiques: dans le dialecte de Verviers-Malmedy: on ne peut dire à premiere rue si $\hat{a}$ est un an dénasalisé, on si an est une nasalisation arbitraire do $\hat{a}$. Même ineertitude pour les finales -de. -te. On soupecomnera tout au plus que Lobet et Villers ont obéi à l'analogie du fr. grande.

Un premier point est probable. G.. I 1s. ćcrit drâhon (beancoup). qu'il faut analyser d' rihon "de raison " (raisonnablement, passablement ; doù : "à foison "). De mêne. il paraît certain que d(u)grâte représente une locution eomposée. Ln manuserit verviétois de 1759 sépare les deux mots : du grâde qu i-a dés mänates issé "je suppose qu'il a assez de revenus ". ('et exemple montre de plus que notre expression pent devenir locution conjonetive et se mettre au début de la phrase.

D'après Schuermans (1). les dialeetes flamands emploient gerulen ("deviner"; néerl. raden), op het gerad ("en devinant, au hasard. au petit bonheur "). C"est lì (qu'il faut chereher l'origine du w. d' grâde. qui est emprunté de ( $* p)$ 't geraad. ou plutôt de (i)li geraad "je derine». La réduetion de gerand en srâd est normale. Par ét ymologie populaire. la préposition $d(u)$ s'est substituée à l'initiale 't ou " $k$. En nomme, i r'viniè, d' grude signific : "il reviendra. je suppose ". Lané aree une certaine intonation. cela équivant à : "jaime ì le eroire. ce n’est nullement dontenx pour moi ".

(') Algemeen Vlanmseh Idioticon. Kilian a aussi gherasten (conjecturer, deviner). Le luxembourgeois gerôt signifie "hassard, occurrence". 
En namurois. aree le même aeeent, on dit dandjuretis "dangereux" " $(=$ il ricque fort que ce soit rrai, j'espère que e'est rrai) (1). En montois, avird goue le mlême rôle (Sigart, p. 209). Dans le pays gaumais, les formules daime, daimey (devine, derinez) émaillent la eonrersation. exprimant un degré plus on moins grand de probabilité, tandis que th-t', taijèy (tais-toi. taisez-vous) affirment ou nient arec plus de conviction.

\section{fr. s'ébrouer, ane. fr. espro(h)er ; liég. sprognî}

I. Lianc. fr espro(h)er vient du francique sproitan (Meyer-Lübke, no s18s), forme aneienne de l'all. sprïhen. "faire jaillir ". La faęon dont Godefroy en parle prête fort à la eritique. Il fait deux articles au lieu d'un seul et. si dans le second espro(h)er, r. a., "asperger. éelabousser" est défini correctement, dans le premier où il range trois exemples du v. n.. il passe trois fois à côté de la traduetion exacte: (son cheral) esproha signific. nen pas " hemnit", mais "s"ébroua "; chat qui esproe " souflle de colère ", et non "miaule "; de même, en parlant d"un oiseau à qui rous tenez en rotre bouehe le bec jusqu'aux yeux, s'il esproe bien "pres. dont est il sains. il faut comprendre : "si eet oiseau souffle de colère contre rous, e'est 1 indice qüil est sain »(2). De plus, Godefroy oublic la forme sproher, que G.. II 639. signale dans une rariante de Jean d'Outremeusc au sens de "cracher ". Enfin. d'aprìs Godefroy, esproement "exprime l'idée de moquerie "; nous y verrons, au propre, un éclat de gros rire. une explosion de rire qui asperge autrui.

On nia pas encore, que je sache, eherché dans l'anc. fr. espro(h)er l'origine du fr. mod. sébroner. Cependant les hypothèses émises pour expliquer ce dernier sont nombreuses et diverses. Pour l'un, "ébrouer dérive peut-ĉtre de *lron (= brave), l'ébronentent du eheral pourant passer pour un signe de eourage" $\left(^{3}\right)$; eomme si ee neétait pas tout aussi hien et phutôt un signe de surprise et d'effroi ! (') Pour l'autre,

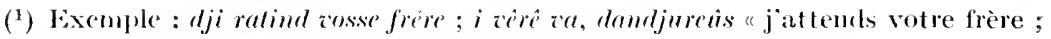
il viendra, jespere" (I'irsonl, I 1s:3) ; voy. anssi Forir, vo elanjreti.

$\left(^{2}\right)$ God. ne donne plas de traduction; eelle dı Lexique de Bomnasd et Salmon : "crier" ne vaut rien. - J) méne le wallon dit qu'un cheval qui šébroue (qui sprogne) (at sain ; sprogmi pent se dirc anssi d'un chat (gni soufle bruyamment de peur et de colère. Le wallon pent ici servir de guide : dans tous les exemples cités esprober répond au we sprogmi, oi noms vosons d"itlents un dérive du franeique sprocian ; voy. ri-apress.

$\left({ }^{3}\right)$ Körting, Dict. d'itym. fr. ; cent lopinion de Diez, adoptée par Littré.

(") Comp. "un cibroucment de cheval soullant de peur ", Zolat, C'ne page d amour, p. 402. 
s'ébrouer se rattache it s'esbroufer et vient done du proveneal exbroufu qui a le mème sens (1) ; mais comment justitier la chute de $f$ ? Pour le Dict. général, "peut-ĉtre s'ébroner se rattache-t-il au même radical que ébrouer, t. techn. : plonger dans l'eau (des tissus sortant du métier), l'ébrouement des animaux ayant pour résultat de faire sortir une sorte de vapeur par les ntaseaux" $\left.{ }^{(2}\right)$. En somme. la question reste pendante, car môme la dernière hỵpothèse. la plus sérieuse. ne va jas sans quclque difficulté de sémaniquue.

Pour moi, il me paraît naturel d'admettre que esproér. au lieu de disparaitre comme on le croit, a donné régulicrement *éprouer, lequel est devenu ébrouer. v. intr. (156t). puis r. rél., sous l'influence du synonyme s'esbroufer. L homonyme ébrouet, ane. fr. esbroer (abbrühen), t. techn., a pu aussi inllucr sur le changement anomal de pr en $b r$. Pour le traitement rocalique, la concordance est remarquable entre (es)broerr, (é)broner . all. nod. (ab)brühen. et esproer, ébroner : all. mod. sprühen. Enfin cette hypothèse a l'avantage de montrer la survivance de esproer dans la langue moderne et la prarenté du fr. s'ébroner avee le w. sprognî, qui a le même sems.

II. L'article de G.. II 390. sur le liég. sprognî est ineomplet et ne dome pas d'étymologie. On le remplacera par ce qui suit.

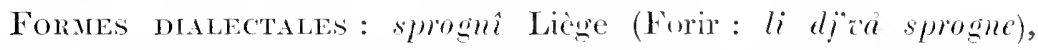
Fléron; -i Starelot, Malmedy, Doneols, Wardin-lez-Bastogne. Gires et daus le condroz: - er Jupille. 'Trembleur ; - $c$ Bande ; sprougni Neuf-

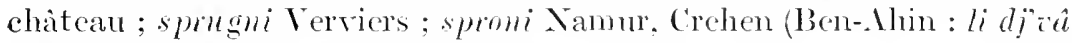
spromile. il a sprogni : c'est signe quil est hitî ou sain). - Les graphies suivantes sont suspectes : sprongni (G.) ; speognet Verriers (BsW 40,

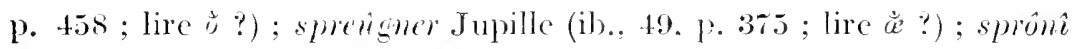
Namur (Pirsoul ; lire spromi ?).

Sigificitions : 1. s'brouer, soufler bruyamment de l'eau hors de la bouche et du nez; se dit surtout du cheral. C"est le sens le plus ordinaire; on le commait partont: de Liège-Verviers à Neutchâteau et à Namur ; - 2. ćternuer, en parlant de l'homme: Doneols. Wardin, Bande et dans le Condroz; - 3. pouffer cle rire au point de s'engoner (Malmedy : Villers), ou mienx : rire en projetant de la salive, rejeter de la nourriture en riant la bouche pleine (Fléron) : - t. soufller de

(1) L. Clédat, Diet. étym. de la langue fr., 1912.

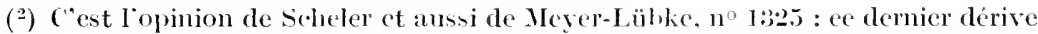
du germ. brôjan : ébrouer (abbrühen) et s'ibrouer (schmauben). Ch. Joret défend la même thèse dans Romamia, 1x, p. 110. Voy. enfin les Fransïsiscke Stuclien, vi, pp. 31-33. 
colère. en parlant d'un chat (Jupille : BSW 49. p. 375) ; - 5. "souffler un liquide qu"on a mis dans sa bouche "(Verviers : Remacle. ro sprugni); "ponsser" un liquide aree la bouche au risage. éc. : imprégner aree la bouche une ćtolfe d"eau. d"hnile" (id. : Lobet, ro sprugni) ; "épandre de l'luile grasse ou de pétrole sur une chaîne trop encollée : lourrier projette le mélange d’eau et d’huile par la bonche ! "(ib. : M. Lejeune, Foe. de lappêteur en draps: BSST 40. p. 4.58): - 6. v. mip., bruiner (Starelot. Malmedy) : i sprogne. il a sprogni : il a toumé one suprognore " il est tombé une légère ondée "; à Faymonville. on emploie dans ce

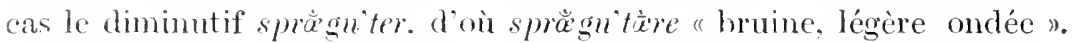

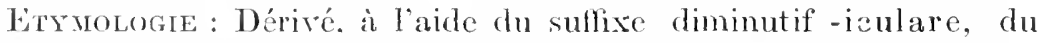
francique sprowan $\left({ }^{1}\right)$. forme ancienne de l'all. sprïhen « faire jaillir, projeter arec force (par ex. des étincelles) \#. Le type schématique

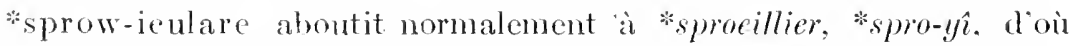
sprognte par épaississement de y en gn $\left({ }^{2}\right)$. - Dans le liégeois Jean d'Outremeuse on lit: " a rote sa lenge et les piechez sprelhoit (var. sprohoit) hor's de se boche " $[=$ il a déchiré sa lanúue et crachait les morceaux] $\left({ }^{3}\right)$ : sprelhoit est sans doute une graphie inexacte pour *sproelhoit. imparfait de *sproelhier. G.. II 639. a deviné dans ce verbe un fréquentatif de l'all. sprüle'u : mais il a négligé de le rapprocher du moderne sprogut. qui en ent pourtant inséparable. - Comparez au surplus le malm. i sprogne. qui répond à l'all. es spräht " il tombe une pluie fine ", et ce que nous disons plus haut du fr. ébromer.

\section{ane. fr. effriboter}

Ce not se rencontre dans un texte de 15t:2:

son ne leust wate de sus moy,

Morde, je leusse effriboté. (Romania, xxxm, :346).

M. Behrens. Beiträge, 1). ss. le rattache à l'anglais freebot "agir en flibusticr, piller ". M. Ant. Thomas déclare eette conjecture peu rraisemblable. mais ne met rien ì la place (Romania, xxxrs, 264). Ne pourrait-on pas invoquer le w. fribote "bribe, lambeau ", difriboter "eflilocher. dégueniller" "? P.mur la eomposition et pour le sens, effriboter scrait analosue an fir. écharper. écharpiller "mettre en pièces ».

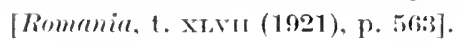

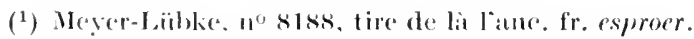

(2) (ompl. homyol (bonte de neige) $>$ homenot. ete.

(3) Wyreur des histors, 1 , :s:3. - Godefor repreduit ce teste sans la variante ct alvee : aroli, an licul de arolf. que (i. corrige : a role. 


\section{ane. fr. embegaré. begart}

Froissart. dans ses Poésies. parle d'm "poreel ort ct cmbegaré" ". Seheler et Godefroy traduisent le deruier mot par : "sonillé "; dams sou Glossaire des poésies de Froissort. Soheler ajoute cette note: Il y a probablement eomnexité entre hegorer. troubler. salir. souiller, et bigarrer ? Cela reste à examin r \% - Ces rapprochements sont hors de propos $\left({ }^{1}\right)$. Le mot dérive de l'ane. fr. be gart ?. que Gudefror ne sait. pas traduire (dans ce passage : "tel eoup li a donć... ke gambes reversees le trebuce el begart n) et que nons ex]liquons sans peine grâce an liég. bègå "purin, jus de fumier "; roy. l'article bèna. Le sens précis de "cmbegaré " est done: "souillé de purin ". La forme *begard a pu donner embegaré. comme dard dome le w. darer "darder". Cependant, le mot rimant dans le texte de Froissart avee regarlé. il faut sans doute corriger *embegardé. Le liég. bigârder "arroser de purin "(lé fosses de houblon) existe eneore à Jupille.

[Romania. t. xum (iפ21). P. jois].

\section{lićợ. èminné}

D’après G.. I. 191, le liég. èmaimé $\left(^{2}\right)$ "guindé. maladroit * dérire de main et signifie proprement "privé de la main "; mais, si l'on compare spaté "éerasé " (qui rép̧ond au fr. épaté" "priré de l'usage d'une patte "). smané (Alle-sur-Semois) "mancliot ". spongntti (Marehe-en-Famenne) "amputé du poing "on eomprendra que cette analyse est impossible. Le préfixe ne peut être que $\dot{i}$ - lat. in-. fr. en-. D'antre part. nous eonstatons que l'on prononce èmé i mé i Bergilers (IIeshaye) et que, prìs de Malmedy, èmé zé signifie "paralýsé. perelus " $\left({ }^{3}\right)$. - Ces deux ćléments noureaux permettent d’établir l'origine du mot. Le radical est le w. mèhin "incommodité. infurmité ". anc. fr. meshain "estropiement. mntilation " $\left.{ }^{4}\right)$. Le dérivé *'e-mèhuin-é a donné. par rédnction normale,

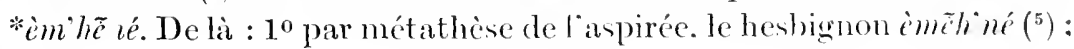

(1) Ailleurs, dans son Eude lexienlogique sur les prósies de Gillon le Muisit, rencontrant desbedarer "soniller", qui vient de bedaire "bune ". Scheler propose de corriger notre mot en *embod wi (Mém. Acal. de B lg. 1sst, t. 3i. vo desbedurer)

(2) On prononee èmẽné (Liège. Ben-Ahin). -i (Vielsahm), imêné (Verviers), èménés

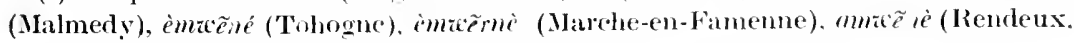
Tenueville).

(3) A Robertville (BI) 1908 p. 31): a Faymonville (BSW 50. p. 54:3).

( $\left.{ }^{4}\right)$ Voy. les exemples dam Godefroy et of. G.. II $10 \geq$.

(5) Comp. liég. mèbuer [moissomer] "glancer = minhlier à Bergilers ; liég. moèhnê "coryza " = mzè̀nh hò à Grandménil. 
zo par chute de l’aspirée et contraction, le liégeois èn êné (1). - Le sens primitif "infirme, estropién" ne survit qu'cu un point extrême de la Wallonic. Ailleurs. le mot a désigné, par hyperbole, un maladroit, guindé dans ses mourements, dont les mains sont gourdes comme s'il arait mal au bras. De même èstroupî "estropié » se prend au sens de "lourdaud. maladroit ". Comparez le gammais a-hachière (ei-après, à larticle hathre).

[BD 1920, 1. 11].

\section{liég. èstèssiner}

Ce verbe signifie: 1 . arreser (un rôti à la broche ou dans la poêle en y versant doucement du jus. du beure fondu) ; 2. par ext., arroser (ce quion mange en burant du vin) ( $\left.{ }^{2}\right)$; 3. r. réfl., s'humecter en buvant, s'arroser l'intérieur, d'où : s'enirrer $\left({ }^{3}\right)$. Il a plusieurs autres formes : estésuner, stéssincr, ètéssiner, tèsiner ; et des dérivés, dont le plus intéressant est stéssinerice, s. f., "cuiller à arroser " $\left(^{\mathbf{4}}\right)$. - Grandgagnage cn parle à deux reprises (I 196, II 399), sans domner d'étymologie. Je ne eommais d'autre essai d'explieation qu'une note de Borgnet, qui pense a l"all. stechen "piquer " à propos d"un passage du Myreur, I $26+$ : "riandes rosties et stechinees de lasmes qui vient d'Egypte"; borgnet oublie qüà l'all. stechen répond le w. stitchî $\left(^{5}\right)$.

Ce verbe appartient à la classe nombreuse des dérivés qui ont le suffixe diminutif -imer. Quant an radical, c'est lane. fr. esteser (lat. * exten'sare) "tcudre, étcndre", représenté d'ailleurs chez nous par lane. w. stesant, stensant au sens intransitif de "étendu, situé " $\left({ }^{6}\right)$. Ni Körting ni Meyer-Lüble nenregistrent un type *extensare; le denier a cependant un article *extentare "étendre " pour expliquer litalien dialectal stentintere "répandre ".

(1) De nucue le líg. mohem (mansionem: maison) a passé par *mºn pour

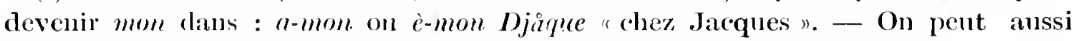
admettre que le licgeois a conou jadis la forme heshignonne èminh'me et que $h$ est

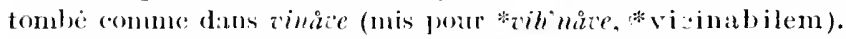

${ }_{(2)}{ }^{2}$ baus une palseguille de la fin du xvate sicele (Choir, p. 187), il est question de bons vivants qui stesinin aron dè zin dès gozâs, dès dorryes "arrosaient de vin des gat (iull des tartes $)$.

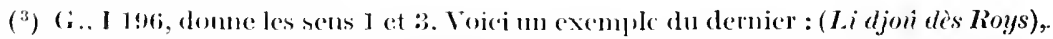

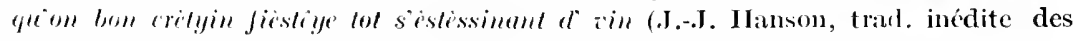

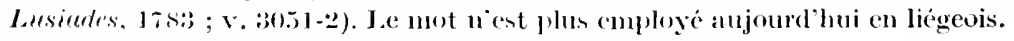

(1) (:.. II :39). Sur le suff. -aricius, vos. lilpendice.

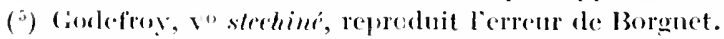

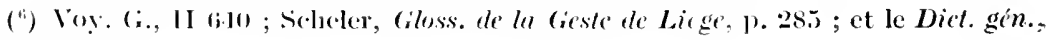
1.Thishlow. 
Le sens propre de èstessiner cut done: "étendre ì petits coups successifs (la sauee sur le rôt)", d'ò̀ : "arroser (le rôt) frécunemment et à petits eoups".-Dapris tensare: w. tizer. et tonsare: w. tozer, on peut objecter que esteser a dû donner istiziner. Telle a ćté assurément la premicre forme : mais. par la srneope normale de la protonique noninitiale (estizner). la douce devient régulièrement forte (istèssner. d'oì la forme refaite èstésiner) : de plus. le groupe st qui précède a sans doute aussi exeré une influence assimilatrice sur la sillante qui suit. - On ne doit pas šétonner de voir une forme istésiner a côté de stissiner : comp. istommalier et stoumalier : istorner et storner ; istra-

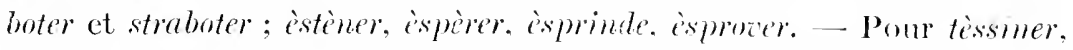
comp. le fr. trésillon à côté de ćtrésillon, le w. trouller à côté de stronaler. etc. Enfin itèssiner est ou bien *intens-in-are ou bien une altération de éstéssiner. par infinenee de ètèsser "entasser", roisin de forme et de sens

\section{liég. ètait, ètêt}

Ladjectif itĥt. archaïque en liégeois. signifie "allège. aise. satisfait" et s"emploie surtout dans azu l'colur itêt "aroir le cour content, être heureux ". G.. II xxir, y voit un dérivé probable du lat. intentus ; mais la phonétique ne s'aecommode pas de ectte hypotliese. C"est exactement le lat. intactus "intart, frais, en bon état ". ane. fr. chtait "bien disposé, aetif, empressé ". I lane. fr. entaitier répond itêt (G. ; Forir ; Villers) "encouragrer, animer ", itêti dans la vallée du Geer : s'ètêti a lörèdje. Quant aux dérivés itêtemint, itêtise. itêtisté "entrain, satisfaction ", ils sont on forgés par nos dietionnaires on ariuellement inusités.

\section{w. fåçon, fauçon}

Dans son Vocabulaire sizétois, M. J. Wasiet note faucom, s. m., "petite botte de paille débarrassée des mauvaises herbes et dont on a ménagé le chaume en ne battant que les épis : fi dis manches acei in. fauson di strin d' swile, faire des liens avee une botte de paille de seigle. J'ai releré le même fauçon dans le Condroz et en Famemne aree des définitions un peu différentes : à Ciner, e’est une "grabe le paille de seigle "; à Marehe-en-Fameme, " une botte de paille toute préparée pour que le courreur en chamme puisse la disposer sur le toit à courrir ". On prononce fảcon à Villers-Ste-Gertrude (même sens quüà Marche) et à Erezée (" paille peignée et arrangée pour servir de liens"). 一 On y verra sans peine le même mot que le fr. fauchon (forme normannopicarde pour *fauçon) "petite faux" ; mais le diminutif a dércloppé ici 
une aceepticn remarquable: 1 . "poignée diépis fauchés. jarelle " (sens. général. aujourd'hui perdu); :2. spécialement. "botte de paille de seigle (Ciner). dentince à fommir les liens (Giret, Erezée) ou à courrir les toits (Fameme, Villers-Ste-Gertrude) ».

\section{w. fèr. anc. fr. ferlier, fernoer}

Dans le Bulletin du Dict. iullon. 1908. p. 39, M. Alph. Maréchal a démontré que le w. tofér ou tot-fèr, "toujours, constamment ". vient du latin firmum et repréente en réalité "tout feme(ment) " $\left.{ }^{1}\right)$; de mòme le sylonyme fin-fír. usité à Viesville (Hainaut). II. Alph. Bayot (ib. 1910. p. 59) a noté dan le Miroir des nobles de Jacques de Hemricourt un exemple an ien du w. fir, lat. firmum : " tant fer chevachoit qu“il n'estoit nin a remuweir , $\left(^{2}\right)$. Enfm. pour eorroborer l’étymolegie de M. Maréchal, jai rappelé (i!.. 1910. p. (60) que fír existe encore, eomme adjectif. ¿ Mahmedy ot i Larcche, dams les expressions : fé on fèr nok a sès solés, loyê sès solés a fèr nok "lier ses souliers à nocud ferme, par opposition à nuud coulant "(Malnedy). loyer ou nolier a fér nol (Laroche) $\left({ }^{3}\right)$. D'oì le verbe afimoli $\hat{\imath}$ lier à noud fermen (Mahmedy). Jaurai pr citer écalement loyi a fér neu (Givet, Ueimont), le gamais farnowity "lier ["s souliers] à nocud ferme ". par opposition à fare in flo ('Tintigny). at le lorrain anfermaté "noué par tous les bouts, difficile it défaire. cu désordre; se dit du fil, de la ficclle, ete. " $\left(^{4}\right)$.

Or l'ancien francais posède les verbes terlier, fermoer, que l'on traduit communément par" "licr de fer. nouer avec du fer s. et où l'on voit des composés analogues i ferarmer. feriestir. saupoudrer, cermoulu,

(1) An point le: rue émintiuge, il est interessant de comparer les emplois de fir

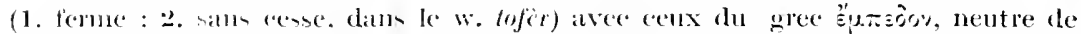
lialjectif

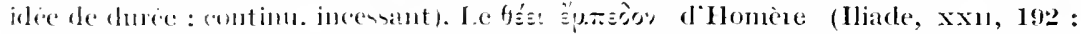

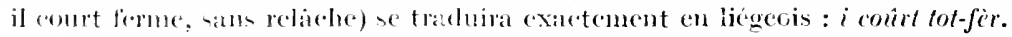

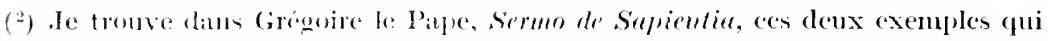

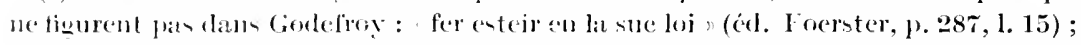

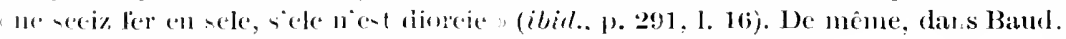

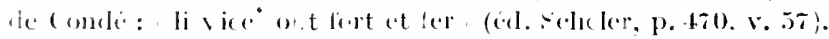

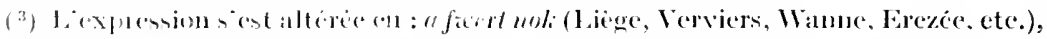

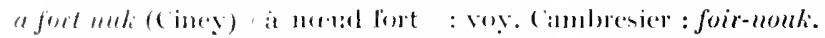

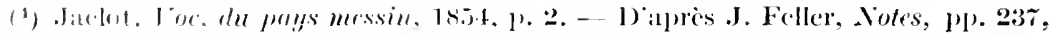

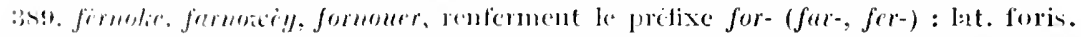

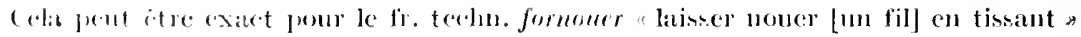

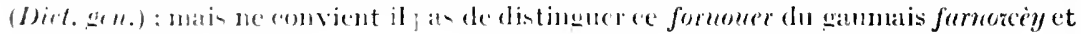
de for melde: 
cloufichier, ete. (1). Etant dommés les termes patois énumérés ei-dessus, je crois que l'analyse traditionnelle est inexacte, et que ferlier. fernoer doivent s'expliquer par fer(m) lier, nert. Al point de rue formel, si la confusion est possible en français entre fer $(m)<$ firmum et jer $<$ ferrum, en wallon le joremier seul aboutit à fír, tandis que lo seeond subit la diphtongaison et devient ficr. (2uant an sen". Ja traduction " lier solidement" comvient parfaitement à tous les cxomples comnus.

[Article paru en premier lien dans Romamia (1911), t, xL, p. 3:25, avec Ja note suivante de II. Antoine Thomas : " Jans l'un ale (as exemples, on lit

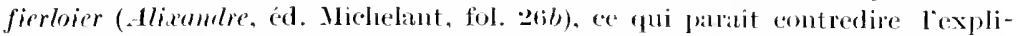
cation de M. I Iaust ; mais cette explication cost par clle-mêne si lumineuse et, dans le passage en question, lantervention du fer (metal) est si invatisemblable,

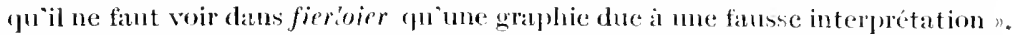
- J'ai trouvó depuis lors dans des textes anciens publicis par Romamin, res denx exemples significatifs : ferm seit sur la plate liee (t. Xxxvin, pp. 498, 535), ferm lievent la stimte prucele (t. xL, 1). 5.t6).]

\section{liég. fiskineû, fiksinê̂}

G., I 20\%. signale simplement : fisline ée.it : fikinê, vétérinaire, maréchal-ferrant qui panse les cheraux, bestiaux ». Forir et $\mathrm{X}$. Lequarré (BSW 20, p. xx) enregistrent les deux formes ; Willem. p. 89, Ia seconde seulement. Près de Liege ('Trembleur, Fléron, Thimister), on ne signale oralement que filisinêt "empirique, rétérinaire ou mérlecin non diplôné ".

C"est fout. Le dossier est mince et ne eomporte aneun essai d"explieation. Il s'agit pourtant d'un terme archaïque et qui, sous son aspeet modeste, peut se réclamer de nobles ancêtres.

Fislinêu - qui tend aujourd'hui à s'altérer an fikineñ - dérive en effet d'un verbe *fistiner, syneopé de *fisiliner, lequel dérive luimême de l'ane. fr. phisiquer "droguer, médicamenter", et de plyssique. ane. fr. fisique "science et art de la médecine ". La prenve en est dans les passages suivants du Myreur des histors de notre J ean d'Outremeuse :

Ill astoit dolaus de chu qu ill ly avoit copeit ses orelless; si print des cyrurgiens et les list fischiner [variante : esgardeir]. mains ilh fist les plaies envynemeir, si l'en convient morir. (I, p. 227:3).

Voilà, bien attestée, l'existenee du r. fischiner (lire fisliner), que l'éditeur Ad. Borgnet s'ingénie à expliquer dans ectte note:

(1) A. Darmesteter, Mots composés, 2e éd., 1) 161-2;. Meyr-Lübke, Gromm. des l. rom., II, $\$ 594$; Nyrop, Gr. hist. de la l. fr., III, \$569; Gonlefroy : femoer. 
Le mot fischiner doit être la truduction du lat. fasciare, entourer de bandes. Il y a amoi le v. fancinare, ensorceler, doù provient le fr.faseiner; mais le prentice de cen deux sens me parait ici le plus convenable.

Lerreur de Borgnet est d'antant plus surprenante qu’il a urait bien dû rapprocher son texte du suivant, ibid.. p. 4\% :

Adont aperchut Josephus la cause de la maladie, si avisat une chouse de phischincrie [variante : phisique] : se dest que toutes ehousen eontraires soy garisucht par :ultres contraires...

An glossaire. ibid.. P. 646, phischimerie (lire fishinerie), - qui a pour rariante et pour synonyme phisique. - est correetement traduit par "mádecine ". Nous sommes done en présence d'une famille fiskiner, -ru(r). -crie. dont la filiation est hor de doute. Le moyen âge n'emploie pliysique (fivique. fusique) que comme substantif et lui donne ordinairement le sens de "médecine"; l’ane. franç. se phisiquer signifie " se droguer, se médieamenter ". Phisique. -er a engendré *phisiquiner, dont le sullixe-iner a me valeur diminutive et fréquentative a la fois, comme dans le fr. trottiner, le liég. ploziner. gotiner "pleuvoir légèrement, le gaumais droguiner. sadroguiner (Si-Léger) " droguer: se droguer ". Quant ì la réduction de *fisilimer en fiskiner, elle n'a rien que de normal.

[BD 1912. p. ตั.]

\section{anc. w.-fr. forece, fueresse}

Lianc. w. fueresse se rencontre dans une charte namuroise de 1248 : "rint boniers ct set rarges fuerenses en terre a le mesure de Liege" (Romania xix. 86). Pour l'expliquer, M. A. Thomas. Nomienn Essais p. 96. propose un type *foere signifiant : "dont on se sert pour nesurer les terres funies (?) ". M. Feller, Notes de phil. wall., p. 200. y voit de

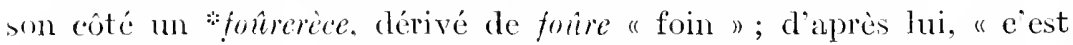
l'átendue de terre. comprenant 20 bonniers $\tau$ verges, qui est qualifiée de fueresse c.-ì-d. propre à donner du foin ". - 1) antres textes liégeois, qui ont échappé a M.M. 'Thomas et Feller, infirment ces denx eonjeet ures et pernettent de formuler une troisiome proposition.

Conlefroy a $u 1$ article forece s. f.. sorte de mesure de terre ", aree ac préciens cxemple: "r bonicrs et $[?]$ xax rergen petites moins con dist fureces (trad. du xume sicele d'une charte de 1265, Cart. du ral-st-Lambert. Richel. I. 10176, fo $\left.61^{b}\right)$. Lat., quingue bonnaria deem 
et norem virgatis parris mimus quam (1) foreces dieuntur n. - 1)ans son Inzentaire des archives de l. Lbbaye du Val-Bemoît. M. J. Cuvelier cite ce texte du 15 juin 1392: "xxir grandes verwes et xv foreche de terre situćes en Bruwier" ; il ajoute cette glose sur forcehe : "nom de la petite verge dans les environs d'Andenne " $\left({ }^{2}\right)$. - - Enfin. dans les registres de la Cour féodale, $37.90 \mathrm{v}^{\circ}$. conservés anx archiv's de Liège, feu S. Bormans a noté : "xxxin verges fowereches de terre erule".

On roit qu'il s`agit de terre arable, ermle, et non de prés à produire du foin. Ce mot féminin en -ece (-eche. esse) qualifie miqucment rerge. (Znant au sens. le texte de 1392 oppose la cerge forede a la grande ierge ; celui de 1265 (= 5 bomniers, moins 19 petites verges appelées foreces) est eneore plus précis. Pour expliquer le rarlical for-, fuer-, fo(w)er-, on sadressera au lat. formm, ane. fr. et anc. w. fuer, foer, feur, four, etc., devenu par exeeption fur en fr. molerne et signifiant "valeur, tanx. mesure. contume ". Le type *iorieia aboutit régulièrement à for'ce (comp. corèlje. mori : couragre. mourir) : cependant force pourrait être aussi bien une réduction de *forerece: *foraricia $\left({ }^{3}\right)$. En somme. zerge forece éruivaut à "verge eourante". Cest la petite rerge, considérée comme étant "de commun fuir ct mesure " $\left.{ }^{4}\right)$, c'est-à-dire eomme unité de mesure adoptée par la coutume du pays. Cette unité variait selon les licux:.

[Romenia, t. Xurn (1921), p. 564].

\section{liég. forlôzer, furlôzer, flôzer}

Forlôser "prodiguer", on mieux furlizer comme on dit aujourd hui à Liège. pourrait venir. d’après G.. I 21.5. de l'ancien saxon forliosan, néerl. verliezen "perdre ". Behrens. Beiträge. p. 295, admet sans objec-

(1) Sic ; il faut lire que (= que). Dans la traduction française et est sûrement interpolé. - Des papiers de feu S. Bormans contiennent ces deux extraits dont la source n'est malheureusement pas assez préeise : " 8 verges grindes et 2 verges fouretes (var. forestes) de terres et de prés "(12it : Charte du Val-St-Lambert); "demey bonier de vingne, vintez petites vergez foreiches moins" (xve s. : ValSt-Lambert).

$\left(^{2}\right)$ Butl. de l'Inst. Arehéol. liég., xxx, 589. Ce texte a paru dans le Cartul. de l.Abbaye du Val-Benoit, édité par le même, p. 697.

${ }^{(3)}$ Les formes fuer-, fo(zc)er- ont subi l'influenee du primitif fuer, four. - Sur le suffixe -aricius, voy. Mappendiee.

(4) Comp. "une ayme de commun fuir et mesure" (Cart. Ste-Croix, 1321). - A Liège, la verge courante est de 16 pieds de St-Lambert ; Ia petite verge $(16 \times 16)$ vaut 218 eentiares; la verge grande vant 20 petites on 4 ares 3.59 milliares; le honnier vaut 20 grandes ou 87 ares 188 milliares. - Dans le langage ordinaire, zerge se dit pour zerge grande; cf. Forir, vo zech. 
tion cette hrpothèse. qui ne va pas cependant sans de graves difficultés de phonctique. Je madresserai plutôt au flamand waerloosen "négliger n ( $\left.{ }^{1}\right)$. en supposant que la syllabe initiale s'est modifiée sous diverses influences, telles que les synonymes forfé "dépenser", forsmimer "négliger", férlanguer, furlanguer "prodiguer". - Quant à fliser "gaspiller ». que G., I 212, voudrait rattacher à forlôzer, il faut plutôt y voir un dérivé de flîse "bourde". où Scheler (ap. G., II 526) recommait avee raison l'all. flonse (nugae, tricac, mendacium); flôzer. outre le sens de "dire des somettes, bourder" (Lobet. p. 562). peut en ellet avoir pris laceeption figurce de "dissiper (son argent) à des futilités".

\section{W. forvîni (Verviers)}

C.. I ?16. a l'article sn'vant :

forvirî (usé jusqừ̀ liı corde, en parlant d’un vêtement, etc.). De l’angl. to aceur (uscer, consumer par l'usage) : comp. lane. 1. all. farwerian (corrumpere), ap. Ziemann, vo ziërn.

Le not est tiré de Remacle, ze éd., qui écrit forrîri et qui donne un exemple de l'intinit if : mi-nbit limince a fortiri "mon habit commence a ctre suramé ». Lobet l'enregistre aussi et je l'ai entendu à Mélen : on doit done le considérer comme propre à la région de Verviers-Iferve. - Quant a l’étymologie imagince par G. clle n’a qu'un intérêt de curiosité : flle nous montre G. enclin à chercher dans un lointain iclione germanique une coplication qui se présente d'elle-même an premier appel. Le liégeois dit forcîli (Forir) "vieillir outre mesure", composé de zîli "vieillir " $\left(^{2}\right)$. Le changement de $l$ en $r$ dans le verv. forcini est dû à lassimilation.

\section{w. foûrèhan (= foûre ìlant)}

G.. II. p. xxr. est le premier à signaler "foûrèhan, fồ̀̀han (printemps) dial. du Limbourg wallon ". Il propose d'y voir un "dérivé inchoatif de forme (linin) ou de fore (pâture des bestiaux) ). - On trouve foûrèthon lam un ante de la vallée du Geer : nouk mu r'vièvè f' fồrèhon, "nul 114 revera le printemps" (BSW 29, p. 540), et M. Fréson, de Glons. mus dune cet exemple qui muntre que le mot est maseulin : on minerè

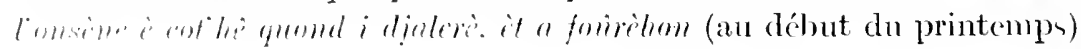

(1) Dirl. lentemico-latimum, Intrappiate, Verdussen, 166r. Voyez Franck-van Wyk

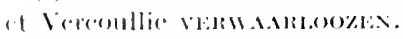

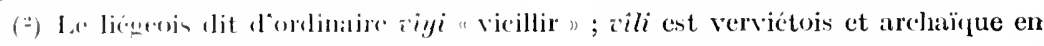
licencois (forir). 
on l' fôyeri-st-̀̀ tère. -- Entin II. A. Horning explique folvihan par "fors issant ". en eomparant le lorrain achi fyen (exire foris) qui signifie aussi " printemps", et le suisse fori (foris ire) ${ }^{1}$ ).

Je crois que Grandgagnage et IIorning ont tous deux partiellement raison : mon prenier est foure (feure. fourrage). mon second ihant (issant = sortant), et mon tout a folive ihant signifie "au moment où l'herbe sort de terre et reeommenee à pousser" " $\left({ }^{2}\right)$.

Pour èhant, point de diseussion posible. C"est le participe du verbe

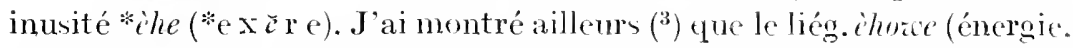
activité) répond littéralenent au frr. "issue "ou plutôt à la forme aneienne "eissue, essue ", lat. "exuta.

Mais four- ne peut sexpliquer par foris, qui a flonné en wallon $1^{\circ}$ l'adr. fou $\left(^{4}\right)$. lequel se place apres le verbe, conme dans le lorrain

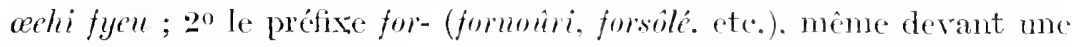
royelle (forcler, G., 11 526). - Pour justifier foris dans notre locution. il faudrait tout au moins l'analyser en foi t vihout (*re-exantem).

M. Horning allègue deux expressions qui renferment un infunitif pris substantivement : "le sortir" = l'action ou le moment de sortir. aprés la claustration d'hiver $\left(^{5}\right)$. Mais, dans son hypothèse. ce serait le participe ou le gérondif "le fors issant" ou "le sortant " qui remplirait la même fonetion en wallon. Cela me parait insolite, tandis que la syntaxe "au feurre issant" na rien que de tris ordinaire et de très satisfaisant $\left({ }^{6}\right)$.

Sans doute, on peut m'objecter que foume désigne l'herbe assez haute. bonne à faucher, et surtout l'herbe fauchéc et séchée. le foin (all. IIeu). Mais le foûre peut être "l'herbe qui doit derenir du foure "sens prégnant). D'un autre eôté, à l'origine, la signification était eertainement

(1) Zeitschrift für rom. Philologie, xvil (1894), p. 218. - Körting, no 3908, et Ileyer-Lübke, $n^{\circ}$ 3431, enregistrent l'explication de M. IIorning. [Or, le présent article ayant paru pour la première fois en 1911, II. Horning, le 17 févier 1912, a bien voulu méerire spontinément : "Pour foûhèhan, je suis entièrement de votre avis ").

${ }^{2}{ }^{2}$ Comp. al cressant del erbage, dans Th. de Kent (God., CRessivt).

(3) Ioc. du dialeete de Stazelot (1904) : BSW 44 ; Projet de Diet. w. (1904), 1. 19, ÈHề (dans un texte de $16: 34$ ) et Ėnowe. - Le verbe emmpose rèche (*re-exere) existe encore dans le namurois et le sud-wallon, an sens du simple "sortir" .

( ${ }^{4}$ Je dois cependant signaler foû-r-eûr ("hors hemre, trop tard "), qui se tronve dans Forir, mais que je nai jamais entendu.

$\left({ }^{5}\right)$ Pour l'ilée, comparez le w. bizahe dans Forir.

$\left({ }^{6}\right)$ Comparez à solo moussunt (Malmedy) " au soleil conchant "; " le clrkit somnant (God., toerses) ; arest issmt (God.) et voy., ci-après, lauticle selumbran. 
plu générale: l'all. Futter désigne la mourriture des animanx, la pâture, at tel est aussi. a mes yeux, le sens premier de fonire $\left({ }^{1}\right)$. Or le meilleur aliment des raches, cest l'herbe sur pied. Noublions pas qu'il s'agit ici d'une région herbagère (rallée du Geer et pays de Herve), ou la srande préoecupation du fermicr est de p mroir nourrir les bêtes. On y attend aree impaticnee le moment où l'herbe pousse. - Au surplus, le foûre chont sallie si bien a l’idée de nourrir les bêtes (forer lès bièsses: leur domner du fourrage) que eette idée a produit la forme altérée fôrihan. notée par Grandgagnage.

Enfin - et eeci me paraît dérisil - un texte de 1556 des Arehives du Ban de Herve (reg. 19, p. 201) contient un exemple arehäque de notre expression : "ne puelent passer parmy le preit synon en wayn temps. voir que a temps de four esstunt ancume fois ". Ce texte est précicux pour p'nsieurs raisons : $1^{0}$ dans "ch mêmes archives, "four ", "foure" se reneontrent somrent an sens actuel de fourage, foin. jamais au sens de fors. lors $\left(^{(}\right)$; — 20 la façon dont le seribe a coupé l'expression montre quil l'analysait comme nous et qu'il ne royait pas dans four un simple préfixe ; - $3^{\circ} \mathrm{si} \mathrm{l}$ lon admet mon interprétation. l'emploi de l'cxpresion est ici d'une propriété et d'une précision remarquables: on permet de passer dans le dit pré à l'époque du regain (e acayin timps). mais on interdit formellement le passage au temps ou l'herbe pousse. paree qualors les tices sont menues et l'ragiles et ne peurent itre brisées sam dommage. ce qui n'est pas le eas pour l'herbe du regain.

[Article paru dans BD, 1911, p. 19. - Depuis lors, on a proposé me autre explication de foùrhan (ibil., 1913, p. s0) : la forme première serait foûrèhon (saison des foins, (e-it-d. satison où le foin eroút) : elle comprendrait la raeine foûr (all. Futter) et le suffixe -ationem. - Cette thèse ingénieuse se heurte à de multiples olyjections: 1 Yon seulement la forme foùrèhan est attestée par le texte hervien de 1550 et par (s. (rui a trop) de serupule, je erois, pour se permettre une "retraunction " sans avertir le lectenr), mais encore elle mia été spontanément signalie par un octogénaire de 13legny-Trembleur (au $\mathrm{N}$. de Liège), II. Ienri Stas, qui prononce foürèhâ (‘-à-d. - -min dénasalisé). — $2^{\circ}$ Les substantif, verbaux du type simhou, firhon (semaison, fenaison) sont féminins,

(') ('omparez le w. atôle (de laall. Il'cide : pâturage), qui, en liégeois, signifie "prairie ", et, an malmédion, surtout "herbe : dul zcêde, de lherbe, on fiston it atide, m brin d'herbe.

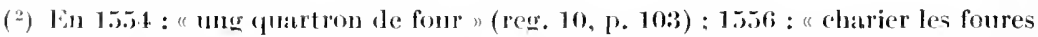
ct wanter les wayns " - " forgangnyt " 1515: "foreomand "15.52 : forclose " 15.55 ;

hors delle soxhe "15:32: "Lorpontaige "15.9: "hol's demince "15.52 ; "hors remlu 15.5 : "hors ambunder "1655. - Je dois la communication de tous ees

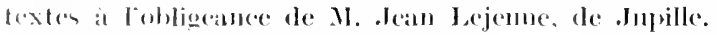


comme le veut le suflixe -tionem; or fohröhon est maseulin. - 30 Ces même; substantifs, qui sont trissyllabes a lorigine, ont perdu régulièrement en wallon la voyelle médiale; seul foutrituon (au lieu de *forithon) ferait exception. $4^{\circ}$ Ils dérivent tous de verbes : tinr $h m$, par exemple, suppose linri (ane. fr. tendrir : devenir tendre). Or fourhom ne peut venir de fôner, lecuel a donné régulièrement fôrkon (syn. de fôrèlje "fourrage ", daprès liouveroy, Dict. hígeois, ms.) : il fiudrat le tirer du substantif foire et en faire cheore une exception. - Je tiens done pour asmuré que å foûrèhar est la forme originelle et -on la forme altéce. Wautre part, le trissylabe ne peut guère siexpliquer que si lon y voit un romposé de deux mots. La conjecture "ibu fuerre issant " présente un sens ecngru ; elle sappuie sur de nombreux types analogues (voy. l’art. selanbran), sur le liég. éhozee, et enfin sur un texte ancien que, jusquà preuve sérieuse du contraire, on doit tenir pour correct.]

\section{gatmmais foûsson}

Le gaumais foûsson (Musson, Ruette, à l'est de Virton), foûssan (Tintigny. Ste-Marie-sur-Semois, Jamoigne, Rossignol), désigne l'âme ou le noyau d'un peloton de fil : c'est le petit objet (eoque de noix, boule de chiffon, de paille, de papier. cte.) sur lequel on enroule le fil pour faire un peloton (1). ('). Maus. Focab. de Trirtom (manuserit de 1850). ne signale que l'acception métaphorique de "testicule ". Adam, p. 253. donne au lorrain foussom le sens de "peloton".

Je crois que la forme premicre était *ohisson ct que ce mot ent dérivé du latin *rolsum (class. volutum "tourne, roulé n). d'oì le fr.

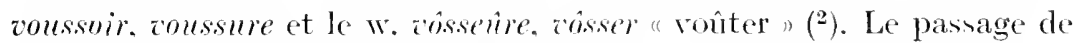
l'initiale $v$ à $f$ s'explique par assimilation régressive. cest-à-dire par l'influence de la forte artienlation suivante $\left({ }^{3}\right)$. - Cette hypothèse s'appuie sur le meusien ecrausm. s. m.. "âme d'une pelote de fil " (Labourasse), dont le préfixe ec-, eg- représente le lat. eon- $\left(^{4}\right)$. et la diphtongue aw le fr. on. comme dams cawper, eouper, caweil, coudrier.

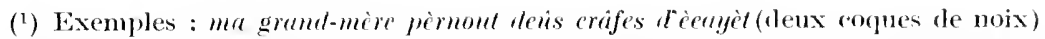

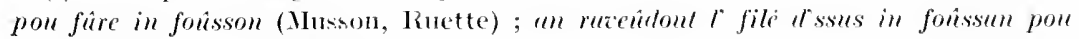
fâre dès lueh's ou pelotons (Ste-Yarie-s.-Semuis); voy. BD 1911, p. 17; Bsw 41, n, p. $160 ; 49$, p. $150 ; 5+1,2.252$.

(2) G.. II tr2, explique zosser fall volutiare, voltiare. Le type *nolsare peut : eul rudre e mpte de la linal: $-e r$.

$\left({ }^{3}\right)$ Comparez gaum. vichue, arl. et nam. iechen > fichan (charleroi, Mons) "putois": aiersè (Nenéc), rièsser (IIazy) > fièssi (IItesbitye) "verser [ia terre]. déehaumer \%, - De même, pour expliquer *pekin >bukin. il n'est pas nécessaire, comme fatit Behrens, p. 20, de supposer me influenee germanique.

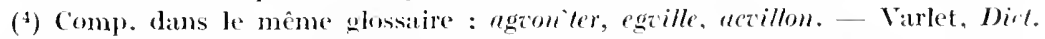
meusien, a un article : iehuousson, cochevean ". Si la forme et la définition de ce mot sont exactes, on peut y voir ectuasm, influeneé par le fr. échereun. 
-.- Le sullixe -onn. lat. -onem. a été surtout product if pour les noms de choses. Il a d'ordinaire la valeur d'um diminutif et s'adapte notamment it des themes verbanx pour indiquer l'instrmment on le résultat de l'action : eomparez le fr. bouchon. eoupon. torehon ; lard. casson. tesson; le gamm. pèton. étineclle. adrmom. entame; le w. dijemon. germe. dièton. pousse. rètehon. crachat.

En somme. le fohtssm ent un petit objet rond quion tourne et qui sert à rouler. un petit "rouleau ". de même que le w. hagnon (dérivé de hagnni. mordre) désigne une partic dlaliment solide, surtout de fruit, quion saisit en mordant. un petit "moreean".

[BD 1911, p. 101].

\section{w. foût'ler, froût'ler}

G., I 205 . cite. d'après Remacle. le verbe frout'ler "tricher ". qu'il tente dexpliquer par le lat. frustrari on méme fraudulare; au t. II, P. 52 t. il enregistre. d’aprés Lobet. foutter. m. s.. où il voit une altération de frotitler. Ces direrses propositions sont inadmissibles.

Jai releré froût ler "filouter (qqeh) " ì Seraing : on mêsse froûtlêu " un maître fripon" : font le" "trichers à IIerve. à Glons-sur-Geer et à Trembleur ; fon̂tlề. fém. fontur"vesse (pour foutul"rèsse) "tricheur, -euse " foûtur'rèye (pour fontul'rèye) "triehcrice " i 'Trembleur. Ce foutter - qui existe près de la frontic̀re limbourgeoise (et jusqu'à Verviers dapeis Lobet. qui domne foudlé et froutlé) - est emprunté du limbourgeoin foetelen "tromper. trichere surtout au jeu" $\left.{ }^{1}\right)$. Quant ì froutter. il a subi l'épenthère de $r$, comme le liég. frumèle (femelle) ot le fr. fromele.

\section{anc. fr. frefel, rouclil fourféle, fouféle, foufète}

On lit phuseurs fois dam Frojustrt lane. fr. frefel "tronble. agitation"

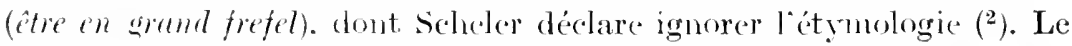
not a smréen en rouchi moderne dans la loeution stre in fourféle (Valencelemon: Hécart). (pui derient in fouféle (Lille : Vermesse; Tourcoing : Wattecuw) r.t. par me nouvelle dégradation. in foufète (Francries: D)ufrane) "ctre en émol. affairé. agitén.

On ne tronve ancune trace dexplieation dans les ghosaires de la région. Il cot lors de donte que nome arous aflaire an moyen h. all.

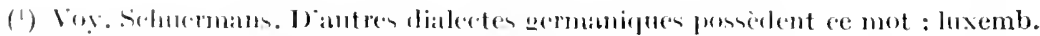

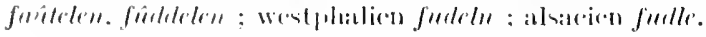

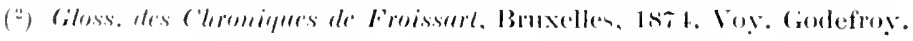


vrečel (all. mod. frevel) "violence, audace. présomption. arrogance, pétulance", néerl. wrëé (dans Kilian : "stomachus. iraeundia"). Je vois, par une note laconique d'Ulrix. n"620, que MI. Genelin invoque de même l'all. frecel pour le rhéto-roman fref̣el ( $\left.{ }^{1}\right)$; mais, comme Ulrix n'étend pas cette explication au groupe septentrional dont on rient de parler, je crois utile de combler la lacune.

[Romania, t. xuvir (1921), p. 565].

\section{anc. w. fud}

Une ordommanee liégeoise de $11.51 \quad\left(^{2}\right)$ termine une ćnumération d'engins de pâche prohibés par ces mots : "le sperwir ou le fud". G.. II 595, traduit le dernier par : "épervier : sorte de filet ". Ce furl (qu'on prononçait sans doute füt') est assurément emprunté du moyen néerl. fueclie, néerl. mod. fuile "nasse" $\left({ }^{3}\right)$. Isa traduction de G. est done inexacte. Dans le texte cité, la conjonction ou margue l'addition. non d'un synonyme, mais d'un terme différent du préeédent. Il faut comprendre quon interdit "l"épervier ou [encore] la nasse".

\section{liég. furtoye}

G., II 22\%. définit ec mot : "1. fressure : 2. lèyî zèy sès furtoyes, se débrailler, montrer ses nudités ». Pour toute explication, il compare lc nam. fristouye ; mais ce dernier. qui signifie "régal ", n’a que faire ici. - Si le liég. furtoye (Cambresier. Inbert), firtoye -ogne (Forir), fèrtoye (Duvivier), désigne des débris de viande et partieulièrement la fressure $\left({ }^{4}\right)$. le mot existe ailleurs arec un sens plus général, où il n'est plus question de viande. Nous relevons firtoye. à Erezée. "bribe " (de toute sorte) ; furtoye, à Starelot, "bribe, lambeau" (par ex., de rêtements : il èst tot-a furtouyes, ș̣n, a brimbades) ; cnfin, à Seraing, dès furtouyes, dans le langage des houilleurs. désignent le matéricl (rails. boisages, ete.) qui a servi dans me taille et quion démonte pour l'utiliser ailleurs. Lacception générale étant celle de "débris. fragment.

(1) Genelin, German. Bestandtrile des rätoroman. Wortschatzes. Progr. Innsbruck; 1900, p. 23. Je n : Elementen in de Rom. Talen, Gand, 190\%.

$\left({ }^{2}\right)$ Texte cité par G., II 611 , vo houcherale.

$\left({ }^{3}\right)$ Pour $k$ final devenant $t$ en wallon, comp. ci-après lart. slièrbalili ; de même G., II 234-5, donne plíli ou plét" "prone ", emprunté du néerl. plecht. Voyez aussi mon étymolngie de lâte ( = néerl. luik), dans BD 1914-1920, p. 97.

$\left.{ }^{4}\right)$ An singulier : dèl furtoye (des débris de viante). on au pluriel : dès firtonyes (à Huy, même sign.). Comp. frétuye à Faymonville Bsir 50, p. s6i. 
bribe ". nous voyons dans furtoye une forme altérée de *frètoye ("*fretouille $)$, renfermant le radical latin fractum (brisé) et le suffixe diminutif -uela. Comparez, pour le radieal, l'ane. w. fratin, fratin, fretin (bris de elôture) $\left(^{1}\right)$, et, pour le suffixe, le fr. pop. fripouille (dérivé de fripe, ehilfon). Ie meusien frapouille (lambeau d'étoffe, persomne de rien), l'ane. fr. drapouille (mawrais rôtement : 1504, ì Valenciennes), et:

\section{w. verv. furzêye, frèzê}

Lobet seul domne le verv. furâye, s. f., "godiveau, pâté chaud de veau épieé. cte. ". G.. II 527, reprend le mot sans l'expliquer. - C'est tout simplemont un dérivé du w. frase "fraise de reau" $\left({ }^{2}\right)$; mais la forme est remarquable en ce qu'on attendrait *ficíye $\left({ }^{3}\right)$. En effet, le w. a tiré de la mème source : $1^{0}$ frèzé (grêlé, marqué de la petite vérole, propr. ridé, plissé ; comp. l'ane. fr. frasillé, ap. God.); - $2^{\circ}$ frèzê (verr. ; t. arch., noté seulement par Lobet. p. 20s) "torehe de paille que les fileuses de laine et de coton à la main entrelaecnt entre les deux poupées de leur rouct "; il faut y voir un emploi mótaphorique de l'ane. fr. fresel "garniture fraisée ", t. de toilette : on sait que le mot fraise (de veau) s'est appliqué, par figure, à la collerette empesée qui fut en rogue an xvie sic̀ele. - A la même famille se rattache enfin l'ard. afürsǚlex " enchevêtrer ", que j'ai noté à Alle-sur-Semois. C'est l'ane. fr. enfresselé (" une dalmatique enfresselee de picres precieuses "), que Godefroy traduit inexactement par "bordé ".

\section{w. nam. hesb. galiène, galziène}

A Dinant at à Ciney. stinde sès galiches signifie "étendre ses jambes, ses gaboles ". De même à Crehen (Hesbaye), où l'on prononce galı,en'. que nous ćcrivons galyimmes. Enfin l'excellont Glossaire de Fosse-les-Namm par M. Lurquin nous domne la forme galsiènes "les jambes. considérées sous le rapport du mouvement : dia tant dansé qu' dii n' sin pus me's galzirnes " (BSW 52, p. 13t) ; on y compare l'ancien français soler "ctre rif, remuant"; mais ce rapprochement,

(1) On tire :ussi géncralenent du même radieal le fr. fretin.

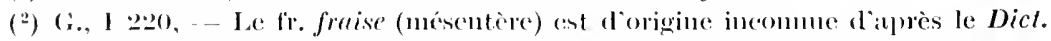

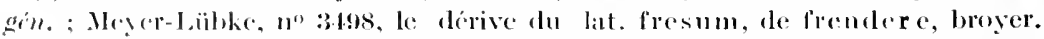

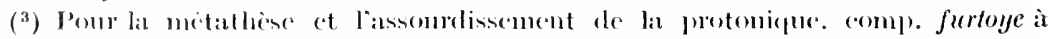

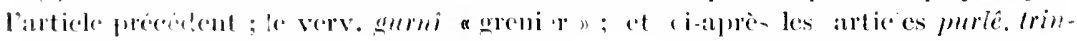
berlin. 
suggéré par la définition, parait inopportun. A Dinant et a Crehen. le mot fait ressortir la longueur des janshes. Partant de là. jöinroquerai l'all. galgen (potence) qui, en dialecte du Grand-Duché de Luxembourg. a la forme gâljen. Liexpression namuroise est due à une métaphore ironique, eonme pour les symonymes fr. quilles. liég. hésses (échasses), skieyes (faueilles). nam. cratives (crosses). Al! point de vne phonétique. le luxembourgeois gatlien explique ì la fois galiene et galicine.

\section{w. gåmète}

La gimète était jadis la coiffe ordinai e de jour et de muit. oì femmes du peuple et paysamnes serraient leur chevelure. Aujourl'hui, ce genre de bonnet a presque entièrement disparu ct nest plus guèr porté que par les viejlles. Nos dictiomairss, qui offrent comme équivalents franȩais "cale, toquet, serrctite " et autres termes approximatifs. oublient "béguin ", qui donne une idée exacte de cotte eoiffure arehä̈qux $\left({ }^{1}\right)$. Le mot existe dans la région X.-F. (Liège, Verviers, Malmedy) ; à l'Ouest. nous le relevons à Bergilers (Hesbaye) et jusqu’a Jodoigne (gomite), enfin an Sud, à Tohogne et à Villers-ste-Gertiude (prov. de Luxembourg). Jusqu'iei. il est resté anu explication $\left({ }^{2}\right)$. J'y vois, pour ma part. une altération de *amete diminutif do came "erinière. ehevelure en désordre, tignasse: $\left({ }^{3}\right)$. Le sens primitif serait : "petit objet servant à serrer la came ou chevelure " $\left(^{4}\right)$. On sait que le ehangement de $k$ en gà l'initiale est un phénomène fréquent dans notre dialcete ; voyez par exenple les articles conmê, gistel. gorlète. gossê. guduc.

(') Le fr. béguin désigne une " coilfe unie, attachée sous le menton, que portaient les béquines, religieuses des lays-Bas; dou, par ext., toute coilie unie s'attaehant sous le menton" (Dict. gén.).

(2) G., I 2:31, se contente de comparer goumite (bomet de nuit), d'après Duvivier ". Mais $1^{\circ}$,honctiquement. il ne peut exinter de rapporf entre les denx mots ; 20 il fut ruyer cet article gourmete de (., 1 239, ot la citition qui en est fuite par Littré, vogourmette. Invivier senl donne gourmete dans larticle confus rue voici : "goumett et gamett, bonnet de nuit et lime ". Apparemment, il a voulu dire que gourmett signilie "liure de gâmett, bonnet de nuit ". Par malheur, méme dins ce sens, le liégeois ne connait pas gonrmète ; il dit toujours loyerre. Je nai trouvé gourmite qu“a Chimay, oì il signifie : rubans qui attachent sous le menton la gorliche on bonnet de nuit des femmes". ("est evidemment emprunté du français.

${ }^{(3)}$ G., I 95 et 3339. donne : crime, crinière ; Condroz câme ; nam. côme ». - Il faut noter que la forme ordinare en liégeois ent cime (rarement litme, kimme), doù : acamer, abêmer, ahimmer "prendre aux cheveux, attaupur ). be rall. kamm, "peigne; crôte (de eoq), crinière (de cheval), etr."

(4) Sur la valeur sémantique du diminutif, dans ce mot et dans certains autres de môme frappe, voyez ci-ilprès la fin de l'article hatrê. 


\section{w. gårmèter (Verricrs). disguèrmètè (1)inant)}

G.. I 234. se contente d'enregistres" "ginimiter. gummander" (d'aprés Remacle. 2e él. : Lobet. p. 214). Il faut lire : su gômèter (Veriers. IIerre. Thimister). r. réfl.. "se quereller. se chamailler ) :

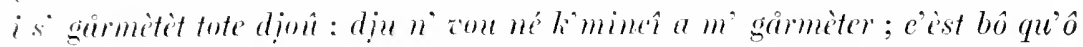
$n$ se ront né girm:ter. sins què $! .$. - La traduction de G. n'est quapproximative et mettrait sur la piste d'une fausse étymologie si on pretendait voir dans garmiter la forme wallome de "gourmander". En fait. ceent le représentant wallon de l'anc. fr. garmenter. forme variée de gramenter (ordinairement rélléchi. an sens de "se lamenter "), lecpuel dérive sans doute du germ. gram "triste. peiné ". Les patois normand. tourangeau. ete. connaissent aussi guermenter. guémenter, ete. (roy. Godefroy). ct lon signale. en dialecte w. de Dinant. le composé si disguèmète "se quereller ". La phrase dinantaise :

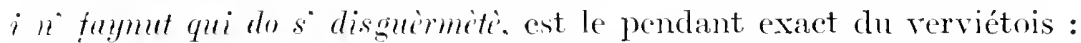
i n' fèt qu" du s" geirmiter "ils ne font que se chamailler " $\left.{ }^{1}\right)$. - Pour la protonique $w . \dot{i}=$ fr. en. comparez agazemiter (Forir) : filouter" (a + gaincnter) : toumèter. tommenter ; parmiti. parmentier ; amèder, amender (roy. p. s).

[BD 1911, p. 103.]

\section{liég. garsî}

Le w. garsi (2) "ventomer ". v. tr.. n'existe plus qü l'extrême Nord-Est (Verviers, Malmedy. enviroms de Liege). Le sens technique tend à se perdere; du moins. je nai jamais entendu a Verviers que la locution : za-s tu fé gurste (ra ten au diable !) ; an sens propre, on emploic la périphrase : on li a miton dés bacites ("boites ": ventorses). - G. I 2:31. ‥ contente dy recommatre l'ane. fr. ganer "searifier ". Or sereer (fendiller) ent la forme moderne de garser. jarser (piquer, searifice). dont len patois de Champagne et de Franche-Conté possèdent ("ureore des clérivén $\left({ }^{3}\right)$. Pour expliquer gereer. Diez. suivi par le Dict.

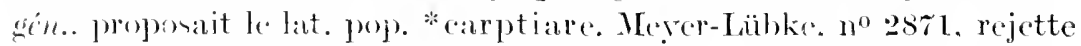
ce type pour des raisoms de phonétique (4) : il admet un primitif

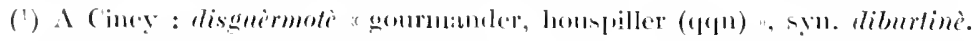

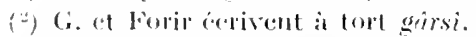

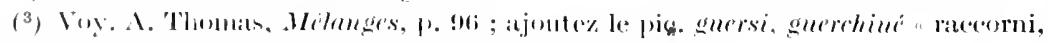

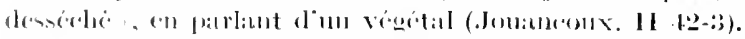

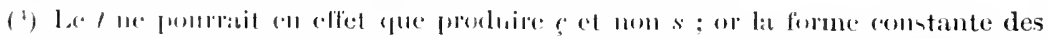

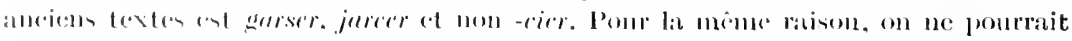

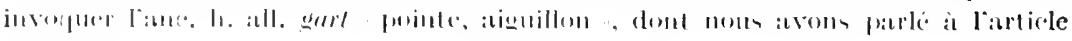
dian this 


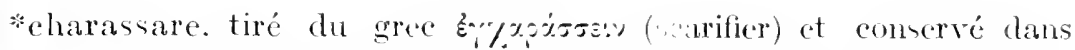

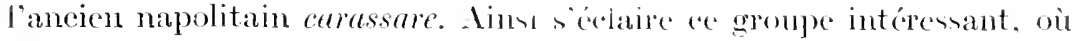
notre garsî mériterait de ne pas ctre oublié. car c"est lui qui reproduit le mieux la forme et le sons de l'ane. lir. saterer.

[Romania, t. xurn (1921), p. s60.]

anc. fr. gistel. w. custèl. cristal. ronehi aguistiller

I. Godefroy a l'article snivant :

gistel, s. in., fuit d'une arme? le monehe? "P'uis prent une [corr. unc] malhe erant de fier par le gistel "(Jeh. des P'reis, leste de Liege, 21795 , ap. Scheler, Gloss. philol.).

L'éditeur de la Geste. L. Borgnet, traduit par "le manche ". Scheler dit à ce propas : "Je ne eomais pas ce mot et je renonce a en préeiser la valeur. II. la professour Le lioy est tenté d'y voir l'all. aestell (monture) ; il a pent-ètre rencontré juste, mais je donte que le mot allemand ait jamais été appliqué an fût d'une ame et que le wallon présente d'autres cas d'application du préfixe allemand ge-n.

Pour le dernicr point. Scheler se tromp. certainement : il existe me bonne poignée de termas wallons qui reprénentent des mots germaniques pourvus du préfixe ge-. Seleder ponvait trourer dan Grandgagnage les plus comms, gnilite. guimène, guimâde ; pour le reste. je renroie à l'Appendice. qui domnera une liste détaillée de ces cmprunts. — Lautre objection ne parait pas phus sériuse. Alunettons que lo germ. gestel(l) n’ait jamais été appliqué an fît l'une armc ; il s'agit de: saroir si telle aeception est possible. Or, lo sens générique : "disposition, arrangement. assemblage ". d'où : "monture, charpente. bâti. châsis, pied ou base ". conduit logiguement ì celui de: "manche (d'mu ontil). hampe (d'un maillet d'armes)". Dans son Glossaire, Seheler note somvent des mots dont Jean d'Outremeure. pour lo besoin de la rime, n'hésite pas à étendre la signification : il dit lui-même que cet anteur " a su enrichir le vocabulaire de son temps par nue multitude de termes, très légitimes de faģon et de sens. qu’il a puisés dans le terrain natal on créés selon le besoin accidentel de sa penséc on l'entrainement de la versification " (préface du Glossuire. p. (i). Gistel est dass ce car. Il signifie " manche "et représente le germ. sestel $(l)$. Noms alloms montrer d'ailleurs que nos dialectes ont conscrvé le mot dams me areption analogne.

II. De Malmedy i Namur. le brancard d’un ehisiot et sutont d'un tombereau, ainsi que l'espace (onmpris entre len deux bras du brancard, 
sappelle entel (Verviers : Lobet ; ard. : Body. Voe. des chamons). crustal (Bormans. Toc. des howilleurs liégeois (1) ; nam. : Pirsoul). eristal (G.. II 515 : t. de min., avee un sens quelque peu différent), cristil (Jupille : BSW 49, p. 3033). Le mot est ancien : braz de erustelles figure dans nos Chartes des Métiers, I $82\left({ }^{2}\right)$, et G., II 573, cite ce texte de $1723:$ : "chevaux attelés, eomme l'on dit, al eristalle". De source orale, jai reeucilli crustel à Dorimne, Ben-Ahin. Marehe et Heure-enFameme, Tohogne, Erezéc, Villers-Ste-Gertrude, Vielsalm ; custèl à Staralot et à Thimister-Clermont; enfin, près de Malmedy : kï̌stö̀l à Gueuzaine, liètel à Robertville. Le genre rarie : maseulin à l'origine, il est devenu presque partont féminin à eanse de la terminaison.

Les dernières formes. originaires de la frontière linguistique, reprodnisent nettement le germ. gestell(l) qui, outre le sens général indiqué ci-ctessus, désigne le train d'un chariot. C'ette signification apparaît encore dans l'aneien wallon braz de crustelles ct attelé al cristalle. Comme le mot s'employait surtout à cette oceasion, il a fini par désigner spéeialement la limoniere on prolongement de l'avant-train $\left({ }^{3}\right)$.- - Pour la forme, on motera $1^{0}$ l'altération de - $e^{\prime}$ en -al(e) sous l'influenee des nombreux dimimutil's en -ale, frr. -elle; _- $z^{\circ}$ l'épenthèse de $r$ après $l i$ initial ; comparez scrène : "échine " (ance. h. all. skina) ; cronzîre, à Siloret, pour comzîe. consîre "amas de neige " crèssôde "pâquerette " (comsolida), roy. p. $59:-3^{\circ}$ le dureissement de $g$ initial en $k$ : voy. l'art. liclìtône.

III. Il faut attribuer la meme origine an montois aguistiller " ajuster. arranger * (13D) 1911, p. 52). Sigart essaie de lexpliquer par *ajustiller, qui serait un diminutif de ajuster. mais cest pure fantaisie. C.e rerbe se décompose en a gestel t ier et signifie proprement "pourvoir de l'appareil con enable, appareiller ". ('omparez l'all. anstellen "arranger" et le fre agiéet. t. de mar., "garnir (m navire) de ses agrès " (de l'ane. holl. soreiden : prépares.)

\section{w. glindis'}

L'ard. glindis" "grillage d'êtang" est signalé it Saint-IIubert par

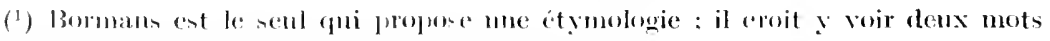
flamismds: lirm!gem, pomsser, traines. st stal (!).

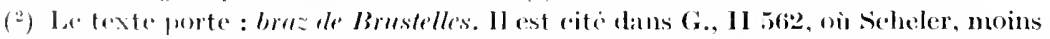

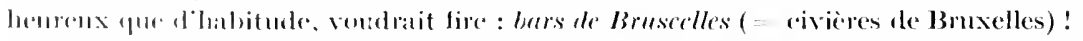

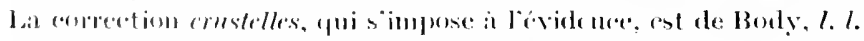

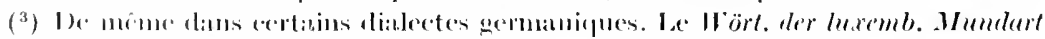

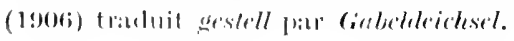


II. Marchot. qui le dérive du lat. clingere " enclore. entourer "( $\left.{ }^{1}\right)$. La tentative cost infructueuse : co mot latin a clû ctre très peu répandu et n’a donné aueun rejeton $\left({ }^{2}\right)$. En reanche, les dialectes framands connaissent gelint "treillis de lattes ou de barreaux de fex " ( $\left.{ }^{3}\right)$ et l'on trouve, en bas allemand. slind "clôturs de planches et de lattes" $\left({ }^{*}\right)$. be li le w. slindis" dérivé sur le type de triyis" "treillis "et du fr. lattis "onvage fait en lattes".

Ce mot a jadis existé à Liège; on le rencontre maintes fois dans les textes anciems avec le sens de "eloture. grillage. treillis". Voici quelques témoignages : (1:311) "juskes a glendice Watclet" $\left(^{5}\right)$ : - (xrre siècke) "ont steppeis et ars [extirp'́ et brûlé] les arbren. userien [portes]. fineistres. bans [eorr. bans: pontres]. weires et lattes. et destruis les glendis entour les vergiers" $\left({ }^{6}\right)$ : - $(1540)$ "muchicr en la scaillic [comr] de la maison et rompre ung giendiee " $\left({ }^{7}\right):-(1564)$ " arat entrée le glendiee pour aller joindre an puits $"\left({ }^{8}\right)$. - Aujourd hui même. glindis survit eomme nom de lieu à (rehen (Hesbaye) : il y dévigne une partie An ruissean qui passe dans ectte eommune et quime cloture longeait. sans doute ì cet endroit.

[Romania, t. Xlvil (1921). p. 566.]

\section{w. gô. gôti : gaum. djô, djwôti ; fr. mugot. mijoter}

Le w. gô. s. m.. signifie : "petite provision de fruits quion tient en réserve pour ses menus appétits \#. G.. I 2:34. parait l'assigner au dialeete namurois; mais on eherehe vainement ee mot dans les glomaires de F. D. (manuserit. 1850) et de Pirsoul. En revanche, gô se lit dans une picee ancienne de Marehe-en-Famenne $\left({ }^{9}\right)$ et se dit surtout à l'Est de Liège, vers la frontière linguistique. A Malmedy. Villers (1z93) a un

(1) Marchot, Phonolggie ditailléc thu patois zevllon (1892), p. 76.

(2) Voy. Walde, Lat. etym. Wörterbuch. - C"est à tort que Du Cange, clisgere, dérive de là le fr. elcuclee et lanc. fr. clicorgue.

$\left({ }^{3}\right)$ Schuermans, Ifioticon et Suppl. : De Bo. - Le rouchi glin "porte à clairevoie "(Luingne-lez-Mouscron) est emprunté du flamand.

${ }^{(4)}$ Cité par Weigand, Gedäxber, comme étant une forme parallèle de l'all. gclände. Nous retrouverous ce radieal à l'article lemslon.

$\left(^{5}\right)$ Cartulaire de l'abbaye du Vul-Benoit, éd. Cuvelier, p. 342.

$\left({ }^{6}\right)$ Jean d'Outremense, Myreur des histors, v1, 67t. - Godefroy, qui eite ce texte, wmine (ehevron), nal pas d"artiele glendis.

(i) Cris du Péron, reg. $\tau 1$, p. 118.

$\left.{ }^{8}\right)$ Rendages proelamatoires, reg. 3. $15 \mathrm{r}^{\circ}$.

(") Li Marièdje manquè (1806), r. 202: Lant qu"i uni anrè dès pomes o gô. 


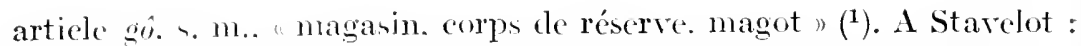
fé $s^{\prime} g \hat{o}$ " faire sa réserve", surtout de fruits $\left({ }^{2}\right)$. A Faymonville-Weismes, où l'on pronume parfois c $\hat{o}$. M. J. I'astin distingue deux sens : « 1. provision de frnit cachée, mise en réserve ; 2. portion de fruits donnée en carleau " $\left({ }^{3}\right)$. I Verviers enfin. Lobet, p. 22:3 : gô " dépôt (d'argent on autre effet en quantité), magot ". Nos autres lexieographes ignorent ee mot, qui d’ailleurs n’existe pas en liégeois. Dans les témoignages cités. nous ne trourons pas l'acecption survante : "endroit d'un bois ou abondent les fruit à eneillir. notamment les myrtilles». 'Tel est pourtant le sens unique que je comnaissais à Verviers dans mon enfance $\left(^{4}\right)$; nou prononcions gon. avec la résonance gutturale propre au verviétois devant royelle ou ì la fin de la phrase, et gô derant eonsonne $\left({ }^{5}\right)$. Cette nasale provient d'une altérution : gô, isolé dans la langue ou du moins paraissant tel, a subi l’analogie des nombreuses finales en -on : $\hat{o}=\mathrm{fr}$. ct liég. on $\left({ }^{5}\right)$.

De la pointe Nord-Est du domaine roman. il faut deseendre an pays de Virton pour tronver l'équiralent de notre gô. A 'Tintigny et à SteMarie-sur-Semois, un $d, \breve{o ̈ a}$, c’est aussi une "provision de fruits tenue en réserve dans une cachette " (BSW 3\%, p. 34t). Le Tocabulaire des enrirons de Tirton par ('l. Maus (manuscrit, 1850) écrit jan, avee la même définition; il a de phus eet article: "sauré, jaunir : mette des peume sanré: syn. jounti, de lì ain jouo de peume ". Au lieu de jau, jouo, jouoti, lisez djô, djou.ơ ou djoure, djouoti on djwôti, comme jai entendu prononcer, près de Virton, à st-Léger ct à Musson. De là le fr. dialectal : "mettre joutir des netles ", que dome le Larousse illustré.

Ce verbe dérive de djô, djouw, qui arait donc à l'origine un $t$ final. Or le liégeois possède un rerbe gôti, dont le rapport avec gô devient par la manifeste ct que nous derons dis lors comprendre dans nos recherches.

On n’a cncore publić sur gôti que des notes incomplìtes ou peu

(1) Gramblgannilge, Etrails de Jillers, p. jt.

(2) Bsill 4. p. jos. I te mène a sprimont et dans toute la région verviétoise.

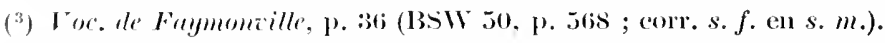

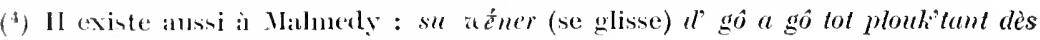

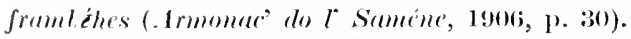

(5) Vuy. Milunges rallons (liege, 1892 ), p. 28.

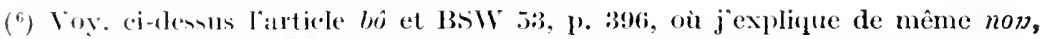
lieu dit il Ireneux, alteration de nô, forme nasculine du fr. noue : *nanda. 
exactes $\left({ }^{1}\right)$. En partant du primitif gô, nous pourons ìcoumer eomme suit le déreloppenent sémantique de ce rerbe intransitif : c’est. i l'origine, un terme d'éeonomie ìurale. conscrvé comme tel dans un eoin extrême de la Wallonic (Malmedy. Faymonville, Viclsalm) ; non loin de là (Liège, Huy). il survit daus des expressions métaphoriques, aree un sens dépréciatif.

I. Proprement, en parlint ales fruits quon met sur lia paille ou dans le fein : "mûrir dans le fruitier" ; sens attesté pour Malmedy par Villers (1z93), pour Faymonville par 1I. J. Bastin, qui donne cet exemple : les bilokes qui-arin? toumé drant trèsse maces, ô lès mèt gôti o foùre "les prunes tombè aviunt d'être mûres, on les met mûrir dans le foin ". [Comme on vient de le voir, éest lonique arception du gaumais djaôti et du fr. dial. joitir.]. | Par analogie : 1. a Vielsalm, les ponmes saluvages, dont on vent lakire du vinaigre, sont mises en plein air pendant denx ou trois semaines pour les laisser gôti, șrı.

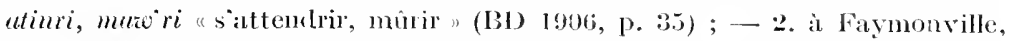
"s'avachir, s'imollir [ = blettir] par un eommencement de dessiccation, se dit des fruits qui n'arrivent pas à maturité, des feuilles, de l'herbe, qui, peu de temps après avoir été coupées, deviennent flasques, surtont sous liaction du soleil " (J. Bastin, l. l. ; roy. les exemples). [L“influence de rôti "rouir, pourrir , n'est pent-être par ètrangère à cette cxtension de sens.]

II. Par métaphore et toujoüs péjorativement : 1. en parlant d'une préparation culinaire qui a mijoté trop longtemps sur le feu : "se dessécher, perdre

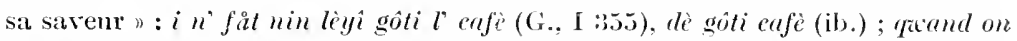
ratint lès-antes po diner, lès crompires gôtihèt (Forir); lé feù u' cu uiu assez mi, lé ljigot gôtih (IIuy); li tuhiar èst gôtèye, li rosti a l gos' di gôti (Liège) : ce goût de gôti diflère du gon̂t de hati "havi, denséché à la surface " et de celui de broûlé "brûlé, carbonisé "; - 2. en parlant d’une persoune qui reste paressensement au coin du feu : "se euire, crompir". Duvivier dome eet exemple : is' gôtih èl coulêye lè feû, où le réfléchi est sans doute amené parr l’analogie de $i s^{\prime}$ rostih, $i s$ " 'ût " il se rôtit, il se cuit ". Je nài entendu à Liège que le v. intransitif : èle gôtih èl coulêye, à propos d'une femme indolente, d'ine crope-è-ciudes; - 3. en parlant de l'eau qui se eorrompt faute de mouvement : "(roupir"; e'est le sens unique que domne le liégeois Rouveroy (ap. G., II 559). Le même a un article gômi, où il se contente de renvoyer à gôti.

Enfin gôti a domné. à Erezée, le diminutif gôtiner, v. intr., 1. "mijoter "; ə. en parlant d'une personne : "traincr (en route), croupir ». -

(1) Trois articles de G., I 239,355 , II 529 , donnent sans explieation deux sens différents que l'auteur ne songe pas à rapprocher. Dans ses Extraits de Villers, il éerit gôtchi (!) au lieu de gôti. M. .J. Bastin. l. l., signale, aver des exemples typiques, deux signifieations dont il faut intervertir l'ordre. IIulyert et G. éerivent gôth (?) au lieu de l’inchoatif gôti. Cambresser, Remiacle, Lobet n’en parlent pas. 
Quant ì solinet. auquel G. compare gôti. sa structure phonétique, malgré la ressemb'ance partielle des signifịations. dénote une origine différente. On dit. à Liège ct à Jupille. gø̈diner. 1. "mijoter. mitonner ":

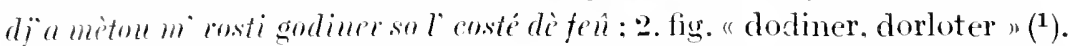
Le verrictois gondiner (Lobet. p. 22t) "gratiner, mitonner ", avec une fause nasale on $(=\hat{o})$. paut sexpliquer par croisement de göliner et d z gôti.

Voilà. en somme. ce que nour sarons aetuellement de gô et de gôti dans le domainc walon. Quelle est lorigrine de ces mots ? ( $\left.{ }^{2}\right)$ Leur habitat purait dénoter une provenance germanique. De même la eomparaison phonétique arce d’autres trames. tels que hô "giron ". hôt'lîye "*gironnée" (du néerl. schont. gothique sliauts) ; pôti "patrouiller, manier indécemment " (Stavelot). dérivé de pôte "patte " (néerl. poot, franeique pauta) ; rôti "rouir". gaum. rouôti (néerl. roten. francique rotian. dune racine germ. raut "pourrir ": Kluge. ro rösten). D’après ces analogues. un tỵpe *gaut. *gôt. expliquerait gô. goti. Seulement, rien dans les langues germaniques ne permet d'étayer cette conjecture.

Un fait eertain - et qui doit déị noms satisfaire - cest que nous reconnaissons gố, gôti dans le sccond élément du fr. mugot, mijoter.

Le fr. mijoter "faire cuire doneement et longtemps" n'a pénétré que depuis peu (Acad. 1798) dans la langue générale. Il provient des dialectes du Nord et de l'Ouest (meusien. rouehi, pieard, normand, maneeau), oì il a des formes et des aceeptions diverses : 1. "faire mûrir " (norm. : migeoter). "mûrir sur la planche " (Haut-Maine : id.) ; 2. "caeher son argent, thésauriser" (rouchi : migoter, mugoter) : 3. "bouillir à petit feu " (boulonnais : mugoter). - Il dérive de migcot "lieu où l'on garke les fruits jusqu’à maturité " (Haut-Maine) ; migoe "provision de pomme d'hiver. ete." (Bayeux) ; mugot "provision de fruits qu'on garde pour liviver et quon laisse mûrir sur la planche " (norm.) ; mijau 1. même sens ; 2. "collection d'objets faite en cachette" (Ard. fr.) ; mi got, mugot " magot, trésor caché " (rouchi, pieard); ete. $\left({ }^{3}\right)$.

(1) Duvivier donne : "godiner, dodiner, dôlotiner, fr. dodiner, dorloter ". Jai entendu en licgeois : si gŏlliner "se dorloter".

()) (x., yuni d'ailleurs ne soupçonne pas de parenté entre gô et gôti, eompare sim-

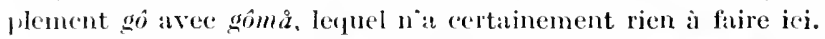

( ${ }^{3}$ Voy. notamment de Montesson, Voe. du IIaut-Maine : migeot, -er ; Goffart, Gloss. du Monzonnais : mijou ; Baudon. Patois de Rethel : migeau ; Sigart : migot, -er ; Herart : mugot, -er, -ell ; (orblet : mugoter ; Jouancoux, Delboulle : mugot ; ete.Jin Willonie, nigo (nam. : G., II 16;2 ; anssi à Charleroi) "amas caché de fruits, d'argent, cte. "s ent altíre de migo sous l'influence de uirl. - Le fr. ungot lui aussi est connideré comme une altération de magot. 
En franẹais moderne. le Dict. gén. admet mugot "magot (d'argent)". La forme la plus ancienne date du xe siècle: musgode, dans la rie de St-Alexis, r. 25t, où le sens est : "provision (de bouche), réserve (d'aliments)" ( $\left.{ }^{1}\right)$. L'étymologic reste ineertaine $\left.{ }^{2}\right)$. Le demier qui en parle, Meyer-Lübke, $n^{\circ} 57$ \%. pose eomme étymon * mungauda (magasin, grenier, réserre), d'origine inconnue. probablement gauloise $\left({ }^{3}\right)$.

Notre étude contient, croyons-nous, des domées inćdites qui peuvent servir à préciser la question. Il en ressort notanment que mus gode est bien un mot composé. dont le second élément - le plus significatif a paru suffisant au wallon et au gaumais. De phus. l'aire de gô. djô. oriente les recherehes vers le domaine germanique. Le premier élément de musgode pourrait bien être le moyen h. all. mos (cibus : all. mod. mus), conme le proposait Storm (*). Le seeond reste énigmatique. Sans avoir la prétention de le déchilfrer. je terminerai sur cés réflexions.

Le groupe gô, goti. djô. diaiôti. putule un type * gautum, antérieur à * (mus)gauda.

On admet gabata (écuelle). * gauta (joue) pour expliquer le fr. " jatte ". " joue " (Meyer-Lübke. $\left.n^{0} 3625\right)$. Notre *gantum y serait-il apparenté ? - J'attire phutôt l'attention sur ce fait que, à côté de gô, le dialecte de Faymonville $\left(^{5}\right)$ posside une forme có. Pent-on en déduire que *gautum est altéré de *eautum ?

Le latin cautum (lieu protégé. enclos: de cautus. cavere), qui a survécu dans le Sud $\left(^{6}\right)$. conviendrait assez pour le sens (endroit préservé : réserve) ; mais les condition géographiques ne hui sont pas favorables : il faudrait qüil êt passé sur le Rlhin ct fût, de là, revemu chez nous, ce qui est bien problématique.

Dès lors, notre * cautum ne pourrait-il provenir de eavatum (endroit creusé : ereux, cachette), accentué à la mode germanique sur

(1) Voy. Godelroy : murjoe, murgoe, mujoe, etc., s. f., " amas, provision ; eellier où l'on conserve les ponnes".

$\left({ }^{2}\right)$ Voy. notamment Scheler, vo mugot; Romanir, in 85 : G. Paris. Vie le St-Alexis, P. 186 ; Z. f. rom. Phil., xxx11, 445 ; xxx11, 433; ; Jouancoux, 11215.

${ }^{(3)}$ Il eite le wallon migo: lisez : rouchi (Sigart), au lieu de : wallon.

( ${ }^{4}$ Romania, II 85. - Comp. le moy. h. all. muoshis, mosteile.

$\left(^{5}\right)$ Ce dialecte, à l'extrême N.-E. du domaine roman (Malmedỵ), est remarquable par labondance de ses formes archaïques (voy. ci-alprès les articles pèri, wahète, z'arbô). M. J. Bastin en a étudié le vocabulatie et la morphologie (BSW, t. 50 et 51).

$\left({ }^{6}\right)$ Frioul, Espagne, Portugal ; voy. Meyer-Lübke. no 178t; Körting, no 2036 ; Diez, p. 442, et Du Cange. 
l'antépénultic̀me ? Ou, ee qui revient au même, de *eavitum (eomparcz *eavitare "creuser ": Meyer-Lübke, $n^{\circ}$ 1792)?

En dernière analysse, si la réponse à ees questions est négative, il ne nous restera qu à supposer à tout ce groupe une origine eeltique.

$$
\text { w. gômå, djômi, ete. }
$$

I. Grandgagnage, I 23\%, 355, II xxrir, traite longuement de gômâ, qui signifie 1. écrouelles, tumeur ; 2. réserre, magot; 3 . jabot des oiseaux ; 4. grossesse ou, en général, mal, ineommodité, à peu près comme le fr. paquet. Il propose diverses eonjectures qui ne méritent pas diseussion. Il a vu juste en $u n$ point, quand il soupęome que $-\hat{a}$ est le suffixe augmentatif, fr. -ard. Reste à déterminer le radieal.

Le primitif survit dans le dialecte de Verviers ; gôme "glande. tumeur à la gorge " est attesté par Xhoffer (BD 1920, p. 49) et, avant lui. par Lobet. qui dome pêle-mêle à l'article gôme, p. 224. les significations : "gomme (végétalc)... glande enflée... gourme... émonetoire, ete. "( $\left.{ }^{1}\right)$. - D'autre part, un manuscrit du liégeois Durivier porte goxime signifiant "gourme ", ct. d'après M. Henri Stas, on dit à Trembleur: : i-a dès goûmes è hatrê "il a des ćcrouelles dans le eou ». A Liège et à Iluy. on emploie sourent dès goum' goumes, forme redoublée is la mode enfantine $\left({ }^{2}\right)$. A Sprimont. daprès MI. Henri Simon, être gômé cest aroir des gômeires, écronclles $\left({ }^{3}\right)$. Enfin, dans la région de spa. gômer, r. intr., "se dit des brebis qui ont une sorte d'éerouelles. gomflement de la granache ou mâchoire inférieure, ce qui est le signe certain de la cachexie chez les animaux" " $\left.{ }^{(\mathbf{}}\right)$.

Il importe de distinguer, au point de rue étymologique, 1. dèl gốme de la gomme s et ?. des gômes "des éeronelles ". Le premier a formé

(1) Je tiem, aluside M. Eug. Boullienne, ancien institutenr à Charneux-lez-Herve,

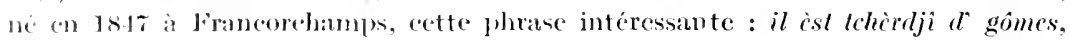

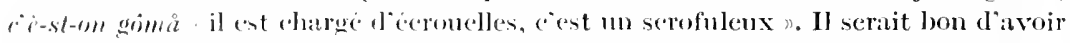

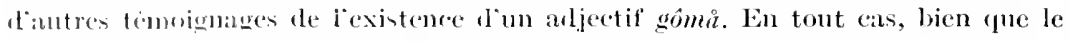
unflixe seit le mêne, il faut distinguer "e gomå (= qui a des gômes; comp. pansio. paumard; "ljoba. *jambard, qui a de longues pat tes) de notre gômå, augmentatif de

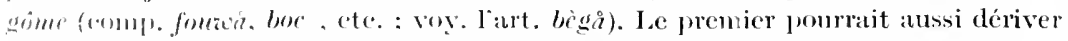

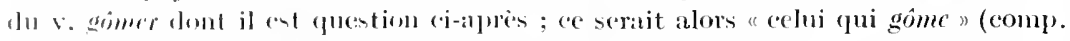

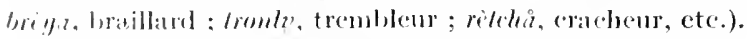

(2) 1) mene, purr designer les icomelles, on dit encore des zizi (Liège), des

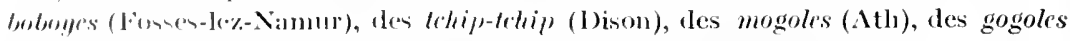

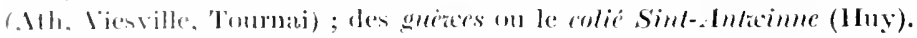

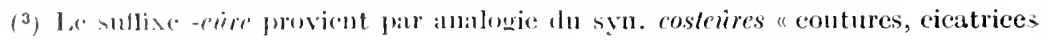

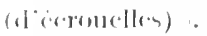

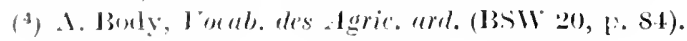


en lićgeois gômer, r. intr., émettre de la gomme, en parlant d'un arbre,

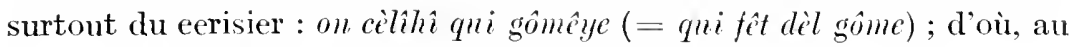
figuré : èle gômêye co, en parlant d'me personne qui aceumule ses raneunes on préventions eontre quelqu'un et qui, un beau jour, éclatera en invectives $\left({ }^{1}\right)$.

Comme on le voit par ee dernier exemple, il n'est pas toujours facile de faire la distinction, et l'on admettra même une sorte de eroisement sémantique. Mais gômes (éerouclles), arec sa forme variée goûmes, reproduit visiblement l'ane. fr. gorme, gourme $\left({ }^{2}\right)$. qui désigne la tuméfaetion du ganglion sous-glossien, le goitre, ou encore les ćcrouelles, alors que le fr. moderne gourme ne désigne plus, au propre, que la phlegmasic de la muqueuse nasale chez les jeunes cheraux. Si, comme il est admis. ee mot se rattaehe au germ. worm "pus" $\left({ }^{3}\right)$, nous devrons roir dans le w. gôme (golume) un emprunt dircet dı fr. gorme (gourme); car, si le terme wallon renait immédiatement du germanique. il aurait eonservé le w initial. Quant aux dérivés, gômé reproduit l'ane.

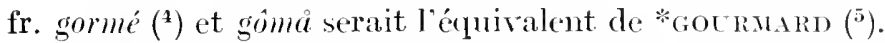

Il serait intéressant d'étudier les représentants de eette famille dans les patois de la France. Bornons-nous ici à quelques cremples. - En Saintonge, les goumons désignent les oreillons, ou simplement des oedimes ou tumeurs molles. - Le Glossaire de l'Anjon, par Verrice et Onillon, signale "goumons, oreillons; gonmouner, v. n., s"enfler, se gonfler ; goumer, v. n. et réfl., se renfler, se gonfler. devenir turgeseent, en parlant d'une bouture qui va émettre des rejets ou des raeines ". Le Vocabulaire du dép. du Doubs, par Beauquier. dome : "gômer, r. n., tremper dans un liquide" (p. 15\%) ; "goumer, gommer, r. n., euire d̀ petit feu, ou tremper ; se dit, dans ee dernier sens. par ex., d'un morceau de pain qui gomme dans l'eau ; fig., gonmer sign. eourer sa eolère,

(1) Inédit ; communication de $111 \mathrm{e}$ A. Gobiet. Le sens figuré pourait aussi, comme on le verra, se rattacher à lautre gômer (dérivé de gômes écrouelles).

( ${ }^{2}$ Pour gourme : goüme, comp. le w. fohme = ane. fr. foume (lat. forma), $i$ toune $=\mathrm{fr}$. il tourne.

(\$) Meyer-Lübke, no 9570. Voyez dans Romania, xxxvil (190!)), р. 584, l'article de M. Ant. Thomas sur lanc. prov. zorm, oì l'on corrige avec raison Godefroy, qui traduit lane. fr. gourmons par "goitre " au lieu de "écronelles".

(4) God. donne à gormé le sens de "goîtrenx "; e’est plus probablement "scrofuleux n, comme dit II. Ant. Thomas.

(5) Pour la ehute de $r$, comp. le maln. mófier (màchonner), qui équivaut à l'ane. fr. morfier (manger goulùment), rouelıi mourfoyi (a Ellezelles ; m. s.) ; du germ. morfjan, moyen néeri. morfen ; (f. Meyer-Lübke, no 5682. - De même bi̊bể = pic. barboir (masque) ; aianaye = zearmaye (*verminalia), ete. 
se contenir à peine " (p. 1.59) : "jomme, s. f., la mousse d'un liquide ; joumer. mousser. fig. écumer intéricurement de colère " (p. 182) $\left({ }^{1}\right)$. Il y a là des indications préeieuses pour l'explication du w. gômer, dont nous arons parlé plus haut, et du w. djômi que l'on verra ei-après.

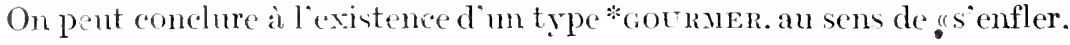
se gonfler $)$.

II. Ce sens mène logiquement à eelui de "jeter sa gourme. se vider, se purger de ses humeurs exerémentiticlles ": puis à celui de "vomir ", sens que possède, par exemple, gormar en espagnol et en portugais. Ainsi s'expliquent le pieard gomir "vomir". qu'on lit dans Corblet, et le namurois gomi $\left({ }^{2}\right)$ "romir" "que G.. I 23\%. enregistre laconiquement.

III. Nom tromvons encore dans ce qui précède la clef d'un autre terme. djomi, dont G. I 25\% fait deux artieles et qu'il laisse d'ailleurs sans explieation satisfaisante. Voici ce passage. dont je rectifie seulement la graphie wallonne:

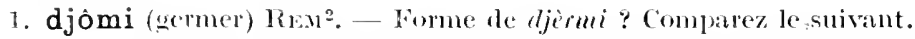

2. djômi (couver, en parlunt du feu) ; Nimurois it. - l'eut-être le même mot que le précérlent : un feu gui couve est un feu qui n'existe qu'eu germe, qui ne fait eneore que germer : comp. l'exemple suivant domné par Zoude : li fêt

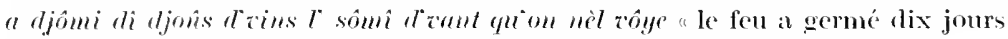
daus la poutre avant "fuion ne le voie, avant de paratte all-dehors " $\left.{ }^{3}\right)$.

En róalité. diemi "germer " n’a que faire ici et les deux articles de G. doirent be fondre en un seul ; djomi est une simple rariété de gômi (1) : tou deux se ramènent à un type *GOURMrr, dont le sens propre serait "commenecr à go(u)rmer (e.-ì-d. à gonfler. enfler, devenir turgescent)". Licxamen de quelques exemples va le montrer avec précision.

(1) Beancunicre tire jomme de l"all. schumu "écume (!) et ne songe pats ì rapproaher les troin atricles que molle ditons.

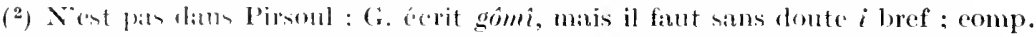
lr pie. gommir et lit mote suivante. le dictiomaire liegeois manuserit de Rouveroy

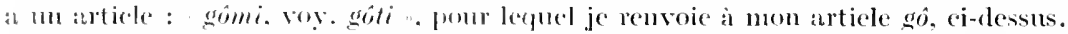

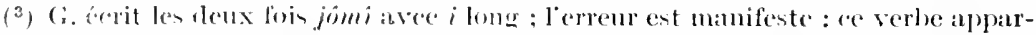

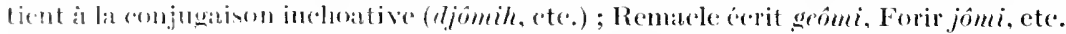

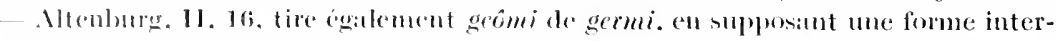
mediatire * joermi (!).

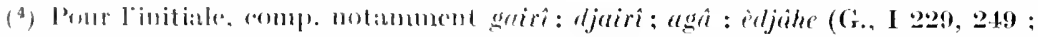

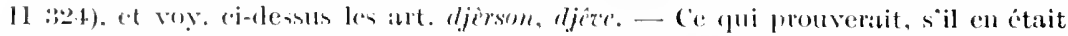

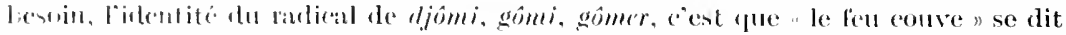

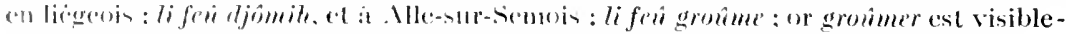
alwat altére de *agmermer. 
En parlant de l'amour naissant qui tommente son amie, une jeune fille dit, dans une pièee liégeoise de 175\% : i u' fat jamay... lèyî djômi on s'-fêt mèhin. Bailleus (1) traduit en note : "il ne faut jannais laisser couver, germer, un pareil mal" ; il est. lui aussi, guidé par la ressemblanee extérieure de djomi et de "germer s qu’il souligne ; mais on doit comprendre : "(laisser) grandir insensiblement, se développer par une aceumulation lente ". - De même lès plî̀es ont fêt aljômi lès à assins (Rem. ${ }^{2}$ ) les pluies ont fait germer les scigles ". Ici, comme dans d'autres phrases analogues, djôni équivant à dị̂mi "germer ", du moins pour le résultat. Ce fait, outre la ressomblanee des formes, explique la eonfusion ordinaire entre les deux termes ; mais au fond. pour le rarlieal et pour le sens exact, rjomi se rattache à l'angerin goumer et an w. gômer de tantôt. - Forir ne dome que l'expression djômi so l' coûr "rester sur l'estomae. causer un embarras gastrigue ") et de fait on dit à Liège : l'a-magnî m' djomih so li stommac'. dj"a on djômihìrje so li stoumac', mais, de plus, je tiens de vieux Liégeois les phrases suivantes : li eolére lì djômihére è coù dispôy lontins, la eolère s’acemunuait depuis longtemps dans son eaur ; li fen dionmith. le fen conve ; et aussi,

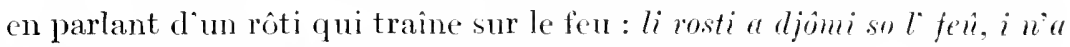
pus ni gos' ni sazoura, il n'a plus ni gô̂t ni sareur $\left(^{2}\right)$. - Dans son beau livre Li pan di bon Diu. p. 112. Ie liégeois Henri Simon nous fait voir que, sous la terre enscmeneée, qui semble morte, on résse di vèye

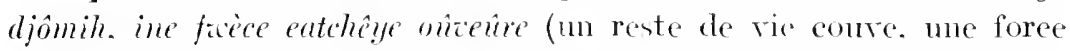
eachée travaille) : le second hémistiche commente à merveille le premier. Ailleurs, p. 98, le poète montre m ruisselet qui djômihíze dans le ravin et que la pluie fait bondir hors de son lit : la traduction «sommeillait "ne peindrait pas la lente acemmlation des eaux $\left.{ }^{3}\right)$. - Enfin.

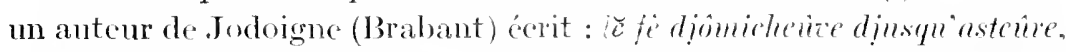
la qu'é blalic por bou. Ie féu courait, roilà qüil flambe tout de bon ( $\left.{ }^{+}\right)$.

IV. Il nous reste à parler de deux diminutitis de djomi.

1. Type *aotruixer. - Le verviétois Lobet ne comnait pas djomi (5); en revanche, il est le seul de nos lexicographes qui enregistre djominer

(1) Théatre lifgeois, ad. de 1s.54, p. 6s.

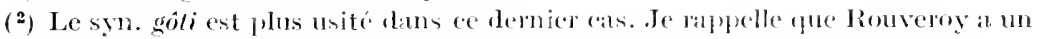
article : "gomi. voy. gôli et que nous avons traité de ce gôli a l"article gô.

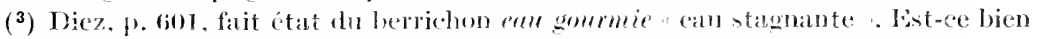
le sens exact ? Ne serait-co pas plutôt : "eau qui sacemmule lentement" ?

() Echmond Etienne : Bsill :3, 1). 235,

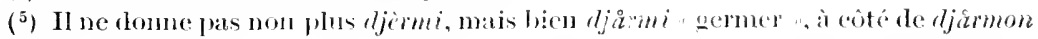
"germe ", 1. 216;. 
"agir lentement" (p. 654). Ce verbe est sigualé à Huy daus cette note que je tiens de M. W. Gorrissen : " 1. eouver, en parlant du feu : lé fêt pout co èsprinde, é djômine déspôy on quêrt d'éûre; 2 . faire un séjour trop prolongé. en parlant d'une ehose soumise à nue opération queleonque qui ne marehe pas assez rapidement : lé tchâr djômine so l' fề ; lé s'mince djôminè è tére déspôy trờs samivinnes". - Il paraît qu’à Liège (Cointe) djôminer existe aussi: li feû (li tchîr, li s'mince) djôminêye.

2. Type * *ourunller. - On signale à Dinant djoûmyi, à Vonêche djômyi "couver" (en parlant du feu). - De même, à Stave (prov. de Namur), d'après M. L. Loiseau, diômi (où - $i$ est une réduction de -yi) signifie 1. eouver, en parlant du feu; 2 . geindre, gémir [?] : gn-a s' vinte qui djômiye "il y a son ventre qui gémit ". Le seeoud sens paraît bien improbable ; M. Loiseau se laisse sans doute influencer par la ressemblanee avec le fr. gémir. On comprendrait mieux : "il a le ventre gêné, distendu ", état de malaise qui peut d'ailleurs s'aceompagner de gargouillements.

C'est encore mu type en -iller que nous tronvons dans le gaumais djômîr "couver" (une maladic. cte., toujours en manvaise part), terme inédit que j’ai noté à Ste-Marie-sur-Sennois et à St-Léger ( $\left.{ }^{1}\right)$. - Il faut (1) rapprocher eet article du Vocubuluire sumais ales environs de Virton, manuscrit de 1850, par Cl. Maus : "chanmé. couver sous la cendre, travail latent : les simeur ly chumain da el eouer. la bile le travaillait d'une maniere invisible ". On lira tehômer (dont l'initiale, si elle est bien sîre, est altérćc de dị): lès-imeurs lî tehômint da èl cacor se traduira littéralement : "les humenrs lui gourmuient dans le eorps", c.-à-cl. saeeumulaicnt sommoisement. Nous retrouvons ainsi, pour muir, le type gomer : GoUkMER, d'où nous sommes partis.

\section{lićg. gorlète, golète}

D’aprè des Liégeois que jai interrogés. gorlite. s. f.. signifie : 1. en t. de boucherie : con (du bocut) : on bokit d' gorlite; 2 . fanom. peau qui pend sous la gorge d'un animal. surtout des bovidés; 3. par eomparaison de dénigrement et toujomrs arec nue épithite on un complénent déterminatif : con débordant (d'une persomne grasse). on vaste gosier

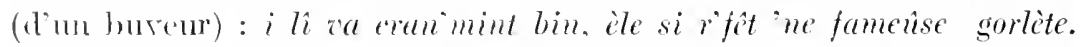

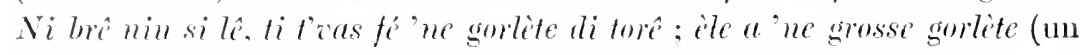
goitre); c'i-st-ine fire sorlite (un fier cntomoir, un grand buveur).

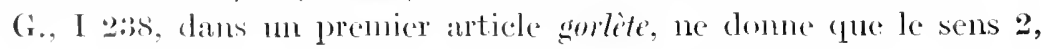

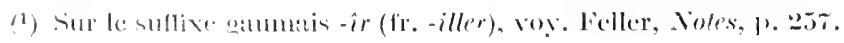


avec cette explication : "Peut-être un diminutif de gorế (collier de cheval)". Il fait, sans explication, un sceond article pour gorlite, "t. de min., manteau de euir que les chargeurs mettent pour se préserver de l'eau $\%$.

La conjecture de G. porte à faux. On ne peut admettre non plus sa division en deux articles. - En réalité, gorlète est mis pour *gol'rète, diminutif de golé "collier ", et répond littéralement au fr. "collerette ". Cela ressort du sens "ajustement de femme, gorgerette", que met en première ligne le Diet. malmédien de Villers (1793). De plus, on relève dans les archives liégeoises du xvie sièele : "une golerette de noir velu " à côté de : "une gorrelette de drap noir servant à une femme " $\left.{ }^{1}\right)$. Dans ee sens, le liégeois moderne n'emploie que le doublet colirìte. colorìte, qui est un emprunt récent du français ; mais la pièce de cuir qui courre les épaules des chargeurs et qui s'attache au cou, a gardé le souvenir de l'aneienne gorlète, objet d’ajustement. Les autres seus s'expliquent d'eux-mêmes. Le fr. collier, t. de boucherie, désigne aussi la partie du bœuf ou du reau comprise entre les épaules et la tête. Quant an fanon, on $y$ a vu une ressemblance avee la "collerette ».-- Au point de rue phonétique, rien de phus fréęuent que la métathèse réeiproque dans les mots du type gorlite, où voisinent deux liquides intérieures : firlisse (Choiv, p. 114), pour fil'rése "fileuse" ; livlêye (verv. : G.. II 511). pour lill'rêye "cuillerée "; ôrliyge (à Crupet). pour ôl rîye " huilcrie "; purlê (roy. cet art.), pour *pil'rê " petit pilier ") sarlète (et non stirlite: G., II 341). pour *sal'rite "salière"; tcharlen̂s. pour *tchal'rêts " chaleureux" ; trahèlèye (Esneux). pour trahil rèye " eohue "; roy. aussi les articles dièrmale, hèrnale.

On ne peut séparer gorlite du mot suivant, dont G.. I 137. II xxvir, parle en ces termes:

golète, fressure.- l'araît signitier proprement : con, d'où l'expresision rapportée par simonon : tot passe po $r$ golite. De là : collet (en t. de bouch.), boutsaigneux : enfin, par extension : mon : poumon de certains animax. Golète est donc probablement un diminutif du latin gula.

La conjecture de $\mathrm{G}$. ne ressort guère des signifieations qu'il détaille avec tant de soin. Nous royons dans golite une forme féminine de " eollet ", qui se dit en français, comme terme de boucherie, au même sens que "collier" ci-dessus. D’après Semertier, Toe. de la boucherie

(1) Reg. anx arrêts, 1580-33. - Godefroy, t. ix, colenete, eite aussi eorlerette (à Spa, 1606). 
(BSW 35. p. 44). le liég. golé " collier " est symonyme de golète "fressure". En certains endroits (Vottem-lez-Liège), golite remplace gorlète au sens de "double menton diune personne grasse". Enfin Durivier domne la phrase : $\grave{e} \grave{e}$-st-ine fomense golete "un grand bureur ". Ces concordances promrent que goléte dérive du lat. collum au même titre que gorlite du lat. eollare (eollier). Au surplus, Godefroy a un exemple de l'ane. fr. collette, s. f., diminutif de cou.

Il existe clans notre dialecte m autre golite, diminut if du lat. g ula, qui répond au fr. gonlette (entrée en entomnoir) et qui se reneontre dans des noms de lien (BSW 49. p. 24 ; 52, p. 216). Il peut être parfois diflicile de distinguer entre les deux: un croisement de collum et de gula est des plus naturels.

[Welenges Kurth (1908), t. II, p. 320 : art. remanié et dévelopué].

\section{liég. gossê}

J'ai entendu ee mot $1^{\circ}$ à Amp,in et à Bergiler, (Hesbaye liégeoise) : on gossê d'ansine "un petit tas de fumier déposé sur le terrain à fumer "; $2^{\circ}$ à Jupille et à Liège (Cointe) : on gossê dr foule "une veillote, un tas de foin plus petit que la hom gnite ". Le sens générique est done "petit tas arroudi ". I part Body. Ioc. agr.. qui atteste ansi le $1^{\circ}$ en Heshaye, et G.. I 2:35. 35t, qui attribue les deux sens an hesbignon gossiă, gochă [nam, -ya = liég. - $\hat{\imath}:-e l l u m]$. nos lexieographes se taisent à ee sujet $\left({ }^{1}\right)$.

Hubert, Duvivier et Forir doment le terme suivant que, personnellement, je n ai pas retrouvé : goss lé "chargé. bien rempli "s syn. de hop lé. ho: lé. On anrait tort d'y voir l'altération de ce hoz'lé, hoss'lé, qui dérive de hose "houscau ( $\left.{ }^{2}\right)$; soss le provient de gosse. eomme hop'lé de hopế. Le sems propre est : "tassé. bourré ".

D'ou vient ¿nsiê lui-mème? G. n'en atit mot. Body compare le hesb. gossê à lard. cossit "veillote", et eette indication se trouve ctre juste: il nous reste ì le démontrer.

Le liég. crosist. nam. conchet (de même somehe que le fr. coche, cochon), signilie proprement "petit pore s et, au figuré, clans certains villages

(1) Forir a denx artides gui ne méritent auteme confinne : gocha veillote net "gocal sorte de gerbe de ble ". Liun reproduit me erreur de G., I 235. que G. lui-

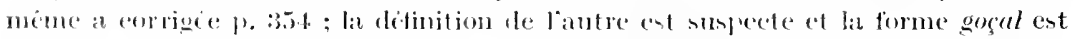

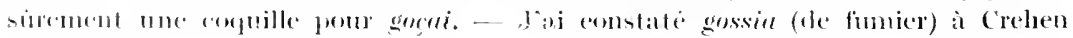
(I)ethaye).

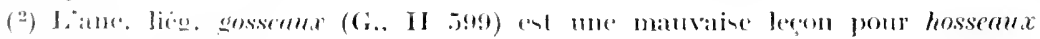

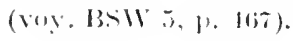


ardemnais (Vielsalm, Jalhay, Sprimont). "veillote". petit tas de foin qui ressemble au dos arrondi du cossit $\left({ }^{\mathbf{1}}\right)$. A I Ierve également: mite lu foùre a cossèts, fé lès cossèts. Du même radical, à l'aide d'un suflixe équivalent, on a formé cossế, dont l'initiale s'est ensuite adoueie $\left.{ }^{2}\right)$ : une eharte liégeoise de 1396, relative aux habitants de Jupille, eontient en effet cochcau, forme francisée qui atteste au xive siècle l'existence du type wallon cossé $\left.{ }^{3}\right)$; nne autre charte liégeoise de 1403 porte la forme gocheau $\left(^{4}\right)$.

\section{lien dit Grétry (à Bolland)}

On sait que la famille du célebre compositeur liégeois a eu pour berceau le hamean de Girtry (commune de Bolland. au X. de Liege), d'où elle tire son nom $\left(^{5}\right)$. Les formes anciennes sont Gretry. Grettry, Grefterix $\left({ }^{6}\right)$. Nous y reeomaissons unc de ces nombreuses désignations toponymiques eomposces de ri (ruisseau) et d'un premier élément représentant un nom d homme : ainsi Gobri (a Tilf-Beanfay : BSW 52. p. 206). aneiemement Gobieri. Signitie "le ruisean de Gobert ". De

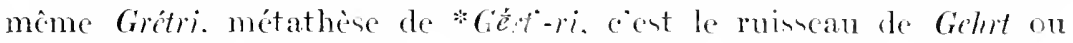
Geert, forme entractéc du germ. Gerharl. nom d'homme.

w. grimon (ard.. hrab.)

G.. II 530, signale sans explieation l'ard. srimm (sorte d'esprit malfaisant), que Borgnet a noté a Muno, a l'extréme Sud de la provinee

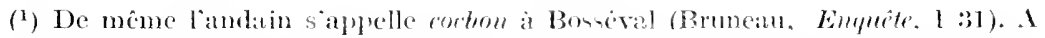

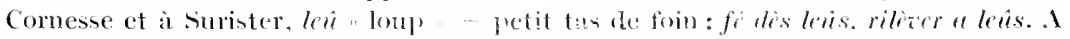

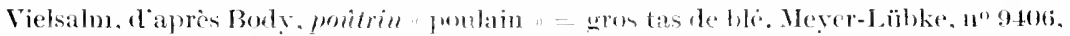
admet que ceillste derive de vituhns vean (comple rependint Thomas, Mr-

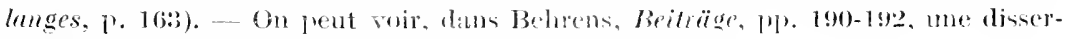
tation sur les noms dimimaux pris metaphoriquement.

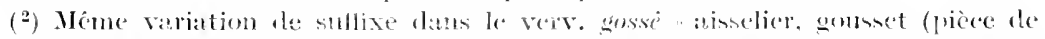

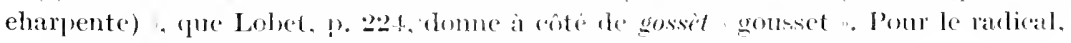
ce gossê $n$ 'a évidemment rien it deméler aver ke notre.

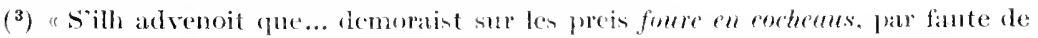

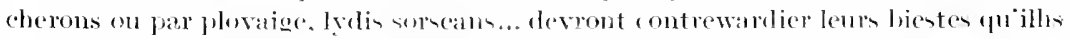

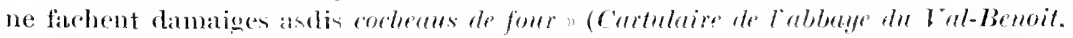

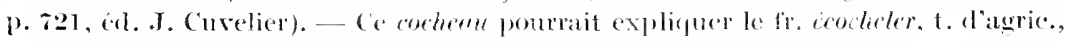

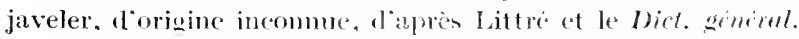

$\left({ }^{*}\right)$ "Pour le dit four aidier fencir et commonrmeir phis le mettre en grans go-

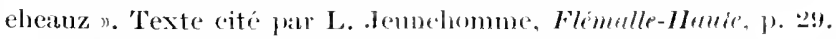

(5) Wallonil (Liège), xil 29 , xir 132.

$\left({ }^{6}\right)$ En 1552 : Henry de Gretterix (Arch, du Ban de Iferve, s, 205). 
de Luxembourg $\left({ }^{1}\right)$. D’autre part, Edm. Etienne, de Jodoigne, dit dans

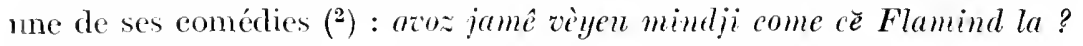
il a Grimon i coar! " "il a Grimon dans le eorps" = e'est un mangeur insatiable). - Les nasales $a n$ et on ayant une tendance à se confondre ct à permuter: je vois daus notre mot une prononeiation dialectale de * griman. forme altérée du fr. nécromant, négromant, de même que nécromancien devient groumancyin, grimachin (Liège), grimancyin (Gembloux, Stave. Givet). Du sens de : "soreier, magieien ", le peuple passe aisément ì eelui de : "esprit malfaisant". C"est ainsi que Simonon définit le liég. égrimancyin: "lutin. loup-garou "(G., I 18s), et que l'on dit ì Jodoigne : àl èst cotchissi dèl gormuancén " il est tenté du mauvais esprit ».

\section{malm. guduc}

Le Dictionnaire malmédien de Villers (1793) a un article : "guduck, adj., perdu, eonfisqué, tombé en commise", qui, dans les Extrait ${ }^{s}$ publiés par G., est défiguré en guzlick $\left(^{3}\right)$. C'est le fr. caduc, avec le sens que lui domnent les jurisconsultes (bona caduea, quibus nemo suceedat heres: Du Cange, $\mathrm{r}^{\circ}$ caducum; sỹn. "main morte)). Cuduc s'est altéré en *gaduc (eomme le malm. gabrigole "cabriole ": Villers) ou *guèduc (comme le malm. lienon "canon" : id.), puis en gutuc; comp. gumune (pour guimène). gommi "grenicr", hugunot "lugguenot", harkubohizer "arquebuser " ct autres formes malmédiemnes. Au reste, ce guduc est un terme archaïque de droit, tombé aujourd hui en désuétude.

\section{w. guèdin}

On lit dans le BSW 5:3. p. 405 : "mète li guèdin (Seraing). e'est serrer le menton entre le pouec ct l'index; mais quel est le sens propre de guedin? On rit con Iesbaye mete lis guingons".

Hin I Iesbaye même, j’ai noté à Bergilers mète lès guètins, avec des détails circonstanciés qui ćelairent l'expression : pour faire rire un coliant. on lui serre latéralement le menton entre le pouce et l'index,

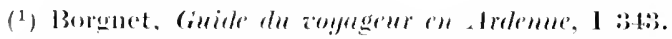

(2) Nos meriuns Cadir, sc. xi. - Gremon est ici devenu nom propre. I)après $\mathrm{MI}$.

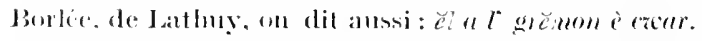

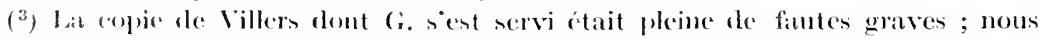

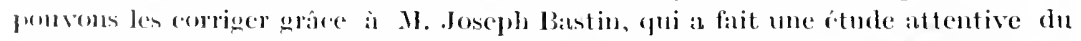

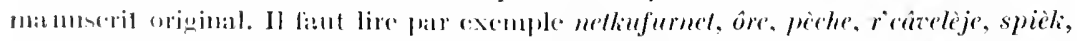

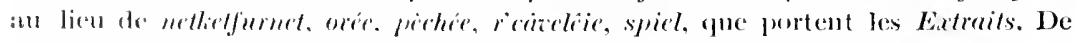

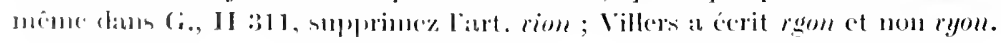


et on le lui seeone de haut en bas $\left({ }^{1}\right)$. Si le lecteur veut bien se reporter à l'article guingon, il n'aura pas de peine à reeonnaitre iei l'onomatopée

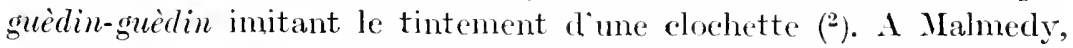
d'après Seius, gnèdin signifie " hochet. jouct d'entant ", ce qui confirme notre interprétation.

J'expliquerai de même cet artiele assez confus de Lobet :

guèdin, moulin à eafé ; se dit aux femmes, faire tourner le moulin à café, par plaisanterie; boire une tasse de aufé à l'insu de leur mari.

Le guèdin que les femmes font tourner quand clles reulent godailler entre elles, rappelle à l'imagination ironique du peuple le hochet qui amuse les enfants !

\section{liég. gueûte}

D'après G., II 530. ee terme de batellerie, qui signifie "bois servant à soutenir les écoutilles ", est probablement le même mot que coyeûte, t. de houillerie, "sorte de pièee de bois " $\left({ }^{3}\right)$. Proposition malheureuse, que la phonétique écarte d'embléc. Le liég. guente n’est autre que le néerlandais dialectal gent. forme variće du néerl. goot "canal, eonduit, gouttière ". La pièce de bois quil désigne est en effet creusée de façon à faire eouler en dehors du bateau l'eau de pluic qui tombe sur les couvertures ou rimes $\left({ }^{1}\right)$. — Sur la Sambre, on dit göte (lés gotes dès couvèrtûres).

Le néerl. goot " canal" a passé de même dans le w. de Ste-ILarieGeest (lez-Jodoigne : Brabant), où jai cntendu : lếte djolie èl gôte, l'eau est arrêtée dans le drain $\left(^{5}\right)$.

\section{liég. guingon}

G., I 248 , 35j, II xxvi, eonsaere à ce mot - qüil laisse sans explieation - trois articles. dont roiei le résumé :

(1) Cela sappelle à Liège fé dés crosses di doríge faire lles cronten de tarte"; à Nivelles : fé "ue crousse de gruml-mère.

$\left({ }^{2}\right)$ Comp. dans Lobet : guelin-guclin "tintin, lruit d'une somnette, d'un grelot", et le fr. drelin, dimlin dans le Dict. générul.

$\left({ }^{3}\right)$ Sur coyeûte (lat. colle eta), voy. BD 1914, p. St.

(4) Voyez ci-après larticle slièrbuliti.

$\left.{ }^{5}\right)$ Il he faut pas confondre ee mot avec le w. gofe (aoutte) qui est bien connu en toponymie liégenise et luxembourgeoise ; loin le Iininutif gotale "gouttelle". Cf. BSW 53. 1. 391. 
guingons, plur. (momus joyaux a lisage des femmes, tels que pendants 1 orejllas, etr.). - Ce mot mignilie amssi : 1. (au sing.) la substance charnue qui pend au con des diufloms, ct: :2. les glandes qui pendent également au eou des

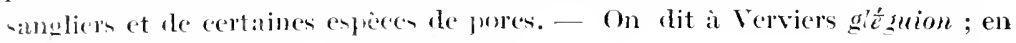
combroz glingom.

Le plus aneien exemple se lit dans me pasquille darant 1650, sur les jemmes filles coquettes (13SW 11. p. 24.5) :

\section{Si varont-èles dèn fièrs d'ardjint}

Et ales guingons qui vont si bin.

L. mot prénente des formes et des sens multiples. Durivier éerit shimugom "clinquant. joyau : mites tos zos ghthmgons" (lisez guingon, arec in = ing allemand). - Forir a trois artieles : gaingon "menus joyaux des femmes" ; gaingun "elinquant, petite lame d'or, d'argent ou de cuirre quon met dans les broderies : falbalas, oripeaux" ; kinclau " elinquant; roy. guing-gan ». Ce dernier faisant défaut, je suppose qüil sagit de gaingan. que Forir prononcait gaingan et

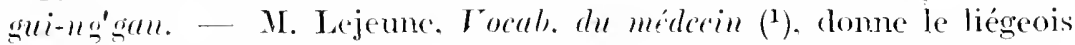
slin-glan "sécrétion solidifice restant attachée à dés poils, ete. (à cause de la forme en battant de cloche ?) ). - I Liège même, jai entendu gli-ns"slan "joyau : ile riglatih come ine catidrale. ile a miton tos sis sli-ng glans. Un auteur de Seraing éerit danns ce sens : dés guign'gons (BSW 51. p. 6s). ct Willem, Dict. des rimes, a : suinglon "pendeloque ". On ne tromve rien la-desus dans Cambresier. Remacle. IIubert. Pour le malmédien. Villers dome : "glinglan. s. m.. du clinquant".

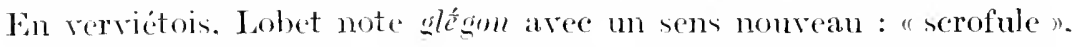

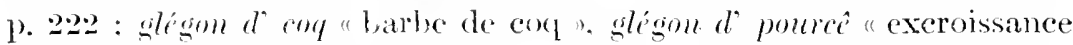
charnue sous lo eou du cochom ". p. 6.5.5. - Enfin. pour achever la

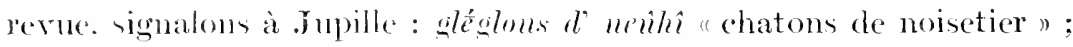

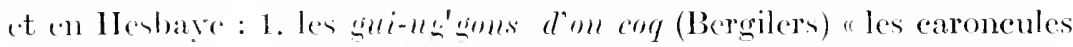
dim con": "2. méte lis guingons "serere le menton entre le pouce et l'index, (13SW $5: 3$. p. 10.5).

Fin resmmé. on distingure daus cette variété de formes: $1^{\circ}$ les suffixes

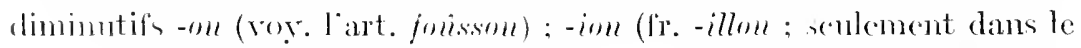

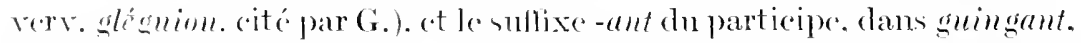

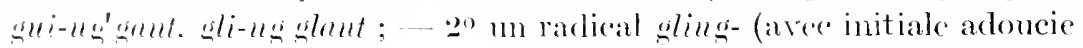

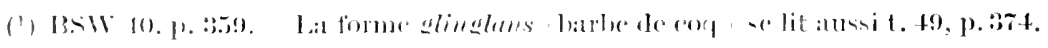


pour *cling-). où nous recommaissom liall. klingen "résonner " (l) : gling- pent s'altérer en guing-. glingl-. guingl-, tandis que la royelle pent aroir eing états différents: im. ign, in. $\widetilde{e}$. $\tilde{e}^{\text {. }}$.

Proprement. le glingon on gli-ng'sant cest le petit objet somore qui pendille (elarine ou sommaitle au cou des vashes. pendelopne. joyan de femme) ; de là. par extension : caroneule de con $\left(^{2}\right)$ on de dindon : glande de sanglier ou de p.ore ; scrofule : scéétion solidifiéc : ehaton de noisetier ; enfin. pour faire rire $u$ enfant. on lui met les suingons (Hesbaye). quand on fait le geste de somer en lui sorrant le menton entre le pouce et lindex (royez p. 122 lart. snidin).

Le seus primitif apparait eneore nettement dans len dérivés suivants :

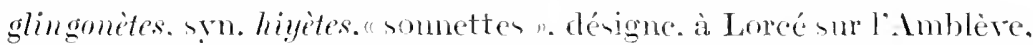
les clochettes du fuchsia ; 一 altéré en sningnignite (Vottem. lez-Liège) "petit objet que les enfants fabriquent à l"aide de l"infrutenecuee de la bourse-i-pasteur : ils obtiement aim m hochet dont la elinnetis les amuse " (Bull. de Follil.. II 1:1).

glignter (Licge. Huy), r. intr.. somner. cliqueter "se clit p. ex. d'un carreau qui casse. dime fenctre que le vent seence - gl liter (Verviers : Lob. ap. G.. II 528) " copter. faire aller le battant t"une cloche d'un seul eôté " - glinsoter (Malmedy : Vill.) "somer les eloches. brimbaler" ; doì : tuglingoter. lans cette jolie expresion : lètê d'glingoter lés clolies "attendre que le clochen aient fini de tinter comp. l'all. ausklingen $\left({ }^{3}\right)$.

d(is)guingonè (Ciney). r. tr.. "délourser". propr. "laire somner son argent».

\section{malm. guzouhe, galguzouhe}

Le Dict. malmédien de Villers (1793) donne l'expression azeûr al guzouhe "avoir à sa discrétion" : de même Scius (189:3), qui écrit aussi cuzouhe. A Farmonville, M. J. Bastin signale : arểr al liø̌ssouhe "avoir à sa diserétion (p. ex. son patrimoine), gaspiller (quch), tour-

(1) Comp. le fr. climpume, de lanc. fr. climquer, neerl. klinlen. Le linelun de Forir est altéré fle clincant, emprunté du français. - Le verbe glingarer existe a Clatirvaux (Jura) avec le sens de "leturter des objets sonores, en tirer du bruit"; roy. Behrens Beiträge, 1). 214 .

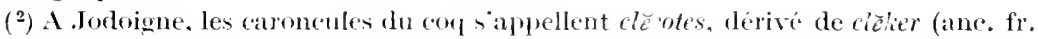
cliquer "faire du bruit "). Ie liég. clicote a le sens général de : lambean (qui clique), chiffon $)$

(3) De même, en meusien : glingoter (Varlet), glingloter (Labourasse), v. 11., "résonner, sonner, en parlant de vitres". 
menter (qqu)"; mite al luésssoulhe "détruire (p. ex. un habit)"; djozer al kierssouke, t. areh. du jeu de billes, "joner à la poursuite avec de grosses billes). (BSW 50. p. 575).

Grandgagnage. dans ses Extraits de Villers, se demande si le malm. guzoulie n'est pas l'all. sesueh "demande, requête". On pourrait en effet alléguer le moyen h. all. gesuch "action de poursuirre (le gibier), droit cle disposer (d'm pâturage) \%. Ccpendant, il faut plutôt s'adresser au moyen h. all. gezoc (subst. de gezichen), qui désigne notamment l'action de tirer it soi, d'enlever violemment, d'attaquer et de piller. On comparera souke it Faynonville, où nous recomnaissons le moyen h. all. zoc, sog (roy. ei-après l'art. sohe). Pour le changement de g initial en k. eomp. licklitône, lièstèl (à l'art. gistel).

A guzoulie peut-on rattacher le malm. galguioulhe, que Villers définit : "baliverne. sornette. fleurette, fable" ? Il est fort tentant d'y voir le préfixe péioratif gal-, cal-, et le même ge signifie aussi : "action de tirer qqch en longueur, de perdre son temps". Ce serait toute espèce de propos oiseux, de contes frivoles. On dit galguzoûde ì Stavelot; galguizoñde à Cherain, Liège, Vervicrs (aussi

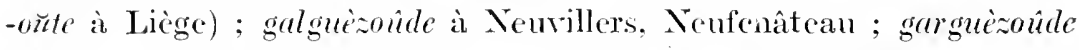
à Namur (G.. I 231). Nous trourons même golglguesouille ì Mons en 1812 (Delmottc). Cette dernière forme aurait subi l'infuence de couille (Sigart : couic "mensonge"); les précédentes, celle du w. boûde "bourde ». (Quant à cullizite (Glons-sur-Gecr), la finale serait empruntée à clüte (néerl. liluit), dont on a parlé à l'article bougnèt.

\section{liég. håbiêr, anc. liég. halbier}

Ce mot a des formes a des signifieations très diverses. Il se reneontre seulement daus la province de Liège (Hesbaye, Condroz. Verviers. Starelot), dans la région de Malmedy, au $\mathrm{X}$. de la province de Luxembourg (Vichalm. Cherain) et jusqu au centre de cette provinee (âbièr ì Lavach(rie, Inoircy-St-IIubcrt). L'aire d'emploi fait présumer une provenanee germanique ; le traitement du $h$ initial change ectte présomption en certitude ( $\left.{ }^{1}\right)$.

Xons croyons que hâbiér ćquiraut à l'ane. fr. herbere (mase.) et qu'il dérive de l’anc. h. all. heribergá (f'́m.), ane. nordique herbergi (neutre) $\left({ }^{2}\right)$.

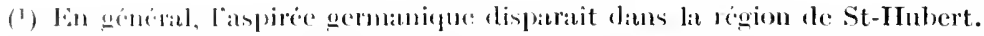

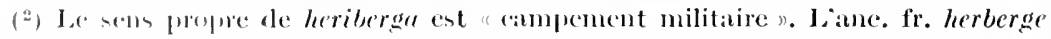
en a tire les significations de "ampement, tente ; logement, habitation ; hôtellerie, aulserea 
Il serait done le frère du fr. anberge, aneiennement herberge, héberge. On peut dater sa naissanee de la fin du xime sièele, ear e'est alors que se manifeste et se généralise la diphtongaison en $i \grave{e}$ de $\boldsymbol{e}$ entraré $\left({ }^{1}\right)$.

Essayons de justifier cette proposition en passant en revue les formes et les sens du mot.

I. - G., II 603, eite quatre formes ancienmes qu'il a recueillies dans les chartes liégeoises : halbier (1440), qui rappelle l'anc. fr. helbere; - habier, habiert (1568); - hazier (1593).

Parmi les formes modernes, on distingue deux groupes :

a) celles de la région de Nalmedy, qui sont trissyllabiques et qui représentent un stade plus aneien et mieux conservé : hâribiêr à Steinbach-lez-Weismes, Robertville ; huilibiêr à Thirimont-lez-Weismes ; hâdibiêr à Faymonville-Weismes; Malnedy : Villers, Seius ; Spa : Body, Foc. agric. $\left({ }^{2}\right)$. - La forme de Steinbaeh rappelle très nettement herberg: la chute de l'r primitive a été empêchée par la royelle $i$, qui s'intercale assez fréquemment, en malmédien, entre $r$ ct une eonsomme suivante $\left(^{3}\right.$ ). Dans les autres, le passage de $r$ à $l$, puis à $d$, s'explique aisément $\left({ }^{4}\right)$.

b) les formes dissyllabiques, dans les provinees de Liège et de Luxem. bourg : hawbiêr (Verviers : BSIV 44, p. +21); habièr (Liège, Verviers); hâbièr (Condroz : G., I 356 ; Stavelot : BSW 4t. p. 509) ; lấbic (Hesbaye : G., I $\mathbf{2 6 0 )}$; $\hat{\imath} b i \hat{\imath} r$ (Lavaeherie, Noirer-St-Hubert : centre dı Luxembourg). - La forme rerviétoise hawbiêr rappelle et explique l'ane. liég. hawier. Les autres se ramènent à hâribiêr avee chute de la protonique, phénomène fréquent qui réduit d’aneiens trissyllabes en dissyllabes. surtout dans le liégeois $\left(^{5}\right)$. Elles se rapprochent du fr. auberge, emprunté, au xrie siècle, du provenẹal aubergo, anciemnement alberga. Au reste, si nous comparons serpillière : saipire ct serpelette : saip'lète, nous admettrons sans peine le passage de herberc à habiêr.

(1) Wilmotte, Etudes de dialect. zoall., dans Romania, xvi 55\%. - Dans l'all. dia!. d'Eupen, le mot actuel est härbereg (auberge), qui a passé en w. de Malmedy sous la forme hèrbêrich.

() G., I 35\%, note, d'après Simonon, un hädlibiè qui ne nous parait pas liégeois et qui a probablement été recueilli en Artenne.

${ }^{(3)}$ Comp., à Malmedy : kirimusse, kemesse ; assêriminter, assenmenter ; sêrimint serment, ane. fr. sairement.

( $\left.{ }^{4}\right)$ Comp. 1. angolà, cèlihe, colidồ, moloze (angora, cerise, corridor, morue) ; 2. dacheron (Hécart), pour lacheron, laiteron ; Diopôl pour Liopôl, Léopold.

(5) Comp. l'all. Leberwurst : verv. liv'go ; ane. w. cortisen : cot'hé ; malm. mreargunê : liég. mzè̀thè ; *tchaformè : tchaf'nê (l. rl. de Jupille), sans eompter les mots plus connus bol'dji, tehì 'th, iol ti, cof'tcui, cor'ci, ete. 
II. - I) uens propre de l'anc. haut all. heriberga, "campement militaire". dérivent les significations que herberge a prises dans la suite en moyen hant all. et en aneien franęais: 1. campement, tente (=installation quon établit à l'étape) ; 2. hôtellerie, auberge (= installation quion troure toute prête i l'étape); 3 . logement. habitation (= installation en général).

Or habiề se présente chez nous avec le sens de "exploitation rurale, ferme (surtout comsidérable)".

On comprend facilement cette dérivation de sens si on se reporte au temps où le mot a dû passer de l'Est et du Nord dans nos dialectes. Au moren âge. la grande préoceupation des rouliers et des royageurs était la herberge. le gîte pour la nuit. En pleine campagne, loin des villes. cette auberge derait pouroir se suffire à elle-même, à la façou des villas gallo-romaines ou mérovingiennes. C"était. par l'aspect extérieur, un grand établisement rural. capable de loger nombre de persomnes et de eheraux. ("est par laspect extérieur. en laissant de côté la destination de l'établinsement, que habiêr en est venu à signifier une exploitation rurale.

Ce point de départ admis. abordons l’étude des significations assez éloignées que notre habiêr a prises au cours des temps. Pour cela, il convient de eomidérer à part les formes anciennes eonscrvées dans les chartes liégeoises ot les formes modernes on orales.

a) G.. I I 60:3. définit les premières : "droit que les officiers des métiers nourellement élus payaient pour leur entrée " et il présmme, - avec sagacité. seton nous. - que ce mot pourrait bien ctre eomnexe avec le hesbignon habic.

Ciest en 1 140. dans la Charte du métier des Febrres, que se troure la premiere mention du halbier:

Item avom, ordomué... que queileonques serat esleu Olficier de nostredit Mestier alle sainet Jiacfues soient temus de payer pocr lect IIALbier, assavoir Gouverneurs et Jureis chancon deamlx deux grillons et ceux qui aroient les

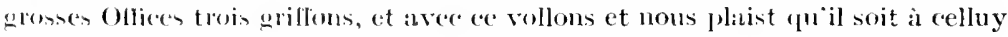
jour payeit et debourseit par nostreelit Rentier aux frais dudit Mestier hit

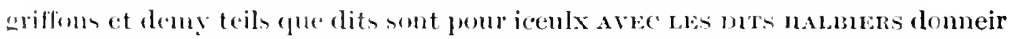
(t partir aux vinaves dhascun a son marmontant pour les compagnons dieeulx stler beire cuscmble à leurs bons plaisirs sens framle ( $\left.{ }^{1}\right)$.

C'as larmences araient lien aussi dans les autres corps de métier. En 15ist. la chate des chandelons fixe la somme que les ofliciers "seront

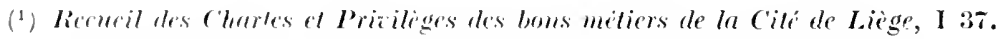


tenus, incontinent l'álection faite, PAYER POUR LELR MABIER, en profit duclit Métier ». Ft elle ajoute :

I.esquels dits mamiens des dits Officiers, soy deveront partir et divider aux vieux Officiers et compagnous dudit Inctier, ayant ¿té présents à laulte éleetion, à faire selon le nombre et quantité dicenx : entendu foutcfois que les Officiers devront avoir double droits, ne fuisse que ì ee jour ledit Métier tenisse table à disner, lors deveront les dits namers être tournez en diminution des depens qui soy foront.

Fat le cas alvenant qu'il sont ou luise comnus avoir par tels dits Oliciers payé pour obtenir destits Ollicicrs phus avant que lerlits maburss, icenx tomberont à l'amende, etc. $\left({ }^{1}\right)$.

Do même, en 1598, la Charte du métier des Chailicrs stipule ce qui suit :

Et comme d'anchicnneté sont été usez à l'élection des gros offices payer

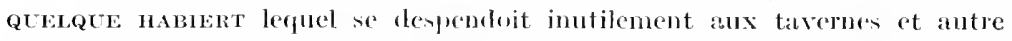
parte, pour pourvoir à tel abus, est ordomé que doresnatrant TELs mabiers soy payeront sur notre (hambre et semt convertis en meubles ct aut re chose, a la plus grande utilité et profit du dit bon Mestier $\left({ }^{2}\right)$.

Le fond de ces textes est assez clair. Mais que signifie proprement le mot halbier? Et eomment rattacher la définition de Grandgagnage à celle du wallon moderne?

Le demier texte cité nous paraît domner me indication précicuse à eet égard. Nous y voyons que ces gratidications ne doivent plus se dépenser à la taverne. mais qüelles doivent servir à lachat linbiets utiles an Métier. Or. a nos veux. on ne faisait la que revenir à l'mage primitif.

En effet. le halbier du Métier. c'était premièrement l'avoir de la eorporation, immenbles of nuatériel. Cela adnis, il est naturel de smpposes que le nonvel éln devait payer une cortaine somme pour angmenter cet aroir : toutes proportions gardées, cétait à pen près comme si. de nos jours, un noureau député repait de l'argent à la caisse de son parti.

(1) Ibid., II 30:3.

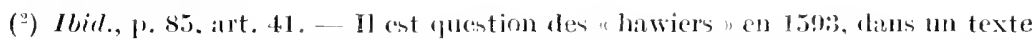
qui ne nous apprems rien ainteressunt (ibid., 1) 145 , art. 5 de lat (harte du mótier

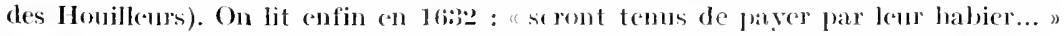
(ibid. II, 92, (barte du metice des Corbesiers). - Cf. S. Bormens, Le bon métier

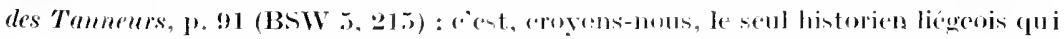
ait souligné celte particularité curituste. Cohlert, Rues de Liège, HI, 560, ne fait que rés uner le" dommées de Burmans. Poncelet n'en dit not dano ses Bous métiers de la Cité de Liège. 
L'expression "payer pour le (son) hallier") s'abrégea en "payer le (son) halbier " et l'on put dire môme " payer quelque habier ". Dans cette formule comrante. qui somnait comme "payer la (sa) bienvenue", le sens premier du mot s'obseurcit. d'autant plus que, par la suite, le Métier étant sans donte assez prospère, cette somme fut détournée de sa destination primitive et dépensée en réjouissances publiques.

Bien que notre démonstration ne repose guère que sur une série de conjectures, nous croyons pouroir aflirmar l'identité de l'ancien w. halbier et du w. moderne hibiêr et. à la définition de Grandgagnage, nous substituerons la suivante: "somme que les officiers d'm Métier nouvellement élus payaient pour le habbier du Métier, c'est-à-dire pour l'exploitation en commun, pour la corporation et son matériel " $\left.{ }^{1}\right)$.

b) 1. Mabiér a pris tout d'abord chez nous le sens général de "établissement. installation. cxploitation. comprenant tout l'attirail et le persomel nécessaires ". Dans ce sens, notre mot est surtout employé à la campasne, précédé de l'adjectif geand. C'est à l'expression on grand hâbiè que s'appliquent la définition de Body, Toc. des agric. : "grand attirail de labour, mobilicr de forme, exploitation rurale qui compte beancoup de bétail et de serviteurs" $\left({ }^{2}\right)$, et - pour la ville - celle de Forir : "grande boutique, commerce étendu". Exemples : ine einse wice qu'i-a an grand habii (Hesbaye) on hâbiè (Condroz, d'après G., I 260, 356). Gn-a on fî̀ habiè è cisse cinse la (Forir, ro hâbiair). Dỉnme mîs d'sse mâsse d'ine pritite botique qui dè d'pinde d'on grand habiêr (ibid.).

Un exemple curieux nous est fourni par le poète verviétois Martin Lejeune, qui parie, dans me de ses satires. dis hiyimes (haines) it dès

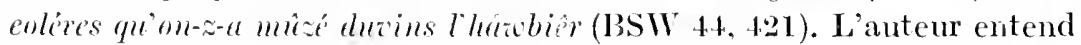
par la l'usine, la labripue. On ne pent cependant traduire aussi simplement, car la mance de dénigrement, que ce vieux mot revêt ici, est trop pronencée : cest ì peu pòs eomme si on disait : "dans le bazar "ou " dans la harague ".

(') Notons aussi que l'all. herberge peut signifies : lieu de réunion des gens de métier, matison de laborporation ; le herbergsvater, cest le père des eompagnons, liublergiste diun corps ale mitier. - Comme il est possible que habier ait, dans les promires temps, conserve le sens originel de amberge, nous pourrions expliquer plus

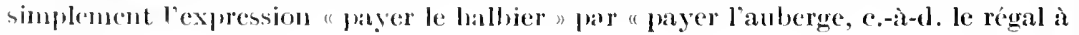
liubleren.

(1) Anjourdhui re sens tond à disparaitite daus les Ardenues : on dit à Stavelot

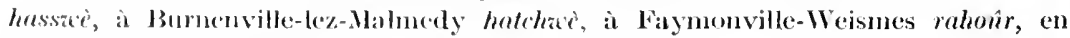
pay's gatmotais hensica, ete. 
Au sens général de "exploitation (surtout rurale)" se rattaehent étroitement les emplois que G., I 260, donne en premier lieu pour haibie en Hesbaye : " 1 . district que, d'après eonvention, chaque berger se réserve exclusivement sur le territoire d'une eommunc ; 2. aler å hibiè : aller travailler dans les ehamps". Cette dernière expression coneorde avee le renseignement que nous trourons dans le Dictionnaire manuserit de Bailleux : "hâbir' (Hesbaỵe), hâbièr (Beaufaỵs), dépendanees d'une ferme, eulture ". Même sens à Polleur. -- Quant à Lobet, qui défnit haubiair : "appondice d"mne ferme, d'me maison, ce qui y tient, ce qu'on y a ajouté ", il nous parait avoir confondu "appendiee" avee "dépendanee " (').

2. Ailleurs la signilieation s'est restreinte de plus en plus ; le mot a désigné telle ou telle partie spéciale de l'exploitation. a) A Jalhay, a Ster-Franeorchamps, ì Esmenx, habièr est synonyme de atiltye et ne se dit que du bétail de la ferme : On mâu habiêr = dès biésses qui n' sont

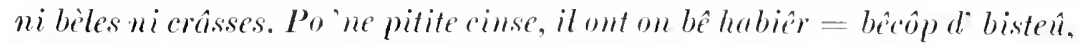
one bìle atèlêye. - b) A Vielsalm, tot l' hâbièr l'ine cinse. cest tot l' mèube, tout le mobilier de la ferme.

3. Enfm, à mesure que les eonditions éeonomiques se transformaient, ce mot - rappelant un état de ehoses ancien. une forme désuète d'exploitation, un ensemble d'instruments dont on avait perdu l'utilisation, qui étaient devenus cneombrants et que l'on mettait an raneart a pris tout naturellement un sens dépréciatif, qui est le plus généralement répandu aujourd'hui dans nos campagnes.

a) Précédé ordinairement de l’adjeci if $z \hat{\imath}$ (vieil), il signifie: "vieillerie eneombrante et de mulle valcur $\%$. Sens relevé à Cherain, Bodeux, Cherron, Villettes-Bra. Stavelot. Sery-Abée, cte. Exemples : I n'ont qu' tos rîs habiers (Cherain). Nos nos-azans fait quites du tos ès rîs hâbièrs (Stavelot). Tot çoula " n'ist qu" dès habiêrs; ci manèdje la "lèst qu'om habier (Nessonvaux). On rî âbiè di batimint ou onc rîye mâjon (Lavachorie). Vî hudibièr! (Body. Ioc. des poissartes), t. d'insulte adressé à une vieille femme. Comp. hadibies dans G., I 2 (61.

b) Procédé ordinairement de l'adjectif grand. il signifie : objet d'une grandeur démesurée et encombrante" : hâribièr (Steinbach-lezWeismes. Robertrille), "quclune chose de grand. de désordonné, de disproportionné, qui n'a mi esu mi tièsse : qué grand hâribiêr! dit-on

(1) Ce mot était sans doute inconnu à Verviers ; Remacle ne le signale pas, non plus du reste que les liégeois Cambresier et Hubert. 'Toutefois ces trois auteurs sont précisément si ineomplets que l'argument a silentio n'a pas iei grande portée. 
d'un bitiment de forme ot de proportions extraordinaires " (1) : grand

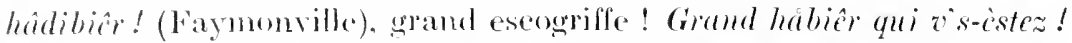
('Trouz). Çu telifan la n'est qu'on srand abiar (Moirey-St-IIubert).

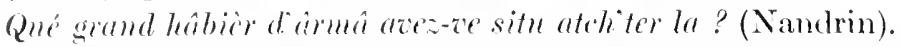

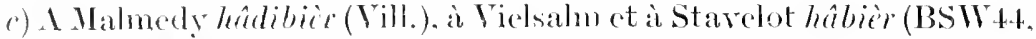
509) déxignent "un engin. me machine queleonque ", avec un sens dénigrant qui apparaît dans les excmples suivants : Qu'ést-ee po on

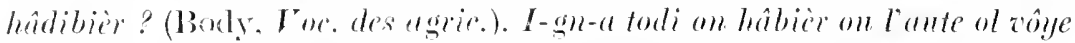
(Stavelot). C'antial. hima, hâbier it calmlemint, anle ont l'ante (ibiel.)

[Résumé ct reminiement particl de BD 190\%, p. 66-ri.]

\section{liég. hadrê. hat'ou hate}

Lobet signale le verviétois hadrai "baille, baquet fait de la moitié d'un tomean scié en deux ), e.ì-el. ce (ne le wallon appelle ordinairement on côpé (un eoupé). Bodyr. roce. des tomeliers, définit le même not par : "moitié d'un tomneau ; tinctte de luassem " (le second sens est aussi liégeois. au dire de G., II 3 H.t). Villers domme le malmédien hadrê d'bothe "assicttée on pelote de beurre" $\left(^{2}\right)$; de même Body. Tor. des

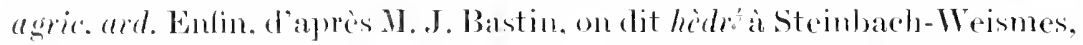
hudré a Faymonville-lez-Malmedy. On a roulu roir clans ee mot la même raeine que le moyen laant all. hader "zerrisznes heugituck" ; mais an lieg. hadep répond le nam. seadria (G., II 314) et, des lors, l’initiale postule un sétymologinge. Ceseadria - somvent altéré en seadia - se reneontro dans les provinces de Namur et de Brabant, où il signifie: "covelle de brasseur (nam.). petite envelle peu profonde dans lacpuelle on lave le beurre (Chastre-Villeromx. Thorembais-

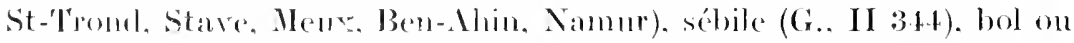
sébile pour donner dugrain aux pombes (Meeffe), someompe (Momale)".

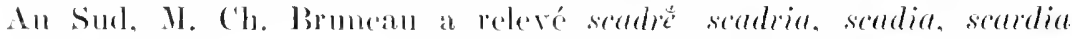

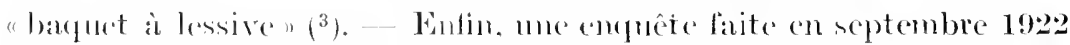

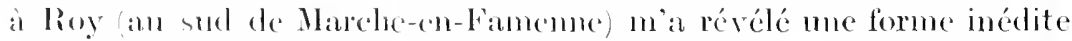

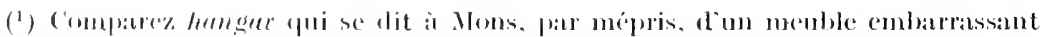

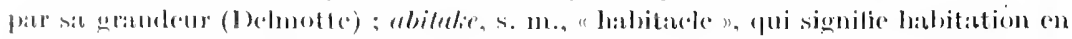

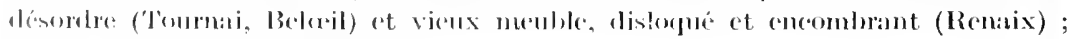

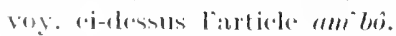

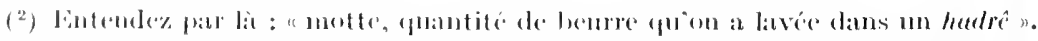

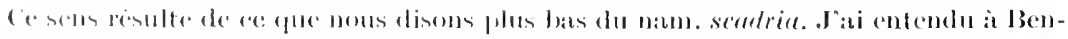

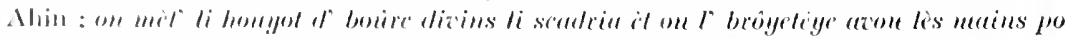
fe sorlit teriri (jedit-latit).

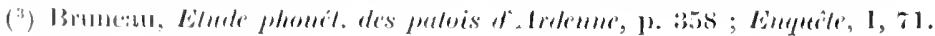


chadrê, avec cette acception unique. également inćdite : on chalrê

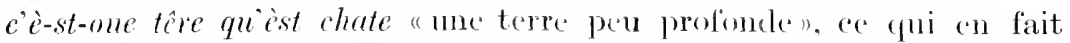
le synonyme de hadiene dont nous parlerons à l'articie suivant.

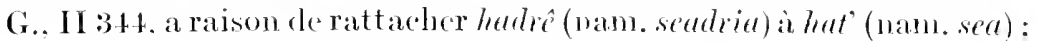
mais il ne parvient pas à dégager convenablement loorigine de ce groupe.

Il faut, selon moi, sadresser an germ. scheiden (got. skaidun) "séparer, diviser". Weigand a les artickes suivants: $1^{\circ}$ gescheit (gesehaide en 1494 ) "mesure pour les maticres sèches. un huitiène clu seticr (=2 litres)", qui vient du moyen. h. all. gescheide. n., "limite", ane. h. all. gesheite, n., "division, ligne on point de séparation ". anc. h. all. gascait. siseceid, secit. m., "division. séparation, section" : - 20 holbscheid. f., "la moitié, ce qui est partagé cu denx ", has rhénan hulffselecit (xives.). ane. h. all. hulpgisceid.

I mes yeux, hat représente l’ane. h. all. seeit (1) et le cérivé hathê: - (uui répond pour le scus à halbselecid - représente un type* skaidarieium, clont la finale a subi loinfluence des diminutifs ch -ellum (2). Le hadrê ou scadria a chî désigner prinitivenent unc petite mesure, puis il a pris le sens général de : "récipient pen profond servant à divers usages". D'où, par métaphore, à Roy le sems de clendrê "torre peu profonde, maurais terrain de culture".

Quant à hat' ou hate, conformément ì l'origine indiquée. ce serait proprement un substantif signifiant "ligne de démareation. limite ". Ainsi s'explique lexpression artrerbiale a hate (Malmedy-starelot), a hate (Robertville, Faymonville : $a=h y, z$ ). a deate (Honffatize) : 1. "jusqu"à (la) limite (extrêne), tout juste" : :. "trop juste. avec

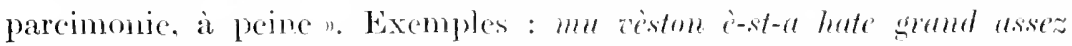
(Stavelot) : i-a dol sutofe a hate po fé one taye (Malnody) : via'ons azou fét a hute devant l' solè mousstunt (Guenzanine-lez-Malmédy). I renuarquer peserer al hute (Huy) " peser trop juste ". aij so-st-al hate (ibid.) "jo suis à court d'argent ". oì late est considécé comme un substantif l'éminin. Une autre forme intéressante existe à Iwenne : ni metes nin l'assicte l'ascute ("trop au bord "). ile toumele. Noms y verrons une expression primitive a-scate ("à [la] limite "). traitée ensule eomme un substantif, et nous éerirons a l'u-scente.

(1) Pour le traitement phometigue, comparez le moyen nécerl. geil qui a domné le w. gate (chèvre), diminutif gadru (chevrette). - On ne peut ohjectcr le w. liég. hète, nam. chète, skète (écharte), qui dérive de l'anc. h. all. scît, m. h. a. schât (écharde; all. mod. Scheit).

(2) Voy. Feller, Notes, pp. 179, 201; et, ci-après, à l'Alpendice. 
Dans la région liégeoise, au lieu de a hate on emploie adverbialement hate seul : i penise hate (ou too hate, ou si hate), ci murtchand la! $i$ côperen̂t s" deût! ("èst hate pèzé "c'est pesé trop juste".

De là. hute, chate $\left({ }^{1}\right)$ est devenu adjectif au sens général de "strictement mesuré " : $1^{\circ}$ peu ample, en parlant d'un vêtement : in-abit qu'ist tro hute (Liège); - 2o peu épais, mince, en parlant d'une tranche de pain : côpez-l' tote hate (G., II xxx) ; d’une terre légère, pierreuse : ine hate têe (Esneux), on chute tèrain (IIoul'alize) ; lig. dij so hat' it tène (Glons) "je n'ai phus le sou " : - $3^{\circ}$ peu profond. en parlant de l'eau : lès-êtees sont chates, le pěs' èst chate (Pellaines: Iesh.) ; de chaussures : dès chates subots (Pcllaines), sabots de femmes; des hates solés (liég.), souliers Molière, opposés à dès hôtes botlienes ; d'un récipient à bords peu élevés : hate assiète (Inuy). chate assiète (Awennc). on hate pot (Glons), hate banse, hute banstê. manne ou panicr peu profonds, d'où le substantif on hute (Stoumont), un petit panier servant à la cueillette des fraises, des myrtilles, ete.

Dérivés : hat'mint (Liège, Chapon-Scraing), strictement, trop juste ; ahati (Stavelot), ahati (Viclsalm), rendre trop mince on trop court, rogner ; ef. BD) 1913. p. 81 .

\section{lićg. art. hadrène}

\section{G.. I 201 , a ces deux articles :}

1. hadrène (" haut-fond, endroit d'une rivière où il y a peu d'eaı ; se dit surtout des endroits oi les rochers, ete., sont presque à nu ") Remacle, a éd.

2. hâdrène (partic pourric, cndommagée, dans un toit de chaume) Simonon.

Plus loin, p. 357, il rejette la graphic hadrène et réunit les deux mots sous un même hef, arec eette glose :

hâdrèze. Le sens rudieat dı mot est: endroit où le fond se montre à décottvert; de la : 1. terrain dépourvu de terre végétale et où le roe se montre ì la surlice, et ensutite les denx acepeptions que nous avens répartics sous les deux articles 1. halrène et 2. hâlrène, qui doivent par conséquent n'en former qu’un. J'ajonte que 2. hâdrène aurait lù être délini : partie d’un toit dénudée de chatume.

Enfin. II 3.t, il range haderène 1 et 'z parmi les dérivés du liég. hat', nam. sea "trop plat, mince, étriqué " à côté du liég. hadiê, nam.

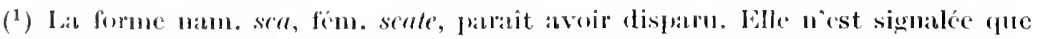

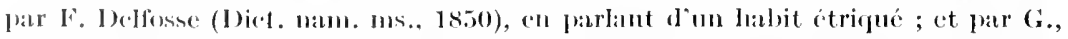

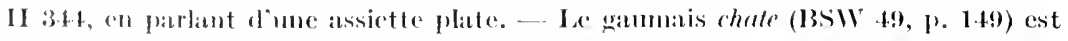
pen usité et frovient salns doute de laudennais. Le gaturais hate (BSW 41, 1, 169) est suspect. 
scadria et, pour tout éclaireissement, il compare le rouchi scater "écraser ".

Cela est sujet à eaution, tant pour la graphie que pour l'étymologie.

L'a bref de hadrène "haut-fond, gué, banc de sable ou de roche dans un fleuve ou une rivière" est eertifié par Remaele, Lobct, Forir, ainsi que par des bateliers et des pêcheurs liégeois. C'est bien le même mot que l'ard. hadrène "terrain pierreux, dépourvu de terre régétale. oì le roe est presque à nu ", que donne Body, Foc. agr., et que nous arons

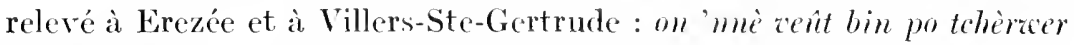
lès hadrènes! " on en voit bien pour labourer les champs pierreux" $\left.{ }^{1}\right)$. Or Du Cange signale hardin(e)a. latinisation de l'ane. fr. hardine "gravier", dans des ehartes picardes de 1197. 1255, 134s (voy, aussi Godefroy). Ce hardine. s. f.. — d'où le w. hadrème par métathèse $\left({ }^{2}\right)$ reproduit manifestement l'ane. h. all. hartin (*hardin). s. f., "sol pierreux" "dérivé de l'ane. h. all. hart. hard, s. m., "sol de sable ferme " $\left({ }^{3}\right)$. Bien que chadrề à Roy (p. 133), soit synonyme de hadrène, on ne peut voir dans ee dernier un dérivé de hat', puisqu'il n'existe pas de suffixe -erène.

Reste hâdrène " avarie dans un toit de ehamme ». signalé par Simonon, Body. Voc. des courreurs (BSII 11. p. 158) et Forir. Ce terme nous est ineonmu et il n'y a guère de chance qu'on le retrouve en vie : les toits de chaume disparaissent des villages les plus reeulés, entraînant avee eux, malheureusement, les bons vicux mots du teroir. Je tiens la graphie - $\hat{a}$ - pour suspeete $\left({ }^{4}\right)$. Il faut éecire hedrène et y reconnaître ume aceeption spéeiale du préeédent : sur les toits de ehaume, les parties dénudées, suivant la remarque de G., rappellent le terrain picrreux, dépourru de terre végétale.

(1) Dans le Condroz (à Sarte-à-Ben, oucst de Huyy), j’ai entendu récemment hadrile (altéré de -ine), même signification.

(2) Comp. liég. tådrou "tardif", ponr *ti̊rdon ; ehaudrè (Awenne) : lićg. hi̊rder "ébrécher "; ard. tehèdron : lićg. tehèrdon "chardon"; tehèdré (Herve) : liég. tchèrdin "chardonneret".

$\left({ }^{3}\right)$ A. Carnoy (Hélanges Ch. Hoeller, pp. 308-17 ; Louvain, 1914) explique par le suffixe germ. -ina un grand nombre de noms de lieux en -ine, entre autres des nons désignant des terres arides et sc̀ehes: Wattimne, Custime, Dorime, Landeme, etc. ; voy. aussi Roland, Top. mam., PP. 519 et suiv. - Notre hadrène a sa place marquéc dans cette famille : il serait surprenant qu'il n'eût rien donné en toponymie.

$\left({ }^{4}\right)$ Cette graphic pourrait très bien n’avoir pour elle que le témoignage de Simonon, copié par Body et Forir. Aı surplus, la prononciation $\hat{\imath}, \grave{a}$, fut-clle même réclle, on l'expliquerait comme celle du lićg. migriyète, paivion, eråmignon (voy. p. 56), où à remplace un $a$ bref prinitif. 
w. hahîre gaum. a-hachière, hach'rôle, ctc.

1. Le Bulletin du Diet. iollon (1910. p. :20 : 1911. p. 78) a signalé, an Sud de la province de Luxembourg, alhathiere "estropié. percius. cache "s qui se dit surtout d'un hoitems. d'une persome qui marehe

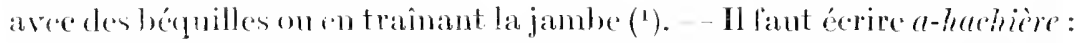
locution composée de la prép. "(lr. em. lat. in) et du subst. hachióre, anc. fr. hetsehiere (de lane. hant all. harmskara, peine, angoisse, tomment). Comparez le w. B-marminee "en marrimence", d'oil : "embarrassé, en suspeus". - Liane. Ir. huseliere ne s'emploie au Sul que dans eette rxpression : mais noms le retromvons an Nord sous les formes bien wallonnes halhere (Erezée). hachire (Cherain. Lutrebois). arec le sens de : "indisposition. légere maladie (épidéniques)" :

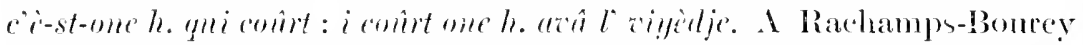

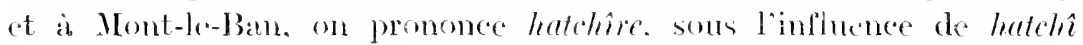
"tirer, tran̂ner".

II. Le même Bulletin (1910. p. 19) enregistre : acheraule (Virton : Cl. Mans), hutchirmle (Ronsignol). 1. dillicile à manier ; ‥ qui se remue dilfreilement n. - I a forme huteli rôle est. en cffet. signalée à Rossignol et i Chiny ; mais on y reconnaitra la mône altération que dans hatchôre ci-dessus. Ia forme pure est hach rôle. que jai entendue fréenuemment dans mes engutetes con pays gammais ( $\left.{ }^{2}\right)$ et qui se rattache d'elle-méme au subst. hachicere. Cette épithète sapplique $1^{\circ}$ à un objet encombrant. gênant [in arö tupin (vase) qu'est leteli"rôle]. ou dillieile à manicer [in

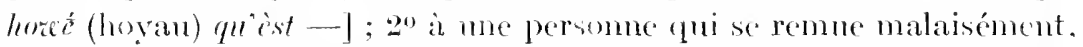
par suite du grand âse. d'm mal on d'une infirmité pueleonque. on par gancherie naturelle [e't - qu'il ist! i n' s't bomdii : eomme il est engourdi! il ne sait bouger ; il is en mon - - dit-on d'un culant qui marehe eneore

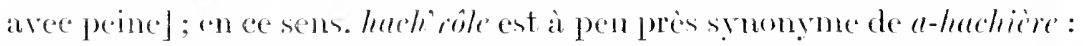
le promier semploie de préfépente pour marquer une gène momentané résultant dim aceident peu grave, on bien me maladresse due an temperannent de l"individul ; lautre se elit d"une allietion physique plus gratre et permanconte.

Le sullixe latin -abilem, lr. -able, clonne en gammais -ôle, qui

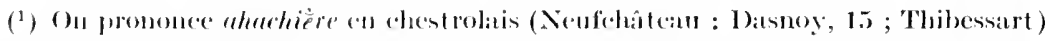

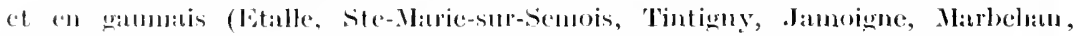

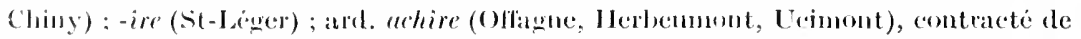
allachire.

(2) Marbedau, Buzenol, Ste-Marie-sur-Semois, Virton, Rnetle, Musson, Mussylat-lille, st-liger, ell. 
s'ajoute i des thimes rerhame (1) ct aussi it des thimes nominaux $\left(^{2}\right)$. Il exprime proprement une possibilité active on passive; de là. il peut marquer simplement la qualité et devenir synonyme du suff. - ềs :

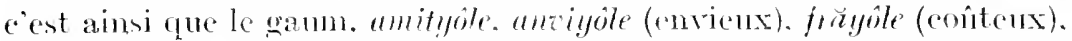

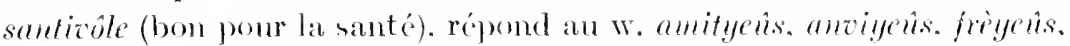
santiaens. Il nest donc pas étommant que hachiere ait produit $11 n$ adj. hachrôle, signifiant: 1 . (ohjet) yni eause de la gêne ; 2. (persome) qui épronve de la gêne. Le pendant. fon mé avee le suffixe parallèle -osum. mous est fourni par le menion hachurene " malingre, qui eroit diflicilement, cn parlant d'un enfant $"\left({ }^{3}\right)$, - Eu chestrolais. Dasmoy, p. 249. dome un autre dérivé intéressant : hach rizen r. caduc. cassé, maladif. faible" ; pour le sullixe -izents. conte. dans Dasnoy : baidizene, chai-

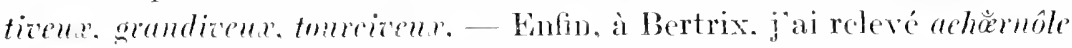
(syn. de siröpi. aflidii : estropié). qui devient uchămês à Offagne (II) 191:3. 1. 21). Jípenthese de n, formant le groupe -rn- kevant la tonique. est sams doute amenée par des raisoms d'amalogice.

[BD) 1913, P1. 98-101.]

\section{all. fi. hamestoc. w. halmustok. amèto}

Godofroy enregistre un mot hamesten quiil ne peut définir. Lexemple

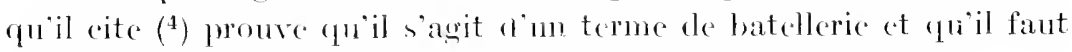
y voir le néerl. helmstnle " harre on timon du gourernail " $\left({ }^{5}\right)$.

Le vocabulaire du hatelier wallon étant, pour les deux ticrs, formé de termes nécrlandais. rien d"étommant quoon retrouve dams nos dia-

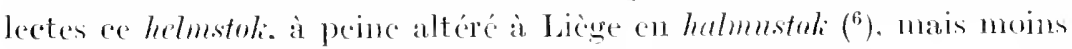

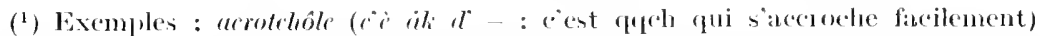
atrimetole (qui se met lacilemont a toute sorte de metiors sans les avojr alpris),

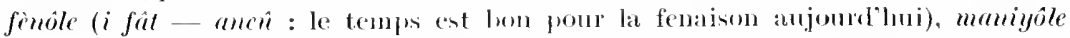

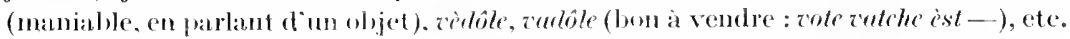
Tous ces clérives, rerueillis à site-Mlarie-snr-scnois et à sit-léger, sont inćdits.

(2) Exemples : udrèssôle, adroit ; amityôle, aimable : frăyôle, (personnc) dépensière, (chose) dispendicuse.

$\left.{ }^{3}\right)$ Cordier, Joe. du dép. de ln Mense (Paris, 1s8:3); Virlet, Dict. du pulwis mensien (Verdun, 1896).

$\left.{ }^{4}\right)$ Douai, xne siecle : les nes ki leveront leur euwages li signeur con puent oster te hamestoc et arienter le nef ('Tailliar, 1) 46it).

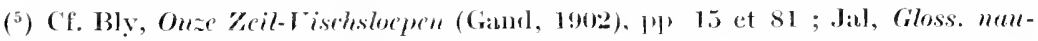
tique; Littre neatue :- Le's dict. Ctym. de Kluge, Fanck, Vercoullie distinguent helm (poignée, manche) de hrlm (castiuc).

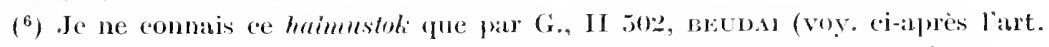
reâdé). On dit ordinairement hrmimle pour désigner la barre du gouvernail. 
facile à reconnaitre sur la Sambre dans amèto $\left(^{1}\right)$. M. Emile Ouverleaux m'écrit que dans le I Iainaut belge et dans le département du Nord on dit ausi aminto (et. avec l prosthétique. laminto) : -in-est iei, eomme en d'autres mots, um prolongement nasal de $-\grave{e}$ - dans la bouche des gens de Lille, Valencienne's et environs.

[Article paru en 1911, daus Romania, xL, 325. - Dejuis lors, j'ai vu que Behrens, Bciträge. p. 142, explique le fr. jaumière et le fr. archä̈que heaume (barre du gouvernail : Littré) par le nord. hjalm, all. néerl. angl. helme "poignée, manche, barre "; à la fin de son article, il cite le w. halmustok. Meyer-Lühke, $n^{\circ} 4102$, admet que ce dernier vient de l'all. helmstoek. La question est done tranchée sous ce rapport. Reste l'ane. fr. hamestoe, que Behrens, p. 130, voudrait expliquer par l'all. hemmstock "barre d'arrêt". Le donte n'est pourtant pas possible : le w. amèto (Charleroi, Thuin) et l'exemple de Tailliar (cité ci-dessus, n. 2) montreut clairement qu'il s'agit, ici eneore, de la "barre du gouvernail"; ham-est altéré de holm. Ajoutons que la souree est plus sûrement néerlandaise quallemande.]

\section{w. hamuslåde (Verviers)}

Lobet domne ce mot 'qu'il définit : "paillon, paillette de fer qui éclate en le (sic) travaillant ". G., I 270 , reproduit l'article sans explication $\left({ }^{2}\right)$. - On prononce à Starelot hamuslade. C'est l'all. hammerschlag. néerl. hamerslag "cuup de marteau; d'où : battiture, écaille qui se détache d'um métal sous le martean de la forge ". La finale s'est modifiée sous l'influenee du suffixe -ade. Pour la protonique -us-, comp. le malm. rômuslirith (G., II 3:3, éerit à tort -îrihe) "branle, danse populaire ", qui vient apparemment de Römerskirchen, nom d'une loealité de l'Eifel.

[Mélemges Kurth, 1908, t. II, p. 330.]

\section{gaumais handé}

Ce mot. qui ne s'emploie guère qu`au pluriel, désigne sur la Semois supéricure, notamment à Tintigny, "les linges des enfants au maillot ct. rarement, par extension, tous autres linges ct retements : alez

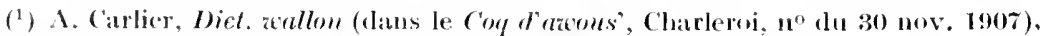
distingue cutre l'amito "tige rectrice mobile du gouvernail "et l'aminte (= liég. hamimle) "tige rectrice non mobile du gonvernail ". Entendez par lá que l'amèto est mue harre on poignce cui s'aliapte a l'mminte et qui peut s'enlever an besoin. benx batcliers de Thuin que jai interrogés ne connaissent pas rette distinetion ; ils appollent ameto la barre, et ralonge de l'ameto la poignee mobile que l'on tire et repousse it volonté en lat fatisant glisser sur l'ameto.

(2) Dans l'article de G., il fant lire "paillon " au lieu de "papillon". 
r'cude lès handés, allez recucillir le linge quion a mis à sécher; il è eo mins sès bons handés, il a eneore mis sès bons rêtenents " ('). J'ai noté andés "habits" a Alle-sur-Semois. Labourasse donne le meusien "andie, linge d'enfant, lange". et Jaclot le messin "haml'e, chiffon ". On ne trouve nulle part l'explication de ce terme, qui est un diminutif en -ellum. L’aspiréc initiale, caduque dans certaines régions, atteste une origine germanique, de même d'ailleurs que l'habitat du mot. Dès lors, le radieal ne peut etre que l'all. hemd (chemise), ancich frison hamede, dérivé do l'ane. 1. all. hamo "enveloppe" ("2).

\section{w. hanivêr (Neufchâteau)}

Le Dict. acellon de Dasnoy (Neufehâteau, 1956) est le seul qui donne: "hanizers, homme grossier, rustre, qui a le sens de travers, allobroge". J'ai relevé, pour ma part, anivêr à Alle-sur-Semois : in drŏle d'anizêr " un singulier persomnage". Dans cette région, comme en namurois, l'aspirée germanique disparaît ; mais elle se conserve en ehestrolais. Nous admettons done que hanizer est la forme exacte, la graphie -vers de Dasnoy provenant de l'analogie du fr. tracers, chers. - Or nous trourons, dans le Wörterbuch der laxemb. Mundart (Luxembourg, 1906) un article : hamnevir $\left({ }^{3}\right)$, adv. "hinten vorn, verkehrt" (= litt. derrière devant, à l'envers, de travers) ; s. m., Narr (= fou) n. C'est là, selon toute apparence, la sourec du mot wallon.

w. hårber, haurbè, haube, cte.

Famille intéressante, que tous nos dictionnaires passent sous silence. Voici les renseignements que j'ai pu recueillir :

haube (Awenne), s. f., haie, di spènes on d' fi dêrlè.

hảrbèye (Erezée), s. f., haie ; - l'aurbéye do couchèt (Lustin), petite enceinte de claies autour du ran (étable), où le pore peut prendre l'air et se remuer.

aurbeù (Ciney, Doriune, Vonêehe), s. m.. ouvrier qui s'oecupe des haies: dcs mofes (monfles, gants) di aurbeñ. [En Ardenne : clöyeñ.]

hårber (Erezée, Jurbuy), -1̂ (Scry-Alée), haurbè (Awenne, Narehe et Ileure-en-Famenne), aurbè (Ciney, Dorimne, Vonêehe), v. tr., enclore (un terrain) de haies : huurbè on pachi (Famenne : BSW „, p. 152) "enelore

(1) BSW 37, p. 337. Voỵ. anssi Bruneau, Enquête, I 527.

${ }^{(2)}$ On sait d'autre part que le bas all, ham-, hamel "arrière-faix " a donné le liég. ham lìte " coilfe que certains enfants portent à leur naissance"; ef. G., I 270, 357, II 604, et surtout Behrens, Bciträge, p. 129.

$\left(^{3}\right)$ Composé de hamcn (ałl. hinten : derrière) et de vir (all, vorn : devant). 
un pâquis" ; empl. alsol. : faire more haje, on même : la réfarer, en bouchant les trous : mats, dans ce lemier ats, on emploie miens le suivant.

rihârber (Erezéc), -è (Ortheuville), rihaurbè 'Iwenne), v. tr., renclore

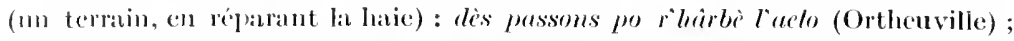

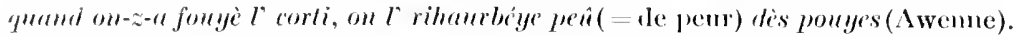

dihârber (Erezée), r. tr., légarnir (ım terrain) dỉ baies.

Les exemples aneiens dont nous disposons ne remontent qu'ì la fin duxre siede. En 1591. les arehives liégeoises parlent de "jardins

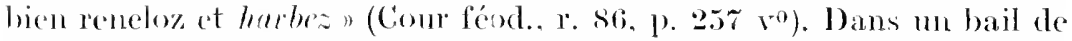
frrmage conelu en 1643 a Dinmot. il est questione de "jardins quon at aecoustmó de habber" et d'arbres qu'il fant "haber [ale peur] qu'ils ne soient gastés " (Sorvais, II ist. de Dorimme. p. 90: Namur. 1910). Une charte de Ciney, en 1586 , eite les "amendes de forehermwaiges et forharbnigess (") ; une antre de 1602 défend de "desharber jartins, encloz, preitz, paxhis et autres pieces de labeur. refermé des haies ou sor $1,(2)$.

Je n’ai ricn trouvé d’andogne ni dans les antres dialcetes wallons $\left({ }^{3}\right)$, ni en ancien francais. ni dans les langues germaniques qui se parlent an Nord et ì l'Est de la Wallonie. Un fait est eertain. Pour me raison de phonétique, heurbe ne peut itre de souehe vomane: $h$ latin aurait disparu. comme lans hippicem, ipe (herse) ; lat. se-, exs-, ere- ou

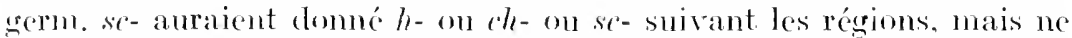
pourraicnt disparaitre sans laisser de trace. comme éest le cas dans aurbe. Il fant un li gemanique pour expliquer l'aspirée initiale. D'autre part, hambe est il humbe eomme banbe (liegs. babe : lat. harba) est is bankbi (barbiex) ; dianbe lieg. djabe. gerbe, provient de l'ane. h. all. garlad. On est donc conduit à proposer eomme seul étymon possible un type germanicfue *harba.

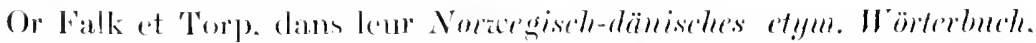

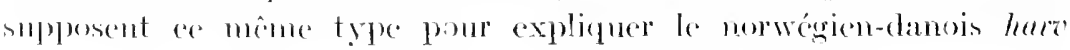

(1) .J. Borgnet, Carlulaive de Cimey, p. 125. Léditeur voit dans forharbaige "le fait de comper le loin ou de farie paturer sur le termin d"antrui "(!). Il fant com-

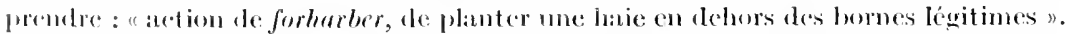

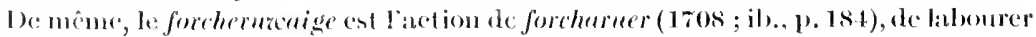

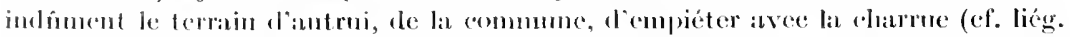

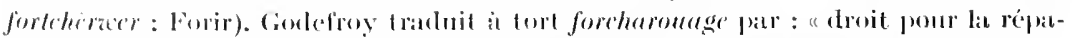

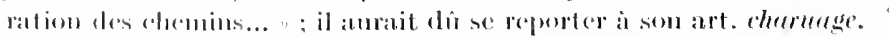

(2) Ihid.. 1). 152. Bolgnet explique deshurber par : "eomper les légumes" (!) ;

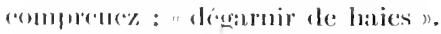

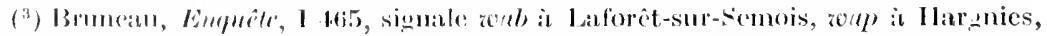
sinniliant " laibe $)$ II n’est gnère possible que ce soit notre not. 
" herse " (d'où est emprunté l'angl. harrow). Cette coüncidence appuie, scmble-t-il, ma proposition. I)u scms primitif "hersen dériverait sans peine celui de " claie. cloture, haie " (comp. le lat. crutes. le wall. hahe hahê, ance fr. harce hareel, hasean).

Sans doute une dillienlté subsiste : comment se lait-il qüil faille comrir si loin pour tronver un parent du w. haube ? *harba est-il venu directement de la Scandinavic lors des invasions normandes ? (1) Arait-il en Germanic. jusque prés de chez nous, des représentants qui ont disparu? En a-t-il cncore ailleurs? (2) Je dois laisser a dautres le soin de répondre à ces ruestions.

[Remaniement de B1) 1912, p. 99. Cf. Meyer-Liiłke, no 405t-6.]

\section{w. hatrê. anc. fr. ha(s) terel}

Ce mot désigne soit le eom (d'm lomme. d'm animal), soit une partie du cou : la gorge ou la muque $\left({ }^{3}\right)$. Létymologie reste obseure. Meyer-Lïbke, no foss. rejette — sans indiquer d'antre solution l'hypothèse de Diez (anc. h. all. halsâdara : veine du com. muque). (qu'il tient pour "plométiquement imponsible ", et celle de CrandgagnageBugge (ane. haut all. harst: breclese rôti), "invraisemblable pour le sens $)\left({ }^{4}\right)$.

Un fait certain. cest que le primitif est germanique et commenee par h. L'aspirée liégeoise. qui tombe en namurois (ntria. atia), suflirait à l'établir. L'airc géographique oriente anssi nos recherehes vers l'Est. - Cela étant, je propose de sadresser au germ. halte? "licoun "qu"on trouve en llamand. ch anglais, dane les parlers du Luxembourg. de l'Eifel de la Saxe ete.. et qui est une forme dialectale du haut all. halfter. nécrl. halster. Par dissimilation. I intérieur a disparn: *ha(l)ter-el $=$ haterl. La forme hastorel poiurait résulter de *ha(l)ster-el $\left(^{5}\right)$.

(1) Ce n’ost guère possible, ct puis le scamblinave le plus aucien a déja $f$ ou $v$, et non $b$.

$\left(^{2}\right)$ Un s. f. urpo (herse) existe en dialcete d'oc du Puy-de-Donne : Revue de phil. française, xxy (1912), 1.. :32.

(3) En ane. fr., cest gencralement la murue. Dans les patois walkons, c'est le com. A Neufchâtean, daprès basmoy, cont le fimon du bouf. - A Ellezelles, nateuriau (aver $u$ prosthétique) "murpue ".

(4) Et aussi pour la lettre. En effet herst a domnć lane. fr. hesticr, liég. hassti

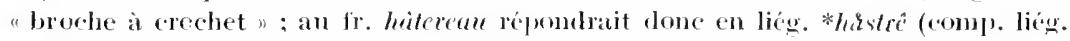
ulustêt : rouchi mutiau). - Sur l'otymologie de haterel, ou peut voir liez $61: 36$, 65 ; G., I 281 ; Romania, iv :360.

$\left.{ }^{5}\right)$ A moins que $s$ dans husterd ne soit adventice. - Halster se trouve déjà en moven néerlandais. Cette famille na rien de eommm atvec hels ni avec halle" ; elle remonte à lanc. h. all. halb "poignce, manche". 
Dans cette hypothèse, le mot se serait dit d'm animal avant de s'appliquer à l'homme. Mais comment de "licou" passer à "cou"? Denx roies sont possibles.

La métonymie d’abord. Grâce aux expressions fréquentes " prendre, tenir, attacher un animal par le haterel", la confusion entre "licou " ot " cou " pouvait se produire. Inversement, l'anc. fr. canole, chenole (* (annabula), qui signifie "canal de la respiration, trachée-artère ", puis " eom, nuque", a pris dans maint dialecte les sens de "lieou, tribart, gorge (espèce de porte-seaux). "te. "Au reste, col ct collet euxmômes désignent non sculement le cou, mais aussi ce qui garnit ou entoure le cour.

1) 'antre part, le diminutif haterel peut avoir signifié dès l'origine, non pas " (petit) licou ". mais "(petite) place ou partic du corps qui est en rapport avec le licou. où s'attache le licou ". Cette valeur du diminutif n'a rien d'insolite. Ainsi le liég. zondion (diminutif du germ. wand "muraille" + -illon) désigne l'insecte qui est en rapport avec la muraille, qui s'y cache, à savoir la punaise; comp. l'all. zeanze, zandlaus. Mieux cneore, l'ane. fr. oreillette signifie 1 . petite oreille; 2 . pendant d'oreille; ce dernier sens est aussi celui du w. orilièze et s'explique par l'ellipse : "petite [chose ayant rapport avec l'] oreille". Voyez aussi l'article gamite.

A Givet, l'atriyote (dérivé du nam. atria + -ot) est une partie du hamachement, la eorde qui se met à la tite du cheval $\left(^{1}\right)$ : un noureau sullixe diminutif a permis ce retour au sens primitif du radical.

[Romania, t. xur (1919), p. 180.]

\section{anc. liég. herbatte; w. èrbate (13rabant)}

La (harte de $152 \%$ relative au Métier des Drapiers de Licige preserit aux Evardeus (inspecteurs) d’aller aux wendes (rames, séchoirs) "depuis le grand Caresme juscu'à la herbatte chacm jour deux fois et depuis ladite licrhatte jusquan grand Quaresme une fois le jour " (Chartes ct Priviliges. I 241). G.. II 6os, avoue ne pas comprendre le mot lerbutte et se demande s'il n'a pas quelque rapport avec l'all. herbst (moisson, automme). Conjecture négligeable. La herbate était ainsi

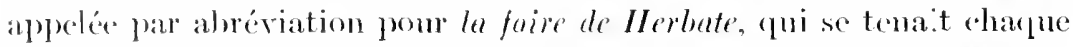
anné anx prés de II forbatte, vaste espace de terrain vague sous Namur pres de Malonne: elle avait lien en oetobre et durait quelques jours. bies le xure siecle, les produits de l'étranger, et surtout les draps,

(1) J. Wibslet, Vocab. ratlom (dialecte givitois). 
aflluaient à eette foire franche. Il y arait une halle particulière pour les draps fabriqués à Maestricht. Ilasselt. Liège. Huy, Dinant. Tournai et autres villes $\left({ }^{1}\right)$.

Le nom de lieu Herbatte s'employait done, par métonymie, pour désigner la grande foire namuroise. Le Brabant wallon en a eonscrvé le souvenir. Dans certaines localités de cette région, èrbate (sans aspiration, à la mode namuroise) désigne une foire partieulic̀re : ainsi, à Huppaye et à Noduwez, on appelle "l'erbate de Jodoigne "le marehé au bétail qui se tient à Jodoigne le jour de St-Lambert (17 septembre). Ailleurs, le mot n’a plus qu'un sens métaphorique, par exemple à

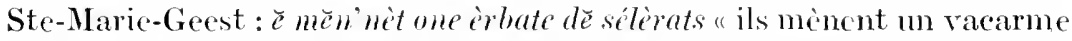
de seélérats ") d'une maison tapageuse on dit : c'è-st-me irbute ; l'aception de "foire animée et bruyante " ne survit plus que dans la eomparaison : c'èst eo pîre qu'ome èrbate ( $\left.{ }^{2}\right)$. A Aische-en-Refail (Liernu), l'èrbate désigne l'octave de la fète paroissiale.

Il resterait à expliquer l'origine du lieu-dit namurois Herbatte. Les premières mentions datent de 1192 : Herbata, ct de 1214 : pratum IIerbatarm, eontenant environ 25 bonniers et demi $\left({ }^{3}\right)$. Ce herbata ne doit évidemment pas être eonfondu aree le type latin qui a donné en namurois yèrbéye "herbes eoupées pour nourrir le bétail ". L'aspirée initiale, - disparue anjourd'hui en namurois, mais eertifiée par le texte liégeois de $\mathbf{1 5 2 \% ~ " l a ~ H e r b a t t e " , ~ - ~ a t t e s t e ~ q u e ~ h e r b a t a , ~ n o m ~ d e ~}$ lieu, est une forme latinisée d’un mot germanique. venu sans doute du Nord. Ce primitif pourrait bien etre *hêr-bate "le chetel seigneurial, le profit ou la dépense du souverain " (4), ì moins que - ee que j'ignore - Ia situation topographique ne permette de voir dans le seeond

(1) Cf. J. Borgnet, Recherches sur les aweiemmes fêtes nammroises, p. 48 (1854; t. xxvil des Mimoires conromés de l'Acarl. royale de Belgique). On lit dans les textes cités : dedens les 8 jours del Harbates "(XIV'e s.), "ceste Herbatte proehain " (1417), "a le Ilerbat " (1459); et plus souvent : "le feste Ilerbatte, le franeq fiest Ierbatte, le fieste a Herbattes, le fourre de Herbatten. - Voy. aussi un acte de 15\%1, eité lar Bormans, Le bon Mćtier des Drapiers de Liège : à Malone à la fore eondist Herbatte... audit Malone en la ville de Namure " (BSW 9, p. 229).

$\left({ }^{2}\right)$ Renseignements reeneillis sur place, de la bouche de M. Zénon Meunier.

(3) Miraeus et Foppens, Op. diplomatica, I 294; Borgnet, Cartul. dc Nammr I, 11.

( ${ }^{4}$ Borgnet, op. cit., p. 1\%, parle d'un édifice (la grange le Comte) "situé sur la petite Ilerbatte... et qui, dans l’origine, servait à l'emmagasiuage des provisions du souverain" (d’après les Comptes du domaine, 1355). - Sur le germ. bate, voy. Weigand, vo batten, Schuermans, vo bat. Le néerl. baat "protit, gain "vient d'une forme primitive à voyelle brève. 
eomposant le mot wallon bate. batte. symonyme bien connu de " digue, quai, bâtardean ". V'oyez ci-après l'artiele herent.

\section{lieu dit Hercot ( $\left.\mathrm{Hur}^{\mathrm{r}}\right)$}

Le bel onrage de R. Dubois sur les Rues de $I n y$ (1910) signale le Chemin de II ereot (vers 'lihange). qui doit son nom à une propriété voisinc, appelée dans les actes Herot, rarement IÍrieot. Malhemreusement l'auteur ne cite à l'appui ni texte ni date. ni même lappellation

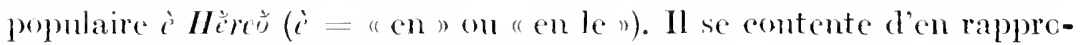
cher Florieot. nom d'me propricté de Tihange.

D’après Fellor, Notes, p. 3.t2, ce Floricot, terme assez fréquent en toponymic wallomne, correspond an nom de lien Verlorenkost. commun en pays llanand, et signific : "dépense perdue, terrain ingrat, où l'on perd sa peine ". Quant ì hireo, je présume qu’il se eompose de deux mots german:ques hêr-cost; le sens primitif serait : "le ehetel seigneurial». On aurait ansi le pendant dn nom de lieu namurois hirbate; voyez l'article précélent.

\section{w. hèrnale (lIự)}

Mot inédit de la région hutoise, qui n'est employé que daus une seule "xpression : la hemule di com (du eul) "le bas de l'épine dorsale, la soudure des deux hanches, juste au-dessus du cocerx " djé t' l'a upici

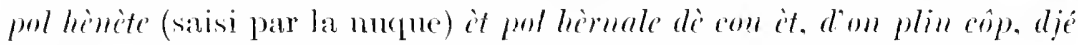
t'la hine "l'oulh (laneé à la porte). - Si l'on détache le sulf. -ale, fr. -elle. il reste un radical herh-. d'origine olseure. On ne pent penser ì

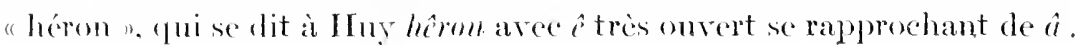
Je conjecture que ac mo" provient de *herviale par métathese (roy. l'article gonlete). Le suffixe est -erelle, comme dans bumliale (tumeur ; G., II 50.5), homprale (chomette : G., I 311), ete. ; comparez diermale, que noms supposoms issu de * dirmi rale et qui présenterait donc le même "as phonctique que notre himale. Le radical appartient a la famille hime "petit morecau de beis fendu ", hivlete "écharde ", himer "fendre". ()n salt que ce grompe dérive de l'ane. h. all. skzna, moyen h. all.

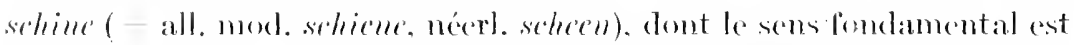

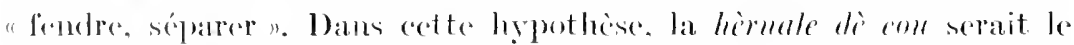

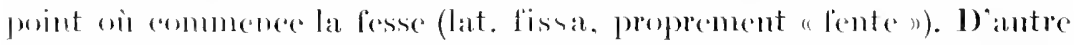

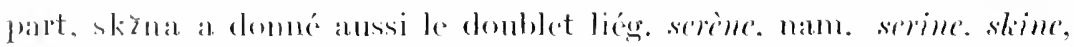

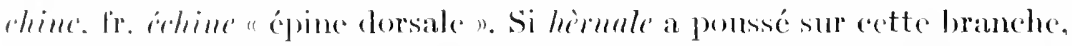
il désigne properement le point ou dinit l'échine. le bout (inf'éricur) de 
l'éehine, l' "*échinerelle" $\left.{ }^{1}\right)$. En somme, les deux sens de l'ane. h. all. skina légitiment l'ume et l'autre de ees interprétations.

Sans donte la phonétique pent élever une objection : sur le type de hin'lite, on attendrait *hin'rale. "himale : mais l'influence de $r$ a pu clianger $i$ en $i$.

\section{liég. heûler, må-heûlé}

G., I 293, donne d'après Dejaer "henler : aeconeher " (sens inusité aujourd'hui). Il pense que si "aceoucher " est iei transitif, héler dérive probablement du holl. heul "seeours, assistance". Nest-il pas plus naturel de prendre " aceoueher " dans le sens ordinaire, à savoir intransitif? De rieux Liégeois me signalent hểler "hurler lugubrement, comme font les chiens la nuit " $\left({ }^{2}\right)$. N'est-ee pas le même mot et le sens de "aceoucher " n'est-il pas dû à unc métonymie ? J'ineline à le eroire, d'après l'analogie de eertaines expressions pittoresques qui se disent de la femme en travail d'enfant : laccer (Herve ; propr. "abover "), brête as broeales (Dison), brêre as nozês harins ou as pouris pèhons (Liège) : allusion aux marchandes qui erient pour annoneer leur marchandise, des allumettes (broeales). des harengs frais on d'autres poissons "pourris " !

Il existe un autre henler. que nos lexiengraphes ignorent at dont je ne trouve de trace que dans un artiele du Vocubulaive des Courvenes par A. Body $\left({ }^{3}\right)$. A ee que m'apprend II. Jean Lejeume. e'est un terme arehaïque d'un de ees petits métiers que l'industric moderne a tués, le métier de platineni. "tôlier" on fabricant de platènes as dorîyes, de

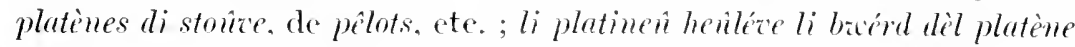
"le tôlier cmboutissait. travaillait an marteau et au repoussoir la plaque métallique pour l'arrondir en forme de bassine, de cassorole, ete." - Nous y verrons sans peine un emprunt de l'all. höhlen " ereuser, caver " (eomp. das Silher hohl schlagen : emboutir l'argent).

Ce heîler "emboutir " nous domne la elef de ma-henili "mal élevé,

(1) De même hiuon (Huy), ehinon (Ciney, Dorinne, Yvoir), skinou (Namur) "ligne séparative de champss forméc de gros huissons " parait se rattucher à ce sens de "échine".

$\left({ }^{2}\right)$ Comp. néerl. huilen, all. heuleu. Nos dictionnaires ne donnent que la forme plus usitée hoûler ; comp. moyen bas all. et moy, néerl. hûlen.

$\left({ }^{3}\right)$ Voici ect article : "poûhen̂, puisoir, ustensile conposé d'un bassin qui est une platine en fer heulé, embouttéc [corr. : heullège, emboutic], et d'une quene en fer plat avec croehet au bout „. BSW 11, p. 172. 
rustre" (1). que G.. II 55, voulait exphiquer par heûler "accoucher ". Il sante aux yeux que le sens propre est : "mal embouti ", d'où, au moral : "mal tourné. grossier". - Enfin nous rattacherons à ce terme technique le composé ihnler "enfoncer", commu à Huy dans cette phrase satirique : él a l' boke si gronde qu'on î̀ ihul'rut on proin d'amolicion! "il a la honche si grande quon y fourrerait un pain de munition!n

\section{liég. heûre (fr. hure) et dérivés}

G. I 293, cite les expressions liégeoises : 1. avu (ou prinde) ̀̀ heûre "aroir (ou prendre) en gripje" : 2. i m' prote hêue "il me porte malheur. je ne puis le souflrir " : 3. quivi heîre "chercher noise ". De ces expressions archaiques, la seconde est sans contredit la plus vivace (2). On la trouve dans le Théâté liégevis du xvine siècle

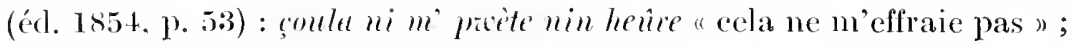
dans Simomon, pp. 138 et 181 : èle mi pwite hềre "elle me chagrine "; dans Bailleux, Fâes, 1856. p. 53 : çoula m' prite hêtue "cela m'inspire de l'éloignement, cela me met en défiance "; dans Hubert, p. 107 : prierter helue "inportuner, tracasser". C"est la seule aussi que j'aie relevée dans le parler moderne : ia-i- fon d' ehal, ti m' proites heûre!

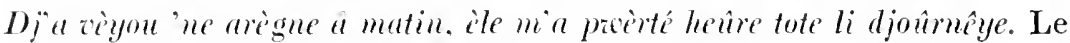
sens est ici : "porter guignon".

G. ne donne pas d'étymologic. Pour expliquer paìter hêre, Simonon, l. c.. invoque le fr. lemert. tandis qu'Altenburg. I 16, propose l'anc. h. all. seùr (all. mod. Schaner : giboulée). J'y reconnais, pour ma part, un emploi figuré du liég. heîre "hure" $\left({ }^{3}\right)$. L'expression "porter hure ¿ qqu " est anssi logique que l'ane. fr. porter bon zisuge à qqu "lui faire bon aceneil " $\left({ }^{4}\right)$. Le sens premier est : "faire manvais accueil ", d'où : "inspirer de l'éloignement, de l’inquićtude. de la défiance, de l'effroi ", et enlin : "porter gnignon ». - L'expression "avoir (ou prendre) en hure "se comprend sans peine. La dernière ("quérir hure "), plus rare

(1) "Mal embouché, grossier" (Duvivier) ; "morose, bizarre, morne, misantlirope "(Inbert); "malintentionné " (Forir). Manque dans Remaele et Lobet. La forme mâthúlé est dans unc pièce de lamonx, euré de Glons, mort en 1826 (Choix, 1.. (93).

$\left.{ }^{2}\right)$ C'pridant Remacle, 20 'd., dome seulement 1, et Forir seulement 1 et 3.

${ }^{(3)}$ Iorir, II, 1.21.-- On sait que le fr. hure représente un type * hûra, d'origine inconnue.

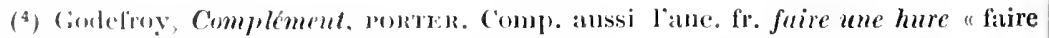
une mine saurigen. 
d'ailleurs et moins correcte que les deux autres, résulte sans doute du croisement de quèri miáre et de pwirter heùre.

On aura remarqué l'absence de l'article, qui dénote l'origine ancienne de ees locutions. Il en est de même dans lonki po d’as höre (Stavelot, Cherain : BSW 4t, p. 5:37; 50, p. 5:31) "regarder par dessous hure ", c. à d. "en dessous, sournoisement" ( $\left.{ }^{\mathrm{l}}\right)$.

Le mot n'existe plus guère à Lìge $\left(^{2}\right)$. Il est mieux conservé au pays du sanglier, dans nos Ardennes, où nous relerons : hure (Malmedy), houre (Cherain, Ortheurille), heure (Villers-Ste-Gertrude. Erezée ; en Famenne et en pays gammais). Il y prend d'ordinaire le sens figuré de "mine renfrognée» : faire une (laide) hure. Spécialement, de deux vaches qui penehent la tête d'un air menaẹant en se portant l'une vers l'autre, on dit à Villers-Ste-Gertrude qu'elles font lìs heures. Enfm, en pays gaumais, heure a le sens ironique de "tête, eerrelle" : il è ène heure du bû (il est têtu comme un bounf); i n' su mèt mi ça dès la heure, $i$ n'è m' ça a la henre (il ne se met pas cela dans la tête, il n'entend pas de cette oreille).

Le mot hure, en raison même de sa force expressive qui devait plaire à l'imagination du peuple, a procréé, surtout dans les dialectes, une lignée nombreuse qui mériterait d'être étudiée de près. Je ne puis aborder ici l'étude complète de cette famille pittoresque. Tout au plus trouvera-t-on, dans les notes suivantes, quelques faits nouveaux concermant le français et les dialectes septentrionaux.

Amuri, en anc. fr. : " (qui a une chevelure hérissée " ; en fr. mod. : "troublé \#. De même le liég. ahuré, dans un texte de 16:31, et le ronchi ayuri (roy. BD 1902, p. 97). - Le gammais ahemèy (ibid.) "étourdi, écervelé " et aheuray "entêté " (à l'est de Longwyon) représentent plutôt un type *Exhurí.

Huron, ane. fr. : "personne à la tête hérissée, à l'aspect sauvage"; d'où, en fr. mod. : "individu grossier et malotru ". [Sur un autre w. huron, voyez la note à la fin de eet article.]

*Hureux, *inurir. - Le liégg. hourevis " se dit de celui quui souffre du froid, de l'humidité, surtout des oiseaux lorsqu'ils hérissent leurs

(') Èle mi réle po d'as s' houre (Ortheuville), po d'zos sa heure (Lavaeherie); louki po d'zos l' houre (Buret-lez-Ilouffilize).

$\left({ }^{2}\right)$ Signalons iei l'expression arehaïque hure de pierre, t. de honillerie qui signifie "le roeher, la pierre même " (G., I 317 ; Bormans, Voc. des houill. liég.). G. compare l'ane. fr. heurt (roeher, tertre); mais l'analogie de houra ou houreye di pire (voy. ei-après) et de vizidje di pire, t. modernes de houillerie, montre qu'il s'igit bien du fr. hure. 
plumes" (G.. I 313). Il signifie "frileux". mais désigne un état acei dentel. passager. tandis que fronlens (= lat. frigorosus) marque une disposition habituclle: dij so si homrenss ony. dji n' mi rilinoh nin, ca dii "'so min frohlen.s. On dit aussi : i fêt hourens ony (il fait aujourd'hui un froid noir. un temps humide et froid) ; mais on ne dira jamais : i fêt tronilểs. - De même, en chestrolais, hörềs ( $\left.{ }^{1}\right)$ - G., I 314, invoque le lat. horrere (!) ; plus loin, II xxxr, il rattache houretts à homri "frissommer, grelotter". Ce verbe. recneilli par Simonon. ne se trouve dans auem autre de nos lexiques ( $\left.{ }^{2}\right)$. Je l'ai, pour ma part, entendu à Jupille, où homri est synonyme de fruzi (frissomner), et à Alle-sur-Scmois, où ări signifie "frémir, aroir un haut le coeur " : die üri tout! - Il faut rattacher ces mots au fr. me et à l'ane. fr. hmerer "hérisser sa crête. ses cheveux". Le verbe hon i, a terminaison inchoative. signilie proprement : "(eommeneer à) se hérisser " : rous voyez eelui qui homrih. les cheveux hérissés. la tête baissée et rentrée entre les ćpanles, les bras serrés contre la poitrine ; le frissomnement n'est qu'une circonstance aceompagnante. Le fr. almirir recèle une image analogue : celui qui est ahmri a l’air de s’ébouriffer de surprise. De même homents signifie an propre: "qui a la tôte hérissée "; il fait songer aux oiseaux soulfrants dont le plumagge s'enfle et s'ébouriffe. Appliqué à la température. il prend le sens de : "qui rend hérissé "; comparez i fit malade. il fait un temps à vous rendre malade, une chaleur étouffante $\left({ }^{3}\right)$.

*Iukand. - Liég. lmra "trogne. mine refrognée" (Forir) ; fé in sbaré hura "faire une mine cfarée" (Simomon. pp. 54 et 181). — Nos honilleurs, à Seraing notamment, appellent houra d' pire un bloe de pierre qui fait saillie et qui menace de tomber; ș̣n. honrêye di pîe. Comparce hure de pieme. p. $147,11.2$.

*Herard. - Le liég. lurr "hure de sanglier" n'est attesté que par Forir ("). De là sans doute le nom de famille IIurard (Liège, V(rviers).

(1) Dasnoy, 1). 256, écrit : "herreux, frileux ; froid pénétrant ". - A Forrières, li houreñs - la bise de mars.

(2) Voyez la note à la fin dle cet article.

$\left({ }^{3}\right)$ Compurez hompients "frileux, qui se tient tout ramassé patr le froid " (Liège : Forir) ; crmfiêss "lowsu "(Liege), "frilenx " (Cherain, Rohertville), proprement "ramasse (n boule, recrogueville comme si on avait une bosse sur le dos". Ces anjectifs derivent de hompe (homple, hupe) et de croufe (bosse) par lintermédiaire

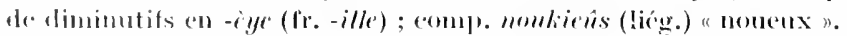

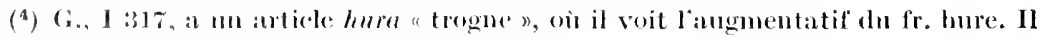
laut donx apparemment lire hrori et non $-\check{a}$. 
*Hurasse. - Wall. hourase (Cherain, Buret-lez-IIouffalize), f., "ehevelure épaisse et hirsute"; syn. tignusse.

Hurer. - Ane. fr. hurer, heurer "hérisser la crête, les eheveux" (God.) ; messin heuré "qui a les cheveux hérissés" (II. Lorrain). J'expliquerai de même l'ane. fr. hower, dans : "dez arrestez [de paille d'orge] qi hourent les bouehes dez ehivalz ». Godefroy, qui cite ce texte du xiIf siècle, traduit inexactement par : "déehirer". - Une description de la dîme de Hensy-lez-Verviers, en 166\%, énmmère des prés hurant sur tel chemin ( $\left.{ }^{1}\right)$. c.-̀̀-d. situés en contre-hant de ce chemin. Ajoutez le w. hourer (Cherain). r. intr., en parlant des raches. même sign. que ci-dessus fé lès heures : et le gaunais su heuriy "se ruer tête baissée (sur un adrersaire)" $\left({ }^{2}\right)$.

Hurée, participe du préeédent employé eomme substantif au sens général de : "une hérissée ". On troure ainsi 1. l'ane. fr. heuree (faire une h. ; dans Chastellain, xve s.), que God. traduit par "révolte "; 2. l'ane. fr. hure ("revers d'un ehemin ereux". dans les chroniques de Froissart et de Molinet; voy. God.). Aujourd'hui encore, le w. hourêye (Seraing). -éye (IIuy), hurêye (Liège). uréye (nam. ; rouchi) désigne le bord d'une route plus élevé qu'elle. un tertre. une éminence $\left({ }^{3}\right)$; 3. heuréye (w. ehestrolais. Dasnoy. 159) "toulfe, trochée, faisceau de pousses (de pommes de terre. de lleurs, ete.)" ; - t. huréc (Rethel : Ard. fr.) "nuce. averse" ; au mase. hewés "enmulus, gros muge noir " (mensien: Labourasse, chamut): onrée (rouchi) "ondée" ( $\left.{ }^{4}\right)$ : aeception figurée se rattachant au sens ?. Comparez le gaum. horlé "monticule", à côté de horlâye "averse ". et le mensien huôme 1. "tas de terre. petit montieule ". 2. "nuage en forme de monticule" (Varlet). De même à Viesville (Hainaut), une nuée. une ondée passagère s'appelle un tacha, e.-à-d. un petit tas (*tassia) ; - 5. le verviétois hourêye possède, d'après

(1) Bull. Soe. vere. darchéol., XI, pp. 2:39-240(Verviers, 1911): "Derrier le Hougne huraut sur le voye de Jehanster, la waide Collas... ; la haye voisinne huraute sur la cornette en haut... ; Devant la Hougne, hurant sur le grand ehemin... la waide Lina Melen..."

$\left({ }^{2}\right)$ Le meusien heursé, hirsu, hourse" (" hérissé "), se heurse'r ("se rebiffer, se hérisser neontre qجn), à Rethel hourissie, hurissie (" frissonner "), a subi évidemment l'influence de hure, huer. Cette même influence me parait nécessaire pour expliquer l'aspirée du fr. hérisser. On tronve en ane. fr. hurucier à côté de hivecier, hevicier.

$\left({ }^{3}\right)$ Les conjectures étỵmologiques de G., I 318, de Sigart, p. 2212, de P'. Marchot, Revue des lang. rom., 1891, t. 35, p. 4.40, nont aucune valenr. C`est biez (jui le premier a rattarlié hutée (berge) à hure. - Comp. ci-après l'anc. fr. hurel.

(') J’ai aussi entenru à Lantremange-lez-lVaremme le w. hourêye "averse , syn. nûlêye, houssê. 
Lobet. p. 25.3, des aceptions singulières que je ne trouve nulle part ailleurs et qu'on pent résumer comme suit : 1. avalanche, éboulement ; 2. poussée, effort d'une foule qui pousse. Le sens 1 peut se ramener à hourêye berge, talus; le sens a à l'ane. fr. heurée, ruée, loggarre, révolte.

Le type nuET me parait avoir subsisté dans le nom de famille bien comnu. C"est lui anssi que je recomnais dans le fr. houret (d'origine ineonnue: Dict. gén.) "mauvais chien de chasse ", et dans le pie. houret (Jomaneoux) "petit domestique de ferme". Pour le sens, ee serait un enfint, un petit homme ou animal à la tète hérissée, à l'aspect sauvage; comp. hurom et, ci-après, hurel.

Huretre se retrouve, à mon sens, dans les deux cas suivants : $1^{o}$ hourete (w. de la Famenne), urite (nam. : G., I 318), ourete (w. de Barvanx-Condroz; rouehi et pieard : Sigart, Héeart et Jouancoux, qui ćerivent hourette). s. f., "bourrée, sorte de fagot", ainsi nommé d'après l'aspect hérissé qu’il présente. Rattachez iei l'ane. fr. houreste, que Godefroy ne peut définir. - 2o hourite, hurète (ard. : G., II 538), heurete (Chestrolais : Dasnoy, 370), hourite (gaumais, meusien), horite (Ste-Céeile, Izel : Brumean, Enquîte, 1 185), s. f., " ehonette". Le liégreois dit houlote (a Stavelot, Vielsalm : houlete) et le franç is a hulotte. qui serait proprement picard (Rolland, Fume pop., IX, 72). G., 11 538, dit que l'ard. hourite = honlote, hulutte, ce qui est possible. Selon Diez 336, le li. hulotte viendrait de huler, forme ancienne de hurler avec influenee de l'all. heulen. Mais le w. houlote, à côté de hoûler, contredit cette dérivation, qui est pourtant admise par Meyer-Lübke, $n^{\circ} 9039$, non sans réserves : "L'ane. fr. huler, dit-il, est la torme piearde de hurler et non le m. h. all. hiklen; d'autre part, le tr. hulotte pent aussi venir du lat. ulula inflnencé par hurler plutôt que par l'a. h. all. hucila ". Pour le Dict. gén., hulotte paraît se rattacher à l'all. enle. altéré sous l'influence de huer. On voit que la question est loin d'être tranehée et que la voie reste ouverte aux conjectures. Pour ma part, je serais tenté de dériver tous ees mots de hure. Le groupe homite, lä̌rite, horite ne présente ancume difliculté ( $\left.{ }^{1}\right)$. Le gronpe hulotte, houlote, honlite serait altéré directement de *hurote, etc., a moins qu'on ne parte de la forme hurlote lumelote, qui existe dims le Pas-de-Calais, la Somme et la HanteHanne ${ }^{2}$ ) et (pui représente un domble diminutif nurelote.

(1) La härile hoüle (la chouctte hurle, crie), dit-on en chestrolais; il ne peut exister de ripport d'origine entre ces denx mots.

${ }^{(2)}$ Rollind, F'mue pop., IX, 72. A noter, p. 73, les formes : houran (Vosges), chahouran, chatheran (Lorraine, Champlagne), chaturon (Aisne). 
*Iuratlle, collectif de valeur dépréciative, expliquerait le pieard heuraillis "bruit confus et tumultueux" (Jouancoux) et le fr. hourailler "chasser avee des hourets", houraillis "meute où il y a beaucoup de hourets" (Dict. gén.).

*Huru (?) : norm. héru "hérissé " (Corblet) ; comp. hurelu.

Hures, type très répandu, dont le sens général est : "petit (individu, objet) hérissé ". Il revêt trois acceptions différentes que nous arous déjà rencontrées dans ce qui précède : $1^{\circ}$ l'ane. franç. hurel (dëfni : "bouffon", par God.) ; c'est proprement un enfant ou un homme qui a les cheveux hérissés. - $2^{\circ}$ L'ane. franç. huel "levée d'un. ehemin" (God. ; Reims, 1431) survit dans le w. ouria (Givet) "talus, terrain en pente, peu étendu) et dans le diminutif hourlê (w. de Comblain-Fairon, Wellin-St-Hubert), horlé (chestrolais, gammais) "talus d'une route ou talus séparant deux pièees de terre " : type *uUreseat. Conp. ei-dessus l'anc. frane. huree. - $3^{\circ}$ Le rouchi ourian (Hécart: houriau) "sorte de fagot"; comp. ci-dessus hourète. L'ane. franç. hourel, défmi : "osier?" par God.) et l'anc. franç. hurel (non défini par Gorl.) se rattachent assurément ici : il s'aggit du petit fagot nommé bourrée ou cotret. - De là, les dérivés : houreler, qui signifie "mettre en fagots", dans ce texte: "houreler et copper au ferment ung bonnier de joisne buis" (Lille, 1445), et : "gans hourlois" (Lille, 1596), co-a-d. gants spéciaux qu'on met pour faire des fagots et aussi pour réparer les haies. Tyjes : *hureler, *hitreloir ( ${ }^{1}$ ).

Nous rangeons en outre dans la famille de ce diminutif :

*Hurelée : gaum. horlâye "averse" ; voy. ci-clessus hurée 4.

Hurelote: pic. hurlote. lumrlote "chouette "; voy. ci-dessus hurette 2; - meusien hourlot "hanneton" ; voy. ci-apres hurelon.

Hurelin: le sobriquet messin hurlin "qui a les ehereux hérissés".

HURELARD : picard hurlud ou hulu, s. m., "harle huppé »; e'est à sa huppe comparée à une hure pue cet oiseau doit son nom (Jouancoux).

Hurequ : outre le pie. huelu quion rient de voir, il laut ranger ici le fr. hurluberlu "personne extravagante, brusque, étourdic". Le Dict. gén. dit que l'origine en est incertaine ; Schelor y voit une onomatopée, Littré un mot de fantaisie. Pour moi, e’est m composé dles deux adjectifs : hur(e)lu (qui a les cheveux hérissés) et berlu (qui a la berluc). Cela peint à merveille la tête hirsute et l'ail hagard d'un individu mal équilibré qui se jette inconsidérément à travers tout $\left({ }^{2}\right)$.

(1) Godefroy traduit houreler par "tailler "; il laisse hourlois sans traduction.

$\left({ }^{2}\right)$ En chestrolais, à Neuvillers-Recogne, in hurluve : " un hurluberlu ". 
IIUzELON : picard hourlon, heurlon, hurlon, gaumais hourlon, -an, "hamneton ". Meyer-Iübke, no 9039, rattache ce mot au lat. ululare (hurler). de même que le fr. Inlofte (chouette), comme on a vu plus haut. Je crois que l'hypothèse hure est pour le moins aussi défendable. Si le hamneton fait entendre un bruissement quand il role, pent-on dire qu'il hurle? Au point de voue phonétique, il y a de plus divergence entre hourlon et le pieard heuler (hurler); de mone le meusien humblon (hanneton), que j’ai noté près de Longwyon. en même temps que hurlay (hurler), hä̀rau (hurlemr). Enfin. eomparez hurillon ci-après. - Varlet dome le meusien homlot (lameton) et Jaclot le messin heulo (hanneton; tourbillon de vent). Ce second sens pourrait ctre invoqué en faveur de l'hypothèse "hurler" ; ecpendant. ne serait-ce pas aussi bien un "comp de vent qui vous ébomriffe"?

Hurillos. - Ce type apparaît $1^{0}$ en ronchi : hurion, hurlion "hamnetou ", d'après Hécart qui l'explique par "me onomatopée du bruissement que cet inseete fait en rolant ": hypothèse que l'on jugera moins vraisemblable encore que pour hourlon; - 20 en ane. fr. hurillon. Godefroy traduit ce mot par "sauterelle"; mais il y a méprise. Il eite un seul texte : "La vine plaie d'Egypte sont loeustez, e'est-ì-dire laoustres et hurillons " (Valenciemnes. xve sièele). On doit évidemment mettre une virgule après laoustres et comprendre que la vin plaie d'Egypte "sont sauterelles et hannetons" ( $\left.{ }^{1}\right)$. - $3^{\circ}$ Dans nos Ardemes, à Erezée, j’ai noté l'expression : il a r'çù dès bês hǚrions "de beaux coups ". J'y vois le sens propre de : "coup violent de denx têtes qui s'entrechoquent" (2) ou de : "comp violent qui rous enfle la tête" $\left(^{3}\right)$. Il y a là une indication intéressante pour expliquer le fr. horion, dont l'origine est incomme Les plus anciens exemples montrent clairement que horion désigne un "coup porté à la tête" ; d'où. an figuré, le sens de "gros rhume ". qu`il a en no"mant. Ce serait une forme dialectale pour hmillon. Voy. Godefroy, moniox 1 et 2 ; Diez, p. 616 ; Scheler, ete.

P.-S. - Ce qui précòde. sanf de légères modifications, a paru en 1919 dans Romania, t. xur, p. 1st. Depuis lors je me suis convaincu que deux autres groupes de mots wallons, dorigine contestée, pourraient avee avantage rentrer dans notre liste.

(1) Comp. dans God., Laouste, un exemple du Lib. Psalm. : "Et locouste et haneton lindrent sans conte, at grant foison ".

$\left(^{2}\right)$ Comme les coups que se portent deux vaches qui "font les hures vou qui "se hurent "; voy. ci-dessis.

(3) Comp, lespagnol hura, abeès, enflure à là tête. 
A eôté de houri "frissonner " (p. 148), il existe en ardennais un autre verbe intransitif honri (Malmedy, Jalhay, Sprimont. Lirezée), hori (Stavelot) "s'abriter (contre la pluie) ". sỵn. s' mite a hourisse (Erezée), a houriche (Cherain. Houflalize), a ouriche (Ortheuville), a oriche (Bande); dérivé homriha (Erezée). s. m., "moyen de s'abriter ou d'abriter qqeh". syn. abatou "appentis". - A première vue, on pense ì l'anc. h. all. scûra (mod. Scheuer). "pui a donné le w. heûre "grange ", et G.. I 305. ne manque pas d'invoquer ce type germanique pour expliquer le liég. si horer "se garer" ${ }^{1}$ ). Nais la phonétique ne permet pas de confondre heîre ("grange") et hmeri : ì Faymonville, où l'on dit $\chi^{\ddot{\alpha} r}$ " grange », on prononce honri (et non yonri) : de plus he est cadue dans certaines formes du Sud (muriche, miche). Je crois done que ce houri est formellement identiclue à houri "frissonner "; il a seulement revêtu une acception figuréc toute diflérente : "se blottir ou s'adosser contre une haie, un buisom, qui forment eomme me hure au-dessus de la tête \%. Le dérivé hourisse. -iche répondrait ì un type nurss, fém. -isse, ou serait le dérerbal de nurrir.

C'est aussi. je pense. un rejeton de hure qu'il fant roir dans l'ard. huron (Forrières), honron (Laroche) "gros glaçon " on micux "gros cube de glaee ", dont le sommet fait saillie sur l'ean. On lit hurou dans Jean de Stavelot (2), mais ce pourrait ittre une erreur pour huron $\left(^{(3)}\right.$. Quant à hèrô (IIuy), hèrau, huran (G., I 289). hixô (Méry-sur-Ourthe ; Liège : Forir), qui a le même sens, on pourrait adnettre que e'est le même mot altéré par influence de hirî "déchirer" ; mais c'est plutôt un mot différent. dérivé de ce hirì et signifiant " débâciè " ( $\left.{ }^{+}\right)$. qui s'est substitué daus le Nord à hourom "bloc de glace ", alors que ee dernier a survéeu dans le Sud. Il eonvient de remarquer que l'initiale de

(1) Si horer n’a rien de commun avec notre houri ni avee le germ. seu ra. Il s’agit d'un emploi figuré de horer 1. creuser au moyen d'une hore (m. 1. all. sehor, pic), 2. drainer, éliminer les eaux. Comp. sêwer (exaquare) et si sèzer "s'esquiver". Sur hore, voy. BD $1914-19,1$, 95.

$\left({ }^{2}\right)$ " Et quant ilh relingnat, les hmouz des glachons furent si hisdeuzement grans, et eressirent si grandement les aiwes, "qu'il habatirent le pont de Gemeppe..." (éd. Borgnet, p. 113).

${ }^{(3)}$ Comp. hongnete (J. de Stavelot, P. 190), qu'il faut surement lire hougnete. De même zuendićs (id., p. 190) = acuulićs (vidés), conme le prouve zeudurent (p. 191).

$\left({ }^{4}\right)$ A Stavelot hirô signifie "rupture de lil glace, débâcle ": hirôder "se rompre:

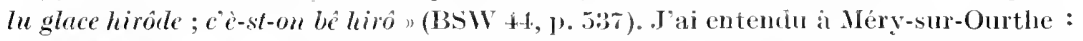
n-a lêre qui hirôléye. "A présent, me disait le viemx plasseur d'eau, il n'y a plus guère de hirôs (gros glaçons), ils se brisent contre les barrages ", Voyez ei-après les articles sizin, trèssìrin, et G., I 289, où l'ordre des sens doit être renversé. 
houron à Laroche ne peut s'expliquer que par un $h$ germanique ; scgermanique ou latin y domnerait ch (voy. l'article horon).

\section{w. horon}

I)'après les dietionnaires liégeois (Cambresier, Remaele, Hubert, Forir, Dnvivier), horon signifie "madrier, planehe épaisse de chêne ". A Mamedy, Starelot, 'Trembleur, Neurille-sous-Huy, ete., on entend par là une " losse, la première et la dernière planche d'un tronc qu'on refend "; tel est aussi le sens (que j’ai noté près de Iouffalize ('), oì l'on prononee choron. Enfin, d'autres auteurs (Lobet, Body, Rouveroy) donnent les deux acceptions. - Pour G., I 305, horon appartiendrait à la même famille que le terme de batellerie hore (= fr. écore, de l'anglosaxon seore). Sans doute. l'ancien liégeois xhorm et l'ard. choron postulent un primitif ayant sc-à l'initiale ; mais la suggestion de G. parait des plus contestables. Je préfère invoquer un type *ex-eor-onem. dérivé du lat. cor "ceur "à l'aide du sullixe dimimutif -on $\left(^{2}\right)$. Un horon, cest, suivant le point de vue, ou bien la croûte, la partie extérieure détachée du trone, de façon qu'il reste l'intérieur ou cocur de larbrc (une dosse), ou bien une partie tirée de ce cour même (un madrier). Ainsi s'expli(querait l'hésitation ertre les deux aeceptions traditionnelles.

\section{liég. hotche, hotchì}

G., I 300-1, traite séparément hotche "cosse, gousse" et hotchi "casser net". Plus loin. II :319, il refait sur hotche un artiele plus nourri, sans aboutir à une solution satisfaisante: il y voit le fr. (ć)cosse, ce qui est phonétiquement inpossible. Pour hotchî, il ne donne rien de sît $\left({ }^{3}\right)$.

(1) A Bonnerue et à Buret. La prenière planche après le choron s’uppelle li arvèzine, puis viennent lès plantches dè milan. - A Cherain, on prononce anssi choron; à Laroche horon.

$\left.{ }^{2}\right)$ Pour le radical, compl, l'unc, fr. coral "caur de chêne"; w. corâ, t. de houill. "bojs de renfort placé dans certains boisages " (a Seraing; (f. Body, Voc. des Charrons, vo ábon). - Pour le sullixe, comp. le w. et anc. fr. coron (bout), dérivé de l'anc. fr. cor, coru, w. czoèr, lat. cornu . - X IIalleux (d'ilpres Body, ibid., vo horon), on dit horion, aver un sulfixe -ion, fr. -illon; voy. ci-dessus une note à l'art. cakìló.

${ }^{3}$ ) Il renvoie au l'r. escocher, t. de boulanger, "battre la pâte avec la paume de la main pour en former une seule masse ". Ce not est elans Littré, mais je n'en tronve l'explication nulle part. - M. Semertier, I'oce w. dhe bonlanger (BSW 34, 1) 268), attribue par crreur au w. holchi le sens de ce fr. escocher; il a ual compris Grandgagnage; cette alcecption est en réalité ineonnue. 
Dans l'ane. fr. escouchier, fr. éconcher $\left.{ }^{1}\right)$, M. A. Thomas a reeomu le lat. *exeuticare (de ex et de cutica, forme allongée de cutis "peau, écoree »). Ce type se retrouve dans l'ane. fr. eskolice "briser, rompre" et dans le pieard écoquer "casser". Nous allons voir qu'il rend aussi parfaitement compte du liég. hotchî.

Le sens premier "dćeortiquer" subsiste $1^{\circ}$ dans : hotchî dès pen̂s, dis féves "éeosser des pois, des fíves ", expression que nos dietionnaires ne connaissent pas $\left({ }^{2}\right)$. mais que je tiens de vieux Liégeois; $2^{\circ}$ dans : dji u' l'i a nin hotchî $\left({ }^{3}\right)$, proprement : "je ne le lui ai pas écossé, je le lui ai fait manger hotches èt tot (avee les eosses) ", d'où, au figuré : "je le lui ai dit sans adoucissement, sans préparation"; comp. le fr. " ne point mâcher une ehose à qqu ". - De là, le déverbal hotche, f., " eosse (de pois, de fèves)"; dans le Brabant oriental : scotche di proès, di féves, près de Jodoigne, à Ste-Marie-Geest, Noduwez, Marilles. De là aussi, avec le suffixe - ellum : -ia, le diminutif hotcha (Huy), m., "pois mange-tout", scotcha (Namur), "1. sorte de pois ; 2 . gousse de pois" " $\left({ }^{4}\right)$.

Par analogie avee le hris de la eosse que les doigts font éelater, hotchî a pris le sens de : "casser net (un éni, une branche, un os, ete.) ", puis de : "trancher net (la fane des lolés, des earottes, des betteraves, ete. " $\left.{ }^{5}\right)$. Cette aceeption dérivée est devenue la plus fréquente; elle a supplanté la premic̀e et obseurci, comme nous l'avons vu pour Grandgagnage, l'origine du mot. En voici quelques exemples : lès pôtes sont totès hotchêycs (Liers-lez-Lic'ge) "les épis sont tout cassés " ; hotchî on bic's. si hotchî l'ohit dil djambe "easser net un bois, se fracturer le tibia "; au fig. tot hotchî "tout net, recta, brièvement ": dji l'i a dit tot hotchî " je le lui ai dit sans détour, sans ménagement" $\left({ }^{6}\right)$. - Souvent

( $\left.{ }^{1}\right)$ Eeonelier le lin, le chanvre (= w. spimlji), e'est frapper la filasse avee une baguette, dite ćcouche ( $=$ w. spinlje), pour en faire tomber les fragments de la tige qui y sont restés adhérents. - Voy. Thomas, Mélunges, p. 64; Romania, 1910, p. 222 ; Neyer-Lülke, $1^{\circ} 2999$.

$\left.{ }^{2}{ }^{2}\right)$ G. et Forir ne donnent que di(s)hotehi "écosser". Comp. le uam. splossi ou displossî (Pirsoul) "écosser") ; le fr. plumer ou déplumer.

${ }^{(j)}$ Remaele, $2^{\circ}$ éd., et G. ont tort d'expliquer directement par : "mâeher, sens figuré dérivé de : easser net \%。

(4) G., II 349, scocus; Pirsoul scotia.

(5) Lobet, p. 247 : "effaner... effeuiller ". De mêne à Stavelot: hotchî dès rècines, dès pétrâtes "décolleter des earottes, des betteraves" (BSW 44, P. 538 ; BI) 1910, P. 12). Ce sens pourrait aussi bien se rattacher plus directement à celıi de "déeortiquer».

${ }^{(6)}$ Cet exemple devient ainsi synonyme de : lji " l' $i$ a uin hotchi. J'ai entendu aussi à Liège : dji v' plak'rè (ou col'rè) çoula tot hotchì so vosse tèyêt (assiette), ee qui pourrait aussi bien s'expliţuer par le sens propre. 
intransitif : li lame dè contê a hotchî (éclaté) comme dè vềle (Liège) : de bave's quèst sudjèt" a hotehî, de hotchant batès "du bois qui casse faeilement, qui n"est pas coriant (flexible)". - Enfin, nous rattacherons ici le nam. seolect (Fosse-lez-Namur : IBSW 52. p. 158), m., "épi brisé de sa tige". seoketer (ib.) "briser", dont le $k$, an licu de $t$, atteste l’influence du ronehi : eomp. le pic. écoker cité ri-dessus.

\section{lićg. hotchèt, fr. teehn. hochet}

Le liégr. hotchit, nam. ofchit, signifie "boule de menue houille pétrie avee de la terre glaise ". D’après G., I 300, ce mot "vient peut-ctre de lotchî (casser net), les hotchets étant faits de bouille concassée ". On doit écarter cette eonjecture pour deux raisons. Nons venons de voir que l'aspirće initiale de hotchî représente le lat. exe- ; loin de tomber en namurois. elle y deriendrait se ou ch. De plus, hotchit a une acception moins spéciale que G. ne le pense. Linsi. le malmédien comaît hotchit d' bohre ou d'vicier "polote de beurre, de neige". à côté de hotchit a broiler "motte [de charbon] à brîler " ('). En chestrolais, hotehit se dit d'un "tas de foin. meulon ou reillote" (Dasnoy; p. 159). A Stave, au S. de Namur, si cavfe a otchit, cest " se coiffer en faisant un toupet bombé sur une tempe". Le sens général est done "petite masse arrondic $*$; il a subi la même restriction que le synonyme clute (Verviers, Malnuedy ; du néerl. liluit "motte ") ; roy. l'article bougnèt.

Pour expliquer hotchit, il suffit d'en rapprocher holiet d'ansène, qui désigne, dans le Condroz, le petit tas de fumier déposé sur le terrain à fumer $\left({ }^{2}\right)$, ainsi que le eluestrolais holicte "petite butte, éminenee isolée, motte" (Dasnoy. p. 2(i3). Ce grompe se rattache au moyen h. all. hoveler "bosse ", moyen néerl. hocke, flam. hok, ail. hocke "tas de foin ou de blé dans le champ " $\left.{ }^{3}\right)$. Il faut tirer de la même source le non de

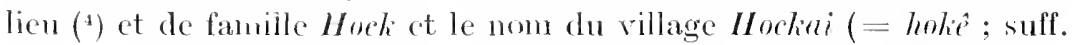
- cllum), qui signifie done "petite émincuee" $\left.{ }^{5}\right)$. (Quant à hotehit, il dérive de holi, horke. conme botehit "boncle ou meche de ehereux" du

(1) lixemples tiris de Villers (179:3), qui ajoute: "(être) come on hotchòt "gras et

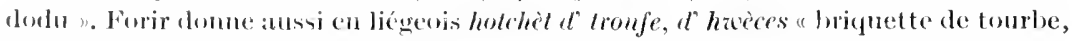
le tall n.

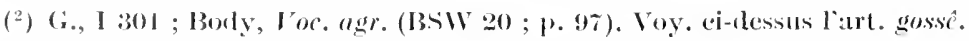

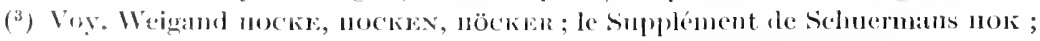

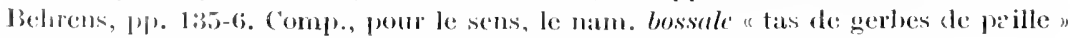

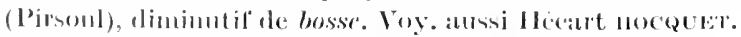

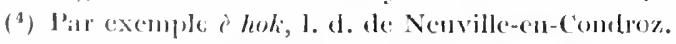

(5) Comnsom, Toponymir de Franconchamps (BSSW 16, 1). 225), dérive à tort Jockai du gerns. haug. 
néerl. lok, all. locke. Pour holìt à côté de hotchìt, comparez le liég. flokit, flotchite "petit noud de rubans" : crohit (Stavelot), croteliet (liég.) "erochet" ; stoliê (lićg.) "petite sonche "stotchit (Faymonville) "tige [de ehon] ": polie, polite (Liège. Verviers, Malnedy) "pustule ", potchit (ib.) "petit tas".

Le fr. teehn. lochet - différent de hochet " jouct " - signifie, d'après Littré : " $\mathbf{1}^{\circ}$ sorte de bêche usitée dans les terrains légers ; $\mathbf{2}^{\circ}$ forme dans laquelle on moule la houille : $3^{\circ}$ (au supplóment) charbon préparé avee eette expèce de moule " (1). Littré tire ee mot de hocher "seeouer, remuer n. Il a peut-être raison pour le $1^{\circ}$ que nous ne connaissons pas ; mais on retroure dans le $3^{\circ}$ notre hotchèt, francisé à Liçge même en hochet (de charbon). Le $2^{\circ}$ en dérive naturellement $\left(^{2}\right)$. Il faut done renverser l'ordre indiqué par Littré ct rejeter son ét ýmologie $\left({ }^{3}\right)$.

\section{liég. hoûr, anc. fr. heulle}

A côté du liég. hoûr, nam. mîr. m.. " tréteau de scieur de long, ete. ", dont l'origine n'a rien de mystérienx ( ${ }^{4}$, on comait à Liège un autre mot hoikr. m.. "dos (d"un eouteau)". que l'on n'a pas encore éclairci jusqu’à présent. G.. I 31 . le rapproche de l’anc. fr. hoole, qui a le même sens. et dn w. homel $\hat{\imath}$ "talus". Ce dernier n’a rien à voir icei (") mais l'antre indication est a retenir. I) not ansien français nous avons trois formes différentes : "le hule d’un coutel " dans le Ménagier ; "le heulle d'une hache " en 1:395; "le hoole d'un eoustel * en 1.26 ( $\left.{ }^{6}\right)$. Ces textes du moyen âge doivent aroir mienx gardé le tyje primitif que le dialecte moderne; je tiens done home pour une altération de *houl ( $\left.{ }^{7}\right)$. Or Schnermans enregistre le flamand hoesel. m.. " los d'un eontean" ( $\left.{ }^{8}\right)$, qui serait, d'après lui. le même mot que houdsel. Anx germanistes de nous dire s’il a raison. (2noi qu’il en soit, le flamand hoesel (prononcé houts'l) rend assez bien eompte de l'anc. fr. hule, ete. et, partant, du mot liégeois.

(1) Voy. aussi le Larousse illustré. Le Dict. gén. n’admet pas ce groupe.

$\left({ }^{2}\right)$ Ce sens $2^{\circ}$ n'est pas connu, je crois, à Liège. D'après Morand (ét. de 1780, II partie, \$502), les Liégeois appellent lutetle te moule à hochets. Je nai, pour ma part, jamais entendu que le terme général foùme "forme».

$\left({ }^{3}\right)$ Le fr. hocher se traduit en liégeois par hossî (du bas all. hotzen) ; hocher et hossî n'ont ancune parenté d'origine; noy. G. nocnì, nosi ; Dict. gén. nocnen.

$\left.{ }^{4}\right)$ C"est le fr. hourl, dorigine gemanique; roy. le Dict. génicul.

(5) Sur hour'lê, voy. ci-dessus p. 151.

$\left({ }^{6}\right)$ Voy. Godefroy, neclue. Niez, Körting, Meyer-Lübke n’en parlent pas.

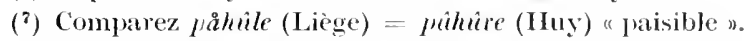

$\left(^{8}\right)$ De même De Bo donne hocsel, ocsel (en Flandre française hoczel, oezel). 
ane. fr. hovalon

Godefroy ne peut traduire ee mot dans le texte suivant : "que toutes les eompaignies et troupes estrangeres eussent a sortir, tant les Espagnolz que Neapolitains. lanquenetz et hovalons" (1594, Journal d.Olier, dans le c'ab. hist., t. xxvi, $1^{\text {re }}$ part., p. 156!. - Il faut lire houalons = Walloms. On commait le rôle joué par les gardes wallonnes dans les guerres des xvie et $\mathrm{xvI}^{\mathrm{e}}$ sièeles : e’était un eorps de troupes des armées d’Espagne, levé dans la partie wallonne de la Flandre. La graphie houa $(=w a)$ est analogue i hui $(=w i)$ dans le fr. huile et dans l'ane. fr. huihot, huigner, huillebrequin. De même aujourd'hui $o u=w$ dans ouest, ouate, ouaiche.

[Romania, t. xurn (1921), p. 56\%.]

\section{liég. hoye, fr. houille}

Dans sa eurieuse Lettie à Ch. Grandgagnage, datée du 13 juin $1856\left(^{1}\right)$, J.-H. Bormans regrette l'oubli du liég. hŏye dans le Dietiomuaire étymologique de la Langue zollomme. Lui-meme rapjorte ce mot au thiois schol, scholle (défini par Kilian : erusta soli vel terrae) et eompare le liég. hăye "ardoise ", qui vient de schael: "schol et schael sont en effet des dérivés du verbe schillen ou schollen, peler, éealer, s'écailler, ete., et signifient écaille, ćelat, motte de terre, schiste, ardoise, ete. " - Tel est aussi l'avis d'Atzler (eité par Diez), qui rattache houille ì l'all. scholle, ane. h. all. sholla. Sans se prononeer ouvertement, Diez laisse entendre que cette opinion lui parait fondée. - Enfin Scheler, Dict. stym. fr. propose timidement l'all. kohle "charbon ", tout en reconnaissant que "scholle expliquerait l'expression charbon de tere en houille dans un texte de $166 \mathrm{t}$ : ce serait du charbon en bloes "; il cite même, i l'appui de cutte opinion, la forme anglaise secole dans Palsgrave, p. $260(2)$.

Bormans rejette délibérément l'explication par le thiois lool, all. hohle. pour une raison de phonétigue : "le changement du li initial d'un mot tudesque en h wallon est peut-otre sans exemple ". Ft, de fait, j’ai passé en revue la série des mots wallons eommenẹant par $k$ et par h, et je n'ai recueilli qu'un exemple sans grande valeur : hik'hose "copuelurhe" (Clemont-'Thimister ; G., II 536), du néerl. kinlihoest,

(1) Bull. de l' Inst. archéol. lieg., t. ㄹ, p. 556.

(2) Dans son Ciloss. des Chronique's de Froissart, Scheler voulait expliquer houiller par foniller. 
all. keichhusten ( $\left.{ }^{1}\right)$. Quatre cas, qu'on pourrait, à première vue, invoquer, à savoir cougnot : hougnot ; comlot : houlot ; corote : hotote ; cotchit : hotchit, ne doivent pas ctre mis en cause : hougnot "quignon " n'est signalé que pas Simonon (dams G., 11 5:37) ; e'est une altération de cougnot. gougnot d' pan (Forir). sous l'influenee de hougnot : houyot dont nous parlerons tantôt ; de même le malm. hougnèt d' pan (Villers, $1793) ;-c o n l o t=$ "culot ", tandis que le verr. houlot = "*éeulot. "; le verv. horote est un diminutif de hore (canal), tandis que le liég. corote dérive de cori (eourir) ou provient du croisement de horote avec cori ; enfin cotchit et hotchit ont sûrement un radical différent : cotchit se rattache peut-ître à l'angl. colec ; pour hotchit, voy. l'art. ci-dessus. On ne peut done s'appuyer sur ces mots, et la formule "germ. $l_{i}=h \grave{a}$ l'initiale "reste eneore à démontrer pour !e wallon $\left({ }^{2}\right)$. Au surplus, nous verrons bientôt que le sens premier de longe n'a pu ctre " eharbon".

Au point de vue phonétique. le passagse de l'ane.-lı.-all. skolla au liég. hoye s'explique aisćment. Le sc initial, latin on germanique, suivi d'ume royelle, devient régulièrement $h$ dans les mots populaires de ce dialecte. Pour le monillement de $l$ et pour la réluction de $l y$ à $y$, il suffit de comparer le traitement du lat. pulla, cui dome liég. poye, montois pouye, fr. poule ; de même ğ̋̆la = lićg. guềye, gueule ; möla = liég. meñye, fr. meule ; comparez eneore ala = malm. éyé, liég. éle, fr. aile; tela = ard. teîye, lićg. tenle, fr. toile ; *s tel a et stipula = ard. stêuye (Wardin-lez-Ioulfalize), lićg. steûle, fr. étoile et éteule. On roit que, pour expliquer ly dans houille, il n'est pas nécessaire, comme fait Diez. de supposer (si la forme franẹaise est de provenance wallonne) une forme ane.-h.-all. *skolya.

De ee eôté, done, nulle diflieulté. Mais, si l'on se place au point de vue des formes différentes que doit revêtir un mot passant d'un dialecte à l'autre, une objection assez grave se présente. Ce n'est que dans la région de Liège et du N.-E. que $s c$ devient $h$. A l'Ouest, et notamment en namurois, il devient ch : chame, chanle, chète, chache, chupe, ehover, chonter, chume, churer, chilite, chou, ete. En montois, il reste sc : skète, scar, skite, scou, skièle, scoupe, ete. En français, il donne éch, éc : échasse, échelle, écoupe, écume, ćconter, ete. C'ette gamme dialeetale s'observe par exemple au complet à propos du liég. haye, ardoise : ard. chaye,

(1) La forme w. peut s'expliquer par l'influence de hiliter (hoqueter), par dissimilation, ou par influence assimilante du second $h$.

( ${ }^{2}$ ) La première édition de cet article (BD 1907, p. 125) porte à cet endroit une diseussion rle textes, qu'on juge inutile de reproduire ici. 
nam. (par execption) et mont. scaye. rouchi et franç. écale. écaille. Or, partont en Wallonic. dans son rayomement au Sud et à l'Onest, le germ. skolla aurait produit la forme unique höye, à peine nuaneée en houye (à Mons). Comment expliquer eette anomalie ?

D'abord, il ne faut pas perdre de vol limportant facteur chronologique. La loi qui a présidé aux divers ehangements phonétiques dont nons venons de parler, a exercé son action à une époque reculée et a domné naissanee anx différents phénomènes simultanément et indépendamment. 1)ans les temps postérieurs, en tout eas au xiI sièele, eette loi arait cessé d’agir, de sorte qu'un mot a pu et même dû passer dès lors sans altíration d'mu dialecte dans les dialectes roisins. C'est, eroyons-nous, ee qui cut lien pour notre mot. Alors que haye - chaye seaye - escaille étaient nés de bonne heure et en même temps sur différents points du Nord-roman, hoye, vers l'an 1200, passa sans changement de l'list-wallon à l'Ouest ; la forme liégeoise s’imposa aux autres dialcetes et, par suite. au français. Diez a done raison de définir honille : lütticher Steinkohle... getciss ein uraltes locales Wort.

Les données historiques que nous possédons sur la déeouverte de la houille justifient-elles cette manière de voir ? Assurément. puisque le premier texte qui en fasse mention de façon péremptoire, date de 1195 ( $^{1}$ ) et que Liège est considéré comme le bereeau de l'industrie houillère sur le continent. "On ne tromve pas, dit II. Gobert, une seule charte antérieure an xume sicele dans laquelle le charbon de terre serait mentiomé. Après une étude eomplite de tous les diplomes et chartes imprimés eomms. eonecrmant notre pays, l’érudit archiviste de la ville ale Bruxelles. M. Alphomse Wauters, est arrivé aux mêmes conchusioms que nous".

Ainsi done - pour reprentre l'expression de Diez - hoye est un "très ancien mot liégeois". Et voiei comme j’expliquerais son évolution sémantique. Bien arant la déeourerte de la houille, ce terme existait dans rette pointe extrône de la Wallonie, avee le sens général

(1) Hoe ammo lerru nigra ad focum faciendum optima per IIasbanimm in multis locis est incenta (. Immless Sumeti .Inobbi Leodiensis, publices par M. .J. Alexandre, pour la Socirti des Bibliophiles, p. 52). Ce texte famenx est de Reinier, moine de St-Jactues,

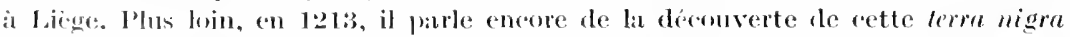
corbomem simillimn quae fabris el fabrilibus of panproibus al ignem faciendum est ntilisimu. Il est a noter ane l'annaliste designe par deux fois la houlle an moyen d'une póriphrase. Nous empruntons ces textes anx Rues de Lirge do Gobert, Il li:3, yui a fait de la ruention un exposé très intéressint. Voyez ci-après l'ar ticle teroute. 
de "petite masse, motte" $\left({ }^{1}\right)$. On disait en liégeois des hoyes de glaee, de pierre, de neige, de terre, de heurre, ete., avant de dire des hoyes de charbon. Lorsque le eharbon de terre fut déeonvert, ee dernier emploi, devenu le plus important, fit oublier tous les antres : de là, des hoyes (sans complément) ne désigna plus que "la houille en morceaux" $\left({ }^{2}\right)$. ("est sous cette forme et avec ee sens restreint que le mot sortit, vers l'an 1200, du canton oì il avait vécu juspu'alors, pom voyager - arec la chose - vers l'Onent et le Sud et faire la fort une que l'on sait ( $\left.{ }^{3}\right)$.

A l'appui de eette thèse, je erois que l'étude des dérivés - où le sens générique que j’indique plus hant s"est nettement conscrvé - foumira un argmment de sériense valeur et, cn tout cas, inédit.

1. Parmi ces dérivés. je range tout d’abord houyot (Liege. Verviers; altéré souvent en houguot) et houyè (Spa), qui signifient "pelote (de neige), motte (de beurre. d'argile, cte.) ". Grandgagnage. I 30s, déelare tout à fait ineonnue l'étymologie de houyot et du v. homyî, jeter des pelotes de neige. - Il faut y voir le diminutif (-ot. $-\hat{\imath})$ de hoye, au sens originel indiqué ci-dessus : ou houyot d' niraye, e'est une pelote de neige, pressée entre les mains $\left(^{*}\right)$; ou houyot $d$ boùre, c'est me motte de beurre. G. eite la jolic expression be hw a houyots, boire a tire-larigo, à grandes lampées, eomme qui dirait "par bloes". Entendu aussi : l'êue fêt dès houyots "l'ean fait des vagnes".

(1) On dit encore à Stavelot dès hoyes du hreaces "des mottes ou gâteaux plats d'écorces de chêne mouhues " (BSW 5, p. 37t).

$\left.{ }^{2}\right)$ Encore anjourd'hui, l'idée de plurnlité sulsiste dans l'esprit du peuple. Le w. dira : broùler tot plin dès hoyes; i ra zimle dès hoyes so lès viyèdjes. Dans le vocahulaire de nos houilleurs, hoye signifie: 1 . bloc [de eharbon fossile] : ine grosse

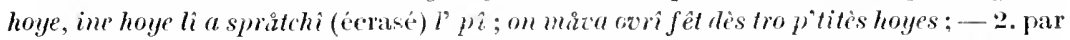
ext., charbon fossile : dèl prope hoge (syn. zomme "veine "), del erisse hoye (syn. dè cris tchifèdje ou tehirbon). - I.es marehauds ambulants crient dans nos nes : ¿s houges! C"est le seul cas où l'on rencontre cette prononciation houye en liégenis. On prononee hoye à Verviers comme à Liège, alors qu'au liég. foye, poye, coye, eorrespond le verv. faye, paye, caye.

( $\left.{ }^{3}\right)$ En français, la plus ancienne forme que cite Godefroy dans son Supplément, est oille en 1510 ; à remarquer l'expression oille de charbon, en 1511. On trouve ouille en 1665 : la suppression de la forte aspiration wallonne n'a rien que de régulier. Enfin l'Aearlémie admit houille en 1718. - En liégeois, nous trouvons dès 1278: "lovrage des hulhes d"une piece de terre" (cité par F. Hónaux, Iouillerie du pays de Liege, p. 111); en 1295 : "Wulhes ou cheobons" (Chartes de St-Lambert, no 448), et en 1340: "ouvraige de huilhe "(ib., $\left.n^{\circ} 630\right)$. Voyez Gobert, Eane et fontaines à Liège, p. 27.

(1) Dans ee sens, le plus fréquent, on supprime d'ordinaire le déterminatif : lès-èfants s' tapèt dès houyots. 
2. houyì. I. z' tr. et réfl. Assaillir en lanęant des pelotes de neige:

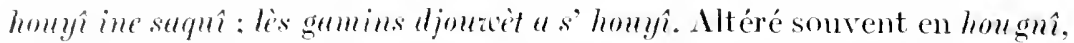

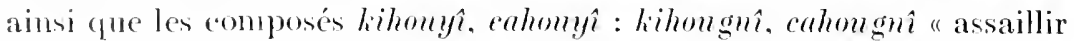
i eoups de projectiles, lapider n. - Pour la forme et le sens. comparez le fr. motter (m berger qui motte ses brebis) ; lapider, mitrailler, ete.

II. ¿. tr. "Herser avee la herse renversée et quelquefois garnie l'épines. On lomye également ar ec me traîne d'épines, sans herse.

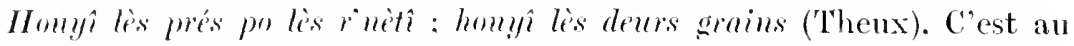
printemps quion homye les gazons et les céréales d'hiver " (Body. Foe. des agrie.). De môme. ¿ Fléron. Trembleur. 'Thimister, homŷ signifie : "éparpiller le fumier dans me prairie ". - comp. le fr. émotter (un (llamp).

III. $\tau$. ti. Exploiter (la veine de charbon fossile) : houyî on drèssant, ine pluteir (me veine en dressant, en plateur) : in-ozri qui houye bin s' zomme (un onvrier qui "houille " bien sa veine. ee qui consiste ì détacher le charbon (on gros blocs : i fêt dès grozès hoyes); - a passif :

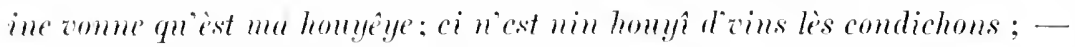
v. réfl. : ine zomme qui s' homye bin (qui se détache par bloes sans donner trop de menu) ; - r. intr. oll sems.s compl. : homyi al zomme ou a tèye (travailler à détacher la lonille dans une taille)': homyî al pîre (enlever le stérile dans une veine en étreinte. strince); rola l'mint qu i fait houy

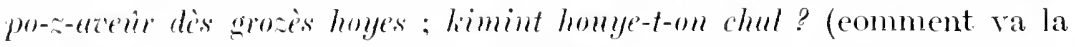
besogne ici ?). - Le composé dishougî " léhoniller " existe à Fléron : crist tot dhomyti il n'y a plus de eharbon à extraire.

Conclusions:

Les dérivés homyot. homyt et homyî (sens I et II) prouvent que hoye avait primitivement lacception de "fragment. éelat. moreeau. motte. bloce "et confirment létymologie par l'ane. h. all. skolla. all. seholle. néerl. schol. Fe sems III de homyî est postérieur et dérive de hoye entoloyé aver la signification restreinte de "charbon fossile ". - Lorigine dn fr. homille (emprunté du dialecte liégeois) n'est done pas aussi ineommue que le dit prudemment le Dictionmuire général.

[131) 190\%, 1. 123. - Meyer-Lübke. no 8005 a (fasc. 8, jaru en 19].4), admet sims restriction que lanc. h. all. sk olla a domné le w. hờge, doù le fr. houille (yuli a donne à son tour leesp. hulla et le port. ulha).]

\section{w. hroûler}

Ire liégerois (?) "lrouler, tamisce" "st dommé par bailleux et repris

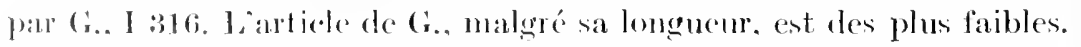

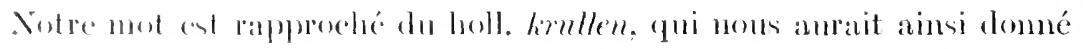


deux verbes différents : eroler "boucler. friser" et hrouler ! - Il faut lire hrouler, qui se rattache an latin cribrum, devenu eriblum (d'oì le fr. crible) et représenté en wallon par croile ("égrugeoir " : Lobet ap. G.. II 516), crille (" crible " : Verviers. 'Tremblemr, Jalhay, Stavelot, Bra, ete.), crîle ("crible" : Villers-Ste-Gertrude. Namur, Hondeng) ( $\left.{ }^{1}\right)$. Le verbe croûler (crîler, erîler) signifie "cribler, tamiser "; d'où le composé hronller. proprement "faire sortir en tamisant ". qui répond au type latin *eseriblare (*écribler) ; comparez lrou "écru ). hlôre "ćelore ", ete.

[Mélanges Kurth, II (1908), p. 321. Remanié. (f. Meyer-Lülse 2322, 2324.]

\section{ane. fr. huricle}

Godefroy a cet article :

huricle, s. f., sorte de plante : "De la huriele dient les maistres qu'on la doit mangier pour aler a chambre (Liz. de fisig., ms Turin, fo $\left.10 \mathrm{r}^{\circ}\right)$.

On recomnaîtra dans ce mot le moyen néerl. helevicl, hericl" "rapistrum arrorum " (Kilian), qui est l'all. hederich "rave sauvage, faux raifort; moutarde saurage, erysimum ; lierre terrestre. cte. ». Des dialectes flamands (Brabant, Limbourg) eonnaissent eneore herik, larik, ete. "moutarde saurage. relaret, sénevé " (Sehnermans, De Bo). Enfin Kramers fait de lerril le synomyme de dolik, irraie. - Quant à la forme. huricle se laisse ranener sans peine à herili : la protonique s'est assourdie en $u$ et $m l$ parasite s'est ajonté a la fin. eomme dans l'ane. fr. bouticle, musicle triacle, ete. Voy. ci-apres l'art. tîlie, ane. liég. ticle.

[Romania, t. xLvil (1921), p. 568.]

\section{rouchi juverne (Mons)}

Le Glossaire acallon de Philibert Delmotte, écrit en 1812 et publié à Mons en 1907. dome sans explication l'artiek suivant :

juverne : kequn de juzerne. Daus un attelage de chariot ou les chevaux sont deux à deux, cest cehui qui est à la droite du cheval que monte le couducteur et que les Wallons nomment kievau de perriau.

(1) G., I 141, dérive à tort le nam. evile du lat. eribellum. - Comp. tribula > trûle, troûle (truble); tribulare $>$ trûler, tioûler, nam. trîler (émietter); extribulare > strúler, stroûler, nam. strîler (émietter): nelula > mûle, nam. mîle (hostie, oublic); nebulata > liég. mâlêye, verv. noûlêye (nuage); affibulare > afûler (alfubler). - A Thimister-Clermont, j’ai noté troủlen "crible ", qui peut venir direetement de troûler "émietter", ou ćtre altéré de *croûlể (*cribloir) sous l'influenee de troûler. 
La forme juzerne est due à une erreur d'analyse. Il faut écrire jus verme, èest-à-dire "en bas de la verne ou du timon". On distingue de même à .Teneffe (Hesbaye) li dj’ó dia panê (le eheral de gauche qui porte un panê "panneau ". courerture ou selle rustique) et $l i$ djvi di djus vièue; ì Perwez (Brabant), le tch'fó d' pagna et eelui dě d'zos vèdje, le eheral de droite, qui se trouve au-delà (= au-dessous) du timon par rapport an conducteur, lequel s'assied toujours sur le cheval de gauche ;

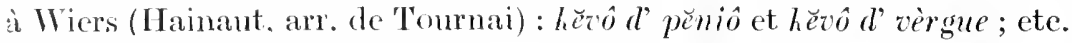
Le montois jus répond au liég. dịks. Poun le sens de rerne, viène, dans eette expression, voyez l'artiele vierna.

[BD $190 \%$, p. 122.]

\section{liégg. keûre, mèskeûre}

Ces rerbes nont pas de eorrespondant français et ne sont connus que dans la province de Lickge et an pays de Malmedy. Ke ûre signifie: "voir de bon gré (qu'un autre obtienne un avantage, le lui souhaiter, l'en féliciter", en all. : "gönnen ", en fr. du cru : "gréer ", qui est au

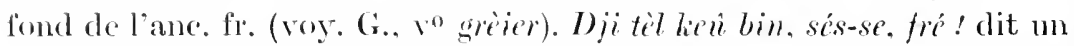
un voisin à 'l'âtî qui vient de gangner le gros lot ; dji v' lien tot l' bin dè momde "je vous veux tout le bien possible ". Souvent ironique : dji li leể bin! " je suis charmé de sa mésaventure, c'est pain bénit ! " Employé négativement, il équivaut à mèslêre (mès-= lat. minus), et le fr. offre ici des équivalents plus exacts: "envier, domner à regret ou chichement, refuser, leprocher, plaindre » (all. misgömen) : mi mêsse ni m' lient nin (ou mi méslient) lếce qui dj’ bề "mon maître me reproche l'ean que je bois a $i$ s' meslent l' pan qu'i magne "il pleure le pain qu“il mange ": i n' si mesligyet rin "ils ne se refusent rien "; nos n' méskèyans nin uos pônes "nous ne plaignons pas notre peine".

Pour (A., I 105, hêtre " est ćvidemment le même mot que le dial. de la Suisse rom. cordere. (souhaiter cordialement queh à qqu), lequel dérive du lat. crir. eordis". Malgré cette conviction, G. ajoute: "D'ailleurs on ponrrait penser à l'all. kïren, bas saxon koren, holl. keuren, ete. (choisir, approuver, avoir pour agréable) n. - Altenburg critique ces deux propositions ot imagine une variante de querre, lat. quaerere ${ }^{1}$ ). M. (ieorges 1)outrepont, trouvant ces ét ymologies peu heureuses, réfute la dernicire et se demande si un verbe *arere, pour curare, ne répondrail pas mieux aux domnées du parler moderne : "Il conviendrait four le solns : aroir sonci, prende soin de, faire des voux pour. On

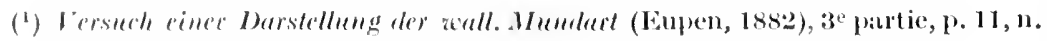


admettrait alors que le radical tonique lie'ti s'ent adonci à l’atone en $\grave{e}$ : lìyans, lièya, kìyou. futur kentè" " ( $\left.{ }^{1}\right)$.

Peu satisfait de ees diverses eonjectures, qui tendent toutes, même la dernière, à établir un radical léne-sans expliquer comment $r$ du radical aurait disparu de la conjugaison. jai naguire $\left(^{2}\right)$ cherché à résondre la question en partant d’un type lat. * quêtăre (rendre coi, apaiser ; d'où : quitter, abandonner; d'uù : aceorder, cte.). Je renonce aujourd'hui à eette hypothèse - phométiquentent correcte, mais compliquée et soulerant des difficultés de sémantique. Je recomais que II. J.-J. Marichal, qui, dans som étude sur la phonétique de Weismes $\left({ }^{3}\right)$, dome laconiquement : "lienre" = lat. cupere", a vu plus juste que ses devanciers. M. Marchot $\left({ }^{4}\right)$ i l'aplui de eette équation, allègue l'ane. fr. covir (<*eupire), qui est dans le Saint Léger: pour expliquer la conjugaison, il suppose que l'analogie des rouples crente,

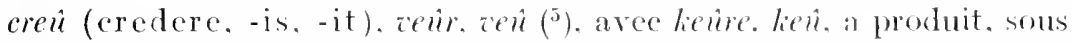
l'influence de crèyans, evèyon, rèyans. íyon. la coujugaison anomale kèyans. kìyou (au lieu de *enans. *eñou). - Pareille influence est, en effet, des plus vraisemblables. Une molification analogue s'est bien produite pour scrire. nos scriyans (an lien de verizans : sans doute à

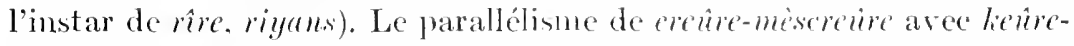
mèsliếre a dû contribuer ćgalement à rapproeher la conjugaison des deux verbes. Enfin. autre preuve que cremere et lie uire marchent de pair, on conjugue en IIesbaye (Borgilers. Oleye) : i crevithe i miskinit (= liég. crèyèt, mèskiyèt).

En somme. la question peut être considérée comme résolue. Liextrême N.-E. wallon aurait done l'hommeur de posséder le seul représentant roman du latin eupere $\left.{ }^{6}\right)$.

(1) Tableau de la conjugaison dans lo w. lírgeois, 1892 (135W 32, p. 102). - Si l'auteur parle d'un radical kể-, c'est sans donte par analogic avee hềre (dji hêu, nos hoyans, du lat. excutere) ; nais, si keure venait de *aure, le rad. verbal serait keur- et $r$ devrait passer a toutes les formes de la conjugaison, comme dans cori, mori, quèri.

$\left(^{2}\right)$ Bull. du Dict. w., 1911, p. 104.

(3) Die Mundart ron Guenzalue-Weismes (Bonn, C. Georgi, 1911), p. 40.

(4) Zeitschrift f. franz. Spr. unt Litt., xxxix (1912), p. 246.

(5) Veûr (voir) est la forme régulière (cf. G., I thi1 ; G. 1)outrepont, l. e., 1. 95) ; mais on n'entend guère que ż̀̀y, vèzi, zen̂y.

$\left(^{6}\right)$ Neyer-Lübke, $n^{\circ} \mathbf{2} 403$, ne cite que des représentants de cup i r e, entre autres le provençal cobir "gönnen " (syn. du w. keûre, que labuteur passe sous silenee). 


\section{liégg. kich'tôn'}

Ce terme d'argot liégenis. qui n'est pas dans les dietionnaires. figure

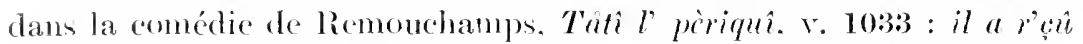

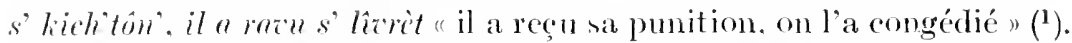
J'ai entendu a liège les phrases suivantes : diji ha a d'né s' kieh'tôn' ou die la èroŷ fé lich'tôn' "je hui ai clomné son congé n. en parlant d'un ourrier, d'un amourenx ; dji hi donrè (s') kieh'tôn' "je le rosserai ";

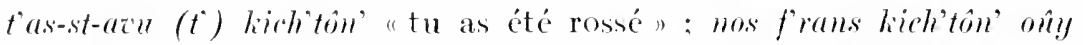
apres l' diner "nous manquerons aujourd'hui après-midi (à l'atelier, à l'école) ", diront des apprentis, des écoliers. En réponse à qui réelame un paiement. kich"tôn'! a le sems du fr. "bernique. sansonnet ! "Enfin, je tronve dans une pièce manuscrite : i fait torli qu'on lin (= aux Soeurs de la ('harité) diase de bon Diu ; sins què, c'sist brimique po l' lichtôn'; cette dernière expression est synonyme de po l' bètele "pour le paiement ". du néerl. betalen "payer".

Les deux sens principaux : "punition " et "paiement " se retrouvent dans le flam. gestarn (2), (qui signifie "ctre puni. être mal arrangé " (dans : er gestatu hebben : Sehuermans) et aussi "payer, satisfaire, s'aequitter "(De Bo). Le flam. staan devient clitôn' comme dans le liég. eanifieh'tôn' (ik kan niet verstann). L'initiale gs se dureit en $k$, comme dans d'autres mots de mêne origine.

\section{w. landon, andon}

En Picardic. Ie landon est un fort bâton de $0^{m}$ so de longueur, qui, suspendu au con des vaches en pâture. les empêche de courir ; c'était aussi. jadis, le billot mis en travers au cou des chiens pour les empêcher de chasser $\left({ }^{2}\right)$. Dans le Iainant, c'est la volée, pièce de bois transversale attachée an bout du timon pour y attacher denx eheraux de volée; a chaque bout de ee landon s'accroche un lamian ou palonnier $\left(^{4}\right)$. Le mot d'ailleurs prend soment wne aceeption particulière : à Wiers-lezPeruwelz. par exemple. le palonnier à deux cheraux s'appelle bat'niére ("bâtomicen") et les jetits palomiers timèsiers ("traversicrs"); l'ensemble y eomstitue le landon.

(1) Voy. le commentaire de cette pièce, BsW 48 , p. 3339.

(2) c"est lall. gestrhen, luxemh, geschtôen.

$\left({ }^{3}\right)$ Jouancoux et Devauchelle, Gloss. étym. picerrl. - C"est le sens du w. lamé, d'ilprès (:., II 10.

(4) Dednotte : loudon; Sigant : landon, rache. - Epinglons en passant les délinitions fantaisistes de Pirsoul, Dict. nummrois : "lomdon, landau, voiture a deux chevaux ; lamia, laudau, voiture il un chevil, landaulet "(!). 
G. n'enregistre que andan (I 3:6 ; sans lieu d'origine) et le hesb. ondon (II 529, 514), synonyme de lame : grand palonnicr anduel sont attachés les petits palonniers". Jai recueilli andom à IIcure-en-Fanemne et à Dorimne ("grand palonnier "; le petit sappelle lame à Heure, lamia à Dorinne), ainsi qu’à Erezéc ("grand palonnier à trois cheranx"); pour deux chevaux. on dit lame; pour un cheval : lamê ou cope). A Marehe-Lez-Eeaussines, an contraire. on dit lame pour trois on quatre chevaux, landon pour deux, lamia pour un seul ( $\left.{ }^{1}\right)$.

On voit que landan et lame sont an fond synonymes. Sigart allait jusqu’à croire que landom "derrait peut-être séerire lamedon (!) et serait ainsi une variété de lame" ; cela se passe de discussion. Jonaneoux et Devauchelle y roient le diminutif de l’ane. néerl. la'de "pieu, bâton "; mais laede. lude. que l'on rattache à l'all. et fr. latte. ê̂t domné *ladon. Il faut rapproeher lamdon du meusien lamd(r)e "perche ou traverse servant à cloturer une propriété ". lecpuel dérive du moven h. all. lander, bavarois lande "perche" $\left({ }^{2}\right)$. Lc sullixe $-(m)$ a la valeur diminutive. Dans andom (omdan. andan). l initial ast tombé parce ru’on l'a confondu aree l'article $\left({ }^{3}\right)$.

liég. leûvrê. anc. liég. leuve. anc. fr. lovier

Grandgagnage, Duvivier et Body signalent le liég. lentrî "petite luearne" $\left(^{4}\right)$. Le premier dérive ce mot archaïque de leu ("loup "), par l'intermédiaire de Vane. liégr. leme. s. f.. terme de eomrenr de toits $\left({ }^{5}\right)$. Là-dessus Scheler écrit cette note : Selon moi. leviru at le diminutif de l'ane. fr. luver. loner (lucarne) qui, à son tour. pent dériver d'un simple luze. loze ; quant à ce dernier. on pent le rancener à l'all. lulie (lucarne. écoutille)...". - Il faut écarter cet all. hle mon moins nette-

(1) BSW 55, p. 380. - Voy. ci-après l'article trèp’sin. - A Ben-Ahin lez-Huy on distingue li k゙mougna (*ommun-ellum ; (f. G. I 12:2) ou groul lamia, et les p’tits lamias.

(2) Voy. Weigand gelïnner et, ci-dessus, l'art. glindis'.

$\left({ }^{3}\right)$ Ne pas confondre cet andon avee l'anc. w. amdon "andain " (G., II 550), qui existe encore à Fosse-lez-Nanur et qui devient laudom à Iazy et ì (hastre-Villeroux : on a dit soyi è laudous (faucher en andains) par eorruption de èn-andons.

$\left({ }^{4}\right)$ G., II 25 et 614 ; ef. aussi p. 496 , vo airuge. - I)uvivier (dont Forir a transerit larticle) définit leûrê : "petite lucurne en plomb sur le toit ". Body, looe. des Conceurs, ajoute quelques détails. - Tú glossatre manuscrit du batelier liégeois donne aussi lêtrêे "fenctre de cabine ".

${ }^{5}{ }^{5}$ On ne le trouve que dans le Règlement de 1561 du Métier des Couvreurs de Liège : "pour la doublure [= couverture] d"une leuve, [ce sera] it la disposition du Ifesureur [des toits], ainsy qu il les trouverat gramdes on petittes". 
ment que le leî de Grandgagnage. A part cela, Scheler a vu assez juste. Lanc. Ir. lovier, lozer, lucer (lucarne : God.) et le diminutif liégeois lemerê sortent de la même souche ; et. pour moi. e’est la même que celle qui a donné l"italien loggia et lo fr. loge, à savoir le germ. laubia " toit servant d'abri. galerie ouverte autour de l'étage supérieur d'une maison, ete. "Parmi les représentants de ee primitif. on distingue le moyen néerl. lone (projectum teetum : un auvent ; néerl. mod. luif, luifel), anquel je rattacherai lane. fr. lovier. Laue. liég. lence reproduit le bas all. löre (i Cologue leuv "grenier". fré(uent dans les doemments des $\mathrm{xvI}^{\mathrm{e}}$ et $\mathrm{x} v \mathrm{I}^{\mathrm{e}}$ siceles ; en Westphalie lörce "galerie ". etc.) ('). Bien qüil soit dificile de préciser le sems de notre leure d'après le texte unique qui mous en garde le sourenir ct où il est seulement question de grandes et de petites lemes, il sagit probablenent d'une loge on galerie extéricure située à l'étage ( $\left.{ }^{2}\right)$.

Meyer-Lïbke, $n^{\circ} 5151$, a deux propositions qui sont franchement inaceptahles : $1^{\circ}$ il rattache au lat. Incublum (crépuseule, faible

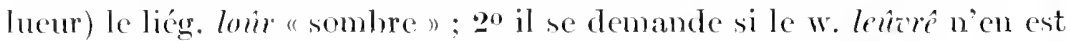
pas dérivé. Or le liég. lohir étymologiquement ne diffère pas du fr. lomel $\left({ }^{3}\right)$ : un cicl lourd. un mal lourd = qui vous alourdit. Pour lentere la source germanique lew paraît bien assurée.

\section{liég. lifer}

Durivier, Rourcroy at Forir doment le participe lifé "lisse, poli, uni ": ass dj'ès sont bin lifés (Dur.) "ros chereux sont bien lisses". Lc mot manque dans Remaele. Lobet. Hubert. Je ne l'ai jamais entendu à Liège ni ailleurs : mais il fignre dans des pasquilles liégreoises inédites du xvir siècle : $1^{\circ}$ an propre, en parlant de chats, (qui sont bien soignés, bin fièstîs, bin lifés, bin fahîs (17+3. Pasquèye MI. J. Pondant, v. 66:3) ; $2^{\circ}$ au fig.. en parlant du caractire de certains hommes souples et obséquicux (1735, Paspuèye du jour des Rois, v. 99) :

Rind'pus poli, rin d'pus lifé :

On-z-è f'rent dès nâlis d' solé (4).

(1) Voy, notamment le Wört. der Eupener Spr., p. 109 ; Blumsehein, Aus dem

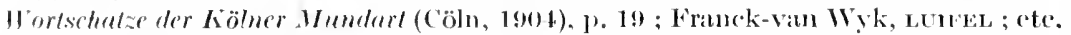
- Le llam. Ince'r disigne une "fenétre au haut d'une porte "(De Bo). Serait-ce le mêne not yue l'ane. lir. locier, luser?

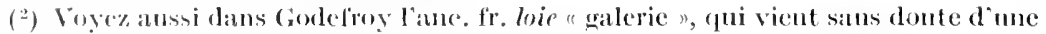
forme lodic " porticue ", domnéce par Du c'ange.

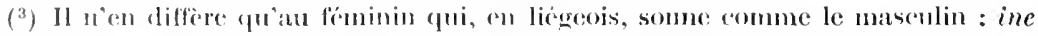
lonir sice, mut, djinl, :u lien de fitire regulièrement loûde comme en verviétois.

(अ) On en l'erait des cordons de soulier". 
G.. II 25, domne l'infmitif lifer "polir, lisser", pour leqnel il invoque le languedocien lifre "potelé, dodı, beau" et le lat. lềis ; mais il est hors de doute que lifer dórive du moyen h. all. slîfen (aiguiser, polir en frottant; all. mod. schleifen). La voyelle tonique $\hat{\imath}$ devient protonique eomme dans : strütchî (étriquer). du moyen h. all. strîchen, mod. streichen; malm. rever (rîjer), du has all. (w)rizen, all. reiben; ete. (1). Pour la rédnetion du groupe initial sl à l. voy. Lotia, et eomparez le liég. lakier " détendre (par ex. une corde tendue), cesser (par ex. de pleuvoir)", qui rient du moyen néerl. et moyen bas all. slâken. même signifieation.

\section{rouchi linche, linse}

A Ions. linche. terme du jeu de contan $\left({ }^{2}\right)$, désigne le lieu oì on se plaee pour commencer la partic. Sigart compare lallemand link, ganche (!). Plus prudent. Héeart ne hasarde ancune conjecture ; il définit simplement lince. "terme du jeu de bonque $\left({ }^{3}\right)$. au moyen dnquel celui qui l'a prononcé peut recommencer un coup qu’il a manqué... Si le joucur dit lince du pas....e est pour pouroir se placer à l'endroit oì le jen a eommencé n. 1)e mème linese on a lincse (à Wicrs), d-aler al lise ou a lise (a Nivelles). i Braine-l Alleud. (quand un joucur. placé sur la ligne servant de pas (lince). touche violemment la bille d'un adversaire, on dit qu'il la pète d'a lince. L'abbé Renard, dans ses épopées nivelloises, emploie l'expression métaphoriquement : piter d'a lince, répondre finement, en touchant juste; dii pète è zers d'a lince je réussis le vers, je rime facilement $\left({ }^{4}\right)$. Enfin. à Court-St-Etieme (Brabant), des arbres plantés en lignne droite sont dits plantés d'a léze. - Il me paraît hors de donte que ce mot est cmprunté du nécrl. lijst (all. leiste) "barde, lisière, bord, eadre ". Le sens convient parfaitement. ( on sait que nos dialcetes de l'Onest nasalisent fréquemment $\grave{e}$ ou $i$ tonique : par exemple le montois grinque, cerise aigrelette, vient eomme le fr. crèque du néerl. livich, all. livieche ; princhề. qui. à Mons. désigne le hanneton, signifie proprement le "prêcheur"; i prinche, il prêche, cte.

\section{nam. lotia}

G., II 3\%, est seul à signaler ec mot namurois. Il lui attribue deux

(1) Voy. ci-après les art. mirou, pirou, strifler.

$\left.{ }^{2}\right)$ Ou courtiru, bille de terre cuite, proprement "petit objet aui court "; de cour-t-eau, dérivé de courir (Behrens, Beiträge. p. 62).

$\left({ }^{3}\right)$ Bille de terre on de pierre ; emprunté du néerl. bonk, os, dont le diminutif bonelet, osselet, a donné le montois bouquette, bouquiau (Belırens, Beiträge, p. 45).

(4) Jean d' Nizelles, 3 éd., p. 211 ; L'Argayon, p. 113. 
sens: « 1. arbre auquel on a recoupé la tête pour servir de borne daus mb bis: : " petit fossé ereusé pour empêcher le passage sur une terre ". Il décide - on ne sait d'après quels arguments - que la signifieation première est "arbre ébranché ", ee qui lui permot de rattacher ce mot au néerl. lont "rejeton. scion, marcotte". "Ia seconde aeception, ajoute-t-il. doit s'expliquer par eeci, gue l'on aura fait abstraction de ce quim lotia ctait un arbre pour ne le eonsidérer que sous le rapport de son usage comme borne $).$

Persomnellement, je n’ai relevé que le sens 2 de lotia. Près de la frontière flamande. à Ste-Marie-(ieest lez-Jodoigne (Brabant). om appelle ainsi un petit lousé de $0^{\mathrm{m}} 50$ de profondeur. (que l'on ereuse au-devant de la trazéye (tronce ou breche faite au flane d'm talus pour permettre aux attelages de monter sur un (hamp plus élevé que la route) ; le cultivatenr qui a fini de se servir de sa trucéye. y pratique un lotia de peur que d'autres ne passent par le même chemin aree leurs bêtes ou leurs attelages (1). Il me parait infiniment probable que ce lotia est un dimimutif en - ellum du néerl. sloot "fossé " $\left.{ }^{2}\right)$. Si le sens 1 de Grandgagnage existe réellement. on peut à la rigneur en faire un article à part et invoupuer le néerl. loot " rejeton " (il y a pourtant belle différence entre un "petit rejeton "et un arbre. même ébranché) ; on peut aussi y voir une acception clérivée de lotin "fossé ": la filiation des sens serait, dans ce cas, l'inverse de ce que présume notre anteur.

\section{w. loton, lôton roton; anc. fr. louton. roton,}

G.. II 38. domne le namurois loton "solive qui soutient le plancher " et. II 616. l'anc. nam. Lotener "tranner des solives, des merrains, des trones d'arbres. ete. ou lien [plutôt] se servir de solives, de rouleaux, pour déplacer et pousser de grosses pieces de bois". Pour tout essai d'explication, il suppose que loton est de la même famille que le nam. lotia et compare l'anc. flam. loote. holl. loot, lot "rejeton, seion, marcotte ". On ne pent souscrire à ces propositions : amment. en effet, passer du sens de "petit scion" a celui de "solive"? Au surphus, voyez ci-elessus l'article lotiu.

Avant de proposer autre elose. il comvient de compléter les domnées sommailes ae G., fui ne combat notre mot que par le namurois.

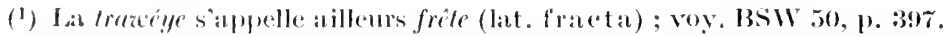

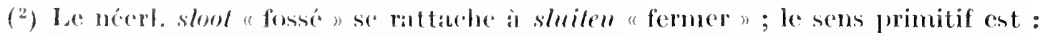
"fosse pour separer des pieces de tere " (Franck-vin Wyk). - Le exrompe initial sb se reduit normalement i $l \mathrm{en}$ wallon ; voy. lifer. Pour ž protonique = néerl. $o o$, comp. clotel " houle "(131) 1910, 1. 222), dérivé du néerl. klool, hiluil " motte". 
On lit dans les arehives de Seraing-sur-Meuse (10) avril 18:0 : admin. des Eaux et Forêts) : obtenir dans la eoupe ordinaire 1820 du bois de la Veequée so bois dits lótons pour la réparation du chemin... "(Communication de M. Nicolas Pirson).

Près de Malnedy, a Faymonville, on comnait encore lôton "solive qui soutient le plancher" (BSW 50, p. 5\% 5 ). Nous en rapplocherous s' lichotiner (il.. p) 5\%5) "se trainer, fainéanter " : c'est, avec un préfice intensif. le nam. lotemer, an sens liguré de "léplace péniblement ». Nous verrons anssi un emploi métaphorique daus le malno. lóton, que Villers (1793) définit : homme bonasse. de bome pâte, cordial, franc cour ". Comparez ci-dessus l’article canepin.

Au Nord de la Semois francaise. M. Ch. Bruneau a relevé loton "pièce de bois suspendue an con des vaches n (Énquête, I 32:3).

Un texte francais de 15:32. publić dans Romania. xxxill, 1).560, parle d'un "corps de louton de la longueur d'onviron deux pieds". Behrem. Beiträge. p. 15\% se demande si c'ent le môme not que le nam. loton. Cela ne parait pas douteux.

Endin, à ces trois formes loton, loton. lonton. il faut joindre la suivante:

Godefroy : roton, m., poutre: "pour" une estake et un ruton, pour justichier dardoir" (13r3, Compt., Areh. mun. Valenciennes).

Brixhe, Essai d'un répertoire... en matiere de mines (Liège, 183:3), II 515 : rofton. m. pièce de bois non équarrie qui se pose en travers du sol d'une voie de roulage [daurs la mine]. Les rottous sont ainsi placés à lat distance d'un demipied l'm de l'autre pour faciliter be trainage $\left({ }^{1}\right)$.

(2ue loton = roton, cela ne parait wucue contestable. Dès lors, on tiendra pour primitive la forme rofon. attestée en 1373. Le changenent de $r$ initial en $l$ est incomnu en francais. nais assez commun en wallon (2). Nous ponvons en inférer que loton a passé du Nomol an Sud. et que, partant. il est d'origne germanique. On doit. je pense sadresser au moyen h. all. ruote (all. mte) "verge ou verguc. perehe, barre. rouleau ". qui convient pour le sems non moins que pour la lettre.

(1) Mêne définition dans Bomans, Gloss. des honilleurs liégeois, qui donne roton comme hors d'usage. On se sert aujourl'hui de rails alpuyés sur des soris d' gatides (seuils de ails). - A n'envisiger que l'article de Brixhe, on pensera naturellement a un dimimutif en -on de rote "route" (tomp). rotis", t. de houill.); nais le texte de 1373 et la forme lôton sopposent à cette dérivation.

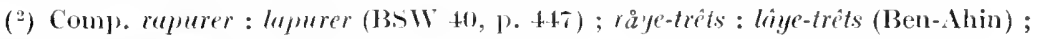
ruhin : luhin (voy. (i-ilprès l'art. rouhin); ete. 


\section{w. louwète (Vcrviers), rouchi loète (Maubeuge)}

Un réceptaire hu pays de Herve, datant de 1775 et publié dans Wallomia, t. x (Liège, 1902), présente eing fois le mot louette (pp. 14t-1; : une louette de fort poirre, 33 louettes de sel : ete.). L'éditeur, ne comprenant pas ce terme. le corrige hardiment cn locette, avec cette note : "cuillère de bois à long manche, diminutif de loce, lonche ". Il suffit pourtant d'ourrir le dictionnaire wallon du verviétois Lobet, à l'article lourett (reproduit par G.. II 540), pour trom er ectte définition : "maille, 6.4 partic de la livre de 16 ones, ou un quart d'onee \%. Il s'agit done d'un petit poids aneien, valant $\tau$ grammes 65. Quant à l'étymologie, il parait naturel de voir dans louziete un diminutif du moyen bas all. lôt, lode "plomb; poids de plomb d'une demi-once" (néerl. lood, all. lot), que Kluge ramène à un prototype germanique *lauda ( $\left.{ }^{1}\right)$. - Pour la protonique du mot wallon, comparez le w. pacène (三 * ponzène, dérivé de l'anc. l'r. poue, germ. *pauta; voy. ci-après l'article pawène) et le w. touzcê : l'r. tuyau. diminutif de l'ane. néerl. *tûta (néerl. tuit).

Je suis fort tenté d'attribner la mêne origine à un terme rouchi, inexpliqué jusqu’ici. A Maubenge, lòte signifie : "un rien, un pen : domez-m”en une loète" $\left.{ }^{2}\right)$. Le sens précis s’étant perdu, le mot aura survécu avee l'aception vague de "quantité minime" ; comp. liard, maille. (Quant à la forme. elle ne fait, je crois, aueune dilfieulté.

\section{liégg. lûrê, anc. fr. lureau ; fr. luron}

G., II 43 et 525, signale le liégeois likê qui, d’après Simonon, n’est

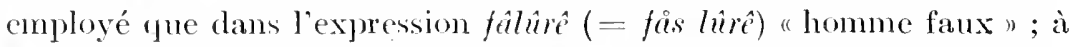

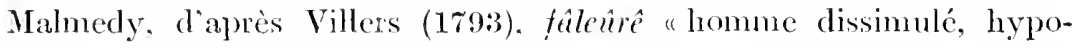
erite ». - Ponr tonte explication, G. renvoie an liég. lurer "leurrer "; mais la quantité différente de la protonique (likế, lurer) fait diffieulté $\left({ }^{3}\right)$. 1)e plus, Villers a lurer à côté de fâlêurê. Enfin, on ne pent séparer le w. lüêdelane. fr. lureau.que Ch. Nisard défnit comme suit: "un bon eompagnon, qui... vivait de repues tranches, trompait les femmes, volait les marchands, un fripon, maitre dans l'art de la pinee et du croc " $\left({ }^{4}\right)$.

(1) Le germ. lôt a passé en malmédien archaïque sous la forme loûte, s. f., " une demi-once" (Villers, 1793 ; encore anjourd'bui a Faymonville, BSW 50, p. 577).

(²) I'ocab. manbengeois (Maubeuge, 1889). D'après Ilécart, loéte, lohéle ou loǘle signifie, dans la même localité : "petite quantité qui se dome en sus de la mesure ". Voy. ci-après l'article razcile.

( ${ }^{3}$ Forir seul incrit harer "leurrer" ; hurer a pour lui Villers, Cambresier, Lobet, 1) nvivier, Bonveroy, Gindgagnage.

(1) Ch. Nisard, C'uriosilés de l'́lym. fr., p. 78 ; cité par God., Lunbau. 
Il fant $y$ voir le diminutif du moyen-haut-all. lûre (all. mod. lauer) "homme rusé, soumois", liken (all. ltuern, néerl. loeven) "gnetter, épier ». Pour le traitement phonétique, on pent eomparer le moyen hant all. bûr "maison " (all. bauer " cage, volière"), d'où provient le diminutif ane. liég. burom. ane. fr. buiron, buron "cabane, ehanmière" $\left(^{1}\right)$. - Le pléonasme fảs hî̀ s"explique aussi naturellement que fais Djudas, fas Pilate. fás Giadilon = Ganelon).

Le Dict. général tient pour incommue l’origine du fr. hrom. Scheler, entre autres conjectures, cite l'all. lauer (ane. lir), qui paraît en effet l'hypothèse la plus plansible. Entre lureau et hron, la différence des significations est aussi légère que eelle des suffixes.

[BD 1920, p. 12.]

\section{ronchi magnon (Harmignies)}

Dans le Bull. du Ditt. it. 1912. p. 59, on signale. i I Iarmignies-lezMons, l'expression obscure: fé magnum parmi djukite " marcher de telle sorte que le pied droit blesse la cheville ganche et le pied ganche la cheville droite $"\left({ }^{2}\right)$. La locution eorrenpondante employée à Bourlerslez-Chimay explique la préécdente : taper Mayon parmi Djakite "s'éeoreher les chevilles, forger", en parlant des elovaux" ". 1)c même à Wiers lez-Tournai : taper Jetn contre Jine. On a donné plaisamment aux jambes des noms de personnes : Jean. Jeanne, Jaequette. Marion ou Mayon, doù Magnon par épaisuissement de $y$ en gn $\left(^{3}\right)$.

\section{w. manote. manoque}

G., II 5+1, a eet article: "3. muite (petite nef ou nef lat'rale d'ume église; en nam. asente [lire acinte]) ). Le mot venant après manoî, la graphie manite est simement une crreur pour manote. Le chiffre 3 qui précède ne pent scrir d"indication; il faut le smpprimer ou le eorriger en 2: un premier article manote (menotte) se trouve en effet p. 7\%. - M. J. Peuteman, Promenade di Striron. pp. 109 et 111 (Verviers, 1902), eite ees textes d'arehives manuscrites: "le pavé de la nef et des

(1) L'ane. liég, buxon se rencontre en 1620 zos maisoms, vos burons : BSW, t. I, p. 139) et en 1634 (nos grègnes, nos mohons, nos burons ; B. et I)., Choix, 1. 106) ; la graphie bûron est sans doute préférable. - Voy. ci-dessus l’article beûr.

( $\left.{ }^{2}\right)$ bans le Haut-Maine (France), on dit eousiner dans le même sens. En fr. fanilier : battre le briquet (voy. Diet. gén., BriqueT); en liégeois : bate (ou fé) dè fể. On dit aussi à Liège : sès pîds ś frotèt lorèye, on encore : sès pùls s"apicit po l" bìteh.

$\left(^{3}\right)$ Voy. bougnou, erimignon, dognon, efe. 
deux manottes" (1726) : "dans les manottes de l'église " (1727). L'éditeur traduit en note : "nefs latérales". - D'autre part, Bormans et Body, Glussaive roman-iallon (partie inédite), doment eette phrase: "les manocques de l'Fglise St-Servais [à Liège] étant mal construites. il faut les refaire " (17\%t. Conscil Privé) ; ils proposent de traduire ee terme d'architecture par : "encorbellement?"; mais il est clair que le mot a le même sens yue ci-dessus. Enfin je troure eet artiele dans le même Glossaive : "bogge. habitation [?] : Messieurs de Stavelot. suivant le reeord de la Cour de Ferier [= Ferrieres] de l'an 1406, doirent detenir le bogge du mosticr de fond de chy en eomble; item la grosse cloche et les manoeles: xrrie siècle: Stavelot. II $n\left({ }^{1}\right)$.

La forme dialectale mamoque (pour manote: "menotte ", diminutif de main. se rencontre fréquemment en rouchi (roy. Héeart et Diet. gén.), oì il s'applique notamment à une poignée de fenilles de tabae, à une manne ou à un panier munis d'une anse ou poignée. ete. $(2 /$. Dans le cas présent. les nefs latérales ont été eonsidérées eomme les "mains " d'une église. par opposition au trone (logge : lisez bodje et royez p. 32), qui. dans le texte eité du xrise sièele. désigne le vaissean principal de l'église du monastère, et non l'habitation.

ane. fr. manser

Liane. fr. mamser, r. tr.. est un mot rare $\left({ }^{3}\right)$. Il figure trois fois dans

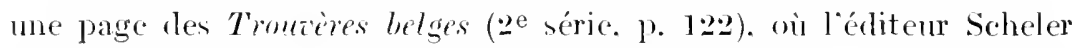
ne sait comment le traduire. Dans son édition du Jeu de la Fenillée d'Adam le Bossu ${ }^{4}$ ). M. E. Langiois a corriggé de façon très heureuse un passage altéré. en y rétablisisant le verhe manser, que les éditeurs précédents n'araient pas compris. Il rient de eonsaerer à ee mot obscur un article (Romamia. t. xur. Pp. 259-261), où l'on trourera toms les textes an quention : sa conchusion est quon peut hésiter entre le sons de "ćtreindre " et celui de "griffer ". On va voir qu'il faut sams hósitation choisir le premier.

Je signalcrai d'abord un article de Grandgagnage. II 5 H1. qui traduit l. ronchi momser par "ctouffer "; une note de Seheler y rceomaît que "e scons "comvient assez bien "pour le passage qui l"avait embarrassé.

(1) Indication pen précise; je nai pu retrouver ee texte aux archives de I,iège.

(2) J“ignore si lon pent rattuchere à la mêne famille l'ance. fr. manoque (1. petite

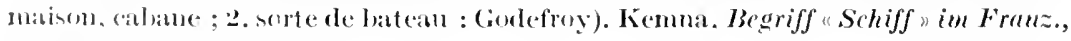
ne: pritle pas ile ce mot.

(3) liarticle de cioklefroy (manser - peigned !) est satus valeur.

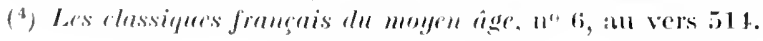


J'ajoute que, dans le nord du Hainant belge, mansè (Lenze), -̋̈ (Ath), - $i$ (Ellezelles) s'emploie eouramment avec l'acecption de "prendre (qqu) a la gorge pour l'étrangler n: on est mansi par me main qui étreint la gorge ou par un eol qui serre trop fort $\left({ }^{1}\right)$. Le sens de notre mot dans les textes du moyen àge se tromve done pleinement assuré.

Létymologie de ce verbe dont la signifieation exacte était si mal comme, n’a tenté persomue juspli ici. Je me eontenterai de remarquer que des dialectes allemands ont une expression analogue, notamment le bavarois mänsen "tenir (qqu) en bride ou séverement " (2), l'eifólien manssen (bezwingen: dompter, subjuguer), le luxembourgeois sich mânzen "se défendre, faire assant * $\left.{ }^{3}\right)$. En west-llamand, pour expliquer minsel (virole, all. zwinge). De Bo suppose un verbe minsen. dont il ne peut préeiser le sems et qui me parait ne faire qu'un arec l'eifélien manssen (bezwingen). C'c chaînon rattacherait le ronchi manse' aux dialectes germaniques.

[Romania, t. xwri (19:21), 1. 568.]

\section{w. manûhe, manowe}

Le w. mamihe.s. f., "articulation du poignet s existe dans la région de Mahmedy, Stavelot, ('herain $\left({ }^{4}\right)$. La seule explieation quon en ait risquée est le latin manipulus "poignée " $\left(^{5}\right)$. ee qui n’a évidemment aucune valeur. - Il faut on rapporoher le w. chestrobis ine mĕnúche. la minuche " eentaine on sentène, hout de fil qui arrète l'éeheveau " $\left({ }^{6}\right)$, et ceei nous amène au synonyme liéneois manowe " eentaine : brin de fil

(1) Lexpression douc 'ne mansure (Lèze) signifie de même "serrer à la gorge,

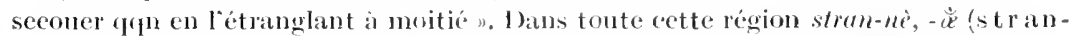
gulare) signific $1^{\circ}$ égorger (un chat strun-ri une souris), $2^{\circ}$ engoner.

${ }^{2}{ }^{2}$ Cité par G., II 135, v" mouse. - Comparez anssi Meyer-Lübke : mansus (apprivoisé).

${ }^{3}$ ) Dérivé de l'adjectif mamms (Eifel). mâns (Iax.) "adulte, fort "? Ou bien laadjectif est-il tirè du verbe?

(4) Malmedy, Faymonville, Guenzaine. Villers le donne en 17983(voy. G., Extraits,

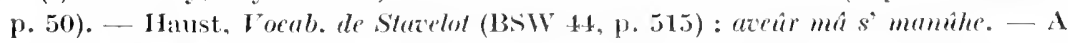
Cherain : "lji m'u stoké one munche : dji m' a metou one munote al manuche "je me suis foule un poignet; je me suis mis une menotte au poignet".

$\left({ }^{5}\right)$ J.-J. Mariehal, Dic Mundert zon Gueuzaine-Wtismes, P. 35 (Bonn, 1911).

${ }_{(6)}^{6}$ Relevé à Neuvillers-Recogne. - Dasnoy, p. r4, donne menuche on épeule. Ce dernier est le gammais peüle, qui signitie (a Buzenol et a Ste-Marie-sur-Semois) 1. la fin, la lisière d'une pièce d'ctolle ; 2 . la fin d"une pièce cle fil servant à la lier ;

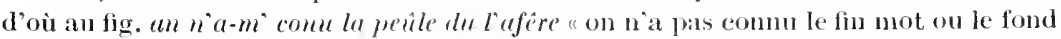
de lalfaire ". La définition domnée RSW +1 , p. 195, est sujette à cantion. Peûle dérive, comme le fr. espole et le w. spoñle, de l'all. spule. 
par lequel on commenee à dévider l'éehereau et qui lie celui-ei " $\left.{ }^{1}\right)$. G., II $\%$ s, inroque à ee propos le latin vulgaire manua " poignée "; mais on peut ĉtre sûr que manowe représente exactement le latin minuta " elose menue ", de mîme que mankhe reproduit le latin minutia, anc. fr. memise "menu moreeau" ( $\left.{ }^{2}\right)$. Dans le w. moderne manîhe "poignet", survit le souvenir de la jolie expression le menuisse du pir "cou-de-pied ". qu'on ne trouve que dans Aueassin et Nicolette (fin du xile siècle) et dont Godefroy a tort de faire un artiele a part : ee n'est au fond qu'une aceeption spéciale de memise.

Pour la semi-tonique é qui derient a sous l'influenee d'une labiale, les exemples abondent: man'cî: *minaciare, menacer ; marinde: merenda (G., II s2) : mazindje, mésange ; mahî: *mixtiare, mêler ; ete. Voyez an surplus p. 43, n. 4. et l'article wahite.

\section{liég. mayeté}

G., I 2.43. se contente d'enregistrer grimaieté "bigarré ". oì il sonpçomne la présence de gris ; mais on eherche vainement, dans son dietionnaire et dans eeux: de Fonir, de Hubert, ete., un article mayeté. Il faut écrire gris mayeté, de même que nèir mayeté, blêi mayeté, rolje. mayeté "[pigeon] gris, on noir, on blen. ou rouge brum, présentant un mélange de teintes claires et foncées " $\left({ }^{3}\right)$. - D'apris Forir, mayeter signifie "mailleter, marteler"; mais ee verbe doit cttre mis hors cause. On pourrait eroire que nous arons iei affaire à la forme wallonne de l'ane. fr. maillenter " tacher, souiller", lat. * maculentare. [Pour la syneope de la protonique non initiale, eomparez flam'ter ("flamanter, baragouiner en flamand), fiè'ter ('I'rembleur) "tailler an fièmint (courbet), ct d’antres exemples eités p. 69]. Mais le sens préjoratif de maillenter fait difficulté. Il vant mieux rattacher le w. mayeté à l'ane. fr. maillette " iche, marque », maille "tacheté, marqué ". Le primitif maille se lit encore cu français de la moncheture sur le plumage d'un oiscaus.

\section{liég. mèh'tèle}

G., I 102, emprunte à D)uvirier le mot meltell "servante", qui serait,

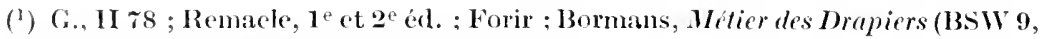
p. 2273) ; i Verviers manate (ib., 39), p. 273).

${ }^{2}{ }^{2}$ (comp. Le fr. menniser, memisier. Meyer-Lübke, $n^{\circ} 5598$, eite le normand

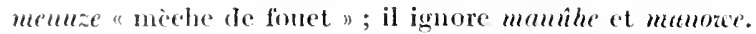

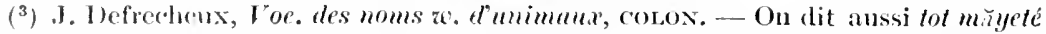

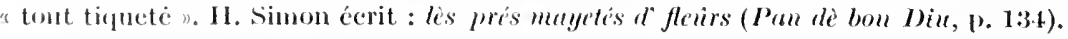


pense-t-il, un diminutif de l'all. magd, ane. holl meeghd. En réalité, e'est le prénom flamand Machteld. all. Mechthild (= Mathilde ; cf. Weigand, s. v, et ro Metie "fille de joie "), devenu nom commun de la même façon qu'en wallon Mayom, Matôye (Marion. Marie) ont pris le sens de "maitresse, amante"; voyez G., II 59. s9. et ci-dessus p. 85.

\section{liég. mènesik. mèn'zik}

Grandgagnage seul donne minesili, dans l'expression : c’ést-on loyâ. mènesili" "c’est un grand paresseux" (II 106). Comme e’est loyi (= néerl. luiand) qui signifie "fainéant, paresseux" ", le sens de l'antre not reste indéterminé et notre anteur en est rédnit à cette eonjecture aussi sompliquée que fragile: "De mène (mine) et de l'ane. flam. sick (malade) ?" - De mon côté. j’ai entendu ì Liège mën'zili, employé avee le sens de "individu, apôtre ", dans des expressions telles que : c'r-st-on drole di mèn'ilk! Qu'ist-ee qui c'ést coula po on min'sik?

Mèn'zik provient apparemment de min'sili : s forte s'est adoucie, conme dans beaneoup de mots $\left({ }^{1}\right)$. Pour pénétrer la strueture du mot, il faut comparer le ronehi bourl'nil" "bouchou (nui sert i jouer à la galoche" (Vermesse) et le liég. roclinil" "petit saligand": mots hylurides formés du fr. bouchon. cochon et du sulfixe diminutif flamand -lie. On voit par ces exemples que le wallon insèr e zans un groupe de consonnes germaniques dont la prononciation est malaisée pour lui $\left({ }^{2}\right)$. Partant de là, nous verrons dans min'sile une adaptation wallomne du llamand mensehle (prononc'é méns\%le), diminutif de mensch "homme ", au sens péjoratif de "individu ». - Il faut expliquer de mêne Flam'zil qui, à Liège et à Charleroi, désigne les Flantands avee me munce ironique: c'est le diminutif de Tlaamsch (Flamand). Tlanmsehke = T'lams'le = Flam'zil (proprement : petit klamand).

\section{liég. meûre èt make}

G., II 60, enregistre. d'après Remaele et sans explication, eette locution qui signifie "tête-bêche ". Cambresier. Hubert. Gothier la négligent. Forir. vo make, ne la commait que sous me forme altérée:

(1) Comp. conzoler, conzulter, hoûzer, paẑ́ ; iei, d’ailleurs, l’influcnce du fr. pop. zigue est probable.

${ }^{(2)}$ Voy, les ant. âih, skierbalik. - A propos du sullise flam. -ke, il faut noter la forme -ètè que le liégeois moderne ajoute à mu substantif pour en faire un diminutif de sens péjoratif : dè boûr-étè (de mauvais beurrs) ; on doeten̂r-ètè (un maurais médecin) ; in-oäri-èlè ; ine molon-èliè (une petite maison délabrée). On lanjoute

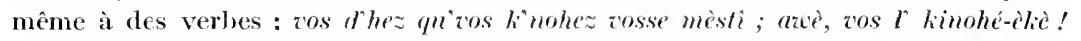
(oui, vous le connaissez à moitié, tellement quellement). 


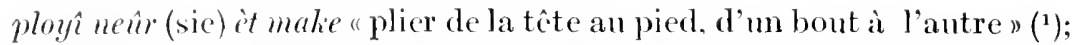
il ignore les expressions divirmi mêtre it make "dormir tîte-bêehe ", mete dès botriyes. dès djuibes men̂re it make "mettre des bouteilles, des gerbes tête-bêche ", qui sont bien vivantes, du moins à l'Est de Liège. A Liege mîme. on ne eomnait plus guère que le synonyme ponte ìt make. littéralement : "pointe et tête " ("). Par bonheur, deux de nos préeieux rocabulaires technologiçues $\left({ }^{3}\right)$ nous ont eonservé le mot men̂re, s. f.. au sens de: "pointe: petit clon sans tîte ", et je tiens d'un vieillard de Blegny-'Trembleur l'adjectif meniriant (pointu, effilé), qu'il prononce

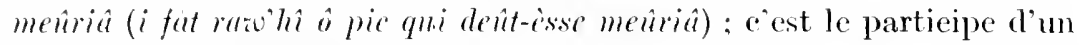
verbe imusité "merirî " tailler en pointe "; comp. athiunt (Lobet) "pointu" ( $\left.{ }^{4}\right)$.

Ce mot men̂re, dont voilà l'état-civil bien établi, nous le retrouvons dans l'ane. fr. meure. forme apoeopée de amenre (amore, amure), pointe de l'épicu, de l'épée, de la lance (voy. Godefroy). Littré identifie eet ancien mot avee le terme de marine umnre "cordage fixant le point d'en bas. nommé point d'amure, d'me basse voile qui se trouve au vent ", et telle est aussi l'opinion du Glossaire muntique de Jal ; mais le Dict. gén. distingue ces deux mots, entre lesquels il n'y a en effet aucun rapport de sens.

Si, comme il est rraisemblable, la forme pleine *amen̂re a existé en wallon, elle a dî s'altérer en mevire à eause de l'expression *ameûre it make, où l'a initial somnait comme une préposition qui pourait se

(1) Meur at mak est dans Iohet, p. 676 (V'rviers); cependant, aujourd'hui, dans la vallice de la Vesdre (Tronz, Nessonvaux, Dison), on ne comnat plus que neûre èt make "tête-bêche ": tu dones mà lès ezcia djeûs (cartes), i v'nèt turtos neûre èt make. Le primitif meüre s'est altéré sous l'influenee de ueûr (noir) et aussi par dissimilation (comp. bique-it-bone "hermithlurodite ", eorrompu en brique-it-bouc: G., I 54). A Il twe, outre ectte altération de forme, lexpression a subi une altération de sens : maker nênre-et-make = "jeter pêle-mêle ".

(") Forir, vo poxtr, domne poute ir make "pointe et tête, jen d'épingles ", et, ro Maki, l'expression muldes èssomme "tîte à tête, t. du jeu d"épingles". Ce jeu ne figure pas dans le Giloss. des jenc zeullons de J. Delaite. Il est appelé a teste bechezel daus Gargatuu, I 22: Burgaul-Desmarets et Rathery, dans lenr note, disent que re jell consiste à fatire deviner si denx épingles qu'on eaclue dans sa main sont plaries tête-hôdhe on dans le mêne sens (Littré : tête-bếehe). Ce qu'on appelle en

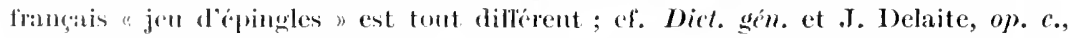
vo ATticiti.

(3) .J. Kinable, Gioss, ze, de cordonnier (Bsw 2.4, p. 287) ; J. Trillet, Vocab.

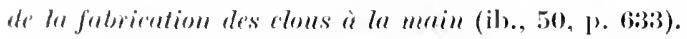

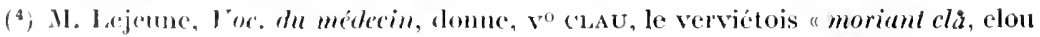
avorte, al tetr moirilie". 
supprimer ; eomparez le fr. "mettre à tîte-bêche " ou "mettre tête bôche".

[Romenia, t. XI (1911), P. 327.]

\section{w. mirou (Verviers, Malmedy)}

G., II 120, donne sans explication: " mirou, sorte de gâteau que l'on fait a Verviers et qui a la forme d'un $S$ ". Cet artiele a sa souree dans Remacle, 2e édition. Lobet définit: "pâtisserie, gâteau en $O$, en $S$ ». A Malmedy, d'après Se'ius. le miron est me "sorte de gâtean qu'on fait pour la Saint-Nicolas " (roy. aussi Wallomid, I 5 ; II 286). - Pour expliquer ce mot. on ne peut invoquer le fr. mirer, miroir. qui se dit murer, mureñ i Liège. Verviers. Malmedy. alors que mirou a toujours $z$ à la protonique. Le suffise est apparemment -ou. diminutif comme dans gadon "peijt de la elière (w. gäde) ", spirmu "écureuil n, tehivrou " cherreuil ". Pour le radical, on peut penser à l'all. schmieren " beurrer (du pain, faire me beurrée on tartine) " et an dialecte luxembourgeois schmier (= all. Butterbrod). Lall. schmieren " étendre de la graisse sur qqch "s'est introduit ì Malmedy sous la forme schmîrer "frotter avec du beurre ou de la graisse " (Villers, 179:3), et, d'antre part, le néerl. smeer. smieren a domé le w. smér, t. de bat., "mélange d’huile de lin et de colophane ". smếrî on brets" "enduire un batean de cette graisse " (G., II 368). Mais ces emprunts sont visiblement de date récente ; mirou (pour * smîrou) aurait une origine plus ancienne, attestée par la ehute de $s$ initial $\left({ }^{1}\right)$ et par le passage de $\hat{\imath}$ protonique ì $i\left({ }^{2}\right)$. Le sens primitif de mirou serait : "petit (gâteau) beurré ", c'est-ì-dire fait arec da beurre.

\section{W. moûhî̀, èmoûhî}

G.. II 141. 523, a les articles suivants:

èmouhi (tavelé) Simonon. De mohe; propr. moncheté.

mouhî ou moûhî (se dit d'un poil oud'un plumage dont la couleur est mêlée inégalement de hlane et de noir : ou blan mouhi est un blane bronillé de noir ; on neûr mouhi, un noir broullé de blane ; ei dj'zå la èst tot mouhî àl tièsse di vîyèsse, ce eheval grisonne ì la tête de vieillesse). Mouhi paraît être une forme de mohî (moueheté). Cependant la forme moûh séearte de eette étymologie,

(1) Comp. lane. w. meppe (bécassine), du néerl. sneppe (Cr., II 622), et voyez ei-dessus les articles lifer, lotia.

( $\left.{ }^{2}\right)$ Comp. lièg. bžzer, rouchi bizer (à Wiers), de l'all. biesen ; liég. püter : anc. fr. pieter (frapper ‘lu pied) ; lićg. spitrut, art. spitant (à Alle-sur-semois); voy. aussi les art. lifer, pirou. 
et puis le sens de notre mot est phutôt : mêlé on brouillé (de blanc ou de noir) que moucheté. Peut-ĉtre ce mot et monhin (taciturue par lourdeur d'esprit) ont-ils une racine conmune.

Le premier est omis par tous les autres auteurs. Cependant, à Liège, Ougrée, Scraing. jai relevé ces expressions : in-èmoûhî vizìdje " un

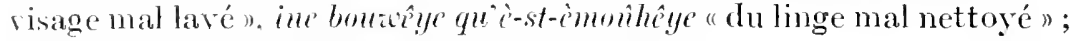
i fêt-st-èmonhî̀ è ci mumedje la "il lait malpropre dans cet intérieur". Sur monhlî. on ne trouve rien dans Cambresier, Duvivier, Rouveroy, Iubert. Forjr signale un sens figuré dont nous parlerons tantôt. Lobet dome seulement : "mouhî, gris condré, en parlant des eogs et des poules » ; de niêne Remacle. 2 édit.. avee ces exemples : blankès' moûh

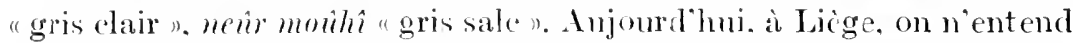
monhî. - $\hat{y} y e$, quen parlant du pigeon marqué de noir et de blanc; mais. jadis. il arait l'emploi gén'iral que hui attribue G.. eomme le prourent ces textes: "ung cheral mouhy lyar " (1552: Eeherins de Liège, reg. 19. fo $\left.282 \vee^{\circ}\right)$; " whe vache de poil brun et veau mouhy " (1686 : Cour de Seraing. reg. 1, fo 1:31). "un toreau mouhy " (1714: Spa).

Il faut écarter l'étymolugie de G., non seulement à cause des diffieultés que $G$. hi-même reeomait. mais eneore parce gie nous relevons $1^{\circ}$ à Alle-sur-Semois : môiji "vermoulu" (avec $;$ et non ch. ee qui éearte le lat. muscu. mouche) : 2o a Stavelot et a Malmedy : mouhi "moisir ": lu pan mouhile. ist mouhi $\left.{ }^{1}\right)$. - 'T'el est bien le sens premier. L'étymologie du fr. moisir, lat. *măcêre, convient exactement pour le wallon, qui a eréé le composé liég. èmû̉hî (*omoisi). Pour la quantité de -ồ-, comparez ponhhî (puiser : de păteus. puits. liég. pus') ; eette quantité est du reste variable: -ou- est href à Stavelot-Malmedy, et même certains Liégeois prononcent: $m$ mouhî " in piggeon au plumage mélangé de noir et de blane " $\left({ }^{2}\right)$.

Forir, a l'artiele mouftî $\left({ }^{3}\right)$. donne les synonymes moukt mouhin " lissimulé et taeitmne; halourd : misanthrope ". Ce sens figuré de moulî̀ n’a rion que de naturel; comp. sourié "séché ", corée "eouré ", qui signilient aussi : "taciturne, dissimulé ". - Quant à mouhin. qui est archaïque en liégcois, on le trouve encore dans Cambresier, Lobet.

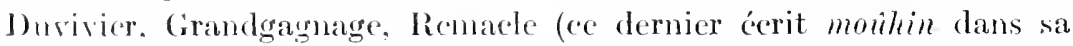

(1) Villers (Malmedy, 17983) domne: " mouhi, v. n.. moisir, se chancir ; mouhihèdje,

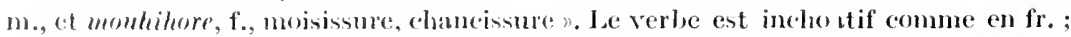
lat finale - $\hat{\imath}$ du liagoris provient sans donte de lanalogie de bouhî, poûh, diloûhâ.

(") Dans les (amplagnes françaises, moisi se dit de même des viaches qui sont de

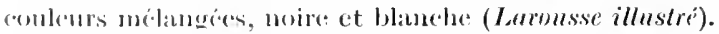

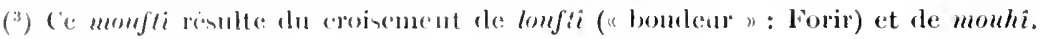


$1^{\mathrm{e}}$ édition). Comme G. le devinait. on ne pent isoler mouhin de monhî ( $\left.{ }^{\mathbf{1}}\right)$. On y reconnaît l'idée prenière de "moisi ", qui a ćroqué cluez nos Wallons eelle d'incrtic sournoise, de mauraise grâce. d'humeur maussade.

Peut-être faut-il ausi expliquer par momhin le verbe dumouh'ner "déranger de chez soi " que nous trourons à Starelot $\left({ }^{2}\right)$, dans ces deux excmples : des houletes quu ciste aredje arent d'monh'ué (BSW 4. p. 355) "des chouettes (que ce tapage avait dérangées"; [au petit matin] s' dumoulin'net lès manedjes (id.. 55. p. 179) "s"úveillent les maisons". Le sens propre serait "dé-moisir" : tirer de linertic, du silence et de l'immobilité.

\section{w. mwèh'nê machuria, cte.}

Dans le Bulletin du Dict. $\pi 0^{\circ} .191 \%$, 1. 7\%-92. J. Feller a consaeré une savante ćtude aux mots wallons qui désignent le eoryza ou rhume de ecrveau. Il établit que les multiples formes dialectales se classent en deux groupes, dont voici les principaux représentants : 1. maièrgunê (Vielsalm), mzargunê (Stavelot, Mahnedy), mà) mê (Goury, Manhay, Villers-Ste-Gertrude), maih'nè (Liège, Verviers); _- B. mateh'rê (Cherain), matchusé (cn Famenuc), machurin (Namur), matehurnia

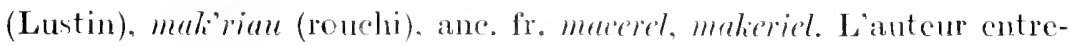
prend ensuite de démontrer $1^{\circ}$ que ces deux wroupes se rancònent à l'unité par l'équation macurgmmè = motchumia (c) passant par un hypothétique *macarchunia) ; - 2o que le tout est d'origine crermanique et s'explique par le bas allemand mork, à Eupen mors. noyen h. all. mure, synonymes de l'all. mürbe, morsele "mon, blet, friable, pourri, c.te.". Etymologiquement, le math" $e^{3}$ se caracterise done par "le ramollissement des parties tuméfićes "; le suffixe - $\hat{e}$ (lat. -ellum) marque "le résultat concret, visible et localisé de la tuméfaction".

Il est ecrtain que cette explication eonvient pour le groupe macer gumêmweh'nê $\left.{ }^{3}\right)$. 'Tout au plus pourrait-on alléguer la forme danoise morlien,

(1) Le suffixe -in peut, à l'origine, avoir désigné le resultat de l'aetion (ici : le moisi, la moisissure); voy. p. 41 , note 2.

${ }^{2}$ A A la vérité, moukin n’est plus commu à stavelot (on le remplace pal moúfrin "mufle, misantlurope"); mais il a dû exister daus cette région qui est voisine de Verviers.

${ }^{3}$ ) Pour la réduction nomale de mzì̀rgunè à mziètuê, voyez p. 127, 1. 5. Mrè̀h'uê est mis pour mzè̀g'tê ; comparez milih'uaut, issu de milig'uunt (G., II ro); rahmêsse (Forir) " vaguemestre "; tah'ucure (G., II 413) "grattin ", pour talineure; dringuèle et ılrinkèle, etc. 
ancien nordique morkinn " niirbe, morseh" (1), qui rendrait eompte du thime de moverguê plus exaetement qu'un substantif verbal das morschen: mais ee détail n’a pas urande importanec.

Quant au groupe B, est-il bien sûr qu'il se confonde avee le premier ? J'ai peine ì le croire. A mes yeux. matchurnia on -gna. loin d'être la forme la mieux conservée, est altéré de matchuria par épaississenent de yen gn: on trouve en effet spurgna, ta(h)urgna. pour spuria, tahuria, dans la région où matchurgua remplace matchuria, par exemple à Dorinne $\left({ }^{2}\right)$. Je me résoudrai done à disjoindre les deux groupes malgré leur air général de parenté. Match'ró. matchuria. mak(ć)riau représente plutôt untype *mucearellum (nis peut-être pour *nucearieium ; roycz ci-dessus hadrê) ; il se rattache au lat. pop. *muccare, dérivé de muceus, variante de mucus: c'est littéralement un *mouchereau $\left({ }^{3}\right)$. De même le ronchi inmakermé "enchifrené " se ramène à l'ane. fr. emmacrelé $\left.{ }^{4}\right)$. par altération de -crelé. -kerlé en -liernć. En suisse romande, à Plagne (Berne), II. Urtel signale s'úmotchemè "s'enrhnner " $\left({ }^{5}\right)$. qui présente la même déformation de la finale et qui est exactement notre mot rouchi, sauf que la-bas le radical a micux conservé la voyelle primitive. Pour le ehangement de o protonique en a. les exemples foisomnent dans nos dialeetes: manonye (momnaie), massuere (mousse:

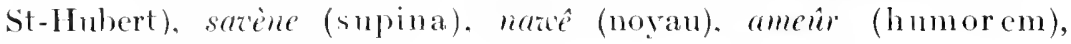
rastignom (rosignol), etc.

\section{ane. liég. oirzelle}

Une charte liégeoise de 1527, imprimée dans les C'hartres et Priviléges des Métiers. I 238. prescrit anx teinturiers l'cumploi de "bomnes et lealles

(1) Falk-Tory Norw.-dlänisches Etym. Wort., sonkex, tire ee mot d'une raeine * merli (liat. ma recere), tandis que morseh vient d'une vaulante * mers. - Au même tlic̀me que mà̀gumê parait se rapperter le w. monrcugne, mourgagne, s. f., "morve,

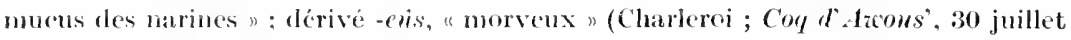
$1916)$ : morgagnedis (Givet) " hargnenx ".

(2) Pour symria, voyez ci-après la rticle sperial. Le nam. tahuria, -rgana répond atu liég. tahourê " nulage l'orage ".

$\left({ }^{3}\right)$ Sur le w. mokî (moucher) et ses dérivés, voyez G., II 130. Ajoutez motchas' "bourux " (Houssines; BSW 5.5. p. 387) ; mokê (Malmedy) "bont de chandelle ".

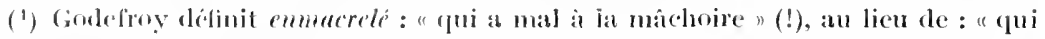
ai le mucerel mu le malieriel, qui est curhumé n. - A rillezelles (Lessines), jai noté :

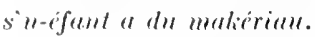

$\left({ }^{5}\right)$ Dians 1 un article très intéressint intitule "Autour du rhume " (Bull. du Gloss. des putois de le suisse romeule, 1913, p. 12). Launteur exphique émotehermè par

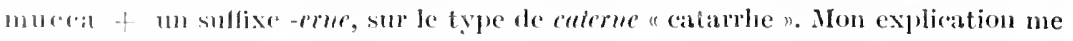
parait jolus simple. 
denrées, sens user de nois de galle. eoperoise ofizelle, brusille. berekmoese... n. Plus loin, dans me charte de 15\% maeque, oriées ( ${ }^{1}$. bois de Brésil " ct: "coperose. sumack. orzées, brusil " (II 321, 32\%).

Le sens de oirzelle, orwée n'est pas douteux et G.. II 623. l'a bien recommu : il s'agit de l'orseille $\left({ }^{2}\right)$. dont le nom figure pour la première fois en 1461 , dans une charte de Lille relative au mone objet : "Les taintures de ... poupre. beequemonls. orseille, bresil, sont taintures faulses et deslealles " $\left({ }^{3}\right)$.

Cependant, Bormans. dans son Bom Métier des Drapiers ì Liége. émet. à propos de nos textes liégeois de 152\% et de 15\% inattendue : "oirselle [sic]. s. f.. oirseille [ric]. noir de fumée que l'on obtient par le bois de vigne... Comparez le flamand arontsel. suie. noir de fumée " $\left(^{(4)}\right.$. L'anteur, on le voit. prend notre mot pour le liég. warsèle (1. noir de fumée; 2 . cirage de botten: G.. II 18\%), qui vient en effet du fl. zwortsel. mais qui n’a évidenmont rien à roir ici. Si je relève son erreur, e'est surtout parec qu'elle a fait récemment une vietime. Après avoir eité l'article de Bormans dans ses Beitrëgé. p. 189, II. Behrens affirme que l'ane. W. oirselle [sic] provient du ll. zicortsel : "On pourrait le pronver, rlit-il. par le w. warsele qui a le même sens (!) et dont G., II 482, domne l'étymologie exacte. tout en négligeant de le rapprocher de l'ane. w. orirelle. qüil mentiome éga'ement p. 6:3. La graphie oirzelle a-t-elle subi l’influence du fr. éerit mir ? on ne peut l'assurer. "Tout eela porte à faux : Grandgagnage ne doit pas ctre taxé de négligence pour n’avoir pas conlombu l'orseille arce le noir de fumée.

Dans oirzelle, la graphie oi a le même $i$ parasite que oir fréguent dans Hemricourt et Jean d'Outremeise) = w. or : aurum, or ; oirfeure (Henrieourt). w. orféce : orficre : coperoise (charte de 1527 , eitée ci-dessus). w. ếprôse : couperose. ete. Elle indique que " est fermé, ce que confirment les formen d'anc. fr. onmolle. ursolle. L'ane.w. orzée se prononęait done ôrzéye.

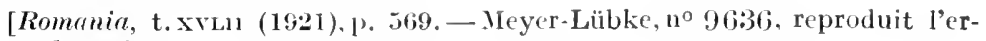
reur de Behrens.]

(1) Le texte porte par erreur : "sumae, queorzées".

(2) Liehen qui donne une belle conlenr violette. An xive siècle, orsolle, oursolle (God.), d'origine incertaine, qui a morlifié sa terminaison d'alprès oseille (Dict. gént.). "C"est la même ehose que l"orchel ou l'ursolle, qui croit dans les Canaries "(Furetière).

${ }^{(3)}$ Godefroy, qui cite ee texte vo bectuemould, l'oublie vo orseille. Il en résulte que, pour le Diet. gén., vo orseille, le phus ancien exemple du mot est de 1518. - On trouve ozeille en 1464 (Rommia, t. xxxin, p. 56t: Behrens, Beiträge, 1. 150). Voy. aussi, dans Godefroy, fuel, fuelle, et, 1. 2:3, notre article berchmoese.

(4) BSW 9, p. 276 (1867). Liarticle de G. na a paru quen 1880. 


\section{w. ongueçon (Roclengre)}

Je tiens de M. II. Frenay, de Roelenge, que, sur le Geer, " $\grave{e}$ lomkson" est synonyme du liég. il conlêye "au coin du feu "(litt. "en la culée "). si l’on compare è djairlin "en-le jardin ". lonlisom pourrait être considéré comme un seul mot: e'est ainsi du reste qu’il est perȩu, au dire de II. Frenay, paree qu'il n'existe que dans eette seule expression et qu'on le met peut-ctre ineonsciemment en rapport avec long. Mais il est hors de doute qu'on doit éerire i longueçon, altéré de angneçon. anl'som (1), diminutif de angue, aule "angle ". C"est l'ane. fr. angleçon "petit angle, petit eoin " $\left({ }^{2}\right)$. Le suflixe est le même que dans ab'çon (plançon, à Eben-Emad), am'çon (hameçon), claw'çon (clou de girolle, fleur du lilas). lip'çon (lippe, grosse lèvre), djèr"çon (voy. cet artiele).

\section{fr. orin ; w. neûrin (eûrin, leûrin)}

Le fr. orin. t. de marine (eordage qui at tache une anere à une bonce), est d'origine inconnue pour Littré et pour le Dict. gón. Le wallon aidera, eroyons-nous. à éelaireir le problime.

Godefroy, t. $x$, ne cite que trois exemples anciens. dont chacun écrit différemment notre mot : min. horym, hoypin. Les deux dernières graphies ne sont que des variantes fantaisistes de la première. Le dérivé oringuer "soulever une anere au moyen de l'orin " (Littré) prouve que le mot derait primitivement se terminer par me gutturale. L'espagnol-portugais minque (= orin) eonfirme cette hypothèse. Cela nous reporte à unc forme *oring.

D'autre part, nos batelicrs de la Meuse comaissent un mot w. neirin (avee len variantes enim, lemin). (que nous n'hésitons pas à rapprocher du lir. orin.

Au dire rle cenx que nous avous interrogés, le ne him. e’est "la chaine pour suspendre l'ance sur le beaupré ". ou encore "le cordage attaché a l'anneau de l'anere jour retenir la bonée n. ou. plus raguement,

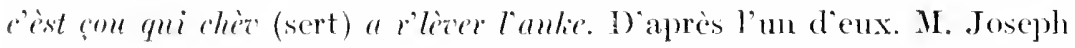
Gilman. vieux maitre batelier à fui nous devons la première de ees définitions, li neûrin se dit. par abrériation. an lien de l'expression

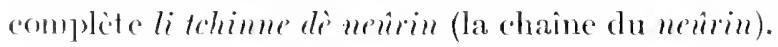

Jes hatelines llamands, auprès de qui nous arous poursuivi notre confuete appellent, ce cordage de neuring-ketting ou simplenent,

(1) Dans la prof onciation locale, an se ronfond atvec on.

$\left({ }^{2}\right)$ Voy. Burmans at Body, Ciloss. romm-lieg., vo anglechom. Godefroy cite l'excmule: "loger en 11 chambre en w1x angleçon" (Eust. Deschamps, Poésies). 
par abréviation, de neuring. Quant a la signification propre ou originelle de ce dernier terme, aucun d'eur nat pu nous lournir de renseignement. Les dictionnaires flamands sont également muets à cet égard. Réduit à interpréter nous-mêne l'expression flamande neuringketting et l'expression wallonne tehimne di nevirin, nous ne royons qu'un sens possible: neuring est la boucle ou anneau (ring) qui termine la tige de l'anere du côté des deux: Jras ( $\left.{ }^{1}\right)$. C'est en cffet à cet anneau que s'attache la claîne (ketting) qui sert, suivant le cas, à relever l'ancre ou à retenir une bonée.

Pour eomnatte la valeur possible de neu- dans le flam. neuring, nous avons interrogé un germaniste distingué, M. P'. T'ack ; sia réponse confirme pleinenent, semble-t-il, cotte conjecture: "Nouring peut etre $1^{\circ}$ une altération duflamand neutring; nent est une forme dialeetale de noot et désigne la partie de la verge de l'ancre où est l'oeillet

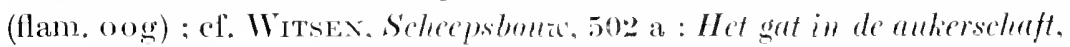
anders de XEUT ; - - wo une altération de oogring (den oo(g)ring devenant, par agghtimation ou prosthese de $\%$ de-nooring. puis

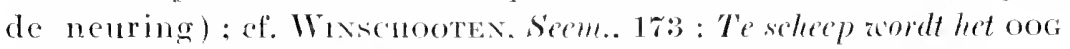
genomen zoor het gat in de anlier sehagt. daar de ring in dratid."

Il sagit maintenant d'explicfuer. - suivant que l'on adopte l'une ou l'autre de ees hỹpothèses. - K's tranuformations phonéticpues qui se sont produites :

I. Le flamand dialectal (limbourgoois?) nenring (= neutring) est devenu mevin dans la bouche des Wallons. On dit ordinairement li neirin ; mais, on nevirin somnant à pen près comme on-elirin, il en résulte quion a pu dire, par déglutination, l'eirin. lecuel, à son tour, par agglutination de l'artiele, a produit li leirin, on leirin. Comparez, en français. le lendemain. le lierre, ete.

Le flamand nooring $i=$ nootring). forme usitée sans doute. à l'Ouest, aura domé en français " un nôrin ". d'oì " un ôrin ", "un orin ", l'us'étant confondue, comme ei-dessus. avec la funale de l'article indéfini.

Quant au changement de voyelle. notons la mône transformation du flam. on en frane. o. wall. eî dans le flam. hoofdband = franc. hoband (auj. hauban), synonyme du flam. looteltouw = wall. heutô.

II. Dans la seeonde hypothèse, le france orin sexplique encore

(1) Ce sens propre de neuring et du w. newrin est perdu. II. Gilman appelle cet anneau lorindje di l'amlie, dauntres simplement lonê. On dit te même lès orindjes dè mastê pour désigner les ocillets en fer fixés sur le plat-bord et servant ì passer les cordes du mat on haubans. Nous expliquons orindje far le flamand ocringje (petite boucle d'oreille), synonyme de onê (anneali). 
plus aisément par le flam. oo(g)ring. - Pour le wallon néirin, voir ei-dessus la note de M. 'Tack.

III. Une troisicme hypothèse eneore possible. c'est l'existence simultanée de oo(g)ring à l'Ouest et de neu(t)ring à l'Est. Le premier aurait donné naissance an franç. orin; le w. neurin serait direetement emprunté du second.

Dans tous les cas, nous pouvons, de ce qui préède, tirer les conchusions suivantes.

1. Le france orin. le w. metrin. le flam, neuring sont identiques, du moins pour la finale : la première syllabe des mots français et wallon pourrait hien itre d'origine différonte, tout en représentant deux synonymes : oog. neut.

2. Ces termes désignent proprement l'ammeau inf'érieur $\left(^{1}\right)$ de l'ance. puis, par extension. Ie cordage qui part de cet anncau. On a dit d'abord "le cordage de l'orinn". puis orin a été considéré comme le nom même du cordage $\left({ }^{2}\right)$.

[Résminc du BI) 1907, I小. 62-66. - Veỵer-Lübke, no 5958 (6e fasc., paru en 1913). tout en renvoyant a notre article, tire le w. (l)eurin $\left({ }^{3}\right)$ du flam. noring ( ${ }^{4}$ ) et le fr. orin du west tham. ooring $\left({ }^{5}\right)$.]

\section{nam. oubouye (Stare)}

II. Louis Loisean signale a Stave (canton de Floremes. prov. de Nomur) le subst. fém. oubmye "glanage organisé ", avee eette explication : "Lorsqu"ori a préparé me partic de la moison jusquà la mise en gerbes, à un signal domné les glaneurs se précipitent en masse pour méchì (glaner): cola s'appelie runfle ou faire une roufe. Cette ruée se répète chaque fois qu'une nouvelle partic de la moisson est préparée. Liensomble des ronfes sappelle nue mbomye: dimain on fra ène

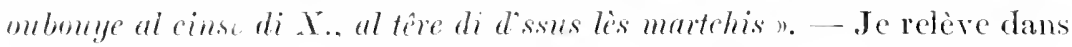
Franck-van Wyk, vo heibei. le néerl. dial. hoibui qui. dans le sud des Pays-Bas, signifie "besogne pressante, foule, presse " $\left.{ }^{6}\right)$. De la le w. (h)oubouge, dont l'aspirée a disparu, comme c'est la règle en namurois.

(1) Nonis cmployous re terme pour distingiler eet ambealu de l'organeau, gui se tronve a lat partic supérienre de ba tige de l'aucre, en flam. kabelring, ankerring.

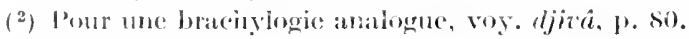

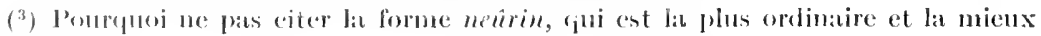
("onervine?

(1) N"est-e [ais me coreur pour nootring neutring ?

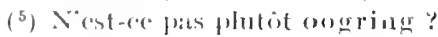

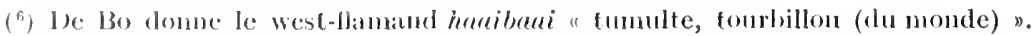


nam. ouyot "bardane "

Le Dictionuaire namurois de F. Delfosse. manuserit de 1850, porte "houyot, bardane, glouteron ", qui manque dans echui de Pirsoul. G., I, 309, consacre un de ses articles les plus faibles à ce mot, qu'il écrit houjo, huio (1) et qu il identifie avece ie liég. hougnt "pelote (de neige) ": cela ne supporte pas la discussion $\left({ }^{2}\right)$. On prononce ouyo à Namur, Lustin, Vitrival, Dailly-Couvin; iyo a 'Tourimne-St-Lambert; uro à Berzée; uyo à Houdeng. Thuin. Jamiouls: yuyo à Viesville. Tilly : types de structure peu ferme et comme "dévertébrée ». qui se dérobent à l'analyse. Le gaumais bihots " piquants du fruit de la bardane" (BSW 49. p. 148) est d'me charpente plus solide et plus claire. mais le $b$ initial étome et le $h$ n'est peut-ĉtre qu'une simple graphie d'hiatus. Pour Neufchâtean. Dasnoy. p. 401, domne wout. En Irdenne méridionale, II. Ch. Bruneau a noté arec précision des formes curienses, qu'il classe comme suit : $1^{0}$ anyo, n. pl.. Doisches, Montigny-sur-Meuse et, sur la Semois inférieure. Bohan, Rochehaut. etc. : nuyon Laforêt. Mousaive;

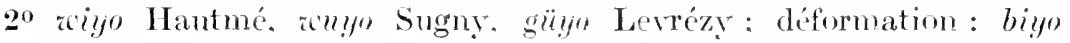
Cugnon, Lacuisine $\left({ }^{3}\right)$. Enfin. à Ben- Ahin at à Gives (entre Andenne et Huy). jai eu la chance l'entendre wihn. forme complète. dont toutes les autres - on le perçoit d'embléc — sont des altérations.

Nous retronvons ce wihnt dans le patois rouchi. En 1812. Ph. Delmotte signale "zihot-campion, s. m., grande bardane, glouteron " ("). Sigart écrit wiot et renvoie à son article io io. dont le début ne manque pas d'intérêt :

io io campion, s. m., bardane; ne se dit qu'en ville [= à Mons]; ailleurs on dit io. uio, zio. Don vient ce campion? C"est un nom de famille assez répandu ; il sera probahlenent arrivé à quelgue Campion, avee les fruits de barlane, une petite aventure aujourd lui oubliée... $\left(^{5}\right)$.

Or wihot est un diminutit de Wilhehn (Guillaune), lequel. conme tant de noms propres, pent prendre un sens péjoratif: en ancien français, en rouchi, en wallon archä̈uge. wihnt signifie " eocu " $\left({ }^{6}\right)$.

(1) Lire onyo, uyo. L'aspirée n'existe pas en namurois. Voy. l'art. bihot.

(2) Sur le liéeg. houyol, voỵ. l'article hoyge, ei-dessus.

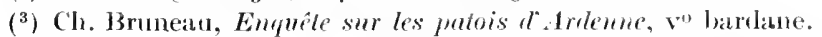

(4) Essai d"un glossaire zeallon ; édité en 1907-1909: Minons, Bolland.

(5) Diet. du zcallon de Mons, 1866, 1. 214. Cette explication de camprom parait tout à fait plausible. P'our io io, lauteur résume, en les accentuant, les propositions de Grandgagnage.

$\left({ }^{6}\right)$ Voy. G., II 488, et la note de Scheler, ibill. - De même, le w. wiyême signifie $1^{\circ}$ Wilhelm, Guillaume; $z^{\circ}$ benêt, curur patient (G., II 487); $3^{\circ}$ cocu (Forir). 
Serait-ce le même que notre aihot "bardane" ? En rouchi, les deux significations enexistent ( $\left.{ }^{1}\right)$. Il est rai que les glossateurs ont soin de les distinguer en deux: artieles séparés. A prenière vue. le contraire serait surprenant : quel rapport peut-il y aroir entre un mari trompé et la bardane? Pourtant, lisez avec attention Hécart, Dictionnaire rouchi (3) éd.. 1s:34) ; (ce qui n’était qu’une lypothèse en l’air deviendra certitude :

wio, lleur de la bardane, avant son epanouissement. Les enfants... eneillent ces boutons qu'ils jettent après les passants en criant wio. 11 parait que cet usage a également lieu en hangueloc...; ; wio peut être venu d'écile (aiguille), d'où wil:, willot, puis zeio, à cause des crochets dont ces fruits sont armés.

wio, cocu. be méme en Picardic...

N'est-il pas plaisant de roin lo brave IÍcart chereher au loin la elef quil tient en main? Sachons-lui gré plutôt de nous avoir. à son insu, donné la solution du problème. En réalité. nous avons affaire à un seul mot pris dans deux acceptions différentes. On appelle whots les capitules de la bardane parce quils accompagnent l'épithète malsommante que les enfants lancent apris les passants : en d'autres termes. ce sont les projectiles an'on lance apres les wihots ( $\left.{ }^{2}\right)$.

I propos du nam. onyo. on me demande ce quil faut faire du nam. yoyo. que Pirsoul ćcrit ioio et défuit : "amiggo $\left({ }^{3}\right)$. lieu où l'on enferme les irrognes on les ragabouds avant de les diriger vers la prison ". J'y rois le diminutil du prénom W'ihot (wiyo. onyo), formé par redoublement de la finale : cest apparemment le nom d'um ancien habitué du violon ou du p'omier yui oceupa ce local ( $\left.{ }^{4}\right)$. Du moins c'est, paraît-il, pour eette dernière raison que la pison commumale de Virton s'appelle barbazili.

[BI] 1914, p. 34. - "Votre étymologie trouve une celatante confirmation

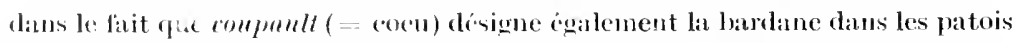

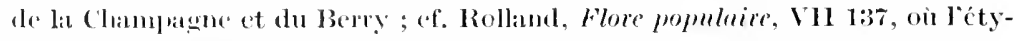
mologie de aette démonimation nest pas indiquée ". (Lettre de M. Antoine 'Thom:as, 12 juillet 1922).]

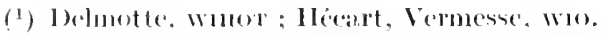

(2) P'antroe, pour s'amuser, jetait des glatcrons (aujourthoi glouterons : bar-

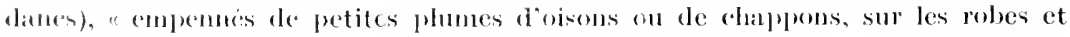

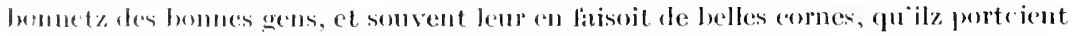

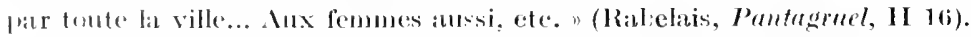

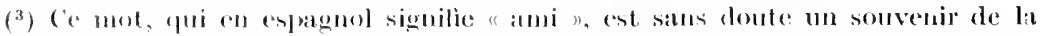

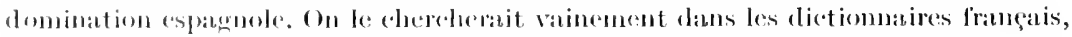

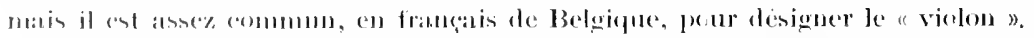

(4) Par une nouvelle extemsion de sens, le nam, alpelle anssi yoyo la voiture cellulaire servant an transport des prisonniers. 


\section{w. pariou}

Pour G., II 195, ce mot dérive de petreinse. paroi. I défaut de démonstration formelle. la filiation sémantique quil étaldit tend risiblement à justifier cette explieation : 1. proprement. un des carrós on pans d'une

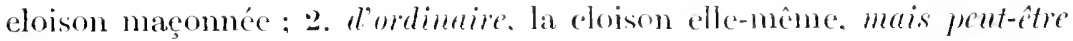
seulement lorsque, au lien d'ùtre maçonnée. elle est faite en lattes de torehis ; 3. plâtras. " - Remacle, ze éd.. qui rémait dans le mîme article parion et payou, no domne que le deuxième sens: "eloison ; ne se dit que des eloisons lattées on tressées que lon conre avee le mortier qu'on appelle torchis, quand on fait des bierques de boue et de (rachats n. Remacle ne songe gucre a faire de l'ét ymologie; sil rapproche parion de payou, e’est qu'il a entendu les denx mots cmployés indifféremment l'um pour l'autre.

En réalité, ces deux synonymes ont une origine commume : payou, e'est l'anc. fr. paillenl (mu' de bange. de terre mêlée avee de la paille hachée), dimimutif de paille: parion (ou parion. eomme on prononee notamment à Stavelot) ent mis pour * payeroli. La métathèse du yod. placé devant me liquide, est des plus fréquentes: ploygeron (bag uette ployé, pour tendre anx grives, ete. : dérivé de ploŷ, ployer, plier) pent devenir plorion (1): squgeloter (attifer, rendre saly) = gilioter : côyenis' (comemenx. coriare) = côngnès: haygener (ćtaler sur des hayons $)=$ hagner. hagn gneer. ete. - Sans doute. les dérivés en -eroù se rattachent d'ordinaire ì des verbes (ef. gittroù. samirom, de gliter, baver, samer, essaimer) : mais. pur expliquer *payeroù. on pomrait supposer en wallon l'existence d'un verbe paŷ. répondant an verbe fr. pailler (eourrir ou garnir de paille) : ou cheore. de même que l'ane. fr. linereul, w. lign'roni (linot), se rattache à linière (champ sené de lin). on pourrait dériver pelyerou des substantifs ane. fr. paillier (grenier i paille; paille), pailliere (chammicre).

A l'appui d'um prinitif * mayeron, on alléguera ces graphies de 1540 et 1552 : les poilherour. pailleroud, pailheroud (d'une étable). dans les Cris du Perron $\left(^{2}\right)$. - En somme, pariou, de mêne que payou, est à l'origine un nom de natière. On définira done ees deux mots : $1^{0}$ torchis, c'est-à-dire mortier de terre grasse eorroyée aver de la paille hachée, employé dans les constructions rurales pour lier les pierres

(') Par exemple, à Stavelot (BSiW 44, P. 518).

$\left({ }^{2}\right)$ D'après Bosmans et Body, Ciloss. raman-zorllon (partie inédite). 
d'm mur. garnir une eloison. ete. (1) ; $2^{\circ}$ pan de mur en torehis; $3^{\circ}$ (d'après G., II 195) plâtras. - Voyez l'article cowayoû, p. 66, où nous arons abouti à la mème définition.

[BD 1918, p. 62.]

\section{W. pawène. fr. pote}

G. Il 204, signale, sans l'expliquer. le liégeois-namurois pazéne "gaucher ". Notons d"abord qu'on dit : "il (elle) est parcène", et aussi : "jouer, jeter al pawène" (i la main gauche). Le mot a done deux emplois distincts. qu'il faut aimsi classer : 1. main guche; d'où : 2 . (persomne) qui se sert habituellement de la paciène. Pour la filiation sémantique, on eomparera le gaumais clitehe-pute "gaucher". propr. gauche patte; le rouchi gambite "boiteux". propr. jambette. jambe trop courte ${ }^{2}$ ) ; le liég. pongnote (Forir) et le rouchi manote (Quevaueamps) "qui n'a qu'une main, manchot ". propr. petit poing. petite main, moignon ; le lićg. pôt lète (G.. II :0:3) "manchot", propr. petite patte ; ete.

Les deux sens de pariene se retrouvent à Givet ct dans la provinee de Namur (Dinant, Denće ete.) : jadis. ils ont dû avoir eours partout oì l'on ne signale aujourd'hui que le premier (Vonêche) ou le seeond (Stave). Le mot tend à se perdre : jai eonstaté sa disparition à Bertrix. Chairière. Gros-Fayss, où il est remplacé par gôtchîr. l'opposé de dravtîr $\left({ }^{3}\right)$.

Le sullixe -ina. fr. -ine. w. -ène, a la valeur diminutive et péjorative (Nyтop, II $\S \S 264-\tilde{\tau})$. Le radical est l'ane. fr. poue, poe. porce " patte", qui représente le frameique *pauta, lequel a domné en néerl. poot. en all. pfote. Pawèue était sans doute primitivement adjectif, eomme le fr. chanmine. terrine; on a dit : "la [main] *ponine m. ee qui signifiait : "la main qui ressemble à une petite patte".

Le mot wallon peut. je erois. ćchairer l'ćtymologie du fr. pote. J'avoue que l'article du Dict. gén. ne me paraît guère satisfaisant : ect adjectif féminin y est déclaré "d'origine inconmue "; il est défini : "gourde " (sens vieilli) ; puis. par extension. "gonflée ». Or. en aneien fianęais. on

(1) Dans la vallé du Geer. parion nai que te sens : on meur di pariou, syn. ou pariouterlje (mur fatit de bois entalatés et de mortier). Jai entendu à Liège paliouterlje, puliotidje (avee $l$ pour $r$ ), payoutidje.

(") I)"apres Ilérart. "on donnat le nom de gambelle au baton dont les boiteux

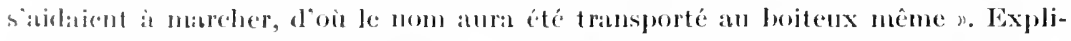
rattion ingenicuse, mais inexilcte. Lellipse ent la même yue dans le fro pied bot, w. pil-a-bile.

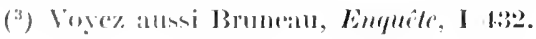


ne le reneontre que dans l'expression main pote ou pote main, ee qui s'explique parfaitement si l'on y roit un enprunt du moyen néerl. pôte "patte" ( $\left.{ }^{1}\right)$. La main pote. c'est. dans ce cas, la main pataude, gauche et lourde comme une patte. A l'origine, le mot n’impliquait pas dénigrement (comme le proure notamment l'exemple du xne siècle, cité par le Dict. gén.) ; mais la nuance péjorative "gourde, lourde, grosse, enflée, dont on ne se sert qu'avee peinens y rattache tout naturellement et subsiste seule aujourd'hui. Voyez ci-apris l'article potelé.

[Romania, 1914, t. xum, p. 4:34. - Dans le manuserit de Lille, Olla patella

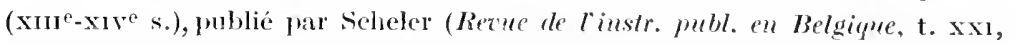
p. $269: 1878)$, ehiragua est glosé patr pouette, où nous voyons le dinimutif de poe "patte ".

\section{w. pèkène (V'erviers)}

Lobet seul signale "pikim, jet ("), ustensile de brasseur. espèce de timbale dont il se sert pour jeter leau ou les maticres dans le bae ". G., II 207, insère le mot sans l'expliquer. - C"est me altération de l'all. becken. néerl. bekle'n "bassin ". Pour le changement de l'initiale, eomp. bichet, pichet dans le Dict. gén. ; pour -kine, roy. ci-aplès tapliene. - J. Kinable. Gloss. des brassemes [liégeois] ne parle pas de notre mot ; mais il a un article chandron "petite cure pourve d'me anse (BSW 20 , p. 299). D’après un brasseur de Liegge que j’ai interrogé, ce tchôdron. sert au mêne usage que la pelieme de Verviers.

\section{W. pèri (Faymonville)}

L'exeellent Trocalulaire de Faymomille (13SIV 50, p. 583) est seul à noter le r. unipersomel perri au słns de "ĉtre imputable à. dépendre de ". L'anteur, M. Joseph Bastin. n’indique pas d'étymologie: mais il nous apporte des exemples précis et significatifs, entre autres : i perit a consèy " cela dépend du conseil communal "; i périhiè a més s'mèles si dj s冖̆ pulté "la faute en sera à mes semelles si j’ai les pieds mouillés "; etc.

En réalité, le w. pèri est identique au fr. périr, dans l'acception. disparue aujourd'hui, de "manquer, ne pas se faire". Seheler a relevé dans les Chroniques de Froissart trois passages qui attestent ce sens:

(1) On retrouve ee mot germ. dans le rouchi pö̀'e " main sale " (Ellezelles) et dans le w. pôte "patte" (G., II 202), diminutif pôt'lète (ib., 203 "manchot ». - L'ane, fr. empotement, adv., "sans soin " (God.), vient-il aussi de lì ?

$\left({ }^{2}\right)$ Ce sens de jet n’est pas dans les dictionmaires françeais. 
ne say ì quoy che demora ne peri, x, 2S6; il ne sacoient pas en quoy il perissoit (ì quoi cela tenait). Ix, 483 ; moy et mes eompaignons sçaurions zoulentiers ì quo! il perist. xrr. $290\left(^{1}\right)$. Godefor ne cite que ce dernier texte.

Lancieme langue, du moins dass le Nord. pourait done user de eette maehryogie énergieque : "A quoi périt-il?" pour signifier : "A quoj tient-il que cela ne se fasse pas? "I’oir. dans le dialeete moderne de Faymonville. le sens positif : "A quoi tient-il que cela se fasse?" Nent-il paseurieux de retronver dans un coin perdu de la Wallonie cette survivance de lancien français?

[Romanir, 1911, t, xL, P. 328. - Voici trois antres textes du pays wallon : y ue pery nient en yenz quil ne nons entissent fait une grande vilonie et injure (1101: J. Grandgagnage. Giloss. des eoutumes de Namur, P. 28, ro mater): et priat al the quith se tosist apparelhier dedtus ledil jour, si quith ne perist mie en li (.J. dOutremeuse. Myreur, vi 365 ) ; ill perist en entu que nos n'arons pria (ib.. p. 70). - Le Cutholicon de Lille a aussi un article : "caleo, valoir, joroir on perir ", qui a embarrassé léditeur scheler et qui s'explique par ee qui precide.]

\section{w. pilaine (Verviers)}

D’après Lobet. pilaine signifie "loquet. laine de la enisse des moutons w. Le Cilossaive des Drapiers de Bormans (BSW 9. p. 2S0) enregistre ce mot vervictois sans l'expliquer et sans signaler que pillaimes est dans mu texte liégeois de $1639\left({ }^{2}\right)$. G.. II $2 \mathbf{2} 4$. voudrait y voir un composé de $\hat{\imath}$ (pied) et de laine: mais $i$ est bref d'après Lobet. Pour moi. pilaine ent aitéré de *pilaine $\left({ }^{3}\right)$ et se rattache à piler "peler n. C"est une forme féminine tirée de pélain (*pellâmen, fr. plain) sous l'influenee de laine et des adjectifs en -anus (dimmin, -aine "demier. -ère n). Piluin, qui est ćerit ei ancien wallon pellin $\left.{ }^{4}\right)$. désigne me espèce inférienre de laine trop eomrte $\left(^{5}\right)$. Comparez au surplus l'ane. fr. pelaine (God.) "peau, fourrure ).

(1) Gurres de Froissart, t. xix, Cilosscaire (Bruxelles, 187 t).

(2) Chartes des Meticrs de Liege, II :321 (Charte des Flockeniers, 1639) ; voy, re

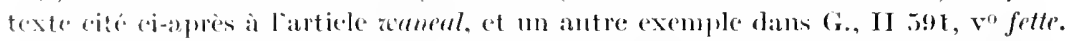

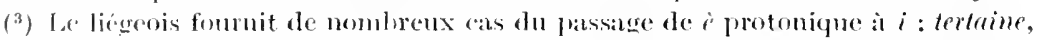

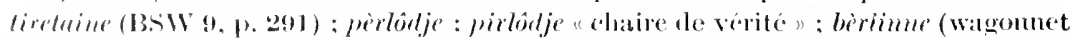

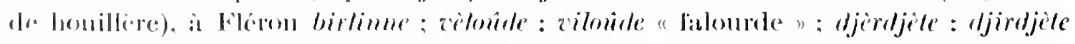
(chuir, p. 1:30) "gosier "; voyez ci-apris larticle terothe.

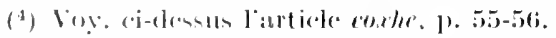

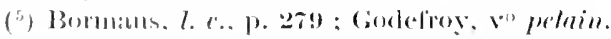




\section{liég. pirou}

De la meilleure raehe du troupeau, on dit au pays de Herve : c'est l' pirou d' l'atèlêye. En liégeois, c'èst l' pirou dè créartì signifie : "e'est le premier, l'homme le plus en vue du quartier ". Cette dernière expression se lit daus T'utî l' périquî, v. 606 ; pour l'expliquer, je disais ee qui suit dans le commentaire de eette pièce: "Sans doute une déformation de Pérom (le payss de l'or, l'Eldorado, d'oì le pays par excellenee), comme tend ì le prouver la forme nomuroise pèrou: vos n'èstez nin l' pérou (un phénix. un homne supérieur) " ( $\left.{ }^{1}\right)$. On pourrait ajouter, à l'appui, eet exemple de Forir : ci n'èst nin l' Pèrou. (en parlant d'um, objet) "eela n'a pas grande valeur" $\left({ }^{2}\right)$, et surtout cette phrase que j’ai entendue à Liège : il a trozé l' Pirou (sie), en parlant d'un homme qui, subitement, prend des allures de richard.

Je crois toutefois qu'il existe ici demx termes différents, qui, d'ailleurs, comme le montre ee dernier exemple, tendent à se confondre dans l'usage actuel : le nom propre Pèrou (= Pérou), et pirou.qui est d'origine flamande. Voiei en eflet, du Westrtaamsch Idioticon de De Bo et Samyn, un article dont je traduis ee qui eoncerne notre sujet:

piro, piero, m., dimin. pirootje. Propr. fr. pierrot, e.-ì-d. sorte de pierrot qui, aux fêtes de village, égaie le peuple avee Paillasse et Arlequin. II porte d'ordinaire un long habit blane avee de grands boutons, et il est toujours le dindon de la faree. - Dat is de piero : "e'est la fleur, l'élice", se dit proverbialement des personnes et des choses: " ("est le piero des poètes. Personne ne pent le surpasser sous le rapport du ehant, e'est le piero. Ce vin est le meilleur, c'est le piero $n$.

Tel est exactement le sens de notre " pirnu du troupeau, du quartier ». On y verra done un emprunt du flam. piro, piero, qui lui-même reproduit le fr. Pierrot (comp. à 'Toureoing. pireut = pierrot : .J. Watteeuw). On expliquera de même pirou "terme enlantin pour appeler le ehat " (G., II 227 ). Comparez le flam. piro "nom qu'on donne à un grand chien" (De Bo), le fr. pierrot. qui se dit du moineau. et le picard pirot. pirou " oie ", pour lequel Corblet. Gloss. étym. du picard, invoque froidenent... le celtique pirou.

\section{fr. potelé. liég. potale, pot'ler}

Pour le Dict. géméral. le fr. potelé (gras et rebondi) "semble clérivé

(') B.SW 48, p. 326.

( $\left.{ }^{2}\right)$ De même en lorrain, en parlant de personnes : ç' "' at m' lo Pìrou (Zeligzon, Teates lorrains, pp. 396, 405; 11et $\%, 1912)$. 
de l'adjectif pote " $\left({ }^{1}\right)$. Te crois. pour ma part. que ees deux mots n'ont aucun rapport ct que le wallon peut nous indiquer une piste meilleure.

Le liégeois pote. s. f... "fossette dans le sol " - qui vient. comme on sait. du germanique $\left(^{2}\right)$ - a domné les dérivés suivants : potê "flaque d'eau ": - putule "fossette n, c.-ì-d. 1. petit enfoneement dans un mur, uiche de statuette $\left({ }^{3}\right)$. 2. petit creux sur certaines parties du corps : cet enfant est tout ì potales. re n'est qu'une potale de tout son corps : pot'ler "faire une potale, surtout dans le sol ou dans la paroi d'une galerie de mine pour y introduire le bout d'un étançon" $\left(^{4}\right)$. - G.. II 250. croit devoir eu séparer pot'ler. v. n., "bouffir " et si pot'ler "se froncer. se crépir, se gripper ". En réalité, il s'agit du même mot. Une étuffe qui put'léye ou qui s' pot'lêye (on dit les deux en liégeois) forme des ereux et des bosses : trop tendue, elle se boursoufle à certaines places et se ereuse iu côté : fossetter et bossettes sont inséparables $\left(^{5}\right)$. Le mot pot'ler tire son origuine des fo settes que présente l'objet ; il est naturel toutefoic que cette image primitive puisse ensuite, dans eertains cas, seffacer devant l'image complémentaire, qui a plus de relief $\left({ }^{6}\right)$.

ce qui précède s’applique au fr. potelé. qu’il est difficile, à mes yeux. de séparer dı lién. poteler. Si notre dialecte ne semble pas connaître ce participe-adjectif au sens partieulier que le français lui attribue, cest pur aceident et eaprice de l'usage $\left({ }^{7}\right)$. Que le français dise d'un enfant gras of rebondi qu'il est tout potelé et le liégeois tot-a potales (tout is fossettes). roila qui est signifieatif et de nature a faire supposer

(1) Sur l'étymologie dn fr. pote, voy. ('j-dessus l'art. parcène.

(2) G., Il 24!1. De l'anglo-saxom p'yll, moyen néerl. put, putte, moyen bas all. pulle, ete., ou l'on veit généralement un emprunt du lat. puteus : puits.

( $\left.{ }^{3}\right)$ En ronchi, d'après llicart, potelle "petit enfoneement dans un mur (qui en indique la propriété) \%. - (iodelroy, vo postele, eonfond deux mots différents. Son

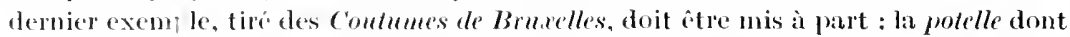
il y est question est celle de Héant. w. polule. Voy. anssi Delmotte : baide, potelle.

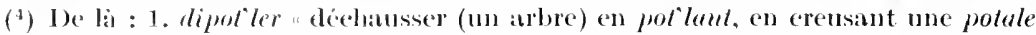
antour du pied "; il faut distinguer ee verbe de ‥ dipot"ler "déhancher, luxer ; proprement : oter de la polule"

(5) D'un torrain acedidenté, on dira : e' n'ent que fosses et bosses. En parlant d'un

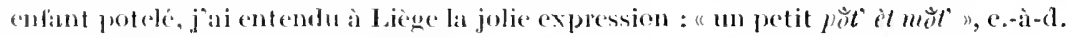
plein de potes (fouses) et dre mottes.

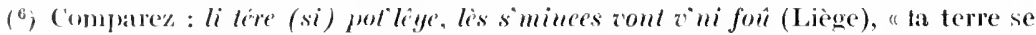
bemisomlle, les semences vut germer" : [on voit que le tabate est mûn quand]

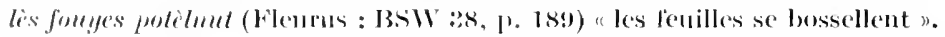

(7) I. Dict. awllon (vervictois) de Idroet seul a un article : pottle "potelé, gras et plein . Je erois que a n'est pas conforme a l'usage wallon, du moins à Liège. Ce dest ettre un gallic ismler. 
une origine commune; je ne puis croire à une simple coïneidence. Autre argument : pote éveille une idée désagréable qui ne pent eonvenir à potelé. Au contraire si l’on explique ee dernier par : "plein de fossettes ", l'image est délicate ; elle convient aux exemples les plus anciens et domne à l'expression plus de frâicheur et te grâce $\left({ }^{1}\right)$.

[Romania, t. xLy (1919), p. 189.]

\section{W. prat'ler (Verviers)}

Le vervictois prat'ler. d’après .J. Franck (BSW 5:3. p. 120), signifie "bavarder, faire la causette". Un pocte du terroir. Martin Lejeune.

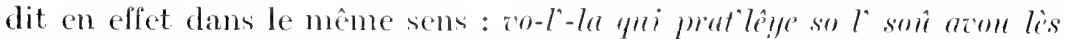
atènes (ib. 40, p. 15:3). De mône, à Clemont-Thimister, d’après le Dr liandaxhe, prater signifie "bavarder a tort et à travers. faire de longues causeries " : un prot levi on, en parlant d'un homme aussi bien que d'une femme. une prateule, cest un moulin i paroles, un havard ineonsidéré. qui invente souvent pomr le plaisir de campeter : cila. éèst

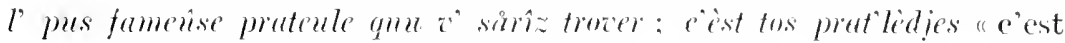
tous commérages". l)e même encore à tremblenr, ò l'on prononee bradler, i bradleye "il bavarde de choses insignifiantes". D'antre part,

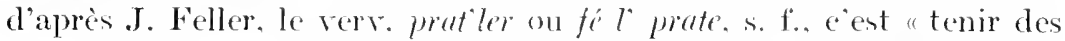
propos flatteurs et caressants " : mo vines né co fé l' prote atoli d mi, suzez! "ne venez pas encore me flatter, me earesser pour obtenir quelque chose $n$. - ce dernier seus ent dérivé : l'idée de flatterie intéressée s'attache naturellement à la lonuacité qui s"excee "autour" d'me persome, en vue de la eireonvenir et de la gagner. Lu surplus. le verviétois a emprunté ce mot au dialecte d'ilix-la-Chapelle, où prattele (bavarder bruyamment) se dit surtout du parler des Wallons $\left(^{2}\right)$. - On sait que le néerl. maten (havarder). prat (bahil. caquet) a domé d'autre part le nam. prante (conte. quolibet). manteler (dire les eontes; G.. II 255).

\section{liég. prèhale}

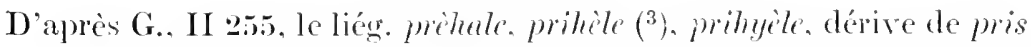
(= eaillé). La phonétinue contredit cette assortion : on aurait eu

(') Godefroy, $\mathrm{x}, 38 \%$. Le premier exemple, d'itprès le Dirt. gén., est de Christine de Pisan (xrye-xye s.) : "Si ne furent ne noires ne halees, Mais comme liz blanches et potellees". - Voy. aussi God., vi, 340, vo potelet, dont la linale a subi l'influenee probable de grandelet, grasselet. tentrelet.

$\left({ }^{2}\right)$ Müller, die Aachener Mundart (Aachen, 1836i). - A Eupen, prattele signifie "euire bruyamment, bouilir" ".onme le néerl, muttelen.

$\left(^{3}\right)$ G. écrit à tort prihele. Si cette forme existe, ae ne peut être que par une fausse aualogie avec mihon: prison. 
*prizale, *prizale, enmme on a prèzeûre, prizếre "présure ". Au reste, il suffira de compléter les données de G. pour que l'ét ymologie se dégage d'elle-même.

Outre les trois formes susdites, dont les deux dernières surtout existent avee le sens de "fromager "à Liège. Visé, Verviers. ThimisterClemont (BD 1910, pl). 52, 55), il faut considérer : prèhyale (Faymonville). priyale (Malmedy, Stavelot) "curelle " $\left(^{1}\right)$; - prihale (Huy), t. de vigneron, "récipient avee trous quon place au-dessus du pressoir"; - prèchale (Awenne). prihyele (Thimister) "presse pour extraire le jus des fruits dont on fait le cidre. le vinaigre et le poiré " $\left({ }^{2}\right)$. Cette dernière aeeption éelaire suffisamment l'origine de notre mot: une prihale, e'est tout d'abord un instrument pour pressurer les fruits ou le fromage $\left({ }^{3}\right)$ : e'est ensuite le réeipient de la presse. et enfin une sorte de eurelle.

On objectera que le diminutif de presse serait *prèssale. Cela est rrai ou du moins vraisemblable $\left({ }^{4}\right)$. Mais, en tenant compte surtout de prihyale, qui est assurément la forme la plus aneienne, nous pourons invoquer un type latin *pressia. qui lève toute difficulté $\left({ }^{5}\right)$.

Normalement, le groupe-hy-derient ch au Sud et perd son élément palatal y en liégeois-verviétois $\left({ }^{6}\right)$ : prèhyale, prihyèle se réduisent ainsi à prèhale, prihile. Reste la singularité de prihyèle. Je crois que cette forme provient de prihyale, dont la finale s'est modifiée sous l'influence de hyèle (écuelle) : la protonique à son tour sera devenue $i$ par dissimilation et aussi sous l'influence de pris (eomp. prèzềre : prizeûre).

(1) Villers écrit prèule (G., II 255); mais on prononce prèyale. La chute de $h$ est remarquable.

(2) La fuantité pressée c'appelle la prèch léye à Awenne.

( ${ }^{3}$ On peut le w. frehul (!), ' a il rattache au lat. fiseello "fromager ", ane. fr. feisselle. 11 y a sans donte erreur de lecture pour prehul.

(4) Le wallon présente toutefois des exemples eurieux de ss traité comme ssy. Tels ces dimimutifs de bosse et de fosse : bochale (Awemne) "avoine dressée et liée à lis tête seulement "[bossale ì IIarche-en-Famenne]; - fohule (G., II 526) "partie erense d'une ondulation de terrain" [foehule, m. s., a Marehe-en-Fam., tandis que fossule y ignifie" fossette à la jonc "]. - En revanche, $x>$ ss dans : assi, massule, passê, prasson, tèsson (axilem, maxillam, *paxellum, *paxonem, taxonem).

$\left.{ }^{5}\right)$ Le type *pressia (presse, eohne) est dans Meyer-Lübke, no $6 \mathbf{6}+3$, pour expligner litalicn prescia. - (omp. *arassia (graisse) $=$ w. crahe ; *grossia: ance fr. groisse ; *latia : anc. fr. laise; *spissia (anc. fr. espejsse) = w. spèche (Stavelot) " "patisseur d"un bois, fouré ", dout le non de fimille Delsupexhe.

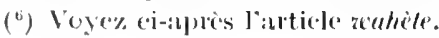




\section{liéng. purlê}

Dans le langage du houilleur liégeois, un purlê e'est un petit massif (stok) ou petit pilier (pilé) de eharbon. qu'on laisse dans la couche pour soutenir le toit de la galerie ou pour une autre raison. Nous y reeonnaissons le diminutif de pilé (latin pilare. ane. fr. piler : pilier). La forme régulière serait "piler̂́, qui répond à l'anc. fr. pilerial (dans un texte du xis ${ }^{\mathrm{e}}$ sièele : Dict. gón., $\mathrm{v}^{\circ}$ cimuise). De là. * pirlê par métathèse (roy. l'artiele gorlète); puis purlê, par assourdissentent de la royelle protonique ${ }^{(1)}$.

ane. fr. racueudre, racheudre (!)

Godefroy fait un article : "racueudre, racheudre. v. a.. atteindre rejoindre ", pour deux exemples du chroniqueur liégeois Jean d'Outremeuse, Myreur des histors. I 188. II 403. Nous y lisons : [il le] rachusut ou racusut ; ee qui signifie en effet . "il latteint, il le rejoint "; mais Godefroy a tort d'y roir un composé de cueudre (cueillir). Il oublie que, trois pages plus haut, son article raeonsuire enregistre deux autres exemples du même auteur $\left({ }^{2}\right)$, oì ruconsuit et ruconsent ont exactement la même signification. En réalité, rach husut n’est qu'une forme wallonne de raconsuit ; le liégeois moderne dit eneore rak'sut. du verbe rak'sûre "rattraper (qqu)". Il faut donc rayer l'article racueudre et ranger sous raconsuize les deux textes qui en sont arbitrairement séparés.

[Romanit, t. Xlv11 (1921), 1. 571.$]$

\section{w. rakète}

G., II 272, a ces deur articles:

1. rakète (ratissoire) Lobet. De rucler,

2. rakète (fruit qui ¿n'est pas parvenu à maturité) Lobet, Villers. Ainsi nommé parce qu'il racle le gosier ou les boyaux.

1. Les dietionnaires français ne comnaissant la ratissoire que comme outil de jardinier, il importe de noter que Lobet développe sa définition par eette glose : "outil de boulangrer... qui sert à détacher la pâte du pétrin ". - L'explieation de G. est aussi troj) sommaire. Si le terme signalé par Lobet vient de racler (qui se dit racler en w. et que Lobet écrit rauklé), ce ne peut être que par l'intermédiaire du fr. dial. raclette

(1) Voyez (p. 104, n. 3) rartiele furzéye.

( $\left.{ }^{2}\right)$ Myreur, II 492, Iv 192. Ajoutez 11111 : "ilh ont raconseus les Huens n. 


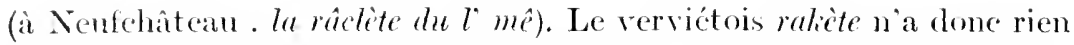
d'indigène : cent du français estropié $\left({ }^{1}\right)$. Les termes proprement wallons sont rèzre (liég.), rìî (verv. : Lobet; = "*rasière "), razite (nam. et rouchi). raclite (chestrolais).

․ Liexplieation de G. est plus ingéniense que solide. Il ne s'agit plus ici d’un terme are, on du moins isolé. emprumté du français, mais d'un mot de frappe bien wallone, attesté dans la région de Mamedy. de Verviers et de Herve. Nous y voyoms une forme variée du liég. -ard. cralicte "fruit mal venu, rahougri" (G.. I 135). De mime. le w. eralite "potite vache chétive" (ard. : Body. Iroc. des Agrie. : pays de IJerve) devient en rouchi raquéte "génisse fort maigre" (Hécart). De môme encore, crakite "crécelle" (verv. : Lobet; picard: (orblet) se réduit ì ralite en namurois. Pour $c r$ initial se réduisant à $r$, roy. une note de l'article ranse.

\section{hesb. rakiner, nam, rascrakiner}

G.. II 282. note sans l'expliquer l'expression namuroise : rascralizuer lis-ouchas "ronger les os". et M. Boigelot. de Namur : rascraliner "se restreindre en tout pour faire des économies ". Pirsoul ćcrit : rasealiner "ronger les os : retourner ses poches pour retrouver quelque chose qui doit sy trourer". I Stare, ratraskine signifie: "ramasser mimutieusement" : à Iluy, racraliner ou racaliner : "reeucillir les dernières grappes oublices dans les vignes ». I)e môme, rascaliner (Gives, BenAhin. Falmignoul), racasliner (Mecfle), racastine (Givet, Marche-enFancmuc). raculincr (Crehen). racaliner (Bleret, Bergilers) : "recueillir les dernicrs fruits restés sur l'arbre " $\left({ }^{2}\right)$.

Cette dernicre acception est générale dans les campagnes de Namur et de la Hesbaye. Elle doit ctre eonsidérée comme primitive. Il faut en effet partir de la forme pleine ro(s)craliner, altérée çà et là en ra(s)cal- (chute de r par dissimilation), racrasti- (métathèse de $s$ préfixe), rac(r)ak- (assourdissement de a. résultant de lamuïssement de cet s). Nous y recomnaissons le thème crati- des mots suivants : liég. et ard. crakite. a Ben-thin crakine, ard. crakin "fruit mal venu, rabougri" (G., I 135 ; Body, Vor. des lgric.). nam. cratchot, ote "rabougri. en parlant d'un fruitn $\left({ }^{3}\right)$. Sur le type du nam. rasgoter (oì ras- = lat.

(1) Pour la réduction de cl à $k$, roy. lauticle akinaige.

(2) Jai notic cu 1890 racrolimer it IIannut ; influence de croc" "eroc".

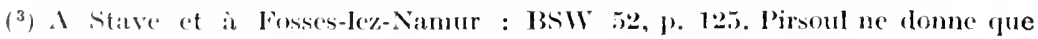
cratchole "petite pommen. Cratchol vient de *erak-illol, *aral-yol. - Ajoutez crakion (Alle-sur-Simois) " trognom de pomme ou de poire" = rakion (Fauvillers). 
re-ad-ex-). liég. ragoter "égoutter, recueillir les dernières gouttes", s'est formé, à l'aide du suffixe -iner. le verbe ra(s)crukiner, qui signifie proprement : "reeneillir les fruits de qualité infór ieure qu'on dédaigne dans la hâte de la grande cueillette" ; d'oǹ, en grénéral : "ramasser minutieusement"; puis. spéeialement : "ronger (des os), rogner (sur la dépense), vider ses poehes ou le fond de sa bourse".

M. Alph. Maréchal a noté à Racour (Lincent: Heshaye) une forme rakiner. qui s'éloigne des précédentes. Est-ce une réduction plus forte de racaliner on doit-on invoquer le néerl. ralien "râteler "? Nous admettrons plutôt qu'un verbe simple craliner $\left({ }^{1}\right)$ a subi l'aphérèse du $c$ initial. Sur ce phénomène, roy. ci-apris une notede l'article ranse.

\section{w. randje, rindje, rindjète (Malmedr)}

G.. II 311, a l'article: "ringeite (Malmedy) "une bouche " (sie), qu'il tient sans doute d'une manraise copie de Villers. Le manuscrit original de Villers (1793) porte : "rinjette. s. f., une boucle". Il faut érire rindjete, dimimutif de rindje, ramlje "boncle [de soulier] "qui vient de l'ane. h. all. hringa (boucle); voy. G. II 27\%. — La forme première rindje arehaïque à Mahmedy. survit a Faymonville : dle est devenue randje à Malmedy (Scius. 1s93). Ce passage de $\tilde{e}$ à $a$ mérite d'être remarqué : eomp). l'article sîprêt. - Les mots wallons rimlie (randje), rindjète derraient figurer dams Merer-Lüble. no 120 s. à côté de l'ane. fr. renge (range) "boucle du eeinturon ".

\section{lićg. et anc. fr. ranse}

G., II 279, définit le lién. ronse par "erêpe : étofle" $\left({ }^{2}\right)$. Pour préciser, disons qu'une ranse désigne une bande de crêpe noir qu'on porte. en signe de denil, autour du chapeau ou autou du bras gauehe. Le mot est bien eomnu à Liège, Verviers, Mahmedy ; on le cherche vainement dans les glossaires des autres régions. - Nous y recomnaissons le germ. leranz, krans "couromne" $\left({ }^{3}\right)$, avee réduction de $\mathrm{cr}$ initial à $r$,

(1) Signalé à Rosoux-Goyer par A. Xhignesse : qucand on-z-a codou (cueilli), lèsèfants vout crakiner.

$\left({ }^{2}\right)$ Pour toute explication, G. ajoute: "Comp. reize : linon clair?" Nais le rouchi reize n'a que faire ici; voy. Hécart, aux mots reize, réze, reche.

$\left({ }^{3}\right)$ Villers, Dict. malm. (manuserit, 1793), donne le diminutif cransliène, s. f. " tour de chapeau en or on en argent, une ganse; alentour d'un plat de choux, aceompagnement de saucisse ou enehonnarte, relevé ". Terme aujourd 'hui inusité. Bormans, Vocab. des houilleurs liégeois, as le verbe cranskiner "combler la coufâde ou la berlaine à la main avec de grosses houilles au des pierres ". Terme aujourd'hui 
phénomène qui a lieu surtout dans des nots d'origine germanique ${ }^{1}$ ). On a dit d'abord : une ranse de erêpe ". puis le déterminatif s'est sousentendu. - Le sens primitif ajparaît encore dans l'ane. fr. ranse, que Godefroy dome sans traduetion, avee ee texte de 1.480 : Atour rond, a la façon de Portugal. dont les bourreletz estoient a maniere de ranses et passoient par derriere ainsi que pattes de chapperons pour homme " (O. de la Marehe, ap. Laborde, Emanx). Nous expliquerons les mots soulignés par : "disposés en guise de couronnes "; voyez la figure, dans le Larousse illustré, ATO'R, et eomparez le fr. crancelin, qui vient de l'all. kränzlein.

[Romunia, t. xLy (1919), p. 191.]

\section{liég. råtchå ; nam. rinkinkin}

Le liég. râtcha signific " diseours long et fastidienx" $\left({ }^{2}\right)$. On trouve les formes rontchá (Liège : fé on - BSW 23, 214), rantchair (d'où le verbe rautchamer, à Jupille : ib.. 46, 202), ratchair (Verviers, Nessonvaux : miner on grtud - "grommeler beaucoup à propos de rien ": G.. II 264), et enfin rintchär à Blenny-Trenbleur, au N. de Liège. Le mot, eomme on voit, appartient à l'extrême N.-E. de la Wallonie. Pour expliquer le radieal, G. invoque le type latin raseare, qui a domné le w. răhî (raeler, frotter, crisser, bruire) ; mais il est manifeste que rahî et ratcha ne peurent avoir même origine. Je m'adresserai plutôt an néerlandais archaïque ringhen (sonner, faire entendre un son ; diminutif moderne rinkelen, joner avee de petits morceaux de métal peré. d'oì : faire du bruit ; eomp. l'angl. ring. faire résonner,

inusité, qui signilie proprement "couromer" (le euffat, le wagonnet). - Enfin, crunse, à IIabay-la-Nenve, désigne un "gateau rond à centre vide" (BSW 34, p. 290), et kranz, prononcé a lallemande, signifie a Nalmedy : "pâtisserie en forme de couronne ". Ce sont des emprunts de date moins ancienne que runse.

(') Comparez C'rnusiun, au vome siècle. ruisseau appelé aujourd'hui Ranseme (Chartes de lablatye de Stavelot-Mlalnedy, I 60$)$; fr. cracher : liég. rètchî ; erâtue et râzê " erosse " (Body, loc. des tounclicrs : BSW 10, p. 24:3); fr. crufe, ruflc (1)ict. gén.) ; fr. riquet (Littré), un des noms vulgaires du grillon, sans doute pour criquet (Scheler); voyez ci-dessus les articles rakicte, ratiucr et comparez encore le w. grimite, rénate "crevette" : llamand gcruat (Defrecheux, F'tume, p. 109).

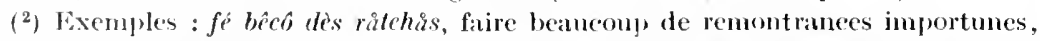
de rérimination monotones, ou de contes ridieules; llji " hoûte nin tos vos ritchis, je n'écoute pas tous vos bavardages ; cèst todl l' minme râtchi, c'est toujours la ménce ritournelle. Le mot mancfue dans Canbresier, Remacle, Lobet, Ilubert, Forir, ete. 
retentir; voy. Franck-ran Wyk. RIXkEL). Ce radical, eombiné arce le suffixe augmentatif et péjorat if -ard, aura donné d'abord rintehoir, forme que j'ai notée à 'Trembleur. près de la frontière linguistique. Pour le passage de la protonique $\tilde{e}$ à $\tilde{a}$ (d'oì $a$. $\tilde{o}$ ). voyez une note de l'article sâprề. Le sens prenier serait done : bruit monotone, semblable à celui du hochet que l'enfant agite sans eesse ; d'où : llux de paroles insignifiantes ou agaçantes.

En tout cas. le verbe néerl. rinkinken (faire du tintamarre ; forme allongée de rimkelen) a passé comme substant if dans le dialeete namurois: fé do rinkinkin (G.) "se rebéquer". fé di s"rinkinkin. (Pirsoul) "faire de ses embarras". Liexplication donnée par G., II 311, doit ctre éeartée.

\section{w. ratére (Verviers)}

Il faut rapprocher ees deux articles de Grandgannage :

S'Rater 's'abstenir) Lobet. Même mot (que fr. rater (manquer son coup), dont l'étym. nest pas certaine; voy. Soheler.

Sraté (s’abstenir) Lobet; = si rater ? avec ine acception analogne à celle du fr. ratier? donc propr. sabstenir par caprice.

Les deux articles se répètent (II 285 et 391 ) sans que le lecteur en soit averti. Mais eette inadvertance n'est rien à côté de l'erreur urave qu'il était pourtant facile d'ériter. Le Dict. (verviétois) de Lohet écrit "srater "qu'il faut lire s" retéce ; quand le r. est de la $1^{\mathrm{e}}$ eonjugaison, Lobet écrit $\dot{e}$ : "sratrapé. sravisé $=$ s'ratraper, s'raciser. Le mème lexicographe enregistre p. 58 : "ater [lire atére], attraper, atteindre, saisir ", et p. 57s : "ter [lire tére]. tenir ". On dit alussi à Vervicrs ratére (retenir), quil oublie de noter ì la lettrine R. - La conjecture de G. tombe ainsi d'elle-nicme.

Tére, doublet reviétois de t’ui (tenir), s’est probablement dégagé du futur térè, liég. timrè, ou s'est formé sur le tỵpe lére. lérè (lire, lirai).

[1élanges Kurth, 1908, t. II, p. 322. — G., I 3:3, commet une antre errenr à propos du verv. atére ("atteindre "), qu"il tire du lat. attingere. C"est un doublet de $a t^{\prime} n i$ «tenir i, toucher is ", ane. fr. attenir.]

\section{iv. ratro}

1)'après G.. II 285 , le malmédien rutro signifie " 1 . raceroc, retard; 2. mereuriale, réprimande ". La souree de G. est le dietionnaire manuserit de Villers (1793), lequel porte textuellement: "ratrot, s. m., buffe 
mereuriale, réprimande : il signifie amsi retardement, hanieroche". G. a modifié loordre des significations parce qu il considère ratro comme une altération de racroc. Il faut semarquer toutefois que Villers a auni un article racrot "accroc, obstacle. cmbarras dans une affaire", et quen définissant ratrot. il ne songe pas à "raceroc" : il tient môme pour seeombaire le sens de "retardement". De son côté, Dasnoy ne roit dams le chestrolais mftrot qu"um srmonyme de chatou "semonee ". Le mot est ou du moins était aussi liégeois. On le trouve dans les IIypocondes (1750) : i cint dacu on bon ratro "il vient davoir une sévère leçon ". L’éditeur Bailleux, qui ajoute en note : Il faut probablement lire racro " ${ }^{1}$ ). verse dans la meme erreur que Grandgagnage.

Il faut roir en effet dans ratro (ou mieux ratros) le déverbal de rotrossî. Villers ne domne que le malm. su rutross? "trouser sa robe. ses vêtements : mais rutrossî est comnu $1^{\circ}$ à Soraing et à Fléron (près de Liège), au sen propre: "relever" (un tas de honille. de briques. de bois. ete.), le rendre plus élevé et moins ćtendu. par ex. pour dégager un passauc $\left({ }^{2}\right)$ : - - 2o à Liège. au sem figuré du fr. pop. "ramasser " qqun. le maltraiter de coups ou de paroles : i s'a fît ratrossî d'on mêsse gos" "il sent fait rosser d’importance ". litt. "d"un maitre gônt" $\left({ }^{3}\right)$. Le w. rutres répond donc au fir. pop. et dial. "ramassen. Pour la lormation, comparez embaras. ramas, rabais. tracas. etc. Le sems de "retardement". domné par Villers, pent rénulter d'une confusion avec racro.

\section{liég. rav'rouhe}

\section{G. II $28 \%$, a cet article:}

raverouhe, razerou, rubrouhe (la rave sauvalge, ravenelle des moissons: raphanun raphanist rum, ru la moutarde ou sénevé des champs : sinapis arvensis; Rouchi razeluque, razeluque (sorte de sénevé), pieard raceluke (rave samvage). Il est vatiscmiblable que ce mot picard est composé de raze + LuQue, Leuque et que ce deruier éloment répond à lock daus laangl. charlock (m. sign.).lequel lock rejrésente lakll. lauch, néerl. look ( porreau, ail), d'oin la finale liég. -rouhe.

Autres formes wallonmes : racelusque. -nsse (Mons: Sigart), réceluche (Cherain), rabrouche (Laroche. Bonillon, Namur. La Hestre), revelihe

(1) Thiatre lirg.. éd. de 18.54, P. 12t: IIypocondes, acte 11, sc. 5.

(2) 1، fr. retrousser a un sens analogue. - A Verviers, ratrossì lès liènçles, t. de

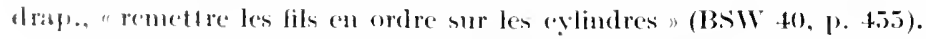

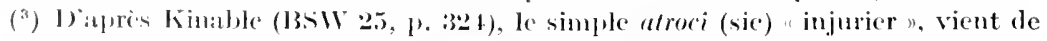

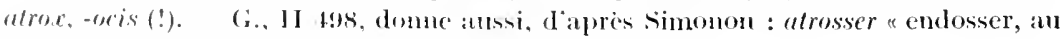
lig. Je nia pas retrouve ce verbe en liegeois moderne. 
(Wallonie malmédicune. G.. II z9s), rizelihe (Hockai), récelouhe (Spa), rèzelohe (La Reid) ( ${ }^{1}$ ).

Ein France : rencloche (Ilante-Marne), refoluche (Aisue), raveluque

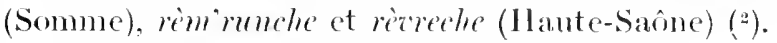

L'analyse que G. tente de ce mot n'est pas conforme à la réalité des faits linguistiques; clle est controdite notamment par la forme montoise razchusque. M. A. TYhomas. Mélunges d'étym. framé, p. 9s, signale dans les langues romanes l'existence d'un suffixe -usea (labrusea. asimusca, molluseu, ete.). et il ajonte qu'il fant pent-ître ansi reconnaitre ce suflixe, an moins a l'origine, dans le fr. dialectal rachseche.

Outre le montois, qui a conservé intactes les comsomes latines. les formes wallonmes en -ombe et en -nhe confirment rette supposition (ef. bûsea : bouhe. fr. buche; musca : mohe. fr. monche). A Liège reclouhe s'est altéré d'abord en rev'mule, puis en rubroule. Liaspiration funale est tombée dans rar ron, eomme dans mbrou, aceident (G., II :63). pom rubomhe : roudm (panicr ou toile sous me charrette: Forir). pour romdouhe. ete.

[Mélanges Kurth. 1908, t. II, p. 3322. - Voy. Meyer-Lübke, 1n 7050. A noter le flam. hazerruische qui, d'après Schuermans, designe dans le Hageland la montarde sauvage, et le west-flam. aremische, huzrmische qui, d'après De 130, désigne le colza jusqu’à sa ilomisom. (ce sont de nouvelles altérutions du type roman (influence du néerl. hezer, avoine).]

\section{w. rawète}

Le Toeduleire de Cheresin. par $\mathrm{A}$. Servais. a un article ainsi libcllé (BSW 50, 1. 5:3:3) :

rawète, s. f., 1. Ce fuion donne en sus d'un achat : zos mi l' dinroz pol razcite, vous me le donnerez par dessus le marché. - 2. Lien de paille pour lier les gerbes de céréales.

A première vue, on pourrait eroire que l'anteur a raison de rémir sous un même chef ces deux simifieations. Un examen plus attentif montre toutefois qu'il s’agit de deux termes différents, dont la ressemblance est fortuite.

(') Feller, Flore populaire scullome (Bull. de Folkl., II 117 ).

${ }^{2}$ ) Roland, Flore popmluire, II r2. (On y lit que le w. rozerombe vient du lat. rapa eruer, ce qui est phonétiquement impossible : - ûca dome -oze en wallon; voy. ci-après l'art. sohe. 
1. Ruarte "lien de paille pour lier les gerbes de céréales " n'a pas, en wallon, une aire bien étendue (Cherain. Marche-en-Famenne, Fanvillers. (iney. Sery-lbée); on emploie d'ordinaire le terme général loyin, licn. Cependant, en pays gaumais (Ste-Marie-sur-Semois, Tintigny : PI) 1908 , p. 75 et 79$)$, nous relevons la forme rociète.

C"est le lat retorta, participe féminin de *retorquẽ re "retordre, tordre en arrière on en sen' contraire ". Comparez. pour la tonique du w. razète, le w. paìte, fàte. màte, càde. tàde (porte forte, morte, corde, tordre); le $t$ intérieur entre voyelles a disparu suivant la règle, et la protonique $e$ est devenue $a$, probablement sous l'influence de $r$. La forme graumaise est d'explication plus délicate. Le gaumais dit pồte, fôrte, môrte, côrde, tôrde, avee ô provenant de done admettre les étapes suivantes: *reworte, *rezote. *rocote, d'où rocite par dissimilation et sous l'influence des nombreux diminutifs en -ite. Il est d'ailleurs probable que ces deux raisons (plus l'influence de l'homonyme ravète) ont également agi pour transformer *racute en ravite dans la région Cherain-Marche, où l'on prononee caude, ficate, et non ewède. facite. comme en lićgeois.

Quoi qu'il en soit de ces diflicultés phonétiques. jl est impossible de séparer notre mot $1^{\circ}$ de l'ane. fr. rente "lien formé d'une branche souple et pliante tordue sur elle-même ". dont Godefroy énumère les formes redorte. romte, raertie, roarte, ronite, rotte, riotte, et dont l'étymologie retorta est pleinement assurée; $2^{\circ} \mathrm{du} \mathrm{fr}$. dialectal rouettes, $t$. de forestier, "brins de taillis dont on fait des liens ". où Littré voyait un diminutif de mue. "le lien étant tordu en rond ", et que Tobler rattache aree raison au lat. retorta ( $/$. für iergl. sprachf., xxm, +1s).

2. L'autre rocète" surcroit, petit supplément gratuit de marchandise" existe, sous la même forme et avec le môme sens, à peu près dans toute la région wallonne proprement dite. Outre racète. il importe de constater que le llamurois dit aussi acite, d'après G.. II 208. et Pirsoul ; ee

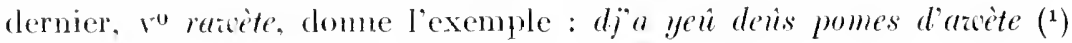
"j’ai reçu deux pommes en plus " $\left({ }^{2}\right)$.

(1) On trouve la même expression clans J. Cohon, Chansons, p. 119 (Namur, 1862).

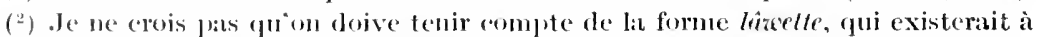

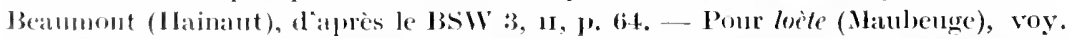
ci-rlessus, p. 172, liurticle lourete. - Quant au montois royete, ancuel sigart parait illentifier le w, razete, rest bien, semble-t-il, le diminutif de roye (ratie) ; voy. Ilécart :

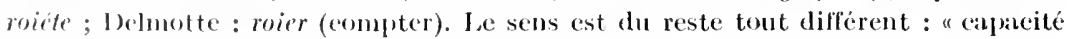
d'un valse ; lation, pitincen". 
Grandgagnage n’indique pas d’étymologie. Scheler, son ćditeur, émet deux eonjectures : racite lui paraît signifier au propre "la petite rave que les légumières ajoutent an marché "(!) ; tandis que ariète répondrait à " un diminutif de noe, oie " (!). 1)'autres rattachent notre mot au w. raceke, race' "ravoir" (1), ou au lat. re-adepta "(la partie) obtenue ou gagnée" $\left({ }^{2}\right)$, on encore à un type lat. *aredd-itta $\left({ }^{3}\right)$.

Pour moi, il est ćrident qüil faut partir du simple awète. conservé en namurois. Il me parait hors de doute également que ect awite correspond à l'ane. fr. noite (augmentation; avantage, profit ; du lat. *arlaucta), fém. du part. passé de aovie, lat. *adauge ére ( $\left.{ }^{4}\right)$. La rawite (lat. * re-adaneta), c'est donc ce qui s'ajoute à l'emplette. la radjoute, eomme on dit, exactement dans le même sens, ì Hugies (Borinage), le surjét à Ath et à Mons, le dézeir à Wiers et a condé (comp. l'all. obeudrein; luxemb. beilocht. de beilegen: ajouter). L'emploi du préfixe intensif $r$-est bien conforme aux habitudes de nos dialectes (eomp. ratinde, attendre ; ricevi. enérir ; mầ. oublicr). - Godefroy ne donne pas *ranite, mais il enregistre sornoite "suraugmentation, surcroit n. composé avee le préfixe sor-. - Voyez ci-après l'article tanarite.

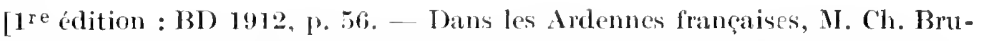

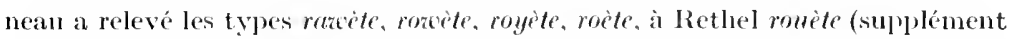
gratuit). Or, dans cette région, - ancta donnerait -zote. Il faut done admettre que le primitif *azute y at devenu razète sous l'influence des diminutifs en -ite, puis rowète, rouite sous l'influence de rone, lat. rota.]

w. rèni, roni, runin ; arèni ; rune; ete.

G., II 294, ne donne pas d'explication. Voici son article:

rèn1 (Vetviers, Malmedy), rien, oljet sans valeur. babiole, fanfrehuche ; [(1.jège : Forir) homme de néant, vaurien] ; (Namur), rènan, rènin, ronan, ront.

(2) .T. Delbouf, BSW 10, p. 14t ; P. Marchot, Le patois de St-IItbert, P. 37 (1990); Ad. Grignard, BSW 50, p. 415.

(2) P. Marelot, dans lia Rezue des langues romanes, t. 35 (1891), p. 438.

( $\left.{ }^{3}\right)$ Niederländer, Mumdart zon Namur, \$ $3: 2 b$.

( $\left.{ }^{4}\right)$ God., $v^{0}$ aoite, eite aussi la forme atoite. J'en rapprocherai l'article araite, que Godefroy ne peut traduire, dans le Règlement des boulangers d'Arras en 1355: "nuls ne poct vendre pain fors le denrée un denier sans domer bort n'azaite". Le sens est que le pain doit se vendre un denier et que le vendenr ne peut donner de supplément gracieux, de (r)azichte. Le syn. bort (bord?) n"est pass noté dans Godefroy. 
Ce dernier mot est employé par les marchands de bestiaux pour désigner une bete sills valeur $\left({ }^{1}\right)$.

Rèni, arec $i$ bref $\left(^{2}\right)$, appartient à la régrion de Verviers, Herve, Spa, Stavelot. Wamedy : on p’tit rini, dès p’tits rinis "de menus objets ",

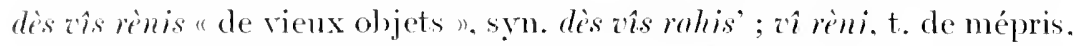
"vieille femme ". En dépit de l'assertion de Forir, le mot paraît ctre incommu à Liège. - I Erezce et à Bovigny, on zî̀ réni désigne " un vieux réduit ", et amssi ce qu'on y remise : " mu objet de rebut "; à Vielsalm, on rini. c'est " $m$ manrais lien, $m$ repaire de vauriens " $\left({ }^{3}\right)$.

cette forme isolée ne peut. à elle seule. nous liver le secret de son origine: on ferait fansse ronte si l'on prétendait, par exemple. y voir un dérivé du lat. rem, rien. wr rin. Iei encore, la méthode eonsiste à reeneillir le plus grand nombre possible de variantes dialectales. G. noms donne bien quatre formes namuroises. mais ses renseignements. du moins en ce qui eoneerne rènan. roman. ne sont pas confirmés : Pirsonl ne signale que le nam. rumins. s. m. pl., "ordures, saletés, balaymres". I'après M. Mph. Maréchal, les vieux Namurois ne conmaissent que rinim, avee le sens général de "rebut " crickyoz qui dïa

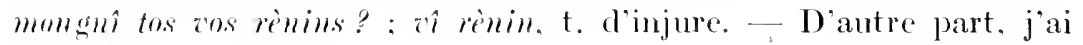
recueilli :

dès rèmins (I)orinne), des saletés : ș̣n. dìs mamuèstès;

dès mmins (Ben-Ahin, solières). des objets de rebut : (Barvanx-Condroz, Tohogne) de menus déhris ;

dès mmis (Perwez), des saletés. des détritus ; lès ronis (Ste-Darie-Geest, Jodoigne), la vicille paille (pui reste, d’année en année, au fond dn gerbier ; doù, en géméral, vicille paille :

lis rognis (Marilles, Noduwez). les debris de paille hachée par le hattage; tès rognis (Ambresin-W.asseiges), des débris, des détritus ; on rogni, un homme te rien.

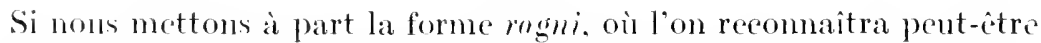
linfluence de "pogner", il reste un radical rè-, rom-, run-à identifier. Or le verbe bien conmu qui signifie "rouiller" présente des variations

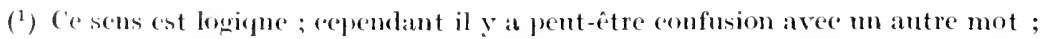
du moins à Jodoigne, P'erepot-('haussce (Brabant), rône s. m., désigne un animal "hitif ; cent propr. un "ruines 1. 2.28(i).

(2) A Herve on prononce anssi $-\hat{\imath}$, sims donte soms linfluence des nombremx mots it sullixe -î, fr. -ier.

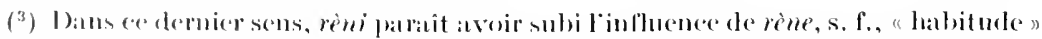

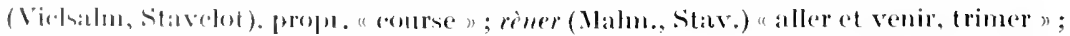

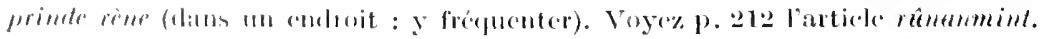


semblables, dont la répartition géographique concorde en général avec l'aire des formes précitées :

arèni, èrèn Liège, Vervicrs, spa, Stavelot : èmi Ciney : arem favec a- = lat. in-) Vonêche ;

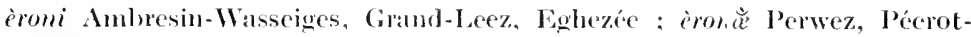
Cluanssée ;

aruni Awenne ; irmi Ellezelles (Lessines) : irmi (1) Namur, Wavre. BenAlin, Villers-Ste-Gertmde, Roy, Marehe-en-Famenue, Erezée, Tohogne ; (anc. w. enrunie dans .Jean d'Outremense ; enmgni Froissart, xv z9o, var.) ; dérivé èrum'té Odeigne, -tié Lasoche (pour *èrmulté, tić) ;

arigni stave; èrigni Namur ; dirivé : arignté Warlin, Houlfalize, Laneuvilleall-Bois, Warisy : arègritè lauvillers :

arougni Oisy, Gros-Fays : dérivé de rougne (ib.) "rouille "; èroyi Dorinne ; irouyi Ioncean-sur-samble ; aromyi Vonêthe (= ane. fr. enroillir) $\left({ }^{2}\right)$.

Certains villages, an $\mathrm{N}$. de la provinee de Luxcubourg. notamment Villers-Ste-Gertrule, Hrezée. Odeigne, comnaisent même runes. s. f.

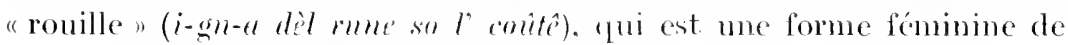
l'ane. fr. ruyn, représentast du lat. robîginem. D'autre part, l'ane. w. ruinins an sens de "roulle se reneontre trois fois dans les sermons de

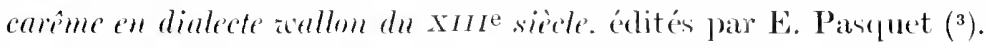

De cet ensemble de laits nous pourons tirer les conchusions suivantes.

Les rerbes èmi, èmi, imini. armi. arini, ete., sont composés dn radieal de robîginem et des prétixes in-ou ad-, - ct non tirés de aeruginem. comme le prátend G.. I 25.5. De même le liég. dirini (nam. dismui) "dérouiller" n'est pas mis pour distrimi (G.. I 175). mais se lécompose en di-rin-i $\left.{ }^{4}\right)$.

(1) D'où, par métathèse réciproque, èmuri à Farciennes.

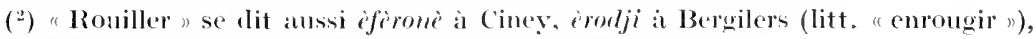
druhtiner à stavelot-Malncdy (voy. l'article rombin).

${ }^{(3)}$ Extrait du t. xu des Mémoires conromes's de I'Acad. royale de Belgique, 1888, p. 25. Le mot n’étant pas dans forl., voici res passiges: "N"asembles mie les anoirs en terre que li ruinins et le uermissicl delissent [?] et manjuent... mais assembles les trésors en chiel la u ruinius nel porat contrir... la 11 ruinius ne vers ne l'empiront". - L'éditenr et le rapportenr Sicheler s'évertuent, p. 19, it expliqpuer delissent. Ne serait-ce pas tout simplement une erreur du scribe pour démolissent? Conparez dans God., t. x, p. (;00), Ruldes, Ja traduction du même texte sacré daus un sermon de carêne édité en 1519: "en terre la on la renillie et tigne demolit".

( ${ }^{4}$ On a forgé par plaisanterie dizarèn, dizèremi daus les "scies "suivantes : nosse crama (ermail) ì-st-arèni, $i$ "' si dizarenirè nin (Vonche); gn-a m' fier'mint (serpe) qu'è-st-èrèni, nèl dizèrènichì rín nin bin? (Ciney'). 
Les substantifs muin. rènim, romi, rèmi sont dérivés du même radieal a labide des suffixes -in. - $i$ (fr. -in, -il). Les variations de la protonique sont len mêmes que dans les verhes préeités. Bien que le sens de "rouille" ait complètement disparu et que, dans certains cas, la filiation sémantique ne soit pas des plus claires. il fant admettre que le sens primitif de "objet rouillé " a clonné. par extension, celıj de "objet mis aiu rebut", puis echui de "détritus. déchet, débris sans valeur".

[Remaniement de BD 1913, p. 55. - Meyer-Lühke, no 7348 , eite le $w$, "rmme [?] "rouille ", qui ne nous est pas comm.]

\section{w. rê̂dê}

G., II 502. éerit : "Brudai. t. de hat., hois qui relie le halmustok ou timon du gourernail avec la partic postérieure du gouvernail ". -

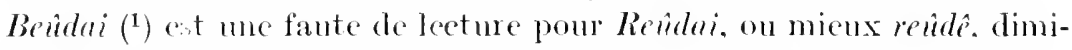
nutif en -ellum de rigidum : ren. lir. roide, raide. Le batelier de la Mense dé igne par là une perche arpuée destinće a renforeer le gouvernail lorsque le safran se trouve à un nivean phus bas fue la barre. ce qui est le cas pour le hima et la mignole. anciens bateaus de Meuse i poupe en retrait et non verticale.

[Romrania, 1911, t. xL, ]. 329. Cf. Meyer-Lübke, no 7314. - G., II 297, donne reûd "perche bien droite " d’après Simonon. I'iti relevé aussi ee not dans le vocabulaire des houjlenrs, à seraing : il designe une tige de fer disposce horizontalement dauss la mine à l'entréc d'un chargeage : on abaisse ce reùrlê pou $\mathrm{r}$ tirer le cible, crèrelí, qui actionne une sonnerie placóe à la surlace. - Entin, a Esneux, d'après M. Edgar Renard, reüde (" roideau " en 1766i) survit dans un num de lieu déngnant un talus, un raidillon. A Alle-sur-Semois, rèdé "raidillon" ent encore du langage commant.]

\section{w. rêvioûles. wêroûles}

G.. II 272. dit simplement : "raicimile, rêrimule, à Malm. an phur.

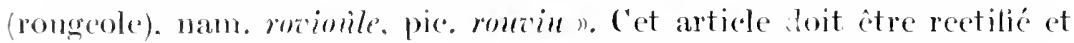
rompleté comme suit.

Dans le domaine wallon proprement dit. Ies termes désignant la moneole se repartissent en trois groupes:

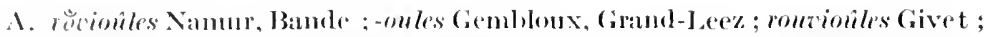

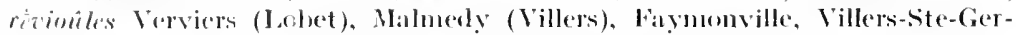

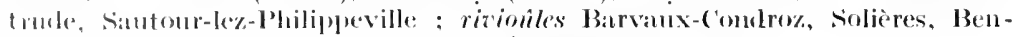
Ahin, Hentre-en-Finneme, Dorimne : rĕ́cioules Wissejges.

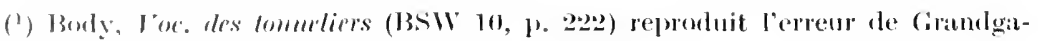

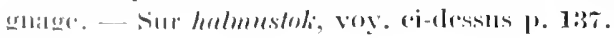


B. rêvioûles Liège, Trembleur, Ambresin, Wasseiges ; révioûles Eghezée, -oule, Ste-Marie-Geest ; rêrvioûles Noduwez, Marilles.

C. zeêroûles Stavelot, Vielsalm, Bovigny, Lutrebois, Ramont, Wardin ; wéroûles ( $\dot{\varepsilon}$ ?) Cherain ; wè̀roûles ( $\hat{e}$ ?) Doncols.

Le mot est toujours an pluriel. eomme beaucoup d'autres noms de maladies que le peuple désigne d'après leurs multiples manifestations extérieures : lès crèhiohles ou crèhinces "les adénites de la eroissance ", lès mowcites "Oreillons ", lès rainnètes "le muguet ", ete.

Le groupe $A$, dont rovionlle est la forme le mieux conservée. vient, comme on sait, du lat. *rubeola (1). qui a donné également le fr. rougeole. Au Sud de la province de Luxembourg (Ortheuville, Sibret, Fauvillers, Bertrix, Chairière, Gros-Fayss; gaumais), on dit lès rondjètes, ee qui logiquement équivaut à rozioûles : le hennuyer dit lès roudjeûrs (Beammont, Chapelle-lez-Herlaimont.) - Dans ee groupe A. la protonique reste brève, tout en présentant des variations aisément explicables. - Ce qui earactérise le groupe B. e'est la longueur de la protonique $\grave{e}, \stackrel{e}{e}$, où l'on attendrait la brève. Je pensais nagruèrc (IBD 191:3, p. 5s) qu'il faut sans doute attribuer cette anomalie à des influences analogiques; quant au groupe C: clont je méeonnaissais I'importanee. j’y royais une métathèse de rêteintêles. Je crois aujourdhui que le contraire s'est produit: wînôles représente sûrement Ic lat. variola ( $\left.{ }^{2}\right)$ (anc. fr. zairole. fr. zérole. proprement "maladie qui tache la peau ").

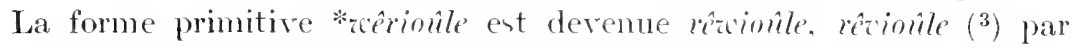
métathèse. sous l'influenec de *rubeola. qui a dî exister aussi à Liège, Ambresin. etc., puisquion le retrouve it Verviers. Masseiges, etc., e'est-à-dire dans le voisinage immédiat.

\section{W. rihîre (Malmeḍ̂)}

Le dictionnaire manuscrit de Villers (Malmedy. 1793) a l'article : "rixhire, f., bonne chère, frérie, festin, fricot, frigousse ". que G.. II :30\%, voudrait expliquer par riche chèe. La phonétique ne peut admettre eette conjecture. Il n'est pas non plus pernis de pencer au préfixe re(liég. ri-), qui serait $m$ - en malmédien. Il faut se reporter à un autre

(1) Niederländer, Mundurt zon Namur, st1. De son côté, M. A. Thomas (Mélanges d'étyu. fi., p. 134) tire de *rubeolus le fr. rouvieur (espèce de gale) et le pic. rouviu (rougeole). - Meyer-Lübke, $n^{\circ} 7405$, ne parle point de nos termes wallons.

(2) Sur $v$ latin $>$ w wallon, voyez l'article reére.

( $\left.{ }^{3}\right)$ Pour le ehangement de w en $z$, comp. a lawire (Iatmedy) : a l'azire (Liège) "au hasard, an petit bonlseur", du lat. *agurium "heur". 
artiele de Villers : "xhirire. f., un grand frieot, une frigousse " : lisez hirîre proprement "*(dé)chirière ". ce qui vaut bien en énergie le fr. crevaille. De là, rihîre par métathèse réeiproque. Voici, au surplus, quelques exemples de ec phénomène fréquent dans les parlers populaires : imuri à Farciennes. pour èmmi (voy. l'art. rèi) ; hourin pour rouhin (roy. l'art. rouhin) ; adaglé pour agad'lé "accoutré " (G.. I 7 ) ; rabada à côté de barada "bavolet" (ib., +6) ; guèdaine "dégaine » (ib.. 247) ; gabare "bagarre" (ib., 22 7 ) ; erotale ou trocale (Ciney) "crotte "; lânî (Faymonville), à côté du malm. nâlî "corclon de soulier "; le gaumais easmarâde "mascarade"; le meusien queubie "béquille" ; le pieard fichomner "chiffomer" ; le flamand begaren pour gebaren (De Bo, p. 82) ; le w. gladio on djaglo (Givet) "rosean, glaïeul "; aignh, wignter (Liège) "glapir ". gnizer (Ovifat) ; désmâhoné (Neurillesous-Huy), pour le liég. dishamoné "dépenaillé "; etc.

\section{liég. rimbion, rêbion}

G.. II 292. donne sans explication : rimbion. rêbion "léger sillage produit à la surface de l'eau par une pierre qui est un peu au-dessous de la surface $\left({ }^{1}\right)$. ou par le passage d'un poisson ". — C'est, avec un sems figuré, une forme altérée de ringuion ("sillon qu'on pratique en rinsuiant. e.-à-d. en déchammant, en domnant le premier labour à une terre ": G., II 294). De même le rouehi strunguion, nam. strunguion "étranguillon ". devient le liég. strambion, mahm. strombion (G.. II 4o6). (Quant an verbe ringû " déchaumer ", c'est l'anc. fr. renguillier (labourer). quion dérive du francique ring de môme que le fr. rang; royez Clrix. $n^{\circ} 1690$.

\section{w. rouhin. rouhis'. luhin. èruh'tiner}

G.. II 329, a les articles suivants :

rouhin (mare de café, sédiment en général). Saus doute pour *drouhin $=$ drousin, par aphérèse de l'initiale.

rouhis', Limbourg (portion de terrain qu'on ne pent mettre en culture à (ause de sa mauvatise qualité). — Prob. dérivé du flam. rusch (jone) : propr. marèatge où ne vient que du jonc.

La second de cer termes se retronve $1^{\circ}$ à Lineé-Sprimont, oì rouhis" désigne un terrain ineulte et broussaillewx (syn. un romhis' : "roneis"); 20 à Jupille. comme terme arehaïque de culture : rinetî lès rouhis' dèl houbire " enlever les fenilles et débris de saments qui jonchent la hou-

(1) C"est-ì-dire : "qui émerge à peine ou même affleure ". 
blonnière apres la cueillette ". - I Liège nème. jai noté dés rouhis' on dis rouhins "du mare de café n. Ce dernicr est bien comm de non lexicographes (G.. Lobet. Forir. Gothicr. Willem). Par métathèse réciproque, il s"altère parfois en hourin (1).

Les conjectures de $G$. ne nous retiendront pas. I] ra de soi que rouhin et roukis" ont le même radical, lequel s'explique au micus par l’ane. h. all. rûh (all. r(mh) "âpre, rude, raboteux". D)ans tous les exemples recueillis. il sagit de matières rugueuses et sales. de résidus qui encrassent, se déjosent et forment erôte. Pour le traitencnt de la voyelle, on comparera houbite. houmer, qui dérivent de l'ane. h. all. hûbe, scûm (all. haube. schaum).

Les crô̂tes de lait qui courrent la tête de certains enfants s'appellent dés mhins à Stavelot, dès luhins à Malmedy. Je tiens cette dernière forme pour altérée. et ruhin pour une forme variće de rouhin ( $\left.{ }^{2}\right)$. avee un sens spécial qui paraitra naturel si l'on part de l’étymon proposé. Pour l'alternance on : $u$ i la protonique. eomparez droussin, froulin (Liège, Verviers) : drussin. fruhin (Stavelot).

Fnfin la même région de Starelot-Malmedy posède en propre ces deur mots : èulitiner "rouiller" durulitiner "dérouiller ". Selon toute vraisemblance. nous avons affaire au mème radical pourvu d'un suflixe diminutif ; le sens premier serait done : "enerasser. décraser " $\left({ }^{3}\right)$.

\section{anc. w. rûlâve}

Godefroy ne peut définir mlame (sic) dans ce texte liégeois : "quant les massuirs veullent pessier $[=p \dot{h} \hat{\imath}$. pèeher $]$. se pessent d'autres bons harpatz melanes" (1+51, (h. den finanees. xr. p. 20. Arch. Liège). Il faut lire harnati (liég. hèrna. ane. fr. harmois. "engin. outil ") rulazes, ou mieux rultâes. Ce mot d'aneien liégeois, qui ne se reneontre que dans ce texte, dérive à l'aide du suflixe aze. fr. -able. du w. rhle. ane. fro rieule, lat. regula. Il signific "régulier, conforme à la règle, légal ". Pour la formation, comparez raisomable "conforme à la raison". Voyez rînammint.

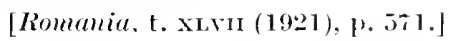

(1) Forme employée par certains auteur's liégeois, notamment Jean Bury ; voy. larticle rihire. - Chose curieuse, G., II 328, donne routins dh houbion "débris de fleurs de houblon " et, dautre part, je relève à Liers-lez-Liège dès roudis" "du mare de eafé ". Ces mots dérivent-ils directement de roudi (G.. II 32-7) ou sont-ils altérés de rouhin, rouhis" sous lintluence de ce rouli?

$\left(^{2}\right)$ J'ai entendu à Coo-lez-Stavelot dès rouhins (" croutes de lait ").

$\left({ }^{3}\right)$ Godefroy a un exemple de l'anc. fr. enruhir " rendre rogue, arrogant ". Faut-il le rattacher à la même famille? 


\section{w. rûnanmint (Malmeḍ̣, Stavelot)}

G.. II 33:. a cet artiele:

runant-chemin Malm. ("grand chemin"). D'un verbe rumer = rèner (courir) ; comp. angl. rum ? - runanmint Jalm. (" communément, vulgairement "). Du même rèner (courir, être en cours) que le précédent.

La forme runer n'existe pas. Villers. en 1\%93, donne seulement le malm. renes "aller et venir". reneur "couratier", renajuit "le juif errant ". à côté de runan climin. runanmin. Il faut éerire rèner, rènêur, rinâ $[=$ rinant $]$ djai . et manant tchimin. runammint. Il ne peut y avoir de rapport entre rèner et les deux derniers mots.qui ont $\hat{u}$ long $\left(^{1}\right)$. Dans mon Focalulaire de Staielot. jai noté muammint ou mulammint. d'après deux vieux stavelotains. J'aurais pu écrire âlong : si je ne l'ai pas fait. cest qu'en réalité cet u ust de longueur moyenne : il tend à s"abréger sous linfluence du préfixe m- (fr. re-). Quoi quil en soit, la forme mikumint parle assez clairement : il faut partir de mile "règle " (roṿ. l'art. mîläe). De là. l'adjectif rilant "régulier, conforne à la règle " et l'adrerbe riklammint : " 1 . régulièrement. normalement, 2 . commmnément. vulgairement. couramment". Les liquides $l$ et $n$ permutent sourent : ainsi. Villers écrit ralonsue. altéré du liég. ranonke "renoncule". Le malnuédien archaïque runant telimin. que Villers traduit par "grand chemin "ans donner d'exemple. doit etre pris au sens moral plutit qua au propre; on pensera au vers de Regnier : "Je vay le grand chemin que mon oncle maprit. ("est le chemin régulier. la roie droite et naturelle que suit le commum des mortels. par opposition aux dítour. alux chemin de traverese.

[Rommine. t. xLvis (1921). p. 5is.]

$$
\text { W. rûte }
$$

I Faymontille-Weismen. près de Malmedy. rute. s. f.. désigne "un ensomble dr plusiems javelles davoine drensées sur le champ et liées is la partie supériente, gui forme pointe : lorsque les javelles sont liées

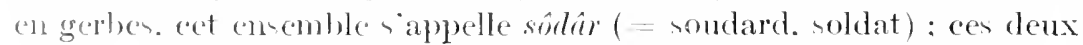

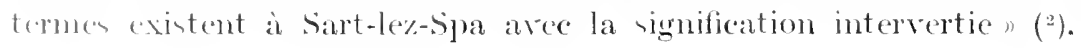

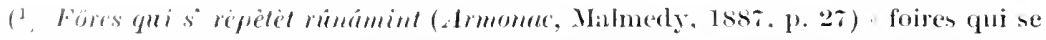

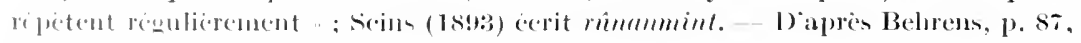

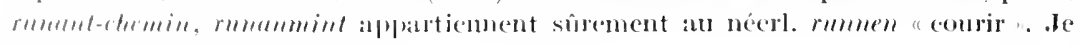
ne puis batiager celte opinion.

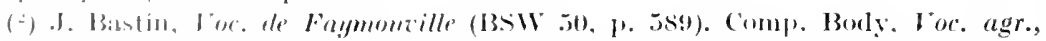
worlir (il).. 201, p. 1s0). 
Le nom de ces faiseeaux est en eflet très variable : on dit, par exemple, à Heure-en-Famenne, des hoùîr.s de seigle ou de froment ; à Bande, on distingue les hoûârs de froment et les sôdârs de seigle. En réalité, rute évoque la même image que les deux mots préeédents : il répond au bas all. rüter, que le Wörterbuch du dialecte d'Eupen traduit par reiter " eavalier" $\left({ }^{1}\right)$. Le sens primitif s'étant perdu. le mot. sans doute à eause de la terminaison féminine, a changé de genre en wallon. L'ane. w. rute se rencontre dans un texte apoeryphe du xul ${ }^{\mathrm{e}}$ sièele : "Leas Rute vinont gaste et broule leur Clostre " $\left.{ }^{2}\right)$.

Il existe encore à Péerot-Chaussée (Brabant) un s. f. mîte "espèee de grand rosean qui atteint souvent la hauteur d'un hommc et au sommet duquel flotte une sorte de plumet". Des dillieultés de phonétique ne permettant pas d'invoquer le néerl. mede (verge) ou le néerl. riet, flam. rijt " roseau " $\left({ }^{3}\right)$, j'y vois un nouvel emploi figuré du vieux mot préeédent, d'autant plus que ee grand roseau s'appelle dame à GrandLeez (Gembloux).

\section{gaum. samousse}

Ce mot ne figure pas dass le Lexique du patois gaumajs de Er. Liégeois (BSW, tomes 37, 41 et 54). ('l. Maus, dans son Vocab. des environs de Virton (manuserit de 1854). enregistre simplement : "samonsse, lisière ». Il s'agit de la lisière d'une toile ou d'une étoffe, comme j’ai entendu dire à Ste-Marie-sur-Semois. Pour la lisic̀re du drap (employée aneiennement pour faire des jarretières et des chaussons), ainsi que pour l'orée d'un bois, on dit la lijiçre. - Forme rare $\left({ }^{3}\right)$ d'm terme bien connu en aneien. franẹais et dans les dialectes du Sud $\left(^{4}\right)$. M. Ant. Thomas, qui s'en est oecupé dans ses Essalis de philologie française, pp. $\%$ s et 84, tire l'anc. franç. cimois, cimosse de *eimussium, *cimussia. dérivés de cimussa " eorde ". On peut roir aussi dans Romania, xxxix, 164,

(1) Malgré leur ressemblance, reiler et rüter sont d'origine différente : reiter (d'où le fr. reitre) vient de reiten "chevaucher "; rüter csi le moyen bas all. rûter, néerl. ruiter, all. reuter, du moyen latin mutuarius.

${ }_{(2)}{ }^{2}$ Manifeste des droits de l'abbesse de Robertmont, Liège, 1633, p. 41. -- Rute se prononce rût' et se traduira par rêtre. Comp. le nom de famille Jamin le ruyte (1555, Arch. de Herve); aujourd'hui Leruitte, Lerutte, Leruth.

( ${ }^{3}$ Le roseau ordinaire s'appelle à Péerot-Chaussée roja (= rozia à Grand-Leez).

${ }^{3}$ Labourasse donne aussi en meusien samouec, à còté de soumoee, soumouce.

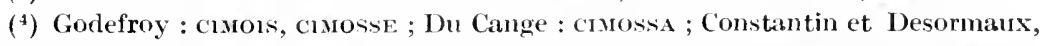
Diet. saroyard : sEmossA ; D'Hombres et Clarvet, Diet. languctocien : smous, smousso ; Puitspelu, Dicl. lyonnais : cumoussa, ete. Voy. Romania, xxv, 384; xxxil, $21 \%$. 
Im article de TI. Blondheim sur cimols. "hord d'un vîtement ". que doment des sourees rabhiniques.

[Romeria, XL (1911). p. 329.]

\section{w. sanke. sankis'}

Le primitif samlie, 4. f. (1). "bourbe rase " existe en namurois, à Marehe-en-Famemne. Awenne, Tohogne, Erezée, Wave, ete. Dans la vallée du Gecr : di l'assmle (Roelenge. Glons) "de la rase ". est sans doute le déverbal d'un r. assonl:î. - De là : $1^{\circ}$ sankis", adjeetif en namurois. bourbeux " (G.) et ausi en liégeois : i fêt sumlis'; ou substantif manculin. ord. au pluriel. "bourbe. vase. endroit marécageux " $\left({ }^{2}\right)$ :

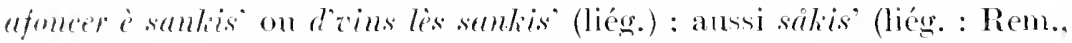
Hub.). sonticis (heshignon : Noduwez: liém. ? : G.). sakise (Malmedỵ); an fóminin : dil sankĕsse (Ste-Marie-Geest) on des sanliĕsses (Perwez. Marille-). On (lit à Bergilers (Hesb.) : di lassonlis'. li pré èst tot coziè d'ussonlis:. - go suncè en chestrolais (" courrir de vase ": Dasnoy. 1. 4.5\%): essanere' ('Tourinnes-St-Lambert) : essanlî̀ (nam.) : lès prés

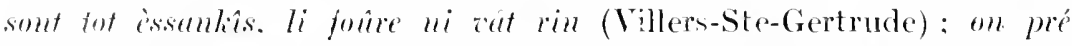

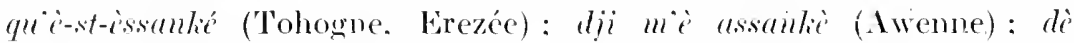

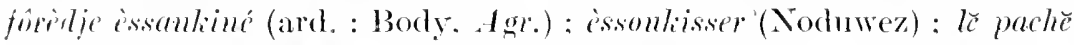
i-st-essanliessé (Ste-Maric-Gest) "le patis est envasé. courert de terre cluarriće par les phuies on par l’inondation ". -... $3^{\circ}$ stucrěue ('TourimnesSt-Lambert). s. f'.. urine des vaches et chevaux, qui constitue le purin.

Le plus ancien exemple que je comnaisse se lit dans une charte de 1353-64t. oi il est question d'me "ierre aecuise et assankie" $\left({ }^{3}\right)$. cestà-dire - d'après le contexte - d'un terrain d'allurion.

G.. II 3 40. compare des mots du Dauphiné. du Berry. du Languedoc et le t. de marine sum (couler à fond). Scheler éearte avec raison ce dernier : mais les antres ue valent pas mieux. "est aller chercher loin l'explication d'un mot qui ne se rencontre que dam la région wallome proprement dite. Il faut sadresser au dialecte flamand de la Campine ot du Brabant, où l'on appelle somk. s. m.. une dépression dans une terre ou sur une route : "de strait higt rol zonken " : lo west-flamand zoulec. s. l'.. signifie de plus "bas-fond " $\left.{ }^{4}\right)$. La confusion des nasales on et an

(1) Ordinarement au phuriel, comme les expressions synomyones lès brotis lis bronlis, lis brigulies, lis berdomyes.

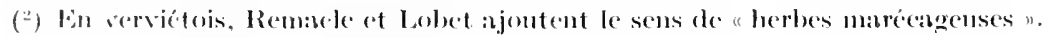

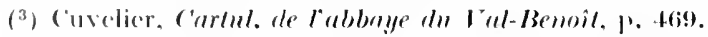

(4) I, flanand zomli se rattauhe a zimben, all. simben. Kilian donne le terme al-

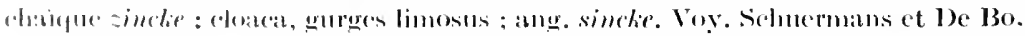


est fréquente dam nos parlers; an liég. sankis" (pour somlis"). comparez le liég. song (sang). stronler (étrangler); royez ausi les articles grimon. ong gle?toll.

\section{W. sâpreû. sampreû. simprê̂ (V'criers)}

C.. II 340 a larticie suivant :

sâpreûs (alfèté, mannéré ; an fém. mijaure) [Remacle]. Lohet a sanprê̂ (maniéré, verqognenx). f'om. sanj)rềze (pimbêchc). ('omp. l’anc. fr. saffre (mignon), safrette (friande, agréable). Remarquez que le liégeois dome tune signification analoguc à scrourếze (pimbêche, précieuse). - Pour la terminaison. comp. selatereis.s. - [Note de Scheler : "sâpreûs prurrait bien être une formation faite dans le monde des cleres et tirée din lat. saporosus (qui at (li1 gotit); comparez l'italien saporoso (charmant). Le sens propre ser"tit : difficile an goût].

Mot du dialecte rervićtois. unité surtout au féminin. F́l sampreñse a le mime sem que le fr. "faire la sucrée, la sainte-nitonehe " : aroir des manières affectées, jouer l'imnocence, la modestie, le serupule. Dam nu noël de Verviers, une jenne fille, qui prêtche le dédain tes salants. sattire eette réponse : a quà bon fé l' samprelise? (1). Et Remacle. ro choufté. nous sert eet exemple : li sâpreùse ! ele bahe lèsoûys : porbî. éle si lît tchoufter it ratchomfter! "la mijaurée ! elle baisse les reux; derriere. elle se laisse embrasser à bouche que veux-tu! ").

La nasale pure n'esistant pas en vervićtois. les graphies sti-s sanont mîme valeur : um. a long mi-nasal $\left({ }^{2}\right)$ et non un a bref comme dans le nam. safiète "gamine. fille de peu " $\left({ }^{3}\right)$ ou dans le liés. satontêuse propr. "saroureuse ". que Lobet domne ausi en vervićtois avec le sems de "pimbêche ". Cela suflit pour éearter les eonjectures on comparaison de G. et de Seheler. Le $p$ fait aussi difficulté; Scheler parle bien de formation savante. mais il faudrait. dans ce cas. powroir eiter des exemples ( $\left.{ }^{4}\right)$.

Je erois qu’il faut partir d’une forme inédite sěprêt. que je retroure dan ten noten prises a Verviers vers $1885\left({ }^{5}\right)$. Ce simpren nous met

(1) A. Doutrepont, Noëls acallons (1909), p. 250 ; voy. aussi BSW 2. p. 291.

(2) Lobet le note arbitrairement de quatre fapons différentes : hanté, franbauk ;

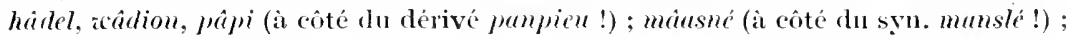
anaie, plason, gagni.

(3) BSW 52, p. 15\%. C"est l'anc. fr. sufrette "fille égrillarde, frétillante", cest-à-dire tout le contraire de notre sampreùse.

(4) Voyez Meyer-Lübke, no $\mathbf{7 5 8 \pi}$.

(5) Et provenant, je pense, d'une conversation avec fen .J.-S. Renier. Ce vieil

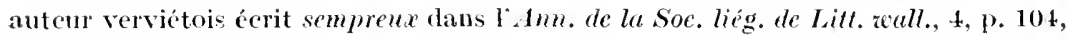
en parlant du " délicat " poète Nic. Defrecheux. 
sur la voie de l’all. zimpern "faire le délieat, le préeieux, mignarder".

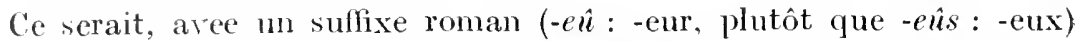
"celui qui fait le délieat "; eomparez zimperlich tun "faire la précieuse", zimperliches Mädchen "mijaurée " $\left.{ }^{1}\right)$. Le passage de $s \hat{e}$ - à $s \tilde{a}$ - ne va pas sans quelque difficulté $\left({ }^{2}\right)$; mais, pour le sens, zimper(lich) et simpr(-eû) se eourrent si exaetement que je ne rois guère le moyen de les séparer.

w savenê (Malmedy)

Le dietionnaire de Villers (Malmedy. 179:3) donne savenai " grand filet de pêeheur" $\left(^{3}\right)$. Seheler (ap. G.. II 3 t3) prend ee mot pour un dérivé de l'ane. fr. saene ( $=$ w. saŷ̉me), avec $v$ épenthétique; mais eette conjeeture n'est pas heureuse. Le malm. sacenê ne fait qu'un avec l'ane. fr. sacenel ("instrument de pêehe"; dans un texte de St-Ouen : Godefroy) et arec le norm. savignian "filet formant une espèce de poche. aree lequel on preud les truites quand la rivière est trouble " (Iittré, Slippl.). C"est le diminutif de l'ane. fr. sacene "espèee de linge ", lat. sabanum.

\section{nam. sclèyî. liég. disclèyî}

G., II 3+8, domne le nam. scliŷt " (se) fendiller," (s')entr'ourrir, en parlant des douves d'un tonneau ". Il cite le liég. risclèyî " resserrer les doures d'un tonneau qui se sont disjointes ". Ce eomposé n'est pas dans Forin, qui dome en revanehe disclèy " disjoindre ". J'ai entendu à Liège : li tonể s' va disclèŷ̉ å solo; l'infinitif est aussi en $i$ bref ; à l'indieatif présent on dit seulement : li tonê si disclèyil ; au participe : on tonê qu'èst tot disclèyon. - Dans la Famenne, où j'ai noté : ci tonế.

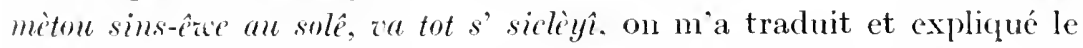
dernier mot par "devenir comme une claie" : bel exemple d'étymologie populaire! — G. rattache sclìyî à l'ane. h. all. slîzan (all. mod. sehleissen. "fendre"); mais z naurait pu disparaitre. Le Dict. général dérive avee

(1) Le haut all. simp, zimper et le danois dialeetal semper ont le même sens que zimperlich et viennent du bas allemand; ajoutez le moyen néerl. zumperlie, simperlic, m.. s. ; l'angl. simper " sourire avee affectation "; le soube zumpfer, zemper " modeste, timile "; etr. Voy, Franek-van Wyek sip ; Falk-Torj sipe.

$\left({ }^{2}\right)$ Voy copendiunt les articles randje et råtchì, et comp. de phus : èsse è lamburin (Liège) : limburin (Petit-Rechain) "être dans là débine "; kidansî (Duv.) : kiłhainsî (Inbert), kidacî, kidincî (Forir) "mâeher "; pramljîre ou prindjîre (Duv.) " mérillemne"); luntir-magique (liég.) : intèr-mugique (Huỵ); crunkî (liég.) : erinkigî (Stave) "tortiller" ; cte.

${ }^{(3)}$ Le bronillon de Villers porte sâzenai, mais $a$ doit être bref. Le mot $n$ 'a rien à voir avec le nom de famille Sanzenuy (Liège), qui vient sans doute de sâvion (sable). 
raison le fr.éelisser de ce slîzan, ct le syn. éelier du franeique slîtan. Cette dernière forme rend un comple exact de notre mot ; comparez le w. sclèyon (malm. splèyon, lićg. sployon) "traîneau ", qui dérive, comme l'anc. fr. esclaon. de l'anc. h. all. slito.

\section{w. selanbran (Neufchâteau)}

Le Dict. icellm de Da\noy (Neufchâteau. 1856 ; p. 453) est le premier à signaler : "sclanbran, angélus du soir ". Les auteurs du Projet de Dict. wallon ont consacré un article à ce terme cheitrolais : il fant y supprimer la forme s'lanbranle. qui n'existe pas, ainsi que la partie étymologique, qui est fondée sur cette forme apocryphe. - On clit s'lanbran à Orgeo, Thibessart, oi la nasale on se prononce an (manter, mancé : monter. monceau). Il en résulte que la forme s'lonbran est la phus pure: clle existe notamment a Libramont. Neuvillers. Recogne, Witry. dans l'expression archaïque : on sone s'lonbran. On derrait l'écrirc s'l ombrant (pour s'lo ombrant), ear elle se traduit littéralement par "soleil ombrant. projetant de l'ombre ". Comparcz, au sud de cette région. les expressions analogues : v'la s'lo boutant on chitchant (Ste-Marie-sur-Semois.Buzenol) "le soleil se couche, on sonne l'angélus"; s'lo boutant n' tard"rè-m" a somnèy (ib.) "l'angélus du soir ne tardera pas ì somer; a s'lo l'vant(ib.) "à l'aurore" ( $\left.{ }^{1}\right)$. - Le dialecte chestrolais a donc conservé, dans ce eas unique, l'anc. fr. ombrer (= ombrager), qui vient du lat. umbrar e et qui diffère, comme on sait, du fr. mod. ombrer. terme d'art. emprunté de l'italien ombrare. D'après une communication de II. Jean Lejeune, les archives manuserites de l'Arouerie de Fléron (lez Liège) portent l'expression : iers soleil mblrant (avec le sens local : "à l'ouent"). dans un texte de 156.5 .

[B D 1912, p. 94.]

\section{w. sèron, cèron}

Ce mot archaïque. que nos dictiomnaires écrivent sèron, signifie "écheveau de lin ou de chanvre "(Villes's, Lobet). "tresse de chanvre ou de lin qui a passé par le sćran" (G.). "quenouillée" (Hubert, Forir) (2). Meyer-Lübke, no $r 8+1$. le tire du lat. sero (au soir)! Quant à G..

(1) En meusien, d'après Labonrasse, s'lo meussant = soleil conehant, et, à l'inverse du gaumais, s'lo boutunt $=$ soleil levant. Cette contradietion s'explique par le sens général de bouter; en ganmais, on sous-entend : "dedans" ; en meusien : "dehors".

(2) J'ai noté à Erezée boule di sèron (syn. b. di tchène, b. d'èsse) "boule de chanvre"; à Leignon lez-Dinant et à Awemne, le sèron désigne le fil le plus fin. 
II 3.56. il y roit une modification du fr. séran.qui désigne l"instrument pour sérancel : mais ee neest là qu'une hypothèse spécieuse. Il faut cerire ceron et rapprocher le mot wallon dil norm. chérion. diminutif du norm. cet ou cher. où M. Antoine Thomas a reeonnu le lat. cirrus. proprement "boucle ou touffe de chevenx. de plumes" $\left.{ }^{1}\right)$.

$$
\text { w. }(\mathbf{s i}=\text { lat. sic })
$$

I. A propos de ladr. si (= lat. sic). Scheler dit, dam son Dict. d'́lym. frauc.. que ce mot s'est substantivé avee le sens de "condition" daus lancienne locution par un iel si. Godefroy eite de nombrenx exemples des locution par (un) tel si que "ì condition que, pourvu que". sous tel si "à eette condition ". ete. Cet emploi de si était également comnu de l'ancien wallon. Dans nos parlers modernes. on en retrouve Ja trace à l'Est du Brabant (région de Warre-Jodoigne). où subsiste

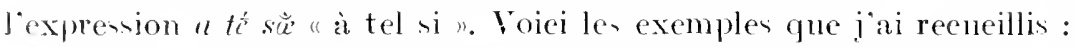

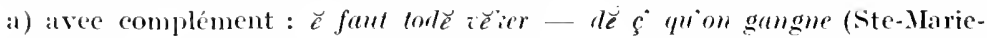
Gecst) "il faut toujours vivre selon ses gatins " : rjĕ la fât - dẹ (ChastreVilleroux) "je lai fait en vue de cela ".

b) saus complément : ljĕ la fêt-(St-Gérỵ, Chastre-Vill., Péerot-Chaussée, $S^{t e-M a r i e-G e e s t) ~ " \hat{a}}$ dessein. avec intention ": s'arindji - Ste-Mizrie-Geest) "s arranger selon les eirconstances "; arindji one têre - (Pécrot-Chaussée)

"arranger une terre d’après ee qu'on veut y semer".

I Court-St-Etienne (Brabant). on dit : ll è-st-aricé a tés si "il est arriré juste à temps, au dernier moment utile ". C'e sens dérive naturellement du précédent.

II. G.. II 361. ne peut déehiffrer le nam. sik (" cn qualité de..."). qüil donne d’après une eommunieation écrite. C'est l'ane. fr. si que ("ainsi que ") : comparez : "que personne ne se présume sique maitre ourrier user de notre Metier " (Chaites et Prizil. des Métiers de Liege. II 59) ; et. en 15.5\% : "Lian et jour smsdit fut ledit IIenry sic marit et menbour de Maroie son espeuse advesty..." (Areh. de l'Etat à Liège : Ban de IIerre. s. ?ol $\left.\mathrm{r}^{\circ}\right)$.

[Rematuicment d'un article paru dams BD 1912, p. 9\%. - J:avais eru alors pwuvoir expliquer de même la plurase : a sn trarayi, i dôrt (Ucimont, Botassart) " au lieu de travailler, il dort ". J'oubliatis que, daus lat région de lat Semois, "represente le latt. in alussi bien que le lat. a d. Nouss avons icei affaire à la locultion en sus de "Ioin de n. bien conmue on aneien français et en meusien.]

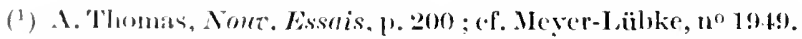




\section{liég. (?) sindrèse}

Ce mot ne figme pas dans les dictiomaires wallow et n’a surement jamais été populaire à Liège. Jo ne le troure que dans un manuserit du curé Durivier (1850) : "sindress del maer. agonie ". C"est le fir. syndérise. terme didactique emprunté du spee. "reproche que nou adrese notre comscience " (roy. Godefroy. t. X). 'Tel est le sem qüil a daus ce passage de Mélart : effrayé de la perte de son sang. ou touché de quelque sinderesise sil y en pent aroir dans unc ame effrontéce et inique " ( $\left.{ }^{\mathrm{I}}\right)$. Les sindièses dil mẩrt désignent donc. plutot que l'agonic en général. les angoisses de la comsence à l'approehe de la mort.

[Romania, t. xust (1921), 1. 578.]

\section{w. sizin}

G. II :360 : sizin (glaçon, glaee détachée); nam. it. ("éelat de glaee qu'on a ('oupé "). Si cette définition est exacte, notre mot appartiendrait prob. an même radical que sizai [Iire cizế, ciseau]. - Sizener (charrier).

Le mot manque dans Remacle. Hubert. Villers, Lobet; ce dernier donne cependant le rerbe sisné. Cambresier défnit : "glaçon" ; Gothier et Willem : "glaçon mince. menu" ; Forir. I 194: " petit glaçon :

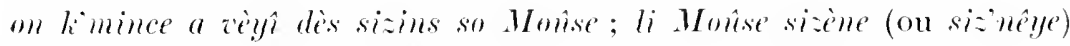
dedia ". - Le mémoire de M. Fouarge sur Ia Batellevie liégenise $\left(^{2}\right)$ nous apporte une forme siz rin. aree cette définition circonstanciée : "slaçon qui -e forme pendant la nuit le long des rives et a la surface des eaux. se détache pendant la journce et s'en va au fil de l'eau ". - Enfin, vers 1890. en conversant avec le vieux passeur de Méry-sur-Ourthe tandis que les sizins descendaient la rivière, j’ai noté ce qui suit. d’après le earactère qui me frappait dans l'olsjet : "glaẹon très mince. formé de lanclles rayonnantes, différent du hirô ou gros glaçon: l'êue sizène. ile za siver" $\left.{ }^{3}\right)$.

Pour en revenir a laticle de (i.. on trouvera sans donte suspecte. daprès ee qui précède. la définition qüil tenait d'un eorrespondant namurois $\left({ }^{4}\right)$. (elle que j’ai notée ì Méry pourrait à la rigueur étayer son hypothèse d'une parenté entre sizin et cist. 'T'out efois, notre not

(1) IIist. de la rille et chastean de IIny, p. 38 (Liège, 16+1).

$\left({ }^{2}\right)$ Inédit, eouronné en 1910 anx coneours de la Société de lit térature wallonne.

(3) Lienu charrie des sizins, elle va serrer ", e'est-à-dire se prendle, geler : comp. Cambresier, vo cizin, et voy. ci-appè̀s larticle trèssèrin. Sur hirô, voy. p. 153.

(4) Pirsoul la reproduit sans indiquer sa souree. 
n'appartenant qu’à la Meuse (de Namur à Liège) et à l’Ourthe liégeoise (Méry-'Tilff), je lui chereherais plutôt une origine septentrionale. Il me cemble que le germ. zeisen, forme dialeetale du néerl. zeis "faux" (moyen néerl. seisene), pourrait expliquer littéralement le w. sizin. Le sens propre serait : "lame de faux ou en forme de faux, lamelle coupante ". d'où. an fig. "glaçon qui présente eette forme ". - Pour la forme isolée siz"rin. comp. liég. mossê "mousse ", ard. mos'rế; verv. pissine ou piss'rine (Lobet) "piscine, etc. "; liég. sîzèt "tarin ". verv.

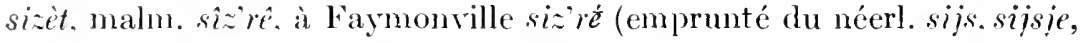
m. h. all. zîse. m. néerl. sîselien, all. zeischen, zeisig ).

\section{liég.s. skèrbalik}

G.. II 366, domne sans explication skerbalite (sie), t. de batelleric, "bois soutenant le toit de l"éeoutille ". D'autre part, nous lisons dans le mémoire inédit de II. Fouarge sur la Batellerie liégenise : skèrbalik. m., sommier longitudinal sur lequel s'appuient les guentes on chenaux $\left({ }^{1}\right)$, qui supportent les rumes (éeoutilles ou panneaux) ; ce sommier repose lui-même sur une charpente appelée tchèyîre ou ehaise ". Cette cléfinition détaillée est d'ume elarté parfaite, autant que la forme shèrbalik, oì nous reeomaissons le flamand scheerbalk, t. de eharp.. "entrait, traverse " $\left({ }^{2}\right)$. Pour la phonétique. on notera l'altération de -ike en -ite $\left({ }^{3}\right)$. l'insertion d'un $i$ d'appui dans les mots d'emprunt moderne présentant un groupe de consomes diffieile à prononeer ${ }^{4}$ ), et enfin le traitement différent du germ. balk dans ba (royez eet artiele) et dans-balili, ce dernier dénotant une adoption plus réeente.

\section{liég. skèrì}

G.. II 366. note l'expession slièri mn batê. t. de batellerie, "ponter un bateau en poupe et en proue "; e'est probablement. dit-il. le fr. "équarrir ou plutôt le w. *squèrî " mettre d'équerre ". Le w. squêrî "équarrir. mettre d'équerre " existe notamment à Fosse-lez-Namur (BSW 52, 1) 161), squérer à Ambresin-Wasseiges. Mais skërî - que. pour na part, je ne comnais que par Grandgagnage, - n'a rien à dó-

(1) Voy. ci-rlessus larticle gucúte.

(2) Voy. sehcerbulk ( = néerl. dwarsbalk) dans l'Idiotieon de De Bo ; et dans Van Keirsbilek. Ambacht san den Timmerman (Gand, 1898).

( ${ }^{3}$ Voy. (i-après l'urt. wite. - L'inverse est plus fréquent : néerl. gelid $=w$. guilite, -ike (G., 124\%); gastrite, clématite, cantharide =w. gastrike, clématilie (Lobet, $280)$, centuritie (Duv.), etc.

(") Voy. les articles ârih, bêrih, mèn'sik, hamuslâde (à propos de rómuskirih). 
mêler avee squiêrî. Il est emprunté du néerl. (een skip) scheren, t. de construetion navale. "dresser les comples et clouer les lisses ", qui se rattaehe à l'ane. h. all, skerjan. skarjan "diviser, partager"; néerl. scheeren, scheren (Kilian) "arranger, préparer".

\section{rouchi soçon}

J'ai entendu à Stambruges (Hainaut) : i n'a pus soẹon dé rié "il n’a plus cure de rien ". On eherehe en rain dans Delmotte. Héeart, Sigart et autres ce mot soçon. dont la forme et l'aceeption sont dignes de remarque. C'est l'ane. fr. sospeçon (appréhension. inquiétude; fr. soupçon), qui n’a survéen que dans cette exprescion négative. avec le sens de: "cure, souci ".

\section{w. sohe (Verviers)}

Lobct domne le verviétois sole du tehèrète "chable [de charrette], forte corde formée de + à 6 torons sans âme qui sert à lier la charge de tomneaux, ete. " (1). De môme à Liège (?). d’aprè Forir. D’autre part, à Faymonville. tont contre la frontière germanique, on appelle soule. s. f.. le eâble dont se servent le bincherom pour f'aire tomber l'arbre du coté roulu $\left({ }^{2}\right)$.

G. If 371. dérive solie du moyen latin soca "eorde " $\left({ }^{3}\right)$. mais la phonétique s'y oppose. Derrière les royelles vélairen o. u, la gutturale latine $e$. g. suivie de $"$. 'cffaee completement en francais et en wallon. Le wallon diffère seulement clu francais en ce que ". u développent it en hiatus: carrutea : chorrue, therowe: sanguisûga: songsue. sansonté ; dôga : ane. fr. done (fr. doure). w. dèce : a uca : ance fr. ome

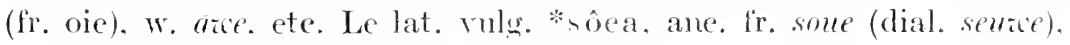
donnerait done *soue. * séze en liégeois.

Le mot n'étant signalé qu'à Liège ("). à Verviers et près de Malnedy. il est naturel de lui asigner me origine germanique. Nous y verom un emprunt du moyen h. all. sos. has all. soch $(=$ h. all. sug " traction. trait "; aree le scns supposé de zugseil "corde pour tirer, trait "). Pour la forme, royez l'article dighe. oi now avons recomn l'all. joch. et l'article gusmulie.

(1) Lobet a tort évidemment de confondre dans le même article ee mot avee sohe, f., "rigole ", qui vient de l’anc. h. all. suochat "sillon "; voy. ci-après zohile.

(2) Communication de M. .J. Bastin ; voy. son Voc de Faym., vo souhe.

(3) Diez, 1. $29 \tau$; Neyer-Lübke, $n^{0}$ 8051. - Voy. ci-après l'article sozie. 


\section{liég. " sônandin " (Forir)}

Forir définit ce mot par : "onclin. croque-mitaine qu"on suppose habitcr les canx ". La traduction "ondin " pourrait mettre sur une fause piste. En réalité, on doit écrire li somnonts-dints $(m=\tilde{o}$. dénaralisé parfois en $\hat{\theta})$. abréviation de l'ome as somnants dints "l'homme aux dents saignantes, ronges du sang des victimes qüil dévore" ('). Vers $18 \% 0$. à Cointe (l. d. de Liçue). pour empêehcr les enfants d'aller jouer du côté des quate roucules, carrefour malfamé, on leur disait que l'ome as sommants dints ou as rodjes (rouges) dints y demeurait. - I) ordinaire on entend par là un esprit des eaux. A Souverain-Wandre. on recommande aux enfants de ne pas sapprocher de la Meuse en leur disant: lome as rodjes dints vis hièrtch iè d'vins "vous entrainera dedans " $\left.{ }^{2}\right)$. De unême à Glons: nalez nin tro près d' Djêr (du Geer), l'ome as rodies

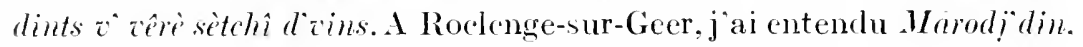
que lon comprenait comme étant un nom propre ct qui n'est que aphèrèsc du précédent $\left({ }^{3}\right)$. I Jupille. on dit aux enfants qui reulent

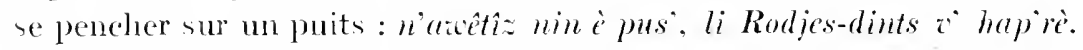
Un des noms du Croque-mitaine à Stavelot: lu rodje-betch ( $\left.{ }^{4}\right)$ à $\mathrm{Na}$ mur Colau-rolje-bitch, s'expliquera de même: c'est un hommc ou un oiseau géant au bee rouge de sang.

Forir, vo bonname (bonhomme), donne lexpression bomame as rodjes dints "espèce de eroque-mitaine ", et nous voyons. par un pamphlet de 16r6. que le's Liégeois se moquaient de l'Empereur

Tot l'loumant l'boumame âs rodjes dints

Et li p'tit borguimêsse d'Allemagne (5).

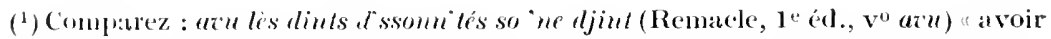
me rancune contre qqu"- A Verviers, "le personnage imaginaire quion dit aux "nfunts ctre au borl de leau pour les en ćloigner" sappelle sainaulin d'après Xhoffer, $\because$ est-it-dire sreinamls dimls "dents saignantes " (.J. Feller : BD 1920, p. 66). Lat forme stbintunt est étrange, ar le wallon distingue entre simut, v. tr., "sitgner (un malidle) " ct sommer. v. intr., "avoir un deoulement de sing ".

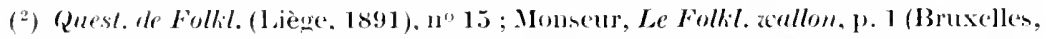
kozez). - Marie-('roeleet (St-Hubert), Thowme' an crochet (Huy), IIenri-('rotehet ('Tintizuy), Djom-Crochet (Ste-llarie-sur-Semois), le père Cent-croehets (Longwyon),

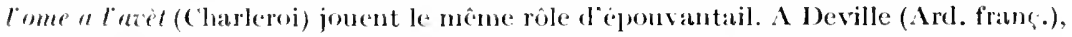
la bete atrutor vit dans la Mense ; efle an des cornes avee lesquelles elle entraine les ('llicunts (IBI) 1910, p. 20(6).

(3) Comparc\%, à Cincy : Wazthgrawe ( = lome a-z-agrazes "l'homme aux griffes"); $n i$

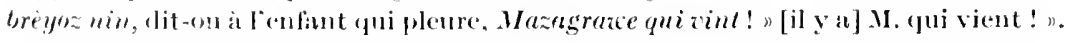

(3) Ioce de stazelot : $135 \mathrm{SW}+4,1,501$.

(5) Henuen, Panuphlets politiques du XVIIe siecte (1913, Verviers), 1). 260. 


\section{W. sondje (Roclenge-sur-Geer)}

On lit dan le Gloss. du chapelie' en peille par Marchal et Vertcour (BSW 29, p. 2+1) : "songe. s. f.. neuviène partie d"unc bosse di stom. e.-à-d. d'une gerbe dont les épis noont pas encore été coupés ». Je tien de M. H. Frénay que ce terme, prononé smolje, n’existe qu’à Roelengesur-Geer. avee le sens suivant : "double poignée de stou (paille à tresser). quon lie sous l’épi avant de la peigner ou serancer : après le peignage. on coupe les épis; sept ou lunit somljes, ainsi décapitées et nettoyées. puis liées ensemble aux deux extrémités, forment un ia (une gerbe) n. - Pour décourrir l'origine de sondje. l'orientation est aiséc : Roclenge se trouve à l'extrêne limite linguistique du N.-E. et le vocabulaire du tresseur de paille foisonne en termes germaniques. Il suffit de consulter l'Idioticon flamand de Sehuermans. vo zang "glane, poignée d’épis (Kilian : sangh) ": roy. aussi lall, sange dans Kluge, Weigand. Le diminutif *sangje ${ }^{1}$ ), aura domé *sandje. devenu sondje dans la vallée du Geer, où la nasale an s'altère souvent en on. (voy. l'art. sanlie).

\section{liég. sorblèsseûre}

G., II 375, a eet artiele: "sor (dans sure-blesseure: meurtrissure) Remacle, 2e éd. Comp. l’angl. sore (blessure) \%. - Altenburg (II. 1:2 : Eupen, 1881) eonfirme ct développe cette explication : "sore clans sore-blesseûre, dit-il, est l'anglo-saxon sâr, ... et adj. (wund, sehmerzlich), comme dans starum vordum (Beowulf), angl. sore, anc. h. all. sêr, n. h. all. sehr, cersehren, etc. "Voilà beaucoup d'allemand dépensé en vain ! Au fond, notre sor n'a rien de germanique.

Remarquons d'abord que le not n'est signalé nulle part ailleurs et que Remacle éerit sor-blesseur : la graphie sore ent inventée par G. en conformité de l'explication qu il imasine. Je tiens pour eertain qu“il faut éerire sorblessêre, composé à l'aide du préfixe sor- "sous". Ce préfixe a le sens propre dans sorlèer (Lobet) "soulever ", sorfa (ard.) "bâton pour soutenir le laix sur l'épaule ", sorlon "selon ", sortini "soutenir", sorpacoster (Malmedy) "1. soutenir qan : 2. porter [um habit] après autrui "; $(2)$ - il est atténuatif dans sordroci "entr"ouvrir ". sorrèy "entrevoir " (Malnedy, Bovigny. Lutrchoic, ete). Un verbe *sorblissî "*sous-blesser : blesser légèrement, meurtrir " s'expliquera comme les deux derniers, ou bien, en prenant sor-au sen propre. par : "blesser

(1) Voy. ci-après lart. zeadje.

( $\left.{ }^{2}\right)$ Ajoutez sorsèyemint, dont nous parlons à l'article sou. 
sous [la peau] "; le mot serait, dans ce cas, d'une justesse parfaite. On relève d'ailleurs : $1^{0}$ au Sud de la Wallonie, sourbature, "ampoule "( $\left.{ }^{1}\right)$; $2^{\circ}$ à Mons, $s(o)$ urbature "douleur du pied assez eommune ehez les eharbonniers qui travaillent dans l'eau "(Sigart). Nous y royons de même une *bature (contusion) " en dessous". ou eneore une " eontusion légère ".

\section{w. sot-dwèrmant "loir "}

G., II 3r\%, note laeoniquement "sot-doirmant : loir, à Liègè et à Xamur ", eomme si le mot s'expliquait assez de lui-même $\left(^{2}\right)$. La Faune acallonne de.J. Defrecheux dit bien que le loir s'engourdit pendant l'hiver et que de là vient le nom wallon de eet animal; mais on peut se demander pourquoi une telle partieularité lui vaut l'épithète de sot. En réalité. et cela mérite d'être remarqué. sŏ cst altéré de š̋ et représente le fr. sept. On sait combien fut populaire au moyen âge la merveillense arenture des "Sept Dormants", de ees jeunes gens d'Ephèse, qui. arrêtés au nu sièele comme chrétiens. réussirent à se réfugier dans une eaverne. où ils s'endormirent pour ne se réveiller que deux sièeles plun tard. Ln souvenir de la légende pieuse a survécu dans l'all. Siebenschläfer et le néerl. zezenslaper. qui désignent proprement "un des sept dormanty ". puis. an figuré. "un grand dormeur ". et enfin "un loir ". Le w. sn-dicérmant n’a eonservé que cette dernicre signifieation, le terme sétant altéré chez nous par ét yomologie populaire et aussi par disumilation. On a dit d'abord set datrmants. puis. comme dans d'antres eomposés traditionnels $\left({ }^{3}\right)$. le $t$ s'est annuï : enfin la royelle i de la syllabe initiale est derenue ò. conme daus sersai (Lobet. p. 5.34 )

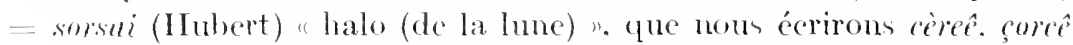
(= cercoan. lat. circellus) : sèrdjant = sordignt "sergent ": Diè-acale = Diw-tade "Dien (rous) garde ! " (G.. I ?.54) : ane (Malmedy) = imê

(1) Brune:u, Enquêle, I 2:3. A rapproclier du gameis sourbate "battre superfi(iellement, sans delier les gerbes" ; sourbatin (Alle-sur-Semois) "gerhe battue". -

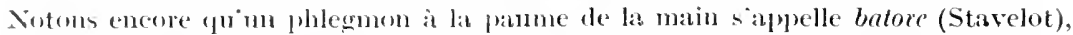

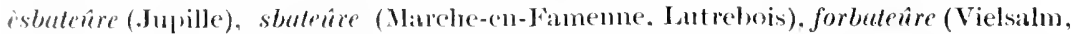
Buringy), fourbatrute (Ste-Mirie-sur-Semois). Voy. Godefroy, sobaterere.

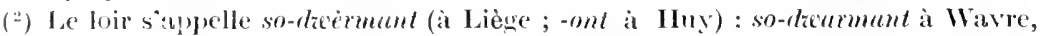
Nimur, Dorinne (Pirsoul domne le nant. sordzarmant, forme peu sûre) : soltelzarmaml is thave, Alle-sur-Semois ; sou-dormuml is Neufehâteat ; lou-rlormant à Virton ;

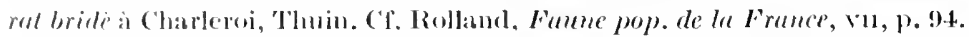

(3) (omp. âs si-fuze " aux sept hêtres " (1. d. de Neuville-en-Condroz); abarin, t. de lonill., proprement nobut $\operatorname{rin}(131)$ 1914-19, p. 68). 
(Faymonville) $=$ omê (Liège) $"$ anneau $" ; *$ sèlogne $=$ sologne (Liège) "chélidoine " ; ete. - Le changement inverse est du reste tout aussi fréquent : sofolier, sífoliér "suffoquer"; Tchodôre, Tchèdore "Théodore"; èstèner "étonner "; pèturon "potiron "; popionile (à Liège, comme les mots précédents) = pèpimile (Sprimont) "têtard ", proprement "petite pope ou poupée "; corcêye (liég.) = himcêye (Esneux) " eorvée "; pèrlôlje (liég.) " ehaire de rérité " (lat. prologium), ete.

\section{w. sotré}

On appelle ainsi, à Villettes-Bra. la litière de paille ou de bruyère qu'on étend à terre pour y déposer les gerbes de blé $\left.{ }^{1}{ }^{1}\right)$. Cette forme curieuse et inédite répond à l'anc. fr. smustré "litière ", qui vient du lat. substratum. Pour s'en convainere, on lira avee intérct l'article que II. Antoine 'Thomas a consacré au fr. soutre "sous-main " et au saintongeois soutrer "faire litiore " $\left({ }^{2}\right)$. - Le w. sotré est encore remarquable si on le compare à sortini "soutenir net aux quelques autres mots wallons où sor-représente le lat. subtus $\left({ }^{3}\right)$. On peut se demander s'il ne faut pas ici partir d'un type *subtus-stratum, d'où *sortré, qui aurait abouti à sotré par dissimilation.

[Romania, t. xL (1911), p. 330.]

\section{w. soû ("seuil ") et clérivés}

Pour expliquer son. Niederländer invoque un type latin *solum, mis pour solium $\left(^{4}\right)$. Il se trompe assurément. Tout eomme le fr. seuil,

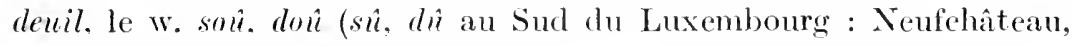
Laracherie, Faurillers, et pays gaumais) rient de solium, dolium. Ce qui earactérise les formes wallomes. c'est la perte de l'élément palatal $y$, phénomène des plus ordinaires dans nos dialectes. Conparez le liég. cièrfou " eerfeuil ". mifon "mille-feuille ", à côté de foye. fouye "feuille"; le malm. à à eôté du liégr. mùy (1. ail ; ״. aujourd'hui); le traitement du suffixe -aculun, qui aboutit au w. - a, fr. -ail; ete.

(1) De même, à Tohogne, c'est le fond du gerbier (stèlêye) ou la base de la meule (môye). - "A St-Géry (Brabant wallon), on fait un sotrait sous la mréye (meule) en disposant à terre 25 ou 30 gerbes "(Communication de M. l'abbé Courtois). Ce sotrait est sans doute une graphie inexaete pour sotré, que j'ai entendu non loin de là, à Gembloux, Grand-Leez. En pays gaumatis, j’ai noté soutrèy à Musson : "gerbier au nivean du sol "; eomp. Labourasse, p. 512.

(2) Mélanges d'étym. fr., p. 147. Voy, aussi Meyer-Lübke, no 8396.

(3) Voyez Projet de Dict. zeallon, [. 26, sorfa, et ci-dlessus l'article sorblèsseûre.

(4) Mundtart von Namur, \$ 43 a. 
"Senil " a donné ehez nous certains dérivés intéressants. Le liég. soyou, terme de houillerie. "lit inférieur d'une eouehe de houille ", se rattacherait, d'apris G.. II 3\%2, a sôye "seie". Nous y trouvons un diminutif *soliolum; comparez le liég. foyou "feuillet ", et royez, pour plus de détails. BD 1914. p. 105.

I Roy (en Faneme), lis sơ̆yemints désignent le soubassement. le mur has sur lequel on place le son̂. cent-à-dire la sablière, la pièce de bois soutenant la charpente de l'ancienne eonstruetion ardemaise. En 15t6, un texte liégeois parle "des vieux soillemens " d'une maison (1). Le composé sorsèyemint (pour *sorsoyemint: lat. *sub- ou *subtussoliamentum) est beaneoup plus commun dans ee sens $\left({ }^{2}\right)$. On le relère notamment ì Stavelot. Malmedy. Erezée, Bande. Ortheurille $\left({ }^{3}\right)$. C'est apparemment le même sens qu'il faut attribuer à l'ane. fr. somsueillement $\left(^{4}\right)$. Comparez le fr. ensewillement.

Entin G., II 380, cite sans explication le nam. souwe "pièce de bois plate que l'on met sous une eloison pour la supporter ou sur un mur ". Cette définition. qui est reprise par Pirsoul, ne doit pas être exacte. J'ai noté somâyye à Ste-Marie-Geest. 'Thorembais-St-Trond. smen à

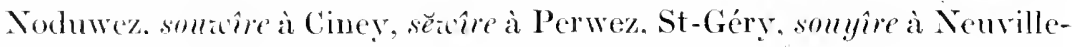
sous-Ifuy soymre à Denće. Partout. ce mot désigne le petit mur bas qui sépare le zerbier de l'aire $\left(^{5}\right)$ : quand on bat au fléau sur l'aire.

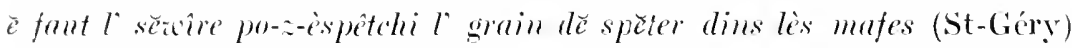
" il faut le petit mur pour empêeher le grain de sauter dans les gerbiers ou travéen de la grange $" .-$ - A mon sens, le type latin *soliat a aura

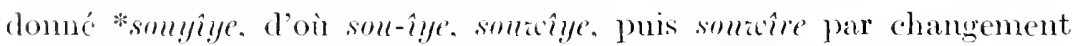
de suflixe. ("ent proprement la partie "sevillée ", le mur bas eourert d'un "senil "ou picee de bois qui supporte les piliers de la grange. Godefroy dome - sans traduction - trois textes toumaisien du xve siecle portast smmlie (-llie, -llye), oì nous reeomaissons notre mot

(1) Citć par Goul. soleme'ml. qui dome à tort la date de 1566. Ajoutez ce texte, (que je tiens de 11 . ocan Lejeune : le haulte paroise... estoit sains soul et soyemens at an plussicurs licux estoicnt les dits maisonnaiges mal entretennus de placepuemens "(15.51. Avonterie de Fléron, 8,1733$)$. - De même, dans les Ardennes françaises : "sonillements, fondations fune construction" (Batudon, Patois de Rellel).

(2) Pour sub- ou subtus- > sor-, voyez les articles sorblèssétire, sotré.

$\left({ }^{3}\right)$ Pour Stavelot-llalunedy, voyez BSW 4.4 , pp. 523 et 540 .

( $\left.{ }^{4}\right)$ Voyez sorchielle, sourcillier, sueller, sucillissememt, soursueillement, resenreiller, dans Godefroy, qui ne trahuit pas ou traluit inexactenent la plupart de ees mots.

$\left({ }^{5}\right)$ A l'extrême Onest, ce mur s'appelle plouyé (Ellezelles), bené (Luingne), bunc "luire (Wiers). - I Tohogne: li soû d'li stèléye "le seuil du gerbier". 
wallon. On sait que, dans le dialecte de Tournai, le partieipe féminin des verbes soumis a la loi de Bartieh-.Musafia est en -ie (1).

ane. fr. soudre (lat. soliere) et dérivés

Des chartes namuroinen de 1328 parlent de derle sorderesse. de derlière sordrevesse ou somdresse. Godefroy, qui cite les texten anx artieles derle et sordreresse. ne peut définir cette épithète. Dans son étude sur le suffice -arieius. M. Antoine Thomas dit a propon de ces expressions : La tlele est de la terre glaise; faut-il rattacher l'adjectif sorderez à sourder, souiller. ou d̀ sometre. ou même chercher une autre étymologie?" $\left({ }^{2}\right)$.

Il faut prendre le dernier parti. Nous sarons que les batteurs de cuivre utilisaient eette sorte d'argile pour leurs ereusets a fondre le métal $\left({ }^{3}\right)$ : cent ce qüindique le passage suivant du mone texte de 13:s : "une derliere, c'est a savoir ou on prent terre de coi li hateur ourent [ = travaillent] a Dignant et a Bouvignes" (Gol.. DERLIERE). Il sagit done de la terre plastique dont les fondenss de Dinant faisaient les creunets à fondre ou soudre (lat. solvere) le cuirre. Les mots derle, devliere qui précèdent l'épithète ont amené. par assimilation. l'épenthèse de $r$ dans le type primitif souderes. sofleres $\left({ }^{4}\right)$.

La famille soudre (fondre, dissoudre) na pas en de chance aupris de Godefroy ; il l'a méeonune ì pen pris partont. Il ne sait pas définir l'adjeetif soldeis. somdis qualifiant un nom de métal ; le sens ent éridemment : "fondn" : un. fomleis, fontis. - On est étomé de ne pas trouver à l'art. soldne le senu propre de : "fondre (um métal)". I l'art. sOLDRE 2. nowelle surprise : somble (um battant de cloche. un lion dargent) est traduit par... "souder" ! - Il fait deus articles sOCDER : 1. "r. a.. discoudre " : ?. "r. n. (!), aroir à faire (!). aroir de

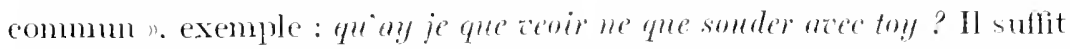
de rappeler le vers de Villon : "luy nayons que faire ne que soultre, pour voir que souder signifie : "résoudre. débrouiller. traiter (une question)" : roy. Diet. gén.. sornes. - Enfin Godefoy traduit smoleresse, sodresse par : "femme d"un soudeur" " et. an t. x. soudeur par :

(1) Ch. Doutrepont, Notes de direlectologie tourmaisiemes :3 (Z.f. frz. Spr. U. Litt., xх11, $\mathrm{P}, 68)$.

(2) A. Thomas, Nonzerma essuis de philologie franç̧ise', 1). 100).

(3) Dony et Bragard, Cocub. du tireur de terre plestique (13SW 50, 1). 611).

(4) La voyelle o représente $u n$ son moyen entre $\hat{o}$ et ô, qui existe encore aujourd'hui dans le dialecte namurois. 
"eelui qui soude". Les textes eités étant de Bourignes (1:311). il faut conprendre : "fondeur ". "femme d'un fondeur (de euivre)".

[Romania, t. xur (1919), p. 192.]

\section{gaunt. sowe. assowèy : w. sawe. assawè (Neufehâteau)}

Nour arons ru, à l'art. sohe. que le lat. vulg. *o ôca (eorde) n’a rien donnć dans le nord-wallon. En revanehe. nous le retronvons au Sud, dan la région de Neufchâtean et de la haute Semois. M. Ed. Liégeois note le gammais assaureye. v. tr.. "passer une ficelle dans les oües d'un poisson capturé pour le conserver vivant dans l'eau " (1) ; il souligne oües pour indiquer apparemment que le verbe en est dérivé. Erreur évidente. Le mot est du reste mal ćcrit : on prononee. non pas au $(=\hat{o})$. mais o ouvert, long. bref ou moyen : jaai pu entendre ees trois sons. et jamais $\hat{\prime}$. - De son côté. Dasnoy enregristre à Neufchâteau : saice "corde, chaine à l’aicle de laquelle on attache les cheraux quand on les faît paître au piquet ; attaehe "; twsazier "attaeher. mettre au pirquet. à l'attache"; dessazer "détaeher " $\left({ }^{2}\right)$. - Voiei cnfin les notes que jai recueillies sur place. On prononee en ehestrolais t'ne săaf. assăàe. dussătié; dju m' à mơnè mu tch'fô a saże "je vais mener mon cheval [pour le mettre] en entraven. En gamiais (Ste-Marie-sur-

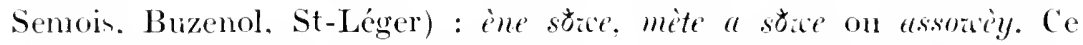
dernier verbe s'emploie dans trois eas : 1. asstrièy (in telifô. in zié), attacher avee la soñe (le cheval, le veau). au pàturage dans lenelos, ou dam l'étable après la médịeture (mangeoire) ou la bôtchîye (cloison); -2. ligoter l'animal en lui jiant la tête et une ou deux pattes de derant ; 3. assuaté l'uch du l'ètôle, barrieader, lier solidement la porte de l'étable. - Je nai pas retronvé le sens indiqué ei-dessus par M. Liégeois ; il existe assurément. mais ee n'est qu'une aceeption figurée ou analogique. la "sonc " étant arant tout une eorde assez forte.

anc. fr.-w. sperial. spurel. spier ; w. spurê. -ia

Godefroy donne sans définition l'ane. fr. sperial dans ce texte liégeois de 1 130 : "lis, scrins, stranaires, sperials et atutres menues fustailles". Behrem. 1). 83. Y roit le west-flamand sperel. sperrel "barre de bois pour fermer une porte ou une fenctre ". Pour moi. sperial ne fait qu'un area spurel. que Godefroy donne. également sans définition. dans eet autre texte liégeois de 1401 : "Les eseherins... puissent entrer ens

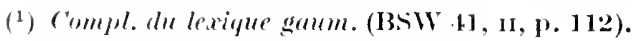

(2) ('f. cionlefroy : stuwe " corde". 
maisons des bollengiers querans en leurs spurean et autre part pain et ce peser" $\left.{ }^{1}\right)$. G., II 639, pour ce dernier passage, renroie arec raison à l'ard. spurế "armoire". diminutif de l'anc. liég. spier, lat. spicarium "grenier". On peut roir quatre exemples de spir (136\%). spier (1406), cités par God., ro spier "petite chambre pour mettre les provisions on conserver les marchandises " ( $\left.{ }^{2}\right)$.

A propos de spicarium (> anc. h. all. spîhhâri, mod. speicher). qui est dans les Glosses de Reichenau - et déjà. au ve siècle, dans la Loi salique - Diez croit que "ce mot ne s'est maintenu nulle part. parce que le lat. granurium suffisait: mais, ajonte-t-il, d'après notre glosse, il faut supposer qu’à côté de granier, grenier. un syn. espiguier, espier était usité en France " $\left({ }^{3}\right)$. Si Diez avait conmu l'anc. liég. spier. il aurait ru que spicarium s"est maintenu longtemps en Wallonie. Au reste. le souvenir en survit dans Spy. commune du canton de Namur-Sord $\left(^{4}\right)$ et dans le diminutif spurê.

Ce dernier, qui a disparu du liégeois moderne. subsiste an Sud et ì l'Ouest de Liège. Nous relevons spurp (Marche-en-Famenne) " armoire à serrer les provisions ": spurét (Iwenne) "petit placard. ordinairement au-dessus de la porte. oì l'on serre le pain. le beurre. ete. " spuria (Namur. Giret) "armoire" $\left(^{5}\right)$ : enfin le nam. spurgna. m. s.. qui présente un cas remarquable de durcissement du yod $\left({ }^{6}\right)$. Lassourdisiement de $i$ en ù̀ la protonique est réçulier; voỵ. l'article purlê, p. 197.

[Romania, t. xwri (1921), P. 573.]

\section{liéng. spinå. néerl. spinaal}

Voici, sur ce sujet, ce que disent nos auteurs :

G., II 386 : spinà (sorte de fil de lin), rouehi ji llespinal (sorte de fil blane). De l'all. spimen, filer ? on dérivé de spène, épine?

(1) Ajoutez eet exemple de 1393 : " les espirias qui sont en le cuisine "; clans un testament cité par Bormans, Cartul. de Dinaut, t. J, p. 135 (Nanur, 1880).

( $\left.{ }^{2}\right)$ Ajoutez dans BSW, t. 5, p. 408, un acte de 1406, qui contient six fois spier, une fois spiet; - Chates et Prizil. des Métiers, in 14t et 1.49: dedans maison, spier on lieu secret " (1521); etc.

( $\left.{ }^{3}\right)$ Diez, Anciens glossaircs romans, trad. Bauer, p. 17. - Ni Körting ni MeyerLübke n'ont d'article spicarium.

(') Spricarium en 840 ; Spiers, Spies en 1228, 1278, etc. ; voy. Roland, Toponymie namuroise, p. 561.

$\left({ }^{5}\right)$ Le mot tend à disparaître devant armzcêre et drèssé ; à Givet, par exemple, il ne survit que dans l'enfantine : zoup-zou-zoup su li spuria...

$\left({ }^{6}\right)$ Pirsoul, rs, 273, écrit spurnia ; j’ai entendu spurgna à Dorinne. - Pour $y>g n$ après $r$, comparez, en namurois, machuria, tahuria = machurgna, tahurgna, et voyez l'article mwèh'nê, p. 182. 
Forir, Dict. liégeois, $11655: \mathbf{s}(\mathbf{i})$ pinâ fil de lin pour les cordomniers : on loukê di-, une pelote de fil de lin : tchètê $d i$-, ligneul de fil de lin ; voy.fi-ı-djonde. | fi-a-djonde ( 366$)$ ] = "fil à joindre "], fil d'Epinai, fil gris pour ligneul fi-blanc (I :3ir), fil de Cologne, fil blane pour ligneul.

Lurquin, Glossaire de Fosse-lez-Numur : spinâl m., fil, ligneul blane. Scius, Dict. mulmédicu (manuserit, 1887) : spinâte 1. épinard; 2. fil de lin pour les cordonniers.

Le nam. spinâl est la forme première. altérée d'une part en spini sous l’influence de spina "épinard" (Liège), d'autre part en spinâte sous l’influence de spinâte. all. spinat "épinard" (Malmedy). Nous la retrouvons dans le néerl. spinaal "ligneul ". qui. en Flandre occidentale. désigne aussi, d'après D. Bo. le "gros fil retors dont on fait des bas et des chaussons"). Chose curieuse, ni Vercoullie ni Franck-ran Wyk ne comnaissent l'origine de ce not néerlandais: on nous dit sculement que l'Etymolugicum de Kilian ne le mentionne pas. L’introduction du mot dans les Pays-Bas est done postérieure au xve siècle. Nous croyons que le néerlandais le tient du wallon. qui le tient lui-même du Sud.

Les eonjectures de G. portent à faux; il aurait dû plutôt tenir compte du rouchi fi d'espinal. que Hécart définit : "fil blanc à l'usage des cordomicrs : on ben sert aussi pour la bounetterie ". Le Glossaire wallon de Pl. Delmotte ${ }^{1}$ ) confirme ce témoignage ; il traduit fil d'Espinal on d'Espinard par : "fil de chanre " et cite un inventaire manuscrit de 13\%1 : "iten autres fringes blanques de fil d'Espinart". Forir traduit fi-a-djonde par : "fil d'Epinai " [sie ; lire Epinal ?]. A monsens. du spinâ(l). c'est du fil provenant de la ville d'Epinal (jadis Espinal. Spinal), qui possède des filatures renommées. Comparez. en français. tulle "tissu trìs fun de fil "; boldue "ruban étroit de coton " $\left({ }^{2}\right)$; turcoin "poil de chère file " $\left({ }^{3}\right)$; en allemand. Rasch (= Arras) "tissu léger de laine ". C'est l'histoire de calicot, de zerdin, de bougie et de tant d'autres mots $\left({ }^{1}\right)$. - Pour d'autres nom dialectanx du ligneul. vogez ci-apricis laarticle tohìte.

[Romaniu, t. XI.vir (192]), ]. 574. - Depuis lors, Mr. Vercoullie m'a simnalé qu'il avait de son côté, dès $1919\left(^{5}\right)$, traité du néerl. spinual dans marticle qui

(1) Licrit en 1812 et publié en 1907, à Mons, chez L. Bolland.

(2) I) Bois-le-I)uc, ville de Hollande (et non de Belgique, (comme dit le Inict. gén.). Voy., ibid., le 'Truiti de la formation de lt l. fr.. \$ 36, ou manque bolduc; en revinnelse,

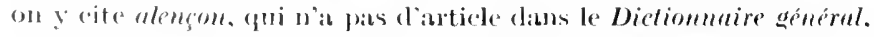

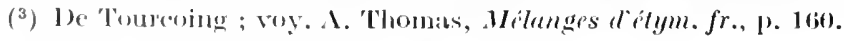

(4) Voy. ei-dessus l'article antecoste.

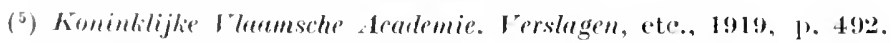


m'avait échappé et dont voiei (en traduction) la demière partie : "Boissière, Dict. anal., vo cspèces, section fill, lappelle en français fil d'Epinay. On le dénomme aussi fil (blanc) bon outrier. Savary, dans son Dict. univ. de commerce, vo fil, parle des "fils blanes bon ouvrier, appelés ordinairement fils d'Epinay, qui se fabriquent à Lille, eapitale de la Flandre française ". De même le Dict. univ. de commerce (Paris, 1805) le dénomme "fil de Flandre... qui se fabrique prineipalement à Lille et aux environs ". Or Elinay est un hamean de la commune de Flers près de Lille. Peut-ĉtre eet Epinay vient-il de Spinogilum, comme Epinay-sur-Seine; sinon, les formes du néerlandais, du wallon et du rouchi font plutôt penser à Epinal... Pent-être y a-t-il en confusion avee la ville d'Epinal, dont le nom était plus connu à cause de son imagerie populaire „.]

\section{w. spougn'ter}

Le nam. sponsnter signific, d'après Pirsoul : "couper le poing; gourmer, domner des coups de poing" ". Cet artiele a le tort de confondre deux mots d'origine différente.

1. spongn'ter (nam.) "couper le poing": spongnitè (Famenne) "amputé du poing ", rient du préfixe $s$ - (lat. ex-) et de pongnèt "poignet "; ou encore c'est le diminutif de spongni, qui a le même sens à Ciney et à Tohogne. Mĉme formation que l'arch. smamé (Alle-sur-Senois) " manehot ". Toyez l'article éminmé. p. 91.

2. spougnter (nam. et liég.) "frapper à coups de poing " est le fréquentatif de l'musité *spongnt. anc. fr. espoingmier. Le prinitif est pougn.t "(em)poigner". dérivé de pogn., poing. De là. lipmugnter (Forir) "houspiller, rosser". Si l'on compare spaimer *expalmare "exprimer [l'eau du linge] en pammant. en frappant avec la pamme de la main ", d'où : "rincer [le linge]". èsbate (Lobet, 64t) "extraire [la erasse] en battant, échanger [le linge] ". on comprendra que spougu'ter a pour sens propre : "extraire [la erasse. la poussicre] en domnant des coups de poing "; d'où au figuré : "rosser".

\section{liég. spricatwére "purgatoire"}

Le liég. purgatwére est du français à peine wallonisé. Personne n’a encore relevé la forme spricatzére, qu'un auteur liégeois. D. Salme, emploie dans deux de ses pièces $\left({ }^{1}\right)$. On est d'abord tenté d'y roir une altération sans importance, une fantaisie individuelle. En réalité, ne serait-ee pas $u$ arehaïsme répondant i l'anc. fr. espurgatoire, dont Godefroy domne une demi-douzaine d'exemples ? La métathèse de $r$

(1) "Ci n'èst nin 'ne vèye, èdon, çoula ? C"è-st-on spricatwére!" (Madame Libert, 11, 12). "Lîdje è-st-on sprieatwére " (Lès sots d Lîdje, p. 65). 
est normale, eomme le montre prudji " purger ". Pour u protonique passant it $i$, comparez scrip'le $u_{\text {s }}$ "serupuleux " et le verv. trik'ter. pour trukiter "tricher", dérivé de truc. Quant au changement plus rare de gु en $c$. il serait dû au roisinage des artieulations fortes sp et $t:$ au reste, Forir dome purcatzére à eôté de purgatzére, et Duvivier zacabondidje "ragabondage ".

\section{w. spruwieû (Verviers)}

Le verviétois spruaicû est, d'après Remacle, synonyme de rigrềs : il signifie, d'après Lobet : "grivois, éveillé, gaillard, bon drôle". G.. II 391, pense à un dérivé de sprècue "étourneau". J'y verrais plutôt un dérivé du flamand spruicen, spruien (Schuemans. De Bo). forme dialectale du néerl. sprocien "arroser, asperger", qui, comme l'all. sprühen. peut sans doute avoir l'aeception figurée de : "pétiller (de vie. d'esprit) ". Le sullixe serait -eu. fr. -eur. Je traduirais done par : "pétillant. qui lance des plaisanteries à jet continu "; comp. le synonyme spitant, qui signifie proprement : "éelaboussant, pétillant " et se dit d'une personne éveilléc, frétillante. Comparez encore djèspruice (à Stave : "gaie, enjonée "), oì dik = le néeıl. geest "esprit"?

\section{liég. stchèrdon. stièrdon}

G., II t00, à propos de stiérdon, tièdon, tchèdon "ehardon". dit simplement que stie et tie sont des modifications de teh. Il y a li un fait eurieux qui mérite de nous arrêter un instant. Beaucoup de Wallons prononcent ty comme tch, eonfondant par exemple tchesse (ehasse) avec tièsse (tête). Par réaetion, ou par une assimilation inverse. d'autres prononeent ty an lieu de tih : de lì, tièdon (ehardon), tièrdin (chardonneret). Mais comment expliquer le passage de tchi- à stie- (et stchi-). qui, chose curieuse, se produit seulement dans stièdon (stchèrdom) ? (1) Pourquoi eette modifieation n'affecte-t-elle jamais teherbon, teherotie, tehipiti, cte. ? Un phénomène aussi restreint doit avoir une eause partienlicre. Si stiérdoner "échardonner" (avec s- : lat. ex-) existait récllenent $\left({ }^{2}\right)$, rien de plus simple (que dexpliquer par l’influenee te ce verbe la forme liégcoise et hesbignonne stièndon, stchèrdon. Mais Forir a sûrement forgé ee dérivé, comme tant d'autres qui encombrent son

(1) Lit aussi, d'appès Iubert, dans stièrlin (chardonneret), au lieu de tchèrdin, qui a suivi sans doute l'analogie de stièrlon.

(5) Forir est seul a signaler ce verbe, que nous n'avons jamais entendu. 
dietionnaire; on ne dit jamais que : stitchî ais stièrdons ("piquer aux ehardons", ee qui se fait à l'aide d'un stitchon "échardomoir "). J'incline à eroire que tchèrdon, employé fréquemment dans cette expression teehnique, a modifié son initiale sous l'influence de stitchî. stitchon.

\section{w. stièrnê (V'ervicrs. Stavelot)}

G., II too, note sans explication le vervictois stienê "eaprice. boutade "(Lobet). On dit de même à Stavclot : qué stièrnê lì print-i la? C’èst dès stièmês qui lì passit ; pur sutiènês (13SW 5t. p. 8t) "par moments, à intervalles n. C'est un dérivé du verbe stiérni. non pas du liég. stierni "éternuer ". qui se dit stiemi à Vervicrs et stiennici à Stavelot. - mais de stiomi "joneher, épandre. éparpiller ". Logriquement stièrné équivaut au nam. spôrdia (eité par G.. II 380) : endroit isolé où il y a des plantes qui y sont comme répandues " : dérivé du nam. spóde, liég. spade. ane. fr. espardre). ("est $1^{\circ}$ à l'actif : le geste brusque de celui qui jette puis ça puis la : $2^{0}$ au passif : la partie du sol qui est jonchée. Une expression analogue existe à Blegny-Trembleur : (faire un ouvrage) a stiènêyes "par à-eomps. en le reprenant de temps à autre, après des arrêts brusques ou sans raison ". Comparez ei-après vièrnê.

\section{w. stinclin}

G., II 401, a un article stinelin (sic), t. de batelleric, "bout de corde servant à l'attelage du cheval de halage ". Pas d'explieation. Le mot reparaît pl. 503 et 521 (anx articles biete et dobe-coice) arec la nême faute d'impression. Il faut lire stinclin. stinlin (e'est ainsi que prononeent des bateliers (que j’ai interrogés) et y voir un emprunt dh néerl. steeklyn "ligne d'amarrage ". Pour le changement $\tilde{e}=\dot{e}$ à la protonique, eomparez en liégeois clédie. clindje (litt. "lef-Dieu" : primevère), égal, ingâl (Forir) "égal "; rôse diundiipe ("rose d'Egylte ": réséda), ete.

On trouvera, aux artieles carasse. guente, shivbalit', skith. d'autres termes de batellerie qui sont de source germanique. En voici encore quelques-uns oì nous reeonnaissons la même origine : dône (G.. II 522). voy. Weigand. Dohne "2; drik ou grek "grappin ", du néerl. dreg ; duwèle, f., faubert, du néerl. diveil ; leviza, m. (G., II 539), brosse. frottoir, du néerl. luizagen ; tif. m. (G.. II 429. altćré de stif. 365), du flam. steefel. néerl. stiff. stijeen (roy. De Bo, Franek-ran Wyk). 
w. strifer (Faỵmonville) et dérives

D'apres Lobet (Verviers), strif'ler. t. de maȩon, signifie "enduire les latten d'un plafond d'un premier mortier : hourder, faire l'aire d'un plancher. d'un pan de bois, d'une cloison, d'un lattis, ete. "(1). G.. II 408 estime que ee strifler vient "probablement de l'all. streifen (passer sur la surfaee de qqeh.) ". Lidée est bonne. mais il rant mieux s'adresser au moven h. all. strifeln (rayer, strier).

De streifen. ou plutôt d'me forme aneiemne et dialectale *strîfen. dérive strifer (Faymonville) "frotter légèrement (par ex. le beurre sur le pain.) " : $i$-a strifé " il s'en est fallu de peu " $\left({ }^{2}\right)$ : strifè dès bagadjes (Doncols, Wardin, Lutrebois) "frotter du linge". De là, strifiou (Awemne). s. m.. "lavette pou la raissclle" $\left({ }^{3}\right)$. M. C'h. Bruneau a noté, dams le même sens, strizim. strucion. à Igimont. Chooz, ete. ; ainsi que strucion qui. à Dohan. désigne le dévidoir : bâtonnet de dix centimètres. peré de deux trous par où passe [en frottant] le fil que l'on dévide $\left({ }^{4}\right)$.

\section{liég. swèlih}

D̋après G.. II $37 \cdot 2$. soilihe signifie " 1 . finàtre : soie de mauvaise qualité; :- toile jaune. gommée et luisante, qui sert à envelopper les pièces de (lrap)" $\left({ }^{5}\right)$. G. croit y reeomaître le fr. soie lisse ; mais il est certain que sailih représente l'all. zicillieh "eoutil" (ane. h. all. zwilih "tissu fait de deux fils"). Pour le traitement de la funale, eomparez ci-après virlih. La protonique wallonne $\dot{\epsilon}$ pour $i$ s'explique par une dissimilation plutôt que par linnluenee de soie. qui. dans ce sens, se dit sôye en liégeois. - Nos Chartes des Mćtiers. II 336. 1. 19, ont en 1534 me forme ancienne zuzulick $\left({ }^{6}\right)$. que G.. II 646. éerit à tort zuwillich et rapporte avec raison à l'all. zivillich.

[Romania, t. xuvin (1921), p. 576.]

(1) De même IBody, Voe. des tomeliers (BSW 10, p. 301).

$\left({ }^{2}\right)$ Bastin, Voc. de Faymonville-Weismes (BSW 50, p. 594). - Pour la protonique $i$, voyez ci-dessus l'article lifer.

(3) Même suflixe - e olum que daus le malm. forion̂ "foret ", fossion " hoyau ".

(4) Ch. Bruseau, Enquête sur les putois d'Ardenne, I $263,516$.

$\left.{ }^{5}\right)$ Le sens 1 n'est attesté que par Lobet, p. 548 ; le sens 2 est donné par Lobet, par hemacle (2ze éd., vo teull ; syn. djòne teùle di sètchê) et parr Forir. Voy. aussi B.SW 40, 3. 458, ou il faut lire soilihe, au lieu de sorlihe.

${ }^{6}{ }^{6}$ Godefroy, vur 34t, aite ce passige sans pouvoir définir notre mot, qu'il estropie en zuzilisl. 


\section{w. tahant}

Ce mot n’est signalé quà la lisière $\mathbf{N}$.-E. de la Wallonie, daus le coin étroit qui ra du S. de Verviers à Harre et Bovigny. Il ne s'emploie guère que dans une seule expression : è tahant (dèl leme), qui signifie : "au déelin de la lune ", à Polleur et à Harre (.J. Feller). à Spa (Body, Yoc. agr.). à Stavelot (Detrixhe), à Franeorehamps et à Malmedy (G.. Extraits de Villers). a Faymonrille (J. Bastin) (1). Pour Remacle seul. è tahant signilie : "pendant le eroissant de la lune" (2). G.. II +13, se contente de juxtaposer ces deux opinions contradietoires.

Je tiens de II. Jospeh IIens. de Vielıalm, des renseignements qui éclairent la question. On distingue à Vielsalm - ou du moins on $y$ distinguait naguère $\left({ }^{3}\right)$ - trois états de lunaison : 1o le crènant syn. li k'mince del lume. li time lume), après la nouvelle lune. lorsque la lume commence à se montrer. puis va cu augmentant : $2^{\circ}$ le tahomt, lorsqu'elle perd la forme de croissant et s'arrondit : après la pleine lune, e’est eneore tahant jusqu à ce qu’elle reprenne la forme de croissant; $3^{\circ}$ e’est alors le discrinant (ou l'difin) del hum. En d'autres termes. Le tahant (deuxième quartier, pleine lume, troisième quartier) comprend toute la période oi la lune est ronde ou presque ronde $\left(^{4}\right)$. Dans la eroyanee populaire. le tahant est la période du bom evè̊ant. e'est-à-dire celle où animaux et plantes se déreloppent le mieux. On sème d'ordinaire en ce moment, alors que la lune est "dure n et exerce toute son influence : les plantes eroissent normalement et sont toujours robustes; de même l'enfant engendré à eette époque "est presque toujours un garçon et est toujours robuste ". Les plantes semées \& crèhant dèl lume (pendant le eroissant, quand la lune est eneore "tendre ") "filent n, e'est-à-dire croissent trop rapidement et restent délicates; ö diseriant. la senence

(1) Exemples de Faymonville: $\hat{o}$-z-êmc mis $d$ sèmer è tahant què crè̊tent ; nosèstans è tahant ; $\hat{\theta}-\approx$-èst foù tahant; lä́ tahant è-st-oute (outre = passé).

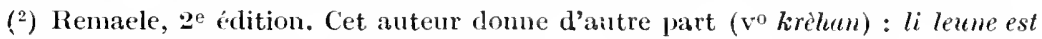
so s' crìhant "la lune est dans son croissant".

$\left({ }^{3}\right)$ M. Hens a pris jadis ces notes auprès de vieillards qui, aujourd'bui, sont presque tous déeédés. Il meécrit à ce sujet : "Les eultivateurs, même frisant la einquantaine, auxquels je viens de m'adtesser, ne savent plus dófinir nettement le takamt. Après hésitation, ils répondent : ayy (oui), c'èst crèłant; mais quelques-uns d'ajouter : c'èst co tahant après l' lune. C'est de ce genre de réponses que vient probablement l'erreur de Remaele ".

${ }^{(4)}$ A Bovigny, une enquête de $\mathrm{M}$. le $\mathrm{D}^{\mathrm{r}}$ Lomry a douné des résultats à peu près identiques : au dire de vieillards, crèchant $=$ nouvelle lune et premier quartier ; tahant $=$ pleine lune et dernier quartier. On ne parle pas du deuxième quartier, qui est sans doute eompris dans le tahant. 
ne l’̀e pas (Vielsalm). Pour guérir les dartres, on les frotte d'onguent ¿ tahant, car è céchant. on ferait coître le mal (Bovigny).

Tahant est sîrement un participe pris substantivement ; comparez ¿ crihant "an eroissant " et le contraire è d'falant (Liège), è d'falihant ou, rarement, $\dot{e}$ d'avelihant (Jupille). On prononce uniformément tahant alors que, dans cette même région - les exemples cités le prourent - crescentem domne, suivant les localités, crihant, erèaant, crechant. Comme il faut un hermanique pour expliquer l’aspirée constante de takant. on sadressera au gothique theihan (croitre, augmenter. progresser; all. mod. gedeihen), d'où dérive l'ane. fr. tehir "grandir, croicle" $\left({ }^{1}\right)$.

La dérivation proposée eoneorde aree le sens propre de tahant: période de la lune qui est favorable à la croissance". le bon croissant. comme dit .I. Hens. Si on lui attribue d'ordinaire le sens de "cléclin de la lume", cela tient à ce que le mot s'oppose généralement à "crois. sant n: on dit, par exemple, qu'il vaut mieux semer i tahant que i crehant.

Au surphus. une expression synonyme, usitée dans la région ardennaise et namuroise oi tahant n’a pas pénétré. a subi la même érolution sémantique. Pour G.. I 165. le nam. deure leune signifie "pleine lune " (opposée à time leune: "le eroissant"). Or une enquîte m'a donné les résultats suivants. Pour le sens attribué à "dure lune ". on est loin d’être daccord. ("est la pleine lune (Ciney. Grandménil), la pleine lune et le décroissant (Erezée, Namur, Lustin. Falmignoul, Ste-Marie-surSemois), le décroissant (Neuville-sous-Huy, Dorime, Wardin). le dernier quartier (Alle-sur-Semois). Sur tous ees points, on appelle "tendre lune "le croissant on premier quartier (2).

[Résumé de 13D 1913, p. 93].

\section{w. ard. tahon}

Le meusicn talum, s. m., signifie "argile, terre glaise ", dont on fait par exemple de la brique (Labourasse). D'après une communication perromelle que je dois à l'obligeance de M. Int. 'T'homas $\left({ }^{3}\right)$. ce mot

(1) Voyez I)iez, 1) 406 ; Godefroy, тепn.

(2) I.e (2uestiomucire de Folklore (Liège, $1891 ; n^{00}$ 902-6) - reproduit par Eug. Ionserr, Le Folklore zeallon (Bruxelles, Rozez, 1892) - prétend que le nam. dl deure leune signific " :u croissant ", et èl tinre leune : "au décroissant ". C"est tout le contraire. I'n octogénatire d'Erezée nous disait à ee propos : $i$ i fä arou noule

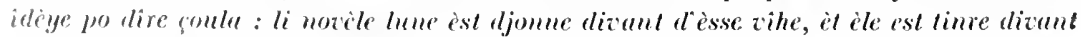
desse dore, lais-se!

(3) lin diste dn 2.5 juillet 1913. 
représente le gothique thâhô, moyen h. all. têher, dâhe. all. moderne thon, qui a le même sens. Cette étymologrie sömpose à l'évidenee, que l'on voie dans tahon un emprunt direet de thâhô ou un dérivé de tâhe. formé à l'aide du suffixe $-m$.

Dans un petit coin de nos Artemnes. à Cherron (Sud de Liège) et surtout au Nord de la province de Luxembourg ( ${ }^{1}$ ). on connait aussi tahom. Il y désigne tout rase de cuisine, récipient de terre cuite, de verre. de poreclaine et même de paille tressée : on tahon po less poyjes (Tohogne) "une ćcuelle pour les poules" ; lis tahons dè manèdie (ib.) "la vaisselle du ménage" $\left(^{2}\right)$ : lís possoms, potits, tryents, ertmeus.s tot çoula c’èst dès tahons (Villers-Ste-Gertrude) : one combène plêne du î̀s tahous (Chevrou). Il est à noter que le moven h. all. tâhe, dâhe arait déjà ce sens dérivé : "pot de terre". - Non loin de cette région. en Famenne, tahom a éélé derant téchom, qui est le fr. iessm. $\left(^{3}\right)$; on dit aussi lès tèch'nis' pour désigner la vaissclle (ynn. lès chilis'. dérivé de chèle "éeuelle". liég. hièle). Enfin. plus an Sud. dans la région de Reeogne et de Faurillers, ainsi que dans le pays gaumais. règne le not tupin, sur lequel on peut consulter Godefroy.

\section{liég. tak'lin}

D'après G.. II 414. tak'lin. t. d'oiselemr. désigne un " jeune oiseau pris peu de temps après aroir quitté le nid ". Pour Lobet, c'est simplement "un petit rossignol "; pour Remaele. 2e éd. : "un jeune rossignol, un jeme linot, ete. "; pour Hubert et Forix : " un griset. un jeune chardonneret eneore gris \%. J. Defreeheux, Famme atellomme. est d'aecord aree les deux derniers et donne de plus ce sens général : "jeme oiseau qui n'a quitté le nid que depuis peu de temps".

Scheler (ap. G.) croit y voir un dérivé de take, fr. tache; nais cette conjeeture tombe devant le fait que les dictionnaires néerlandais ont un article takkeling : "oiseau branehier" (c.-̀-d.. d'après Beseherclle : "jeune oiseau qui n'a eneore que la force de voler de branche en branche ") et que les dialeetes flamands domnent à tolkhe(r) ling le scons de : "jeune pinson qui est pris à l'automne et qui n’a pas encore véen un

(1) J'ai relevé ce terme inédit à Tohogne, Villers-Ste-Gertrude, Erezée, Hotton, etc. Il faut naturellement le distinguer de l'homonyme tahon, tahan "tron".

$\left(^{2}\right)$ Le mot y est en défaveur ; on dit pluss souvent lès helons, dérivé de hèle "éeuelle".

( $\left.{ }^{3}\right)$ Amyot donne encore à tesson le sens de "vasen. - Pour le montois, voyez Delmotte THÉchos, Sigart tÉcnoN. 
hiver " $\left.{ }^{1}\right)$. I a-t-il là un dérivé de tak "branehe " ou du moyen néerl. taclen "prendre. saisir" ? En tout eas. l'origine germanique de notre taklin ne fait ancm doute. Quant au féminin tak'lème, que donne la Faume de J. Defrecheux. il est dî̀ à l'analogie de cusin, -ème, acisin, -ine. ete.

\section{w. tanawète, totènaveûte, etc.}

G.. II 456. laisse inexpliquée eette expression singulière. dont il cite quatre formes arec trois sens différents. Voiei, là-dessus, le résumé de ce que j’ai recueilli dans nos dialecten. Le ehiffre qui suit la localité renvoie au tableau des significations.

Fonmes dalectanes. - A. totinazite Jodoigne 4 ; totanazite nam. (G.) 1. 3 ; ftanaricte Ste-ilaric-Geet lez Jodoigne 3 ; d(i) tènariète $\left({ }^{2}\right)$ en limenne. Beauraing. Namur (Wérotte, p. xxx). Neuville-sous-IIuy 1; d(i) tanawite Awenne 1 ; tènawite Marehe-en-Famemne, Ben-Ahin, Andenne. Ciney. Fos:e-la-Ville. Farciennes 1; tanawete Namur (Pirsoul), Bande. Vonçche. Florenville. Amberloup, Jodoigne, Gougnies 1 ; tenarie lBouftionlx 1.

13. totimazite Robertrille 2. Faymonville 3. Liège $5\left({ }^{3}\right)$; tot nañeute

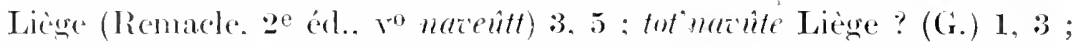
totenuite Starclot, Malnedy. C'herain 3 ; tot nacite Starelot. Vielsalm, bovinny 3 : tot narute Villers-Ste-Gertrude 3.

Siciafficiosi : 1. parfois, de temps en temps (sens le plus usité arec le $3^{\mathrm{e}}$ ) : - 2. à l'improviste (releré seulement à Robertrille-lezMalmedy : i-aconte totinacite : totemarite $i$ récr, il accourut, il vint à l'improviste): - 3. tantot (dam l'avenir), un de ees jours. d'un moment ¿ lanutre. dams un certain tenps (plus indéteminé que quand on dit trit-räle); - t. tantot (cians le passé). il y a un instant (seulement à Joloigne, dans une piece d'Edm. Etienne : nos dijin totenarite'... nous disions tout a thenre... " BSWT 35. p. 223) : - 5. toutefois. néammoim fenlement dams le groupe 13 et daprès les dictionnaires

(1) Voy. De Bu ct le Supplement de sehucmuns. Les dietionnaires étymologiques de Fanck-van Wyk cet de Vereoullie ne mentionnent pas talikeling.

(2) Lat syllithe tot (tout) perd sia voyelle après sylabe maseuliue et devient $i t-$;

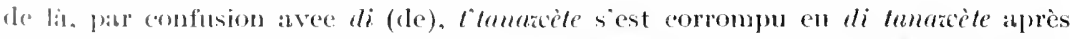

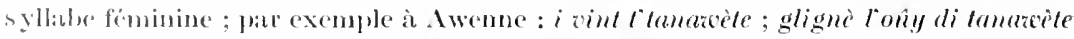
(il vicnt I arfois; rligner l'a.il de temps en temps); et en namurois : dire di tinazede one puler. - Des antenrs liegerois ont rarement use de la lorution nammerise, qu'ils coriscut di time arèle ("le temps en temps"); en réalité elle n'ent pars liégeoise.

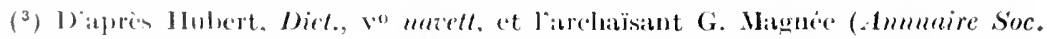

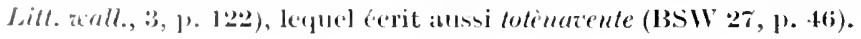


liégeois de Remacle et de Hubert ; sens disparu aujourd'hui avec l'expression elle-même).

Cette diversité sémantique, on le voit, est presque aussi compliquée que les rariations phonétiques de ectte curieuse locution. Essayons de nous retrourer dans ce dédale. Le type le mieux conservé, à mes yeux, e'est le totèntaiète de Jodoigne, qu'il faut décomposer en tot-ènawète. J'y rois le représentant dialeetal d'un anc. fr. *tot en aoite. expression qu'on n'a yas encore reneontrée dans les textes du moven âge et qui derait signifier "tout de surcroît ". J'ai montré ci-dessus, p. 205. que le w. racite "surcroit. petit suplément gratuit de marchandise" vient du latin *re-adaueta et que le simple adaueta survit dans le namurois dlacite "de surcroît". Nous avons ici, dans une locution de frappe originale, une autre survivanee de l'anc. fr. aoite, avoite "augmentation ", participe féminin du verbe aoire. latin *adaugẽre.

D'après des renseignements encore tris incomplets. notre expression serait connue dans me grande partie de la moitić orientale de la Wallonic. La limite quon pent traeer provisoirement serait une ligne partant des environs de Wavre, éeornant légèrement le Hainaut (Farciemues. Boullioulx, Gougnies). sautant à Beauraing et à Vonêche (au Sud de Dinant), puis à Florenville.

Les deux groupes présentent des altérations distinctes. Le groupe it altère surtout la syllabe initiale tot. qui re rédnit à t't- et nồme au simple $t$ final ( $\left.{ }^{1}\right)$ : mais la tonique -riete se couserve remarquablement intaete $\left({ }^{2}\right)$. Au contraire. le groupe B - comprenant à l'extrême NordEst une bande limitée à peu près par Liège. Villers-Ste-Gertrude Cherain. Bovigny. Vielsalm et Malmedy - respecte le premier élément et même en général le second $\left(^{3}\right)$ : mais partout le troisième élément devient méconnaissable : at, si bien comervé dans rewète passe à v $\left({ }^{4}\right)$

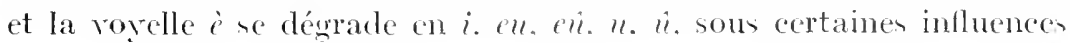

(1) Sur la corruption d(i) $t$-, voyez l'avant-dernière note. - A la deuxième syllabe, certaines formes ont a au lieu de $\dot{e}$; sur le traî́ment du lat. in prép. ou préfixe, on peut voir Grignard, Phonet. de lOnest-ziallon $\$ 36$ (BSW, t. 50, P. 417).

${ }^{(2)}$ Sauf à Bouffioulx : tanaziè in eôp " de temps en temps un eonp ", d'après Grignard, op. cit., $\$ 131$; et à Tohogne, où je relève tènazite aux sens 1 et 3.

$\left({ }^{3}\right)$ La syncope de la protonique non initiale, qui réduit totè en tot est ici normale ; eomparez nam. bolèrljî, tchèrpèt $\hat{i}=$ liég. bol dji, tehèp'ti, boulanger, charpentier.

(') Probablement sous Jinfluenee de nazette. On a perdu de vue la parenté de rawète et de tot-èn-azè̀te. Comparez malm. awoir, a l'azoir = liég. azveuir (*agurium, heur), a l'azîr (au petit bouheur). 


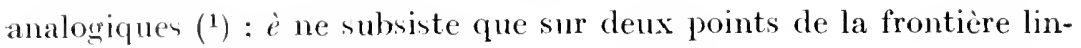
guistique à l'Est de Malmedy.

A première rue. la filiation sémantique offre bien des obscurités. Comment rattacher à l'étymologie proposée les sens énumérés cidessus? En fait, ce n'est pas l'expression isolée "de surcroît " qui, par un processus logique et indépendant. a engendré ces diverses significations : c'est le contexte de la phrase qui paraît la revêtir d'acception apparemment si peu conciliables. Ce que l'on vous donne de surcroît. on ne vous le doit pas et proprement vous ne l'attendez pas. De là : il vient nous roir de sureroît ( $=$ en plus des risites convenues, quand on ne l'attend pas) a pu signifier "parfois, de temps en temps" (sens 1), ou "à l’improviste " (sens 2 , rare). De même : je viendrai vous voir de surcroît (= quand rous ne vous y attendez pas) a pu signifier "un de ces jours" (sens 3). Si cette analyse parait subtile, roici une autre raison : l'ellipse. Lexpression complète est : "par surcroît une fois (ou certaines fois) "; ainsi tanarcè on côp. à Bouffioulx ; tanaciète (it) dès côps qu'i-gn-a. à Jodoigne. Dans l'usage courant. on supprime les derniers mots. en réalité les plus significat ifs. et le sens assez paurre de tanariète s'enrichit de ee qu'on sous-entend. - Le sens 4 résulte évidemment d'une confusion. formelle aree nawếe "nagnère " (2). Enfin, dans la phrase : il se peut que rous n'ayez rien à eraindre, par sureroît prenez vos préeautions, çoula s' pout. tot'nazeûte loukîz a zos $\left({ }^{3}\right)$. on voit qu'un " tout efois " est sous-entendu entre les deux propositions. Le sens propre de tot'nazeûte s'étant oblitéré, on a pris cette expression pour un synonyme de "toutefois".

\section{liég. tap'kène. anc. fr. touppequin}

G.. II 417. note sans explication le liég. tap'liène "tinette " (d'après Durivier). "bassin de garde-robe " (d'après Forir). La terminaison indique une origine flamande. Il faut en effot s'adresser au flamand

(1) Notamment zite as déteint sur to' uazite, et rèzute "laps de temps" (à VillersSte-Gort rude) sur tot inarute.

${ }^{(2)}$ A Viesville, au nord-oucst de Charleroi, tèmacêre signifie "naguère " (d'après 11. Os(ar Pecqueur) ; ici le croisement a dénaturé la forme autant que le sens ; je n' 'n ai jas fait état ci-dessus, paree que notre expression, dont il ne reste phus qu'un débris (tì-), est súrement importée de l'Est.Je relève eneore dans un texte de Malmedy "e 'urieux exemple : il avéze bin l' dotance qu' tot-èt-azè̀re il aléze nos quiter (Armonar do $l$ Saméne, 1889, p. 60) ; ici le croisement affecte seulement la forme ; Je sens reste : "bientôt, un de ces jours" (sens 3).

(3) Exemple (lomué par Remacle, Dît. wallon, ze éd., vo nazeûtt. 
limbourgeois tob "seau". néerl. tobbe "eurier, cuve", diminutif tobbelje "tinette" (ef. angl. tub, all. zuber, zober). Le mot, areliaïque en liégeois, lui rient du Nord : nous relevons en effet sur le Geer tap'liène "tinette" (Roelenge). "demi-seau en bois" (t. areh., à Glons). Pour la forme, on sait que le wallon ehange sourent la protonique $o$ en $a$; l'influenee de taper (jeter) est d'ailleurs iei des plus rraisemblables. Pour le sens, comp. le verviétois til'nète " 1 . cuvette, 2. pissoir " (Lobet), altéré de tin'lète (G.. II 431), dérivó de tène " tine ".

Godefroy a un subst. mase. touppequin dont il ignore le sens. Il eite deux textes de 'Tournai (1446. 1515). oì il est question de touppequins de roisin. Evidenment, il s'agit du même mot, moins altéré qu'en liégeois moderne.

[Romania, t. XLvil (1921), p. 5\%6.]

\section{w. tauye. tôye (Wrarre)}

Ce terme inédit ne nous est signalé qu'à Warre, petite ville brabançomne qui touche à la frontière linguistique : mi tchau èst tauye "ma viande est coriace ": il èst co pus tauye qui l"ûme dè diâle "il est encore plus dur que l'âme du diable " $\left.{ }^{1}\right)$. On y reeonnaît sans peine un emprunt du néerl. taai "eoriace" (all. säh). L’ane. fr. tai "bone. limon "se rattache, comme on sait, ì la môme famille $\left({ }^{2}\right)$.

\section{w. tchal'mê (Fanenne)}

Ce mot n'existe. croyons-nous, que dans la Famenne : fé do tchal'mế y signifie "faire du tapage ". C'eut le fr. chalumcau (flûte ehampêtre), employé ironiquement; comp. l'ane. fr. chalemelev (jouer du chalumeau), qui arait aussi, par ironie. le sens de "erier, bavarder". La phrase : lès tchins sont tot sbarès d'ètinde parèy tchal'mê (Marehe-enFamenne) se traduira done par : "les ehiens sont tout effrayé d'entendre pareil coneert, pareille mu-ique".

\section{liégg. tchèsså - pareûse}

Ce terme, propre à Liège et à la banlieue liégeoise, signifie " eloison, mur de refend, séparation assez légère, formée de bois, de plaeage ou de briques, pour faire deux ou plu sieurs pièces à l'intérieur d'un loeal ".

(1) Communieation de I. Eugène IIeynen, de Wavre.

$\left({ }^{2}\right)$ Cf. Ulrix 2157-8 ; Neyer-Lübke 8531. - On a voulu rapporter à la même source les tazces, nom de lieu à Liège (voyez Gobert, liues de Liège, t. III, p. 576-7); mais la chose est des plus douteuses. 
Daprès un vieux maçon de Jupille, qui prononce tehèzå-parentse ${ }^{1}$ ), c’i-st-ine cuìzm. (cloison) d'ine dimèye brique po té dềs p'titès tehambes foù d'ine grande; on fêt ossu dès d'mèyès tehèsa-pareùses quand le ni montit nin disquiri plafond.

G.. I 156, partant d'une forme tchèsse-ci-l'-parê̂.se que domne Remacle, ze éd., reconnaît dann tchèsse le lat. capsa. Plus loin (II xvin), il précise son opinion : "L'expression chèse-ii-l'-pareùse ou chèse-âzparétisez répond littéralement au fr. châsse-ì-la-paroi ou châsse-auxparois, c.-ì-d. châsue destinée à recevoir les briques qui formeront la paroi, et telle est réellement sa signification propre. quoique dans l'usage ordinaire on s'en serve, sans faire cette distinction. pour signifier l'ensemble de la cloison maçonnée...". Plus loin eneore (II 195), il classe de môme les deux significations qu'il prétend trouver à notre expresion : "1. châsis de la cloison. 2. la cloison elle-même ".

En réalité. la première signification est inventée pour les besoins de l'analyse. et la forme al'où part G. est due à un caprice de l'étymolugie populaire. Il faut écrire tehissa-pareûse et roir san doute dans tehèssa in adjectif du type latin *apsalem, dérivé de eapsa "ehâsse". ce cerait une paró̀ ì châsis ou à encadrement. En tout eas, l'expression est comporéc comme hièrdi-zónge, roie herdale. c.-ì-d. de la herde. tchìri-zôye. voie charale. e.-i-d. par oì peut pascer un char. roya-zôye. voie royale. De même que le lat. regalem. ruya na qu une forme pour

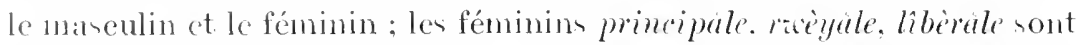

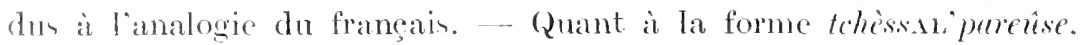
que (r. eomidérait comme étant la prenic̀re. elle prénente un type curienx d'altération. Lorsqu'il ne s'agit que d'une seule paroi, l'expression ine tehesse-puretise a quedque chone de choquant pour eelui qui l'analye comme G. (thisse-as-parentses) : aussi la logique populaire.

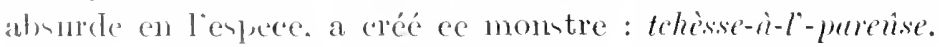

Fin tout citat de canse, les formes anciennes sopposent ì l'interpré-

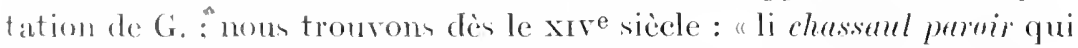
tat cutpe ladicte mayson et le mayson qui..." $\left(^{2}\right)$ : "une chassanz

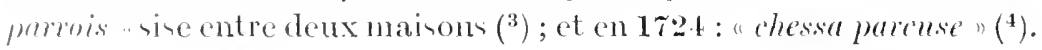

(1) lone landoncissement de -ss- intervocalique, comparer tchèssis (châssis), écrit thriclam Forir, et chetzi (lire tchizi) duns Lollet ; meisse-ozri (en liég. muiste-ovri) que le malucidien prononce mísorri ; pasi (sentier) au lieu de *passî ; cte.

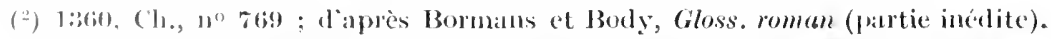

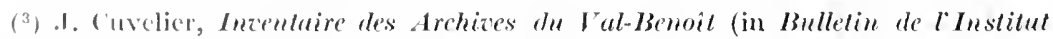

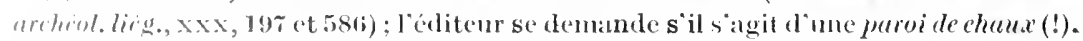

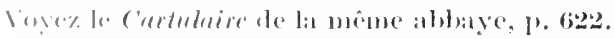

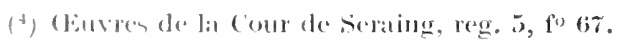


On remarquera que les deux preniers textes s'appliquent à un mur entre deux habitations et non à mo mur de refend, comme e'est toujours le eas aujourd'hui. D'autre part, l'aneicn français ne comnaissant pas chassal (dérivé de chasse), mais bien chesol (dérivé de casa). on pourrait se demander si tehessef ne reprénente pas caralem (1). Cependant la graphie ss des formes ameiennes parait bien exelure ce type et ne laisser de choix qu'entre *eapsalem ou un *aptialem. clérivé de ehasser: ce serait, dan ee dernier cas. une paroi qu'on chasse ou eonduit entre les gros murs.

Dans se. Eaux et Fontaines publiques ì Liège. M. Gobort rénume, p. :280, un texte de 14\$3, oì der religieux proclament que leur résidenee a droit à reecroir de l'eau d'un misseau "autant qu'il peut en passer par le trou d'un cessaul " : p. 27.2. il citc. du 21 juillet 1556. un édit qui défend de mettre empechement "al eawe et rieu deschendante del fontaine aux Tawes... entrante parmi he trau dung chcchal terrier en la rualle en Vingnis ". Léditeur identifie aree rainon cen deux termes, (qu’il défmit : "ouverture dont la eapaeité de réception est maintenant ignorée :- On peut eonjecturer que chechal terrier ou cessanl désignait un chenal où la terre ćtait soutenue par des (duarpentes ou ehâsis de planches. Godefroy attribue ì l'anc. fr. chusse (de eapsa) les sem suivants: " 1 . eadre: 2. ourerture carrée pour l'extraction des pierres" (aree trois exemples de Laon, où il s'agit phutôt de conduites d'eau; comparez. dam God.. chesseguet. i Laon). S'il n'y a pas, dans cet article, confusion entre deux mots d'orighine différente, l'ane, liég. cessaul, chechal (conduite d'cau) sexplipuera de meme par *apualem. sinon. ee serait un dérivé dn verle chasser : opinion que lon pent du reste également soutenir ì propor de tchissa-parcuise.

[BD 1918, p. 59. Remanic.]

w. tchètê. rouchi kèt'fi, keat'fi, ete.

G., I $15 \%$ : tchètê (ligncul), nimm. tchitia $\left({ }^{2}\right)$. On pourrait comparcer Ic rouchi quetefi, m. signit., mais il parait que ce n’est qu'une forme alrégée de lientefi, qui signifie proprement d’après Hécurt : "fil ì enudre".

(1) Voy. God.: chesal "domaine, Iabitation, ete. ", qui répond an lorrain chasau "emplacenent, sol d'un édifice ", à l'anc. wall. chasend "espèce de terre" (1395, Cart. Ste-Croix) et au 1. d. o tchèzu, ozès tchèzâs (par ex. ì Bovigny). - Villers (1793) donne le malmédien "chìzu [lire thè̀zâ], s. m., rente qui se paie an seigneur sur les cheminées, fonage "(G., Extraits de Villcrs, 1).21). C"est l'ane. fr. chesal " habitation ): payer le chesal $=$ payer la rente pour l'habitation.

() G. écrit "1. chetai, -ia ". Le (hiffre 1 doit être supprimé, car lat forme " 2 . chetai (panier...)n n'existe pas : le liég. prononec dins ce cas tchèté, comme le namurois; voy. G., II xvir. 
Ifécart explique en effet lieutefi (chégros. ligneul) par " fil à coudre. de lieute. coudre. et de $f i$, fil $n\left({ }^{1}\right)$ : mais cotte analyse n'a pas de valeur, un subtantif composé sur le type de " eoudro-fil "étant impossible. La comparaison que G. fait entre le radieal de tchitê ct le premier élément de lictefi. lieutefi. en est d'autant fortifiée. Il n'a pu la pousser plus avant, faute d'une docimentation suffisante. Pour résoudre un problème eomme celıi qui nous oecupe. il faut pouroir étudier une série de formes du même terme (série phonétique) en même temps qu'une série de termes traduisant la même idée (série sémantique). Les éléments suivants nous permettront de tirer des conelusions solides.

Ligneul. fil poissé du cordonnier et du bourrelier "se dit :

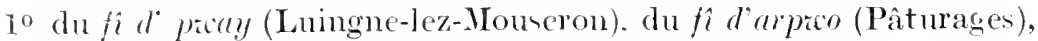
c.-à-d. du "fil de poix" ; all. pechdraht. néerl. pekdraad.

20 du fil gros en rouchi : Toumai, cte. : roy. Hécart. p. or. Vermense P. 244 : - du gros fi à Verviers: Lobet, p. 229:- du fî, en pays gaumair, tandis que le fil à coudre le linge s'appelle filìy "filé ": - du (ou in) bout'fi ou bout di fi à Bertrix et sur la Semois inférieure : Oisy, Chairiere. Gros-Fays, Alle; ete.

$3^{\circ}$ tchité à Liège, Verviers. Ma!medy, Cherain. Warche-en-Famenne, Bastogne. Noufehâteau : -és à Fauvillers: - tchètia, tchèteha à Huy, Namur. Girct, Jodoigne, Charleroi ; par dissimilation : tetia titela ì Ben-Ahin. à Dinant, Philipperille. Luttre ( $\left.{ }^{2}\right)$.

$4^{\circ}$ Enfin. dans le Ilainaut principalement. ainsi quä Couvin et à l'Ouest du Brabant. nous relevons des formes curieuses, remarquables par les variations du premier élément :

djètfi Pâturages : djeutfi Fontaine-l'Erêque. -îy Rognée, -îgue Biesme-ıous-Thuin ;

suitfîy Erquelinnes: gutfi Mons (Sigart. p. 203. écrit : guide-fi); licffi IIourlcus. Marche-les-Ecaussines; lientti Toumai. Ellezelles; liuthone 'Trivicres ;

tchinfi chapelle-lez-Herlaimont, Godarville, Seneffe, Manage; tchinfi Nivellen. Baulers; tchiffi Chimay, Bourlers. Wiers. -n Luttre; tchéfi Thimón. Vienvilie : tche'fi, tiesfi Courin.

Lou doux pronicrs gromper ne prérentent pas de difficulté. Les denx antres. de prime abord, sont plus réfractaires à l'analye. Seul tchèn'fi

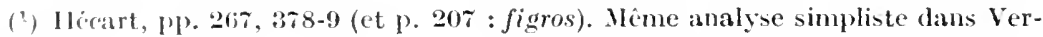
mense, 1. 151 : crulfi; ; et dans Jouancoux-1)evanchelle: "cueugros, (fil) qui eoud gros: (!).

12, Voyez amssi Bruneat, Enquete, I, p. 39\%. 
paraît s'expliquer d'emblée : la composition "chanvre-fil " (= fil de chanvre) serait aussi légitime que celle du liég.-nam. tchène-simince (chènevis : G., I 155) et de lanc. w. lismence (lin-semence : G., II 615). Partant de là, on songera peut-être à voir dans les autres formes du to des altérations de ce type limpide et même dans tchìtê un double diminutif de tchène (chanvre) pour *tchèn'te... Mais on ferait fausse route : *tchèn'tê n'aurait pu phonétiquement se réduire à tchètê ; et puis, si "du ehanrre "se dit en liégeois dèl tchine, on prononce dél tchane dans la région qui a tchinfi.

En réalité, l'explieation des groupes $3^{\circ}$ et to nous est fournie par le fr. chégios, synonyme de ligneul.

"Chégros ", pour" "chef-gros", signifie proprement "gros bout"; il est composé de chef (lat. * capum: elass. caput) et de gros, comme le rouchi fil gros. Nous verrons donc dans tehèt'i, liètfi les formes les plus pures du $4^{\mathrm{e}}$ groupe : il faut écrire tchi-ou kì-d'-fi. e.-ì-d. "chefde-fil ", chef signifiant "bout " et se prononçant comme dans "ehefd'ceuvre". Ainsi tchè-d'-fi répond littéralement à bout-d'-fi (Bertrix, etc.). Toutes les autres formes du t. $^{\circ}$. me tche $\hat{e}^{\circ} f i$ apparaitront sans peine comme étant des altérations de ee type primitif, produites sous l'influence de mots tels que jet, jeu, quene ou coudre, chieu, cul, guide ${ }^{1}$ ) et d'autres encore qu'il est difficile de déterminer. Il ne faut même pas toujours chercher un sens à ces altérations.

Quant ì tchètê, il représente le diminutif latin capitellum "petit bout ". On a dit d'abord un tehite $d$ fi (comme on dit eneore à l'Onest un tchi- $d$ '-fi), puis le déterminatif n'a plus été considéré comme nécessaire et tchètê a perdu son sens général pour ne plus désigner que le bout de fil poissé. Ce proeédé sémantique est des plus communs : ainsi le gaumais fî ne désigne plus le fil en général, mais seulement le fil poissé du cordonnier"; le fr. ligneul lui-même signifie a l'origine "petite ficelle"; voyez enfin nos articles beir, hoye.

[BD 1913, p. 101. - Pour avoir une étude à peu près complète sur les noms dialectaux du "ligneul " en Belgique romane, voyez ei-dessus lauticle spini ]

\section{anc. fr. tenreux}

Godefroy ne peut traduire ce mot qu'il dome d'après un texte tournaisien de $14+4$ : il y est question d'un ouvrier qui "sera tenu de livrer toutes pierres sans fendams, sans cromste ne tenant, et toutes mortures et tenreur mettre hors ". - c cé subst. fém. (et non masc., comme pré-

(1) Voy. par exemple lartiele de Sigart sur guide-fi. 
tend God.) se retrouve dans le verviétois têreûr "partie tendre au milieu de la pierre " $(\mathbf{1})$. Il faut renvoyer le texte sundit à l'article tendror, où manque d'ailleurs le sens technique que nous venons d'indiquer.

[Romaniat, t. Xlvin (1921), J. 57\%.]

\section{liég. tèroûle}

G. II 425 : tèroûle (charton mélangé de terre pour avoir été pris sur les affleurements de la houille) ; dans le manuscrit Orban tharoulle, Malmedy tiroule, nam. lèroule, roneli tiroule. Malgré l'alplarenee, ee mot ne peut être eomposé de terre + houille, qui se dit hoye en liégeois ; ee doit être un dimimutif de ter on $t u r=$ tendre (voy, tinre), peut-être me variété de timrüle, têrüle.

G. a raison de repousser l'étymologie populaire terre-houille; mais son essai d'explication par timrûle n'est pas moins inaceptable : le suflixe -ule ne peut en aucun cas devenir -ôle et la protonique tinrpeut saltérer en têr-. mais non en tèr-avec $\grave{e}$ bref.

On prononce tíroule partout (Liège. Stavelot, Namur. Hesbaye), sauf ver l'Ouest-wallon, oì la finale sabrège (Fosses-lez-Namur, Viesville-lez-Gonelies), et en montois, on elle devient -ouye (de là la graphic incxacte de Sigart : terre-houille). - La défunition de G. concorde aree celles de Bormans. Toe. des homilleurs liég. : "charbon de mauraise qualité quon troure aux sopes (têtes) des veines " $\left(^{2}\right)$. et de Sigart : "tête de reine imparfaitement minéralisée ou altéréc par l'action de l'air et le mélange de substances étrangères ". C"est le sens que Littré attribue aussi au fr. téroulle. qui est emprunté du liégeois. Çà ct là notre mot prend des acceptions spéciales : houille en général " ¿ Crehcon (Hesbaye) et an charbonnage de Gives-lez-Andeme : eharbon menu "à Fosses-lez-Namur : "espèee de terre-tourbe "à Viesrillelez-Condices.

Il fant roir dans timbile un dérivé de tère (terre). formé au moyen du sullixe diminutif -ôle (lat. - cola). C"est proprement de la "petite tarre". de la trerce fine. légère et friable. D’une terre qui s’émiette si

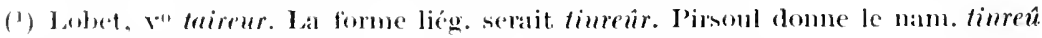
temeretre (de lit viande) e.

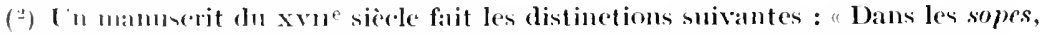
(⿻) ne rencontre dorelinaire que de la tharoule, qui est la moindre de toutes les denrien: prin du fremx charbon, après cela du charbon, et enfin de la houille". - I a liroukte, mélangece avec de langile, sert ì faire des brifucttes ovales à brûler : clûtes

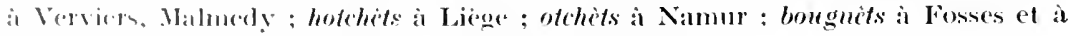

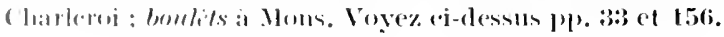


d'hayctêye) aisément, le lićgeois dit qu'elle trûle come dìl tèroûle ( $\left.{ }^{1}\right)$. La forme anciemne taroule rappelle une prononciation de $\dot{e}$ trìs ouvert.

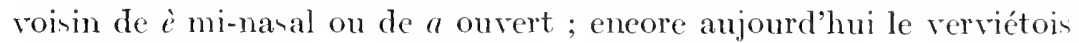
prononee têre (terre) à peu prèn comme timre. La présence de $r$ n'est sans doute pas san influenee sur la royelle précédente. Le franẹais du $\mathrm{xrI}^{\mathrm{e}}$ siècle a de même hésité entre a et è dans tarière ou tèrière. charette ou ehairette, darrice ou derriere. ete. - La forme timle. qui se rencontre à l'cxtrême Est et Ouest (Malmedy, rouehi), a vraisemblablement subi l'influence du verbe tirer. An reste, l'alternance $i: \grave{e}$ à la protonique initiale se retrouve dans tirebule (Lobet) : tèrebale (Remacle $^{2}$ ); viroûle. vèroûle (1. virole. 2. pirot ; G., II 464 et 470 ) ; voyez d'autres exemples à l'article pilaine, et eomparez tirelote.

[BD 1908, p. 49.]

\section{rouchi tévozé}

Le précieux Iroeabulaire de Frameries, que Louis Dufrane a joint aux ourres de son oncle. contient cet article: "téroset ou mieux d'térozé, parfois, quelquefois. Ce mot est d'un emploi très fréquent ; eomparez le tournaicien trévosé". Sigart le passe sou silence ; mais Hécart enregistre tréfosé. trécosé, tizosé. où il roit une contraction de très fois ct, conjecture qui ne donne aueum sens satisfaisant. - Cette exprescion est tout simplement l'ancien franeais tel fois est (= parfois), que le liégeois abrège en téltéye.

\section{liég. tèzi}

G., II 4:2\%, dome sans explication: tìi (syn. de poutener : répandre une odeur bitumineune). tisihedie (exhalaison de rapeurs bitumineuses) ". Ces mots sont ancore bien comnus à Liège. Le verbe ne s'emploie qu'impersonnellencnt : i liminee a tiri; $i$ tizih, syn. de $i$ boutène, i bout'nêye $\left({ }^{2}\right)$ : qué tézihedje qu'i têt chal (iei)!

Pour éclairer le problème. il suffit de comparer, à la même page de G., $1^{\circ}$ le nam. [?] èse tèzé "ctre embarrasé dans les voies digestives, digérer difficilement "; G. se perd en conjectures : Schcler y voit avec

(1) Le liégeois a tiré de terre deux autres dérivés intéressants : tirelote (voy. eet article) et tèris". Ce dernier signifie : "terres amoneelées, déconbles, débris d'une démolition "(Hubert) : schiste, argile schisteuse " (Simonon ap. G.), et surtout " amas de terres, de pierres et de débris de houille, s’élevant en monticules près des fosses à eharbon ". En parlant français, les Wallons prononcent avee raison tèri, comme fait aussi le montois (Sigart : terri. téri). Il est évident qu'on doit, en français, éerire terris (comp). hachis, treillis $=w$. hatchis", trèyis'); la graphie terril, qui tend à s'introduire en Belgique avec la prononciation $i l$, ne peut se justifier.

(2) Voyez (i-dessus l'article bouttener, p. 33. 
rai-on l'ane. fr. tesé (teusatus : tendu) et traduit par : "avoir le ventre teudu »: j’ajouterai : "et I'estomac rempli au point de respirer difficilement ":- 20 le verr.-mahn. téseù "rancher" (Lobet). "attache qui reticnt les ridclles d'une eharrette, traverse " (Villers ; cf. Body, Voc. des charons), lequel équivaut. d'après Scheler. à un type fr. *tenseur, ou micux, me semble-t-il, à un type ane. fr. *tesoir. - Notre tèzi n’a rien d'énigmatique : il reproduit l'ane. fr. tesir "se gonller, s'enfler. se distendre (par une cause morbide, des excès de table. etc.) " ${ }^{1}$ ). Je présume que l'on a dit d'abord : $i$ bontène chal, *on tèih (on se sent alourdi par les rapeurs irrespirables) ; puis que la première expression impersonnelle aura fait dire par analogie : $i$ tinih (il s'exhale des vapeurs qui rous alourdissent). - Sur le radical latin tens-, royez, p. 92, l'article èstéssintert.

malm. tibi-dabò. tibi-warni, ete.

G., II 427-8, a les artieles smivants:

Tibi-dabô, Malm. (sot, niais, butor). On dit à Malm. aussi dabô tont eourt, d'où liég. dabolin (nigaud). L'exp. tibi-dabô prarait être tirée de quelque texte latin, conme quandô-céli (imbéeile), voy. quando. - Tibì-haurni, Nam., raconter one saquè $a t . h$. : raeonter qqch. à tont venant, à qui veut l'entendre. Cp. tibi-carmi. - Tibi-mâreli, Malm. (rustre, homme grossier) ; mâreli signifie marguillier. - Tibi-warni, Malm. (sot, niais, badaud). Warni, isolément, signifie Werner. Cp. nam. Libi-haumi. - [Note de Seheler] Forir : "Tîbi, nom d'un persomage imaginaire : dỉe çoula a Tîbi, a Gĭli, dire eela à droite et à gauche, à qui veut l'entendre ". Sans doute les eomposés ei-dessus ont à laire à ce tibi, mais une explication historique reste eneore à donner.

L'exemple de Forir prouve que Tîbî est un nom d'homme $\left.{ }^{2}\right)$. L'î final est ici la récluction de $i \grave{e}$. comme dans Lambî pour Lambiè (Lambert), Iubì ou IIoubî pour Houbic (Iubert). Robî ou Rubî pour Robì̀, dams Rubîinge. Robertvilie. De même Tîbî est la forme réduite de Tîbie, Thibert. "Dire une chose à Thibert [et] à Gautier " a la même valeur que notre expression familière: "à Pierre et à Paul ", c'est-ì-dire ì tout renant, à qui veut l'entendre, au premier venu. Liexemple

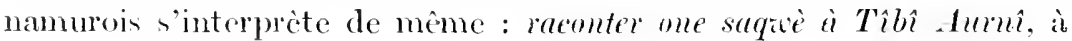

(1) Godcfroy traduit par "ĉtre gonllé "; mais l'exemple qu'il cite : l'ome tesist et enlle du venin de laraigne ", pronve qu il a tort. - Il faut anssi y rattaelier eet antre artirle de codefroy : tusis "rassisié, repu " (Jean d'Outremense, I +1.t). Cette

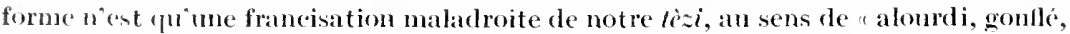
opprensé, (cougestionné (par la nourriture) ". - Enlin je relève à Gembloux le compuce eltize qui a te même sens (BSW 56, p. 162).

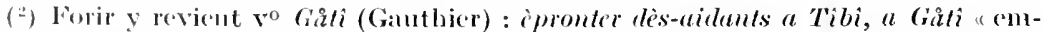

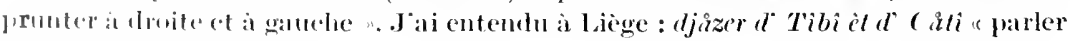
de. P'itruet le P'alll. 
Thibert [et a] Werner. Le w initial tombe en nam. quand il serait suivi du son $\hat{o}$ : aufe, liéw. wafe, all. waffel (gauffre); auder, liég. worder, all. warten (garder). La graphie hammi, qui pourrait prêtcr à confusion, indique un hiatus ou me très légère aspiration.

M. L. Molitor signale à Crehen (IIesbaye) un emploi eurieux de tibi : Dii n' li dhéve nè tîb̂̀ nè dicarmi it i vint miataquer! "je ne lui dinais pas un traître mot et il vient m’attaquer ! " Il est aisé, d’après ce qui

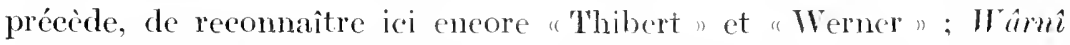
s'est altéré en dioumi (dormir) : bel exemple de corruption pour faire pendant au mâreli cité plus hant par G. ! - Mème emploi à Jupille : i nos-a quité sins dîre mi Tîbi ni W'amî "il nous a quittés sans souller mot, sans nous dire au revoir".

A Malmedy, Tîbî, flanqué de Wiàmî ou de Dabì, a pris nettement le sens péjoratif. Tous ees termes d’inuulte signifient en sonme "niais" ou "rustre ", et rentrent dims la eatégorie assez nombreuse des aneiens noms de personne (surtout d'origine germanique) dont le peuple a fait des appellations sarcantiques.

A propos de dabô, G. a corrigé sa première explication dans les Extraits du Dict. malm. de Villers, p. fir : "Il n'ent pas probable, dit-il, que dabô soit le not latin ; ajoutez par surcroit que le liég. a le syn. dabolin (1) qui semble ôtre un dérivé de dabô. Outre cet argument. l'analyse des expressions similaires et l'existence du mot dans le patois

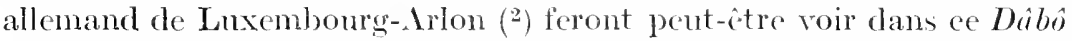
l'ancien nom germanique *Dabold ('Tabold. Tagebold). qu'on aura réuni à Tîbi par une sorte de calembour...

P.-S. - Article paru en 1908. dans les Mélenges Kueth, II 32:3. Je me hâte d'ajouter un mea culpa : j'y fais la part trop large a la survivanee d'anciens noms propres. Issurément. cette influenee existe, mais elle n'est pas exelusive. Le latin de la liturgie, de la scolastique et des tribunaux a laissé de multiples traces dam notre lexique. Le peuple saisit au vol des mots qui frappent son oreille; il s'en empare et les applique avee humour, altérant parfois les syllabes d'une façon saugrenue; les cleres et les étudiants, d’ailleurs, lui ont donné sourent l'exemple. Amsi. je me rends volontiers à l'avis de M. J. Vereoullie (Volkshunde, 191t, t. $\mathrm{xxv}$ ), qui roit dans tibi-dabo les premiers nots de la formule par laquelle le Christ dome à Pierre les elefs du royaume

(1) Forir écrit dâbolin.

${ }^{2}{ }^{2}$ Du buss $\hat{c} n$ dâbô "tu es un grand bêta ". - Comp. un grand dâbô (Alle-surSemois), m. s.; et voy. Adam, Patois lorr., p. 243 : debaue : Héceart : dubo ; G., I 35 : bâban ; Dict. gén., DABO. 
des cieux ("tibi dabo clares regni coclorum ": Matth.. xvi. 19) : et. de mone. dam quando-coli (G., I 1+4: "imbécile "). le début du verset : " quando couli movendi smot et terra " du "Libera me. Domine ". qui se chante aux aboutes et à l'office des trépasién. M. Vercoullie allògue encore le Quasimodo de Victor IIugo et le flam. luudate "femme paresseure et légère ". J'ajouterai da-nobis "individu pitọable " (à Lille: Vermesce) : quoniam on quoniam-bomns (M. Lorrain. Gloss. messin) ; hopséề "individu lourd, paresseux " (Ed. Liégeoin. Lerique gamm.) : quivédo (?) "original "(nam. : G., I 1.tz); plâcébô "lhatteur, rapporteur " (il). II 230) on "niais, dadais " (malm. : Villers). De là. de nouvelles création plaisantes. comme le liég. magnâbô (Dur., For.) "grand mangeur ". du liég. magnî "manger" ; le gaum. flâcébô "paresseux". du gaum. flâ "mou. indolent " : etc.

1)'autre part. M. Colson (Wallonia, 1908; t. xvi. 165) se demande si tîbî mầrlì. tîbî gatî. ne doivent pas s'expliquer uniquement par la rime, comme le fr. à lu tiemme. Etiennc. Cette influence est aussi très possible. Cependant. i la rime a pu agir dams certains cas, tibi dabo ou quando coli. par exemple, se refurent à cette explication. - En somme. on aurait à démêler iei de influences très diverses : aprolications plaisantes de textes liturgiques, compliquées de calembours dont les anciens

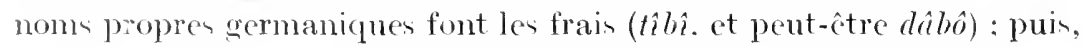
brochant sur le tout. là-peu-près et l’instinct de la rime (dwarmi, mârli. pour Wharmî), déformant de vieilles expressions au point de les rendre parfois mécomnainables.

\section{w. tîdje. pîdje ("chemin")}

G.. II 129. ne eonnait que le premier de ces mots Je résme son article :

tidje, nasc. (1. bande de gazon, telle qu'on en voit le long de eertains chemins, soit parce qu’étint trop larges une partie reste couverte, on paree que les bords sont escarlés : 2., de là : (hemin bordé de gazon). Ancien liégeois : tiege, tye ge. Namurois tirlje (au sens 1)... L'analogie de lidje (lige) venant de l'all. ledig. de Lidje (Liège) de leodicus et de sîlje (siège) de sedium, permet de rapporter notre mot a un primitif liégeois theodic, qui pourrait être le goth. thiudisk, etr. : popularis, c-it-rl. qui appartient à tous.

('ot csal d'explieation neest pas heureux : en elfet. à la même pagre, ponle "xpligner tihe (anc. fr. tiesche. tiois). G. invoque à nomveau diutise, deutuch, qui ont ici pleinement ì sa place.

Liorigine ot to s'ms premier de tîlje nou apparaitront clairement, si nous fudiom alabord le w. pîdje. 
Inconnu. du moins aujourd'bui. à l'Est de la Wallonie. ce demier mot a été jadis ct est encors très vivant dams le centre du I Iainaut (pays de Charleroi). Voiei. au sujet de pilje. "l'un de nos termes toponymiques les plus curicux n. les remeignements préeis que contient la Toponymie de Moncean-sur-Sambre. par Em. Dony et 1 . Carlier ${ }^{(1)}$ :

"On le trouve dann maintes loealités voisines de Charleroi, à Dinant, à Laneffe (Namur), à Felleries (N.-E. d'Aresnes). A Nonceau-sur-Sambre, on ne connait plus aujourd"hui, comme nom commun, que les piljes a Lalmont, olficiellement "rue du Pige ", mais le peuple continne ì dire dèskinde ou rimonter pau (par le) pidje; cótait anciennemont le nom de divers chemins de cette localité, sous les formes piege 14 frit, pierge 1490, pirge 1408... I'étymologie de ce terme a toujours intrigué nos historien lowallx. Clément Lyon, dans son Histoire de Marchieme. 1. 500, y voit un diminut if et l'explique par pichinte, phsinte (pied-sente), opinion que tous les antres acceptent sans disenssion... Or, d'alprès les pièces d'atrehives. Ies piges étaticnt le plus souvent des chemins très larges : le pige herdal menurait a charleroi $\mathrm{r} \mathrm{m}$. de large ; le piege polict est qualifié de zoye cheruable : le piege delle coullure avait $3: 2$ jieds de large a Montsur-Marchieme. En outre, les piges ćmumérés dans len chartes sont des chemins intereommunaux ; clles font me distinction trèn nctte entre piges et sentiers... Chotin, dans ses Etudes itym. et archéol. dn Hainant, p. 1r1, méeonnait notre pige. quil écrit : "Ie tige = la chaussée ,... Bien plus concluants sont. pour nous, un texte du xie siècle appelant du nom de pirgus, à Dinant, la soute royale : cia regia quae znlgo dicitur pirgns, et un autre du xu siècle (1139), où il est dit : ria publica quac vulgo peginm dicilur (Du (ange, vo pegium). Te Pige est bel et bien le conrespomdant de cin jublica et le bas latin pegium a pu donner en roman piege. altére en pierge on piege : cfo au rente pirgins ot pergus dans Du Cange... Par opposition aux chemins pricés et anx sentiers, soumis à des servitudes de passigge, les priges rentraient dans la eatégorie des chemins publics, ouverts à tous comme les heerbaenen, heetstraeten ou heeraegen connus daus tous les pays germamiques."

On me pardonnera diaroir cité ì peu pres in ertenso ect article $\rightarrow$ documenté ; tout y est excellent. sauf la partie ét ymologique. Le pegium. de Du Cange. latinisation gro sière du roman piege, n'a ancune vitalité. L'altération de piege en pierge est. de plus, inadnissible; c’est le contraire. évidemment. qui s'ent produit : pierge est la forme première et suffit ì résoudre le problème.

C'est dans une charte de 9:32. eitée par Du C'ange, que nous relevons la trace la plus aneienne du mot : pergum regium ; puis, successive-

(1) Mémoire couronné par la Sorićté de Littérature wallonne. [Nous eitons ici le texte du nanuscrit. Ie mémoire a eté imprimé en 1913, dann le BSWW 55 . Les auteurs ont corrigé, d'après nos indications, la partie étymologique de leur article pirlje.] 
ment. pirgus regins (Liège, 1131), pirgus (Reims, 1134), in pergis et amiqnis riis (Laudm, 1172), pirgins (1213). Et le sens est nettement indiqué par Carpentier : "itinerarius agger, via strata, regia ; gall. grand elsemin, chentin ferré ". L'article pierge dans Godefroy est tont aussi décisif et pour le sens (ehemin empierré) et pour l'aire d'enıloi de ce terme (Laon. St-Qnentin).

Seul le latin petreum pent avoir donné naissance it pierge et au w. pîlie. La démonstration nest pas malaisée : ềrenm donne "cicrge" (1). sans correppondant wallon : — sororium : anc. franç. serorge. w. sondle: - ferrias: anc. franç. ferges, firges = fers d'un prisomnier : en ganmais mete a fietdles on afiétlii un eheral = l'entrarer à l'aide d'une chaîne terminée par deux anneaux où passent les pieds de devant; — *inferrias: w. ifinljes (Malmedy), d'où èfirdji, ifmedji, ane. fr. enfergier ; - sêrica : serge.

Pour en revenir à tîlje. qui est le pendant de pîdje, on y rerra sans peine le lat. terreum. oì le $y$ s'est de même comsonnifié. I la vérité, les formes *terge. "tiége ne se reneontrent pas, du moins à ma comnaissance; mais la forme wallonne (francisée en tiége) est assez probante par elle-même. Ice sens premier est done "chemin de terre ". par opposition an pidje. "chemin de pierre". Il faudrait renverser la filiation sémantique établie par G., et définir : " 1 . chemin de terre, bordé de gazon : 2. de lì : bande de gazon, etc. " $\left({ }^{2}\right)$. On objectera pent-ĉtre que d'autre, voics. par exemple les sentier's, sont "de terre "; mai le tîlje a pu. ì la différence des sentiers. néeesiter certain travail d'établissement, de terrassument et d'entretien ; ef. Godefroy. TERré.

[Article faru en 1910, dans la Revue de Dialectologie romane, t. 1, p. 378. Jai su depuis hors que Meyer-Lühke, Rommu. Etym. Wört., fasce, 6 (paru en 1!13) domne * pe triea comme étymon de l’ane. fr. pierge, sans doute à cause du provençal phirega. Cela eonfirme on somme mon explieation de pidje, ear petreum ne differe de petricum que par le sullixe : les deux types doivent aboutir an mêne résultat. - Chose étrange, Neyer-Lübke ne mentionne ni lc w. tirlje ni le lat. terreum (ef. Körting 9480). Cependant l'est-wallon tîdje est remarquable ; il fait, pour la forme et particllement pour le sens, le pendant Ic lomest-w. pirlje; il ne pent s'expliquer que par ter reum ou, ce qui revient at jen près an mône, par *terieum.]

(1) Aur. lr. cirge. Dans le fr. eierge la diphtongaison est irréguliève (cf. Meyerläike, (iramm. f. w, p. is, note de la traduction française), mais ee detail na pas d'importance $\mathrm{i}(\mathrm{j}$.

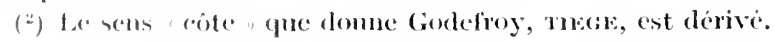




\section{liég. tîke}

G., II 429. dérive le liég.-nam. tîke "taie d'oreiller " du lat. thera, qui a aussi donné le fr. taie. l'all. zieche et l'ane. llam. tycle (eulcita). Mais thêca aurait domné *tôye, comme le gaulois r ea domne le w. rôye, fr. raie ( $\left.{ }^{1}\right)$. On ne peut pas non plus proposer * êcula, qui serait devenu *tùle, *tîle, comme têgula a domné l'anc. fr. tiule. fr. tuile. w. thile, tîle; rêgula : ane. fr. riule, w. rîle, rîle.-- Noun voyous done dans le w. tîke un emprunt du moyen néerlandais tîle (taie). qui lui-même provient du lat. thêca. Le néerl. moderne tijli ne désigne plus que l'étoffe dont on faisait la taie d'oreiller : e'ent le "coutil» au lieu de la "coute". Le w. tîle a mieux conservé la signification prinitive : taie d'oreiller" (Liège, Verviers, Malmedy, Marche-en-Famemne, Stave, ctc.). Le diminutif tîl'lète (Glons), qui derient tîglète à lioelenge-sur-Geer (et à Liège: Simonon, ap. G., II 429). a le même sens de taie d'oreiller à carreaux rouges et blanes". Le diminut if tîlite signifie "taie "à Wardinlez-13astogne $\left({ }^{2}\right)$; ailleur's (par ex. à Verviers, d'après Remacle et Lobet, ap. G.. II +31 ; à IImedy et à raymonville), il dénigne la toile à carreaux, le coutil dont on fait les taies d'oreiller. G.. II 131, a tort d'écrire tikète ct de penser, pour expliquer ce mot. à tiliet "moucheture".Enfin, dans l'ane. liég. ticle $\left(=t \hat{l} \mathrm{l}^{\prime}\right)$. l est parasite, comme dans l'anc. fr. bouticle, musicle, tunicle, etc. $\left({ }^{3}\right)$.

[Romania, t. xLvil 1219, p. 57\%.]

\section{w. tirelote (Liegre?. Vervicrs)}

Ce mot est signalé par Lobet qui le définit : "anthracite : terroulle..., maurais eharbon" ; et par Bormans, roc. des houilleurs liégeois, qui écrit : tire-lote "fonse où l'on n'extrait que de maurais charbon". G., II 4:32, l'enregistre sam explication. - Ajoutons yu'en verviétois l'expression c'èst dèl tir'lote s'emploie proverbialement dans le sens du fr. familier : "c'est de la camelote".

D'après les définitions de Lolset et de Bormans. on pourrait y roir l'altération d'un primitif *tévelote. formé de tère (terre) et du double suffixe diminutif -el-ote (comp. babelote, bourelote, fafelote, fierelote, malielote, masselote, papelote. tchoufelote). Ce suffixe diffère de -oulle

(1) Narichal, Mundart zom Gueuzane-Weismes (Bonn, 1911) croit éluder la difficulté en donnant tike : tecam comme une "formation savante"!

(2) En ehestrolais, Dasnoy, 1). 481, domne "tiéque, tiéquette, paillusse, taie ". On prononce teliètiète à Fauvillers.

$\left.{ }^{3}\right)$ Voy. Godefroy, Tuke, et ei-dessus l'article huriele. 
(roy. l'art. timble) en ee qüil peut avoir le sens péjoratif. De la tirelote, ce serait proprement " de la terre (charbon) de mauraise qualité ». Le sem donné par Bormans serait dérivé : le verbe tirer aurait agi à la fois sur la forme et sur la signification. Le substantif tîre "espèce" (royez l'article atîlêure) a pu ćgalement altérer la forme et le sens d'un primitif *terelote. Au surplus, pour la protonique $i$, comparez $t i$ ron̂le (ì l'article tènoule).

[BI) $1908, \mathrm{P} .51$.

w. tougnoûle, touyon. touǵnon, touyeter, twagne, etc.

G.. II 4:3\%. dome tongnoûle "jabot de chemise ", d'après Remacle et Lobet : Durivier note ce mot eomme étant verviétois. Forir le eonsigne dans son Dict. liégeois, mais il l'emprunte surement à ses devaneiers $\left({ }^{1}\right)$. - Nous r recomnaissons l'ane. fr. toaillole, diminutif de to(u)aille "morcean d'étoffe, servictte. nappe". du germ. twahlja "esuie-main" (voy. tmaille, tarü̈olle. dans le Dict. gén.). La forme prinitive *tou(a)youle est derenue tougnoûle par épaississement de y en sil (2).

On expliquera de même tougnon. que Lobet définit comme suit : "torchon. fenme malpropre ; tougnon. d pan, sros morceau de pain caucé on coupé par (?) havard" $\left(^{3}\right)$. G., II 43\%. reprend cet article de Lobet en comparant. pour le premier sens, tomyon (1. souillon; 2. on gros tonyon. nue femme replete, fort grase). qüil range parmi les dérivó du nam. tomyi " touiller. agiter pour mélanger "(*). On ne peut aceepter cette dérivation. Tomgnom est altéré de tomyon. mais celui-ei (comme le pieard tomillon "torchon. essuie-main ") représente l’ane. fr. twaillon. m. s.. dont Godefroy domne les formes varices tonaillon, trmillon ("). Voiei donc eomme on définira le w. tomyon. tougnon : 1. "mor-

(1) ("est le cah pour bom mombre dauticles de Fonir, dont la redaction laconique et le manque d'exemples montrent que lantenr ue tient pas ces mots de souree persominelle.

(2) Xous avous noté une loule d'exemples de ce phénomène; voy. les articles

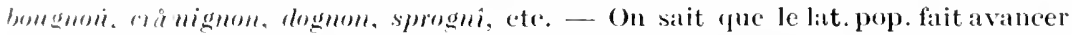

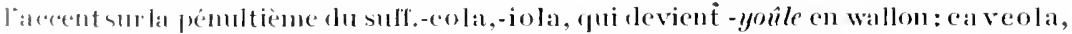

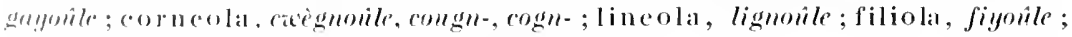

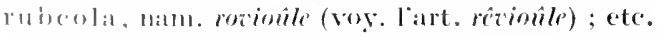

(3) Nons anwns en effet relevé à Blegny-Trembleur : longnô (-ô hervien = -on liepenis) moreaul (de pain) taillé grossièrement".

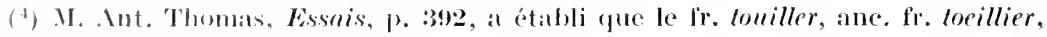
vient dul lat. turliandare.

(i) En revandhe, lank. fr. ne eommat pas *tooillon, qui serait dérivé de tooil, torillier. 
ceau de toile ou d'étolle servant is ensuyer : enuie-main, torchon "; 2. fig., en parlant d'ume lemme sale : "torchon. souillon" ; on gros touyon "une femme grasse et peu appétissante" ; par antiphrase : binami p'tit touyon (Dur.), t. d'alfection à l'adresse d'un enfant (') : 3. par extension du sens 1 : tou suon d' pan " ehanteau de pain coupé grossièrement " (comp. le fr. chiffon de paiu).

Puisque nous sommes à établir la survivance dam nos patois de l'anc. fr. to(u)aille, nous rangerons ici l’ane. w. toëtte (17:34). que Body signale sans pouroir le définir. Ce mot (quion pronongait sans doute touyète) répond à l'ane. fr. tocaillette "serviette "; de là, péjorativement, en parlant d'une femme : "torchon " $\left({ }^{2}\right)$.

A Neuville-sous-Huy, d'après M. II. Gailliard, tomyeter signifie :

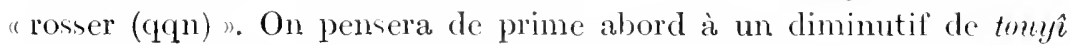
"touiller"; mais ce dernier n'a eonservé, en hutois, que le sens dérivé "lanterner, travailler arec nonehalance", tandis que, dans le môme dialecte, ratonyeter signifie : "réparer grosièrement (un vêtement)". Nous verrons done dans touygeter un dérivé de *touyète "torchon". Le sens propre (disparu ?) est : "torehomner (csisuyer, nettoyer arec un torehon) ", d'oì, au figuré : "rosser".

Enfin, à Ciney, daprès M. Léon Simon. existe l'expression : one twagne di pavin. di tchau "nn gros morceau de pain, de viande " $\left({ }^{3}\right)$. On y retrouve le primitif *tzalye, èent-à-dire l'anc. fr. touille, attesté dès le $\mathrm{xu}^{\mathrm{e}}$ siècle au sens de "morceau d'étoffe". Cette explieation, donnée

( $\left.{ }^{1}\right)$ Il vaudrait peut-être nieux faire rentrer cet exemple dins le $3^{\circ}$, en eomparant l'expression analogue binamé fitit bokìt (bien-aimé petit moreeau).

${ }^{(2)}$ Voici Yarticle de Body, Vocub. des Poissurdes (BSW 11, p. 2:37) : toëtte (hors d'usage), injure qui, comme deux ou trois antres de notre voeabulaire (voy. lodier et malchière), avait une sign. qui nous est aujourd'lui ineonnuc. Nous l'avons reneontrée une seule fois, il est vrai, rlans ect exemple : at esté si téméraire et si oublieux de son devoir que de nommer lespouse du sieur comparant toëtte; conme cette injure est d'autant plus atroce, etc. (1 13.34 , Greffe de spa, reg. 91, p. $161 \mathrm{v}^{0}$, Areh . de la province de Liège) ". Le même Focab. cite drap d' hièle, drapế, tôrchon, wite, qui s'emploient aussi comme injures à ladresse d'une femme. - Lodier n'a rien non plus de mystérieux; voyez dans Godlefroy les deux artieles de ce nom. Quant à malchiere (1573, Cris du Péron), cest tout simplement mùssire, forme ardennaise (à Jalhay par ex.) du liég. måssitte, fém. de måssî "sale ".

$\left({ }^{3}\right)$ On dit aussi trarlague (Ciney). - Le brabançon one czaggnc " une grosse tartine „ (a Pécrot-Chaussée) paraît bien être altéré le twagne (pour tw : ero, roy. l'art. tzè̀zon). - Le Dict. numurois de Pirsoul, $\mathrm{n}$ 300, a un article : tiroigne, s. f., gros moreeau de pain, grosse tartine ". L'anteur aura sans doute mal lu la note d'un correspondant qui avait écrit hroigne. En tout cas, il a un article oncleure (œuf pondu sans coquille), qui est un modèle... de coquille; il faut sûrement lire one (= une) leùse; la même erreur se reproduit à l'article zcespe. 
sans préambule. pourrait soulever des objections. Après ce qui préeède, elle paraitra naturelle : le cinaeien twagne di proin n'est pas plus surprenant que le verviétois tongnon d' pan.

\section{liég. toûlasse}

G., II 438, donne tohlasse (temme tris grasse) et ajoute quelques comparaisons ineffieacen. D’après Hoek, Liège an xve siècle. p. 120, ce mot en vieux liégeois désignait un gros tomneau. On troure en effet dans les Cris du Perron : "une thoulasse de vin du Rhin " (15.40); "une toulas de vin de Franee" (1599). C'est le néerl. toelast (all. zulast) "barrique de vin, tomneau contenant 640 bouteilles" : pour last : w. lasse, voyez G., II 613. Le sens propre a disparu depuis longtemps ; la métaphore a sauvé de l'oubli ce not d'autrefois qui, comme tant dautres, ne survit plus que grâce à une comparaison satirique. Nous lisons dans une pacquille de 17.43:

Il èsteût gros come ine toûlassc,

Il aveût on vinte comc ine basse.

Pour l'inage. comparez tonê "tomneau ". "femme petite et grosse " (Body, Iroc. des Poissardes) : le rouchi confarde (Fécart) et, ei-dessus, l'article conmê. p. 53.

\section{liég. trèfiler}

G., II 4.4. donne trifiler "1. tressaillir : 2. selon Simonon : trépimner \%. - Le mot existe à Verviers. Liège, Huy ct ju^quà Namur (Pirsoul). Il signifie "ćprouver une vive agitation, être en proie à la fic̀re que eause l'impatience joveuse $n$ : dii sin m' côr qui trifèle di djôye dè ày ci binamé (Nö̈ls. p. 242); quand l' djônê zent s' crapande, i trèfèle tot, quand le jonvenceau roit son amie, il ne tient pas en plaee, il est hor de lui, transporté d'une joie fébrile. syn. in' si sint min, il ne se sent pas. Comme on le voit, c'est plus que "tressaillir".

Pas dexplication dam G. ni ailleurs. Il faut éearter tout rapport arec le fr. technique tréfiler, w. trefiler. Notre mot provient du moyen néerl. drevelen ("itare, fréquenter ire": Kilian), qui est le fréquentatif dn néerl. dryze"n (all. treiben) dans le sens neutre de "se monvoir rapidene'nt "( $\left.{ }^{1}\right)$. Le west-flamand commaît encore drezel, drezelen ("trot, trotter : I)( Bo). Liexemple des Noëls eité plus haut pourrait se traduire familicrement par : "le cour me trotte (a la pensée de voir le H(xise) $\%$

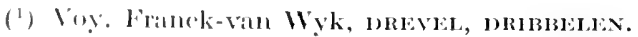


En wallon. le type primitif s'est modilié sou, l’inlluence du préfixe trè(trans) et de filer : mais. ec qui proure que l'infinitif était à lorigine *trèfler. c’est que le liégeoín conjugue : dii trifile. ot le verviétois : dim trifä̀le (comme lunfler. infler. sofler). plutot que : dii trefile (comme filer

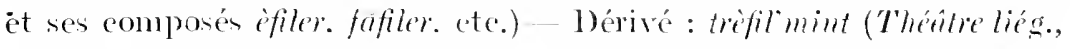
p. 116) ou mienx trifilmint (Forir) "émotion joyen e. qui sc manifeste par une sorte de fierre".

[BD 1920, p. 13.]

\section{w. trèp'ser}

G.. II, 146, note le liég. tripser. nan. tripiser "donner à une terre le troisième labour "s et propose de l'explicuer par tris-passer. Apparemment, e'est la définition sundite qui lui suggère cette conjecture. Bien que tris (pour ter : trois fois) soit insolite. on pourrait à première rue ctre satisiait, surtout si l'on comsidere que "domner à la terre une seconde faȩon "se dit biner en namurois comme en franęais et que, pour la troisicme façon. le nanumois emnloie tîtê (anc. fr. tierecoier) à côté de trip'ser (G.. II «94). Cependant, à supposer môme que la définition de G. soit exacte pour le liégeois, on sera mis en défiance si l’on apprend que. dam certains dialeetes du sucl. trip'ser sapplique it d'autres opérations de la culture. Rien de plus divers d'ailleurs que les noms attribué aux labours suecesifin. Iinsi. à IJeure-en-Famenne,

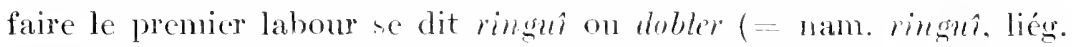

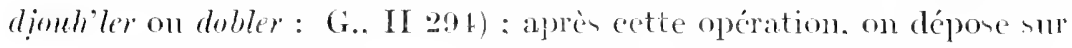
le champ les tas de fumicr et on les épand : retourner le fumier dans la

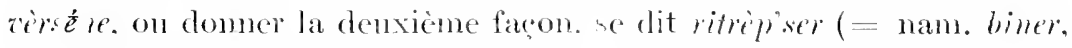

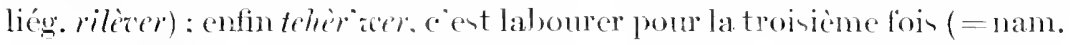

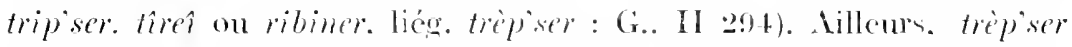
(Ferrières. Villers-Ste-Gertrude), ritrifiser (Havelange. Comblain-laTour) signifient "déchammer en enfouisant le funier : tripiser on

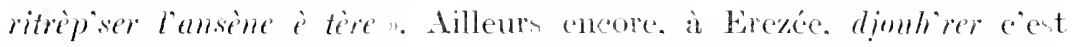
"déchanmer une dịnlhîre on jachìre et trip"ser "donner le deuxionse labour ".

On le roit par ces exemples. notre not doit aroir an à l'origine, me acception plus générale que ne l'indiquent la définition et l'étymologie de Grandgagnage. En r'alité, frép'ser reproduit le lat. trans versarc. On objectera le liég. tricierser. nam. tracièser, qui dérive de la mêne source (G, II +53); mais ce doublet, de sen. général ct d'emploi quotiden, a pu maintenir sou intégrité en s'ilpuyant su l'adscrbe tritid, trucie" traress n, tandi- que be teme technique, vidé de sa sign fication 
preniè̀e et livré à lui-même, a subi l'évolution nomale : *trèvièrser s'ent d'abord réduit à *trèv'ser (comme bol'djî : boulanger, vol'té : volonté. ete.). pour devenir trèp'ser par un ehangement phonétique dont nous domons d'autres exemples à l'article trèp'sin.

\section{w. trèp'sin}

D'aprè G.. II +66, le trèp'sin ou trèp'sî éent le grand palomier auquel sont attachés deux autres petits palomnicrs. appelés copes, coplês, coplîs ou mème trèp sîs et servant à coupler les chevaux (1). Ce terme ent propre à la provinee de Liège et au Nord de la province de Luxembourg : à Rọ (en Famenne), j’ai noté trìp'sî pour deux chevaux, andon pour trois ou quatre ehevaux; de môme. à Ortheuville. trup'sî "petit palonnier ". andon ou balance "grand palomier" (royez cidesus landom. p. 166).

De même que trèp'ser reproduit le fr. "traverner "trèp'sin et trèp'sî répondent respectivement au fr. "traversin " et "traversier ", désignant une pièce de bois qui forme traverse. Au reste, térérsier "petit palomier " existe eneore aujourdhui à Wiers (Péruwelz). ainsi que teréssi "grand palomier" à Ellezelles et à Luingne-lez-Mouseron. Le fém. trezesiere a le même sens dans un compte tournaisien de 1t2s (cité dams God., volés).

Pour la phonétique, le changement remarqual, de ie en plevant s n’a jas encore été. que je sache, signalé en wallon. Le picard comnaît les formes bisen "faisenr" pt p'ser "fesser ". Dans no dialectes, il fant

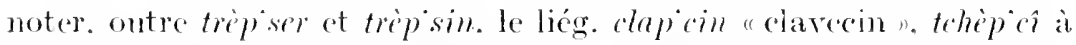
côté de teher'vi. ane. fr. chevecier (G.. II 12s). (t) le diminutif nam. stapsoml. anciennement staresonl, dépendané de la commune de Stave (2).

Grandgagnage a un autre article trip'sin "bail à cheptel "sans cxplication ni indication de source. Ce sens inattendu. que doment aumi Lobet et forir. rénulte d'un confusion grosicere avee tricin. anc. fr. trescens $\left({ }^{3}\right)$.

(1) Le Gilossaire rommu-lígeois de Bormans et Borly, $v^{0}$ areire, donne Ia forme tropsis an xure siecte. - Voy. Borly, Voe. des charrons, aux art. trèpisin, bellanee, copli, aimi que la ligure, planche n.

${ }^{(2)}$ Roland, Toponymie mam., p. 545-6. - Le mane anteur, p. 402, explique Tripsé par le nom propre d'homme Tribo: a pourratit être phu modestement

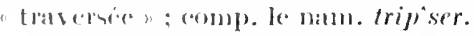

(3) Tricin 11 est pans daus Lobet, qui est probablenent responsable de ladite

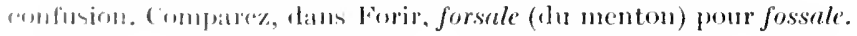


A Blegny-Trembleur. un trejp'sé (-é hervien $=-i n$ liég.). cost une languette de terrain, large d'environ 0mso, qu'on doit lainer inculte le long d'une haie bordant un champ on un chemin. Il y a là un empioi

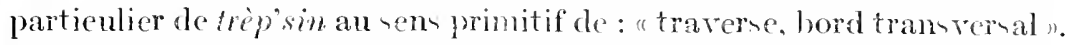
- On expliquera de nêne öl triphimu (lieu dit de Borigny) par : "en la traveraine ". Godefroy donne des exemples de traversaine comme nom de lieu.

\section{w. trèssèrin}

G.. II 4.5. note sans explication : "treserin. débâcle de glacon" " Forir écrit : "trécivin. débâcle des ghees : tapage. vacarme ". De mon eôté. j’ai entendu à Jupille tresscin. avec cette définition : "denecnte eompacte des glaces qui courrent toute la surface de la Meuse n ( $\left.{ }^{1}\right)$. On y reemnait san peine un dérivé de trissèrer (trè + serrer). formé à l'aide du sulfixe - in qui indique le rénutiat de l'action $\left(^{2}\right)$. Ce verbe, aujourd'hui inusité, se rencontre dans un passage de la Geste de Liege, cité par Godefroy : "Muene et altres riviere si forment tresserat... " $\left({ }^{3}\right)$; le simple sèver s'emploie encore aree le même sen : l'ât sizène, ile ra sirer (voyez ei-dessus l'article sizin).

De ce côté done. nulle difficulté. Il n'en ra pas de môme au point de vue sémantique. Le trèssèrin. étymologiquenent, doit désigner la fermeture complète du fleuve. l'embâcle. - ce qui paraît contredirc la définition traditionmelle. En fait. l'embâcle ent déterminé par une deseente compacte de glaçon qui peurent s'amasser et se soudes de facon a interrompre la navigation. Il ent done naturel que le sens propre se soit étendu à cette circonstance déteminante. Comparez sere (= ane. fr. serrail) qui. dans le dialcete de Givet. signific : amas

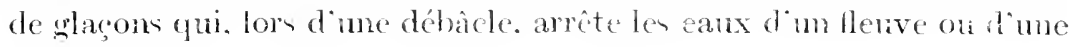
rivière " (4).

(1) Un ditail interesant : les borains (Iny) on borainues (Jupille), de condenr ver-

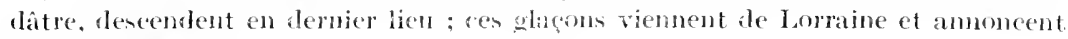
la fin du trèssirin.

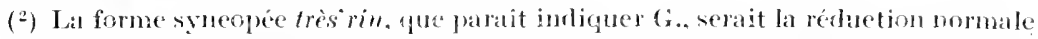
du type primitif trèssèrin. - Sur le suffice -in. voyez farticle butin, p. 41, n. 2.

${ }^{3}$ ) Scheler, Gloss. phil. de la Geste. dit i ce propos : tresserer, se congeler : il se peut que le v. soit à la forme impersonnelle et Murse, ete., au cas regine ". -

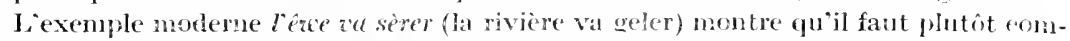
prendre : "La Mieuse - et, à phus furte raison, les autres rivières, - s i fortement gela dans toute sa masse... ". Toutefois, dins le dép du Douhs, on flit impersonnellement : il serre ou il resserre (il gèle), il dessprre (il légèle).

$\left.{ }^{4}\right)$ J. Waslet, roeab. atrallon-framerais (dielecte ginetois). 


\section{w. trèvint, trèvaye}

Le liég. trècint signifie "époque, temps " : è trèrint dè Noyé ; a minme trinint. so l' trivint. "sur ees entrefaites ". Ise mot existe de Malnedy it Namur. G.. II + 48 . qui écrit trizin. domne les formes plus rares trizin, truvin. Il eonjecture que co terme vient de trèc. ane. fr. trize, trince, et compare triange (entretemps, subst.). qui est seulement dans la „2e éd. de Remacle. - I mon sens. il faut écrire tièrint et inroquer le lat. interrentum au sems de "espace de temps entre deux actions"; comp. corint "courent". l's-avints "l'Arent». Pour laphérèse intrè $=$ trè. comp. trèeyi "entrevoir ": malm. tridozri "entrourrir "; nam. trè-oy" "entroür ". Lane. fr. comaît entrevenir (survenir). cntrecenue (chose qui survient). - Le préfice trè-. tri- pourrait encore représenter le lat. trans-, eomme dams téboulhî. trèeôper. triciè. d'autant plus que lane. fr. possède aussi trescenir (venir, arriver). Il est parfois malaisé de distinguer si tiè-vient de inter ou de trans. Ce qui tranche iei la question en faveur de inter, e'est la forme truvint et surtout celle qui a cours ì Viesville (Hainaut) : su én-intrifin la, su cis-intrifins la "sur ces entrefaites". où -rint a subi l'influenee du subst. fin.

Quant à trínaye. les conjectures que Scheler émet dans sa note (ap. (i.. II H.s) sont mal fondées. Il est plus heureux (ib., II xxxi) quand il pemse au fr. interalle. En clfet. la série *intrècale. *trèrale, thèrly' n'a rien que de régulier; comp. le malm. a cazule. verv. a cazaye "à ealifourchon" ; le liég.-verv. pevcuye "percale " (Lobct. Forir) : le montois comécleye "carnaval" ; ete.

[Mílunges Kuxth (1908), 11325 ; remanié.]

\section{liég.-nam. trin-bèrlin}

G.. II H. concegistre simplement le namurois, "trainberlain : tintamarren. Le mot at comnu ailleurs: jai noté à Starclot tim-burlin "Lapage (tim pentr trin par disimilation) et a Liege : moner on trin-

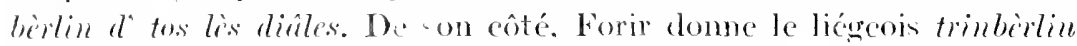
"trantran, cours o"l naire de certaines affaires". - Scheler (ap. (i. II H. 11. ) parait rapporter notre net an fr. tremblement; nais,

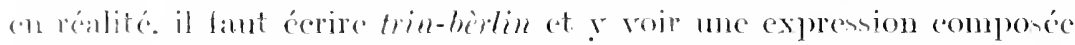

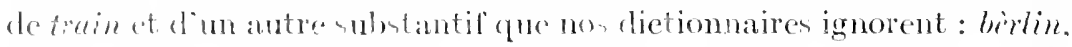

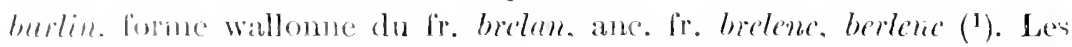

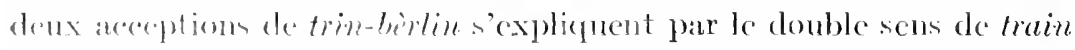

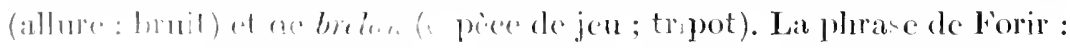

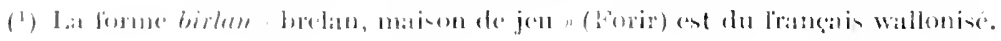


i k"nole li trinbirlin "il comnait le trantran. la routine le telle affaire" a signifié d'abord : "il connaît le train [du jeu appelé] brelan ". D'autre part. "faire du trinbivlin " e"est proprement "faire du train [comme on en fait dans un] brolan, du train [ele] brelan ". - Le wallon a pu domer à bèlin ee sens figuré de "tapage" (1). On tronve burlin isolé ehez un auteur verviétois. $\mathrm{X}$. Poulet, quile trakluit par "tremblement " (BSW 3, p. 3si) : entendez d"après le contexte: "événement qui fait tapages. D’autre part. Body. For. ast.. p. 36. donne l'exprension ardennaise tot lowlin "tout l"attirail rural". en areot : "tout le bazar. tout le tremblement $\%$.

\section{liég. troute. troudale}

Le liég. troute, verviétoin trute. signilie "gomrgandine" $\left({ }^{2}\right)$; d'autre part. à Spa. troute se dit d"une " (vieille) lemme mansade, imsoeiable" $\left({ }^{3}\right)$. Il faudrait plutôt cerire trmule, car c"ent assurément le primitif de trondule. que jai entendu à Liège dam l'expresion : ine céye troudale "une vieille désagréable ". Body $\left(^{3}\right)$ et Duvivier attribuent le même sens à cèye dromedale ou drodale. Fonir à dridule at drondale.

D'apres Seheler (ap. G., II t5ii). trmete est eonnexe aree l'ane. h. all. trût (mod. traut : eher, intime) : mai sehcler ne eonsidère que le sens "gourgandine": de plus. il ignore le diminutif trmedule. dont l'aceeption saecommode mal de a conjeeture. Je madrenserai plutôt à trûtle. forme dialcetale de l'all. drude "soreice "( $\left.{ }^{4}\right)$. qui me parait expliquer les diverses formes et significations des termes wallons. Troudale était. à lorigine. snonyme de macrule "soreière " il s'est conservé comme terme d’injure à l'adrense d'une vicille insociable.

\section{w. twèzon (Liège, Terviers)}

'Terme de serrurerie, ignaló ans explication par G., II 43+ : "toizon, palastre : boite de fer qui forme la partic extérieure d'une serrure (Lobet) ". Même définition dans Forir, avee un exemple où le mot est

(1) Comp. le fr. boucun (1. arels, : lion de debauthe : 2. dénordre, tumulte) et le

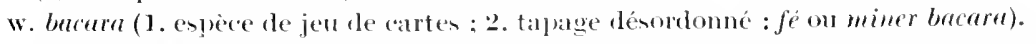

(²) Daprès Remacle. Hubert, Forir, Lobet et (i.. II 45(i. Seul, Duvivier écrit

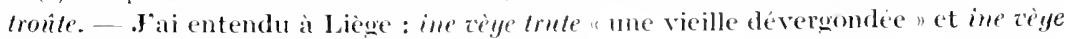
troute " whe vieille princheme ".

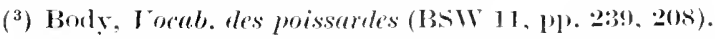

(4) Voy. Kluge. Dmeds. Cet auteur rapproche hypothétiquement drude de trunt, mais Weigand lui dome tort. - Je releve en dialerte all. du (x-1). de I axembourg : drudel. s. f., femme sans ordre, l'extérienr négliqe ; deudeleu. arranger sans soin (Wört. der tudemb. Mundurt, 1906i). 
du féminin. L'artiele de Lobet est ainsi libellé : "toizon. palastre. plaque de fer battu sur laquelle est bâtie la serrure ; - toison. dépouille d'un mouton. ete. ". Ce dernier sens n’a que faire ici $\left({ }^{1}\right)$. Quant à la ticèzon (le palastre: boite d'une semrure). il faut $y$ roir une altération de czò̀on (2). emprunté du fr. cloison, qui a également le sens technique de : "boîte qui renferme la garniture d'une serrure" (Littré). Pour cicè- : ticè-, comparez ticagne : cacigne, p. 255, n. 3.

\section{w. unuses (!)}

G., II 158. a un artiele qui est anu-ant comme une énigme : unuses,

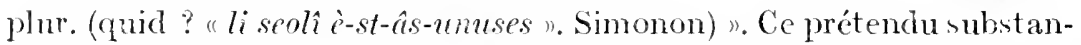
tif pluriel ent un monstre mort-né. Il faut eomprendre âsunus. altération du lat. asimus: l'écolier est asinus parce qu'il est puni cúbonnet d'âne ! - Comparez la locution latine ad recisum (au revoir), devenue en liégeois. par étrmologie populaire ou par plaisanterie : Âs trềs vîs-omes, aux trois vieux hommes). Ilécart. p. 251. a aussi ce curieux article: "j" su aus hanas on hosanna. je suis fort embarrausé. je ne saisque faire". Proprement, sans doute : "Je ne trouve devant la vaisselle à relaver ", les hanas ou anas (fr. hamap. liégr. hèna) désignant tout ee qui a servi au repas (Héeart. p. 24.3).

\section{w. vêre, divêre}

La toi`on sappelle ìne. s. m.. à Faymonville et à Malnedy $\left({ }^{3}\right)$, zếre à Siarclot. à Vervicrs $\left({ }^{4}\right)$. en chestrolais (Dasnoy. p. 295 : rer) et jusqu'en meusien (Labourasse : cerre). I mes yeux, on ne peut en séparer dizêres.s. m.. qui désigne 1. la toison du mouton. 2. toute espèce de récolte. d’ordinaire cneoresur pied. C.. I 17\%. et Remaele. ㄹe éd., domnent les deux significations. qui sont attestées notamment à Verviers (5) et

(1) Le fr. toison (de brebis) se rend en liég. par rote li laime (G.. I 129!) : tatèzon, dans ce sens, est un gallicisme, forgé par Iobet. Voyez ri-après l'article zêre.

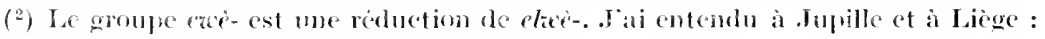

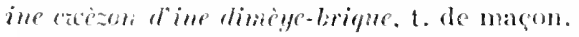

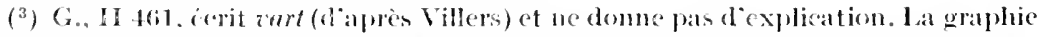

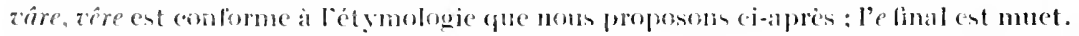

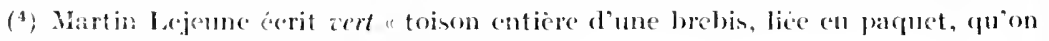

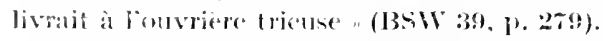

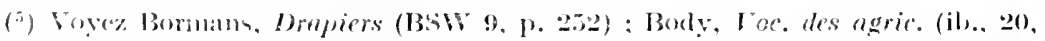

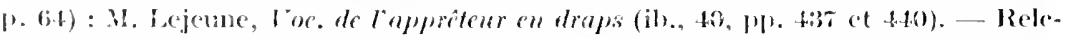

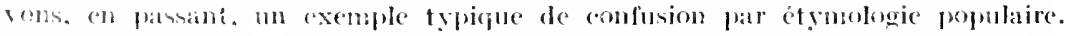

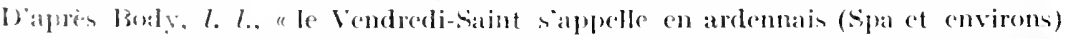

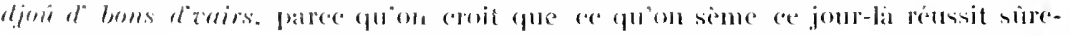


à Robertville-lez-Malmedỵ ${ }^{(1)}$. En général, le second sens ent le seul usité. par exemple à Lì̀ge: on bế d'ồre, une belle réeolte

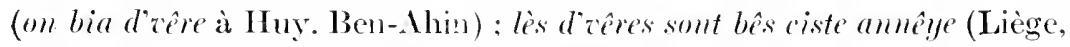
Villers-Ste-Gertrude) ; såcler sès p’tits drềess. sareler ses légumes; lís rotis sètehèt queute on cinq' dizêres d'ine tére bèn-icrihèye, les maraîchers tirent quatre ou cinq récolten d'mue terre bien funcé. On comnaît

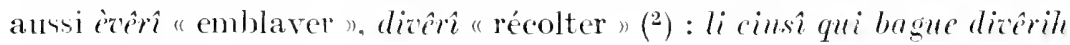
it rếếrh (Fléron). le fermier qui déménage récolte et ensenence à nouveau ; an dire de nos maraîehers, ine bone tére ni déit may èsse divêrèye, une bonne terre ne doit janais être nue. inoeeupée, déponillée de régétation.

Sur l'étymologie de divêre. G. ne donne rien de plausible, et encore moins Delbouf, qui éerit les d'rért. "les verdures ou légumes". en supposant que "e'est une façon de prononcer pour aert" (BSW 10. pp. 143 et 189). Pour expliquer vére "toison ", liehrens, p. 2z2, invoque le moyen néerl. et ancien frison wêr "mouton " $\left({ }^{3}\right)$. Conjecture ingénieuse, mais à laquelle je ne puis me rallier. non plus quà l'opinion de Labourasse, qui en fait l'équivalent littéral du fr. cair "fourrure" (lat. varium). Je tiens pour assurć que notre mot répond a l'ane. fr. relre, venure, ete., s. m., "toison" (lat. vellus. *vellerem). Godefroy fait un artiele itaire. s. f. [sic; lire: 11 .] pour un seul exemple du xve siècle à Mézières : "tous les zaires des laines ". Il n’a pas ru que ectte forme meusienne devait rentrer dans son article velre. où il cite notamment "vianre ou veanre de laine " en 1412 et 147.t à Tournai $\left({ }^{4}\right)$. Il y domne ausi un texte de 1247 : "apres li primier viaure recheu (charte d'Onnaing), aree cette traduction timide : Peut-être récolte de foin, toison du pré ". Le sens n est nullement douteux pour qui sait que nos

ment ". En réalité, il ne s'agit pas ici de dicêre "récolte ". nais de l'aredalique dirêre "vendredi ". La ronfusion nest pas possilıle à Faýmonville. oí récolte se dit diate (comme a Malmedy), tandin que le Vondredi-saint s'alpelle djôr bon dire, en anc. fr. jour du bon divenres.

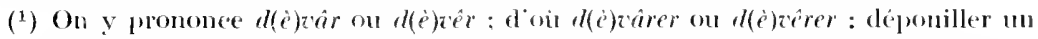
mouton de sa toison ou une terre de ses prohuits. (I'après M. l'abbé Dethier).

(2) A Villers-Ste-Gertrude, diziri = pasce, gatic (par le manvais temps) : ei grain

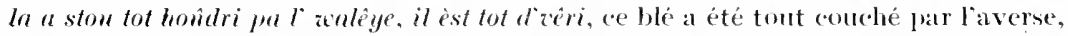
il est tout gâté. - Dans la région de lluy ce sens est attribué à forcêri, -èye, qui se dit aussi d’un fruit trop mîr. d'une pâte fermentée à l'excès, qui ont perdu leup valeur. De même à Tolıgne : nosse foûre èst forcêrî, notre foin a perdu "ses forces" (parce qu'on a trop tardé à le faucher ou à le rentrer); à Tohogne, comme à Liège, d'vêr = récolte; pour la toison, on dit cote di limue.

(3) Voyez (i-après l'article wére.

(4) God. donne aussi "laine zinurice" (= de toison) au xume siècle. à Tournai. 
archive liégcoi-en arignent à zaire la double aeception de "toison " e de récolte". En roici quelques témoignages : 1. "qui renderoit vaire de laine qui ne fuisse entior" (Chartes et Privilèges des Métiers de Liege. I $2+2)$ : "vilain raires non laudaubles " (ib.): "reires ou laine de berbic et d'aigncalz" (13\%t : ('art. de l'église de St-Lambert. Iv 511 ) ; 2 . " doit aroir tout tailhict of reseaweit $[=$ re-ex-aequatum : nivelé] le cear delle ditte faxhe et doit laisser pour chascun bonier Xxr stalons "(13st. à Ougrée : Liber Silvarum. no 190. fo 10) ; tous les iaires et dispoilhe de + faxhes de bois " (1+20 : Echerins. 3, 10\%) ; "foure et iaire d"un pré " (ib.. 16. 194) : "les iturres et emblavurez" (1460: Irchives de l'abhaye du Val-Benoît) (1): "alleir weaidire apres le premier zearre en un preit " (15.58 : (burres de Soumagne. 21, 90) ; "i... alcuns avires, soit arbres, ahans. maisons ou choses semblantes soyent trovées sour le werixhas "(Cout. de Liège. I 306) ( $\left.{ }^{2}\right)$. - Nous n'arons pas encore trouvé d'exemple ancien de devêre (toison) ; mais le sen dérivé se rencontre en $14.5 \%$ : "proces a cause des derers des trincux " (Echevins, 23, 94). Ajoutez-y : "desiearive et defructer" (15+1 : (Eurres de Soumagne, ㄴ1, 17\%) : "une terre encerye" (15\%: : Ban de Herve. 18. 2222 ro).

Pour le traitement phonétique. on peut comparer les mots qui présentent $\breve{l}$ d devant une concome. I part ceux qui ont le suflixe - ellum (bellum : beal. beau. w. bê. bia), les exemples sont rares : germ. *helmu : healme. heame. w. hême (casque) ; *peltrum : peantre, w. pête (fer-blanc): upelt a :́péntre. w. spête, spiate * *ellariarium

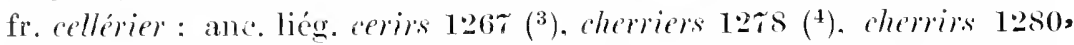

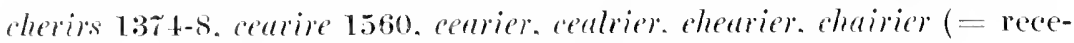
veur der renten en nature: fóm. cherresse $1+72$. cerresse 1501 ; la cearie était l'endroit où l'on devait payer ees renter).

En -omme, "êre a d'abord eu le sens étymologique de "toison", puis a pris le sens figuré de "toi-on de la terre : récoltes, denrées. légunes. arlsren et tous régétaux cultivés dont on dépouille régulièrement le sol " : eette sceonde acception. on l'a vu. est même la plus fréquente dam les archives liégeoisen. I'tré a engendré chez nous èvêri

(1) Cuvelier. Inventaire: : : avril 1460 .

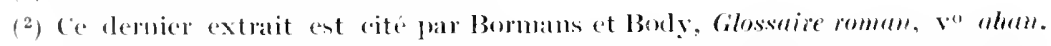

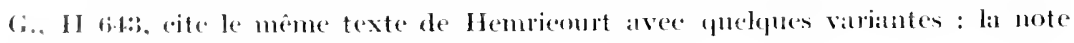

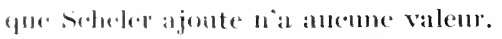

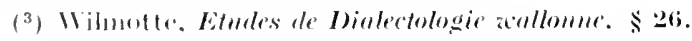

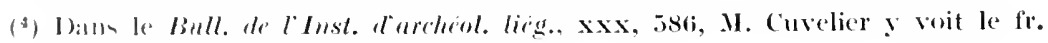
"cirice, fabricaut de aierges" (!). 


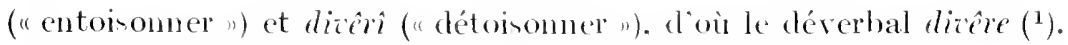
La forme malmédienne ôtere, d'âteresexplique par le fait que rểe, d'ấe, avait - et conserve encole en rerriétoin et en ardennais- un $\hat{e}$ trè ouvert, se rapprochant de $\hat{\imath}$ (royez l'article timule).

\section{w. vièrna, anc. fr. vernal}

G.. II H6z, voit dans aima le fr. goncernail prive de sa première

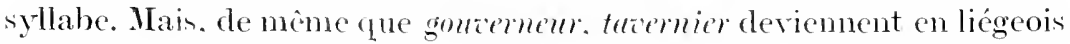

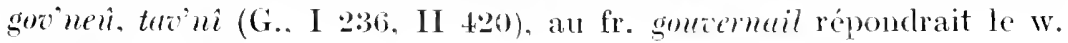
*gov'ma.

Le rièrna d'un batean. e'ent le moyen de le vièner (ane. fr. verner: diriger un bateau). Le sulfixe est - $a$. fr. -ail, lat. - aenlum. Le verbe se rattaehe au $\mathrm{fr}$. dial. verme (amme, espèce d’arbre). De là, le w. viène (solive. panne, pontrelle), l'ane. fr. come (gonrernail) et le montois verne (timon; voyez ci-dessus larticle jucerme).

Le verbe cerner. w. ièmer. s"ent formésur letype barrer. Quant à vièrina, qui devrait s'entendre proprement de tont le méeanisme servant à gouverner, il ne désigne que la partie extéricure qui peut s'immerger et avoir prise sur l'eau. D'ordinaire ce ciérna (gouvernail de rivière) ent assez dércloppé.

[Résumé d'un article, parn dans B1) 190\%. 1) 121, dont Veyer-Lübke, $1^{\circ}$ 9233, adopte les conclusions. Sim un sens métaphorique de cièrma et sur le dérivé vième, voyez l'article suivant. - Gulefroy a un article zernal, s. m., "gaine formée de madriers lixés verticalement, dans laquelle s'mboite le mât du batcaun; il cite deux excmples dont l'un ne peut s'accommorler de cette définition : "Ii vernal furent perhus de la neif " (Dial. dn pape Greg. p. 178; lat. : ex navi clavi perditi). Le lat. clucus et le w. ziemn témoignent quio fiut tradure li zernal par : "les gonvernails".]

\section{liéur vièrnê}

L'article préeédent, sous sa première forme, se terminait aims : "Quant à vieme que Lobet et Forir enreqistrent avec le sems de : boutade, eaprice, et que Scheler ramène à un type latin rertiginellus (G., II 46r), je serais fort tenté d'y voir le dimimutif de rièue. sans pouvoir toutefois décourrir le lien sémantique qui mirait ees denx mots"».

Cette dérivation, que suggérait la phonétique, je crois pouvoir aujourd'hui la tenir pour assurée. De deux côtés différents, on m'affirme qu'au Sud et au Sud-Est de Liège (Esneux. Bra, Chevron). vièrna

(1) Comp. le fr. repaire, de repairer, et le w. lès-êvess dè ljoû "l'aube ", déverbal de éri (= ane. fr. *uirier) "avoir l'air ou l'apparence, ressembler". 
sınifie. non seulement "gouvernail", mais encore "eapriee, changement bru^que d'idée " $\left.{ }^{1}\right)$. Le pont qui réunit ces deux sens d'apparence si différents, cent sans aucun doute : "coup de gouvernail qui porte bruৎquement le bateau à droite on à gatiehe ". Dès lors, viernê appartient légitimement i la famille viène, vièrner. vièrna ; il a signifié au propre : "*petit changement de direction du bateau", d'où, au fig.. "caprice. boutade ". Comparez les articles stièrnê, wandîhe.

Daus le sesis sudit, vièrnê est un diminutif "rerbal ", c'est-à-dire qüil dérive directement de cièner, au même titre que stièrnê. spôrdia (roy. p. 233) dérivent de stièmi. spôde. Il diffère de vièmế "petite poutre" (dérivé nominal de viène). que M. Charles Bruncau. Enquête, I 180. a noté en Ardenne. Il ne faut pas non plus le confondre avec Viumê (nom de lieu, par ex. à Xhendremael). qui signifie : "petit verne. petit aume ".

\section{liég. vilwè. vîlwè}

Foris a un article ciloi "ćtabli de eordomnier. écofrai". A part Willem. qui écrit viloè. tous nos autres glossaires ignorent ce mot ; môme le Vocubulaire du cordomnier par Kinable ne connaît que le terme générique taice "table " (BSW 24). On le retrouve. écrit rîloi. dans une conédie d'Edouard Remouchamps $\left({ }^{2}\right)$. - Nous y recomaissons le fr. teehn. reilloir "table carrée à rebord. sur laquelle le bourrelier place ses outil et ses matériaux" (Littré). ou. comme dit Sachs-Villate : "table où le cordomicr et le sellier travaillent à la lumière ". Comparez le liég. sizen. proprenent : "moyen de sî̀er (veilles. travailler à la lumicre)". et spécialcment : "porte-lampe que les armuriers plantent dans lew établi et qui se compose d'une tringle en fer armée de plu-

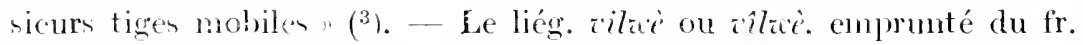
reilloir. nous vient sans doute par la roie de Namur. comme l'indique

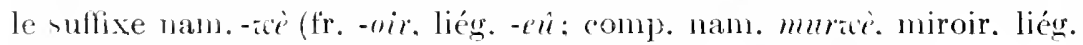
murêu). C'est le seul mot liégeois qui ait ce suffixe ćtranger.

(1) M. Pabbe .J. Bastin pour Bra et Chevom, M. A. Iallemand pour Fsnewx, I.e

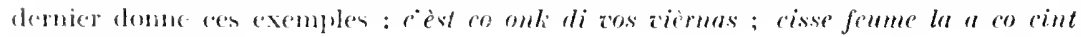
tiermess is liesse.

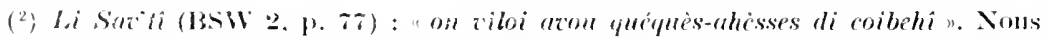
avoms entendu tantôt $i$, tantot $i$ a liege. oì diallenes le mot parait pen usite aujontrothui. On dit dombinaire banc.

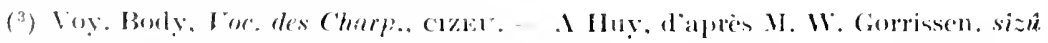
disiene predinment litabli du eordonnier. 
liégr. vinåve. ane. liég. vinable

Ce s.m. équivaut à l'auc. fr. visnage (voisinage); le suffixe seul est différent: -able, lat. - abilem, au lieu de -age. lat. - aticum (1). C'est littéralement : w "(endroit) voisinable ».c.-̀̀-d. où l'on peut voisiner, une agglomération. par opponition aux demeures éparses. - Le radical est le même que celui de vihène (aler al-), vilh mev "voisincr", vih mâhe (aler al-). dérivés d'un primitif* vihin $\left({ }^{2}\right)$, nani. vijin. que le fr. voisin a supplanté en liégeois (rivèin, wèzin). La forme ancienne wih'måre, s. m., s'est altérée en vinaive quand on a perdu de vue la signifieation première du mot, devenu nom de lieu et, pour ainsi dire, nom propre (3). Pour la raison eontraire, aler ai vih mahe (verv. -ive, malm.-stav. -âye, s. f.) s'est conservé intact : quand on prononce ce mot, on pense malgré soi aux symonymes vilinei. aler al zihime.

Il fant éearter la propouition de Scheler : vicinabulum ( $\left.{ }^{4}\right)$ et celle de G.. II 468 : vicenabulum. Il ne pent ctre question d'un suffxe -abul um, qui ria jamais eu de vitalité dans la langue populaire. G. se trompe de plus sur le radieal. It y voit vicena. quil troure dans un texte du moyen âge at qu il présente comme un dérivé de ricus, bourg. Or ee ricena (roisinage. quartien) ne peut itre qu'une latinisation grossiere du w. ribinte.

Sur la foi de l'étymologie de G.. on a prétendu que rinaze, comme le latin ricus, désignait primitivement "un bourg. un quartier, une vaste eirconseription de la eitén et que, par abus, il s'est appliqué ensuite à "la rue la plus importante du quartier" $\left({ }^{5}\right)$. Le contraire ent plus exaet : le sens a subi me extemion plutôt quine restriction. A l'origine, le inâte (I). e'ent l'endroit "où l'on peut voisiner ", le voisinage, c.-̀-d. un enemble dhabitations (disposées d'ordinaire aux deux côtés d'une roie). Hvec le temps, le groupement s'accroit, des rues latérales se construisent : mais, par opposition à ces nouvelles

(1) A lanc. fr. zisnage répond lame cinadje "réunion de tous les hommes du village pour discuter doue affaire commune " (a Cherain : BSW 30. p. 534).

(2) On dit zehin à Cherron. zeygu at Bande, zejgin à borinne.

(3) On peut supposer aussi l'influence analogigue de zini (venir), - Niederländer,

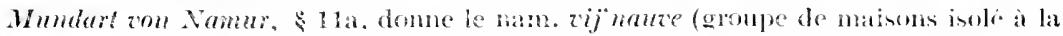
campagne), qu'it tient din octogenaire namurois. Grignard signale vijhoûce " lat

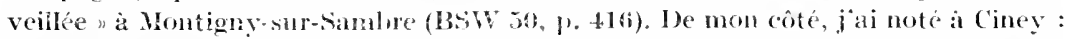

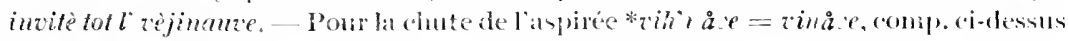
I'article èmimé.

(4) Gloss. phil. de la Geste te tiege (1882), vixablit.

(5) Gohert. Les mes de Liege, 1. Iv, 1. 1838. 
rues. le nom de anấe (II) eontinue à sappliquer à celle qui fut le noyau du groupe : il reste le "nom propre "de la voie la plus fréquentée du faubourg. de la grand rue du bourg ou village. Simultanément, et frar opposition à d’autres agglonération de même espèce. vinẫe (III) "ent dit de tout l'ensemble groupé antour du noỵau primitif. Aimi Liege. an xn e siecle. comprenait six imables ou quartier ( ${ }^{1}$ ) et les citadin de Malmedy appellent cneore inaines les villages ou hameaux qui avoisinent leur ville. Mais. i dam chacun de ces villages. comme dan les faubourg de Liège. zincire a gardé Ir sen II. il i cen faut assurément que ce soit par abms.

[BD 1910, 1. 68 ; remanie et développé].

\section{liégr. v'nou. v'nowe ("nenu. -ue»)}

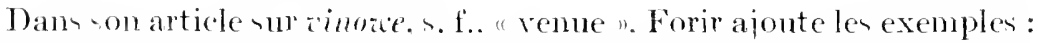

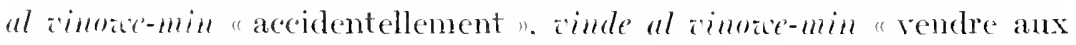
passants". Il y a confusion. Lexpression archäque dont Forir nous conserve le souvenir $\left({ }^{2}\right)$. reproduit. arec une légère altération. l'anc. fr. a la meme main "au détail ". Godefroy. à l'article mem. ne cite qüum seul texte. d'origine namuroise; mais len Chartes des Métiers liégreois contiemnent mainter fois eette expresion $\left({ }^{3}\right)$ : elle était done d'usage courant a Liege. - Du sens premicr : "(rondre. acheter) au dótail ". dérive celui de : "par oceasion. en protitant d"une cireontance aceidentelle : c'ent aims qüil faut interpréter la traduction "aecidentellement " lomnéc par Forir. - ( uant a la forme. la confunion des groupes

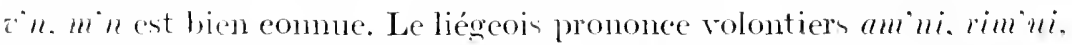

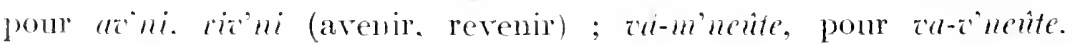
*a-linexte (Val-Benoit. 1. d. prè de Liège). Ici. inversenent. m"uon

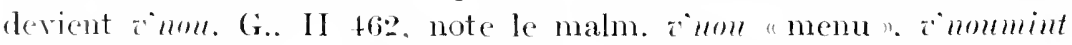

fincment (anc. fr. mennement). Le liégeois di-ait de mêne au

(1) Il avoit. a cely temp. et encors at a present. a ryaules a liege (I Iemricourt. Merrir des mobles de IIesbryge. ed. de 1910, 1. 30:3). Le souvenir n'en survit

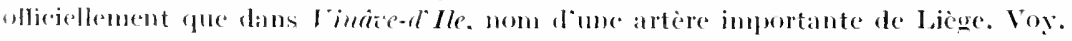

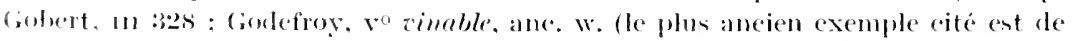

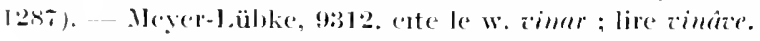

(2) Juvivier la domme anus, avece la traduction : "comme il tombe".

(3) T. I, 1. 2:3.4: rendre en gros, ne à la memuë main p (1525); 1. 24:2 : "les draps

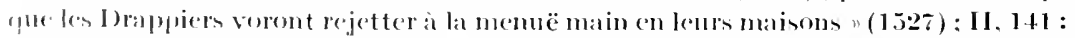

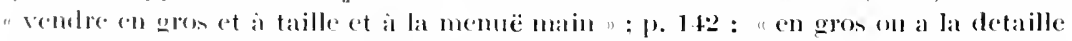

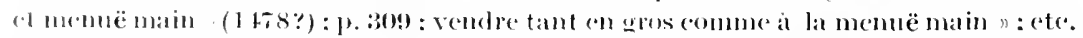

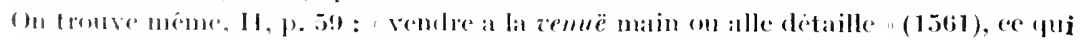

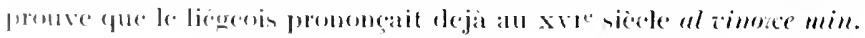


xvire sièele: nous avous de eette époque deux exemples curieux de v'nou employé adverbialement eomme le fr. menu (1). Aujourd'hui eneore, à Seraing. on entend sourent la locution tot v'nou. tot minou, dans le sens de : "eoup sur coup. avec acharnement, d'arraehe-pied " $\left({ }^{2}\right)$.

A Roclenge-sur-Geer. on appelle lís r'mons strins les fétus trop eourts qui se trouvent dans le gerbes de paille servant autressage (13D 1921-22. pp 8 et 36 ). Je erois reeomnaitre encore ici *mmom. qui anrait subi une altération insolite. san doute sous l’influence de l'r de strin. L'expression "les menu strain " a dû exister jadis. comme on dit anjourd'hui "les menues pailles". Comparez. ì ce propos. l'anc. fr. menustin. que Godefroy domne dans un texte de Douai (1536) et qüil faut tres probablement corriger en memu strim.

\section{W. vîrer}

G., II t69, a l'article suivant:

vîr (envie, volontí, surtout volonté abstinee entetement). (ce mot ne peut

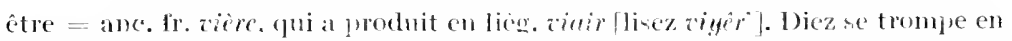

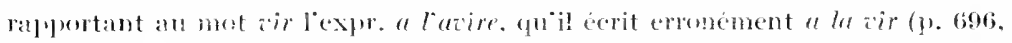

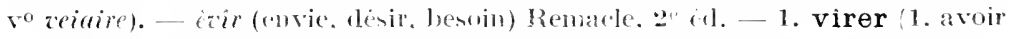

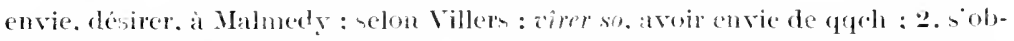

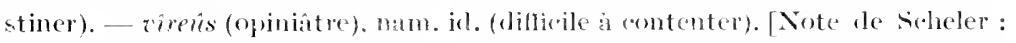

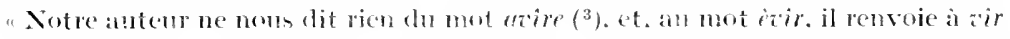

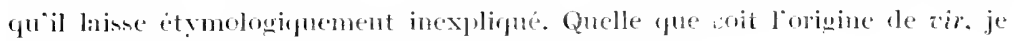

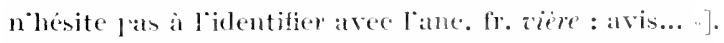

Il n'existe pas, que je sache. d'antre essai d'explication. Q quoi quien pense Scheler. G. a raimn d'écarter l'anc. fr. Fiére : ce denuer noest qu'une forme de l'anc. fr. ataire (ri-age : aris. manicre de roir), lequel répond au liégeois ciycere. m.. "rihagen et vient peut-être du latin videatur $\left({ }^{4}\right)$.

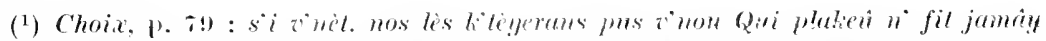

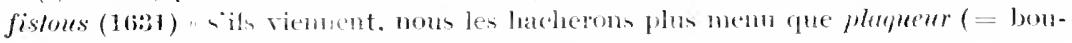

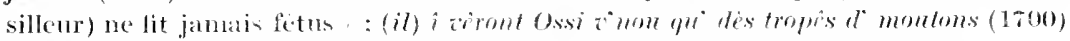

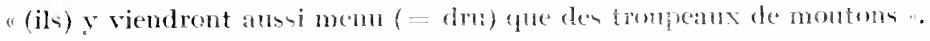

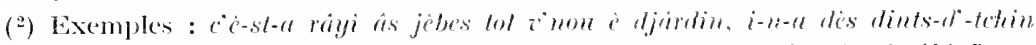

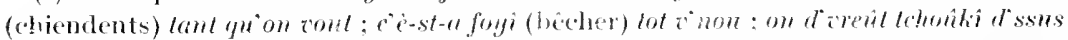

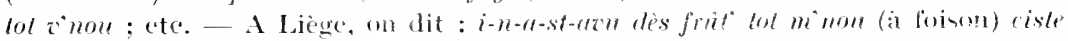
aminue.

$\left({ }^{3}\right)$ Scheler onblie que G.. dans ses Extrails de Villers (1865), explingle correcte-

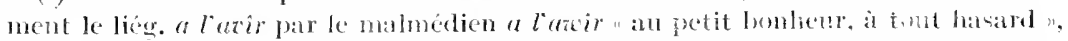

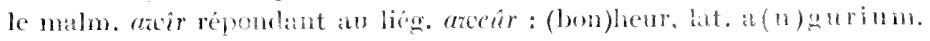

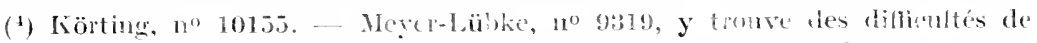

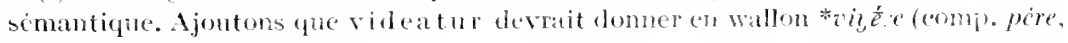
líre. de pater, latrol. 
Firtre ent propere au dialecte ent-wallon (1) : on peut donc lui supposer unc origine germanique. D'autre part. ce terme éveille surtout l'idée d'opposition. de résistance opiniâtre $\left({ }^{2}\right)$. Nous sommes ainsi amené à nou adresser à l'ancien h. all. widirôn. moyen h. all. wider(e)n "résister. sopposer, refuser".

Les excmples suivants montrent que "contredire " est bien la signification fondanentale de notre mot.

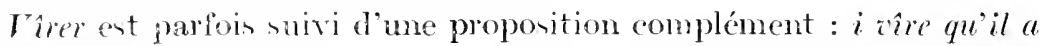

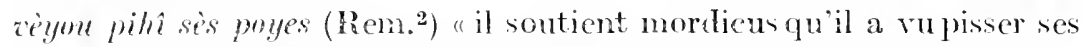

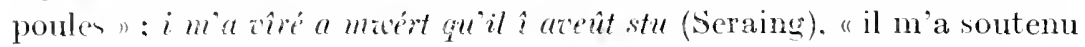
obstinément (litt. "à mort " jusqu’à la mort) (qu'il y arait été ". Mais dordinaire il est intransitif : qui zolez-v" tunt virer? (Forir) "pourquoi contester si obstinément ? " ni virez mî tanf (Bergilers : Hesbaye) "ne répliquez pas tant" ; Cle vint virer so tot (BSW 21. p. 175) "elle rient faire de lopposition à propon de tout". Le sens "aroir envie, désirer" ent dérivé et mêne cette traduction ne rend pas la force de l'expression: un enfant, par exemple, qui zîre su tot çou qui zeùt, "s'obstine, malgré vos refus. à demander tout ce qu'il roit".

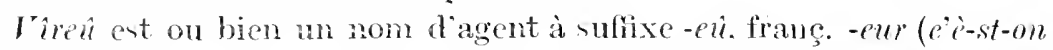

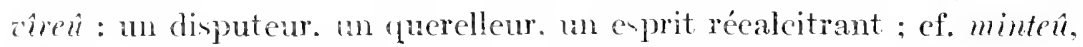
-ense: menteur, -euse), oa bien un atjectif qu'on ćcrira dans ce cas

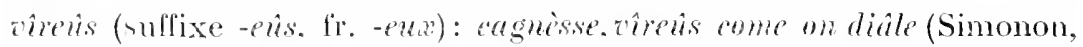

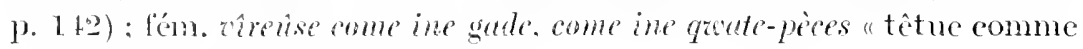
une cherre. comme un lézard" $\left.{ }^{3}\right)$.

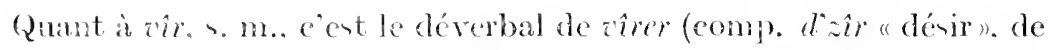

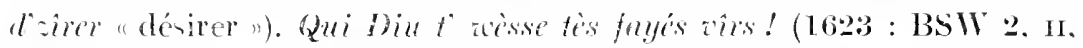

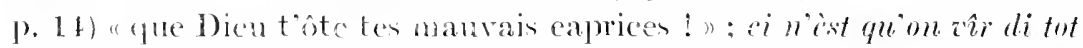

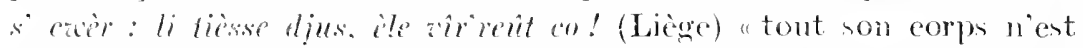
quobutination : la tête coupée, elle solutinerait encore ! " : qui de $\hat{u}-d \hat{j}$

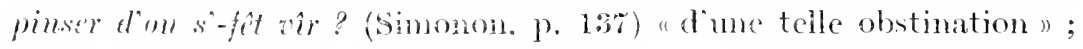

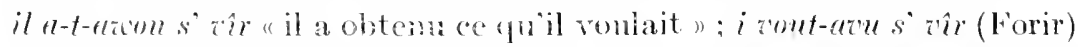

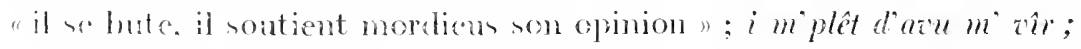

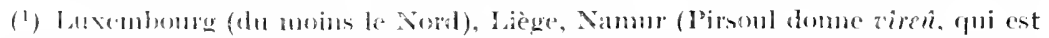

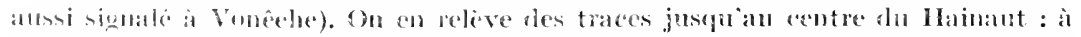

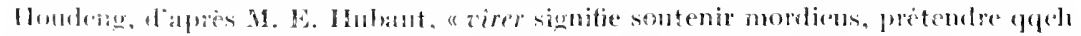

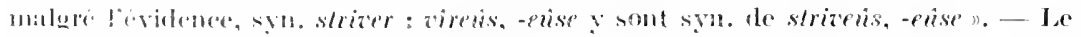

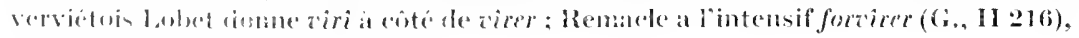

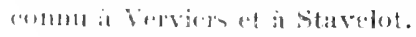

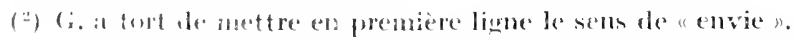

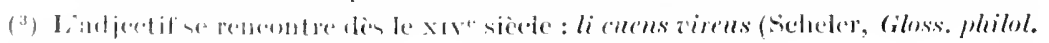

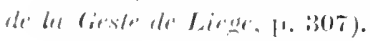




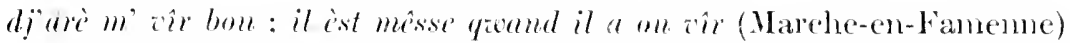

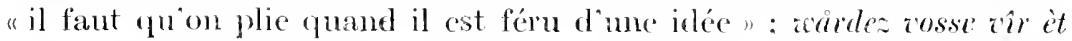
dj acidrè l' mein' (liem²) "gardez votre opinion et je garderai la mienne "; ni hoîter qui s" vîr, ni fé qu'a s' zîr (id.) "n'éeouter que son

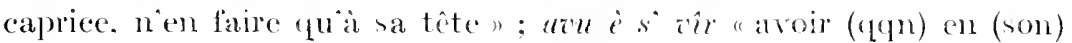
inimitié. en aversion" : locution imolite. dont je ne comais qu'un exemple (Théutre liég.. éd. Bailleux. p. 173). - Liexpression fé a s" tôr "faire à sa tête", sous l'influenee visible the "l'tîr "au hasard ". a engendré (fé. ou diner. ou prinde) ul rîr. que liemacle. ze éd., traduit par : "(agir) sans réllexion. (Aomnce) sans compter. (prendre) sans ehoix". Il est certain. d"antre part, que irîne (liem. ${ }^{2}$ ) résulte du croi-

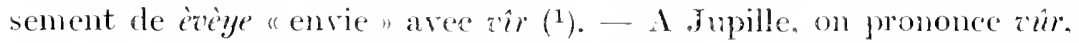

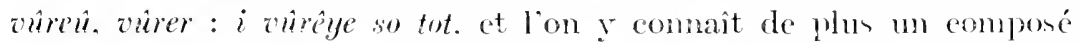

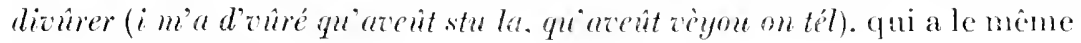
sens (.J. Lejeune).

La dérivation que je propose ci-dessus satisfait pleinement pour le sems. Elle ne fait pas grande difficulté pour la lettre : wideren sest normalement contracté en *aieren par la chute du d intervocal; comp. le néerl. weder : aeer et l'all. widersmu: bas all. wersen (1ix-la-Chapelle). néerl. weerzin (d'oì le w. iérin). Le changenent de w en est rare. mais non sans exemple : eomp. a lavô. altéré de a lawir ; le nam.

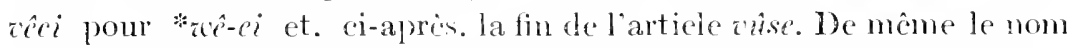
de lieu $V$ isé ( $i$ long). en llanand Weset. dérivé de l'all. wiesé. prairie.

[BI) $1920, \mathrm{p} .14$.

\section{licus virlih}

G., II 470 : virlih (allègre, alerte, vif), nam. verlije (qui aime ì jouer). Comp. dialecte de Bayenx virli ("petite vive " [?]). De virer?

Voici un exemple de 1768 : on mines bin virlihe it bin ricolicsise (Théatre liég.. éd. de 18.it. p. 11\%) "vou ne paraissez bien réjouie et bien gaillarde. "Le mot manque dam Villers. liemaele et Lobet : mais il est dans Hubert. Forir. Millem (2). Nous releroms en outre : rîrlih à

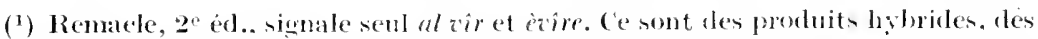
créations individuelles qui, penr être intèressantes conme fous les faits linguistiques,

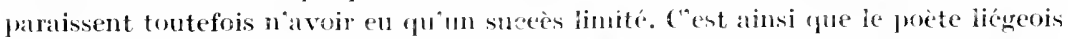

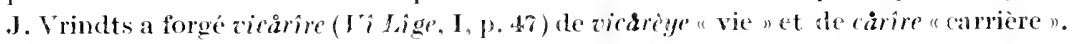

(2) La traduction "viril... " de Willem (Dict. des rimes, p. 108) semble être un essai dinterprétation; essai malheureux, cela va de sui, et plus malheureuse encore lindée de forger, p. 55, un substantif " virlihté : virilité" " - Le glossabe manuscrit du patois de Stavelot par Detrixhe a un article : "ritlik. hubie, enprice, idec bis(cornue ", qui na rien a voir avec notre mot; ('e n'est quitue valliante pell sure du 
Verviers et à Fontin-Emeux : irlih à Glon-sur-Geer (on rapand qu'est bin virlih "un enfant qui est bien dégrourdi ") ; verlih à Bergilers (en parlant d'un petit enfant : "gai. évcillé. remuant") ; vèrlitch à Xamur (Pirsoul!. - Le fr. cirer n'a que faire ici. Il est eertain que notre mot reproduit le moyen h. all. vîrelich, anc. bas francique fîrlic (= festus : devenu en all. mod. feierlich. solemel). lequel remonte, comme le fr. foire. au lat. feria $\left({ }^{1}\right)$. Le sens premier : "qui est en tôte" s'est perdu ; il nou reste eelui de : "riant. réjoui. joyeux. animé ".

[BD 1914, P. 36.]

$$
\text { w. vûse (Verviers. Malmeḍ̣) }
$$

Ce mot est signalé par les auteurs suivants:

G., II 47: : vuse "bruit confus de la voix quand on prie ", "air ") Ch.-N. Simonon ; zuse (" rumeur, bruits confus de voix animées; son. ce qui frappe loüe ") Lobet. - Seins, Diet. malméflien, manuscrit, 1893 : vùse s. f., voix : plorer "haute anse "sangloter".- J. Bastin, Ioe. de Faymomille : vùse s. f., dans lexpr. plắrer a haute zuse "pleurer à haute voix, sangloter ". Comp. tehuse "choix".

Mot rare. de l'extreme Nord-Est (Verviers. Malmedy). qui ne survit gù̀re (que dau l'expression ironique tehouler it plorer a haute àse (2). Ifeureunement. nos vieux nöls nous en ont conscrvé un exemple précieux $\left({ }^{3}\right)$. Liange. chantant daus le ciel. invite "bergers et bergerettes s i quitter leurs hameaux pour aller voir le Nesic ; un paysan dit alors à sa voisine:

$$
\begin{aligned}
& \text { Inoutez, wèzène Liz'hèt" : }
\end{aligned}
$$

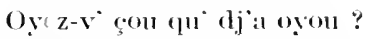

$$
\begin{aligned}
& \text { Cisse bele zâse mi dispicte. } \\
& \text { S" n`a-dj nin bêcô dwèmon. }
\end{aligned}
$$

I. le hau de mélodien s impose $\left.{ }^{4}\right)$. On a prétendu cependant que

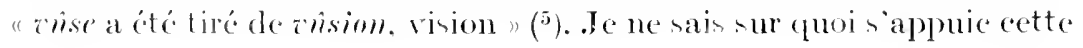

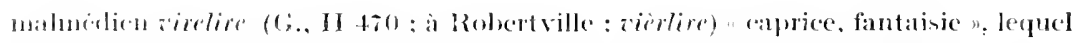

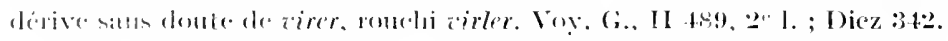

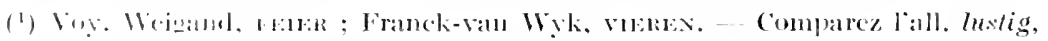

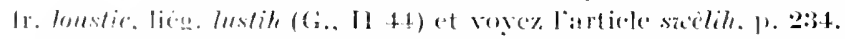

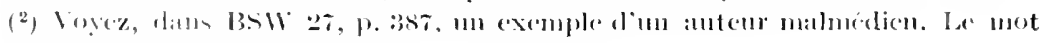

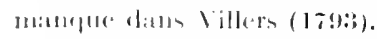

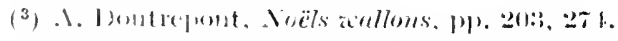

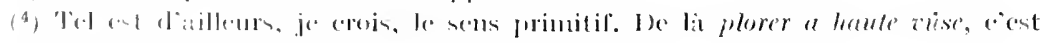

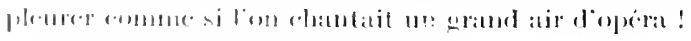

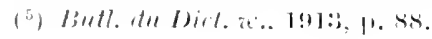


assertion : les auteurs que nous avons cités attestent que le mot désigne "ce qui frappe l’ouïe". et non " ce qui frappe la rue ou l'imagination".

D'autre part, guidés sans doute par certaine resiemblanee extérieure, ees auteurs s'accordent, on l'a vu. à mettre ruise en rapport avec le fr. voix. En réalité, le lat. rôcem n’aurait pu donner que *ênh. *vể (1). Il y a bien le west-flam. roois " 1 . voix ; 2. air d'un chant "; mais eet emprunt du fr. woix - ou zoisse, comme on prononce à Tourcoing, paraît limité à la Flandre occidentale $\left({ }^{2}\right)$. Le w. tchìse, f., "choix " ne devrait pas non plus être invoqué pour établir l'équation v'ûse = voix. C'est le déverbal de tchîzi (got. kiusan, anc. h. all. chiosan, all. liesen, néerl. kiesen; comp. néerl. keus : choix), tandis que le fr. choix est tiré de choisir (d'une forme germanique parallèle kausjan).

Comme il faut bien ehereher autre chose, je m'adresserai à l'ane. h. all. wîsa (all. weise, néerl. wijze), qui signifie: 1. "manière " (d'où le fr. guise), 2. "mélodie ". Ce dernier sens se retrouve dans le bas all. wîs, d'Eupen et d'Aix-la-Chapelle. qui a pu s'introduire à Malmedy et à Verviers. Quelque étrange que soit la forme roùr. elle peut s'expliquer sans trop de peine. Nous arons vu, à l'article virer, des exemples du changement de $w$ initial en $v$. Pour le changement de $\hat{\imath}$ tonique en $\hat{\imath}$, comparez vîr, vîer (Liège) = vîr, viurer (Jupille); hîfe ou hûfe di djèye (Sprimont) "écale de noix", ûlê (ane. fr. islel "îlot"), dans la rue Lulay des Febrres, à Liège.

[BD 1920, p. 17. - Depuis lors, mon collègue de l'Université de Liège, M. Verdeyen, n’a signalé que zoos ou roois existe dans les différents dialectes flamands avec les significations suivantes : 1. voix ; 2. mélodie, d’où le diminutif vooske (züske dans le Hageland) ; 3. suffrage, d'oì zoozen, zozen, voter. D'autre part, il paraît que zeijae n'cst guère employé dans les provinces flamandes. L'étymologie que je propose ci-dessus est done sujette à caution; il serait très possible que le w. ruise fût emprunté du flamand zoois, qui lui-même vient du roman. Nous avons vu un processus analogue à l'article tike.]

\section{liég. wadje, watche}

Ce mot désigne la bretelle ou bricole servant à soutenir les bras d'une brouette ou d'une cirière ; les deux bouts ont une ourerture par où passe l'extrémité de chaque bras du véhicule : li ixutche ist trop strête,

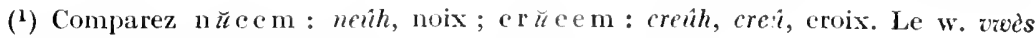
est emprunté du fr. voix.

(2) De Bo, Wesizlaamsch Idioticon, Gand, 1892. On ne trouve pas le mot dans Schuermans. 
èle mi côpe lis spales. Certains. comme Forir, le font du maseulin, sans doute sous l'influence de l'homophone wadje "gage ". G., II +74, qui le dome au pluriel, suggère la eomparaison avee l'all. wage "balanee ".

Dans une enquête sur le langage du houilleur à Seraing, j’ai relevé ee qui suit : la burtèle di hèrtchen $\hat{a}$ batch. bretelle ou harnachement du hereheur-bac, est formée de deux bandes de forte toile. lès watches dèl burtèle, qui se placent sur les épaules et passent sous les bras ; sur le bas des reins, les watehes se réunissent à une manote ("menotte " ou poignée de fer) qui porte un erochet $\left({ }^{1}\right)$. Je erois que le langage archaïque de nou houilleurs a conservé le sens premier du mot et je définirai wadje ou. comme on prononee, zatche : large bande de très forte toile, servant notamment à faire la bretelle du hereheur, du porteur de civière, du eonducteur de brouette, ou même le licou d'un cheval.

Partant de là, on s'adressera au radical germanique wad-, dont le sens primitif est "tissu ", d'où : "rêtement, lien, câble ", etc. $\left({ }^{2}\right)$ : compare l'all. archäque wat. f., pièce d'habillement (ane. saxon wâd; ane. et moy. h. all. wat) ; et le néerl. gewaad vêtement. lijnwaad linge, lijlicade linceul $\left({ }^{3}\right)$. Le w. wadje s'expliquerait pas un diminut flamand *aralje signifiant "petite pièce de tissu ". - De même le w. bodle vient du fl. beulije : le w. bondje, rouchi bonge. botte, faisceau, du fl. bondje $\left(^{4}\right)$ : le nam. deritehe (G., I 165), du fl. duitje; etc.

\section{lićgr. wafî}

G., II 475: wafí t. de couturière (Remacle: surjeter; Dejacr : surjeter, fatufiler ; Hubert : bâtir, coudre à grands points ; Lobet : brocher, surjeter). Le mot tiendrait-il de zafe : gaufre ? [Note de Seheler : Comp. le souabe wiflen : recoudre des parties déchirées.]

1)urivier définit le liég. wafî: "faufiler ". et Villers le malm. aafer: "coudre sans farre d'ourlet", ce qui veut dire sans doute : "coudre sam plier l'ét offe ". A Liers. j'ai entendu ricufer au sens de : ourler ". 1)'une enquête personnelle il rénulte quaucun des termes franęais surdits ne traduit exactement le liég. wafî. Faire un surjet ou surjeter (- ljumle dés costenires, fé on djondèdje) eonsiste à mettre les tissus bord it bord cet à les coudre en passant le lil par dessus les deux bords, tandis que aufi consiste a faire la même opération quand, au préalable, on a

(1) Voyes la figure dans 331 ) 1914, p. 82.

${ }^{2}{ }^{2}$ Jo dois cette suggestion à l'obligeance de M. J. Vereoullic.

$\left({ }^{3}\right)$ Voy. Weigand WaT ; Franek-vall Wyk gewaAd.

(4) Behrens, Beilräge, 1, 2ז. - Voyez ci-dessus les articles bodje et sondje. 
eousu les deux tissus mis bord à bord : e'ent done une opération complémentaire. L'expression ordinaire est : wafĩ ons costểes ; vola in-abit qui n'èst nin bin fini. les costeûres ni sont nin minme wajèyes. Pour traduire wafî. le liégeois dit "surfiler", attribuant à ee mot français un sens qui n’est pas dans le Dict. génévol. mais qui mériterait d'y figurer. On pourrait ausi forger "sureoudre".

Seheler a pressenti l’étymologie de wafí. Cependant. il faut plutôt remonter au moyen h. all. weifen "dévider ( ¿u fil), ane. h. all. ấfan "toumer" ; eomparez l'ane. h. all. weif. waif "ce qu'on tourne ou enlace autour de quelque chose ", ainsi que le moyen h. all. iceifier(e) qui désigne une sorte de point on de dentelle ${ }^{1}$ ). De même que le gothiquefraneique wîpan, weipan. a donné le fr. guiper, -ure, de même wî̀fan, weifen, a procréé notre wafî. Pour le traitement de la protonique germ. ei, voye tahant, partieipe d'un verbe *tahî que nous dérivons du gothique theihan, anc. h. all. thîhan.

\section{anc. fr. wage, waghe}

Dans son Glossaire des Poésies de Froissart. Seheler note trois exemples de zages, s. f. pl., cité parmi les pièces d'habillement que mettent les bergers: "solers takenés..., wans, wouges, chaperons petis" ("2). "Je ne saurais, ajoute-t-il, en préciser le sens; peut-čtre sont-ee des hauts-de-ehausse, des honseaux. leqquels mots ne paraissent pas dans les passages cités". - Dan une énumération analogue, une seène pastorale du xve sièele. dorigine liéggeoise, que M. Cohen a éditée récemment, contient le mot arashe, que l'éditeur interprète par "hautde-chausse " $\left({ }^{3}\right)$. Je crois, pour ma part, qu’il s'agit plutôt de houseaux.

On trouve en moyen néerlandais weggen "perones, fanciae, fr. guettres" (Plantin) ; wagge "tibiale, pero "(Dict. tentonicum, Antrerpiae. 1667) ; et. dans les dialectes modernes d'Anvers et du Brabant : wag. wagge "overkou, slofkous, fr. gutctre " (Schuermans). L'anc. fr. wag(h)e, qui se prononẹait sans doute ioggle et qui ne se reneontre que dans de rares textes de nos provinees wallonnes, provient manifestement de cette source thioise. Il a d'ailleurs laissé un rejeton dans le voeabulaire de l'ardoisier ardemais. A Vielsalm. les zagnètes di gngnos,

(1) Je renvoie pour le détail ì l'artiele très documenté de Th. Braume sur le fr. guiper, dans la Z. für rom. Phil., xvil1 (1894), p. 530.

${ }^{2}$ ) II 319, 28. - Godefroy donne les deux autres passages et traduit sans Iiésitation: "haut-de-ehausse, houseau".

( $\left.{ }^{3}\right)$ Hystères et Moralités du ms. 617 de Chantilly (Champion, 1920 ; 1. 87 ). 
ce sont des genouillères en cuir ; les aruguètes di pîds, de petites guêtres en cuir ; elles servent à protéger les genoux et les pieds des fendeurs pendant le travail (J. Hens : BSW 46, p. 191). De même en Ardeme française, M. Ch. Bruneau a relevé zagates "jambières de louvrier ardoisier " $\left.{ }^{1}\right)$. - Il lésulte de là que l'ane. fr. wage, aughe, n’a rien de commun aree le liég. iádje que nous avons expliqué ei-dessus.

\section{w. wahète (Verviers, Malmedy)}

La variole s'appelle à Liège lès polies ("poehes, ampoules ") ou poliètes; à Namur plokes ou plokètes (avec $l$ épenthétique). Près de la frontière allemande, eette maladie porte un nom qui n'a pas eneore reęu d'explieation : lès wahites, Malmedy : Villers. 1793 ; G., II 475 ; Verviers : Lobet $\left({ }^{2}\right)$; — wahyètes, Franeorchamps, Vielsalm ; wachetes. Bovigny, Lutrebois, Sibret, Wardin-lez-Bastogne $\left({ }^{3}\right)$; zèhyotes, Faymonville : azeûr lès wèhyotes "avoir la petite vérole ", mète lìs àhyotes "vaceiner"; une wèhyote, c'est um bouton. une petite pustule arrondie (J. Bastin. Ioc. de Faym.).

De een quatre formes, la dernière se rapproche le plus du radieal primitif. Nous y recomnaissons en effet le lat. pop. *ressîca, d'où le fr. vessie. malmédien vèhie (le liég. vèssèyc est emprunté du fr.). Le diminutif *èhyète, -ote (= ane. fr. vesciette: pustella) a subi le ehangement de $v$ initial en $w\left(^{4}\right)$. Le groupe - $h y$-devient normalement $c h$ au Sud; il se réduit à $h$ en verviétois (eomparez d'hinde à côté de d'hyinde et voyez l'artiele prìhale). Quant à l'altération de la protonique è en $a$, les exemples abondent.

Une rahite, e'est done une vésicule, une "petite vessie". Au pluriel, e'est la petite vérole qui, en meusien, s'appelle de même les vissies, alors que chez nous le terme roman a presque partout eédé devant poke. d'origine gurmanique.

anc. fr. waibe,-er, -aige; w. wêbe, -î, d'wêsbî, wêsbi

Godefroid confond dans le même artiele raide (pré, pâturage; all. aceide) et le synonyme araibe $\left({ }^{5}\right)$. qui est d'origine différente. Il

(1) Brunean, Etude phonctique des patois d Ardemip. P. 10 h.

(2) Forir dome aussi ce mot qui, toutcfois, est ineonnu à liège.

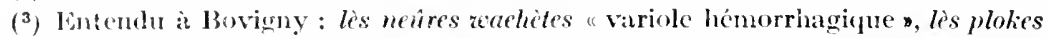

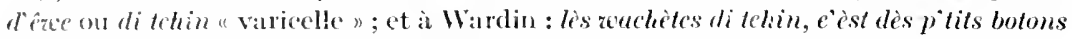
qu' lis-ifants atrapant co bin socint, sans doute une sorte durticaire boutonneux.

(4) Voye\% (i-iprese lanticle ziére.

(5) bans ce textre de 1575 : "portions de bojs converties en zaibes et essarts "

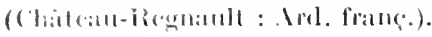


derrait mentionmer acaiber (paiturer), dans une ordonnance liégeoise de $1705\left({ }^{1}\right)$, et axibaige. dars une charte du 19 arril 1450 aecordant aux habitants de Lobbes le "droit de anibaige. passonaige et pasturaige " $\left({ }^{2}\right)$. Delmotte, qui cite ee dernier texte, signale anssi waibier "paître ". waibiage "pâturage, surtout en parlant des poules et autres oiseaux de basse-cour (à Montigny-le-Tilleul)", waibes, aibes "pâturage; fig., lieu oì l'on va d'habitude (ibid.) ", les waibes de Thuin, lieu dit près de cette ville.

Dans les patois modernes: on retrouve eneore:

$1^{0}$ atébes (Ard. fr. : Bruneau. Enquête. I, 99) "bois de la commune " $\left({ }^{3}\right)$. Le mot a survéeu dans des expressions proverbiales : "il marehe sur mes atébes" (= "briséen". à Sévigny-la-Foret : Bruneau, ibid.) ; i n'èst nin daus sès cếbe's (Daillỵ-Couvin : "il est dépaỵé ") ; ĕl ist foû

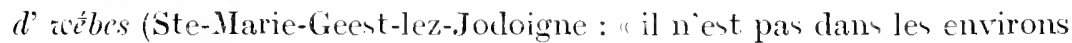
où on le roit d'orlinaire"): dii su dius sis wêbes (Stave: "je suis dans ses bonnes grâcen ") :

¿0 aichlic (Neuvillers-Reeogne) "aecoutumer " v. tr. :

$3^{0}$ ấbètle (Bergilers: Mesbaye) "paturage (des poules), espace où

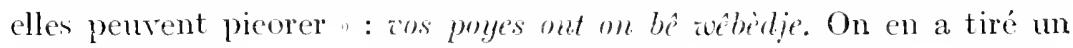
verbe ấbèiji. qui remplace l'inusité ẩb̂̀ et qui ne se dit que des poules : lis poyges ront wểdî "paturer "(Bergilers).

to at bi (Ste-Marie-Geest. Noduwez) "paturer, surtout en parlant des poules : lès poyes atébiygente - En liégeoir. đểbì a la mème aeecption (Simonon. ap. G.. II 475$)$. mais le mot ent archä̈que $\left(^{4}\right)$. Cambresier, Remaele, Lobet, Willem ne domnent que wîli. Hubert omet wî̀

$\left.{ }^{1}\right)$ "Lieux où la herde bannale a aceoutume de zeaiber" (Polain. Ord. de la Prineip. de Liège, 1, 328).

(2) Voy. Ph. Delmotte, Gloss. Tivallon, 1812, publié à Mons en 190\%. - Dans les Doc. et Rapp. de la Soe. archeol. de Charleroi, xin 89, on lit en 14.50 : " de tons pasturaiges, vraibaiges..." (corr. waibaiges).

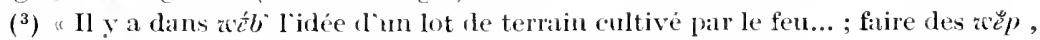
à Saint-Menges, e’est préparer les gazons pour l'essartage ". (Bruneau, ibid.). Il s'agit done rles bois eommunanx, qui sont partagés entre les habitants : chacun essarte son lot pour la culture ou la pature. Ce sens de "domaine partieulier" apparait dans les expressions figurees que nous eitons ensuite. - Dans un autre de ses ouvrages, Limite des dial. en Arl., P. 78, M. Ch. Bruneau signale près de Givet un eertain nombre de noms de lieu formés avee zeibe, lesquels s'appliquent tous à des bois; il y voit un mot germ. wa ber "forêt ", que Kurth, Frontière, 11 90, invorque pour expliquer la Warte (forêt de Woëvre), Warre en Brabant, ete. La conjeeture de $\mathbf{M}$. Bruneau me parait inadmissible. Au reste, l'existenee d'un mot germ. waber est plus que douteuse.

(4) Jai cependant entendu récemment ì Flémalle et ì Seraing : lès poyes weibèt è pré; po qu' lès poyes ponèsse bin, èlai fât on wểbèlje. 
revanche, il a wêt " paître, en parlant du bétail " (?) et wểbî (?) "pieorer, en parlant des poules" (1). Forir fait wêbi (picorer) de la eonjugaison inchoative $(-i h)$, sans donte sous l'influence du synonyme

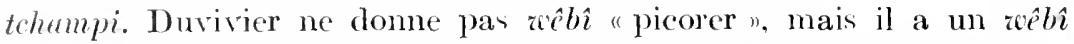
"regarder. bayer aux corneilles. guetter", que Forir lui emprunte et que G. enregistre aussi. sur la foi de Dejaer et de Duvivier. Il s'agit sîrement d'une acception métaphorique ; on ne fera done qu'un seul article âtêt a 1. picorcr... : 2. fig.. aller eomme à la pieorée, e.-à-d. de droite et de gauche. le nez au vent et l'oeil au guet ».

Scheler (ap. G., II 475) se demande si on peut considérer comme une simple corruption de icêdî ou alléguer le bavarois waiben "aller cà ct là ". La seconde conjecture est a surément la bome. Notre mot se rattache au moyen h. all. weiben "se mouvoir ęà et lì ", forme variée de schaciben (schweben). Comparez le liég. hêbî, nam. chêth "biaier, aller de travers". qui rient de lall. dialectal scheib: all. sclicef "oblique" ${ }^{(2)}$. - Quant à wêbe. s. f. (non liégeois), e"est le déverbal de wêlut.

50 G. I 1\%s, laime sans explikation cet autre mot, oil nou reconnaissons un complocé de atêt

diwaisbi, diwaibi $1 . t r$., faire quitter a qqn un endroit ou il a l'habitude de se tenir : si dizcribi : se retirer d'un lieu, d'une maison que l'on fréquen-

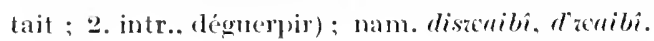

La forme première est d(is)acêtbi telle quelle apparaît en namurois ${ }^{3}$ ) ct chez le liégeois Simonon $\left({ }^{4}\right)$. 1) oì, par métathèse de $s\left({ }^{5}\right)$, d'wêsbî, -i, forme usitée à Verviers. Stavelot. Malmedy. Robert ville. Bovigny, ete. $\left({ }^{6}\right)$.

(1) Léprnthiose de $r$ est fréquente devant $l, l, m, n$; mais je n'en conmais pas

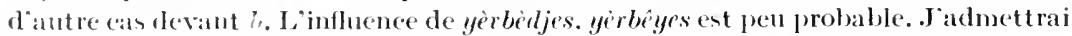

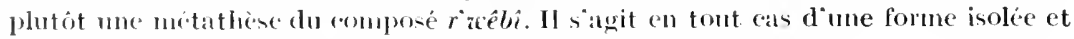
pell sirre.

(2) Ci., I 206:3 : Weigamd, scomer.

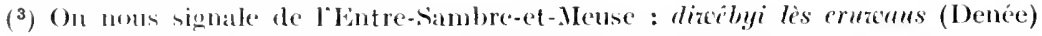
"cxtirpel les naunvaises herbes" ; fè in tomir pou s" diszếpyi (Stave) "faire une promenacle putr se dégonrdir : diszcêbyi (Ciney) "indisposer, attrister, dénoraliser"

(") Poisio's, J. 176. I) mêne, dans Forir : dizaibi (-z) "elfaroncher, étranger, digôter, eloigner, reluter

(5) Voye\% d"antles excmples a l’article dplouslrer, p. 86.

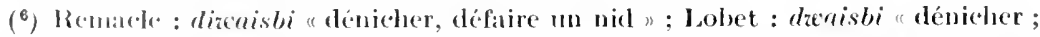

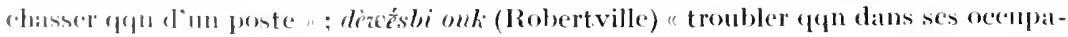
tions ; duatshi (stanchot) "eonduire, faire deguerpir

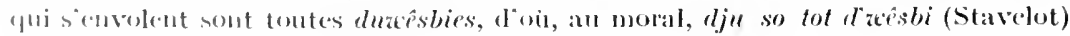

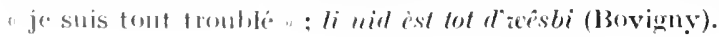


Enfin, pour Malmedy, outre duzuaisbi "désaccoutumer, dépayser ", Villers (1793) a un verbe intransitif waisbi "commencer à faiblir, diminuer de force et de courage, se relâcher ". C'est, en réalité. le môme mot, amputé du préfixe $d u$, fr. dé- $\left(^{1}\right)$. Le synonyme laispi (Malmedy) "lâcher ; commencer à faiblir "n'a peut-être pas été sans influence.

[BD 1920, p. 18.]

\section{liég. wåkî}

Le liég. wailî signifie "coiffer". Dans une pièce de 1733, intitulée Pasquêye mâ pégnêye, mâ wâk̂̉ye, en l'homneur de Delloye, primus de Lourain, on lit par exemple:

Qui tos lès eis qu'ont dès earotehes wâkèsse leûs dj'vâs, mètèsse lès flotehes !

On trouve le composé dizeâkî " décoiffer " dans une pasquille de $\mathbf{1 6 7 5}$ (BSIT 2, II, 30) :

\section{Mins nos n' lêrans nin po çoula dè d'wâkî lès pots èt hènas.}

Ces termes tendent à disparaitre : a Liège même, on n'emploie plus guère que l'expression ironique : zalkî sinte Catrène "rester vieille fille ». - En namurois. G. signale waker (II 478). qu’il faut sûrement lire aunler (II +84). et les composés si ranker "se rattifer" (II 286), maulié "mal ajusté " (II 5.2), contracté de mu aulié ${ }^{2}$ ). De son côté, Dasnoy note le chestrolais zauliêu "cordon écru. espèce de cordon grossier fait de fil écru ", qui servait sans doute à la coiffure des femmes. Il va de soi que ces mots, à Neufchâteau comme à Namur, sont oubliés depuis longtemps.

Pour expliquer zakî. G.. II 4zs, invoque rainement l'anc. h. all. fahs, vahs "chevehure": il ne peut tetre question que d'un thème germanique walk- ou wak-. Ici. je crois qu'il s'agit de wak-. que nous trouvons dans le moyen néerlandais wàken $\left({ }^{3}\right)$. luxemb. withelen "être éveillé "; luxemb. wâch, wâcherech. Mix-la-Chapelle waacher "éveillé ". Un dérivé de cette famille (wacker. zuliker) a développé, en allemand

(1) Comp. dieaee, vautrin, rèné, viglé, par aphérèse pour dédicace, d'vantrin, d'rèué, driglé. - G., II $4 \tau 7$, donne le malm. áaisbi, avee une eonjeeture que nous pouvons négliger.

(2) Voyez ei-dessus, p. 82.

( $\left.{ }^{3}\right)$ De même le $\mathbf{m}$. néerl. et $\mathbf{m}$. bas all. slâken " détendre, se détendre " a donné le liég. låker (ef. G., II 9). 
et en néerlandais, les significations suivantes : "éveillé, vigilant, attentif. rif. actif. frais. allègre, rigoureux. brave. eourageux "; il faut y ajouter. dam eertains dialeetes : "paré, attifé " et, en suédois : "joli" (1). Nous nommes done autorisé à eroire que le liég. si zákî, arant de signifier spécialement : "se coiffer", avait le sens général de : "se donner un air éveillé. plus frais et. partant, plus agréable à la vue, se faire brave, s'ajuster, s'atiffer".

\section{w. wandîhe (Malnedr')}

G.. II 479. dome sans explication le malmédien acandîhe, s. f., "caprice, boutade, fantaisie". Ce mot est tiré du dictionnaire de Villers (1793); il a disparu du parler actuel de Malnedy. - C'est, avee ehangenent de suffixe $\left({ }^{2}\right)$, l'ane. fr. aandie "éehappatoire", dérivé de gandir. acandir "échapper. sesquiver", que Diez tire du gothique wand jan (all. wenden, tourner). Wandihe signifie done proprement : action de tourner (pour s'esquirer : comp. le fr. pirouette) ; mouvement brusque et impréru hors de la direction raisonnable : caprice, ete. Pour la sémantique, comparez bîhêe (biais, biseau ; eaprice, boutade) et royez ci-dessus les articles stièmê. rièrnế.

anc. w. waneal (!), crait (!), etc.

Le glossaire de l'ancien Métier des Drapiers liégeois. par Bormans, a l'article suivant. qui renferme presque antant d'erreurs que de mots:

wanealx de craitz, s. m. ? (suranné). Suin, laine graisseuse du dos du mouton, et qui attire les mites ? (Charte des Drapiers, de 1527, dans le Recueil des Chartes et Prizilèges des Métiers de Liège, t. I, p. 232) $\left({ }^{3}\right)$.

Iu texte de la Charte de $1527\left({ }^{4}\right)$. eomparons une formule analogue qui apparait un peu plus tard, daus les Artieles additionnels de 1542 (p. 2.5. du móne Recueil). Il s'agit de "fause draperie " ou de drap fabriqué saus avoir observé les règlements :

(1) Voy. Woigand wacken; Falk-Torp vakken. - On pensera au fr. dial. braze "qui fait belle figure par la parure, l'ajustement".

(2) Lc sullixe -ihe - fr, -ise. - 1)c même le fr. maludie, lièg. maladèye, malm. -ie, devirnt maldike a stavelot, Favmonville. Inversenent le fr. murflumdise, verv.malno. martchumblike, devient marthandèye en liégeois.

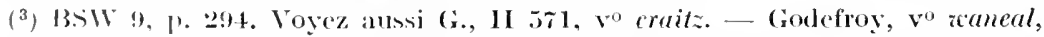
represluit latrtirle de Burnams en supprinant les deux points d'interrogation.

(1) On ne enmant cette (harte que par le très médiocre Recuél de 1730, qui fourmille de fautes. Sur ha façon dont il fut composé, on pent voir BSWW 5, p. 461. 
$152 \%$ : que personne ne présume doresnavant faire draps de laine de deux foixhes de Retomdeur, de wanealx de eraitz, de floekons, de noppes, de retaillons, ne d'autres laines deserables...
1542: [ces drays] se deveront faire de bonnes et loyales laines et étoffes sans ancune faleité, eomne de laines de deux forches de traits de flockons, noppes et retaillons, ne autres laines deserables...

Les mots que nous soulignons manquent dans la seeonde colomne: cette omission, fortuite ou voulue ent suggestive, comme on le verra. De plus, nous n'aurous pas à tenir compte de la ponctuation fantaisiste des deux textes.

Les règlements défendent d'user de déchets de toute espèce dans la fabrication du drap. Flocons. nopes, retrilloms, se comprennent d'emblée (1); mais la détermination des autres matières prohibées ne va pas sans peine.

1. Que veut dire laine de deux foixhes (frrches) ? Le liégeois (e) friches répond au fr. forees, lat. forfices. D’après Bormans. p. 250. les efoixhes désignent "les grands ciscaux avee lespuels on tond les draps", ce qui est vrai, mais incomplet : il se demande s leine de denx foixhes de retondeur (sic) signifie : "laine restée dans len cireaux du retondeur is la deuxiome tonte [du drap] $\%$. Pour moi. dams les textes de 15:\% et 1542. il faut mettre une virgule aprè foikhes ou forches, et comprendre qu'il s'agit des "eiseaux à tondre les montoms". La bonne laine ent fournie par la tonte des moutons qui se fait au printemps. L'expression singulière laine de deux foixhes dé,igne apparemment la laine obtenue par une deuxième tonte pratiquée en été. De fait, au dire de Bormans lui-même (p. 141), on ne pouvait employer que pour faire des étoffes de doublure $\left({ }^{2}\right)$ on des draps de lit. la laine, trop courte. des moutons

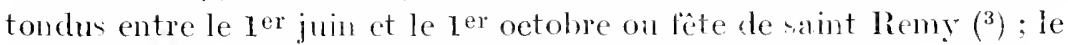
mouton ainsi tondu sappelait toz $:$ agnean. jenne brebis de lannée (").

(1) Voy. Bormans, l. c., pp. 263, 275, 284; nope est dans le Dict. général.

(2) Ceci peut expliquer lartiele de Godefroy : "touset, sorte d'ctoffe (une hupplande fouree de touzez : 1453 , 'Tournai) \%.

${ }^{(3)}$ La même défense existait ailleurs. Du Cange, Tondero, cite ce texte d'Abbeville (omis par Godefroy) : "que nuls ne puist drapper de gratuse ne de pelich fait depuis Tondisons jusques a le S. Remy ".

(4) Bormans, p. 292, vo tosui ; voy. aussi agnelin, p. 240, et dans Godefroy. Le w. tozê (lat, *tonsellum ) signifie proprement "petit [mouton] tondu ". Comparez l'ane. fr. tousel " jouveneeau ", touse " jeune fille ». Meyer-Lübke, no 8 r85, dit que l'on ignore la raison de eet emploi de tonsus. Le w. tozí, dont il ne fait pas mention, ne résout-il pas fa dilfieulté ? Touse aurait désigné d'abord une "brebis tondue ", puis, comme terme d'anitié, une jeune fille. Comp. Meyer-Lübke, $n^{\circ} 2256$ 
Je signale, i ce propos, l'article somertonture de Godefroy, dont la définition : "lei extrémités les moins funes des toisons" paraît suspecte. Godefroy cite notamment un texte de 137 oì l'on parle de "seurtonture d'aignelins et autre; mauvaise; matières [à faire draps] ". Logiquement, surtonture doit désigner le produit de la "surtonte " ou tonte supplémentaire de l'amnée. Ce serait, dès lors, l’équivalent de notre "laine de deux forees".

2. Les mots de Retondeur, de waneald doivent se lire, en supprimant la virgule : de retondeures de wavealdx. - Ce demier est une variante de roalherceal (1437 : BSIV 6. II, p. 113), woulweal (J. de Stav., p. 495), zeleweal (J. d'Outrem., iv 52) et autres formes wallonnes de l'anc. fr. veluel (lat. *villutellum "velours "), qu'on trouvera dlans Godefroy, VELVEL. - Bormans (1) a cru que retondeur désigne ici "l'ourrier qui retond ". En réalité, ce mot est du féminin pluriel et dérigne "les poils que l'on (re)tond sur le drap". Le règlement défend de faire du drap "de (re)tondures de velours" $\left({ }^{2}\right)$. Notez qu'ici, comme souvent en wallon. le préfixe re- n'a pas de valeur sensible.

3. Le lecteur aura déjà corrigé de lui-même erait $\left({ }^{3}\right)$ en traitz. d’après le texte de 1542, qu il faut ponctuer ainsi : "forches, de traits, de... ". II va de soi que de traitz est indépendant de reavealx. On défend de faire du drap de traits, e"ent-à-dirc "de bouts tirés ou ploqués" (ef. Bormans, p. 292). Traits est synonyme de bouts, queues ou penues "déehets de la chan̂ne ou de la rame " $\left.{ }^{4}\right)$; toutefois, on le reneontre rarement dans ce sens.

(1) Suivi par Godefroy, Betoxinetr.

(2) Comparez, dans le même sens, l'anc. fr. tomdures de drap (I588), dans Godefroy, et, pour la forme liégeoise : djondêre, ponden̂re "jointure, piçute" (de djonde, poude : joindre, poindre). - Notre not se retrouve dins la charte des Floekeniers en 1639): "tous drajus de laine, où y auroit chaines ou lilets d'esse, des poillages, flockons plocus, dec noppes [.] des mains [.] des retomdenrs, des pillaines, des nokies,...” (Recueil des Chartes des Métiers, 11 :321): "[défense d']emplir eoussins d'aneienne [corr. ancunne] sorte [.] de poillages avee mais [.] de Retomdeurs de draps, ou noppes ou norkes, pareillement de toutes telles denrces, comme d'oreilliers, tatelettes [corr, tikelettes?], ou s'mblables..." (ibid., p. 324). - Lire retondentes, conme soyenres, (p. 321) : sciures. Quant a mains, mais, ce sont des graphies défectucuses pour mahaing, défunt, tare, vice, liég. mod. mèhin (roy. God., sustians; Bormans, in BSill $9,1,272)$.

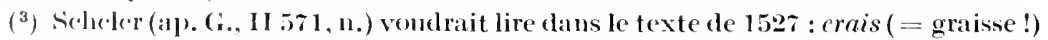
au lien de eruitz. (onlefroy, qui cite ce texte, ritonberr, complique l'altération en transerivint eroitz.

(4) Voy. Bormans. Pl, 242, 279, 282 ; et Renier, Hist. de TImbstrie drapière an pruys de libge, 1'. 217. Ce dernier onvage, estimalle ì certains points de vue, ne nous a di d'aneme utilité daus notre examen critique. L'auteur se fait l'écho des explicatinns fantaisites ale Bormans, notamment pl). 230 et 2.42. 
Reste l'expression "laines deserables", qui n'a pas encore été signalée. G., II 58\%, explique l'anc. w. "playe deserauble " ${ }^{1}$ ) par " desserrer ". On pourrait aussi. à la rigueur. y voir un dérivé de l'ane. fr. desirer (déehirer) ou encore de desentrer (dessevrer : comp. Bormans, p. 255) ; mais l'explication de G. se recommande par sa simplicité. Il s'agit en tout cas de laines qui peurent se relâcher et se rompre trop facilement.

\section{w. warbô}

G., II 480 : warbau (man, gribouri : ver doù vient le hanneton), nam. zaribau (ver bouvier). Composé de zar $=$ zer (comp. warglèse $:$ verglas) et de bau = bouf ? Ou modifieation de zermant, qui en rouchi et en picard signifie la même chose? [Note de Scheler : "I.e thème zurarb- ne serait-il pas = harb = scarb (comp. escarbot) ?]

D'après la Fanme aullomme de .J. Defrecheux. axarbô désigne la larve de différents insecten et surtont celle du hanneton. En général cependant. les eampagnes de, provinces de Brabant, de Namur et de Luxembourg réservent ce nom an ver bouvier ou larve de l'asstre des bovidén.

Les formes dialectales que nous avons recueillies sont assez nombreuses : acerbé Farnonville : -a Tohogne. Erezée. Herve : — iarbê

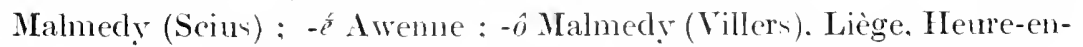
Famenne; — đârbô Namur (Pirsoul), Jodoigne. Marilles. Noduwez: — iearibô Namur (G.) : - ièrabô Borigny : - warobia Vonêehe : waraba Fauvillers, Lutrebois ; - $e^{\prime}$ Oisy. Gro-Fay̌. Chairière. Alle-surSemois ; - $\hat{o}$ Neufchâteau, Léglise. Bertrix. Ste-Xarie-sur-Semois, Dorinne ; nacabô Etalle : influence de noir on prosthène de $(n) n$ ?

La charpente du mot est diune constance remarquable : les variations vocaliques de l'initiale n'ont gutère d'importance : eelles de la tonique peuvent s'expliquer en général par l’influence de suffixes bien

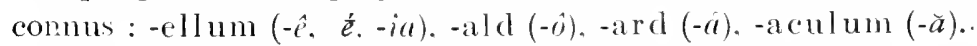

Les eonjectures de $\mathbf{G}$. et de Scheler ne supportent pas la disenssion. Notre mot est dorigine germanique: on doit en effet sadresser $1^{\circ}$ à langlais warble (ver bouvior). qui a de multiples formes : warblet, warback. warbie. warbetle. warmul. etc. : 2o à l'all. werbel. (acker)icerbel (taupe-grillon, courtilière).

Au surplus, le w, warbô a des affinités indéniables avee l'italien du

( ${ }^{1}$ Daus le Règlement de 1424 : "quiconques... quasserat gens à playe ovierte [ou] deserauble ; lat. : laedet vulnere aperto seu aperibili n( Louvrex, I 35). Godefroy, Deserable, ne eite que ee teste de J. de Stavelot, p. 550 : "et ly fisent une plaie deserable en la tieste ". Vọez aussi G., II 580, déférables (lire : deserables). 
Nord barbel (charançon) et avec le fr. dialectal guéribé. garibet, (b)urebec, hubert, heurebeuf, ete. ${ }^{1}$ ). désignant $u$ insecte qui ronge la vigne, le peuplier, le bouleau. ete. Pour expliquer ces différents termes, M. Sehuchardt $\left({ }^{2}\right)$ suppose un type germanique *wer ribel (= all. werbel), qui serait formé par croisement de l'all. werre. grillon, et aiebel, charancon. II. Schuchardt ne parle pas du groupe wallon dont nous nous occupons; cependant le type prinitif s'y montre plus clairement que dans les autres dialectes français. oil les altérations sont innombrables $\left({ }^{3}\right)$.

\section{w. wèrbiyon (Bovigny). wiban (Malmedy)}

1. I Bovigny et à Sam-Château (X.-E. de la pror. de Luxembourg, prè de la fronticre allemande). on appelle le grand palonnier : la balance, et les petits palonniers : les aèrbiyons. Le suffixe est-iyon, fr. -illom. de valeur diminutive. Quant au radical. il faut très probablement le chercher dans le moyen h. all. werben "se mouvoir, tommer, rouler". Le sen littéral du mot wallon est done : "petit objet qui sagite sans cense ". Comparez dautres noms du palonnier tels que l'all. seliaengel, le w. balame' ot le smivant.

2. Miban, qui signifie "palomnier" à Malmedy et à Faymonville, est signalé saus explication par G.. I 48\%. et par J. Bastin. rocab. de $F(! y m$. (BSW 50. p. 599). Je crois qu“il faut écrire wibant. participe subtantif du radical ba: all. wib-forme adoueie de wip-(néerl. wippen: bacculer, se balancer). A St-Vith (Eifel), au Sud de Malmedy, on dit wibeln "s aspiter sans cesse" $\left.{ }^{(}\right)$. qui répond au flam. wippelen, m. s. l'our le sens littéral de wibant. eomparez ei-dessus averbiymn.

\section{w. wére}

Le w. atere (liég. nam., ard.) signifie "cherron ", pièce de bois qui

(1) Voy. Aut. Thomas, Mélanges, p. 92 ; Godefroy : heurebeuf (herboz, en 1400, ì Friburg. = "ver bouvier"); Litté : hubert, hurebec, urebec. - Comparez de plus : garbon (hollanl, Fame pop., xn1, p. 179), qui désigne en Bigorre une larve qu'on trouve sur ou sous la pean des moutons ; barbon (ib., p. 11:3), eourtilière, dép. de I’Nlier ; zaranom (ib., p. 179), ver bouvier, env. d'Annecy ; véranbi (ib.), id., env. de Belfort ; verbli (ib., 1). 180), idl., à Boulogne-sur-Jler et à Saint-Pol. - Littré, Shypl., dome le norm. zerbled (larve du hanneton), qu'il explique par zer et blé (!),

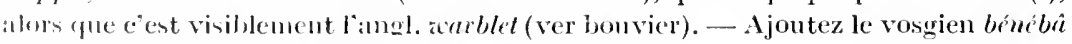
(ver bouvier: Ilingre, loce de la bresse).

(2) Ceitschrift f. rom. Phil., xxvi, 395; Clrix, $11^{\circ} 2419$.

$\left({ }^{3}\right)$ Je signale icei le: w. ararbia (Namul : G., II t80), weirbia (Hny) "lamprillon, petite lamproie", dont j’ignore lorigine. Serait-ce une altération de *aèmia (*vermellum) ?

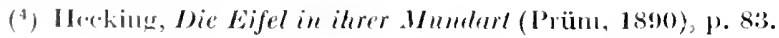


repose sur les pannes $\left({ }^{1}\right)$ et porte les lattes du toit. On trouve zeeire au xıve siècle, dans un texte de Jean d'Outremense, que nous avons eité p. 109 (voyez aussi Godefroy s. v.), Littré aceueille notre mot sous la forme zaire. G., II 486. qui écrit à tort wère. ne donne pas d'explication : quant aux conjectures que Scheler ajoute en note, on peut les négliger. M. Behrens, qui s est oceupé incidemment de âére $\left({ }^{2}\right)$, voudrait le rattacher à l'ancien frison wềr "mouton" (contracté de aceder, all. widder "bélier"). Enfin le latin vâra "pièce de support "paraît à M. Ant. Thomas une étynologie toute simple $\left({ }^{3}\right)$.

Cette dernière proposition est, à mes yeux. excellente sous tout rapport. Cependant elle n'a pas l'heur de plaire à Meyer-Lübke, no 9150, qui trouve surprenant le ehangement du $v$ latin en $a$.

L'objection prouve seulement que la phonétique wallonne n’a pas eneore livré tous ses secrets. Frì réalité. ce ehangement, surtout à l'initiale et devant $a$, est loin d'être une rareté. Sans compter des mots comme z'é, waŷ̉ (vadum, vadare, gué, guéer), wayłme (ragîna, gaîne). rasse. zé̀sse (vespa, guêpe) pour lesqueis une influence germanique est admise à tort ou à raison, on eonnait le liég. atapêtr "vapeur", wahê " eercucil (vasecllum. vaisseau), füarglès' "verglas ", wih'ner (Forir) "voisiner" wionthî "contourner, tordre" (varicare, d'après G., II 480) ; le malm. iłan, waner "van, vauner ", win "vain " $\left(^{4}\right)$, wivrou "verveux", liég. zirrou (rertebolum : G., II 472). D'autre part, nous avons vu ci-dessus wêroulle: variola; wahète : vessica - -itta ; zểrî: variatum; warmaye, à Huyzèrmaye "essaim d'éphémères": *verm-alia; l'ane. w. zareal: *vilutellum (5).

Pour la tonique, comparez clarum, amarum, mare > clér, amèr, mér, Enfin, la forme ancienne aceire confume aussi la dérivation vâra $<$ wéré, que l'on tiendra done pour pleinement assurée $\left({ }^{6}\right)$.

(1) En wallon ziènes (litt "vernes "; voyez l'article vièrna) : lès zéres si clazèt so lès viènes.

(2) Beiträge, p. 273.

(3) Mélanges d'étym. fr. (1902), p. 169.

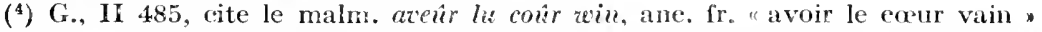
(= abattu, malade), sinti win « sentir le faguenas". Il faut en rapprocher le malm. win-mâ "premic̀res douleurs de l'accouchement ", que Seheler (ib., p. 486) voudrait expliquer par l'all. zeehen "douleurs n: et zeainis" "étiolé ", liég. zeinnis", que G., If 479 , rapporte an w. waine "cric" (!).

(5) Voyez l'Index. - Meyer-Lübke, $n^{\circ} 9376$, invente un mot liégeois wa, qu'il tire

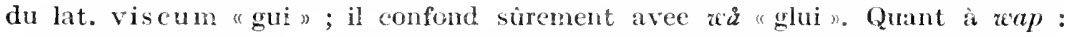
vapidus (?) d'après G., II 480 , le même aliteur, $n^{\circ} 9146$, le travestit en $z u p$.

${ }^{6}$ ) Cf. G. Doitrepont, Etule sur Ilemrieourt, $\$ 2$. 


\section{w. wêri (Verviers)}

G.. II t7\%, dome daprès Lobet : acairi (1. confus; :2. chie-en-lit : vilain masque). Remacle défmit le même not par cette phrase : "il a chié an lit ". On le cherehe vainement ailleurs; il faut done supposer qüil ent propre à Verviers, on cependant, pour ma part, je ne l'ai jamais entendu. Ce terme était probablement linsulte que les enfants lanȩaient jadis anx masques aceoutrés de linges sales (cf. Dict. gén., ro chie-en-lit) : par analogie, il a pu s'appliquer à la personme qu'on réduit an silenee et qui est. comme on dit familièrement, dans de vilains draps. Il faut done renverser l'ordre des significations données par Lobet et Grandgagnage. Si, comme je le crois, $i$ final est long $\left({ }^{1}\right)$, zếvi répont à l’ane. fr. zairié (de diverses couleurs, bigarré, tacheté ; lat. variatus). Pour le traitement de l'initiale $(v=w)$, royez ci-dessus l'article acére.

I Alle-sur-Scmois, jai entendu cette phrase : "le sang zốriye à la peau (par suite d'éraltures) ). Le verbe cếti, me disait-on, équivaut à chmé "suinter "; mais, en réalité, zêri (d'm type *varire?) a plu de pittoresque; il a dî signifier premièrement: "faire tache, mettre des lignes colorces (sur la peau éraflée) ".

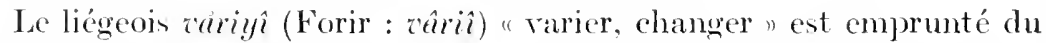
franẹais. De même zuvier, v. n., "divaguer", zarié, adj.. "troublé, délirant ", dont M. G. Jorisienne a traité dan BSW 17, p. ㄴ.40. et qu'il faut lire sans donte arec a et non a. Je troure le réfléchi : $i$ s zariyéve "il se troublait. il se trompait de nom " (ib.. 50. p. . 42 ).

\section{W. wèrlê̂}

Liaraigné fauchenx. Phalangium opilio. porte en Wallonie les noms les plur divers. qui sont surtout des noms de persome. C"est. suivant les régions. un "faucheux", un "herger ". un "vacher ". un "cloutier ", un " cordomier", un " mesurem ". une " dentclière ", une "madane ", me "grand-mère ", un "grand-père ", un "cousin ", un "galant ", ctc.. c.te. I)e plusieurs cotón on l'appelle "loup" (w. lek ; notamment à l'Wht : Glons-un-Gecr. Coo. ILarzé ; au Centre : Houdeng. Monceansur-Sambre; a l'Onest : Mous). Enfin, vers la lisière germanique de l'Est a du Nord, on lui domne un non signulies : werlen ì Cherain (13.5W 50. p. 5.34) ; wellen a Mamedy. Stoumont; wallen stavelot;

(1) Romarele et Lobet nigligent presque constamment le circonflexe. Au sumplus, f'emploi exelamatif du not a pu fare abreger la finale. 
wèlề à Lixhe-Visé: et. dans le Brabant : wèllü à Gembloux. GrandLeez. we'le à Court-St-Etienne et à Chastre-Villeroux (1).

Ce nom ent sûrement composé de "loup" (w. lèu = lèe. li dans la région brabançonne) et d'un premier élément qu'il s'agit de déterminer. Pour ma part. je tiens wèlế pour la forme la mieux conservée et j’y vois une adaptation wallonne du germ. werwolf (homme-loup. lyeanthrope), qui a donné, eomme on sait, le fr. garme dans loup-garme $\left(^{2}\right)$. Cette eonjecture permet d'expliquer en outre un lieu dit de la eommune de Sprimont : la he d' icèrlề, qui signifie, d'après moi, la "bruyère (all. heide) du loup-garou" $\left({ }^{3}\right)$.

De là. deux eorollaires : $1^{0}$ leù, nom de l'araignée faucheux (voir ei-dessus) est probablement une abréviation de rérlèi ; - 2o nos aneêtres, du moins dans le voisinage de la frontière linguistique. ont d'abord désigné le loup-garou sous le nom de wèrle ù, décalque transparent du terme germanique. Dans la suite, sous l’influence du franȩais. le composé pléonastique leû-icarou s'est substitué à aièlèt, lequel a survéeu, vidé de sa signifieation primitive, dans l'appellation populaire du faucheux et dans tel nom de lieu $\left({ }^{4}\right)$.

[BD 1920, p. 21.]

ane. fr. wetterel

Godefroy traduit par : "licon, eollier de cheral ". II cite denx excmples

(1) La Faune zeallone de .J. Defrecheux donne, entre antres noms du fanchenx, celui de zeellen (BSW 25, p. 48 ; zeellèn dans les éditions de 1890 et de 1893). Liauteur n a pu me dire d'où il tentit cette forme, - qui est évidemment me erreur de leeture pour wèlleth. Elle est devenue wèlin dans le Questiomaire de Folklore, p. 5, et dans le Folklore wallon d'Eug. Monseur, p. 9 ; weillin dans le Projet de Diet. w., p. 14. Toutes ees formes sont a eanceller.

${ }^{(2)}$ Il convient de noter qu'un antre insecte, la courtilière (w. leâ d' tére "lonp de terren: Defr., Faume w.), s'appelle lon-turou dans le Puy-de-Dôme (ef. Rolland, Faune pop., xiu, 112). - Pour la forme de zèrlề, eomparez l'all. zerbock (" hommeboue n), qui a donné le w. zirbour dans le Condroz (G., II 46:3), zèrbo au payss de Verviers (Monseur, Folkl. zallon, p. 5), pour désigner un être fantastique, gardien de trésors mystérieux. Le premier composant, qui devrait être wèr, s'est ici altéré sous l'influence de l'adjectif zèrt. - A remarquer enfin que la sauterelle s'appelle vèrbok à Awenne (lez St-Hubert).

$\left(^{3}\right)$ La forme primitive a dû être hé dè zè̀rleî̀ ; eompare\% fond d' gotes, lieu dit d'Ayeneux (BSW 53, p. 391), qui est altéré de foul dès gotes.

$\left({ }^{4}\right)$ Je crois reconnaitre une autre survivance dans une mélopée que chantaient naguère à la vesprée les bouviers de Fosses-lez-Namur en eonduisant leurs vaehes à labreuvoir : warluu, zarluu, lès zatches do sau, ete. (BSW 52, p. 16ĩ). Nétait-ee pas primitivenent une inploration adressée au loup-garou? Wurlau, pour warlen, serait anené par la rime sau (essart). 
du $x v^{e}$ sièele, tirés des arehives de Tournai, oì le mot est au pluriel. Ces textes. le second surtont (" une bride et les deux zeetteraux, pour servir audit eheval "), auraient bien dû lui montrer que sa définition était erronée. Il s'agit manifestement d'un dérivé de zettier, gaitier, fr. guetter. Quant au sens. il suffit de comparer le liégeois icêt'roûle " oeillère ". qui lérive de acitî "regarder". à l'aide d'un suffixe -ariolam, synonyme de -ar-ellam. C'est un petit objet eoneernant l'aetion de regarder ; eomparez bat'roulle "batte à beurre ", plant'roûle "plantoir", ete.

\section{liég. wihète}

G., II 488 , donne sans explication le liég. wihìte " jeune fille, frisque, folâtre, grivoise ». - C'est probablement un diminutif féminin du nom propre Wilhelm (Guillaume), dont nous avons vu le mase. zihot, à l'article ouyot. Wihète répondrait done au fr. dial. guillemette (= sotte, étourdie ; (f. Lobet, vo valtrou). La forme normale wihote, qui est dans Godefroy, aurait ehangé de suffixe sous l'influence des nombreux termes wallons de sens analogue : cåcarète, tchamarète, turhurète, mazète, ha guite, djouguète, frikète, fringuète, ete. Le mot rentrerait dans la liste des nombreux noms propres devenus nonıs eommuns avee un sens satirique; royez ei-dessus l'article tîbî.

\section{w. wisplote (Verviers)}

Nous ne eonnaissons ce mot que par Lobet, qui en parle deux fois, pp. 628 et 675 . Il éerit d'un eôté : wuissplott " haillon, guenille, ehiffon, ete. ", et. d'autre part : mett les wisplott "mettre le voile nuptial que le prêtre tend sur la tête des époux pendant la ećlébration du mariage ». G.. II 490, dit simplement que "zis' rappelle l'all. zeisch, torehon, chiffon ". Le rapprochement parait plausible. Quant au seeond composant, il est diffieile de ne pas le reeonnaître dans cet article que je traduis du Wörterbuch der Eupener Sprache (dialeete allemand ('Eupen, is l'Est de Verviers): "plute, n., vieux habits, vieux linges, chiffons ; néerl. provineial plodde = phunder (néerl., all.) qui, à l'origine, signifiait, sans idée dépréeiative, effets ou ustensiles de ménage " $\left.{ }^{1}\right)$. Wisplote dé onerait donc proprement des chiffons servant à essuyer (comp. l'all. familier wischtuch : mouchoir). Lironie de l'expression mite lis risplnies a bien la note populaire. Reste à voir si on comnait le mot daun la région germanique roisine de Verviers. 


\section{w. wite (Verviers)}

Ce mot wite, s. f., ineonnu à Liège, appartient à la région de l’Eıt (Herve, Verviers, Spa). Pour résumer et clarifier l'artiele assez confus de Lobet, p. 628, il signifie $1^{\circ}$ (sens ordinaire, le seul aujourd'hui usité) "loque, moreeau d'un tissu de rebut, torehon pour laver le plancher "; - 20 (sens arehaïque) " amadou éeonomique ou de ménage ", e’est-àdire loque brûlée qui servait jadis à battre le feu aree le make-fét (briquet, fusil) et un flin (pierre de flin, silex) ; tout eet attirail était serré dans une petite boîte appelée laisse às zites (en liégeois làsse al sitofe "boite à l'étoffe ").

G., II 490, le domne sans explieation. Godefroy, à propos de l'ane. fr. vite "bandelette", eite un exemple wallon qu"il tenait d'Albin Body : "deux wittes à laver" (1790: Arehives de Spa). Cela pourrait faire penser au latin vitta $\left(^{1}\right)$; mais le rapproehement imaginé par Godefroy ne repose que sur une vaine apparenee.

A Trembleur, au Nord de Liège, le verviétois wite est ineonnu. Au sens 1, on dit, comme en liégeois, dra d' mohone ("drap de maison"); mais, au sens 2, j'ai releré wike, s. f., li lasse ais wilies. Or cette forme préeieuse reproduit manifestement le moyen néerl. wieke "eharpie; lambeau, moreean. loque "( $\left.{ }^{2}\right)$. Pour l'altération de wike en wite, eom. parez ei-dessus l'article stierbalik.

\section{w. zgliné (Villers-Ste-Gertrude)}

Mot inédit, signifiant : "battu. rossé " (il a stou zgliné come i fait) et seulement employé au participe passé. On y reconnait le suffixe diminutif -iner et, dans le groupe zal-, le radieal syneopé de zingler " eingler, sangler un coup" (G.. II 491). La forme pleine *zingliner se retrouvera peut-être ailleurs. La réduction du trissyllabe en dissyllahe est normale; dordinaire, toutefois, la syncope afleete la royelle protonique ou médiale ; comp. stèss'ner pour *stèsiner. stèssiner (à l'article éstèssiner), wass'ner pour *wazoner. Iei, pour me raison d'euphonie, e'est la syllabe initiale qui s"est réduite : je doute qu’il existe un autre exemple de ee eas. A noter enfin l'adoucissement fréquent de $s$ fort en $\approx \grave{a}$ l'initiale; royez zoh'lé.

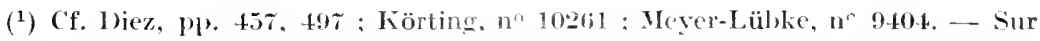
lane. fr. wite on gnite (long voile de femme), voyez anssi Grodefroy, vur, 335, 300.

(2) Voy. Franck-van Wyk. - C'e a'ilie ent le mème mot que le néerl. zciek, qui en flamand signifie "mèche de lan pe" (de meme glac l'all. wieche, wieke: à Aix-la-

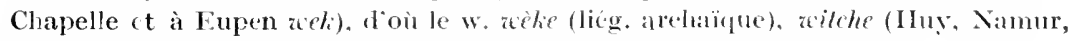
II (sbaye), s. f.. mèche de lampe à l'huile granne "; voy. G.., II 4 8. . 


\section{liég. zîvèrcôf}

1 Verviers. ver 1sso. jai entendu souvent eette expresion singulière : [aller ou envoyer qqu] as (ou ’ less) zéfurcôf. pour dire " an diable, aux antipodes". Longtemps après. j'ai déeouvert dans le Dictiomnaire de Lohet (Vervier, 185 t. p. 659) l'artiele suivant : "I-fur-liof. Indes orientales : se dit des personnes embauchées par subterfuge pour les Indes". Mème forme dans une pasquille du relviétois N. Poulet :

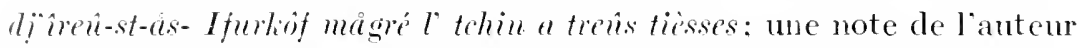
(BST 1860. t. 3. p. 3\%3) nous apprend que tel est. a Verviers. le nom populaire des enfers et. à Liège. celui des Inde, hollandaises. - A Liège, comme à Verviers. le mot est aujourd hui prenque oublié. Je ne l'ai rencontré que deux fois. sous la forme zivercôf, dan les 56 tomes du Bulletin de la Soejété de Littérature wallonme (1). M. Menri Simon comnait l'expresion divins lès zîbèrcôf : "en enfer". De plus, une fiche de feu I-idore Dory porte que cis-ive'erêjfes signifie "aux Indes hollandaises ". et Dory ajoute cette conjecture : "Sous le régime hollandais, o: enroyait anx Inde, orientales comme soldats les jemes gens indisciplinés dont le parents ne savaient que faire; on les vendait pour les iles. comme divait le peuple. L iles et ierlinopen ivendre) parainsent ètre les éléments de ce mot". - Enfin versons au dosier ce texte curieux et quelque peu déroutant qui nous vient de Malmedy :

Cès vìs, sûtis come leûs bodèts, pinsint trover lès "Ziles Ferkoffes" qwand qưi vèyint lès tròs Marèt. lu Ru, lès Pouhons èt lès Gofes $\left.\mathbf{(}^{2}\right)$.

Pour le coup. aurions-nou affaire à un groupe d’îles de la Polỵné,je? Mais on aura beau fouller eartes et dietionnaires géographiques. rien ne nou éclairera. Une seule conclusion ، “impose, e’est qu’il s’agit bien d'me loeution d'origine germanique.

Pour l'expliquer $\left({ }^{3}\right)$, on s'adressera au néerl. sielverlonpen, qui a uignifié jadir : "vendre son âme (au diable) ", et plus récemment : "se

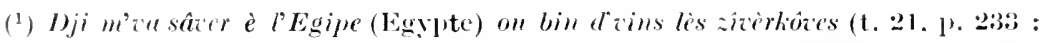

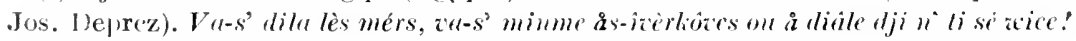
(t. 5:3. 1). 10s: God. Halleux).

(i) Ces vienx, anssi (peu) subtils que leurs paniers, pellsèrent tromver les antiporten quame ils virent les trous .laret, ete. (lieux dits des enviroms de Malmedy) ".-

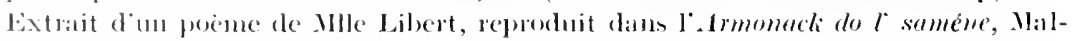

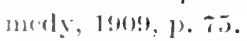

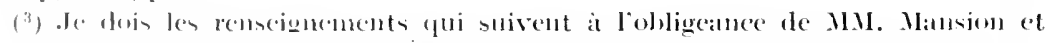

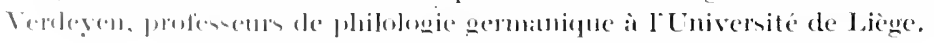


laisser, pour de l'argent, enrôler comme soldat ou (ommme matelot n. Les dictionnaires nécrlandais du xrme siècle. IIalma. Marin, ete., définissent comme suit le subutantil zielierlonper (litt. a vendeur d'âmes ") : marehand de chair hmmaine ; unurier (pui mmmit d'habits et de provision des soldats. des matelots, à raison d'un gron intérêt pour ses avances ; curôleur ou enbancheur de soldats ct de matelots pour les Indes néerlandaisen "(1). Naguere encore, on appelat aimi en Flandre ceux qui reerutaient des remplacants pour le service militaire.

Il résulte de lia que l'expresion primitive "enroyer qun au (ou aux) *silivirlip" signifiait l'enroyer aupres de l'enrôleur on de chrôleurs pour les Indes. Dans la suite. le sem propre s'étant oblitéré et le z initial s'étant confondu avec la finale du pluriel as (aux), on a pris le nom du racoleur pour celui du paý où il expédiait ses victimes; par unc nouvelle dégradation. les Inder sont devenuer les antipoder, puir les enfers : ainsi, an lieu de as (aux). on a pu dire i lix..., divins lés... (en les, dans les). - Q Quant ì la forme, sil-ne s'est maintenu que dans le texte de Malmedy, main l'ćtymologie populaire y a fait voir des

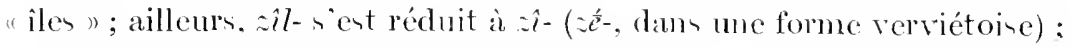
- ièr s'est maintenu en liégeois (bìr rénulte d’une dissimilation) : fir en malmédien = fur en verviétois: - enfin la finale-ipp' est derenue -ôf par asimilation arec arer qui précede. à moins qu'on n'admette me forme germ. *siclierhonfer qui existe pent-etre dams les patois rhénans clu roisinage. La rovelle brive - of de Malmedy est probablement amence par la rime.

\section{lićg. zoh'lé}

Mot inédit, 'que j'ai noté. sams indication de source, dans ce texte liégeois : li coton dèl lampomite est tot zolilé d' crusse "la mèche de la (petite) lampe ent tonte pleine le crasice n. De mêne que ingler (royez (i-dessus agliné) est une prononeiation hien wallonne de cingler, solilé se ramène à sohî, verbe délivé de solhe, s. f.. "rigole, tranchée pour l'éeoulement des eaux" $\left.{ }^{2}\right)$. G.. II 371 , cléfuit sohi : "faire une saignée

(1) Voy. anssi Grimm, qui cite un exemple de lirisch (17+1): "secterhenfer, in Holland, qui navigantibus in fudiam homines adducit ut in navibus serviant n. Le petit Wörterbuch der Eupener Sprache (knpen, 1s!s!), donne : silererlö̈üper "matrehand d'enclaves".

$\left({ }^{2}\right)$ L'étymologie donnéce par G. (lat. sulcus) ne couvient pas. M. Esser a montré (BD 1912, P. 101) que sohe vient de l'ane. h. all. suocha (sillon). -- Voyez an surplus Blumschein, Aus dem Wortschutie der Köluer Mundart (Cöln, 1904), p. 22:3 ; on

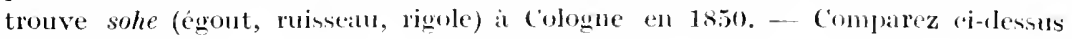
l'article sole. 
pour l'écoulement der eaux ". puis il eite, sans traduction, la phrase : cist-ome la èstêt si plin d' bièsses qu'i sohîue, où sohî a le sens intransitif de : "ruisseler, regorger. déborder" (syn. ridohî). De même : i-èst tél'mint plin qu“i sohe (Verviers : BSW 53, p. 422) « il est tellement irre qu'il déborde ". -- C"est par une figure analogue que le diminutif zoh'lé peut signifier : "inondé, eouvert d'une multitude". 


\section{Les noms dialectaux de la "culbute » en Belgique romane}

L'essai de synthèse qui suit est sans doute eneore bien incomplet; il aura tout au moins le mérite de montrer la riche variété de nos patois, d'offrir quelques explications inédites et d'orienter les recherches ultérieures $\left({ }^{1}\right)$.

1. Certain jeu d'enfant consiste à se dresser sur les mains, tête en bas et jambes en l'air ; le débutant appuie d'ordinaire les pieds contre un mur. Cet exerciee s'appelle en fr. pop. "(faire le) poirier ". les jambes figurant les deux branehes de l'arbre; anc. fr. perier (God.).

C'est ausci le nom qu'il porte dans le domaine exploré : pèrî ou pê̂rî Liège (rare) ; pèrî Bovigny ; pèré Stoumont, Wanne, VillersSte-Gertrude ; pwar $\hat{\imath}$ Namur, Denée, Wavre, Harmignies ; preèr $\hat{\imath}$ Genappe, Péerot-Chaussée, Chastre-Villeroux, Ciney, Chimay, Gilly; proirné Tournai, Wiers, Pâturages. Quevaueamps ; pour̂̉ Ellezelles; pori Toureoing. - L'expression ordinaire est "faire le poirier "; à Ciney " planter le poirier " $\left({ }^{2}\right) .-$ On dit : (faire le) haut puorî Ueimont, Denée ; - iè Thibessart ; -ié gaumais (S.-E. du Luxembourg) ; proarié fourchn Margny-lez-Florenville; arbie fourchn Lille; fè l' fortchöye mèlèye (= pommier fourehu) à lioy en Famenne $\left({ }^{3}\right)$.

A Seraing, M. Nie. Pirson signale le mot hôstal, terme de gymmastique : Li proumî qu'on fêt, qucand on èst jumnasse (gymnaste), c'èst $l$ hôstal. ('e mot (germanique ?) paraît être d'introduetion réeente.

(1) Cette ctude a paru pour la première fois en 1914, dans le Bulletin du Dictionmaire aallon. Elle est ici complétée et remaniée en plusieurs endroits.

$\left.{ }^{2}\right)$ De celui qui n’a plus d'argent, on dit plaisamment à peu près partout : " Il peut faire le poirier, il ne tombera rien de ses poches ".

$\left.{ }^{3}\right)$ Comprarez fouché-paciré dans les Vosges ; planter la pourée en Touraine. De même, en westphalien, den boom stan (= den Bamm stehen) et, en patois d'Aix-laChapelle, der beereboom stohn (= den Birnbinm stehen). 
2. La culbute, à proprement parler. est un tour eomplet sur soimime. On peut distinguer : $1^{0}$ la culbute en arant : la tête est posée sur le sol et l'on tourne cul par dessus tête. de façon à retomber sur le do ; - zo la c. en arrière : on se met sur le dos, on rejette les jambes en arrière, de façon à retomber sur les genoux : - $3^{\circ}$ la c. latérale, moulinet oì l'on fait tourner le corps sur les mains. puis sur les pieds; en fr. "faire la roue "; - to enfin la e. accidentelle, où l'on tombe brusquement à la renverse : d'où. en général, chute. pirouette. eabriole ( ${ }^{1}$ ).

Il va de soi que tou les mots qui suivent nont pas toutes ees significations. A l'oceasion. on indiquera au moyen d'un chiffre $\left(1^{0}, 2^{0}, 4^{0}\right)$ le sens usité dans telle ou telle localité. Les renseignements reeueillis permettent de eroire que le $1^{0}$ et le $4^{\circ}$ sont les plus connus. Rarement on a mentionné le $2^{\circ}$. - Quant au $3^{\circ}$, tout ee que je sais, c'est qu'à Bourigues-Dinant on dit : fè l' ruce di tehaur "faire la roue de ehar "; à Malmedy : fé l' toûr du pacion "faire le tour de papillon "; à Faymonville: fé dès rones d pacion : à Roỵ : fè l'mace : à Pâturages : fé le racé "faire la rone"; à Liège: fé $l$ mate dazie "rone dioie" ou di tchìite "de eharrette".

Les expressions sont groupées d'après les trois grandes divisions dialeetologiques de la Belgique romane :

1. Wallon proprement dit (lićgeois, ardennais, namurois et brabanȩon de l'Eit) :

B. Gammais (dialecte de la Lorraine belge, au Sud de la provinee de Luxembourgr):

C. Ouest-wallon (Charleroi-Nivelles) et rouchi (rariété du pieard: Mom-Toumai).

Dan chaeme de ees divisions. on suit l'ordre alphabétique.

1. WilloN PROPREMENT DIT.

3. bèrdoûse, bourdoûse (Liège : G.). terme enfantin, "eulbute " con sénéral. Duvivier donne bmerdonfe et bourdoulé. J'ai entendu ausi burdoufe (il a fêt "ne bèle bardoufe). Forir ne signale que bourdi-bourdombe "xclann. pour exprimer une chute, me eulhuten. I Verviers, bourdoûssetr. birdonisser (al zalêye) : "culbuter, dégringoler". Il est hors

(1) Il existe cuncore, parmi les jeux enfantins, une autre espèce de culbute, qui

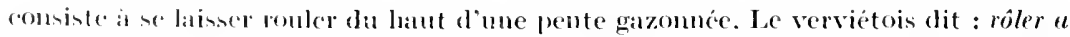
fagnime "muler il [la façon d"un] fagot "; le liégeois : rôler ou djouzcer a si sprăkhth $l$ botronile "ronler ou joner à sócraser le nombril * à .Jupille rôler a fahène on

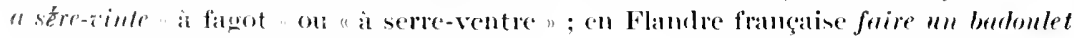

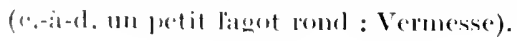


de propos de pensel ì l'all. burseln. comme fait G.. I ï. Cen termes sont de pures onomatopées $\left({ }^{1}\right)$. empruntéen de l'all. bardans ot du nécerl. pardoes, perderes : roy. Weigand. Franck-ran Wyk. - 'Telle ent auni,

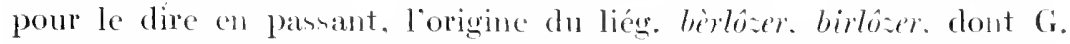
n'indigue pan l'étýmologie. Nous ý verroun l'altération d’un primitif

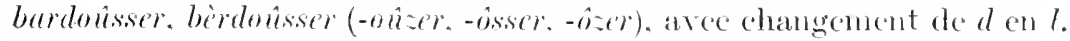

4. bèrwètem à été domméa Lanemillc-au-boir (centre de la province de Luxcmbourg) conme synonyme de compète "enllunte" : cent d'ailleurs le sens quil faut aussi lui attribucr dans l'expresion fé birvite (liég., nam. ard.). bien comne surtont au jeu de quilles : "faire faux bond, coup nul, ne pas abattre de quilles ou mêne mau quer la planche en lançant la boule \#. - On serait tenté dabord d'y voir une aeception figurée de bercite "brouette". par analogic arce ume broucte qui se renverne en avant on de êté ; mais, curéalité. il

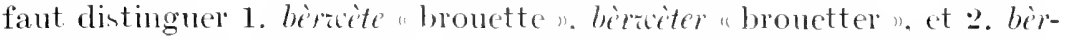

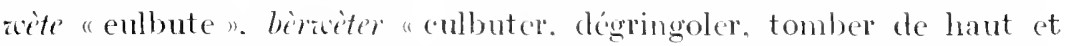
arce des choes; d'où faire fiasco " $\left({ }^{2}\right)$. Le second répond au franeg. pi rouette, -er, dont l'origine. mal connnc junqu”ici. s’éclaire par ee rapproehement. Le franc, "faire la pironette an jeu de mail " (Littré) ne peut en effet cotre séparé du wall. fé birite au jeu de quilles. En meusien - et ailleurs évidemment - faire la péronette signifie "culbuter "(Goffart. Glossaire du Mousmmais). En wallon *pirates. *pèr-

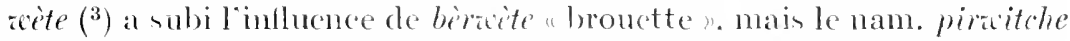
"pironette" (L. Pirsoul) reproduit le radical primitif. D'autre part, on troure. arec le sem spécial cle "toton. moule de bouton pereé d'un trou au milicu ". pirouelle. peromeille $\left(^{1}\right)$ en me-in (II. Lorrain) et. en wallon, perayge Bastogne. St-Hubert; pirivge Laroche, Cherron, Condroz et Famenne: picizye (pour *piritye) Liege. Bergilars : pirweitche et pivitehe Huy, Namur. Fonses; enhin. à Burdinne (en ILeshaye), nous relevom la forme sperivitche. qui rattache elairement tout ee

(1) De même botuluf "patatras" (= flam. parduf). - Comparez ret article de Lobet, p. 253: "houptata, chute d'un enfant sur son derrière et qu'on relève de suite sur pied \%, - Au langage enfantin appartient anssi l'expr. fé toutoume on totome (= tommer. tomber).

$\left({ }^{2}\right)$ Le nammrois dit dan ee cas bèrouler. Bèrzèter "dégringoler " est liégeois et surtout ardennais : vọ, abèrzèter dans B1) 1906, 1.94.

$\left.{ }^{3}\right)$ Pircicte est dans le Diet. liég. de Forir ; mais, sous cette forme, c'est un emprunt rérent du français.

(4) Comp. le meusien péroile (Labourisse) : "s. f., gros son avec lequel on jone au patard (jeu de bouchon, le bouchon lui-même) ". 
groupe au bas-allemand d Aix-la-Chapelle spirewippehe "toton" $\left(^{1}\right)$. La chute de $s$ initiale (sauf à 13urdinne). l'altération de la finale (sous linfluenee des -ufl. -iyge, -ìye, -ite. -ìle). le passage du w germ. à ou fr. (purenent graphique. eomme dans marsouin) sont des phénoniènes ordinaires $\left({ }^{2}\right)$.

5. coubotèye Robertville-lez-Malntedy. Borigny, Wamne : fé coubutéye. syo. fí l' coupèrou "faire la culbute". A Faymonville. fé coubotèye. cent faire la eulbute en arrière. - Composé de cou " eul " et de butiye "bouteille".

6. coupèrou. G.. I 131. n'en explique pas l’origine. C'est une altération de cou-pè $\hat{\imath}_{\hat{\imath}}$ " eul-poirier " (roy. \$1), la finale ou faisant écho à la première syllabe. - Le même type se retrouve en Picardic : cupoiriev (God., $\mathrm{r}^{-0}$ pericr) ; en rouchi : tchupori Peeq. tchupouri Luingne-lez-

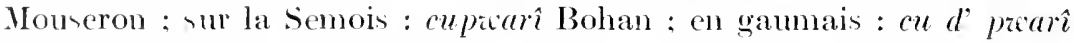
Buzenol, St-Léger (au sens : ${ }^{\circ}$ : pour le $1^{\circ}$. on dit cu d' boviré) ; en meu-

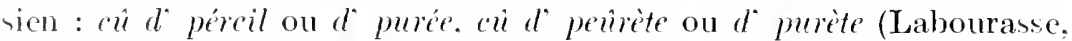
Varlet). - En wallon proprement (lit. il est fort répandu et présente des formes variées : eupaièi Forville ( $\mathrm{N}$-E. de la prov. de Namur); cupèri Awenne. Denée ; coupèri Dinant. au sens $4^{\circ}$; coupèri, copèri Ciney ; copiceri Dorinne $\left({ }^{3}\right)$ : coupirć Neufehâtean $\left({ }^{4}\right)$. Bertrix ; coupèrè Oisy, Laracherie. Ortheurille, Tohogne. Roy et daus la Famenne; cupèrè Freux lez-St-IIubert ; conpö̀è Chairière. Gros-Fay ; coupirou Moxhe ; coupérou Barvaux-Condroz ; cupèrou Eghezée; coupèrô Erezée; coupèrou Liège, Glons. Verviers. Spa. Wamne. Malmedy, Bovigny, Lutrebois. Ciney. Ben-1hin. Vezin. Petit-Fays ; couberou Liège (Duvi-

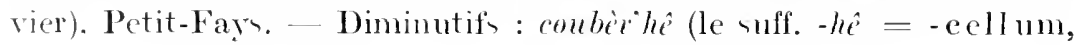

(1) Jon. Müller, Die Aachenér Mumdert, 18:36. Ce mpprochement est stogéré jar (.. II 230. Lorigine de spirewiphche (qui, pantit-il, n'a plus cours aujourd hui is Aix) ent domnée exactement par Scheler : spire "chose prointuen et wippehe, dimiı. de a'ill" "mouvement misle (il), note). (f. Bchrens, Beiträge zur fr. Nortgeschichte, 1) 125.

(2) I propos du w. percigy (lire pèraige). Ieyer-Lübke, no 9.515, exprime une antre opinion qui me parait inaceptable. Il en fait le congénère de gironette et ne parle, ni à ect codroit ni ailleurs, du fr. pironette.

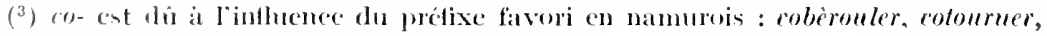

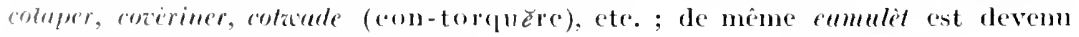
commlit en namurois moderne, \$10.

(3) 1 Nouvillers-Recogne, on a formé de la le ve tr. conpirè ; a certaine épogue (moinon :) ken jemes gens se saisissent d'une jeme tille quils couchent par terre

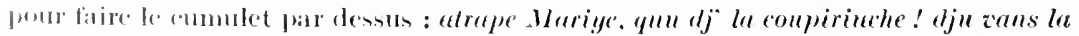
conpire! lac jeunes filles font de mene quami elles peuvent attraper un jeme lomme. (communication de M. G. Collinct). 
-scellum) à Bergiler en Hesbaye ; fi la comperite Lutrebois, one cupètè Rachamps-Bourey. lu coubirète Wardin-lez-Bastogne. Notez, au sens 2o. les expressions couperon d mam'zèle Liège, on d' madame Glons (cumulit d' mam zèle Namur : Pirs.). cupacari ur réér Bohansur-Semois, tchupari a l'ind'iếr Luingne-lez-Mouscron.

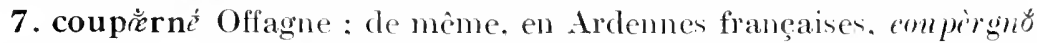
St-Fergeux, Acy lez-Château-Porcien (1). - Le première syllabe ne pouvant représenter eul (à Offague : lï̋), j'expliquerai littéralement par *couperonneun, diminutif de l'anc. fr. comperon "cime. sommet", qui a laissé d'ailleurs d'autres dérivés en wallon : copurnale (-ella), "faiseean de gerbes " Liège (Forir). ou cncore "gerbe d'avoine à un seul lien : dressî lès copnrnales "Licrs ; seopmmi Marehe-en-Famenne, Ciney. Dorimne. "eouper le sommet. le bout ", ane. fr. escoupperomer ; discopurnć Denée, même sens : acopurli (pour -nè) Beauraing, qui a aujourd'hui le sens péjoratif de "emmanehel" ", mais qui a dû signnifier "attacher par le sommet (par ex. des gerbes)" : d'oì acofărmûre Chastre-Villeroux, BD 1910, p. 13t : 1922. J. 35.

8. couribèt, m.. liég., G., I $1: 31$; couroubèt Duvivier. courubèt Rouveroy, corubit Remacle. Forir ; avee changement de suffixe : conroubot à Jupille ; diminntif : courombinit à Liège. crombinèt à Geneffe. - C'est une forme maseuline de "courbette" (rov. God.. corbet), qui a pris le sens de "eulbute". Je relève. dans $11 n$ texte liégeois de 1\%3*, croubèt (BSW 1, 180) et. dans Forir. enmonbite avec le -ens primitif de "courbette .

9. cou-z-å-haut Verviers (Lobct. p. 30.5). litt. "cul-cu-hant " : té dès cou-z-a-haut "faire des culbutes" : royez \$13.

10. cumulèt, s. m.. terme francisé commu dans toute la 13clgique romane, où il passe même pour être français. C'est le seul mot en usage à Namur (avec le verbe cumuleter. G.. I 1+7). dans la région de Charleroi et dans une partie de la Hesbaye : Marilles, Ambresin. Wasseiges. Il a d'ordinaire les sens $1^{\circ}$ et $4^{\circ}$. On fait eependant ȩà ct là des distinetions ; ainsi, à Luttre, il n'a que le sens $1^{\circ}$ (pour le $4^{\circ}$ on dit crumuga ;

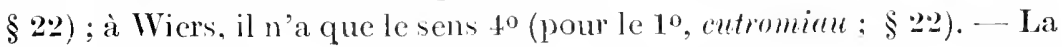
forme la plus ordinaire est cumulit. On prononce liömö̌lit en Brabant (Chastre-Villeroux, Péerot-Chaussée. Jodoigne) et sur la Semois inférieure (Alle. Oisy).

Grandgagnage en rapproehe le rouchi tumite. tumeléte et le normand

(1) Je dois ee renseignement et la plupart de ceux qui eoneernent les Ardennes françaises à l'obligeance de M. Charles Bruneau. 
cumblet. dérivén de lane-haut-all. tômilón (néerl. tuimelen, wall. foumer. tomber), main il n'explique pas sulfisamment le ehangement de $t$ initial en li. M. Antoine Thomas (Romania, 1909. p. 3r9) conjeet ure que lo besin cumblé représente un tỵpe médiéval *tumelet contaminé par cul. Pour ma part, cette influence me paraît asururée et je verrai de neme dans la finale du wall. cumulet lönfluence du fr. mulet : eertaines formes. dont nous allons parler. ont en effet -moulet traduction du fr. "mulet" ( $\left.{ }^{1}\right)$. Le peuple, on l’a dit mainte fois, a le besoin de s'expliquer. füt-ce par labsurde. les divers éléments de son langage; la présente étude, conme touton eelles du même genre. pourrait s'intituler : "le jeu des influences et des combinaisons".

La première syllabe de eumulèt présente çà ct là de eurieuses altérations : on trouve eumoulèt à Crehen. Ambresin (Hesbaye). comulèt à Fonses et dans le patois moderne de Namur. sou l'influence des préfixes

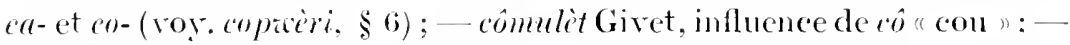
scimmlit Romedemne. Sautour-lez-Philippeville. influenee du nam. si srôyt "s "cotaler les jamber ouvertes. se fendre démesurément " $\left({ }^{2}\right):-$ erammlit Moxhe, eromoulet Bouvignes-Dinant (au sens $1^{\circ}$. tandis que coupriri a le sens $4^{\circ}$ ). Denée. Landrichamps-lez-Givet. influenee de eri, "rô "gras" : l'expression pacarter a crô-cia "porter (un enfant) sur ses épaules". litt. "à gras-veau" (= eomme un reau gras), a sans doute déterminé "gras mulct". La finale du type eul-tumerel (\$ 20 ) a déterminé cumulia (Nivelles: Renard. LArgayon. pp. 32. 138).

Le nanurois appelle aussi cumulit le "pigeon enbutant. (all. tummler, angl. tumbler. ap. G.. I 1+7). qui est dénommé ailleurs culbuten et tourniquit d'après. Defrocheux. Focal. de la faune acallome : royez \$19.

11. mou d'avonne Bovigny : fi $l$-... "faire le muid d'aroine" : culbuter en roilant par terre. Expresion métaphorique. expliquée par coct article du Dirt. malmédien de Villers: "fél' mon l'arône, he dit des chevaux. lorsquils sagitent de droite et de gauche. couchés sur leurs reins. gagner son avoine "I)"un solipede qui se roule ainsi sur le dos. on dit a Imimont. qu'il fait le picotin ; a Genappe. IIoudeng, Stambrugen. qu'il bat ou gagnge son aroine. De même. à Genappe, d'un enfant qui se roule en s'étirant. on dit qu'il se anne. par comparaison arec len poules qui "se vamment" dans la poussiòre à l'approche de loragre.

(1) Comp. con d monlè (Thimister-('lermont) "arrière-train très développé chez 16. jeune veaut, litt. e'ul de mulet".

(2) Vuye G., II :3, \% 
12. pertainne, pirtainne Liège : se dit surtout de la cullute que fait le cerf-rolant qui tourne sur lui-mime. et aussi en parlant d"une persomne. De même pèrtontainne "chute brunque. cullute. plongeon:

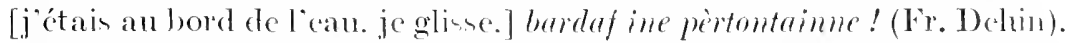
C'est le fr. pretantaine. d'origine ineomnue d'apris le Dict. gin. - Conp. le liég. aler pérti-pètaimme "aller cahin-caha ".

13. scoudro Liège. 'Terme arehaïque et rare. attenté seulement par Cambresier (17s\%) at par licmacle. G.. II 350. l'enregintre d'aprèn re dernier $\left.{ }^{(}\right)$. Origine ineommue.

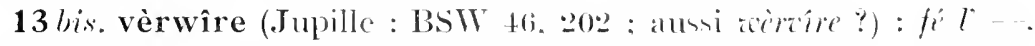
faire la eulbute, surtout le poirier : yru. pirtaime. Comp. le flam. coerceling "tourniquet " (Schucrmans).

B. Gatiais.

14. cu de berceau. Cette expresion est attentée par Maus. Tocab. des emirons de Tirtom. manu erit de 1850. M. Ch. Bruncau nou cignale cubèrsô à Cumières (Marnc). C"ext probablement une altération de *cambrecean eontaminé par cul : "culbute se dit en picard camberseu $\left({ }^{2}\right)$ et emareden (Corblet). en blaisois emberselle $\left({ }^{3}\right)$. Comparez $\$ 1$ s.

15. cu d'boûré Etalle. Tintignyy, Mellier. St-Léger. Virton. cte. ; cu $d$ bacarô Musson. Proprenent "eul de hourean"? M. Ed. Liégeois conjecture que cette exprension date de l"époque où la décollation se faisait par la hache sur un billot : apres le comp fatal. le supplieié eulbutait sur lui-mene d'arant en arrière $\left({ }^{1}\right)$. - S'emploie aux sme $1^{\circ}$ et $4^{\circ}$; au sens 20. On dit cu d' pwarî : roy. \$ $\$$.

16. cutrumé Etalle (archaïque). Willier-lez-Florenville; liŏtrŏmét Alle-sur-Semois ; cutrommé Mouzon (Arol. franç.) : cutrumian liethel (ib.) $\left({ }^{5}\right)$. Le type ent "*ul-tumerel "arce nétathie de $r=$ "cutru-

(1) On trouve dans Wesplanl, Li pluisir dè tchuntêt. p. so : féli seoudrot faire la

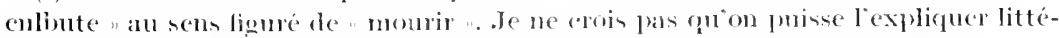
ralement par : faire son cul droit ". car droit se dit dresit en wallon liegeovis.

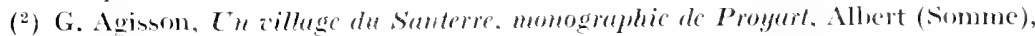
1906, p. 225 : "Pent venir du vieux fr. cumbreselle, action de se bainser pont recevoir qun sur son dos ". P'one l'évolution simantique. comp. s\$.

$\left({ }^{3}\right)$ Thibaut. Gloss. de payss blaisois, dome deux at ymolngies lantaisisten, mais il cite combreselle dans. Merlin coccaie. I 17, et combrecelle dans IRabchis. II 222.

${ }^{4}$ ) Explication dontense, cela val de soi. En voiei une antre de même valeur : "cu-dc-bouri, culbute sur la tête à la façon des canards: bontri, exclamation pour rappeler les canards" (Varlet, Dict. du pretois meusien, vo boûri).

$\left.{ }^{5}\right)$ H. Baudon, Le patois des encirons de Rethel, 190\%. P. 33:3, domne aussi cutrumeler "verser seus dessus dessous. ap parlant des voitures". 
mal : roy. \$2. - Les formes coutrumé Ucimont, coutrëmṕ Corbion (pour cn-, hắtronmé) présentent de plus une métathèse de voyelles. à moin qu"il n'y ait simplement influence du fr. cou.

\section{Oeest-Wallon et Rouchi.}

17. cabèriole Tournai : " eulbute, saut périlleux ". Altération du fr. cabriole. De mîme, dans l'Anjou. capériole. carpéiole "eulbute". En liégeois. cabriyole a le sens du fr. "cabriole".

18. cu d' buzèle Frameries. M. Louis Dufrane, qui signale ee terme, a noté cu de bisclle dans un no de 1896 du journal Les Noncelles du Borinagc. - C'est sans doute une altération du pieard cambrecelle, comme en de bercean $\$ 14$.

19. cud'troncha Houdeng (rer's 1860 , d'après M. l'aroeat Hubaut) ou cud'roncha Houdeng. La Louvière. Brapuegnies (d’après d'autres), cud'ronmia Houdeng (auj.. d'après M. Hubaut). s. m.. "culbute", au sen $1^{\circ}$. - La dernière forme paraît être un simple corruption de *entroumia. \$2.2. Les deux autres sont d'explication plus difficile; je crois pourtant qu'elles peurent se rattacher au mome thème par substitution du suffixe - cha - cia (-cel, -ceau) au suffixe-ia (-el. -eau) (1). On aurait la série *entroum"cha, *cutromm'cha, cud'troncha $\left({ }^{2}\right)$, cud'roncha, ce dernicr, par étymologie populaire, somant eomme "cul d' rond chat - - I l'appui de eette conjecture. j'invoquerai un mot que Sigart. Dist. montois. p. 362. signale sans l"éclaircir : "troumchat, hirondelle. à Frameries n. Il faut san doute écrire troum'cha et expliquer, comme ci-dessus. par troumia ("toumeria. *tumerel) $\times$ suff. -cha. On a ru, à la fin du \$10. que le "pigeon culbutant "s'appelle cumulèt, culbutek. Qüil s'agisse jei d'une hirondelle et non d'un pigeon, cela ne fait par difficulté : en effet. d'aprèn J. Defrecheux (Faune wall. vo colon), "hirondelle" est un terme de coleben emprunté à la langue franẹaise pour dérigner "eertaine espèce de pigeon au rol rapide et très élevé ".

20. cumĕriau Rebaix. Wodeeq. Flobeeq ; mmorian Ellezelles. IDe mône à Reims cumarib $\left({ }^{3}\right)$ et, dams les Ardemes françaises: rumivio à Château-Poreien. cubèrò à lamogne.] - Cén diverses formes

(1) Comp. cumlerche Houdeng, "grand chaudron dans lequel on fait cuire la nourriture des pores. ete. ", qui serait, en ane. fr., *chanderoncel; voy. dans Godefroy, cturemel, coroneel lorroncel, moneheroned, ete.

(2) Ponr liuscrtion de d, comp. le pic. cul de tremel que Jouncoux doune à eòté te cutromblé.

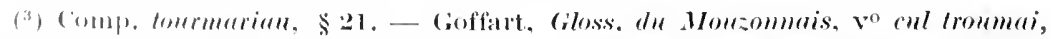

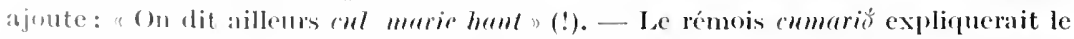

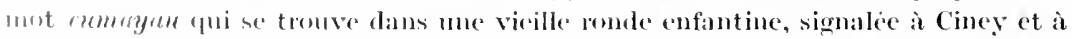
lwome. Les culiunt tournent en se tenant par la main et en chantant a Au romdean, 
dérivent d'un prinuitif *tumeriuu, -eret. contaminé par cul. Voỹ. cutrumian $\$ 22$, tehuberlìre $\$ 23$.

21. cutournia Ouest de Charleroi ; atournian rouchi (Héeart, Sigart); Thivencelles-lez-Condé, (quiérrain. Beloxil. Maubeuge, I Iarmignies. Leuze : coutourniau Borinage : cutrougnau Bcaumont. [Ce type est aussi eonnu dans les Ardennes françaises : cutoumiau Boueonville; cutourgnţ Château-Porcien; cutrougna I Iautes-Rivières ; cutrougnau Rocroi ; cutrugnau Séeheval. Deville, près de Monthermé; cu détournai Mouzon (Goffart); cu détromné Vrigne-aux-Bois. prés de Mézières.] - I.e simple tourniyau se reneontre à Pâturages. tmurnéyau à Flobeeq. Il répond à l'ane. fr. tornel (1). Les formes précitées supposent un diminutif en -ellum de tornum. ane. fr. torn, tour, s. m. : un *toumeau, e'est un "petit tour" (comp. four, fourncau). de mème qu'un *tumereau, qui signifie proprement "petit oljet qui tombe ou que l'on eulbute (cf. tombereau). a pris dans nos dialeetes le sens de "petite chute ou eulbute"); eomp. tumete $\$ 2+\left({ }^{2}\right)$. II. A. Thomas, l. c., se demande si. dan cutourniau. le verbe tourner est prinitif ou s il s'est substitué à une date récente à tumer, toumer ; il est difficile. suirant lui, de répondre avee assuranee. Je me permets de trourer ses scrupules execssifs et d'admettre, jusqu'à preure du eontraire, qu'il s'agit en l'espèce d'une formation parallèle à cutrumiau. - Il est naturel cependant qu'un type ait influé sur l'autre. Ainsi s'expliquent cutourmiau Quevauchamps, Leuze, Ierchies, Ormeignies. et le simple tourmiau Isières. Ostiches. Maflles. Irehonwelz-lez-Ath; twurméyau Lens, Lessines; altéré en tourmomyan Ath, sous l'influenee de mouyau "muet" $\left({ }^{3}\right)$. et en trimonya Nivelles (par métathèse pour

Cumuyau. Ma graud-mère a fait un sant. C'umayan! (C'iney); Rondeau, Cumayan, Nosse grand-mére a fait on saut, Cumayfau! (Awenne). Les enfants s'accronpissent en lançant bien fort le dernier mot. C'ette romfle est ividemment importée du sud.

(1) Godefroy le donne comme adjectif : "qui tourne", et comme substantif" "pont tournant ". - Un " tourneau ", cest aussi un "pretit objet qui tourne"; tel est le sens du liég. tournê "sabot, espèce de toupie".

${ }^{(2)}$ De même encore sauterean "petit être ou objet qui saute "aurait pu signifier et signifie peut-être dans certains dialectes : "petit saut".

(3) Voy. crumuya, \$2:2. - M. Jules Dewert merit ì ce sujet : "L'athois tourmouyau signifie "tour muet "; il s"est dit d"abord les tours de pantonime exécutés sur la rue par des bateleurs : puis, par extension. des culbutes et cabrioles de toute espèce ". Lexplieation est ingénieuse, mais elle n"est pass nécessaire. Nous constaltons ici, comme pour cumulet et pour mainte autre forme, l'action de létyomologie populaire, c-à-dl. de la tendance génerale à transformer un mot plus ou moins obseur sous linfluence l'un antre mot qui offre quelfue ressemblane de sens ou de son; cette transformation lui prête ordinairement une upparence de sens.s ; voy. Nyrop, Gramm. historique. I $\$ 528$, IV $\$ 551$. 
*fommiga). - Reste le rouchi tourmériau (Hécart. Vermenc). tomermalun (Vermesco). On l'expliquera de deux façons. Ce peut ître comérian. enmurime (\$20) contaminé par tomr. D'autre part. on pent -upposer. à eôté de tommiun. un trye *tournerian (formé de tomrner comme tomberen. souterean de tomber. santer) ; le changement de $n$ en $m$. expliquerait. comme dan tmm minu. par l’influence de tumer.

22. cutrumiau rouchi : Arennes. Girault : entromian Wiers (1). Landrecies; entrmmian Dour ; cutrimin Berzée. Sautour-loz-Philippeville: chute de dan, entumia 'Tubize. cutumimu Neuf-maisons, I'Tertre-

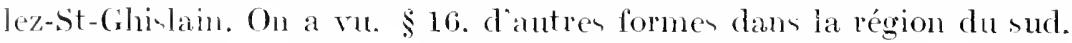
Ellen répondent tonter au champenois ementurée. que I. Ant. Thomas (Romamia. 1909. 1). 379) explique avec rainon par "*ul-tumerel ". Iei nour arom, de plus la métathine de $r$. - Même phénomène daus le imple trumiau rouchi (Vermere. p. 490 : Hécart. p. 469 ; voy. aussi Hécart. vo tommereat. tumereut) : trumia Soignies. Marche-les-Ecaussinnes: trommin Le Reulx : trmm'mia (hapelle-les-Herlainont $\left({ }^{2}\right)$. On a ru. $\$ 20$, que *mmeriau est devenu ailleurs emmerian. Il faut éqalement rattacher ici crumuya Luttre (au sens to ; cf. \$ 10), dont la deuxiome partie a subi linfluence de mmyn "muet ". comme tom-

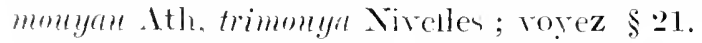

23. tchubèrlîre, ․ f.. Mouseron. Luingne. Toureoing. Peeq " eulbute " (au sens $\left.4^{\circ}\right)$. Riépond à un type "*tumerel-ic̀re ". dont la premiere yllabe sent altérée nou l'influcnce de tchu "cul " (eomp). tchu-

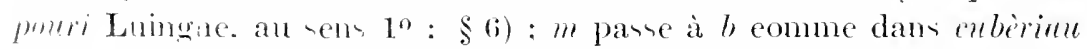
pour cumèrian ( $\$ 20$ ) et peut-ĉtre ausi sous l'influenee de birlire (T'ourcoing : "rhiffon. loque. Jambean "). oì je vois un dérivé de bure,

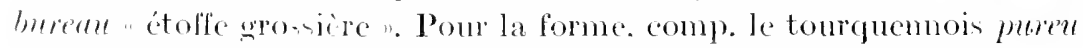

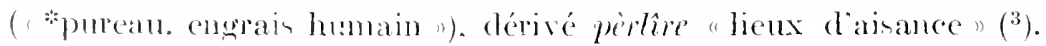

24. tuméte (faire -..) rouchi (Ifécart. Vermese) : tumète Borinage (sigart, p. 3(i3) ; temmite. temmelite (Ificart) : temmelet Peeq ; timblet Lille (Vemente. p. 48.5). faire l'équemmette (ib. p. 490). - Dérivés de lanc. fr. tumer (w. tommer. tomber). d'origine gernanique ; comp. le bournuignon cutimblis (- *al-tumelot) et le norm. emblet. On a ru. $\$ 10$. rumulet issu de *timelet. - Les dialectes flamands et allemands comatiscont amsi des dérivés du môme primitif, employés avec le

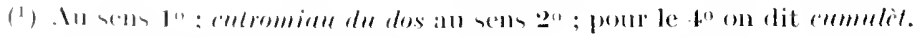

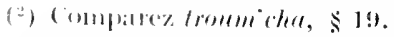

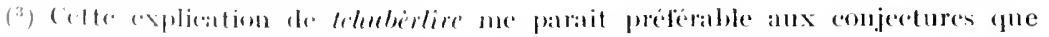

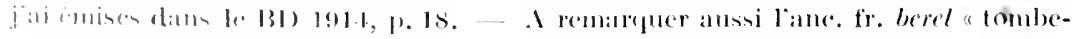

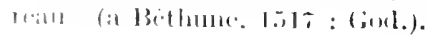


mème seus : tmimelit. -ifte Furnes (Fandre oceid.), tmmmelööt Aix-laChapelle, Eupen. tumeleut Beres (Pruse rhénane) ; tuimelant Anvers et 1'. oceid. ; voyez Schuermans, Fleumsch Idiotienn.

Appendice. - Daus ce qui précede, on s'ent. antant que possille, renfermé entre les limites de la belgique romane. Ene etude du mêne genre pour les autren régions serait assurément intéressinte. Voici, pour y aider, fuelques notes brèves.

I. Dialectes romans de France et de Suisse.

bousen, fatre le - norm., Calvadus (Rezue des purlers pop., 19062, 1. 45).

bousiquet "culbute ", Injon (Verrier et Ouillon).

calpobta, -esse " (rulbuter, -te ", Vongen (.J. Hingre, Patois de La Bresse).

ermberseu, pieard; comberselle. blaisois; voy, 14.

caneoubriyo "culbute, pironette que font les enfauts" (Boillot, Putois de la Grand Combe, Doubs). - La seconfle partie est evidemment "boyaux" ; voy. cantibonelle.

cantibouelle Langres (Jocab. langrois 1822). Companez lernibonclle. - Composé de emuli (comparez cancou-, cuem-, hican- = toume?) et de bouelle "boyanx".

chêne-drette : faire le - (J. Rongé, Le parler tourangeau, 1912, p. 41).

corbiche " eabriole ", norm. (1) Boin et Travers, 1856).

coupricre "cabriole " pieard (Corblet). - Pontrenpière?

courpélé, m., Vonges (.J. Hingre, Patois de La Bresse).

comereleu picard ; voy. 14 .

cuboule, cuboulaye lorrain ; cubouler "culbuter". he cul-boule.

encauboule, kicanboule, f., cullunte (M. Lorrain, Giloss. messin, acrit cumemboule, quiquemboule). - Voy. cartibouclle et licangôle.

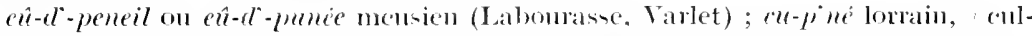
bute (Diet. patois par L. M. P[etiu], cure de St-N[abord, Vonges], Nancy, 18+2). Litt. "eul (de) panier" ; compratez, en dial. langrois, "panice fomrehu "syo, de "poirier fourclus"; $\$$.

culiemêlu, enpessu (Bridel, Collosstrire smisse).

eupellia Lyon, euplot Danphine, enpelié, cupelit Gascogne (Puitspehu).

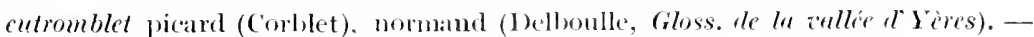

Altéré de *eutombelet, prob. sou l'inlluence de entromlelet.

cutroulelet normand (1)ebboulle, o. e.) ; entromè picard (A. Ladien, Giloss. de

Démuin). - Le verbe trondeler signilie wouler par terre".

kicangôle Naney. - Voy. cantibonelle et cucanboule ; -göle = gueule ?

moulinet norm. (Joisy). En Ilam. bruxellais molelen (Schuemins).

omelette, faire l'-, ('hattean-Poreien (Arel. franç.).

pastouret norm. (Moisy). - Altere saus doute l'un ancien *mstourerez "(jen) propre aux (petits) pâtres ", quon pourait ajouter à l'étude de .I. A. Thomas sur Je sull. - aricius.

pie percée "culbute" (R. de M[ontesson], Ioc. du IIaut-Aaine, 1859); syn. piquechène (ibid.).

pilegatier ou pirgatier, m., ("ulbute (.lontbéliard, dep. du Doubs). 
pique-chene, voy pie-percie.

jiquet "culbute", Anjou (Verrier et Onillon).

sameublète. sumssublète, sautublète norm. (Du Bois et Travers). - Composé de saut ct du norm. enmblet, altéré, $\$ 10$.

tèmibouelle Bomrges, Sancerrois. - Anc. franç. tormeboele; eomparez eamtibouelle. tirebonille. f.. cullute (Irontbéliard, dép. du Doubs).

trimbole norm. (Ioisy) ; pie. foire le tram-boyelle (Jouaneoux, I, 2r6) ; pie.trimboire (Corblet), șrn. capriole de sautriau (id.).

tourne-boîle, tomrnefiehe, tournemoelle, tournevire (de II [ontesson], Voe. du IHautMuine, 1, 451).

11. Langues germaniques.

beereboom "poirier ", Aix-la-Chapelle; voy. $\$ 1$.

botiel, de - schlòn, de - schièrren, Weiswampaeh (G. D. de Luxembourg); bolilibunz, koplibunz, koppedisjen (G. D. de Luxembourg).

burzelbunm, purzelbaum all., "culbute". - Composé de baum "arbre " et du rarlical du v. burzeln, purzeln "culbuter "; le sud-all. borzen signifie "faire saillie "; l'all. bürzel sign. " eroupion " et, dans certains dialeetes, "éminenee de terre, butte " (= wall. croıtèt $)$; voy. Weigand, eol. 312, 494. Même radieal dans kaukelpurz Saxe, koekelpor L Leipzig, burzelbock Silésie, loblzehop Schoppen-lez-Faymonville (Prusse rhén.) "culbute".

burzelbock Silésie et anc. h. all. - Bock signifie propremert "ehute ". Voy. Weigand, col. 260 .

kimlelpurz Saxe, hochelporz Leipzig, kaupelsturz Thuringe ; kơkelbờ, de schlìen ( = schlagen), Montzen-XIoresnet ; hokelebolsch Aix-la-Chapelle (Müller, Die Aachener Mundart, 1836); kokelenbot Limbourg belge (Schuermans) ; kukeleboom siegertand. - Pour le premier élément, voy. Weigand, vo gaukeln "jongler, faire des tours ", Franck-Van Wijk, vo gooehelen.

kobold Brandebourg, Pomćranie; m.-h. a. lobolt, dérivé du v, kobolden "eulbuter"; du danois-sućdois kobolt "lutin " (Falk-Torp) ; voy. Weigand, kobolz.

liolbotte danois : emprunté dı fr. culbute (Falk-Torp).

kop en hisch, de - schlun, Martelange (Luxembourg belge) ; iver kop en asch fảlen (Arlon) "tomber sur tête et cul ".

huchenschass Antriche (cité par Müller, o. c.).

kuitelbuit Limbourg belge ; kwitelen, kwikelen "culbuter" (Schuermans). moleken flam. de Bruxelles (Schuermans). Propr. "moulinet ".

perre, perrebom (Flandre oecicl.: De Bo) : pertelboou (Gand) $=$ all. burzelbaum. trummeleut (cologne) : la finale est probablement heut (Haupt : tête); le premier compusint tumelu altéré sous linluence de trumm, trommel (Blumsehein, Aus dem H'ortsehatie der Kö̈luer Wuemlart, Cöln, 190)t, p. 22(i).

tuimeluter Invers, Fl. occid.; tuimelèt, -itte Furues ; ete. Voy. \$2 2 .

thimelperte, thimelpryss Flandre oceid. (Sthuermans).

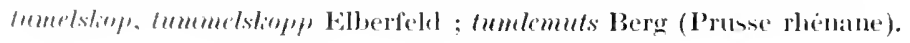




\section{APPENDICE}

\section{Les mots germaniques à préfixe ge- qui ont passé en wallon}

Pour le sujet. je ren voie le leeteur à la note de Scheler, eitée ci-dessus, p. 107. Voiei une liste de termes qui représentent des emprunts wallons de même origine et de même type que l'anc.-fr. sistel.

aguèrôdî (wallon : Vielsalm). p. …

aguistiller (rouchi : Mons), p. Ios.

caliguluk (Malmedy), s. m.. "merveille. objet précieux, chose rare et extraordinaire " (Villers. Dict.. ms.. 1793). -- Emprunté du bas-all. et néerl. lannegeluk " fond de la bouteille, auquel sont attaehées des proprićtés merveilleuses ".

camatche (w. liég.). p. 43.

cristal. custèl. kèstèl. ete. (Liège. etc.). p. 10\%.

d $(u)$ grâde (Verviers, Malmedỳ), p. sr.

geschot (ane. wallon), dans le reeueil de Louvrex, t. I, p. 202. § 7 : "les deniers receuz pour l'amende on geschot ou despens" (15+8). C'est le moven bas all. geschôt. qui avait le sens de "taxe, taille, eontribution \%. Le w. moderne scot. fr. écot. vient. comme on sait, de l'ane. bas all. skot (all. mod. schoss).

ghitalle (ane. wallon), dans une charte liégeoise de 1582. qui fait défense de vendre, en eertains cas, le poisson autrement qu'en gros, "assavoir ... les scolkins par ghitalle appellé vulgairement boirean, eontenant chascun douze vingt" (G.. II 559). Scheler est tenté d'y voir le fl. shetal. setal "nombre" : le mot exprimerait une mesure numérique (ibid.. 1). 597). Cela ne fait aucun doute.

gistel (ane. fr.). p. 10\%.

glindis' (wallous). p. 108.

guèrôder (wallon), p. 2.

guezite (Mons : Sigart). s. f.. figure, bouche ; - guèzike (w. de la Famenne) : djo lî f'rê bin têre si guèzilie (dans une pièce de 1800. Li Warièdje manque, aete II. sc. 7 ) "je lui ferais bien taire son bec" ; guizike (w. de Stave lez Namur) : quéne guizilie qu'i li vint! "quelle 
grose figure il lui vient !). - Emprunté de l'all. gesicht, néerl. gezicht (risage).

guilite (w. liég., uam.) "ligne. rang, rangée. file, série ». Du néerl. gelid. all. glied. On trouve aussi les formes wall. guilike (G.. I 24 ; comp. guezite. -ike). guilite (Lohet. 1). 2:32 ; influence des diminutifs en -ite'). suilih (Lobet. p. 180)). Ljoutons réguilite (Liège). riguilite (Jupille. Vervier $\triangleleft)$, règuilite (Verviers), où l'on peut recomaitre l’influence de rếgue (règle). riglinne, règuinêye (rangée. file). On dit riglite à Seraing, riguinite à Thimister.

guimène (w. liég. : (.. I 247.355 ) " eonseil de guerre, tribunal militaire "; forme franęaise ou francisée guemine (G., II 600). ghemaine (God.) : à Malmedy. gumune (Villers, 1793) ; d'où : gumuner (ibid.), "traduire qqn devant la guemine ". Dérive de l'all. gemeine " eommunauté " (roy. Behrens, p. 122). - Aujourd'hui, guimène est rare en liégeois, où il a pris le seus de : "eoutume. usage. habitude". Nous relevons à Trembleur : prinde ine drole di goumène "contracter une singulière habitude \%. - En ehestrolais, d'après Dasnoy. p. ¿48. guminer est synonyme de halliner "barguigner. hésiter "; il a dû jadisignifier "discuter un projet au sein de la guemine ». De même le gaumais guminèy, que le Lexique de M. Ed. Liégeois traduit par "rouler de mauvais projets " : qué quu t' gumines co ? J'ai noté de mon cóté à Ste-IIarie-sur-Semois : qué quu t' gumines tout-la? "qu'est-ee que tu remues bruyamment par là ?" Il è guminé toute la neûtiye "il s'est agité toute la nuit (sans dormir)».

guinåde (Liège?, Verviers : Remacle', Lobet, Forir), guènâde (Malmedy : Seius), dans : d'mander - "demander grâee. s'avouer vaincu "; brêre - (BSW 40, p. 21s: 44. p. 413), même sens. - Emprunté de l’all. gutude "grâce. merci ", moyen h. all. genâde, néerl. genade (G.. I $2+\pi$ ).

guitîre (w. liég. : G.. II 530) "marée, flux et reflux de la mer ". On lit dans Mćlart. II istoire de IHuy (Liège. 1641; p. 526) : "Il fit donner un assaut général, la guittière estant restirée vers le vienx havre". C"est le moyen néerl. ghetîle "temps déterminé, époque. d'oì : maréc " (Behrems. p. 138). Godefroy donne l'anc. fr. ghethie, itide.

guizèle. dans : ine pitite suizèle "un petit effronté " (Crehen : Ifenbaye), "une persome malieicuse " (Ciney). De l'all. geselle. néerl. "sezel " compannon. camarade", qui a pris la nuance péjorative comme il arrive souvent pour les mots empruntés d'une langue étrangìre (comp. ci-dessus gauezite, guminer). Le genre féminin du w. ent dû à linduence de la termination (eomp. guezilie). 
guzouhe, cuzouhe, kăssouhe, p. 125.

kènouk (gaum.) : gu-i dis crombîres a lienonk (Ructte-lez-Virton) "il y a des pommes de terre à foison "; "das" lienouf (Etalle : BSW 41 , II. 143) "à foison. à bouche que veux-tu" (= all. dus ist genug. cest assez). - Linitiale s’est durcie comme dans kìstil, lichlitône, ete.

kich'tône, P. 166.

netkufurnet (Malmedỵ), adr.. " entièrcnent, tout nct, sans biaiser " (Villers. 1793 ; Grandgagnage. dans ses Extruits de ce dietionnaire malmédien. écrit par erreur netlictfurnet). - Emprunté de l'all. nett geciährt nett (?). c.-à-d. "net garanti net "?

plat'kizak (w. : dire qqch -- c.-à-cl. tout net et tout plat). De l'all. platt gesagt " dit platement". On trouve plat"zuras (. Lm. Soc. zall., 5. p. 73), plat cuzat' (Malmedy : Villers), plak' kizak, plakizak, platezak et mêne, par étymologie populaire : plate casaque ou plalie tès-ali (" eolle tes aetes").

\section{Le suffixe - aricius $\left(^{1}\right)$}

Dans ses Nouceaure Essais de Plnilolngie française (1905), pp. 62-110 et 359-362, M. Ant. Thomas a montré que le clévelopjement de ce suffixe en Gaule était beaucoup plus considérable qu'on ne se le figurait ; il a dressé une liste imposante d'environ zo mots français et dialcetaux où il retrouve ce sullixe. M. J. Feller. dans le Bulletin du Dict. wallon de 1910, pp. 69-73 et 77-121, et daus ses Notes de philologie wallonne (1912). pp. 166-221, complète, an point de vue du wallon et de l'ancien français. Ia liste de M. Thomas, soit par le nombre des termes, soit par une documentation plus précise et plus décisive sur quelques-mo (2). M. Thomas avait montré que le développement extraordinaire du sulfixe composé - ar ̌̌cius résultait notamment cle la eonfusion entre le suffixe féminin - aržcia et le suffixe féminiu - zssa d'une part, et d'autre part entre le suffixe masculin -aržcius et le suffixe masculin - zttus. M. Feller, à son tour, a le mérite d'avoir prouvé qu'une confusion analogue s’ent produite en wallon entre le suffixe masculin - ař̆cius et le suflixe maseulin -ellus.

Après les listes copieuses de MII. 'Thomas et licller. le sujet est près

(1) Nouvelle édition, eonsidérablement augmentée, d'un article paru en 1910 dans la Revue de dialectologie romane, t. 11, 11\%. 379-381.

( $\left.{ }^{2}\right)$ M. Behrens, dans la Zeitschrift für framz. Sprache und Litt., t. xxvir ${ }^{2}$, p. 170, signale aussi une dizaine de mots wallons omis par M. Thomas. 
d'être épuisé : on ne pourra plus que glaner dans le champ qu'ils ont moissonné avee tant de soin. Voiei, pour ma part, une poignée d'épis ramassés de-ci de-là et quelques remarques eritiques sur les articles de mes devanciers.

acheret, ane. fr. - Le Glossaire roman-latin du xve siècle extrait de la Bibliothèque de Lille. publié par Em. Gachet (Bruxelles, 1846). note p. 1.1 : "zenaticus, kien acheret ". C"est une erreur de lecture pour cacheret (propre ì chasser ; du rouehi cacher). Cf. Scheler. éd. dı même Glossuire, p. 25 (Anvers, 1865) : Thomas : chacerez, p. 95.

affeterresse, anc. fr. -- Voyez ci-après eschoicheresse.

affoerece, ane. fr., s. f.. "provision de bois de chauffage ", dans un texte de 1255 des Mreh. Mos. (God.). - Dérivé de affoer (afouer); royez Dict. gén. AFFOUAGE.

aspergerèsse, w.. "goupillon ", dans la revue Jadis (Soignies, 1912. p. 18\%). - Dérivé en -érécé on simple altération de aspèrgès?

ausserot, mcusien. "sorte de raisin uris. syn. affumé "(Labourasse) [?].

balerèces, w. de Hesbaye. s. f.. "hattes de jardinier : planches carrées de $\left(0^{m}\right) 0$ de coté. que le jardinier sattache ans pieds pour battre la terre ensemencée ". - Dérivé de baler ; ef. G.. I 42, 4:3.

ballereisce, variante baeleresche, anc. fr.. épithète decille. dansles coutumes de Beauraisis : "s est dit d'une ville qui n'avait point de charte de commume. cf. bateis " (Godefroy). - Dérivé, comme le précédent. de baller. syn. de battre? Ce serait. dans ce cas, le synonyme de villo batiche.

bènerèce (wall., Erezée). ^. f.. dans : one grande - " une grande toquée "Serait-ce l'ancien wallon bunneresse "porte-drapeau " (Feller, 1). 18.5). employé ironiquement?

boucherot (joner an-). colin-maillasd. jeu oil lon "bouche" les yeux (Beanquier. Toeab. du dép. du Dombs).

bourderesse: "pour un panelet de canchie viers la piere bourteresse ('n allant a point viers l'uis dou mountier " (compte de 1+4. cité dam la revue Judis, de Soignies, t. xrv, p. Ho). Il sagit sans doute de la picre du bourdoir ou galerie (roy. Godefroy). dont parle un autre compte de 1.5:3: : " ponr nu nomvean bourctoir fait sur le marehé " (cité dam le mome reneil et dams Wallomia. Liege. t. xix, p. 315). 1) après M. A. I)(nnenddre. on y faisait les adjudieations publiques. - Voyez pountant lanticle homrderesse.

bouterèce ('Thomas, p]. 95. 101 : Feller, p. 186): rọez. ci-après. ribunterier. Ninuère. dams la enlture du luoublou a Jupille. d'apres 
M. Jean Lejenne, on appelait bout'ree. s. f., un jeune plant de houblon destiné à bouter. à domner des jet. On provignait les boutréees à la St-Jean : deux ans après, elles devenaient des wân'réces. c.-̀̀-d. des tiges propres at "garnir " les échalas (passês) ; l'amée suivante. la wân'ièce devenait une forcân'rèce. co-̀-d. une pousse maîtrese qu'on guidait sur une perche (alon). Enfin. la troisième année de sa production, la plante. qui avait atteint son développement complet, s'appelait pact'ièce ou copitice : elle portait toute sa production de cones (plokites) et était arrivée au sommet $\left(c^{\prime}\right.$ pète) de la perche. - Voy. triperèce.

brasserez, qui sert à brasser la bic̀re. II. Thlhomas. p. 95, cite un exemple de 1456 et, p. 361. m autre de 1250. Je relève candiere biassereche en 1202 à Bapaune (dans 'Tailliar. Recueil d'actes... 1. 20) ; chambre braceresse cn 131:3 (God.. bracenc) ; moulin brasseret en $1+48$ à Corbeil (Delmotte, Gloss. wallon, ro brai).

briserece, ane. fr., brisement (God.). - Comp. bruierece. crierece, croisserece, retenterece, cités par Thomas, pl. 107, 10s, 110.

brôyerèce, w. liég... s. f., pierre à broyer, spatule (Forir). - Du w. broyt. broyer.

carcheresse, anc. fr.. adj. f., t. de drap. : "lirre d'estam ou de traisme - "(Chamy. 1410 : God.). - Dérivé de earchier. forme variće de chargier "charger".

carimadjôrèce (w. ard. : Erezée), roy. p. 46.

carreche, cariesche, anc. fr. (God.). adj. f.. "servant au cheval attelé à une charrette" : "selle cariesche" (1375, Aimont) ; "selle cariesche" (Abbeville).

Chastrès (wall. Thèstiè), conmune du canton de Walcourt (Namur). Dérive sans doute de Castritium, forme latine de $86 s$; cependant le"s formes du xine siècle Kestereces. Chasterece. Chestereche, que Roland cite dans sa Topomymie namuroise. t. I, p. 5+6, postulent, semble-t-il. un type *eastraricium. - L'Allemagne posiède deux Kestrich, dont l'un s'est éerit Cheisteriche (Förstemann. II. 392 ; cité par Roland, l. c.).

cheneverez, dans un texte de $\mathbf{1 3 8 0}$ cité par Godefroy. REToct? ${ }^{2}$; faut-il lire "chanvres"? ou "chanverez". c.-ì-d. "objets faits de chanve "? - Comparer chanerret? (God.).

chevret, s. m., fromage de lait de chèrre (Beauquier, Voc. du dép. du Doubs). Voy. cheverez. Thomas. p. 75.

chicaneresses (affaires -). M. Lhospital, dans God.. Compl. ; "qui tiennent à la chicane $n$. 
cimeratte (memien : Labourasse, Varlet). s. f.. petite quantité de liquide qui a sumté. goutte ; par ext.. petit verre de liqueur on d'eande-vie". De cimer (ib.) "sunter".

cinerèce (wall. Erezće). s. f.. faux provenant de Ciney (BSW, t. 55. p. 4.2$)$.

civeret (anc. norm. : éerit siveret dams me eharte de 1808 : Revue des parlers pop.. 190.2. p. 11\%). s. m.. charge d'me civière.

cloeraite : "roie — " cité par God., TEırERE, dans m texte tournaisien de 1293. Parait bien venir de clore (comme cloiere " enclos. cloture "). Ce serait donc le chemin de clôture; comp. chemin finerot dans Thomas, p. ri. Pour -ai- en tommaicien. comp. zoniturais (God.. Feller) et, (ci-aprèn. sucteruite.

cop't'rèce, roỵez ei-dessus bont ric'é.

croiseresse, anc. fr.. " (roisade " (God.).

cwårèce (wallon : .upille). s. f.. hache servant à éfuarrir et à faconner an pointe le pied des perehes à houblon. A Ingleur-lez-Liège.

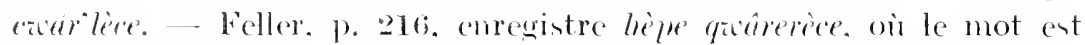
adjectif. A Jupille, il est substantif et les deux $r$ se sont simplifiés.

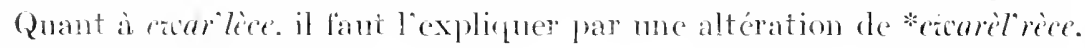
lérivé de "ackler.

eschoicheresse (?). Codefroy (ite mu texte d'archires du c'alrados oì il ent question de brie eschoicheresse et de bric affeterresse. On cherche vaincment un article affeterresse ct. vo brie. l'exemple n'est pas repris. Quid ?

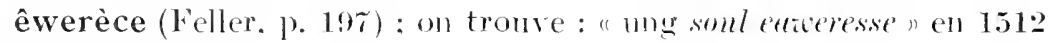
(13SII 5. 11, :30).

faherèce (wallon : Feller. p. 19\%). L Le dictionnaire manuscrit de Villers (Malmedy. 1\%9:3) a mu article "foxheresse. maillotense "(sic). D)antre part. Bormans et Body (Gloss. romem-acollon. partie inédite) ont noté dam les arehives liégeoines: "une banse facheresse".

feurmwagerasse (.J. IIngre, Iroc. de lo Bresse), s. I.. "la lorme où lon égontte le caillé du lait en le retirant de la chandicene.

finih'rèce (wallon. t. de drap. ì Verviers). s. f.. "finiscuse ". machine i) parfaire la tonte du drap. des étoffes. cest-ib-dire à domner les dernicher compen it une picee.

forèce (Goul.). rovez ci-dessur l'ari. fueresse. p. 96.

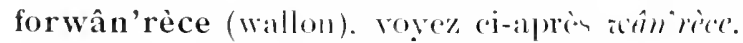

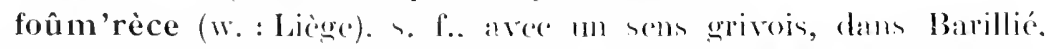

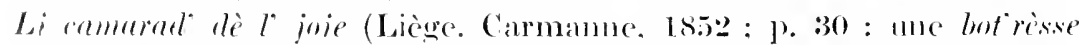
"hotteuse" se vante d"aroir unc formiresse trè profonde). Le sem 
propre : "moule. matrice, destinée à domer une forme " a dû exister dans un métier liégeois ; mais on ne ke trouve noté nulle part. Comparez formeres. 'I'homas, p. 10:3.

fourtch'rế, s. m. -- Feller. p. 199. signale deux sems de re mot gaumain et chestrolais. En voici deux antres que jai recucillis a Mlle-surSemoiv: 1. frelon (ains nommé parce quil porte sur la tìte deux appendices en forme de fourche, ce qui la fait appeler fommèt à Oisy, fonèt en gammais. diminutif de fóme, foine on foume) : - 2 . absès interdigital (comp. Ie w. fotch roule. f.. môme sem. à Verviers. Thimister). On recomait l'altération du même primitif fourcherez dans le fr. moderne fonveleret "autour de moyeme yromenr ", et dans l'anc. fr. fourcherot "qui forme une fourche, un earretour ". employé comme nom de lien en Bomrgogne. - Voy. heivice.

foutrot (meusien : Lahourase), s. m.. " jeu de cartes oì le perdant reçoit. des autres joueurs ot sur le bout des doigts assemblés. un certain nombre de coups avec le paquet de cartes. De même en Berry" Près de Longwyon. ce mot signifie : 1. mouchoir fortement tordu pouvant srvir à frapper ; ’- jeu de valet de pique. ou le perdant reegoit sur la main un ou plusieur coups de foutrot.

fouyerèce (wallon). Grignard. Plométique de louest-zallon. \$ 34 , (BSW. t. 50. p. 415) écrit sempe fouy'resse "beche "et range eet adjectif à côté de vatch'rèsse. cins'résise (vachère, eensière) parmi les dérivés en -issam. - C"est évidemment un dérivć en-ariciam de fount. foniller. répondant à $u$ type foeres.

fringueret (ane. fr. : God.). adj.. "élégant. à la mode ", dans " cordons fringueres ". - Propre à fringuer (folâtrer) ?

frinteresse (anc. fr. : God.), adj.f., retentissante. bruyante, épithète de la mer, dams la Geste de Liege. - Dérivé de frinter "retentir. faire fracas".

fruiterez (ane. fr. : 'Thomas. p. 75 ). - Ajouter : "unc rasiere fruiteraices de puns " (1320 : Charten toumaisiennes, dans Zeitschrift für fr. Spr. und Litt.. xxir, 1(13).

fueresse (anc. w.). rovez p. 96.

hadrê (w. ; Feller, p. :201). voyez p. 1:22.

halboterèce (wallon: Liège. Ans. Glain). s.f..t. d'arm., rabotspécial pour halboter on faire, dans certains fusils, la halbote (canal creusé dans la languette pour y loger le canon). On dit aussi halbotêt. s. nı. (Communication de M. Laurent Colinet).

hantresse (anc. wallon). s. f.. action de hanter. converser : "avons 
eu eonveruation et hantresse ensemble " (1590, Cris du Perron ; d'après Bormans et Body, Gloss. rom., ns.).

hauteresse (ane. fr.. dams Froissart ; voy. God.. et Scheler. Gloss. de Froissart. s. f., arrogance. fierté. — Dérjé de hauteur ? Môme formation que forteresse. secheresse, longueresse, courterèce? Vor. Feller. p. 19\%. et ci-après tchî̀ce.

hètch'rèce (wallon liég.), adj. f., dans tone hètch'rèce. t. de houill.. petit tomeau monté sur me espèce de traineau pour puiser et transporter l'eau dans la mine. syn. tène di hièrtchể (Bormans, Voc. des howill. liég., vo teme). - Doit ctre ajouté à l'art. hièrtcherèce. s. f.. de Feller. p. 20:3.

heûrèce (wallon), "dans fotche hềièce (pour *henteriee). fourche à seconer le foin " (Feller. p. 20:3). - Voiei quelques détails complémentaires : fotche hyetuice (à Burdinne). grande fourche de bois à longues dents pour secouer (hyen̂re) les gerhes battues sur l'aire; fotche scurèce (i Indenne). fourehe pour faire des meulons (et aussi. sans doute. pour les défaire. pour les épandre en secouant l'herbe); fotche tehevirèce (Meux). fotche eurce (Ste Marie-Geest, Arsimont). fortehe entèce (GeestGerompont). fourtehe irce (Stave). fourtche a slieñe (Bourlers lez Chimay), fourche de bois dont on se sert pour seconer les foins. ou encore pour remuer. pendant le battage. les gerbes déliées gisant sur l'aire. - Quant à l'article de Pirsoul "foite cheuzresse" (Feller. 1. 1s9). il faut le rayer sans hésitation : Pirsoul aura mal lu un "fortcheurresse " que lui aura transmis un eorrespondant.

hourderèce. A l'art. de Feller. p. 204. ajontez : 1. pierre hourdresse ou bundresse(?). dont parle Gachet. Gloss. du Chevalier an Cysne (Brux., 1859) ; roy. ci-dessus bouderesse ; - 2. ourderet. s. m., t. de bat. sur la Dendre et l'Eccaut, montant du bas du mât. en flamand sietag (communication de M. Emile Ouverleaux).

hov'terèce (wallon : Erezée). adj. f.. dans lisse hov't'rèce. t. de charp.. lisse ou entretoise allant de la corante lisse au cherron. La corante lisse est parallèle au premier cherron : la lisse di bihere va des montant à la corctute lisse.

jecterece (anc. w.), adj. f. ; "me heppe jectresse ". var. "jectante" (157:2 : Cris du Perron) ; "espées, heppes. jecteresses et aultres bastons offensires" (15\%0 : ibid.) ; textes cités par Bormans et Body. Gloss. roman-icallon, partie inćdite. Il faut, dams le second texte, lire heppes jecteresses, (--a-d. des haches qui servent d'armes de jet. Voy. Thomas. p. 10.t, vo jeterez. 
jostereche (ane. fr.), adj. m.. dans eskill justereche, bouclier qui sert aux joûtes. aux tournois (J. d'Outremeuse. Myreur. I. p. to). Le mot n'est pas dans Godefroy.

larèce (gaumais) s. f., "côté d'une maison qui se trouve cutre deux pignoms: de latus + -arician (Feller. p. 206). Il fant lire lârèce. Il est probable que ce mot représente plutôt un type later-îeia.

lôyerèce (wallon: (Blons-sur-Geer), adj. fém., dans vèdje lôyerèce, perehe horizontale placée dams la haie pour y attacher (loŷ̉. lier) les plants et les piquet (५ym. prime à Jupille. Fexhe-Slins ; cf. G.. II $25 \%$ ).

macherèce (wall. namuroin: cuze macherére. G.. vo faubite). Ce not ne signifie pas, comme le pense M. Thomas, p. 9\% : (eure) qui sert ì teindre. M. 'Th. a été trompé par le graphies équirorues de G. Le verriétois "mache", ourrier teinturier. que donne (r.. II 540, se prononee et doit s'écrire matche. Il n'a ricn de eommun avee macherèce. qui dérive du nam. machî. liég. mahî, mêler. mélanger (ih.. II 5s). C'est done la cuve où le teinturier prépare le mélange.

nètirèce (wallon de V'erviers). s. f.. "planche avec carde spéciale

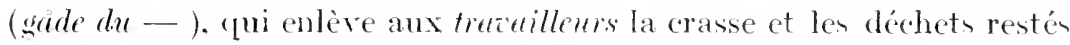
encherêtrés dans les dents "(BSW 39, 27t). Voy. rimet rère.

parfôrèce (wallon : Lièuge. Ans, (ilain), s. f..t. d’arm.. perforewe. très longue mèche à bois pour transpercer la crosse du fusil dit forr ante (Communication de M. Laurent Colinet). - Mis pour * parforerces.

parresce (anc. wallon). adj. f., dans ce texte: " ung droit coutcal. une lunet parresee. vi pamalle pour crespir cuere" (Echerins. 11. 1220 ; d'après Bormans et Body, Gloss. rmmem-ic.. nus.. vo pamalle.) Mis pour *parerice. La lunette ent " montil de corroyeur employé pour parer les cuirs "(Larousse illustré). On pent roir une deseription détaillée de eet outil dans Borman. Le metie'l des tamenes (BSW 5. 36\%). Comp. reparerece dans Thomas. p. 109 : riparerece dans Feller. p. 217.

pastouret (norm.). s. m.. (nlbute (Moisy). - - Altéré sam doute d'un ancien *pastoureres " (jeu) propre aux (petits) pâtres

pèI'ré (w. : Alle-sur-Semois), péleriau (rouchi : Hécart), s. m., chêne éeorcé sur pied. bois pelard. - Peut-ître altéré de *pelérez.

pescherez (anc. fr.). "qui sert à pêcher " : voy. Thomas. pp. 98, 105. 109. Ajoutez-y le pieard pélieret. s. m., 1. bateau de pêche ; 2. petit ver dont on se sert pour pêcher à la ligne (Corblet). — En wallon. l'adj. fém. pèhrèce se lit dans une satire liégeoise de 17.50 contre les femmes: ine feume, c'èst l'vèdje pélirée dès-ènocints, la verge ou la ligne i pêcher les simples d'esprit (BSW :3, II, 3). 
planerèce (w. he-h. : Crehen). plânerèce (rouchi : Ellezelles). s. f.. brique employée en parement. Simple altération de panerèce (Thomas. 91. Feller. 212; fr. panncresc). soms l'influence de plat. plan.

les Planerèsses, lieu dit, haut plateau i Bernister, au-dessus de Malmedy.

plâterèce (ronchi : Ellezelles : platressie dans Hécart), s. f., espèce de truvelle dont le plafonneur se sert pour étendre le plâtre. De là est emprunté le nam. platrice que dome Pirsoul; voyez Feller, ⒕

plazeré (gammais : Buzenol. Ste-Marie-sur-Semois. "1. petite plaee, petit terrain plat ; 2 . prouse devant la maison : 3. clairière ». M. Feller, p. 221. y voit un dérivé de platea " place ": mais l'artiele suivant de Maus. Foc. gamm. des eniroms de rirton (ms.. 1850) infime cette conjecture : "plasic. petite partic de pré clôturée on isolée; placeriè dans certanes loealites: plasie dans nu compte de 1+16, plaisié en $140 \%$ ". Ce mot est asmmément apparenté à lanc. fr. plessis " elôture, enclos", et vient diun type *plaxellum, *plaxarellum ,(altéré de * plaxaricium sous lönfluence de *plaxellum ?). Le sens propre ent donc "endroit entouré de claies". Comparez le w. plis"nîre (à

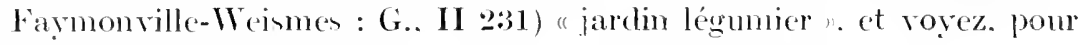
toute cette famille, Meyer-Lübke, à l'art. *plaxmm.

plokerèce, s. f. - I. Thomas, p. 109. a l'article suivant : "plaquerece. outil pour plaquer : plaqueresse. carde pour plaquer la laine (Duha-

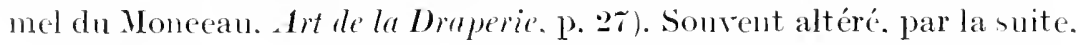
en ploqueresse." - C'est le contraire qui est juste : pla- résulte d’une fausse perception ou d'une erreur d'impression pour plo- $\left.{ }^{1}\right)$. Sur ploquer, w. plokit. dlu bas all. plocken "carpere". rọ. Behrens. Beiträge. p. 306 : Meyer-Lübke. $1^{\circ} 6004$.

potch'trèce (w. : Brensonx-lez-Liège). dans fotche potclirèce. fourche pour faire sauter (w. poteliter "sautiller n) et extrairc les perehes à houblon lichées en terre. Ce levier fourchu s'appelle rayen i Jupille. rayerice à chênée. dérivés de raŷi "arraeher, extirper".

poûh'rê (w. : Ilerve). s. m.. petit puinard clans une cave. Peut-ĉtre un lanx dinninutif en -erế, altéré de *puiseres "(lien)où l'on puise. qui scrt à puiscr ". - poûh'rèce (w. : Jupille). s. f.. racine plongeante dn houblon. qui s 'mfonce junqüà trois mètres de profondeur.

prisseresse, adj. (ancien tommaisien : xive s.). On lit dans les Clatertes toumaisiennes publices par Ch. Doutrepont (Keitselm. f. fro.

(1) De mêne curde est sonvent défiguré en corle. M. 'Thomas, p. 108, en signale des exemples; ajoutez-y Godefroy, skrax, et le Nonceau Larousse illustré, plaqueRESSE. 


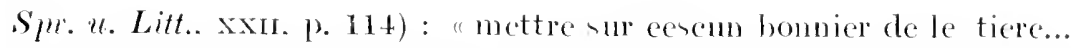
xwr kareez de fiens prisseressez "(13.5.5) : "doit chascun bomier de la dicte tiere fumer bion et denement de xw karees de tions priseraiches" (1398; p. 11\%) ; 1. karees de fiem priseraiches " (139s: p. 119). - (2uid"?

privaret, priveret, puveré, plouvré (picard). s. m.. hirondelle de mer. - Dérivé de pricét. apprivoiser?

pwèt'rèce (w. : Jupiltc). s.l... dérivé de pàèttre porter. Voy. bunterice. rabaterèces (w. : (Insset. Amunerie liés.). s. f.. pinees en acier qu'on place dams l'ćtau pour tenir les pièces. C'f. Thomas, reboteres. p. 105 ; Behrens. l. c.

raboterèce (w. liég..). - f.. machine à raboter les planchen.

rassîrèsse (w. de Verviers). s. l'.. machine qui conche le poil d'une étoffe. qui lustre un drap (BSIl t0. 1) 4.5.5). Pour *assirerèce. dérivé de ressir. rasseoir. Companc\% sirees.

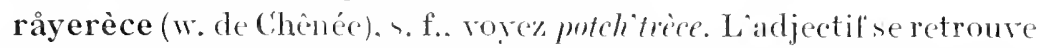
dans have rayerce (Ben- Mhin. Gives: t. archaïque. remplaec aujourd'hui par casse). hone is deux dents servant i l'arrachage des pommes de terre. Dérivé de rayî. arracher.

remouyerèce (w. du Brabant). `. f.. arrouoir.

ribouterèce (w. liég. : Forir. II $52+$ ). s.t.. racloir de jardinicr pour entever l'herbe dam les sentiers. Forir dome ansi bontevece "riangle de jardinier ". Voyez Feller. p. 1si.

ricouderèce (w. de Stave). s. f.. fer recourbé muni d'un manche. servant à ricomde (reconeillir) lon ćpin ct à les mettre en javelles derriere le faucheur.

ricoûrerèce (w. de'Tohogne). S. f.. demi-rarlope: royez conteréce. Feller. 1) 196.

rilèverèce (.I. Wa let, racab. a. de Giāet). s.f.. cognée pour débiter Je bois abattu dans les coupes: elle a mu manche phus eourt que l'abatcréce. C.1. Brumean. Euquête. I. 164.

rinèterèce (w. d'Ampsin-lez-1Iny) , s. f.. platine qui. dans la fabriquc de creusets, sert à nettoyer les outils. Voy. neitirice.

salerèce (wall.). adj. f. ; pire salevèce. 1. (Liège) pierre servant ì broyer le sel ; -- 2. (Tohogne) pierre servant à presser le sel dans le lard on le jambon que l'on reut saler; à Roy. on dit pire di sè.

saurterèce (w. de Gembloux. Petit-Leez), s. f., one [atche] sôrtrèce. une hache propre à ensarter.

sbaterèce (w. : Strée-lez-1Luy). s. f.. t. de carr., ciseau ou pointe servant à sbate (aplanir) la surface d'un bloc de pierre.

serclerette, - rot (Beanquier. For. du dép. du Doubs). sarcloir. 
sirèce (Gorey-(urigny-lez-Longwy : Meurthe-et-Moselle). s. f., erpèce de bae en bois, ouvert d'un côté. que la laveuse prend avec elle et dau lequel elle sagenouille pour laver son linge au bord de la rivière ou de la fontane (Communication de M. P.-D. Navez, de Muson). Dérivé de sir : seoir. asseoir.

so-prèsse. If. Feller. p. ำ 18 . insère dans sa liste un mot quiil écrit superèce et qui désigne une partie du chariot. le lisoir. Il n'en donne pas l'explication et se contente de ponctuer d'un "! " la graphie soms-prèsse de Dasnoy. p. 85. Je erois pourtant que ce dernier a raison et que notre not na rien de commun arec le suffixe - arieius. Si je comprends bien larticle de Dasnoy, il en ressort que le fr. "lisoir " traduit le chestrolais sous-presse et que le lisoir de l'avant-train est surmonté de la pièce appelée en fr. "sellette". Or Body (Foc. des charrons. vo chirai) nous apprend qu'en ardennais la "sellette "se dénomme prèsse. Il ent done naturel que le lisoir de l'avant ait pris le nom de soms-presse. Par analogie, ce nom se sera étendu au lisoir de l'arrière; c'est ainsi qu il est question de leux so-présse. par exemple à Erezée et à Orthouville: li so-presse di drant et li so-presse di drî. à chaque paire te roues. pour supporter les ridelles. J'ai entendu à Grand-Halleux et à BasseBodeux : lu presse' do tchêr. ceist çou qu' les rombes (ranches) su mitit disines.

sorderesse (derle--). voyez p. 2027.

spindj'rèce (w. de Bovigny). s. f., battoir pour spindjî (espader le lin ou le chanve).

spoûl'rèce (w. de Verviers : 13SW 3S, 20s), s. f., t. de tiss.. espice de rouct servant à eharger l’époule.

stèssin'rèce : pour l'ćtýmologie, voyez p. 92.

stopresse (ane. liég.). adj. m.. qui sert à stoper (étouper, calfater; boncher en général) : "rué ung coultea stopresse apres un homme " (15.5.5. Cri du Perron ; cité par Bormans et Body, Gloss. roman. m.).

suweraite, adj.f., qui sert à essuyer: "III petites toulettes suwcraites", 139s. dans un testament de 'Tournai (God.). - Dérivé de *suzer, liég. someer, sécher (*exsueare: essuyer). Il s'agit d'essuie-main. La finale -aite est altérée de aice : le dialecte tournaisien a les formes -efaic:-erais (pour -erez), fém. eraice (pour -evèce). Voyez ci-dessus clorenite. et 'Thomas, ple. 76.77 .94$.

taperet (Vocab. langrois. 18\%2). s. m1.. pistolet àsureau. - tap'rèce (w. d'Angleur). s. f.. nom donné à la copitrèce en avril, c.-à-d. à la plante de houblon arrivée à son maximum de prodnetion. Dérivé de treper. jeter. 
tchèdjerèce (liég.). s. f., grande pelle dont les mineurs se servent pour charger la houille dans les wagomncts (BSW 5., p. 264). - A ajouter à l'article de Feller, p. 219.

tchoquerèce (gaumais : (1. Maus). s. f., combat, rixe, bagarre. où l'on se domne des comps.

tèyerèce (w. : Blegny.'Trembleur). s. f.. t. d'arm.. lime à fendre, espèce de seie qui sert, par exemple. à faire les rainuren dans len têten de vis. - Cf. Thomas. p. 100.

tonderèce (w. de Malmedy : Villers). s. f. petits ciscaux de drapier. forcettes. A Roy-en-Fameme. jai noté lo même mot comme t. archaïque, pour désigner les forces à tondre les moutons. - C. F. Fller. p. 220.

tuteri, tutri (mesuien : Cordier. Labourasse. Varlet). s. m.. goulot de la bouteille on du hroc. en forme de biberon. 1)u r. tuter. qui signifie "téter, sucer" dans la Marne (Varlet). Sym. tutot (id.). Pour le suff. -eri (=-ereau : -erez). comp. pétri (Labourasse) "étincelle lancée par un feu qui pétille ". musseri (Varlet) "troglodyte" (= menssernt: Labourase; Thoma, p. 104). Pour le radical, royez G.. thiter, tuturon.

villerec, -rech, -ré (anc. fr.). adjeetif masc.. qualifiant une sorte de filet. Godefroy eite deux exemples tirés des chartes du Mainaut : un sacqueau villerech (1534). sacqueau villeré (1619); le premier texte se retrouve plus complet à l'art. resque de Godefroy. Le contexte indique qu'il s’agit d'un : ac ou filet prohibé. Quid ?

wân'rèce (w.: Jụille).wâr'nèce (Brenouxx). wågn'rèce (Angleur). s.f., plant de houblon quand il a au moins deux ans et quil ent à même de grimper sur un prsstè on échalas. I'année suivante. fe plant s'appelle forwentice it Jupille : cest alor une pousse maîtresse quion guide sur une perche, alon. On pourrait croire à premiere vue que le radical est aiaghî "gagner ". J'y rois plutôt des dérivés de *aami "garnir". Toy. bouterice.

\section{III}

\section{Le Dictionnaire étymologique de Ch. Grandgagnage ( $\left.{ }^{1}\right)$}

...Il est un homme dont la figure mérite d'être éroquée en ce jour et dans cet auditoire universitaire, $u$ homme que ses contemporains - du moins dans son pays - n'ont pas apprécié à sa valeur, que la plupart d'entre rous ignorent peut-être et à qui doit aller notre recon-

(') Extrait de la leçon inaugurale du Cours de Dialeetologie wallonne, donnée à l'Université de Liège, le 18 novembre 1920. 
naissance, car il est l'aneêtre, le préeurseur, le véritable fondateur de la philologie wallonne. Je veux parler de Charles Grandgagnage, né à Liège en 1812 et y décédé en 1878 .

Son labeur fécond s'est exercé dans le triple domaine de la lexicologie, de létymologie et de la toponymic wallonnes. "Ce fut, - nous dit son biographe, - un savant, an sens sérieux et solide du mot, et savant parmi les plus distingués de son pays et de son temps... A l'étranger, il était comme lincarnation, la personnification des études wallonnes. ('était par excellence le philologue wallon, le linguiste liégeois, auquel l'Europe aimait à rendre hommage... Son érudition solide, sa eritique ingénieuse le fïrent remarquer par les savants les plus éminents de France et d'Outre-Rhin... Les plus brillants représentants de la philologie en Allemagne et en France, Diez, Pott, Diefenbach, Förstemann, Littré, le tenaient en singulière estime et le citent comme une autorité en linguistique..." ( $\left.{ }^{1}\right)$.

Je n'ai pas la prétention de refaire, après un maître aussi autorisé que M. Doutrepont, la biographie de Grandgagnage. Je voudrais seulement souligner l'importance de son effort et, - laissant même de côté ses deux ouvrages sur les anciens noms de lieux de la Belgique orientale, où il fait la critique mimutieuse des sources anciennes de notre toponymie et jette les bases de cette science nouvelle, - je roudrais rappeler l'xuvre magistrale qui mériterait, à elle seule, de perpétuer son nom.

("est en octobre 1845 - il y a juste soixante-quinze ans - qu'il publia la prenière livraison du Dictionnaire étymologique de la Langue wallonne $\left({ }^{2}\right)$.

Tout était à créer ", nous dit-il lui-même dans son Arertissement ( ${ }^{3}$ ). Ft en effet, à un double point de vue, son Dictionnaire apparaît comme une sorte de "cráation ". C'est d'abord un vaste répertoire où l'auteur se propose de "recueillir tous les mots des différents dialectes et des dilférents âges "; il a dû, pour cela, "s'assurer des formes, de la rraie signification (ce qui est bien plus malaisé qu'on ne croirait), établir une orthograplic conséquente sans qu'elle blessât ni l’étymologie ni l'ouil "; il a dû dépouiller d'innombrables textes manuscrits et imprimés, organiser $n$ service de correspondants qui le renseignaient oralement

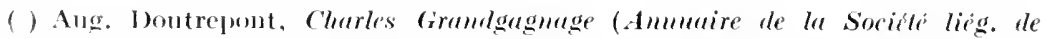
Lill. a'tll., t. 16, Pp. 19-4!); Liège, 1903).

(2) I impression fut interrompue en 1847 , reprise en 1851 et de $110 u$ cau suspendue (e) 185\%. Ia lin ne devait parâtre qu'en 1880, deux an après la mort de lauteur.

$\left({ }^{3}\right)$ I anu e qui suit, les passages cités entre guillenets sont tirés de la mêue source. 
ou par écrit, se livrer enfin à la ehasse aux roeables, chasse aussi passiomnante que hérissée de diffieultés et de déeeptions. Mrant lui, les amateurs de lexieographie s'étaient bornés à noter le parler d'une localité ou d'une région, se proposant presque uniquement de signaler des provincialismes. Il fut le premier à "embrasser le cercle tout entier ".

C'est qu'aussi bien la récolte des mots n'était à ses yeux qu'un moyen : elle lui fournissait les matériaux qu'il fallait dégrossir et disposer exaetement pour l'cuure qu'il rêvait. "Comparer les nots d'abord entre eux, puis avec ceux des autres langues et idiomes romans, enfin reehereher l'étymologie dans plusieurs langues différentes, éparses dans une quantité de livres ", tel fut son principal objectif et, là surtout, dans l'explication historique des mots, il se montre eréateur, esprit curieux et subtil.

Cette ourre commeneée aree enthousiasme, it la poursuivit pendant quinze ans, "sans reeeroir ni louange ni blâme, même de eeux qui, le touehant de plus près, pouraient le mieux le juger ", sans éprourer d'autre plaisir que " eelui que proeure l'aeeomplissement d'un devoir ». Cette indifférenee de son milieu, cette froideur dont il se plaint amèrement, et sans doute aussi d'autres raisons restées obseures, finirent par déeourager sa nature sensible et délieate. En fin de compte, pendant les vingt dernières années de sa vie, il laissa dans l'abandon son précieux Dictionnaire. Près de mourir, il ne vit dans son entourage personne qui fût de taille à parfaire son travail et à publier ses notes manuserites : il dut recourir à la scienee d'un étranger, un Suisse établi à Bruxelles, Auguste Seheler, qui s'acquitta de sa mission avec un zèłe, une probité seientifique, une eompétenee qu'on ne saurait proelamer trop haut. Grâee à Scheler, le dernier tiers de l'ourrage parut en $\mathbf{1 8 8 0}$.

C"est au-delà de nos frontières que Grandgagnage vit son glossaire aceueilli avee le plus d'empressement : c'est à l'étranger que se fonda sa réputation. Au reste, dans sa conception première, le Dictiommire étymologique était "principalement destiné aux étrangers et caleulé pour leur usage ". Persuadé à juste titre que nos idiomes populaires eontenaient me foule de formes aneiemmes bien dignes de l'attention des romanistes, remarquant, dès $18+1$, en ćtudiant les C'eltica de Diefenbaeh, "wn certain nombre de cas oi [pour l'explication des origines] le wallon pourait être cousulté avec plus de profit quaucume autre langue romane ", jugeant que la langue de son pays "formait réellement un chaînon essenticl dans l'histoire générałe des langues romanes", il sunstitua, suivant ses propres termes, "lintrodueteur national du wallon auprès des étrangers". Son plus vif désir fut 
"d'apporter une modique contribution à l'édifice philologique qui s'élevait en Allemagne ». Et, nodestement, lui dont les études n'avaient pas été dirigées vers la philologie moderne et qui dut, par ses propres forces, s'outiller de toutes pièces, il déclare n'aroir en, en se livrant aux recherches étymologiques, "d'autre ambition que celle de déblayer le terrain et de préparer le travail à des savants placés dans des conditions plus favorables que lui pour cultiver ce champ d'études".

Il fit plus cependant : il traça le plan, jeta des fondations solides, éleva la charpente de l'édifice. Si presque aucune partie ne reçut de ses mains l'achèvement désirable, s'il y laissa de nombreuses lacunes, son auvre, que tous les critiques ont jugée remarquable pour le temps où elle parut, est encore aujourd'hui le vocabulaire wallon le plus riche et le plus précieux qui ait vu le jour.

Nous reconnaitrons sans peine que pas un article peut-être de son glossaire n'est définitif et que son ceuvre nc répond plus suffisamment aux exigences aetuelles de la science étymologique. Sa méthode, on l'a dit, est loin d'éviter tout reproche : trop souvent il hésite à conclure, il accumule les rapproehements superflus, va chercher ses étymologies dans de lointains dialectes germaniques au lieu d'interroger les dialectes voisins. Toutes ces critiques sont fondées, et bièn d'autres encore que le maniement quotidien de son Dictionnaire peut suggérer. Mais qu'importe? Pour en juger équitablement, il ne faut pas perdre de rue qu'il n’eut pas de prédécesseur, qu'il dut à lui seul frayer la voie qui, à présent, s'élargit et s’aplanit de jour en jour : il faut se rappeler son mot : "Tout était à créer ", et méditer ce jugement d'un maître : "Ce travail scientifique dépasse de beaucoup tout ce qui a été entrepris antérieurement sur la lexicologic et l'étýmologie des parlers de la France" ( $\left.{ }^{1}\right)$. Il faudrait enfin pouvoir imaginer où nous en serions aujourd'hui si Grandgagnage n'avait pas, comme il dit lui-même, " débrouillé le chaos ", et commencé à mettre de l'ordre dans un domaine oì règnait le désorche le phus complet.

("'est de lui, comme nous l'avons dit, que date la philologic wallonne; mais, on doit bien l'avoner, le grand effort de Grandgagnage a relativement échoué. Faut-il attribuer cet échec à la froideur de l'aceneil que lni fit le public wallon ? Sans donte, mais il y cut d'autres eauses, dont voici, à nos yeux, la principale. Grandgagnage s'était trompé sur l'étenduc et les difficultés du sujet qu'il cmbrassait. En 18+1, il conçoit la

(1) 1). Behrens, Bibliographie des patois gallo-romans, ge éd., traduite par E. Rabict (Berlin, 189:3), p. 217. 
première idće de son wurre : il en conmenee la publication quatre ans plus tard. Eu réalité, éest dix ou quinze ans de préparation silencieuse qu'il lui aurait fallu. Dis 1850, il dut le recomnaitre lui-même : Le fonds ćtant pour ainsi dire inépuisable, les matérianx continuaicnt à arriver peudant que le travail d'élaboration s'opérait, de sorte que l'édifice eroulait souvent avant d'être acheré".

Deux ans après, en 1852, il suspend définitivement limpression de son livre et, en 18.55 , il arrête la préparation de ses notes. Lapparition, en 1853, du chef-d'ourre de Diez, le Dictionnaire ítymologique des langues romanes, hit fit aussi probablement eomprendre tout ce qui lui avait manqué jusque-là. Quoi qu'il en soit, inclinons-nous devant la longue angoisse qui étreignit son co’ur. Il dut éprouver la douleur poignante de l'artiste qui, devant l'ćbauche imparfaite, se sent accablé, impuissant à concrétiser son idéal. Hélas! ! il n’avait plus l'ardeur ni la santé nécessaires pour recommencer un labeur épuisant... Il ne lui restait que la eonsolation de penser qu'un jour pent-être sés compatriotes lui rendraient justice et de se dire, conme le héros malheureux : In magnis zoluisse sut est! Et rraiment, avoir eu la volonté de réaliser une grande chose, n’est-ee pas le signe d'un noble caur et d'un esprit d'élite?...

Celui qui, an 1845, inaugura brillamment les reeherches scicntiliques sur nos dialectes, méritait bien l'hommage que nous hui rendons aujourd'hui. Son Dictiomatire a valu à notre petit pays une place d'honneur dans la philologie française : ce titre suffit amplement pour que la Wallonie n'oublie jamais le nom de Charles Grandgagnage... 


\section{ADDITIONS ET CORRECTIONS}

Page 3 , note 4. Pour el réruit à $c$, voyez aussi p. 202, n. 2.

P. 4. a l'article alon, ajouter: Pour la semantique, comp. le fr. espalier (mur d'ap)pui). derivé le la forme italienne répunale.

l'. 41 , a l'anticle butiu, ajouter bètin 'fui, à Faymonville, signifie "neige boneune an légel (Bstr 50, p. $5+8)$.

P. 12. Le gratte-cul sappelle piculô à Petit-Leez (Gembloux) ; cest sans doute

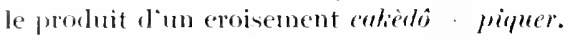

P'. 48. Pom la dérivation de chuom, comp. l'ane. fr. breon (liég. brèyon) "nuscle, morcau de viantle, mollet "qui vient lu franeipue brido.

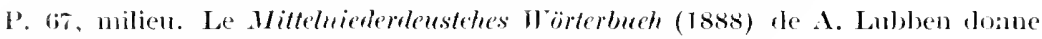

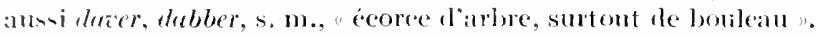

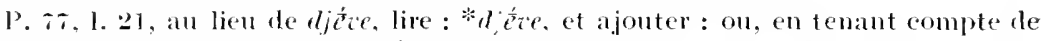

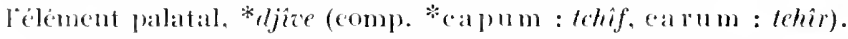

P. 1 13. Herbate est aussi le nom dºn hamean de Wavere.

I'. 150, 1. 2. A Pellaines (I leshaye), on appelle oulote la grande chouette fui se tient dan- les clochers, etc.. ct ourom la petite chouctte qui niche clans les cremx des allites.

P. 171, din. Pour roton, lôton (de rute), comp., p. 261, drorlule, drôdule (de drude).

P. 172 et 190, suprimer lasterintue devant pauta; p. 18, milieu, lire *acu(.ellim.

P. 179, n. "2, ajonter bigit, p. 22, que nous tirons du germ, ing ige.

1'. 191. 1. 1 : pote ent subntantil clans e' pastage de Bauduin de Conde : " I tes eours luas et a tes potes. (Qui sunt grosie, ron alens machues. Et avene noires et crochues (ad. sicheler, p. 16 is ; note, p. hos).

P. 20:3, 1. 2, ajouter abrouche, que jai uote à Petit-Leez (Gembloux).

P. 285, n. 5. sipprimer le (?) après vapilus, qui a très bien pu clomner le lié-

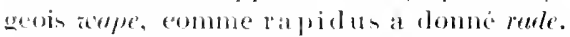

P. :300, 11. :3. A Bourignes-I)inant, les enfants chantent : Al roulumse dès crôs

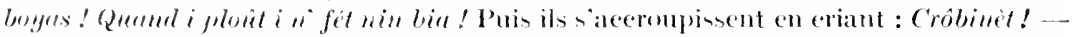

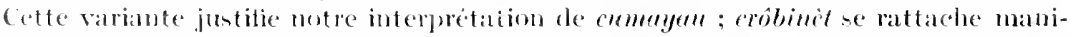

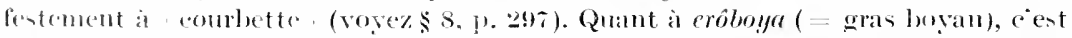
sim dente. par efymologie populare. ane des imombrables deformations du type

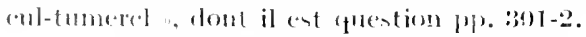

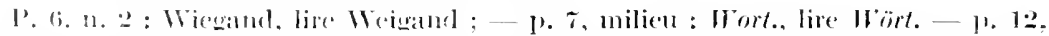

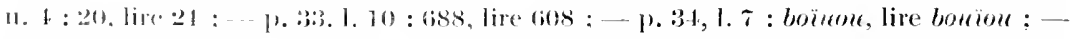

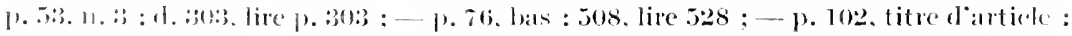

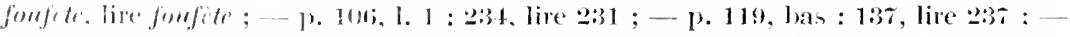

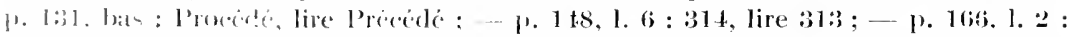

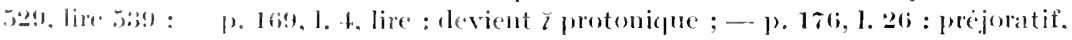

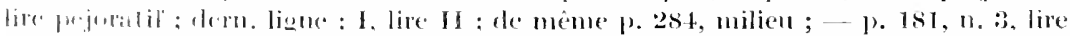

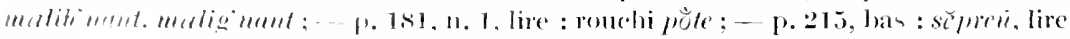

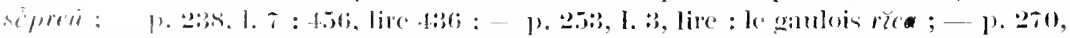

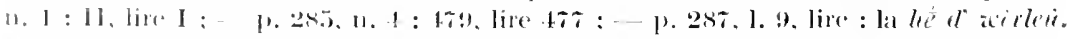

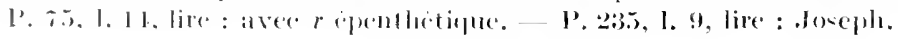




\section{INDEX LEXICOGRAPHIQUE}

\section{Cinique}

bor (?), $26 \mathrm{n}$.

derva, derw, 6 ;

*gaba, *gala, 76, 7\%.

* gabata, *galvita, *galla, it, 113.

*atutum, $11: 3$.

$\dot{x}: \cdots ;, 1$.

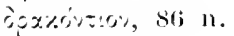

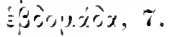

*gavis, \%ร.

*livira, 146 11.

*musganda, 113.

rica, $25: 3$.

\section{Gree}

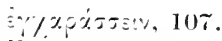

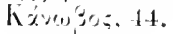

\section{Germanique}

abòken, abóketen, :32. aanbour, anbau, 6.

allne, âne, 12.

alisel, achisel, 4 .

are, areh, aro, aris, arrig, 13, 14.

baat, bate, $14: 3 n$.

baken, bikenen, 21.

bald, 20 .

lalk, 7 1., 20, 31 11., 220.

Jatrdauz, 295.

bark, bork, berck, 24 .

bauch, :3:3.

b:1110, :31.

becken, hekken, 191.

behôf, behoxf, behuf, 29 .

beige, hige, 322.

beost, biest, 42 .

lerg, bёreg, 2:3.

bergfrirl, 2s 1 .

betalen, I 60.

beuk, heukje, :3:.

bi-, 20 .

biesen, $179 \mathrm{n}$.

bilumedan, 26 .

blein, 29 .

Bockholz, :30.

bohren, boren, 21, 26, 27.

bôk, bôken. bôkemaul, :32. bonalje, 2it.

bonk, boneket, 169 n.

bole, 26; 10 .

loro, loolnere, 2-1; 11 .

horstlale, 41 .

bout, loelz, :30.

boxhioren, $12 \mathrm{n}$.

britlo, :32:2.

Iriigcl, -eln, :39.

bramel, -ен, :34.

brijan, 89 $\mathrm{n}$.

briilncen, s!).

buik, :3:3.

birr, batter, 21, 27, 17:3.

cirle, kitle, 48.

carsoulc, corsomle, ist.

coke, $15 !$.

cretto, wette, 60 .

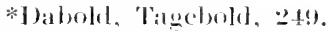

ditver, (it, :3:20.

dich, deikk, deceg, dijk, 르.

rliutisch, deutuch, 2.51).

dohme, 2:3s.

llece, 2:3:3.

drevel, -elen, drijven, treiben, 256\%

lliench, 2:3.

drude, 2015, :32:2.

irutel, -elent, 2061 11. 
duitje. 274.

Iweil, 2a33.

(erfworm, sis 11.

(rute. 150 .

likhen, fingen, vâhen, 2 .

firlie. feicrlieh, virelich, 2:2.

llasehe, $4: 3 \mathrm{n}$.

Hanne. 9s.

forke, fuecke, fuik, 33, 56, 103.

fretclen. 102.

forliosian, 97.

frechoot, (i).

frefei. 103 .

arlgen. galjen, 105.

garloti, 7.4, 1 tol.

giare, wareteg, 73.

and $-\mathrm{ig}, 7 t$.

wart. gard, -e, gerte. 7.t.

gallo, garten. Tt.

gc-. 107.305.

Geert, Gehrt, Gerhamd, 121.

geest. 2332 .

geit, $1333 \mathrm{n}$.

erelid. glied, :306.

gelint. glind, 109).

Eemacht, gemaakt, 43.

acmein, $4: 3$; gemeine, 300.

genate, snate, 306.

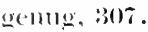

geralen, geraud, $8 \%$.

geraten, gerôden, 3.

grepel, gargel. 80.

gencheit, -eide, 133.

gerenchit. 30.5 .

genclle, gezel, 300 .

gexicht, gezicht, 306.

gentiatll. 16 iti.

gentel. gestell, $10 \%$

gesuch, I26).

extal. :30.5.

[avel, giclod, sol.

exezore. 1206.

whetirle, :\$ori.

glind, 109 .

(rot) 12:3.

erort, -in, grot, $7: 3$.

(2):ant. 106i.

hatek mul matk (parck), tis.

liatler, 1:3:2.

haterelomers, i:.

lailinen, dis.

laill, 1] 11. halbseheirl. 1:3:3.

halsablara, $1+1$.

halter. halster, $1+1$.

ham, hamel, 1:39 11.

hamisch, hämiseh, hem, 6.

hammeln, s.

hammerschlag, 1:3s.

h:mo, 139.

hanebalk, habnembalken, $ォ$.

hangen, hengen, tis.

hannevir, 18!).

*luthas, 1 to.

harmskita, 136.

harst, $1+1$.

hast. hritin, 135.

hederiek, lierrik, lanik, 168 .

heibei, hoiboi, hatihati, 186.

hehmstok. 13\%-8.

*helmu, 20it.

hemil. 139.

hemmstorek, 138.

*hề-late, 143 ; *hêr-eost, 14 .

heriberga, herberge, 13, 126-8.

heul, 14.5.

henlen, hulen, huilen, 145,150

hiefe, lijepe, 12.

hinge, bs.

hingeraten, 3.

hocke, -cer, hok, $15 t$.

hoescl, $15 \%$.

höhlen, 145.

IIondichoote, 9-10.

hoofthand, hoofiltouw, 18.5.

hotzen, $157 \mathrm{n}$.

homlsel, 157.

hout, holz, $\$ 30$.

hringis, 199.

hulle, haube, 211.

huik, 333.

hurd, hürde, 25.

huwila, 150 .

-111:1, $1: 3.5$.

joch, 8:3. 8+ 11 .

juk, sis.

kaai, kam, kidle, 48.

Kalterherberg, 13.

kimmoshe, 43.

kamm, 10.5 n.

kanmegeluk, :305.

kite, liste, 5: 11.

kills.jan. 27:3.

kivelen, 1\%. 
-ke, $17 \%$.

kersoutle, karsoule, 5!.

kerte, kilrte, kritt, (i1-6:3.

kenzelen, $47 \mathrm{n}$.

kinkhnest, keielıhusten, 158-9.

kiusan, kiesen, kiezen, 273.

klingen, 125.

kloot, khuit, 15(;, 170 $\mathrm{n}$.

knaplous, +4 .

kolıle, kool, 158.

kot, -e, -erij. 5211 .

kothe, köther, -ei, 51 .

k'am, kramme, 5\%.

klanz, krans, 199.

krassen, kratzen, 59 .

krite, krete, $62,03$.

kriek, krieelie, 169.

krippe, 60.

kjom, 5 .

krullen, 162.

kwast, 6t.

laede, lade, 167 .

lakmoes, lecmoes, 2:3.

lande, -er, geländer, $16 \pi$.

last, 256 .

laubia, 168.

*auda, loud, lot, 172.

lijst, leiste, 169.

lok, locke, $15 \pi$.

loot, loote, lot, 170 .

lôt, lode, lood, lot, 172.

lôve, löve, lenv, löwe, 1 is.

luiaarl, $1 \%$.

luik. 103 11.

luiwagen, 238 .

lûye, laues, liuen, lanem, Inejen, $17: 3$.

lustig, 272.

luwer, 1 (is n.

Machteld, Mechthild, 17 .

magel, meeghel, $17 \%$.

mänzeแ, manssen, mânzen, 175.

menschke, $17 \pi$.

minsel, *minsen, 175.

mork, morg, murc, morken, morkinn, $181-2$.

muos, mus, 113.

nestel, 4.

nett gewiihrt nett, $30 \%$.

nenring, neutring, 18+-i).

neut, nont, 185.

noring (?), 186.

oog, ongring, 185 . orring (?). 1 sis.

orringje, 18.5 11.

falrioces, pardal', 295.

lalliti, poot, 112, 172, 190.

firn, piero, 1!3:3.

platt gesant, 307 .

plecht, 10:3 $\mathrm{n}$.

plocken, :314.

plisteren, phusteren, sti.

plute, plodde, :2ss.

porlenen, 32.

potteken, 13.

jottick, 1:3.

praat, praten, prattcle, 195.

put. putte, $194 \mathrm{n}$.

l'aken, 1 (s!).

laut, rotjan, roters, 112.

reiter. 21:3 n.

rilly, 2110.

ringhen, rinkelen, „20).

linkinken, ?01.

rinne, -en, 15-16.

rîven, reiben, 1 (i9).

lof, rocf, rouf, ?2!)

Rönersirchen, 138.

rûh, rauh, 211.

rii] ). 4:2.

rumnen, $212 \mathrm{n}$.

luote, rite, 171. 3222.

riiter, $21: 3$.

siluge, zalng, 223.

sceit, selueilen, skiliklan, 1:3:3.

schatel. 158 .

scheethalk, 7 11.. 2020).

scheil), schief, 278.

schellen, schillen, 15s.

scheren, scheren, serjan, 201.

schine, sediene, selicen, 144.

schmier, -en, 179.

schol, scholle, 158.

schoret, skituts, 112.

allow, 15:3 $\mathrm{n}$.

scit, schit, scheit, 13:3 1).

s'ou'ce, 154

seimll. schamm, 211.

scilr, schantel, 140.

sciur(al), scheler, :27, 153.

scrole, 1.58.

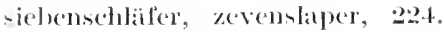

sijs, sijs.je, 22.20.

skajdall, scheiden, $1: 3: 3$.

skina, 144. 
skolla, 158-1(i2.

slâken, 169, 27!) n.

slifen, schleifen, 169.

slitan, slizan, $216-7$.

slito, $21 \%$.

sloot, sluiten, 170.

smeer, smierell. 179.

sheples, $179 \mathrm{n}$.

core, 2203.

spèrel, sperrel, 228s.

spihlıiri, speicher, 229.

spinatat. 2029 230.

sprirewipleche, 206.

sprocien, spruwen, spruien, 23:.

sprowan sprïhen, 88-90, 282.

spule, $1 \% 5 \mathrm{n}$.

stain. 16 (3).

steclien, 92.

steefel, stijf, stijven, 2:33.

steeklijn, 2:33.

stiefel, stievel, 80.

strichen, stroichen, 169.

strifeln, streifen, 234.

suocha, 2.21 n., 291 n.

sîr, saller, 2\%.

taai, ziih, 241.

takkeling, 23:

teig, 22.

thâho, tille, thon, $23 \pi$.

theiluan, gevleiluen, 236;.

tike, tijeke, zieche, 258.

$\operatorname{tir}^{2}, 1 \%$.

tol). tobbe, tul), 2t1.

toelast, zulast, 256.

tritt, traut, 261 .

tirlit, tuit, 172.

timilin, tuimelen, 298, :30:3-4.

twallja. 254.

verliezen, verlorenkost, 144.

verwarloozen, $98 \mathrm{n}$.

Vlatamsclike, 177.

voos, voois, 273.

rrevel, wrevel, frefel, $10: 3$.

vïske, 27:3.

waber (?), 27\% n.

wacker, wâken, wâchen, żo.

wîl, wat, wat, *wadje, 2 t.

walloosen, os.

wage, 27t.

walge, wageen, wag, 275.

wand, wandlaus, wanze, 142.

wandjin, wenden, 280.

warblet, $28: 3$.

weerzin, widersinn, 271.

weilsen, waiven, 278.

wejale, 100 11. 276.

wêr, weder, widler, 263, 285

werbel, *werribel, 28:3-4.

werbell, 284.

werwolf, $28 \%$.

Wezet, 281.

wib-, wipl-, 284.

willirôn, widern, 2\%o.

wieke, wiek, $28 \%$.

wifin, weifen, 275.

Willelm, 187, 288.

wiple, wippelhe, $296 \mathrm{n}$.

wisa, weise, wijze, 273.

wisel, 288.

worm, 115.

zeis, seivene, 2200.

zielverknopen, -er, 290-1.

zimpern, zimperlieh, 216.

zog, zoch, zllg, 2221.

zonk, -e, zinken, 214.

zwartsel, $18: 3$.

zwilih, zwillich, 234.

\section{Latin}

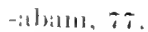

-i1hilinll, 1:36, 2067.

flerlinales, 3.

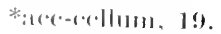

*3141-(0)1/11m, 18, 19.

-itrulu11, 711, 2.25, 265, 28:?.

आll, all-. 2, 12, 2218; voy. in.

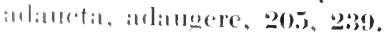

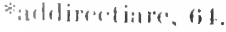

abliarroum, -rotitil, 19.

adjutun, 19.

ad revisum, 262.

aerugine.m, 207.

allibulate, 16 is.

*agurium, 209, 22:39 n. 2699 11.

ala, 159.

-alial, 5t.

amabilem, (i.

-imllen, 41.

-:4111111, 192. 
apothecal, $1: 3$.

arena. 15.

-aricium, 46, 13:3, 207, $2307-831 \%$.

-arium, 25.

asinus, 262.

-ata, 82.

-atia, 12.

-aticum, $26 i$.

-ationen, 100.

auca, 2:21.

*ancellum, 19.

aurum, $18 \%$.

barba, 140.

*)arra, $-1101,21$.

*bassiare, 65.

*hettare, 42 .

1)is-, 25, 26.

bitumen, 3 i.

bullat, 33,34 .

hisca, 203.

*annabula, 142.

(a)itellum, 245.

cappa. 24:2-3.

*apsalem (?), :242-3.

*aptialem (?), 242-3.

* captum, 81, 24.5.

*arpitiare. 106.

carruca. 2021.

casa, -alem, 243.

Castritium, *astruricium. 30)!

cautum, 113.

eavatum, 113.

area, 70.

* caritum, -are. 114.

*cellariarimm 20 it.

-cellum, 19, 296-т.

* charassare, $10 \%$.

cimussa, -ia, -ium, 213 .

cinctum, -orium, -11ra, 49.

cingulum, 49.

eircellus, 224.

cirrus, 218.

* conctiare, 6.t : *eoactirc, dili.

conculat, 67.

coagulum, (i⿱宀⿻三丨口.

collum, -are, 120.

com-, con-, 68, ro, 101.

*commum-ellum, $167 \mathrm{n}$.

conjungula, 55.

consolida, 5!).

cor, 154 .

*comicellum, 19. cornti, $15+\mathrm{n}$.

*ereticellum, 19.

(roxa, 56 .

*assia, 1916 i 11.

* cremarelum, -iclunt, 55.

crescentent, 2y36.

cribrum, criblum. $16: 3$.

(rucen, 27:3 11.

(nlpare, 51 .

(upere, *erpire. 16.5.

cutina, l.5.

de- (is : de-ex-, 12.

digitus, *atum, *ata, st.

dices, 2201.

dolimm, 2:25.

domenticus, $56 \mathrm{n}$.

dominicellum, 19.

dracumculus, dranculus, 86.

ellum, 1ㅇ, 133, 139, 155-1;, 170, 181, .964, 28:3, 288, $: 307$.

-eotum, -eolam, 31, 49, (15, 2:34, 246.

.54 n.. 285.

("x-aentimare, $47 \mathrm{n}$.

exalplatre, $15:$.

*ex-coactare, 61 i $n$.

*ex-cor-onem, 154.

*excriblare, 1633.

exeutere, $165 \mathrm{n}$.

*exenticare, 15.5 .

*exere, *exuta, 99.

*expralmare, $2: 31$.

*extensare, tale. 92.

extribulare. 16:3 11.

faba, 75 .

fictum, 19.

fisciare, 2.

feriat, 27:2.

ferranentum. 199.

ferrias, 252 .

ferrum, 95.

firmum, 94.

tiseella, $194 ; \mathrm{n}$.

sforaricia, *foricia, 95.

forticeme, $633 \mathrm{n} . .281$.

foris, 9411.999.

fortum, $9 \%$.

fractum, 101 ; fract: $170 \mathrm{n}$.

frigorosus, 148.

genellus, 75 .

geminare, gencllarc, $71,75$.

glammatica, toj.

*gromsia, 196 ; 
Eulat, 1200, 159.

hippicem, 140.

homorem. 1\$2.

-jectlit, 49.

-iculare. 90.

-ilia 12.

-imen, 41.

in, in-. 12, 57, 207, 218, 239 n.

-ina. 191$).$

*illeraraintre (i.5.

inetelinem, 53, 54.

inculpare. 51 .

*inferriats, 2.5.2.

ingenium, :3.

intacius. 9:3.

*intensinare. 9:3.

inter-, interventum, 2060.

iter, 11 .

jugum, 8:3.

labarim, 1 и.

later-icia, 31:3.

*atia, 196 ; 1 .

Huentrum, 16is.

*maculentare. 176.

manuiunem, 12 n.

mats-11s, 175 11.

mal'cềre, 18: 11.

merendat, $] 76$.

*minaciate, 176.

minus. .), 164.

minuta, -utia, 176.

*mixtiare, 176.

molal, 1.5!

*monticellat, 19.

muceat, -atre, *mucearellum, 18\%.

nutrerle, 1si).

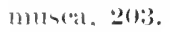

11: livm, 12.

*nalulat. $110 \mathrm{n}$.

navierlla, t9.

hebulat, -alat, 1(3i) n,

писест. 27:3 11.

-(1)

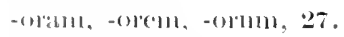

$-(1)-11111,1: 35$.

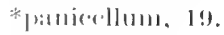

1):3110.1111, :31.

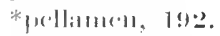

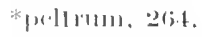

pedret111, * petricum, 2.52.

pilitre. 197.

Hitlail, :31t. *plaxellum, -aricium, -arellum, 314

*presia 196.

frologium, 225.

pllla. 333, 159.

jutêre, -ilus, :36.

futcus, 180, 19.4 n.

quassus, -are, -iare, 65-60.

quingluagesima, 49.

*as(i)eare. 200 .

*re-allateta, 205, 239.

reliellate, 1 s.

*re-exere, go 12.

regula, 2211, 2.53.

reill, 206 .

retortil, 201.

rigid-ellum, 20os.

robiginem, 207.

mubcolus, -i1, 209.

ruptuarins, $213 \mathrm{n}$.

sal)allum, 2016.

*alicedli, jol.

sanguisuga, 221.

sapius, $7 \pi$.

-sc(e)llu11, 29$)(;-\tau$.

sero. 217.

sic, ․18.

simgulum, jo.

*

solium, -iata, -iolum, 225-(j.

solvere, 227.

spicarime, 229.

*spissia, 19! 1 .

*stêla, 15!).

stijmla, 1.50.

strangulate. $175 \mathrm{n}$.

stb-, sulitus-, 2206 n.

sulstratum, 2205.

*smb(tus)soliamentum, 226 .

*stretiatre, $1 ;$ it.

siplinit. 1s2.

legula, 2.jis.

telar, 159.

tensare, 9:; : temsatus, $2+8$.

terlerim, *terlicum, 252.

thê(:) :-5:3.

-tionem. 1011.

tomsatce, (9):

*tonsellum, 281 11.

*10rucllum, :30)1.

toreat, tunc ("at, 5.).

1 1rats-. 2600.

trimsersate, 257 . 
*tratleum, 31 .

tribula, -are, $163 \mathrm{n}$.

-l1ea, 20:3 11.

- llela, 104.

ulula, 150 ; ululare, $15 \%$.

umbrare, 217 .

-แrum, -am, 2т.

-uscat, 203.

*vallicella, 50.

vapidus, $28511 ., 322$.

vala, 285.

veriatus, 286.

valicare, 285.

variola, 209.

varire (?), 286.

velrius, $2(j ; 3$. vasechlum, 19), 285.

vellus, *vellerem, 2iis.

*verualia, *verminaliat, 115 11. 25.5

*vermellum, 28+ 11 .

vertelolum, 20s.3.

vertiginellus, 265.

vesical, 270.

vicinabilen, 9211.

videatur, 2(j!).

*villutellum, 282.

vitta, 28 !).

vitulus, 121 11 .

vocem, 27:3.

*olutum, -i1re, 101 .

volutum, * volutiare, 11) 1.

\section{Frangais el diatereles}

-a (1r, -ail), 70, 265, 28:3.

a (fr. en), 136, 218.

a-, 2, 4, 12, 1:3, 5\%.

-â, -å, -âr, -au (frr. -arrl), 202, $80,85$.

114, 201, 283. -å (fr.-al) ㄴ.2.

Aarschot, 8, 9.

abarin, 2224 11 .

åbarone, 111.

àlicon, 184.

abiljawe, ab'djonwe, 18.

abcrolondè, 1.

â)ièr, 126-7.

ålion, 5 .

abitake, 13: 11.

-ille, 6, 136. 211, 26\%.

abôkî, -eler, -cuer, 32 n.

abrouche, :322.

acåmce, $105 \mathrm{n}$.

acheraule, 136.

acheret (?), 308.

achüruôle, -cûs, 137 .

achîre, 130 \% 11.

aclin, -er, 3 .

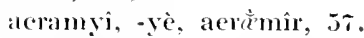

acwassi, 64.

acwati, 66.

adiıglé, agadlé, 210 .

-årle, 138.

årlios', ådiyos', 1.

afagoter, afaliner, 13.

afillant, 2.

afèmoki, anfernowé, 94.

af alleterresse (\%), :30s.

alloerece, 308.

ifoeline, $6: 3 \mathrm{n}$.

afiller, 1 (i:3 11.

agaimter, 60\%.

agăyomèter, 100 .

aginaure (adjonove?), 3.

agio, agialu, agios, 1.

agrafant, algrifaut, -pant. 22.

aguerôdì, 2.

andistiller, 10ה.

a-luarlicère, -îre, 1:30;.

allati, 1:34.

-àlıe, 12.

ince, ålinince, aise, $1 !)$.

aliere. -i, 19.

alumi, - 6 aheurey, $147-8$.

aïle, aider, 12 $\mathrm{n}$.

-aine, -ainne, 15, 16, 192.

ajowe, aiouwe, 19 11.

akêmer, akinmer. acdurer, $105 \mathrm{~s}$

akinàve, 3.

alloo, $\mathbf{b}$.

-ale (fr. -elle), 14t.

ălể, ålul, H.

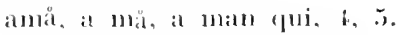

andile, -aule, -auve, ti.

a111 bô, $1 i$.

amiljô, am jour. $\tau$.

amèler, amøler, amader, s.

ameto, aminto, 1:3s.

ameitr, 1s2.

ameure, -ore, -me, 17s. 
amigo, 1 s.

aminter, inmenuler -enr, 8.

antinte. $138 \mathrm{n}$.

an'tehò, -011, ?.

amwinne. !)] $\mathrm{n}$.

-in, -on, 1:2:2.

anal. hanall, 262.

anil anô, alloyere, 12.

inleonte. fulascote, 8, 11.

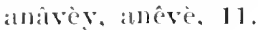

alli), (i.

allule, $1: 39$.

andion andion andoye. 1.

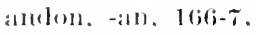

anoleçon, 184.

anivers. 1339.

anki) (?), $5($ ).

anneote. 9. 11.

muspimlje. iz.

all tolio, -(oll, 2.

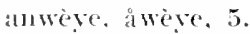

arire arite. 205. 239.

apribter, apaisenter, 69.

apotadji, -gie, 13.

aluteliter, $1: 3$.

alputicare, apotiker, 13.

ate- (fre re-) 18.

arable, areine, 15.

areô, atreoll, 50.

arêne, arime, arenier, aruicr, 15-17.

arèni. $201 \%$.

allete, ariesse, 12 11,

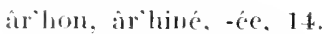

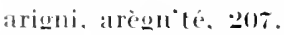

arilı, arilimint, 1:3.

ariler, -i. -eye. 14.

aromeni, arouvi, 207 .

arrisuli. 24.

arurot (?), s, !) 11.

allumi, 2017.

ărvolul, alvolut, $35 \mathrm{n}$.

imeate., 1:3:3.

: larecit (?), 8., 9, 11.

atsperencremer. 836s.

analuki. 214.

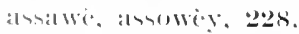

issonke. $-i,-i 4,211$.

at:aliun. T).

at 10 , at ni. 201.

alibrirre. 17.

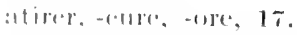

atriat, attis. lattre, 1 t. atriyote, 142.

auberge, 12:.

auké, wåkî, 82.

aulé, -ia, 4.

aurbè, -éye, -ê̂, 139.

ausserot, 308.

avale, avoite, $205 \mathrm{n}$.

-åve, vovez -able.

avèrdondé, avèrgongni, 1.

averlant. avèrlu, 18.

avir, awîr, awềr, 20()9 $11 ., 2339$ 11. 2(i9).

awiljale, awe, 18.

awêbiè, $27 \%$.

awète. 201-5, 2:39.

aw"hê. aw"jale, 18.

-aye (fr. -aille), 54.

áyelie. *ayou, 19.

lı-, hè-, bi-, 2.).

ba, 19, 20, 30 11 .

bilben. barboir. $115 \mathrm{\mu l}$

bactoure, batpuenure, 20.

biale, 20.

barljawe, -owe, To.

bagou, bagouler. is.

bajone. bajole, bajote. 78.

bakine, $21 \mathrm{ll}$.

bakner, -enre, 20.

balrl, batut. 20 .

halerèees, 308 .

ballercisce, baeleresehe, 308 .

balzin, palzin, $101 \mathrm{11}$.

bar, $21 \mathrm{n}$.

bår. bâr, baur, -e, 21.

baradil, rabada, 210.

barlazok, 188.

batrdafe, -oufe, 294-5.

harôtì, 25.

bate, batte, 144.

lat niére. 166.

ball, 20. 30.

balucuel (?), 31.

lie. leet, 41-42.

becefuenouls. :2:3.

belfroi. herfroi. 2s n.

lẹ̀å, -all, begart, 21-22, 91.

Behô. :30.

behorclis, 25

behouriler. 20 .

billteur, belfreude, bellefroit, 28 n

benerexe, 308.

berekmoese, berquemomix. 2:;-23t.

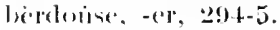


bêrih, 2:3.

bèrlin, burlin, 260-1.

bèrlire, 302 .

bèrlozzer, birlozer, :2!5.

berlu, 151.

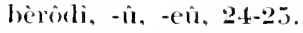

bèrwète, -èr, 295.

bètille, $16(\mathbf{6}$.

bete, -er, -ir, bèti, 41-42 ; hètin, :32.2. béton, :36, t2 ; betun, betumei. :36.

beưlai (!), 208.

betr, betir, 20(j-208.

bentin, 41.

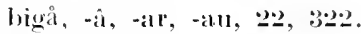

bigårler, 91.

bîhê, biseatı, 2280.

bihot, biyo, 29, 187 .

bilıôle, 2.5 .

bîrå, 2․․

birlan, $260 \mathrm{nt}$.

birôrlî, birlôdì, 22-25.

bittaine, 40 11.

bitume, 36 .

bizer, bizer, $179 \mathrm{n}$.

bleime, blème ; blène, -er. 29. 30.

l)lèti, -in, 41.

bo, $1,0 \mathrm{n}, 30$.

boeå. 끄.

bochale, bossale, $196 \mathrm{n}$.

bodje, -cye, 32, 33, 174.

bôkê, -iiı, 31 .

bok'hô, 42 n.

bôkî, -ìve, -cler, -ener, 31-32.

bondje, longe, 274.

bonian (?), $34 \mathrm{n}$.

boncpue, 1 (i9.

Borain, Borinage, $26 \mathrm{n}$.

borordeis. 20 n.

bort (?), ㅃ05.

botike, 13.

bou, 31 .

boucherot, $30 \mathrm{~s}$.

bouch'nik, 17\%.

bongnet, -ote, -011, 33.

boulue, 20:3.

bouquette, bonquiau, fig n.

bomrleresse, bourdoir. 308 .

bourloitse, 294-5.

bout d' $\mathrm{fi}, \mathbf{2 + 4}$.

bonterèce, 308-9.

boît'ner, -cîre, 35,36 .

bouye, -ote, -ou, 33, 3t; buyou, 3t. bratler, 195.

litagra, :30;.

l,an, brand, -eter. -ir. 38.

hrandi, -iner, 38.

lianvole, bronvolé. :3t-:3!).

hrant, brèroll. 3:22.

J masserez, braferesnc, 309.

hríli, :34 11.

brelene, berlene, 2060.

hicelle. s.2.

brif-lolaf, $: 3$.

lirigale, biguele, 39.

brigatr, 22:2.

brigunae, :38 11.

brioler, briguler, 38 и.

briserece, :30!).

briver, 37.

brivolé, $3 \%$.

brôki, -ièrlje, -ion, 1.5.

hroissequin, 2:3.

l,ron, bran, 39 n.

briverèce. :309.

bruskin, -̀ेne. 2:3.

b11, 206 $n$.

but, huwe, huwo, 20! 11 .

bur, :3:2.

bugatr, 202.

lubot, 29.

bulaine (?). 40 .

lour, bure, 206-2s.

burin, 20; 11 .

frurlin, 260-1.

hron, butron, 28, 173.

birte. 26 .

buntieliupe, busticlape, 40. butaine, -ane, bustane, H.

butin, 41, ;32.2.

butver, :30.

("al-, 4.5.

(abèriole, :300).

(ahouyî, calıomgni, 162.

eaissier, guatisier, 6.5.

cakèto, 12.

raliguluk. 305.

(alkizúte, 1206.

(amitelee, 43 .

(cane, 10,)

canabuse, $4: 3$.

Pancpin, lienpin, $t$ t.

(anilich"tôn", 166.

carabaljoye, earabontela, tio.

earamadjoye, -djoyeló, $t 6$. 
caramateveler, $\mathrm{fig}$.

earrheresenc, :309.

calli-, ("all-, ("ali-, 15 ; (4) al-, gal-, 126. carbintedje, aribodion, 45.

arimarjồe, -dja, - bjorècos, 45, 46. einlioli, 25.

cammadjoye, cranadjôye, 46.

earoveler. 4 is.

(appin (?), t+ $\mathbf{n}$.

earreche. carieselie, 309.

cats. Bio,

rasmarâle. 삐․

catî. autcrève, 520 n.

raำ.ำ.2.

cóveler, 47.

"awe, cowe, 78, 82.

carie, cearier. 26 t.

cellerier, ecrir, rherrir, 206t.

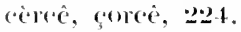

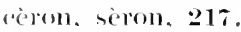

(ossiall, 243.

chate. chake. chakine, -er, ts.

Mrathê, 1:3:3, 135.

chahmean, -cler, 241.

ehancer, $47 \mathrm{n}$

chanon, chä̈m, chö̈on, chon, 4. ts. claseal, 243 II.

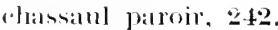

Clatustros, 30).

rlatt, chate, 13:3-4.

chandre, $135 \mathrm{~m}$ и.

(Wiaye, haye, 15!).

clicelas terricer, 248.

(begros, 24t5.

hiele, -iv, $2: 35$.

ellenerverez, :30)!

rlicial, 24:3.

ehenat patrelloe, 243.

rhite, hite, $1333 \mathrm{n}$.

eherret, 3009 .

"hicancer, 45, 19.

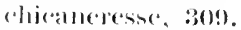

(hicertate, 1!).

rhime, 11t: (chinem, $145 \mathrm{n}$.

(1)

"hipres, st.

-rial, - rollit, :3001.

eicale, rincale, fis.

rimb, rimbulje, rimatise, sol.

(dincer, rimeratte, 300 ,

rimois, rimmse, 2213.

rimefure ( vimgure), cincwène, eîwème, 49.

cinerèce, Ciney, 30!)

cinguc, sangle, 49 .

cintron, ('îtrồ, 49.

(izê, ciseau, 219 .

claper, 41.

clecote, cleker, chicote, 125 11.

cl'́cljè, clindjè, 233.

aligneter, 3 n.

(ditehe-pate, 190.

cloeraite, 310.

(lotet, 1 \% 11 .

cloyombe, 54 .

rlîte, 136.

('ô, gô, 110 .

coche, -î, 6.5.

cochlati, 1:1.

coclion, 120, 121 n.

coch'nik, $17 \%$.

cohå, 22.

whe, 56, 63: : coxlle, 55.

eoke, .54.

comê, ('olmê, 53.

-ron, $76,184$.

congle, co(n)jongle, 5.5.

(onsande, consonde, 59.

consîne, chonzire, 10 s.

coje, -er, 50-51.

copler, $51 \mathrm{n}$.

(сор)urmale, 29\%.

cor, corm, $154 \mathrm{n}$.

côr, côt, quaturt, fis n.

corii, coral, $154 \mathrm{n}$.

(ari, corote, 159.

eron. 5. 15t n.

Corommolise, 5.

cortchai, cortiscall, cortillage. 5l

cortillier, 52.

(r)sse, 154.

crissceler, $4 \%$.

cossèt, 1200.

costant, Constaut, T!).

rotclict, 1.59.

cot'hê. 19. j].

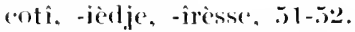

coticr, -erie, -in, eottage, 51, 52.

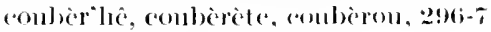

(ou-botèye, anti.

conchale, -èle. (iis.

conchèt, 1:0.

(a)tere. 58.

collet, antette, 52-53. 
coulper, 51.

cougnot, gougnut, 159.

coulot, 159 .

counaye, commatille, $5: 3$.

tommet, $3: 3$.

eompanlt, 188.

coupèrète, 296 .

eomperom, 20\%.

compèron, -i, - $\grave{c}, 296-\tau$.

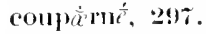

couplire, 29ti 11.

courcelle, (i:3.

couribèt, courlette, 295, 3202.

courtall, -iau, 16!).

eouvaine, $17 \mathrm{n}$.

cour-z-å-hatut, 297 .

eovir, 165 .

covvète, 18 n., .j:3.

côyenès', eơgnès', 189.

coyeutite, $12: 3 \mathrm{n}$.

eovin, -imne, 45.

coyombe, -̀े, coyonke, 54.

crait (!), trait, 280 .

crakite, 198.

erakin, -ine, -ion, 198 ; -iner, 199.

erama, cramail, er⿳亠口冋口, $5 \pi$.

eramiète, crènulete, 5 .

(rantion, cråmiyon, -ignom, 56-58.

cramillon, 5\%.

Cråmoùse, ('rommoûse, ว.

erimse, 20011 .

eranskène, -iner, $199 \mathrm{n}$.

crasse, -î, -erèsse, $5 s$.

eratehot, -c, 198.

craton, creton, $6 * 2$.

créchinnes, 16.

crèliant, 2:35-6.

erèpe, (io).

erètue, grinqule, 169.

erèsse, 59.

crèssode, eri-, eru-, 59.

crètale, 61.

crète, 60-62.

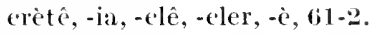

reter, 61.

ereti, -î, 61 n., 62 1.

crètin, kèrtin, 60.

(reton, crèton, 6:.

cretu, crêtu, 61 n.

cristel, -ale, 108.

crôtinèt, :32:2.

croiseresse, 310. croke, crotehet, $15 \%$.

croler, 16 iis.

(rotale, trocaste, 210 .

(rotuli, 102.

croule, -icuis, 1 Hs 11.

"roûle, crùle, crîle, -t’r, 1 (b:).

crumuya, 802.

(rustal, -e, 10s.

"ucluale. -èle, (i:).

ruclie, roher, 6i:3.

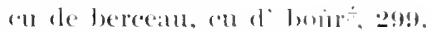

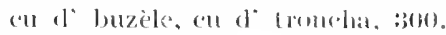

(rilissette, 56.

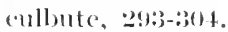

ध०umitriall. \$30).

(ammulet, 2917-8.

('urton, (rèton, 133.

(ustel, 10s.

('utoumiant. 8301.

("utominial. $\$ 300$.

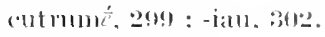

(euzoulue. 125.

cwaci, 6 t.

(rwagne, 255.

cwalle, -ì, cwalle, -er, -i, 64-5.

cwălèce. cwărliee, :310.

eranse, bis.

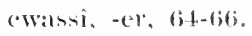

ewate, - $\dot{e}$, ifi.

rwayot, -e. 65 .

(walyoil, ti6s.

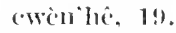

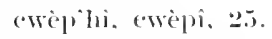

('wèr, 15411.

ewèste, $65 \mathrm{~m}$.

(wezon, (eloison, 201i:-

ditho, dabolin, 24 ).

dambê, damoiseat, t!.

dan, don (= latt. donct), 5 .

dandince, 38.

darm-, 85.

l'aotier, $6: 2$.

(lè-, 1ㄹ.

delissent (?), 207 m.

délningander, degingender, 68 .

dènàyè, *lesmaiver. 11-12.

dervee, tit 11.

descrable, -auble, 283.

desharber, 140.

desmâlınc, dishåmoné, ㄹ10.

despatouwer clispatuer, (is.

despostues, despoester, di!. 
dentaknter, tig.

denvervire, 20 (j4.

denre lemne. 236.

dentelic, 27t.

dive, léve, -er, 6t.

H'frèsti, s6.

diliboter.

diuà, digue, 22.2.

digrite, si.

dilubinc, 67-8.

dilitrber, $t 40$.

diprotler. 194 n.

direni. $20 \%$.

dincleyi 216 ;

ainerimi, -ien, $5 \pi$.

Binguèrnctè, otè. 11$) 6$.

disgungonè, 125 .

dislouvi, 162.

dinwebi, d'vesli, -i, 27s.

ditillti, 59.

divenues, $2633 \mathrm{n}$.

divere, l'vàre, -er, -i, 260-4.

diviner, 21.

diwaki, 273.

dizarèni, dizòrèni, 20 n.

djalce uchlue, it.

djade. $7: 3-74$.

djalfe. T⿰.

djallik. 82.

(jaima. $7(1)-72$.

djanne, -cr, -elége, io n.

djanteler, : 1.

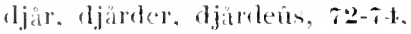

ajordin, jardin, 7 .

d.jimni, djiammon, $117 \mathrm{n}$.

djawyi, ts.

lijelijil. $-\frac{1}{2}$ sol.

djerdjete. Tl;.

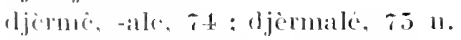

djermi, 11 (i-T.

ljerinler. : :

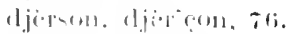

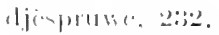

djelli. ajentii. ata...24.

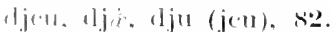

1jen, ljoni (joma). 83.3.

difur, - -

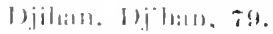

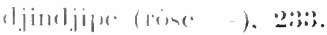

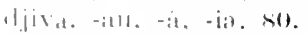

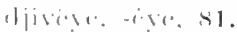

1]ir, 1ju11w, 111). djoiguive, s2.

djohe, -eler, sis.

ajok, s:3.

djomi, djomniner, 116-8

djuntî, 82.

djuî (jong). 83.

djollôti, 110.

djumèle, 7.).

djus, 1 ist.

honon, s4.

doguinne, $17 \mathrm{n}$.

drie, st.

dona, Donat, Bonat, 85 .

done. 2:33.

doì, đû (deuil), 225.

doumbiosse. st; $n$.

dove, doxe, domye, $74,85$.

d) ponstrer, s6.

dpoustrer. duspointrer, 85.

d'rihon, st.

dritoncle, drangle, -ure, sib.

lrèk, grèk, 233.

drodale, drombe, drombale, 2061, 3:2:2.

drongue, -lie. the, s6.

d11-. Iis ; (t11n-, 12.

duelinguter, 1:25.

du griule, st.

dulameniner, 6s.

dumonliner, 181.

durnintiner. 211.

dusnevi, 11.

dussatwe, 228.

dutinclente, s:3.

dilwele, 2:3:3.

i- (fr. (11) $), 91$.

- $\hat{e}^{2}$ - $i_{a}$ (fr. -eill ), 120, 1s1, 233. 28:3.

chromer, sis-go.

-ece, -rehe. -esse, 97.

icale, cualle, 160 .

erlier, cerlinser. 217 .

ierebeler, 121 11.

ereme, enclume, 53.

icrope, ceoper, 51$)-51$.

coopuler, 1.5.5.

(a)e, $15 \mathrm{t}$.

contet, ceonte, 5is.

irranil. -vis', 57.

(") valsoll, 101.

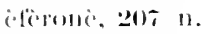

alriboter, $\%$.

cfwehc, 281.

colnunc, exlommin, 58. 
eqrinaneyin, 12:.

ehant, ehowe 9!.

ènîler, 146.

- ekè, 17 11.

-el, -crel, 75.

te, $\frac{1}{c} \mathrm{ve}$ (aile), 159 .

- elete, -elote, 25:3.

èmainé, èninné, èminhoné, etc.., \$1.

embegaré, 91.

embreler, s:

èmiljon, †.

èmoủlî̀, 17!)-180.

empotement, $191 \mathrm{n}$.

ènåhe, ènève, 12.

enelinable, 3.

encoper, encoulper, 51.

-ène, -ine, 190.

cuforcenes, 8:3 11 .

enfressele, 104 .

engenave, enginable, $3-4$.

ellgoner, 78 .

enluacrelé, 182.

empluir, $211 \mathrm{n}$.

(3)runié, -gni, 20\%.

entait, -ier, 9:3.

entriboulé, $37 \mathrm{n}$.

èntıri, èrıni, 210.

envearye, 264 .

éperule, espole, $175 \mathrm{n}$.

Epinal, Epinay, 229-231.

er- (fr. re-), 1 ז.

èrane, erraine, 15-17.

èluate, 143.

-erece, 46, 96, 227. 307-817.

-erelle, 14t.

èreni, èroni, ènni. èrigni, 207\%.

êres, êrì, 205.

erickses, 12 11.

ernier, 15.

erodji, :07 n.

-erou, 189 ; -eroùle, 288.

èrovî, èrouyî, ํㅜ․

erve, errer, erraine. 16.

èrulitines, 211 .

èluñté, -tić, $20 \%$.

ervelenx, 18.

es- (a-), 1 .

èbate. 2:31.

esclloinheresse (?), 310.

esclato, 21\%.

encoleler, $151 \mathrm{n}$.

escot (?), s, 9, 11 . encouthier, boucher, eskokier, 153.

entrami, 5\%.

enpintat, -atrd, 230).

enpirial, 2209 11.

(-)poignicer, 2331.

espolier, esproer, -ement, ss.

crmurgatoire, 22:31.

ésanclét, èranki, -iner, -ines, 214.

wtencr, 9:3.

entensiner, 9:298.

ervereomolé, coverenoignje, 1.

if (eonjonction elidce), s:3.

itat, itct, -i, etc., 9:3.

- it 120-1:-ite, 204, 288.

ition, 248 11 .

efriver, ts 11 .

-(1) (fr. - - (111), 216, 2:32, 270).

-cil (fr. -oir), 260 .

-

- (111, -еiोr, 2т.

enti, eri, 1 ks.

ềin, 18t-6.

-cûs (tr. -cux), 18, 1:37, 216, 2801

crêri, 20:3-4.

evire, $2(1)-251$.

iwerere. :310.

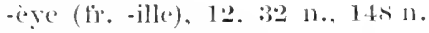

-eve (fr. - re). 81.

ficent, fiment, 98 .

fillerece, 310 .

fialii, faimiele, 2.

fillite. 172 .

fasmowe 94.

fauchatione, 1711.

faturlon, fiturom, 9:3.

firti, fay"lie, fayije, 19.

lex, ferm, 94-95.

lerliner, fornoer, 94-45.

firtore, firtore, 10:3.

fistit. 202.

ferlinwalueratis, 310 .

fi, til. 2.14 .

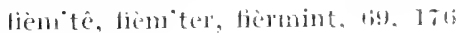

liensi, $101 \mathrm{r}$

fik-inei, fiskineil, 45-94i.

|jleresse, firlèse, 11 !).

lin-fire, 94.

linihrece. :310.

firlatine, tredaine, 46.

lisicfue, linchiner, ete.. 95-9)6.

flam ter, flam"zik, $176,175$.

llane, t:3 11 . 
floket. Hotrbete, 15\%.

Howcer, 9s.

tochlete, $193, \mathrm{n}$.

focke, fohe, forcke, 33, 55, 56.

focr. tiuer, fenr, four (fr. fur), $9 \%$. tohale, fochale, fossale, 1!) n.

folin. $41 \mathrm{n}$.

fonet fonnet. :310.

for-, foil-, 69 .

for-, far-, fer-. ! + n.

forcharonage, $140 \mathrm{n}$.

forece, -eche, -ciche, 96i-9t.

fòchlatu, 9s-100.

forharbaige, 1 to.

forlower, 97.

forveri. 206:3 11.

forvili, forviri, 98.

forvirer. $2 \approx 0 \mathrm{n}$.

torwân rèce, 810 .

fotchioule, 311.

foì, !)!

foimin rece, :310.

tôtre, foimehan, 98-100.

finulcle, fonfèle, foufiete, 102 .

fourcherez, -et, -ot, 311 .

fourtel: $1 \frac{1}{\ell^{\prime}}, 311$.

foimstil, -a11, 1111 .

foit ler. front ${ }^{\circ}$ ler, 102.

foutrot, 311 .

forma: 22.

forrerece, 311.

fraise, frase, frescl, 104.

fratin, fratin, fretin, 104.

frofol, 102-10:3.

frebal (:), 1 !)

liege. $-\hat{\mathrm{c}}, 104$.

fribote, !)

frimgleret, 311 .

frinleresene, 311 .

fromlefis, frileux, 1 ts.

froiteler, 102.

fruitrere, 311.

find, $10: 3$.

fincresere, gri.

fiur, finil, !).

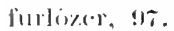

furtore, -(1)

fi1merre, $10 \%$.

fwelues, forees, 281 .

世ำ

מalguizomle, galgutenomille, etc., 120.

sallene, salyiunc, galmiene, 194. gallimatias, Hi.

gamalehe, -isse, 4:3.

Q:แ111bète, 190.

gincte, gomìte, 105.

gatmin, 4i3.

gargosson, -siê, -zial, garguechon, it;.

ginluette, oti.

garmenter, gamèter, 106.

garsî, -er, gercer, $10(;$.

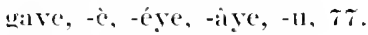

gawe, -1े, 78 .

geschot, :305.

gliethic, :306.

ghitalle, 305.

givere, 81 .

glignter, gléter, 125.

glin, glindis', glendice, 109.

glingon, -onète, glingoler, 12.t-125.

glommia, $5: 3 \mathrm{n}$.

(a), 109-11\%.

gocha, gochear1, 120-1.

gole, golete, 119-120.

gomme, -å, -er, -ê̂re, 111-5.

gômi, gomir, 116.

gorgecoll, 76 ; 1 .

gorlete, $118-120$.

gorme, $-\dot{c}, 115$.

gossê, -iat, -èt, -clé, 201-1.

gote ; gote, gotale, 123.

gôti, -iner, 109-114.

gotterinne, 1711 .

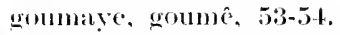

gotime, gollome, etc., $111-8$.

motuminc, 30$)$.

gommigoumes, 114.

gompmie. $105 \mathrm{n}$.

gonmi, -ic, $117 \mathrm{n}$.

gramanter, 106;.

Grélry, 1. 1.. 121.

grimachin, grommaneyin, 122.

grimayelé, 176.

erimoll, 121-2.

grônmer, 116 n.

gurluc, 12:.

guledin, 12:2.

guemine, ghemaine, 304.

Encrololl, (i:3.

guêtôler, -i, :3.

gucrsi, guerehinc, 106 n.

gutersol1, 7 (i).

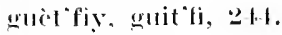

encille, 12:3. 
gucinge. queule, 159 . suezite, quèzike. 305. guilite, ite, -il. $\mathbf{3 0 6}$ guimène, gumune, -еr, 806. guinåde. quinâde. $: 306$. guingon, 12:3-5. griper, -ure, 275. guitire, guittière, $30 \%$; guizèle, :306. guminer, -è, :306. guzouhe. 12:5. hibièr, hâdibièr, etc.. 126-1:32. hachiere, hachiôle, ete.. las;-s hathê. 1:Y:-t.

hadrene, 1:3t-5.

håhiner, 68.

hahise, haschicre, 186.

hallier, hawier. 12\%-180.

halboterèce. :311.

halmustok, hamestor, $1: 35$

hamâle, hamaule, 6.

lambar, 7 .

ham'ler. s : ham'lete, $189 \mathrm{n}$.

haminde, $1: 38 \mathrm{n}$.

hamuslide, 1 iss.

hand $\frac{t}{\hat{t},} 1: 38-9$.

hanguiner, lis.

hanivêr. $1: 39$.

hanoche (?). 12.

hanscote, s. 9. 11.

hantresse, $: 311$.

lataine, helable, 15.

harber, hatber, -i. -eye, 1:39-1 fl)

hatrine, 135.5 .

hàribier, hâlibier, hadibier. 127.

hasterel, haterel. $1+1$.

laantier. hasti, 1+t $\mathrm{n}$.

hat, hate, 1:32-4.

hateh et mateh, tis.

hateherole. hatehioc. $1: 36$.

hatrê, haterel, $1+t$.

haube, haurbè, $1: 39$.

hauteresse, :31:3.

haye, chaye. 15s.

hayou, hạpener, hảener, 1 s!

h'. $28 \%$.

$-1 \hat{c}^{2}, 18,19-296$.

hêh, rhêhi. 27 s.

hèle, helon, hiele, 22:37 11.

heme, heamme. 264 .

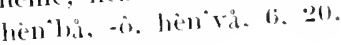

hène. $14 t$
Ilephatte, 1.11, 142-t, 3:20.

herbere, 12ti-t.

herberih, $1: 3$.

llereot. Herient. 1. 1., 1+t.

herenalle. 15.

hérissere, heurser, ("te., 1 t! 11 .

hernale, $1+4$

hèrô. hiros. lutro, 1.:.:

hécu, 1.51.

Hesta, Helstal, 5t.

hetch rece, :31:2.

hite, $1: 3: 3 \mathrm{n}$.

hemeque, hestic, :3:3.

heviler, 14.5.

heulle. houte, hule, 15\%.

heripon. hexpion, t2.

hentaillin. 1,5t.

heire (necoller). 16i, n.

hecile (arange), 27. 153.

hevire (hure). $146-1.52$.

hente - ex eye, 149.

hetrece, :31:2.

heurete. 150 .

henreais, hourewn, 1 ts.

hention, 1.52.

herurlon, 152 .

heciti, haubatl. 185.

hik"hone, 155.

hiner, hincon. hinclete, 1+t-5.

hiri. hire. hireder, 153.

hisire. gihire. 2111.

- bio. :30.

hir. hitteyce 112

hocher. $158 \mathrm{n}$.

hochet. $1.5 \%$.

Hock. Ilockai, 151\%

hoikt, huterute. :3:3.

hoh (si mète a--).

hok. hoket. hokete. 154 .

hemmer jostr. $\tilde{x}$

hore (li. hure). 147.

hore. 15:3 11.. 1.54. 15\%

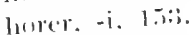

freriele. 150.

haim, 1.52. 1.5t 11 .

horlt, -iser, 14 !) 151 .

horots. 15t.

homerte, (emote, 1,5!)

horver, hyom ner, 51.

hosiannal (?). 262 .

himsealls. 12011 .

lna-i. 1.5 11. 
hoistal, 29:3.

hotehe, -î. 15t-6: hotelat, 15.5.

hotchèt, 156,159 .

houbite, 211 .

lomgnet, lwminot, houyot, 159, 161.

louille, 1.58-162.

hoiler, 145,150 .

loulot, coulot, 1.59 .

lunklor, linulète, 1.50.

lummer, 211 .

Imире. lomplets, I Is 11.

linitr. 2.5. 1.3\%.

lomra, latlal, 14s.

lowabiller, -is, 1.51.

homianse, 14:)

Jomrd, 2.5, 1.58 n.

hourderiece, 312.

lonere (fr. lome), $1+\%$.

hourel. lentrelen, 1.51 .

hominer. 14 !).

lomet, -exte, -ente, 150 .

lonurciis. $11 \%$.

homerere, 1 h!s.

lomri, 14s, 1.5:3.

lomrihat, 1.5:3.

homrin, 2l1.

lonurisos, homriche, 1.53.

lomile, 1.51, 15\%.

horrloi, 151.

ho11,l11, -311, 1.5:-2.

lourlot, -ole. 151.

lanrlote. Lutrlote, 150.

lo110011.15:3.

homle. Intete. 25.

houye, hourot, $11 ; 1$.

homyi, homgni, 162.

lom1001, 161, 185.

hovalon ( wallonl 1.5.

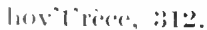

hove, homye, homille, 1.5k-162.

lorei, hozele, 120).

lirniller, 1 (i:2.

huldri, 1:32.

laller, lutrlere, 150.

lıulle, lumilhe, lil $\mathrm{n}$.

litalolte. 1.s0.

linimise, 1 h!).

lutra, -

lowre. 1 16;-1.3.3.

hurees, 149.

latirel, 1.51 .

hurer, lowrel, -i.te, $118-1.50$. litreye, 1 t!).

lnuricle, 16 (i:3.

lnurillon, Intrion, lnmlion, 152.

lnurir, -is, -isse, 1.53.

lourlari, 1.51 .

lunliu, 1.51.

hurlon, 1.5.).

liurlote, lomilote, 151.

lurlu, hurhuberlu, herluva, 151.

hmron, 147, 15:3; huron (?), 15:3.

-i (-il), 201s.

-î $\left(-\mathrm{i}\left(\mathrm{r}^{2}\right), 25,206\right.$;

-ia, -ia11 (-2:a11), 300 ; voy. $-\hat{\mathrm{e}}$.

ifureof (!), 290 .

-ille (-ise), 280 11.

-in, H, H. 1 so 11., 208, 25s-!).

-iner, 4s, 92, 9(i, 117, 199).

inflin, $4 \mathrm{ll}$.

inmakemis, 1s2.

int"ly, 20.

intré-, intrèlin, 260 .

intribole, intrivole, $37 \mathrm{n}$.

inwiye, inw”jale, etc.. 18.

io-jo-campion, $18 \%$.

-ion (lr.-illon), -iyour, -ignoll, $\$ 211,56,57$,

8t 11., 124, $142,15411 ., 254$.

-is (fr. -is), 25 11., 10!1, 214, 237, 24711 .

itide, :30);

-iveris, 133 .

ivo, 185.

jard, jarderus, gatrolems, jatrems, 73.

jecterece, $31:$.

jostereche, :31:3.

jome, jomter, 7т-7s.

jontulc. $\left.-\boldsymbol{t}^{2}\right)^{2}, 116$.

joitir, 110.

jure, jurlucer, s1.

juminile. 7.5.

jus, juverne, 163-t.

Kahèlsrih, 1:3.

kègneter, :3 11 .

kèlotincr, 71 .

kème, kinme, cånne, $105 \mathrm{n}$.

-kenc, 1!1, 240 .

kènpin, eanejuil, 4.

kènouk, kènouf', :307.

kèrtinu, kertelé, 62.

kètin, 60.

kèrton, -๕11, 63.

kớssouhe, 125.

kèstel, 108.

kital'lé, kulalile, b9. 
ketifi, keutefi, 24:3-5.

keûre, 79,16 16-J.

kê̂se (comble), ร!).

kichtôn', 16is.

kihomyî, kihougnî, 162 .

kil"lềye, kir'lève, 119.

kipougnter, 2:31.

kंmougna, 16 is n.

koeme (?) = keive (cuive), 50.

koxlue, colne, 55.

kuhåhiner, 68 .

Laixhealı, Layliıy, 1. (1., 19).

låker', 169, 279 n.

lak'mouse, 2:3.

lame, -ê, -iau, libi-i.

laminto, 127.

landon, landle, lande, 166-7.

làni. nâli, 4,310 .

làrèce, 313.

laıjat, 19.

Lanzelle, Law"jale, l. d., 18 n.

lâtuette (?), ㅁot. n.

lê, lècề, 8:2.

lerquenoux (?), 2.t.

lế. lè (loup), 121 11., 167, 205\%.

leîrin, $184-6$.

leuve, lenvê, 167-8.

le iิwă, 2:333.

liclion, niguion, :2.

lifer, ] (is-?).

lignuou, linot, 189.

Limboùil (Limbonrg) 1:3.

lince, linclue, lise, léze, 1 (69.

livvèhåye, 8:.

lodier, $25 \overline{5}$.

locte, loïéte, 172.

loie, $168 \mathrm{n}$.

lol'ain, -ainne, 25!) n.

lotclièt, 156.

lotener, 170-1.

lotia, ]69-170.

loton, lóton, louton, 170-1, 32.2.

lonette, louwète, 172.

loin, lourd, 168.

loite, $172 \mathrm{n}$.

lovier, $167-8$.

lovå, 17 .

lôyerèee, 313.

luhin, ruhin, 211.

lịrê, lureau, luron, 172.

lurer, leurrer, 172,

lustih, loustie, ⒉2. liile, 10:3 11 .

huver, lover, $167-8$.

mả, ma11, mons, 4-.j.

macerel, malchuria, mak"riau, 181-2.

nitulierèce, :31:3.

magnon (= Mayon, Malion), 173, 175.

maget, matgrmme, 11: I1.

mångiỳ̀te, jo; 11.

må-leùle, $1+5-6$.

malıi, 17t, :31:3.

maille, -e, ette, 176 .

maillenter, ] iti.

main, mais (= mahaing), 2s:2 n.

make, ]77-s.

maldhiere, matsoire, 2.5.).

mal-gneiye, mal-rljeve, s..

man'ei, menacer, 7 to.

mancte (?) $17: 3$.

manote, manoke, 17:3-4, 190 .

manowe, -awe, 175-6.

milnser, -11re, $174-5$.

manthe, -itehes, 17.5-6.

mareschaturer, mascåcer, j1.

marne, malle, mòlex, s.j.

แกลำชj dint, 182.

IIIsssur $\frac{1}{t^{\prime}}, 15 \%$.

matchre, matchurnia, -rna, 181-2.

mauké, 8‥

mareté, -er, 17 ;

mazagrawe, 2023 n.

mazindje, mésnge, ]7r,

mèhin. me-hain, 91, 2\$2 n.

mèhner. mèn'her, 9] n.

mèhlèle, 1 r6.

mène-ik, mèn'zik, $17 \%$.

mellil. -11e, :26s.

mentise, -nisse, -11ze, 17(j.

mès-, mèrkenu'e, 1 (jt.

menustin (?), :26!).

mente èt make, 177-8.

meiriant, moriant, 17s.

meive, meule, 15!).

michleclape, 41 .

migot, ere mijoter, 112-113.

mirou, $17 \%$.

mótier, mortier. mourfeyi, $115 \mathrm{n}$.

mohon, maison, 92 11 .

moisir, 180.

moki, -̀े, motehas', 182 n.

molàvint, 82.

mon, a-mon, è-moll, 92 n.

monesale, +9 . 
ntous, malls. moins, :3.

montelis. 82.

mon l'atronme, 298.

molifrin, $181 \mathrm{~m}$.

moufti. 180.

mouhi, mouhin, moủji, 179-\$1.

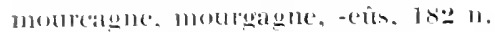

mugnt. muspode, 112-113.

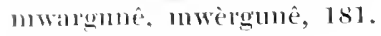

mwènine, y1 n., 181.

maif, maif, maifver, 12.

năle, nasle, mastel. 4.

nålî, 4, 2216.

nateuriau, $1+1 \mathrm{n.}$

havé, -rye, s.z.

nawè, novall, 1k2.

nèçale, nacelle, 4y.

néromant, nécromancient, 122.

neple, 17! 11.

netirece, :31:3.

netkufurmet, $830 \%$

neir et make, 178.

netrin. 1st-6.

nigot, $11: 2 \mathrm{n}$.

niguion. 1 (ii) 11.

nile, 11i:3 11 .

nivit. 22.2.

nô, now, notle. 1 in n.

moultere, mile. -eye. 1633 n.

nwalahic. $2 x: 3$.

wille, mille, $16 \mathrm{il}$ n.

oirzelle, orze, orseille, $180-3$.

-oje (- -iline). 12.

-ible ( -ilble ) (i, 1:3(i-i.

olvive, orlive, 119.

omber *motion. 5.

(m)

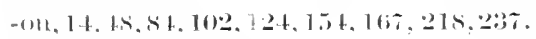
ancleme (2). 205, 11.

(1)1611), 16it.

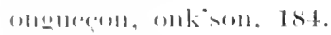

(1)110in, in.

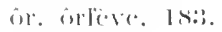

molia, 25 .

oncillettr, oriliete, 112.

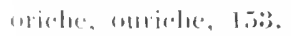

oria, oringuer, 1st-8;.

wimlje. 1,5 11.

mlarle. I1).

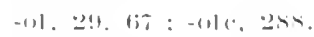

116./1, 1. 15\%.

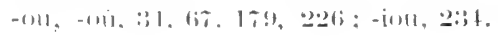

-oile. 253: -joile. 209, 254 11.

oubouye, 186.

othê, risean, 19.

oulote, :3:2.

ôtr, ourláge, oumli, $25,15 \%$.

ourderet, $: 312$.

ouree. 1 19!.

ourete, 150 : ouron 322.

ortit, -iats, 151.

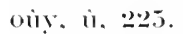

-oye (fr. -onille), 104.

olivet, 187.

paleatre. 8.2 .

pålıtıle, -ûre (juinible), 1.57 n.

pailleromx, poilleroux, 18 s.

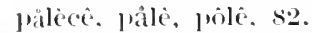

jame. prannat. penió, 164.

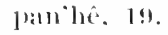

farforece. :31:3.

parion, parom. ls!.

parresce. :31:3.

pasturact. :31:3.

ja) wente, 172. 190.

peluerere. $31: 3$.

pèkène, 191.

pèlain, plain. pelaine, pellin, 192.

pèl ${ }^{\circ} \ell^{\prime}$, péleriatr, :3:3.

fencis, pèm, 18.

beri, périr. 191-2.

pèri. pwari. etc. (poirier), 20!1:3.

pèrlôlje. …5.

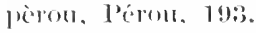

pirtainne.pertontaimne, pretantaine, 20 ?

pìmeye. -ive. 20.5.

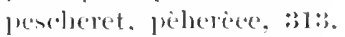

fete, leatutre. 264.

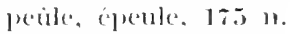

phisiquer, phinghinerie, $95-948$.

piendi). :3:2:2.

pirlje. prege, fricrece. $251-2$.

pilaine, 19:2.

piler. pilerial. 195.

pircul. l'icrotet, 19:3,

pircuetta pirwitele, piweyc. 205.

pit-it-bole pied but. $190 \mathrm{ng}$

piter, pricter, 17!) 11.

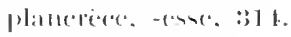

pliterere, : : 1.t.

plat kizak. plake\%ak. ete.. :30r.

plarere. plasic, plesin. : :1t.

plek, plet. 10:i.

plonisure. 
ploki, phokerene, 314.

phoperou. plorion. :31 11.. Is:

ployin, +111.

pi) (ןख⿰丨: l'aul), 31. 7!.

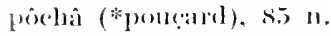

poke, -ete, poteliet, ploke, 15i, 2060.

ponte èt makr. 17s.

ponteris, s'z.

pupionle, pepponic, 20.5.

posiete, posisile, i!!.

purtelle, potelle. $19+11$.

jutile, 1913-4.

potchtrèce. 314 .

pote (main -), 190-1, 194-5, 3202.

pote, -ci, -alle, 19:3-t.

pote. 112, 191 n.

potele, pot'ler, 1!);-t.

jot lete. 1!) 1 .

potepuin, potiket, 1:3.

peticke, 1:3.

pone, poe. powe, 172. 190-1.

protette. 19].

promgunte, 19\%).

joûhice, -ive, :314.

poibi, puiser. 180 .

pouteris, :36.

point ner, -ciole, :3,5-3;1;.

ponitrin, 12:11.

pove. poure. poule. 3:3, 159.

prat ler, prateule, prate, 195.

pralute, -cler, 19.

pribale, frihele, etc.. 1!5-6i.

primes, prunc, :30 11.

princhec̀, 1 (i).

prisiseresse, priscrabelace $314-5$.

privarct, 815.

purle. $19 \%$.

put, puterir, :36.

pwètånctel, s:3.

pwetrece, 315.

qualisier, rainsier, 6i.

quasse, lit 11 .

quilaine ("), quitainc, 40.

'pwanse, -i. -eler, 65 11.

rabaterèce, 315.

raboterèce, :315.

racler, -ette, $197-8$.

raconsulive, rak'sure, 197.

raso, 202 .

manembe (!), rabluendre (!), 197.

raljoute, 20.5.

rallî, 200). lakète, $197-5$,

rakince, 1!s-!).

rakion, I!s 11 .

ramalljive, tho.

lambeller, -is, rambinders, s.

ramelje. 19!s.

ratmonke, -

ralunde, $1919-2000$.

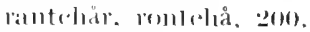

ras-, 19 !sis-!).

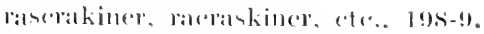

rasgoter, rigeter, 19s-9).

rankignou, mosigmol, 182.

rassirece, :315.

ritaller. ill.

råtullì, -ill, $20(3)$.

ratcer, ratioi, 201.

ratonserter, 25.5.

jatro, ratrosi, 201-2.

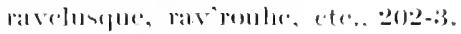

raw witc, 20(13-5, 2:39).

raverere.

rivimue, $17 \mathrm{n}$,

rebion. jimbion, 210.

resater.

reciviler. ecurr, ti.

reche (sortir). (9s).

redl, 20 s.

reguilite, riglete. :3ors.

remulyerenes, 315.

rellatr, rollan (?). 20,i-ti.

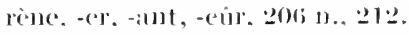

rellace, rallye, 199.

renguillier, ringui, ringuion, 2211.

foni, -i, -ill, 20.5-8.

rente. inorte. 204 .

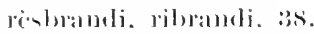

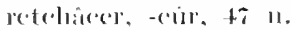

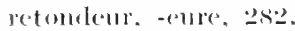

retroki. -incer. eler, :;2.

reude, 201s,

rilipe, er, 12.

reveler, -eus, 18.

rev'louhe, revilie, 2002-3.

rèvioules, ré-, ri-, ro-, 208-!l.

rèvole, $37 \mathrm{~m}$.

l'ewioniles. 20!9.

rixine, 1!s.

lihmuterère, :31,

riciveler, 17.

ricoti, $i 211$.

ricomblemeres. 
rieourerécer, ;3]

riglèter, $; !)$

rihărher, $\hat{e}$, rihaurlie. 1 to.

rilure, hirire, 20(1)!

rileverèce, 315.

rimulje, ète, 1 !ns.

rincterece, 315.

ringemler, э0 11 .

linkinkin, 20101.

rilitelàn. $20(1)$.

vitaliter.

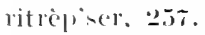

liule, rible, rile. $25 * 3$.

river, 169 (1).

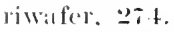

romets atrins, 206!l.

11) lomf, 23!).

rorlje-hetelt, rodjex-dints, 2222.

rolinne. 16i 11.

rimunkirils. 1:Bs.

rimil. 206 ; 11 .

romi, rogni, 2006i,

ronke, ronle. romelne.

roti. monti, l12.

roton, 171, :32.).

lomli. -in, -is: 2211 11.

rumljeter, romljemr, 20!).

mollill, -is?. 210-1.

musiems, rouvin, 20(1)-!).

mate. 201.

rive. rale, 25n: rovete, 2011 11 .

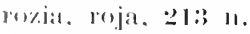

rurcimelele, 17 .

xulin. 211.

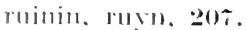

mulane (!), rulise. $\geq 11$.

rilant, rilammint, 212 .

risle. ricule, $211-2$.

rimathl, rimammint, 221‥

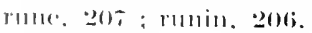

lille. $21 \geq$.

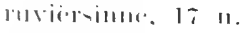

silciale. H!).

ratelitl, $11 \mathrm{H}$.

sailaturlin. 22-2) 11 .

saleriece, :31.5.

silluer.

simere, 17 11.

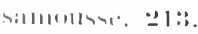

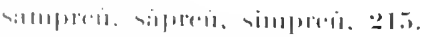

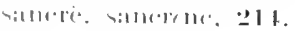

si1he, -iv, 211.

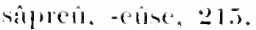

simbjete. sarriette. st.

sirtete. 119.

sisurterece, sortrèce. 31.5 .

savène, 18\%.

savené, savellel, 2016.

salwe, sowe, 220s.

shaterece. 315.

s'at. scate, lat, 1:3:3, 13+ 13., :35:3.

s'adria. hadrê. 132.

salve. lasse, 160).

soleyi. 2lit.

scleyon, spleyon, sployon, $21 \pi$.

scoket. eter, 156.

scotelie. sootelua, 155.

scot. ecot, 305.

scoudro, 299.

scrène, skine, skinon, 1+t-s.

seweri, sewerer, 2ut).

a (a te -). 2.18.

sclanloran, selombran, s:3. 2217.

semale, single, simgle, 50.

rerelerette. serelemest. $: 31 \bar{s}$

serer, s(ma, 2.5!).

sèron. cèront. $21 \%$.

set". sept. $22+4$.

seiils, sciul, str. $2 \pi$.

si (lat. sic). ㄹls.

sik, si fue. 221k.

sindrène. sruclerience, 201!.

sillewe (lat. simenulum), so).

sirece. :310.

sizer, sizeli. 2 (iti.

sizet. siz rex, 2000 .

sizin, sizner. vizin, $21 !$.

skeshalik, -it. 2200 ).

stèri. 22001.

stite, hete, 1:3:3 11,

-mante. !1, 2:11.

smitr, -î. 17!).

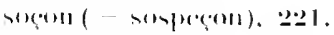

solur. soule. 2.21 .

solic. -i, 리.

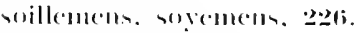

solice, solse. sti 11.

saluleis, soutlis, 2.20.

sologne. reledirloine.

somamelin. 22:22.

solldije. 202:3.

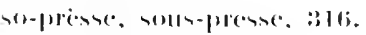

(1)

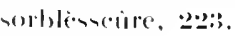


sorderesse, sordrense, 2027.

sorsèyemint, "2006.

sot-dwermint, 2204.

sotré. 22.5.

soû, st̀ (seuil), 20.2.

souder, -corr, -resise, somdre, 2207.

soulic, soullic, :206i.

sourbate, -lure, -in, 220t.

somrueillencent, 릉.

sourtonture, 202.

soustre, soutrer, 20.7.

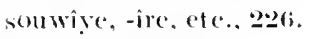

sowe, salve. 202.

soyemint, 2026.

siyen, $11 \mathrm{n}$.

suyol, 22:ti.

spåner, "2:31.

spaté, 91.

spèlıc, 1!96 11.

sperial, 208 .

spèrwitelue, 295.

spete. spiate. epreatute, 2064 .

spicer, spir, 2029.

spinc., -il, -âte, 2020-2:31.

spindje, -i, 1:3, 15.5 n. ; -erèce, 110.

spitant, spitant, 179 $1 . .232$.

spôcle, spôrelia. :2:3:3.

spongni, spomgneter, 2:31.

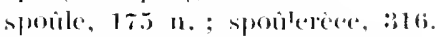

spricatwere, 2:31-2.

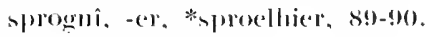

spoher, s8, s!).

spruwicî, 20:32.

spure, -cl, -iir, 2028-2:30.

sipy. 209.

Sitipunoul, 25s.

stchèrlon, stierran, 20:32.

st thal, :30.

stesint, stensant. 92.2 .

stessiner, -rèces. (1).

stenle, steùye, 1.is).

stiène. -i. -ìye, 2:3:3.

stif, 口:3:3.

stinciin (!), stinclin, 2:3:3.

stitchli, stit(lon, 2:3:).

stivillz, 80.

stoke, stutchèt, 1.it.

stopresse, 316.

strambion, strombion, ㄹlo).

stringuion, stronguion, :210.

strumne, 175 n.

streme, :30 n, ; strumer, H. striler. -eler, :2:3.t.

strifion, strivion, 2:3t.

strin, $6 !)$.

stritrlıî, Iris).

stroiler, striler, strikar, $16: 3 \mathrm{n}$.

struvion, strivion, 23.4.

strject, :005.

sis (ell - de). 218.

suweraite. :316.

swelih. 2:31.

talcliat (*tarisial), 14!).

talıant, ㄹ:3is-ti.

taloull. "2:3ti- $t$.

tahource tahurial, tallumenal, 182.

tai, $2+1$.

take, tahe, 5ti n.

taklin, :2:35.

tallawète, tènawète, et( ., 2:35-240.

taperet. tapereere, 310.

tal)kène. $2+4)-1$.

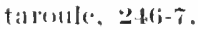

tasis, 24ts 11.

taluye. toye, 22ti.

tawes (le- -). 1. 1., 2+1 11.

tchate, telacant, tchakè. $48-49$.

teluakine, -er, 4.8-19.

trolakter, -treces, $48-4 \%$.

tallal'me, 2.t1.

trhảlêtis, 119 .

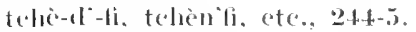

teliedjereces, $31 \%$.

twhètre. telièdront, t:3.t 11 .

trhekete, $2.5: 311$.

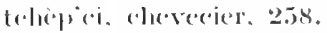

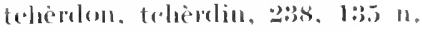

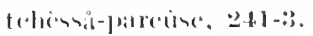

trhense, fhisse, "2t:2.

tulicti, -iat, :342-5.

tuliegit, 2t:3 11.

trliti. 81, sez.

trhivise. eheville, 81 .

tehomer (?). $11 \%$.

freloguerese: $: 31 \%$.

trhuberlire. :30).

teluizi, tohise. 27:3.

techoll, tiolinis", $2: 35$.

telitil, z:sti.

tol foin ent. telfeye, 247.

time (_ tem), :30

tentellx, téreitr, 2t.s-6i.

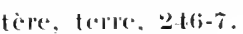

tére. tini, :201. 
teris: teri (tomil !). 245 11.

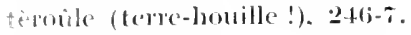

terversicr, 20.5s.

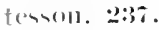

true, teril. 217.

teille, teûse. 1.59 .

triver, tivozi, trifoze, 245.

terereace, :31\%

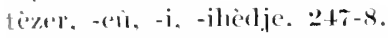

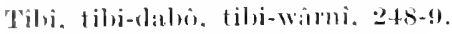

tidje. tiener, $2.50-2$.

tif, $2: 3: 3$.

tihe (ticurlue, tiois), 2.jo).

tike, tikete, tiglite, 253.

tiluete, tinlète, $2+1$.

tin-lomlin. 260.

t-iulini, s:3.

timle lenue, 2:30.

tirci. tieleoier, 25.

tire, tire, 17.254.

tirelote. 247 11., 25:3.

tiroigue (?), 2.5.5 1 .

tiroule. 2th-7.

tiule, tuile, tille, tile, 25:3.

toaille, tomaille, - ette, ezt-ti.

torallon, touillon. 254 .

toëtte. 25.5

tofier, tut-fer, !)

tomblerienes, $: 317$.

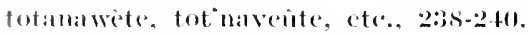

tot liet. tometelet. 51 .

tomenom, tougnoile, 25t-(i.

toulasse, $2.5(\mathbf{i}$.

tomplequin, 240-1.

tommiriam, tommiam, tomrniyam, :301-2.

tolise. - el, -et, 281 .

touwi, tuxill, 172.

tomi (tomilla), tmyeter. tomyon, 2.5t-5. toric, 2st.

traherliye, 110.

traili, 2010).

traveraine. 2.5!).

trawiye, fromir, 170.

tri-. 2 (i).

trierin, trencems, 2.5s.

triflere. -emint, 2.ing-7.

tribes, is.

tripler. tripingr.

trifisin. -i. - $\therefore$. -inne $258-9$.

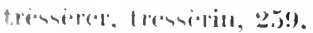

trevilye, 20(i).

trevericter. trevint. trivint, truvint, 2(io.

trîh, :2::.

trin-bèrlin, z(jo).

trintehe. -î. 201).

'Pripsie. 1. 1., 2.:8 11.

trivie. trivicuser, 25.

trio (triu), $: 31$.

trmalale, tromde. 261.

troulle. -er, cil. 16is 11.

tromm chat, :300).

trîler, trîler. troiler, 1 (i:3 $\mathrm{n}$.

trimian, :302.

trite. $2(i 1$.

tumiote, $: 302$.

tupin, $2: 35$.

tuteri, tutri, :317.

twagne, 254-6i: twatagne, 25.5 1 .

twezon, :201-2.

ilie (ilot), 1!), 2־1.

mumses (!), 2ti2.

mite, 1.50 ; mige. 149.

แwo. 1190. Yuyo. $18 \%$.

viçale, valucele, .30.

vail. "20:3.

vairié. vårịi. -er. 280.

valusle, vérole, zos).

veature, viature, viamrices, 20(3:3.

verei (ir.i). $27 \mathrm{t}$.

vielje, verenter, 16 t.

vèhice, viosère, zili.

vèhin, vèvin. vèjin, 31: 11.

vejinauve, vijnanve, 2mis n.

velle, 20 (j:3.

veluel. 28:2.

vidlour, verbo(k), 28s.

vere, ville, vaire, $201 ; 2-4$.

verefe forece.

vêri (vềri ?), ״̈si.

verual, veruse veluer, entis: 16it.

versalintse, $17 \mathrm{n}$.

verwile, 29!9.

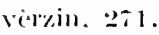

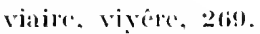

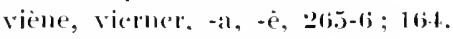

vilienc, vihner, -ahe, 로․

vili, vicillir. 98.

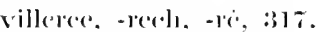

vilwè, vilwè, zoir.

vinalje, vismage, 2068 11.

vinäve, -ilble, 26t;.

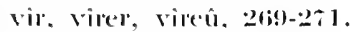

virelire, vièrlìre, $27.2 \mathrm{n}$. 
virer, virler, 27:3.

virlilı, virlih, 프.

vivătoìs. s:3.

Vice. 271.

vonoll, vonowe, vonemmint, zosis. vôster, -eiter, 101.

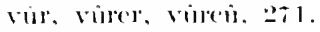

vilise, 272-:3.

vwes, voix, :7:3 11.

wall, wale, 1 t. 11.

wadje, watche, 27:3-4.

wafi, $27+4-5$.

walle, walghe, 205.

walgucte, wagate, 2zisti.

walhe, 1!), 285.

walsete. watehere, whiote. 2zis.

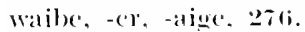

walde, welle, -i, ב-7li-s.

wainis, winnis, 20.5 1.

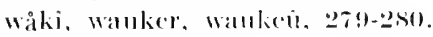

Wallin, 1.58.

wall, wallel. 205.

wamdic, wamdilse, 280.

wameliun, 142.

walleal (!), 280.

will reces, :317.

wal, wale

Wallia, wellia, 25l 11.

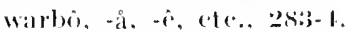

wånder. Ealeler, 7t.

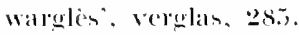

wammaye, wermaye, wămalye. $11511 . .25 .5$

warsele, 18:3.

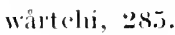

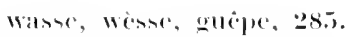

wastarde, Worsterl, 10.

wareal, veleweal, etc., 2x-2.

wayime, gaine, 2x.5.

we. wayi, 20.5.

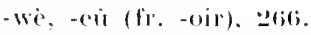

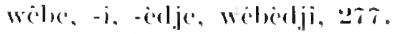

werle, 100 11., 2zij-s.

weke, witrhe, 2us! II.

werlt, - 283.

werbi (?), ״-7.

werbiyol, בהt.

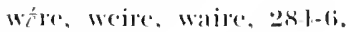

weri, 288;.

werleit, welleit,

wêulles, 200\%.

wenti, 2-7!).

wetremle, wetterel, 2057-s.

wilatl, 20st.

willete, eote. $20 \mathrm{sis}$.

wilgot, wiot, wiye, wuyo, 1 siz-s.

will, vain, 2.85.

wipplete, 2ֵs'.

wite, wike, exil.

wiven, viven, vervelu, 285.

wivente, 18 t n.

-yi, -i, -ir ( ir. -iller), 52, 5i, 118.

Yण्रण, $187-8$.

yul, :3:3.

zuline, zingler, 289.

ziverkif, 2 !)

zolile. 2011.

zumilicl., 2:3t. 


\section{INDEX ANALYTIQUE}

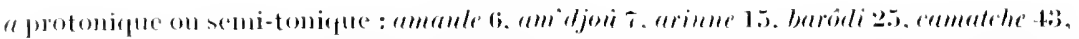

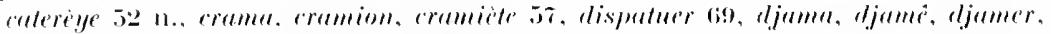

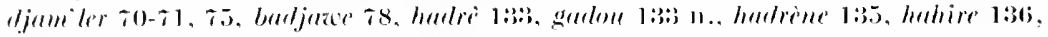

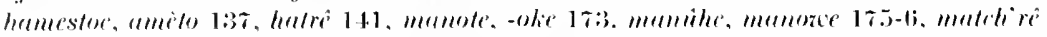

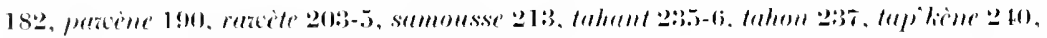

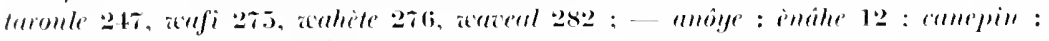
lientrin +t, an'bo : himbi b.

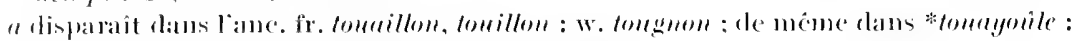

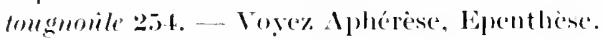

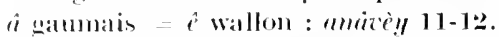

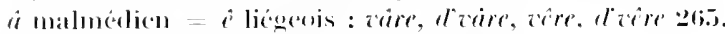

Arectue pris substantirement : si 218.

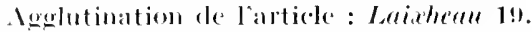

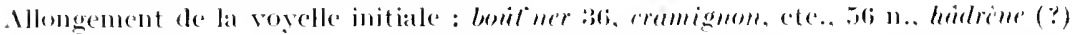
$1: 3511$.

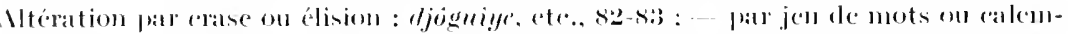

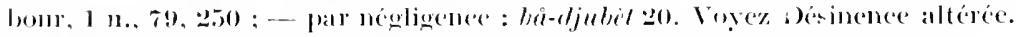

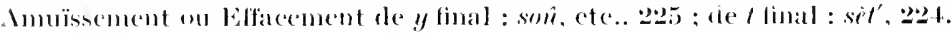

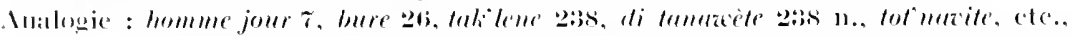

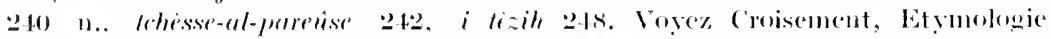

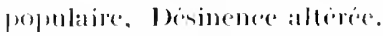

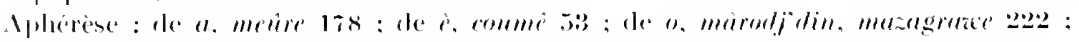

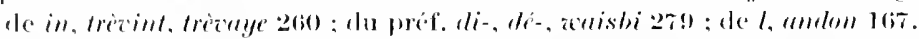

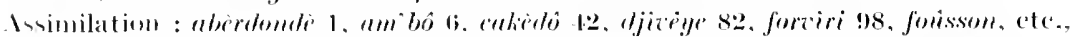

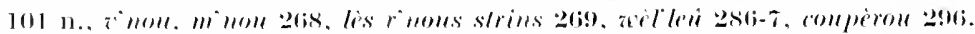

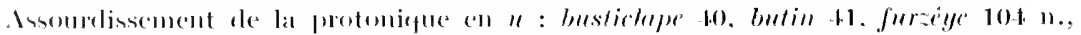

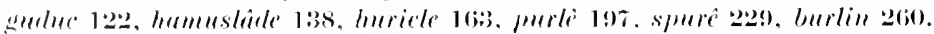

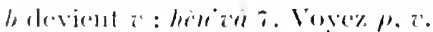

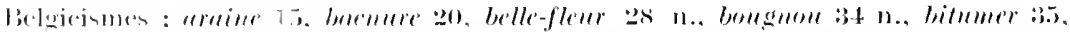

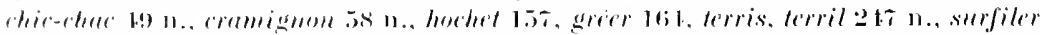

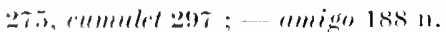

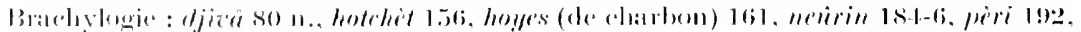

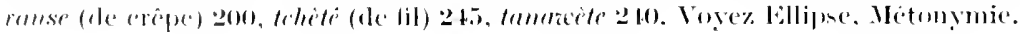

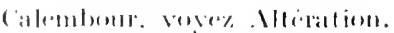

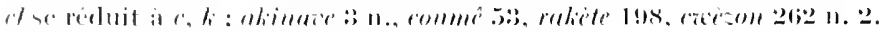

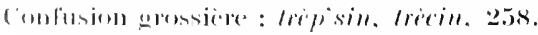

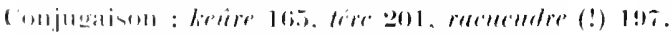

(intamination, vere\% ('m)iscoment.

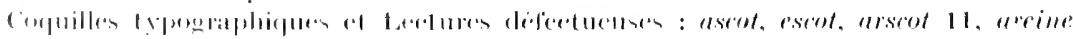

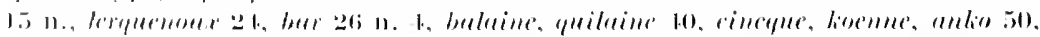

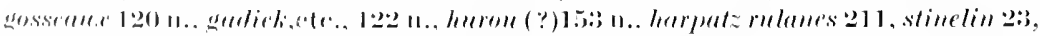

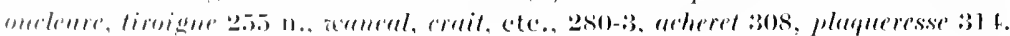

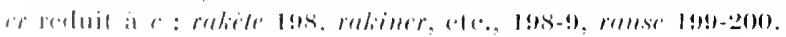




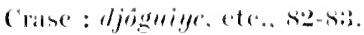

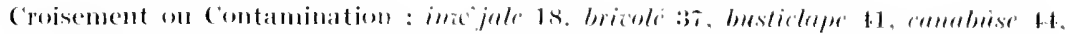

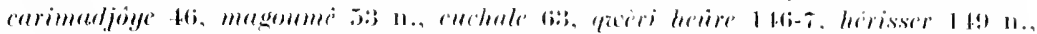

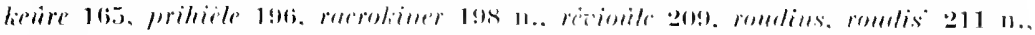

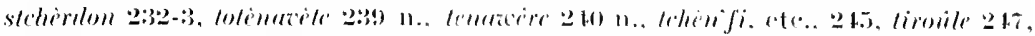

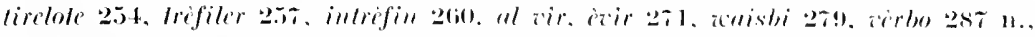

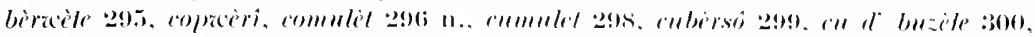

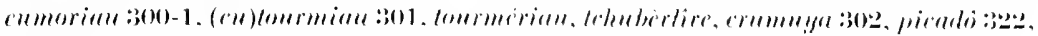

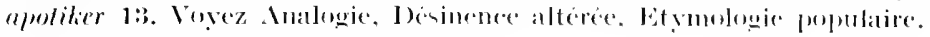

eac devient toc: : 20 (i).

ereer-vicut du latin cos- : (it, dit.

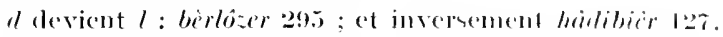

berivation, vogez sullixes.

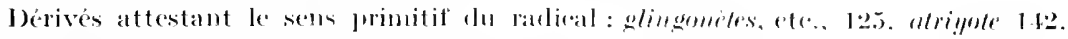
lonlyol, etr. 10;1-2, acogneles $275-6$.

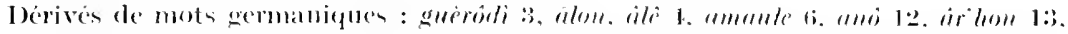

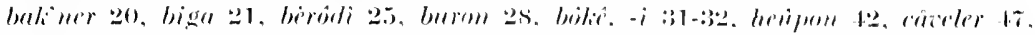

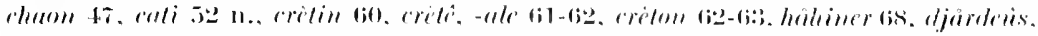

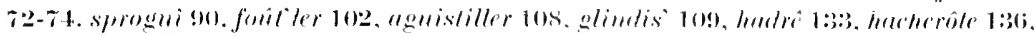

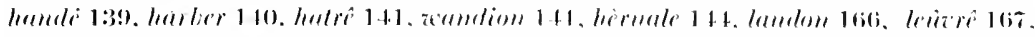

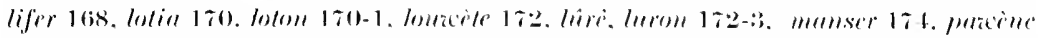

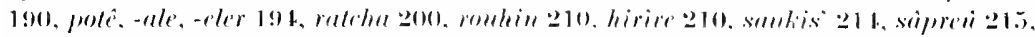

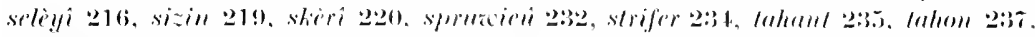

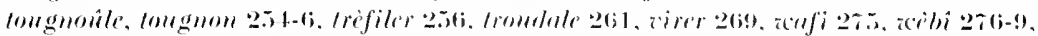

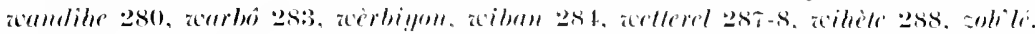

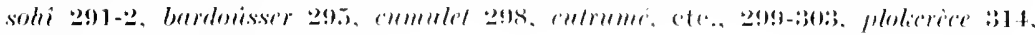
avinice $: 31 \%$, hirot $15: 3$.

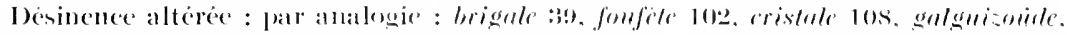

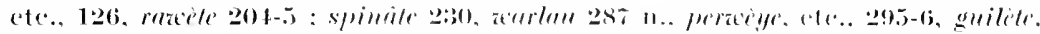

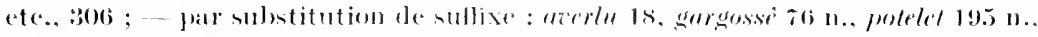

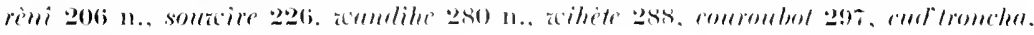

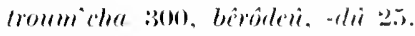

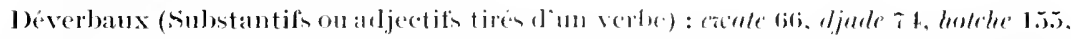

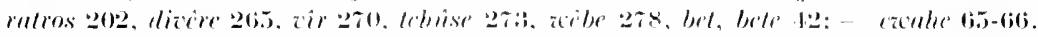

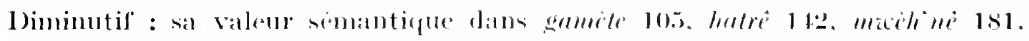

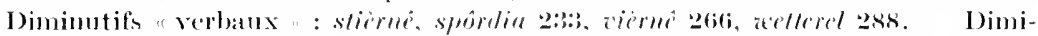

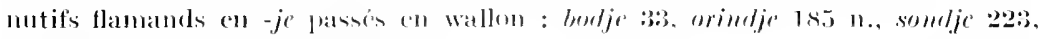

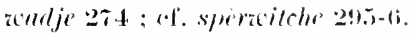

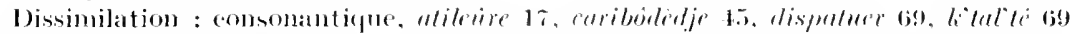

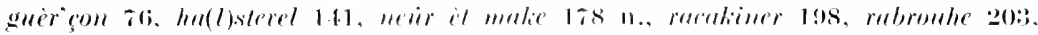

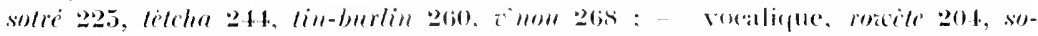

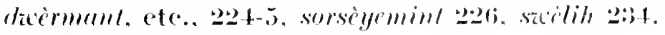

alj, voyez $g, j$.

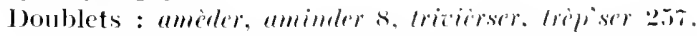

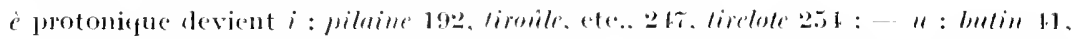

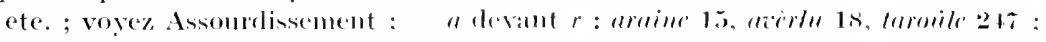
(cf. zâre 26.5 .

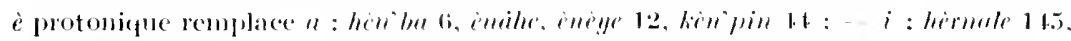

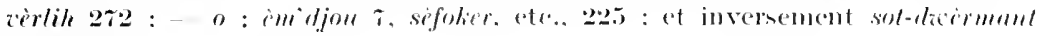
etc.., $2 \geq 4$.

if long protonique se matsaline : stimelin 2:3:3. 


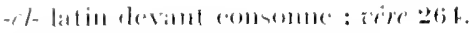

Bllipe, veree Brachylegie.

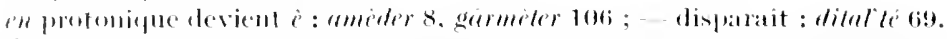

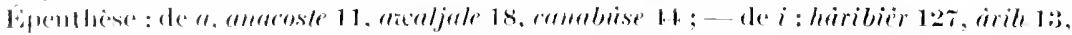

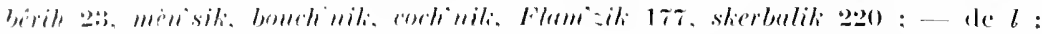

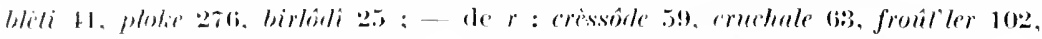

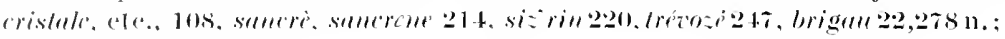
de 11 : achermile 1 .

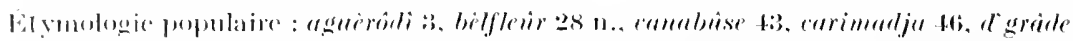

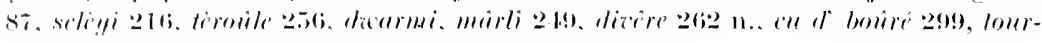

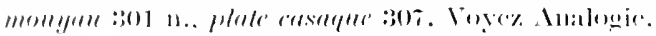

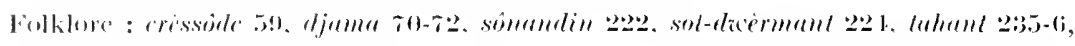

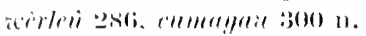

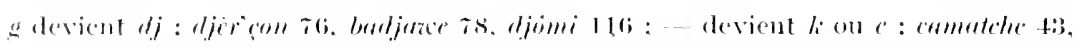

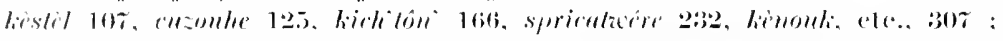

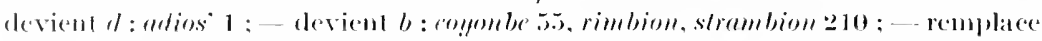

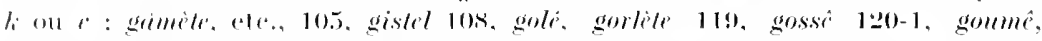

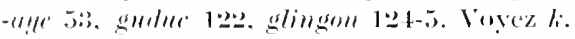

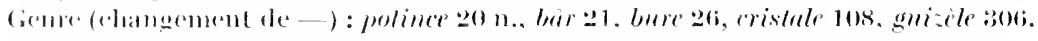

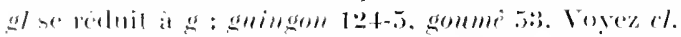

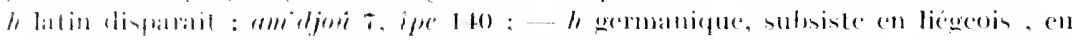

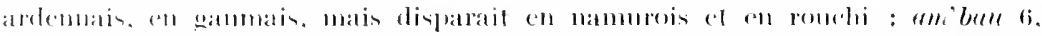

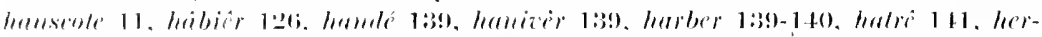

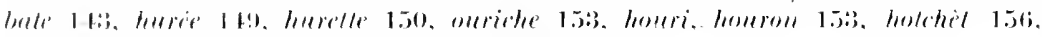

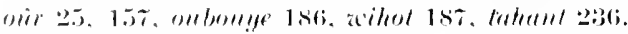

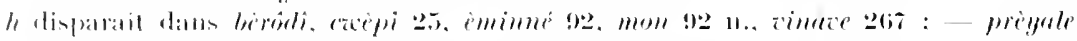
186; 11.: - rmirom $203: 3$.

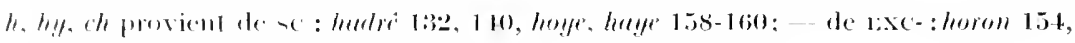

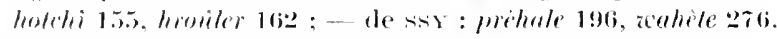

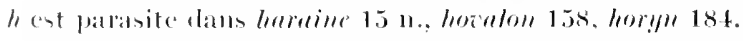

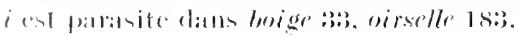

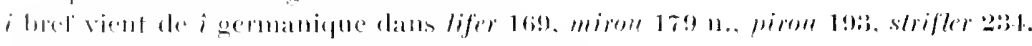

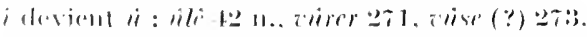

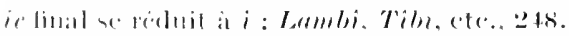

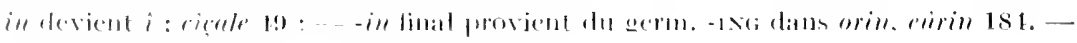
Vines Nabile.

forerion do (2)

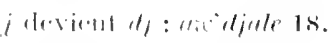

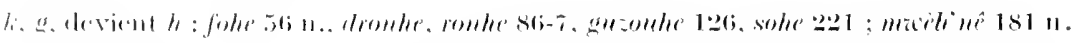
Virver.

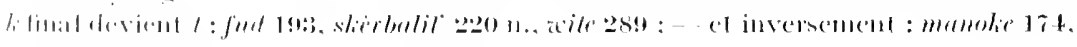
entilite 20.0 11.

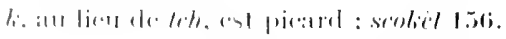

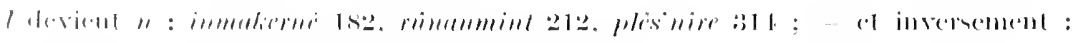

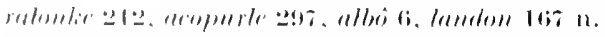

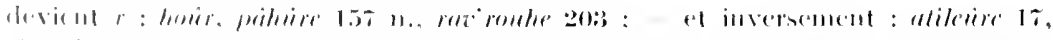

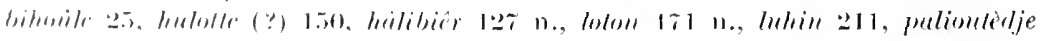
$1 ! 110$ 11. 


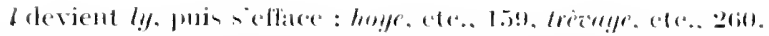

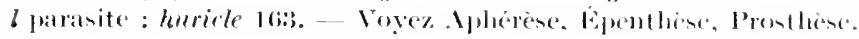

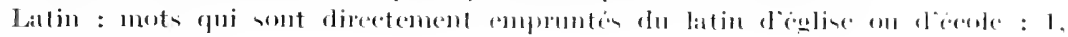
$2+9)-2.50,26(; 2$.

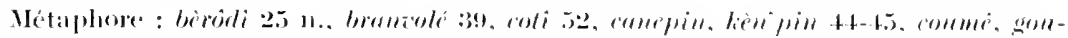

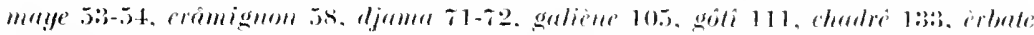

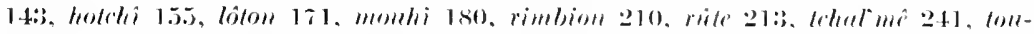

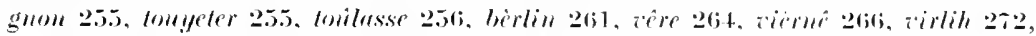

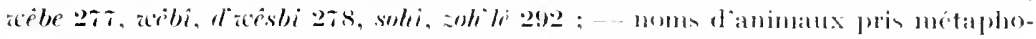
riquenent : 12111 .

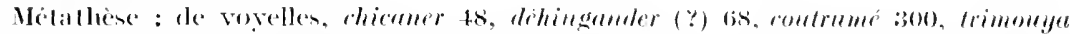

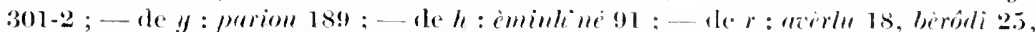

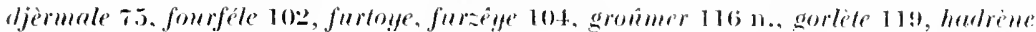

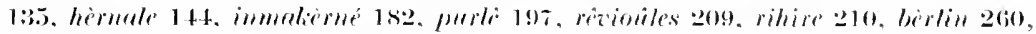

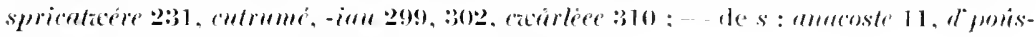

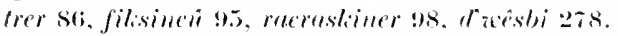

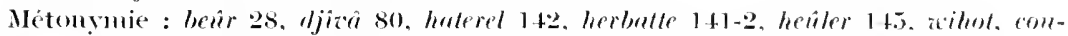

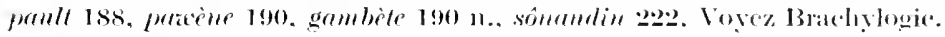

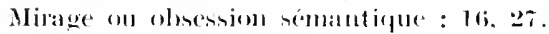

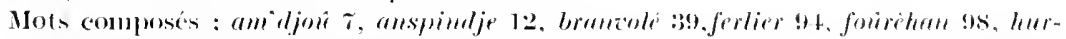

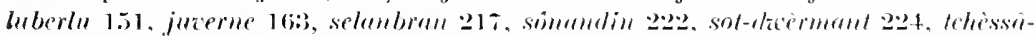

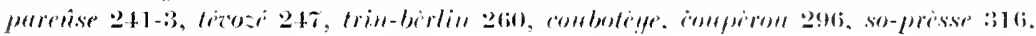

"r, vovez l et l'posthene.

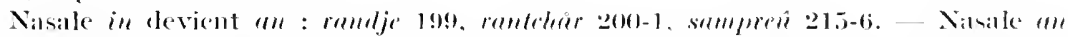

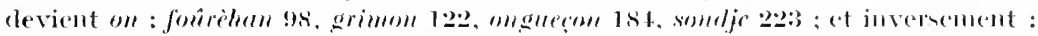

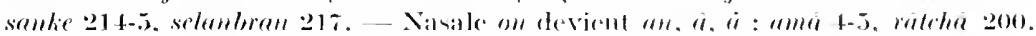

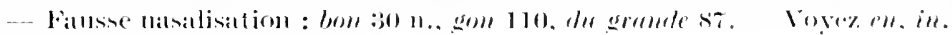

- ne devient -me: bleme $30 \mathrm{n}$.

Noms de liem, vorez Topomyunice.

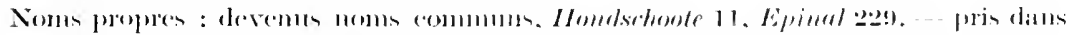

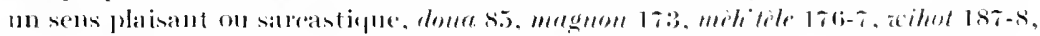

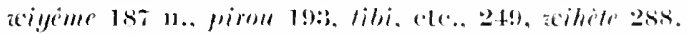

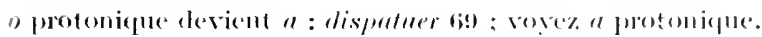

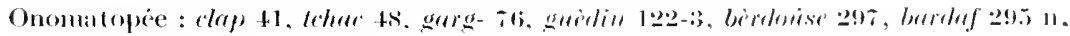

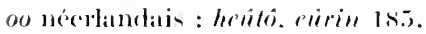

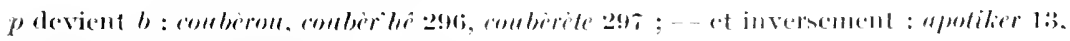
pèline 191. potituer :35.

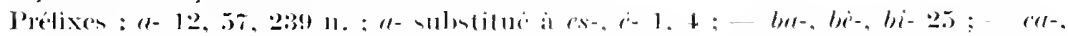

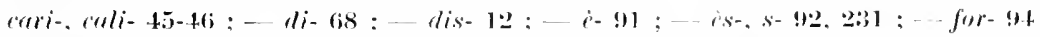

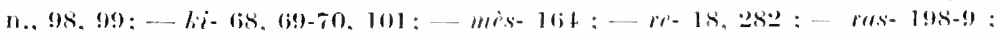
sor-. sour-, sur-, so- 22:3-1; ; - tris-2060:- germ. ge- 107. 305-7.

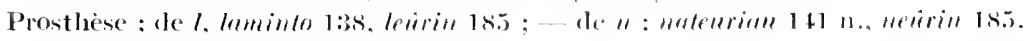

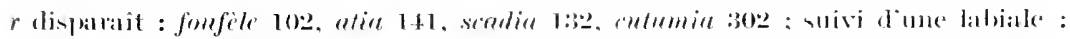

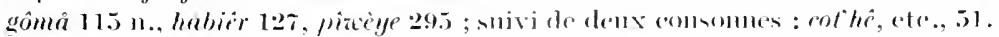

Voyez l et Métatlièse.

$r$ suivi de yod devient dj: prilje, tidje, etc...2.2.

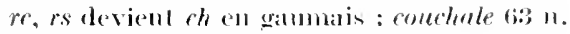




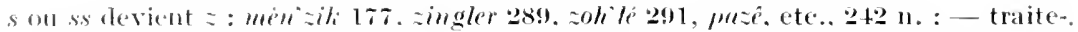
mcont dess. ssy, a' $190 \mathrm{n}$. : - traitement de se initial, latin ou germaniqne, abontissant a $h . h y, c h$, fch. efe. sc : 140, 158-4, 155, 156. 159: - s disparait dans le grompe germanique sinitial : lifer 169. lotia 170. lieker 169, 279 n.; su : mirou 179;

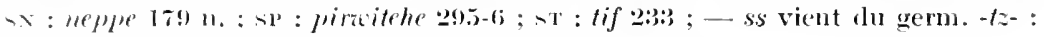
crassî j8, hossî $15 \pi$.

Sultixes germaniques -ima 135, -lie 177 ; -len 1:3. 191. 240.

sullixes latins, voyez pp. :32(i-9.

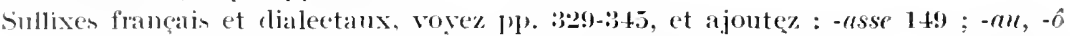

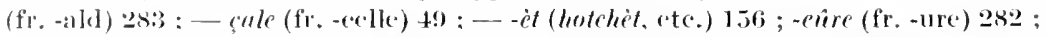

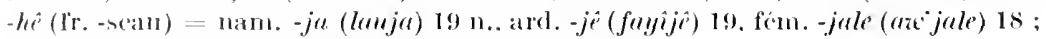

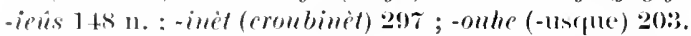

Syncope de la vovelle initiale : cronbèt 297 ; zgliné 289 : - l'une protoniçue non

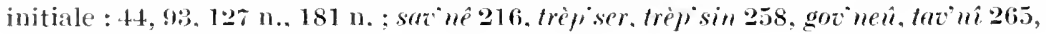

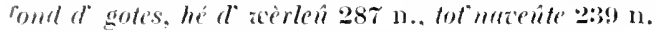

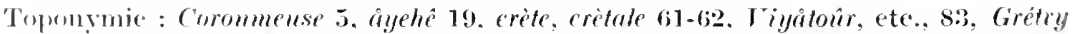

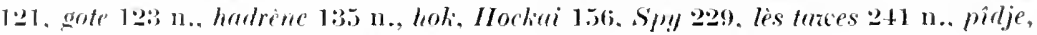

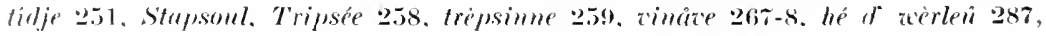
(lowsties :30!).

"devient i spricutacere :2:32.

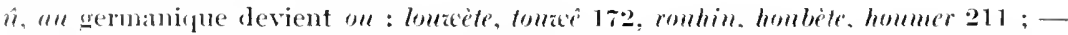

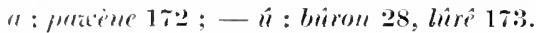

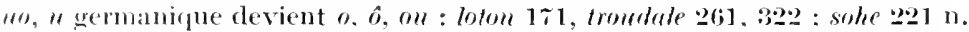

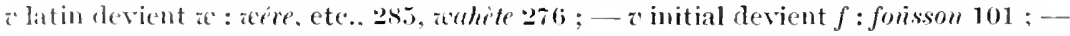

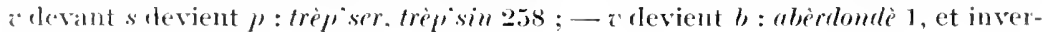
sement : himizi

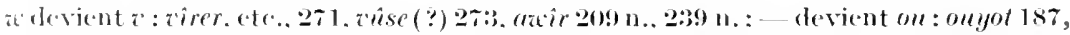

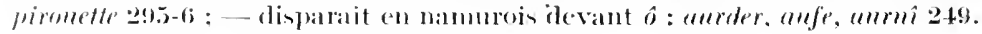

a batin devicut ss: assi, etr.. 196in.

y timal dispallatit : soli 20.5 .5$.

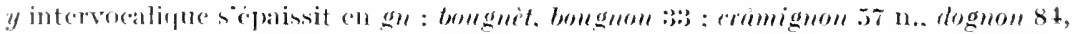

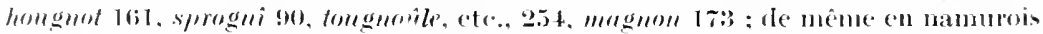

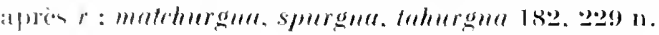

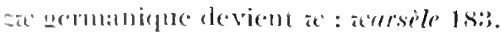




\section{TABLE DES NOTICES}

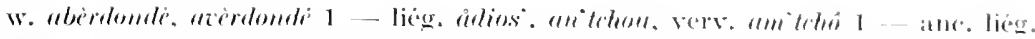

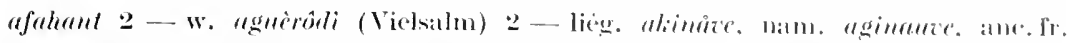

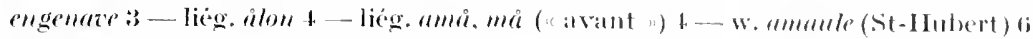

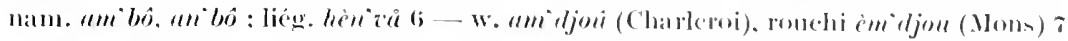

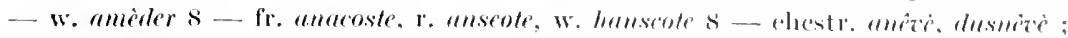

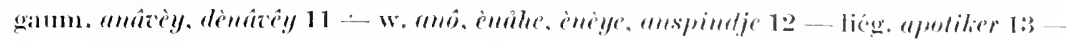

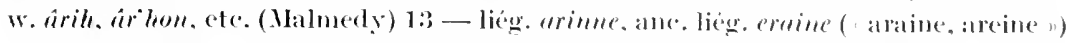

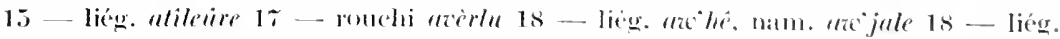

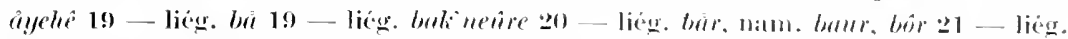

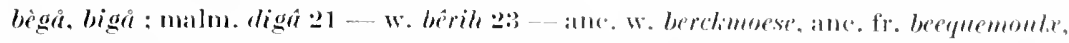

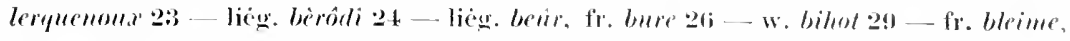

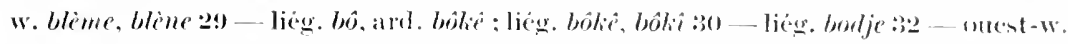

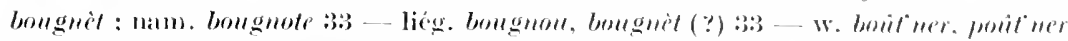

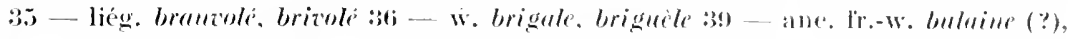

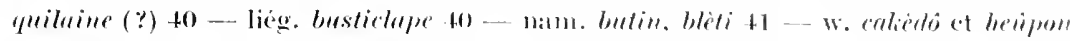

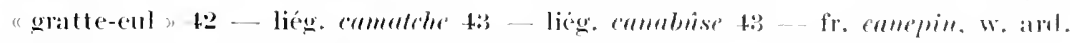

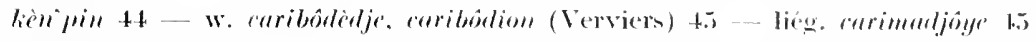

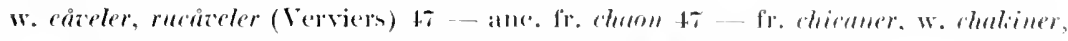

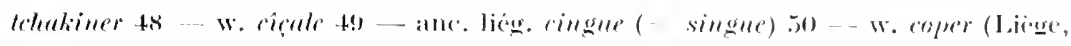

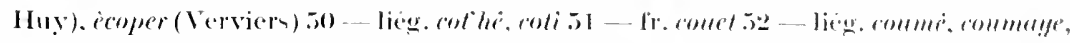

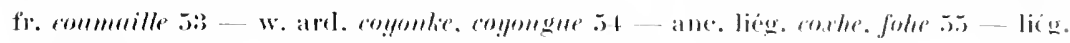

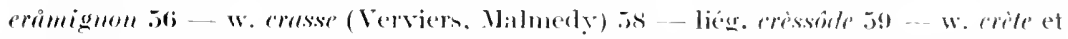

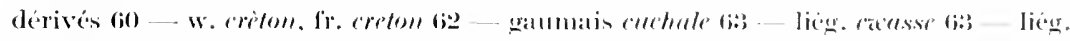

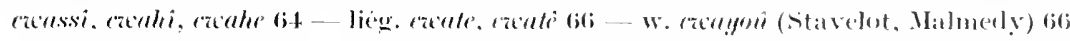

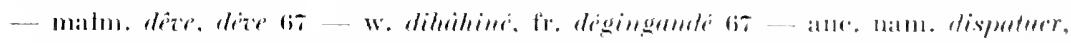

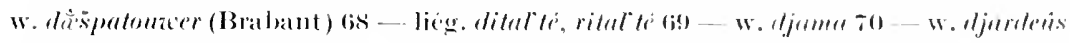

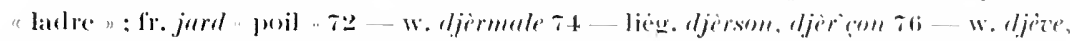

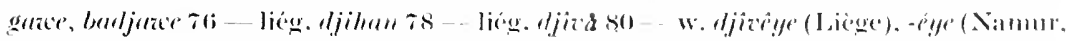

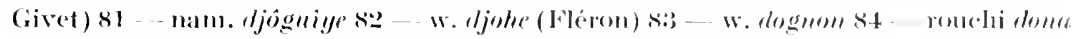

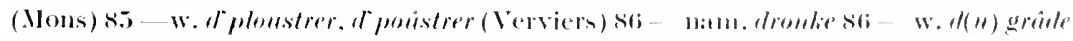

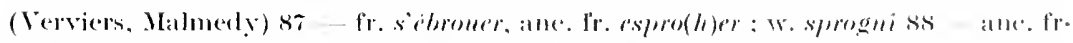

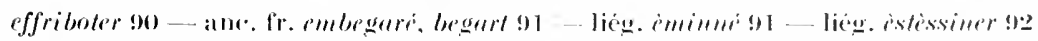

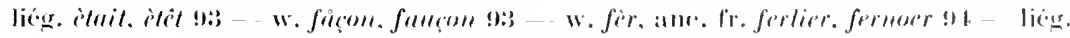
fislimen, filisined

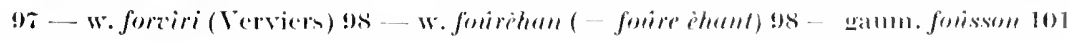

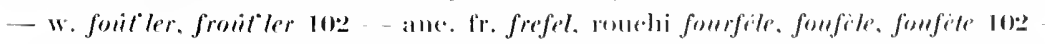

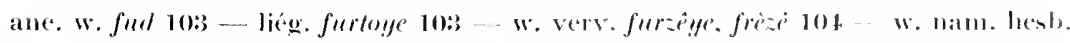

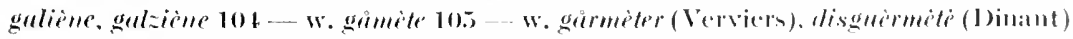

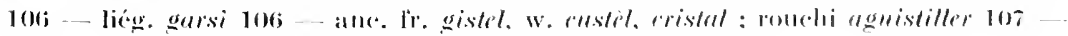




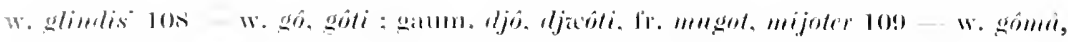

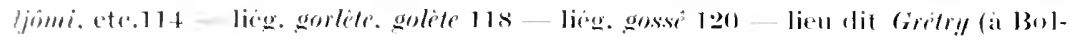

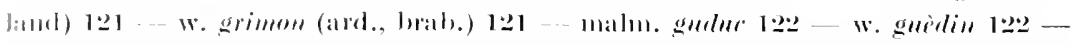

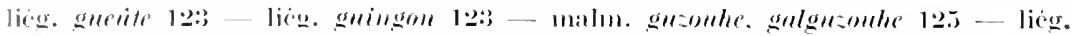

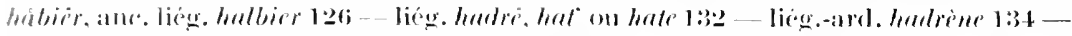

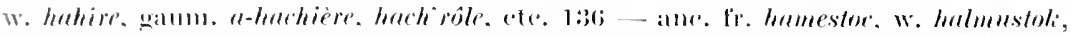

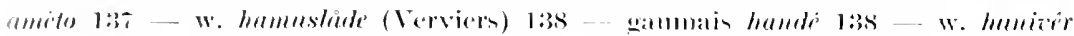

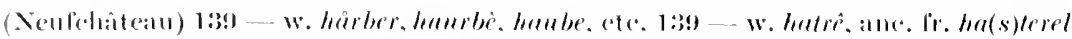

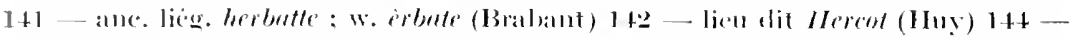

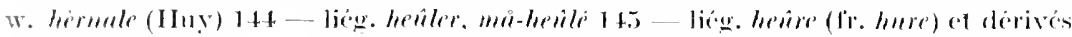

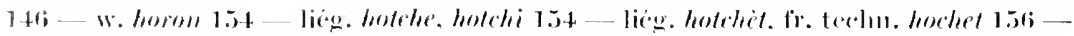

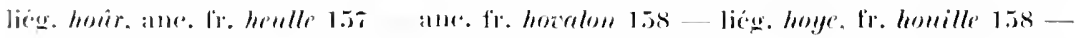

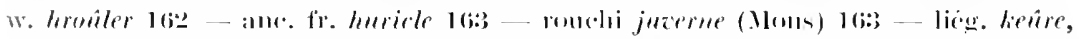

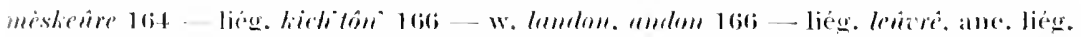

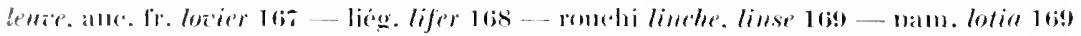

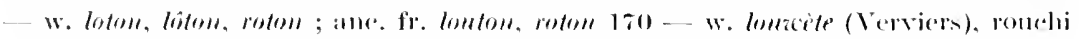

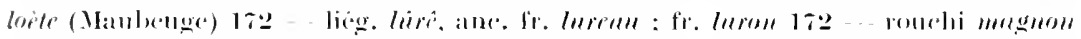

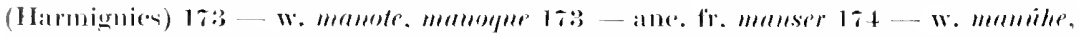

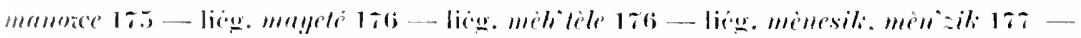

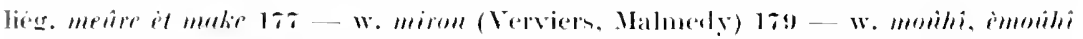

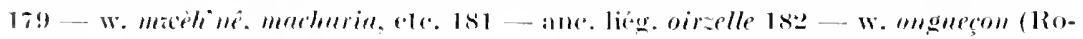

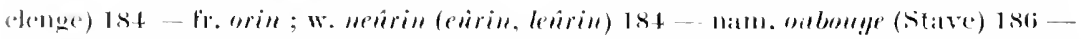

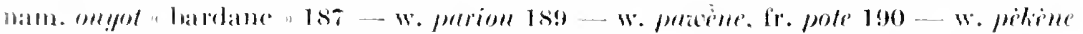

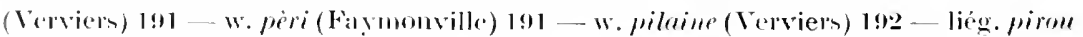

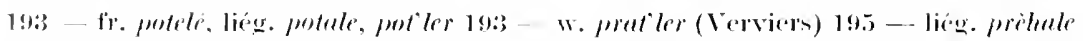

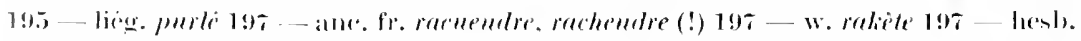

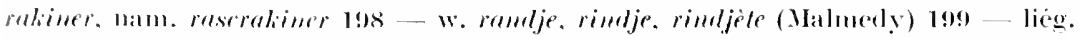

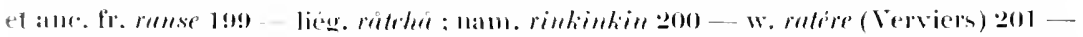

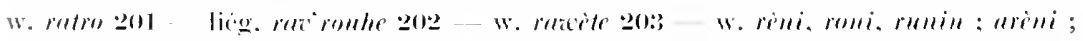

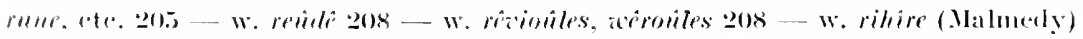

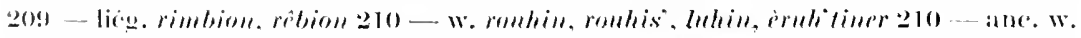

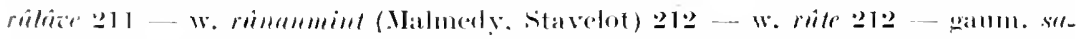

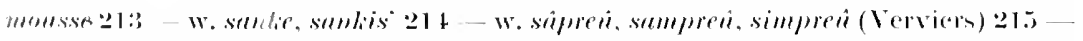

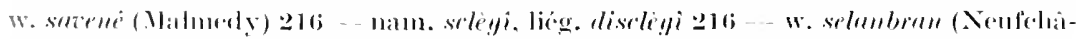

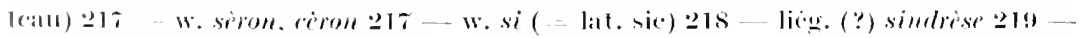

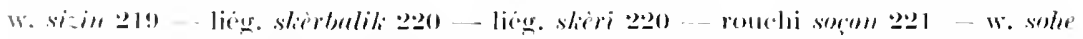

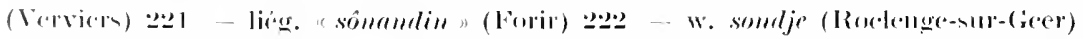

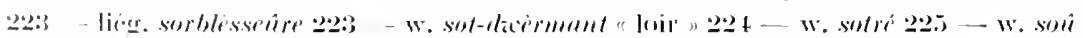

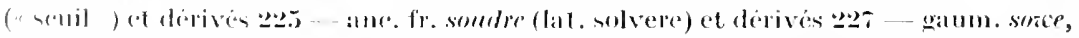

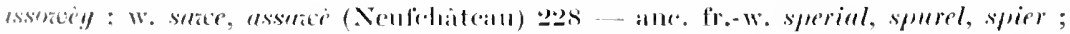

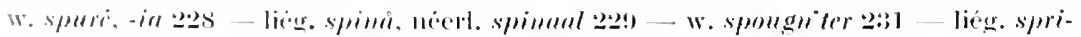

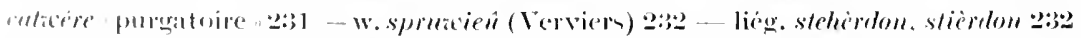

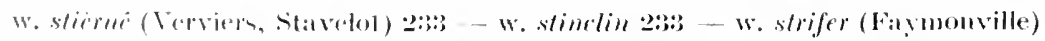

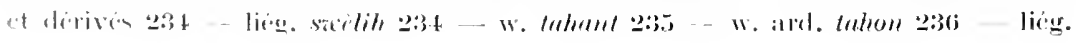




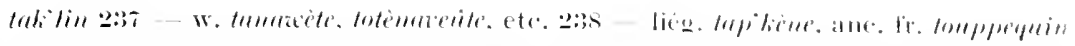

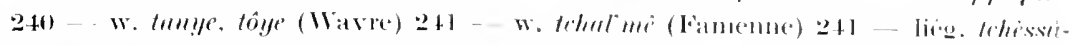

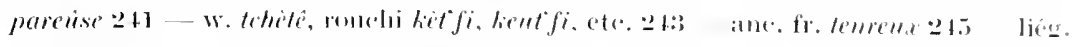

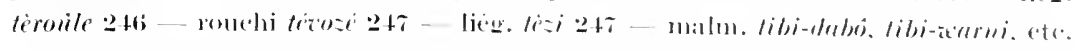

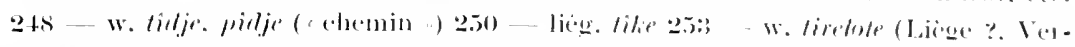

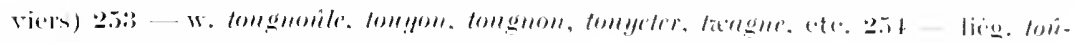

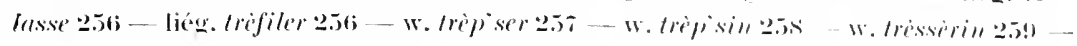

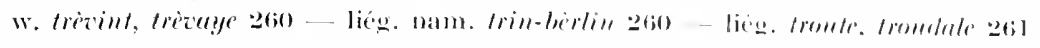

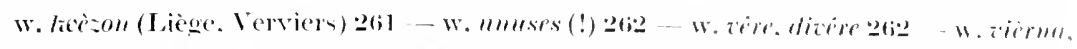

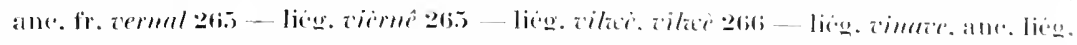

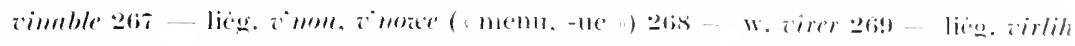

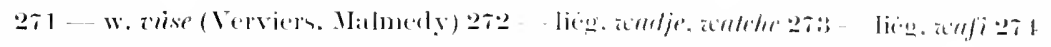

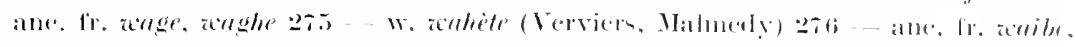

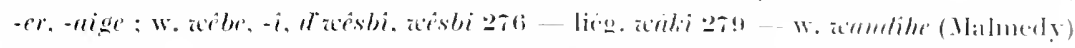

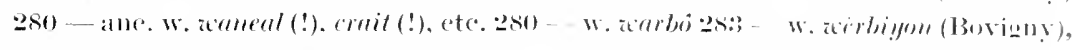

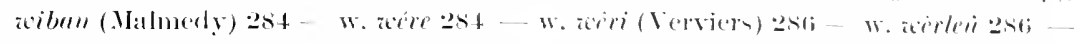

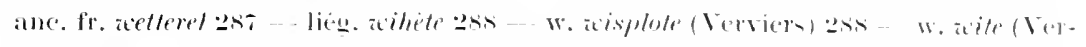

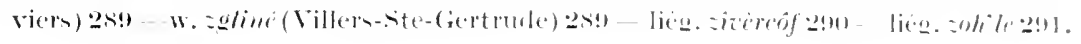

\section{Addition à la page 133 .}

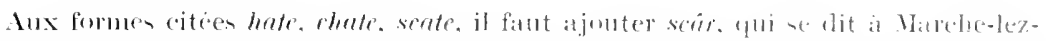

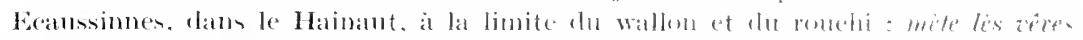

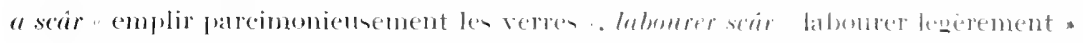

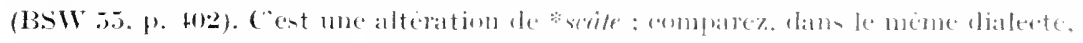

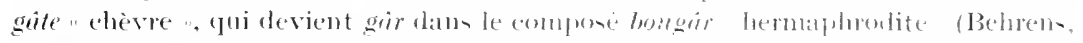

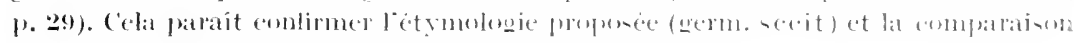
aver le liegeroin gate (warm. geit). 



\section{TABLE DES MATIËRES}

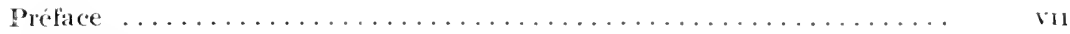

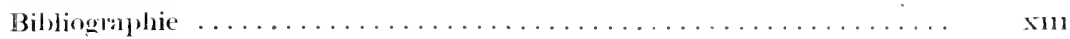

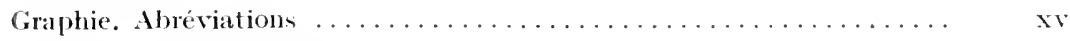

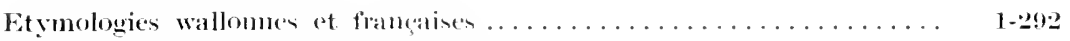

Voir la Table des Notices, 1. 351.

Les noms dialectaux de la "rulbute, en Belgique romalne ........ 29.3

Appendice. - I. Lees mots germaniques à préfixe -gé qui ont patsé (1) wallon $\ldots \ldots \ldots \ldots \ldots \ldots \ldots \ldots \ldots \ldots \ldots \ldots \ldots$;30.5

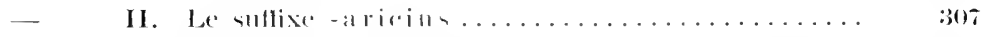

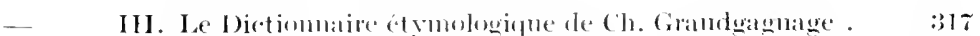

Additions et Correction $\ldots \ldots \ldots \ldots \ldots \ldots \ldots \ldots \ldots \ldots \ldots \ldots \ldots \ldots \ldots$

Index lexicographique...................

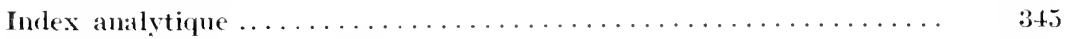

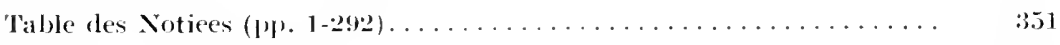

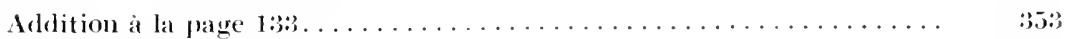

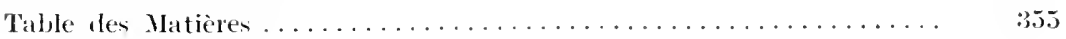






PLEASE DO NOT REMOVE CARDS OR SLIPS FROM THIS POCKET UNIVERSITY OF TORONTO LIBRARY 
